\title{
DESIGN OF HOUSING THAT IMPROVES THE QUALITY OF LIFE OF THE HIGH-NEEDS ELDERLY IN NEW ZEALAND
}

\author{
BY
}

\section{YUKIKO KUBOSHIMA}

\author{
A Thesis \\ submitted to the Victoria University of Wellington \\ in fulfilment of the requirements for the degree of \\ Doctor of Philosophy
}

Vitoria University of Wellington

2020 



\section{ABSTRACT}

The ageing population is growing rapidly in New Zealand, and those with high-care needs are increasing at an even higher rate. Government policy calls for ageing-in-place, staying in their own home as long as possible, without entering residential care. Subsequently, there is a growing need for housing that accommodates the impairments and care requirements that typically correspond with ageing. Recently a reduced quality of life (QoL) was reported in one of the independent living options specifically designed for the elderly with care needs in New Zealand. Without change to housing that offers support and care for the elderly, the reduced QoL of residents is likely to remain a problem.

A review of extensive literature on QoL and the role of architecture identifies a number of gaps in existing knowledge about housing design that facilitates the QoL of those elderly with high needs. These gaps include that, while the role of architecture is already established with regard to facilitating independence and control, there have been only limited insights into housing design that facilitates a wider range of aspects of QoL such as facilitating personal identity, important activities, relationships and maintaining high quality of care. Given the absence of design frameworks that are useful for designing housing that improves the QoL of the high-needs elderly in New Zealand, this research develops a holistic framework for housing design that improves the QoL for this cohort.

Two phases of survey are conducted in three types of senior housing complexes: retirement villages, and both public and private rental housing for the elderly. First, data on the current situations regarding these housing types, focusing on the models of care and physical environments as well as residents' care requirements, are obtained through two types of questionnaires. This preliminary survey is followed by a qualitative, ethnographical investigation for the QoL of residents that relate to physical environments. Through semi-structured interviews and full-day observation of 30 elderly people who receive assistance in daily life and their caregivers, as well as documentation of physical environments, data are gathered on their perceptions and spatial use. Numerous themes for QoL emerge and are categorised into six main headings: Control in daily activities, Meaningful leisure activities, Meaningful relationships, Maintenance of possessions, Comfort and Quality of care.

A number of design requirements are then identified and discussed with reference to existing knowledge, which leads to the development of a design framework. Housing that improves QoL needs to accommodate a variety of needs that reflect diverse individual preferences, circumstances 
and types of impairments. There is a need for reorganisation of space to accommodate caregivers, valuable relationships and personal possessions. The careful design of micro space greatly improves residents' control. The design of a complex is also influential on QoL, particularly for facilitating activities as well as maintaining both privacy and connection. Finally, the potential to implement the framework is examined through designing example models of housing units and complexes. The design framework developed through this research has great potential to improve a wide range of aspects of QoL for the high-needs elderly in New Zealand, thereby helping them maintain satisfying and independent living longer. 


\section{ACKNOWLEDGEMENTS}

I would like to express my gratitude to my supervisors, Geoff Thomas and Jacqueline McIntosh, who consistently supported me in many ways during my PhD study, and guided me throughout this project, encouraging my development as a researcher.

I am grateful to all people who participated in my surveys, who included housing operators, elderly people and caregivers. In particular, I owe my most sincere gratitude to 30 elderly people who let me in their home to undertake the in-depth survey. This unforgettable experience of sharing their day-to-day lives and their hopes and fears, and my appreciation of it, have been of great help in maintaining my motivation for research through the rest of my $\mathrm{PhD}$ period.

I would also like to thank people who provided me with particular aspects of support - Judith Davey, who gave me precise gerontological advice, and Esme Mulligan, who gave me important advice for the development of housing design. Also to Phil Saxby and Nikki Cleary, who assisted me to understand and express myself in colloquial English.

I am grateful for the financial support from Victoria University of Wellington (VUW) School of Architecture, VUW Faculty of Architecture and Design, VUW Faculty of Graduate Research, the Victoria University Foundation, VUW Postgraduate Students' Association, and the International Academic Forum (IAFOR). Their support helped me with conducting surveys and writing as well as enabled me to present my research papers in many conferences and journals.

I owe special thanks to my family. To my mother and late father, who supported me throughout my life. I am also thankful to my children, Miki and Shunya - I appreciate all your patience and support during my PhD study — and Saki, who was born just before I finished this thesis. My final thanks go to my husband, for supporting me in many ways - for being understanding and for putting up with me through the toughest moments of my life. 


\section{PUBLICATIONS DURING CANDIDACY}

The following is a list of publications during my $\mathrm{PhD}$ candidacy, which contain a large amount of work included in this thesis with some or substantial modification.

\section{Double-blind peer-reviewed Journal Articles}

Kuboshima, Y., McIntoch, J., \& Thomas, G. (2018a). Bathroom Design for Assisted Showering that Improves the Quality of Life of the Elderly. The Journal of Aging and Social Change, 8(3), 69-89. doi:10.18848/2576-5310/CGP/v08i03/69-89

- Contribution: $80 \%$

Kuboshima, Y., McIntoch, J., \& Thomas, G. (2018b). The Design of Local-Authority Rental Housing for the Elderly That Improves Their Quality of Life. Buildings, 8(5), 1-13. doi:10.3390/buildings8050071

- Contribution: $80 \%$

Kuboshima, Y., \& McIntoch, J. (2017). Housing Design that Improves the Quality of Life for Post-Stroke Elderly People Living Independently. Hospice \& Palliative Medicine International Journal, 1(4), 59-71. doi:10.15406/hpmij.2017.01.00018

- Contribution: $70 \%$

\section{Double-blind peer-reviewed Conference Papers}

Kuboshima, Y., McIntoch, J., \& Thomas, G. (2018c, 28 Nov - 1 Dec). Designing for relationships: housing and communities that improve the quality of life of the high-needs elderly. Paper presented at the 52st International Conference of the Architectural Science Association Melbourne, Australia.

- Contribution: $85 \%$

Kuboshima, Y., McIntoch, J., \& Thomas, G. (2017a, 29 Nov - 2 Dec). Implications for the design of rental housing for the elderly that improves their quality of life. Paper presented at the 51st International Conference of the Architectural Science Association, Wellington.

- Contribution: $85 \%$

Kuboshima, Y., McIntosh, J., \& Thomas, G. (2017a, 22-23 June). Care, physical environments and dependency: the design of housing for the high needs elderly to live independently. Paper presented at the Cities, Communities and Homes: Is the Urban Future Livable?, Derby, UK. 
- Contribution: $75 \%$

Kuboshima, Y., McIntosh, J., \& Thomas, G. (2017b, 2-5 Jul). Models of care and physical environments of current housing for the elderly: the possibilities of the rental housing for the dependent elderly in New Zealand. Paper presented at the 33rd PLEA Conference, Edinburgh.

- Contribution: $75 \%$

Kuboshima, Y., McIntosh, J., \& Marques, B. (2016, 5-6 Sep). Improving the sense of wellbeing for dependent older people living in supported housing. Paper presented at the Wellbeing 2016: Co-creating Pathways to Well-being, Birmingham, UK.

- Contribution: $75 \%$

\section{Peer-reviewed Conference Paper}

Kuboshima, Y., McIntoch, J., \& Thomas, G. (2018d, 8-10 Jun). Facilitating meaningful activities and relationships: Designing dwellings and communities that improve the quality of life of the high-needs elderly. Paper presented at the The Asian Conference on Aging and Gerontology 2018, Kobe, Japan.

- Contribution: $85 \%$ 


\section{TABLE OF CONTENTS}

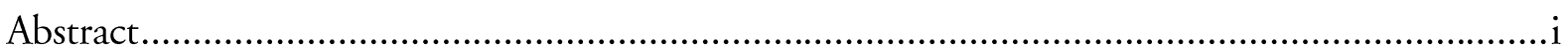

Acknowledgements ……………………....................................................................ii

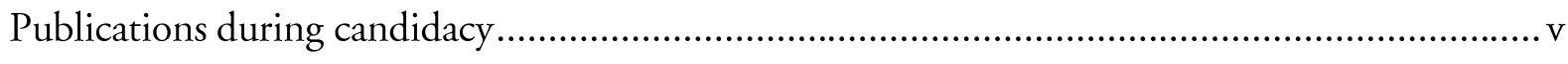

Table of contents........................................................................................................... vii

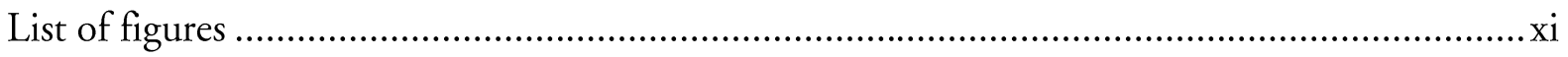

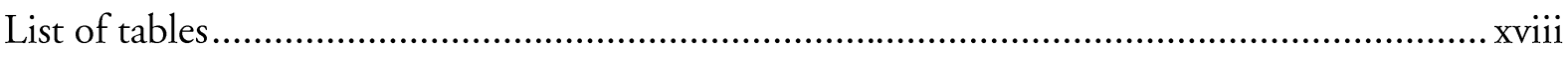

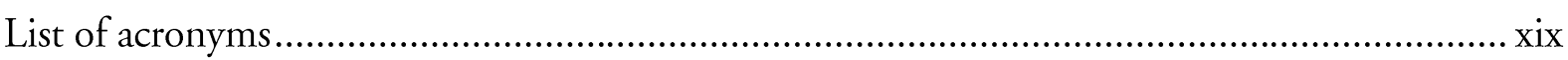

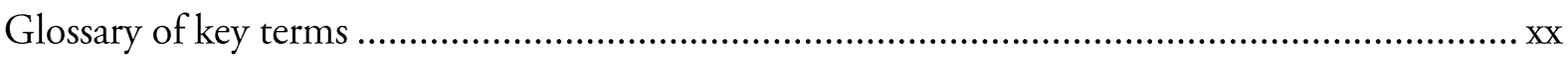

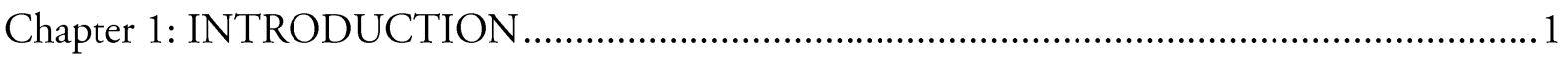

1.1 Research context and problem statement.........................................................................

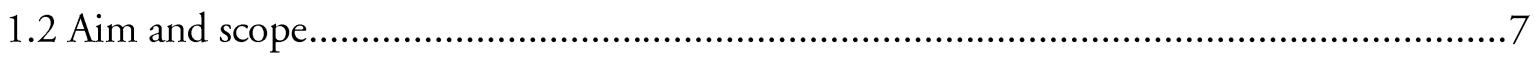

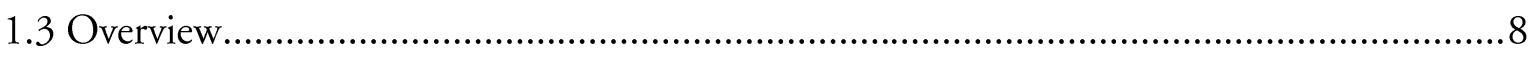

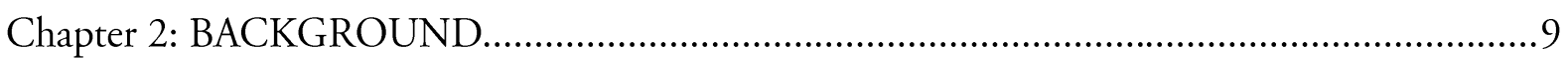

2.1 Socio-demographic features of elderly people in New Zealand ...........................................

2.1.1 Age, gender and living arrangements ......................................................................

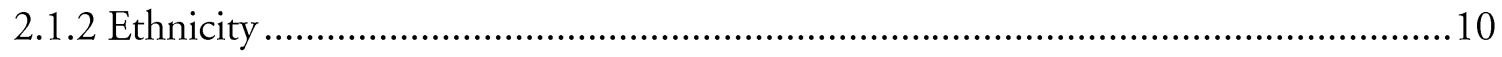

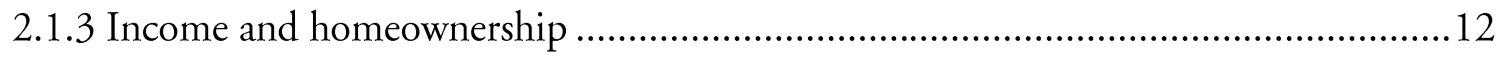

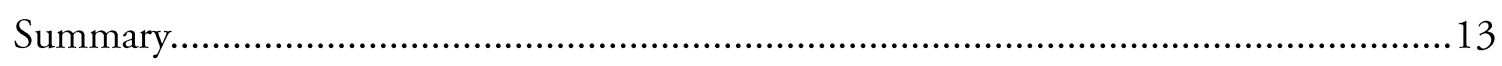

2.2 Ageing and impairments of the elderly and support and care in New Zealand..................14

2.2.1 Biological ageing and impairments of elderly people ……......................................... 14

2.2.2 Care and support for elderly people living independently.........................................15

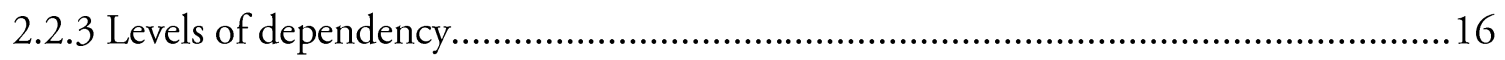

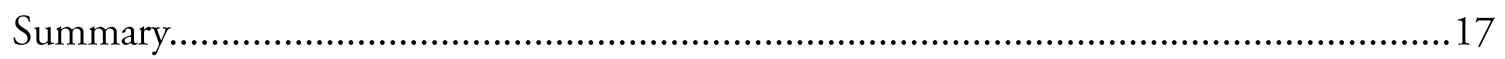

2.3 Housing options for high-needs elderly to live independently ....................................... 18

2.3.1 Housing options for the high-needs elderly overseas..................................................18

2.3.2 Housing options for the high-needs elderly in New Zealand ....................................22 


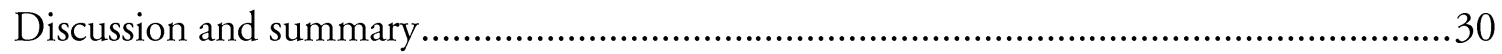

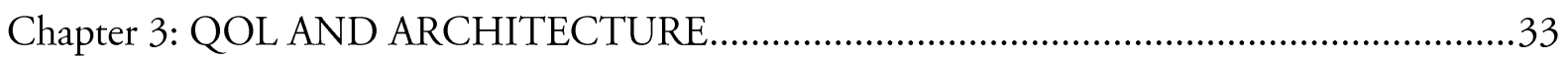

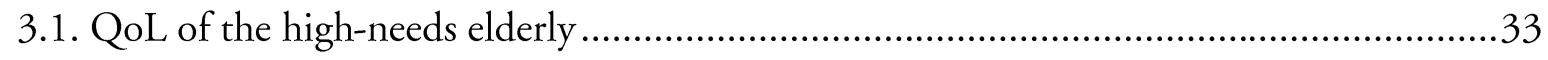

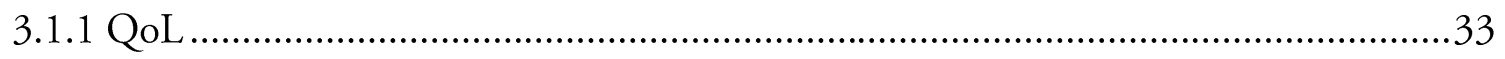

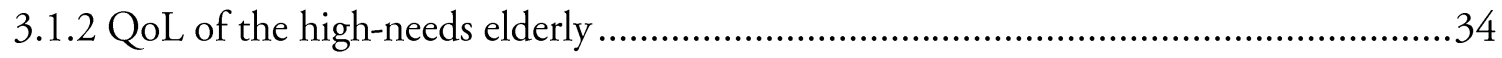

3.2 QoL of the high-needs elderly in relation to physical environments ..............................36

3.2.1 Overview of the expectation of the elderly for their living environments in New

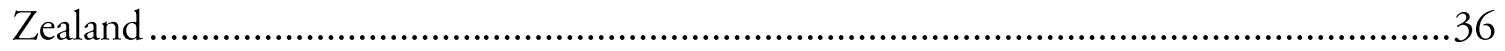

3.2.2 QoL of the high-needs elderly in relation to physical environments .........................37

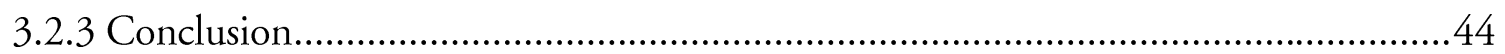

3.3 Existing design frameworks for housing for the elderly that address their QoL ................45

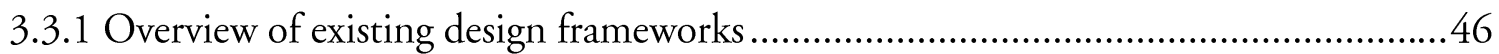

3.3.2 Review of design frameworks by themes for the QoL of the high-needs elderly ........48

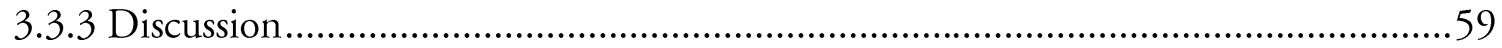

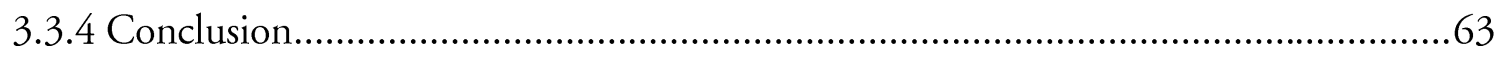

3.4 Conclusion, gaps in knowledge and research questions..............................................63

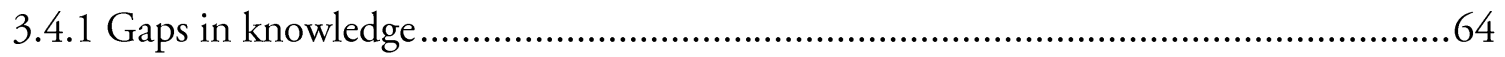

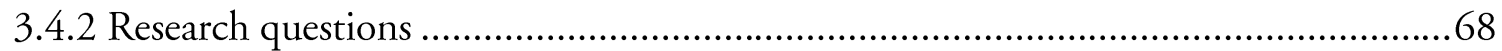

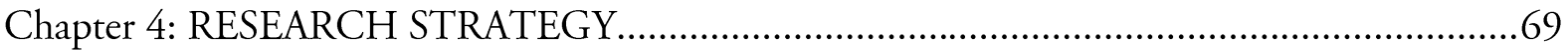

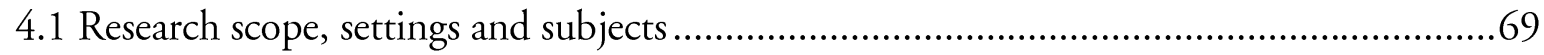

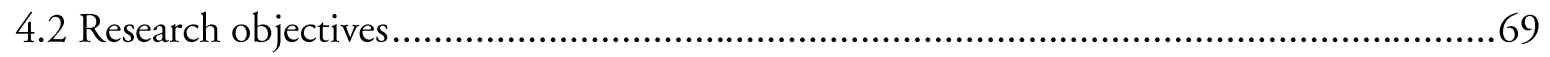

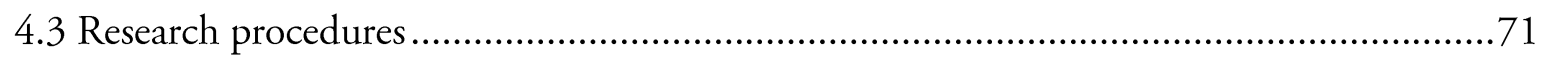

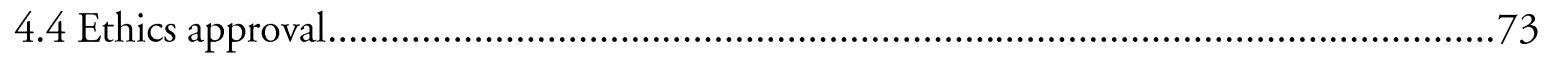

Chapter 5: CURRENT SITUATIONS OF HOUSING FOR THE ELDERLY AND

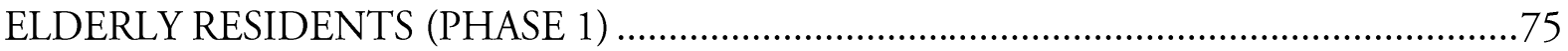

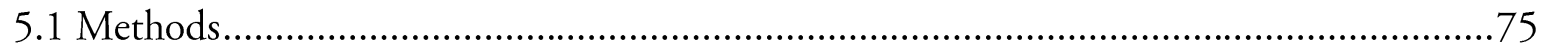

5.1.1 Phase 1-1: Data collection for models of care and physical environments .................75 
5.1.2 Phase 1-2: Data collection for the care of elderly residents......................................77

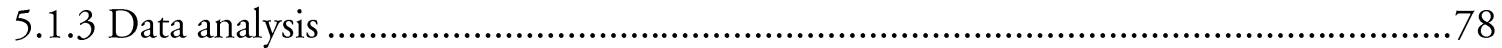

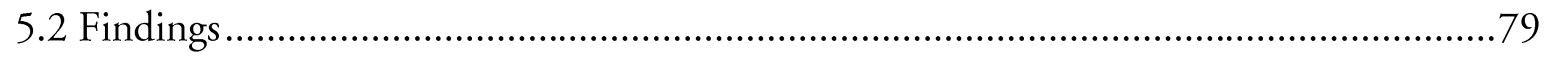

5.2.1 Findings from the Phase $1-1$ questionnaire....................................................... 79

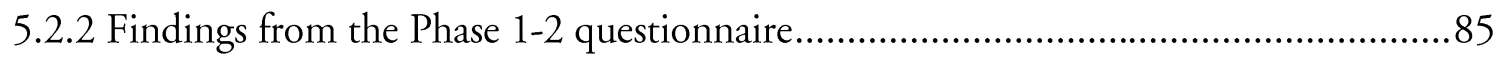

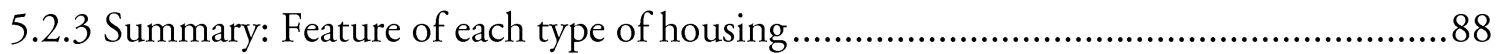

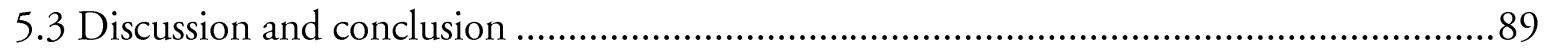

Chapter 6: METHODOLOGY AND METHODS FOR RESEARCH ON QOL IN RELATION TO PHYSICAL ENVIRONMENTS (PHASE 2) ..........................................93

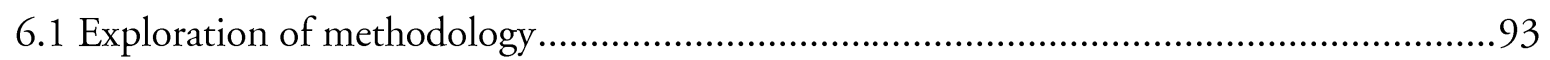

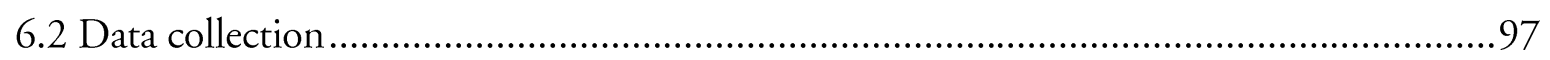

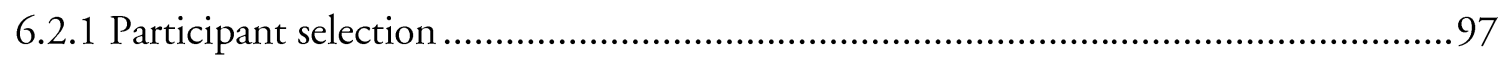

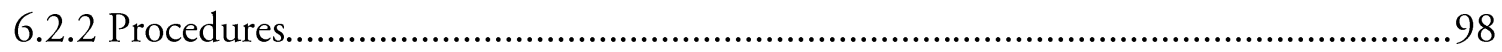

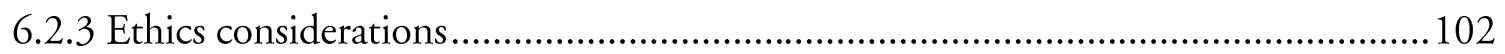

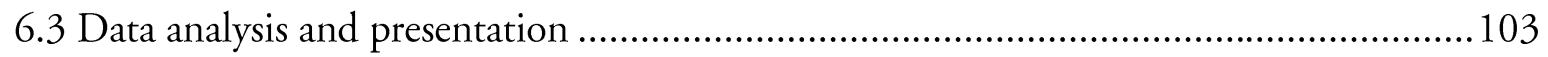

Chapter 7: QOL OF THE HIGH-NEEDS ELDERLY IN RELATION TO PHYSICAL

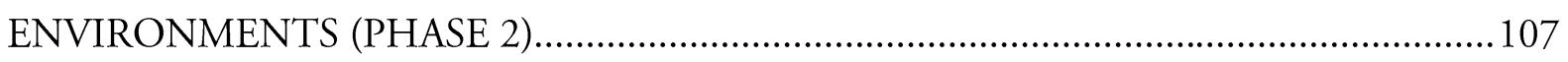

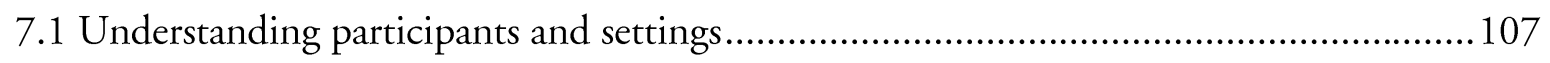

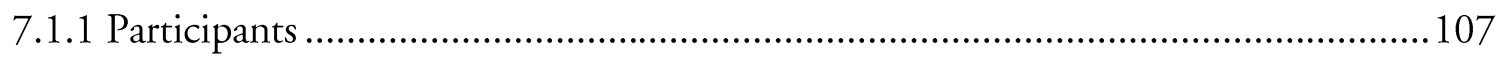

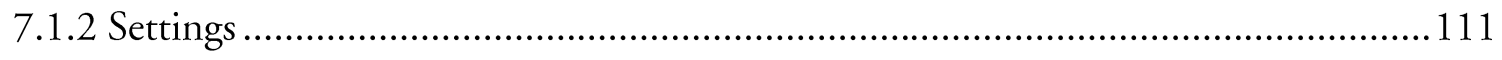

7.2 QoL of the high-needs elderly living independently ................................................118

7.2.1 Theme 1: Control in daily basic activities........................................................ 118

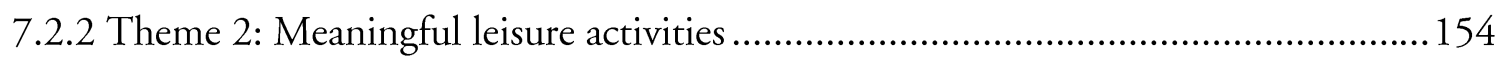

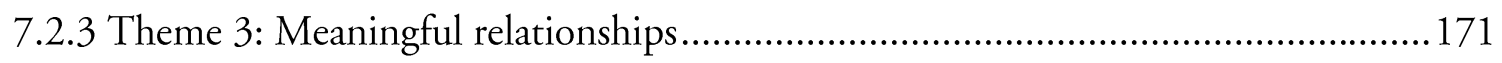

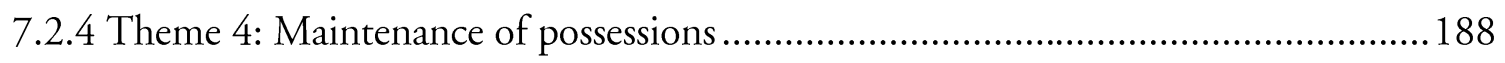

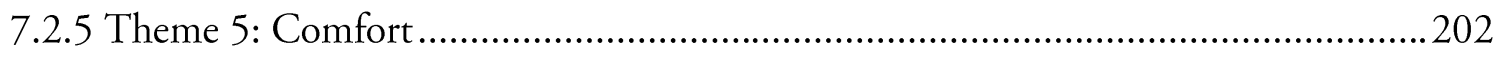

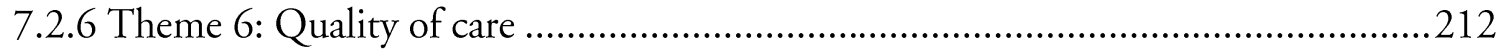

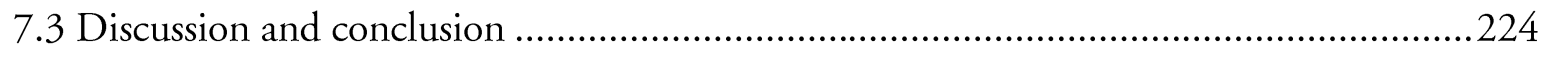


8.1 Establishing the relationships between themes for QoL and design considerations ........227

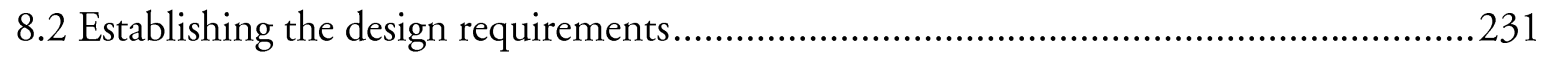

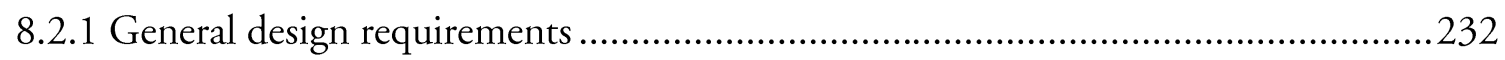

8.2.2 Requirements in the design of the individual unit ...............................................241

8.2.3 Requirements in the design of the complex .......................................................265

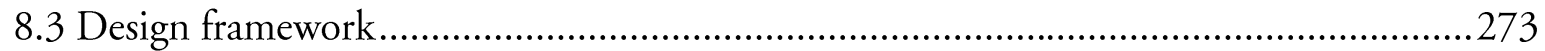

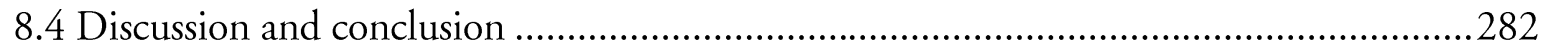

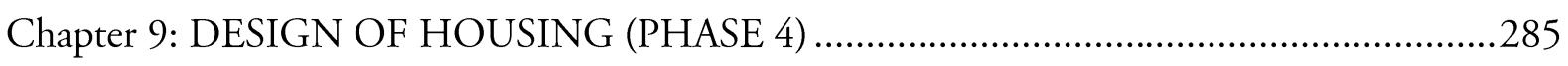

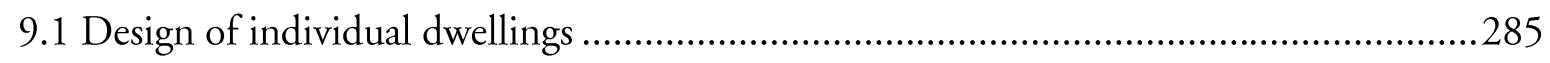

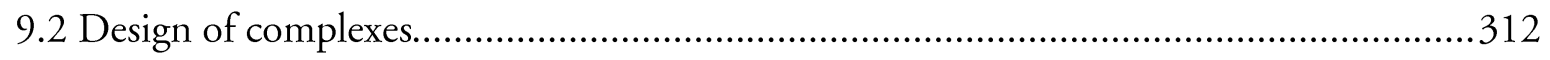

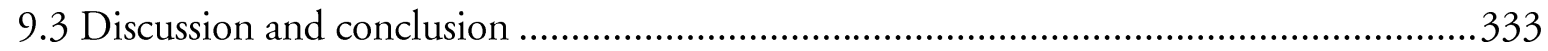

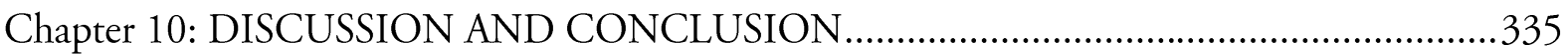

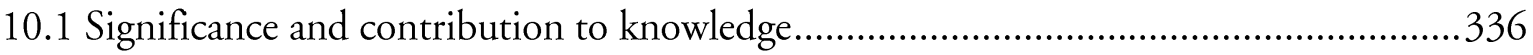

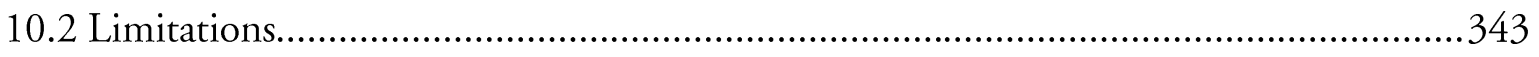

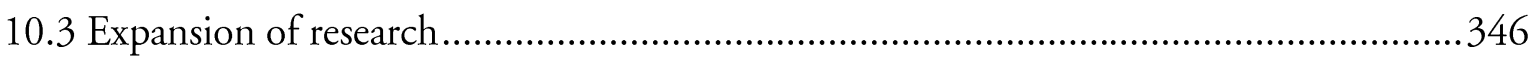

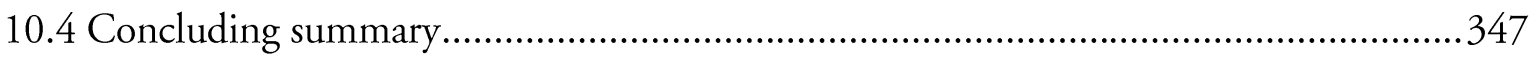

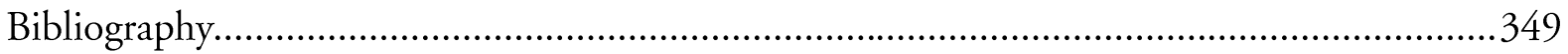

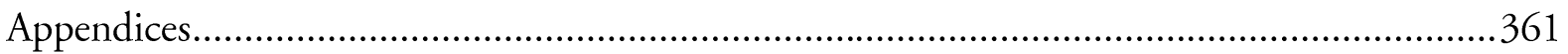

Appendix 1: Ethics approval memo, information sheets, consent forms and questionnaires. 361

Appendix 2: Data sheets in the Phase 2 survey ................................................................383

Appendix 3: Individual preferences in activities and relationships.....................................385

Appendix 4: Analysis of relationships between QoL themes, design considerations and design

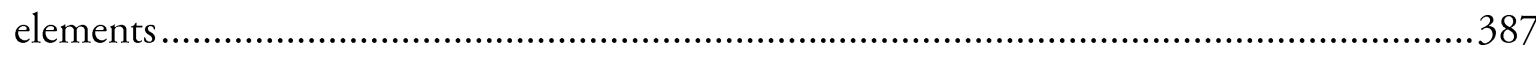

Appendix 5: Design of housing — floor plans ..............................................................397 


\section{LIST OF FIGURES}

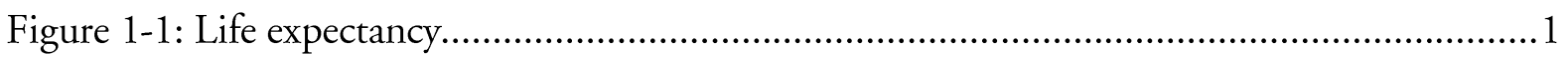

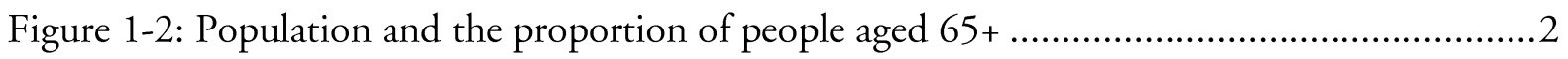

Figure 1-3: Life expectancy and ILE at age 65, 1996 and 2013 ...............................................3

Figure 1-4: Projected number of older people with high-support needs......................................

Figure 1-5: Proportion of residents by dependency levels and length of stay in residential care....4

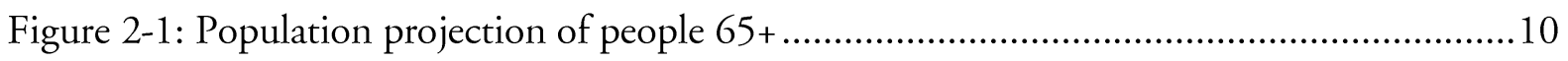

Figure 2-2: Projection of ethnic composition of people aged $65+\ldots \ldots \ldots \ldots \ldots \ldots \ldots \ldots \ldots \ldots \ldots \ldots \ldots \ldots \ldots . . . . . .11$

Figure 2-3: Living arrangements by ethnicity ..................................................................... 12

Figure 2-4: Homeownership rate by age group.....................................................................13

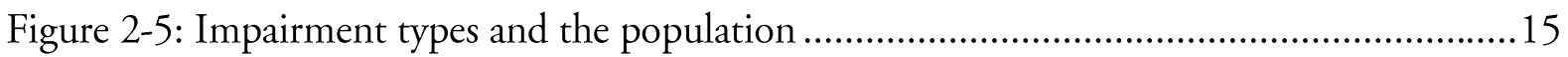

Figure 2-6: Proportion of older people living in their private homes with levels of priority for

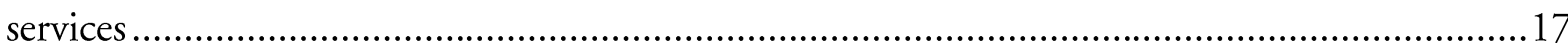

Figure 2-7: Concept plan for assisted living..................................................................21

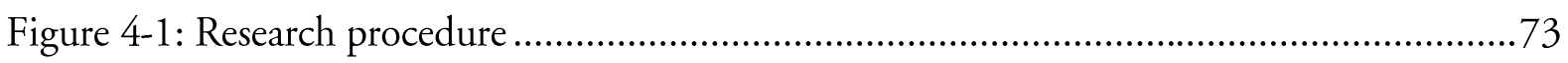

Figure 5-1: Distribution of number of units per complex *...............................................8

Figure 5-2: Unit composition of retirement villages and the adjacency of residential care facilities .80

Figure 5-3: Adjacency of residential care facilities to rental housing complexes. .81

Figure 5-4: Unit composition among retirement villages containing both independent and supported-living units

Figure 5-5: Distribution of the percentage of units that have access and facilities for the disabled

Figure 5-6: Communal areas provided for residents ………………………………........... 83

Figure 5-7: Services provided for residents......................................................................... 84

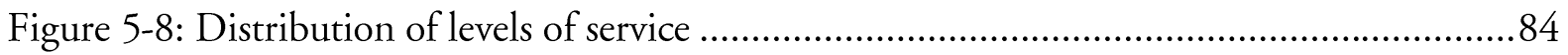

Figure 5-9: Levels of dependency among residents receiving personal care...................................86

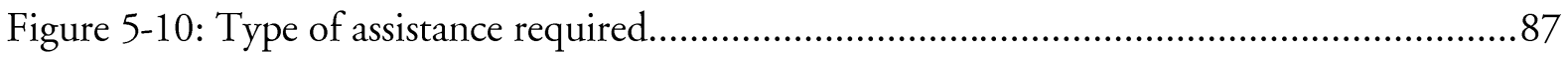

Figure 6-1: Data collection procedures ............................................................................99

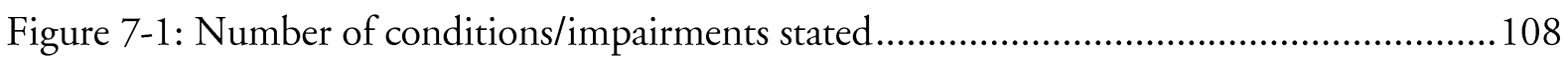

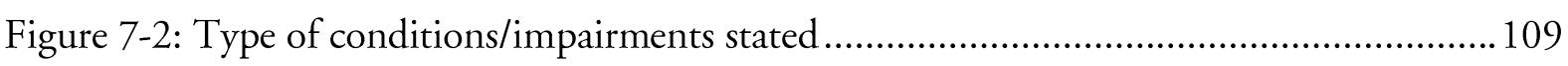

Figure 7-3: Requirements in assistance (personal care) by activities ........................................110

Figure 7-4: Type of mobility aids/vehicles mainly used ..........................................................111 
Figure 7-5: Number of units contained in a housing complex

Figure 7-6: Layout type of housing units

Figure 7-7: Building type of housing units

Figure 7-8: Relationships between the unit size, layout type and type of housing complex

Figure 7-9: Typologies of the shower area

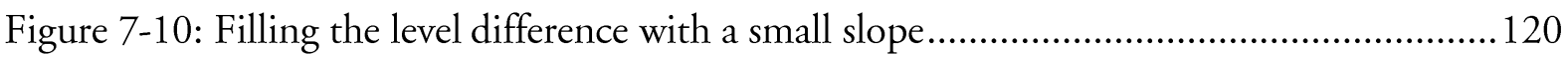

Figure 7-11: Opening of the shower door that was a tripping hazard ..................................... 122

Figure 7-12: The bathroom door was normally kept open.

Figure 7-13: Trouble for a walker-frame user in opening a hinged door inwards (left) and outwards (right)

Figure 7-14: Insufficient space for a walker-frame user in front of a hinged door......

Figure 7-15: Hinged door with a wrong opening direction

Figure 7-16: Having two bathroom doors facilitated a circulation that avoided turning around

Figure 7-17: Wall corner often hit by a walker frame

Figure 7-18: Mobility scooter kept in the bedsit unit ...

Figure 7-19: Procedure of turning around the mobility scooter and the required space.....

Figure 7-20: Toilet seat raiser.

Figure 7-21: 'Picker upper' (and a walking stick)

Figure 7-22: Easier route from the bedroom to the toilet.

Figure 7-23: Space for a microwave that is too high

Figure 7-24: $700 \mathrm{~mm}$-deep table accommodating tools and foods while leaving space for tasks

Figure 7-25: Gathering all foods and tools on the bench before cooking ............................... 138

Figure 7-26: Sitting space for washing dishes with a dishwasher .......................................... 140

Figure 7-27: Inconvenient space for a fridge-freezer ......................................................... 142

Figure 7-28: Insufficient storage resulted in insufficient bench space for cooking tasks............143

Figure 7-29: Insufficient kitchen space, which resulted in too narrow a passage for a wheelchair

user

Figure 7-30: Use of railing and hooks.

Figure 7-31: Bench space fully used for storing various items

Figure 7-32: Too far a distance between the storage for dirty clothes and the washing machine

Figure 7-33: Insufficient space in the laundry room for turning around with a walker frame...147 
Figure 7-34: Laundry cupboard.

Figure 7-35: Lack of adequate space for a laundry rack.

Figure 7-36: Consideration of non-slip floor design (floor sections)

Figure 7-37: Convenient layout of the garage with respect to the kitchen.

Figure 7-38: Letter box that was difficult for a walker-frame user to access.....

Figure 7-39: Plan of gardens (PU3) (left) and the photo of the enclosed section (right)...........157

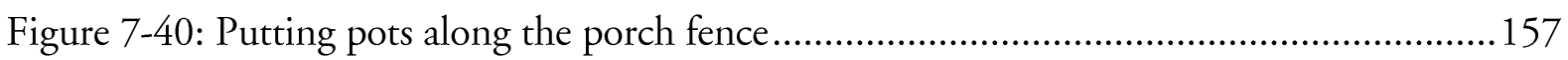

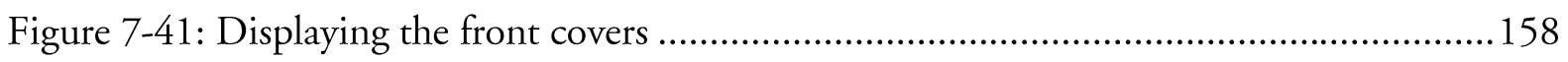

Figure 7-42: Reading in an armchair by the north-facing window....................................... 158

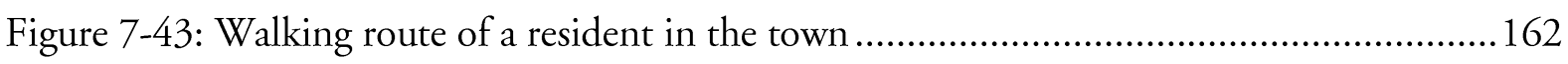

Figure 7-44: Using furniture as 'things to hold onto'............................................................ 162

Figure 7-45: Layout of the sitting space in the alcove within the lounge .................................. 165

Figure 7-46: Layout of a chair and a TV for people with vision impairment........................... 166

Figure 7-47: Posture of the resident with hemiplegia when sitting at the table.........................167

Figure 7-48: Difference in the sitting position depending on the preference for seeing outside 167

Figure 7-49: Layout of the PC screen that can be viewed from the sitting space ...................... 168

Figure 7-50: Sitting space that facilitated engagement in multiple activities............................ 169

Figure 7-51: Hand-made storage for remote controls, pens and so on ...................................169

Figure 7-52: Using different spaces for various activities...................................................... 170

Figure 7-53: Interior layout that hinders the view from the sitting space to the door

Figure 7-54: Open space in front of the unit, which was used for hosting large number of guests

Figure 7-55: Accommodating overnight guests using the garage. 176

Figure 7-56: Insufficient dining space to accommodate ten residents and their walker frames.178 Figure 7-57: Spatial organisation that encouraged resident interaction (left) and their frequent

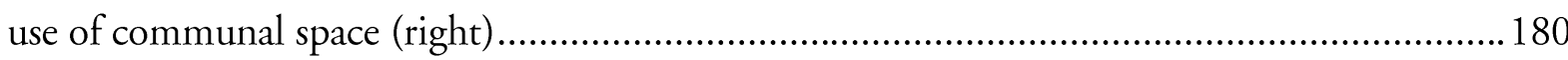

Figure 7-58: Layout of units with consideration to the distance and angles .....

Figure 7-59: Unit layout that contributed to a greater sense of connection while maintaining

privacy

Figure 7-60: Layout of units and windows that affected privacy

Figure 7-61: Level difference as a strategy for greater privacy.

Figure 7-62: Confusing road system with many one-way roads

Figure 7-63: Desk that had been used for a long time

Figure 7-64: Unbalanced space for furniture in the lounge and the bedroom 
Figure 7-65: Inappropriate room shape for accommodating furniture

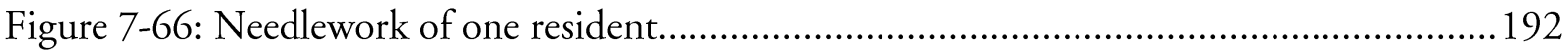

Figure 7-67: Photographs displayed on shelves and walls....................................................... 193

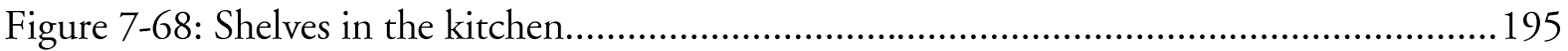

Figure 7-69: The recess under the kitchen bench that was used as storage ..............................196

Figure 7-70: A hand-made stick used to take things down from high cupboards .................... 197

Figure 7-71: Hanging clothes and bags at the front door......................................................200

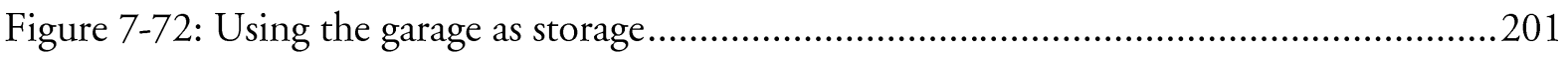

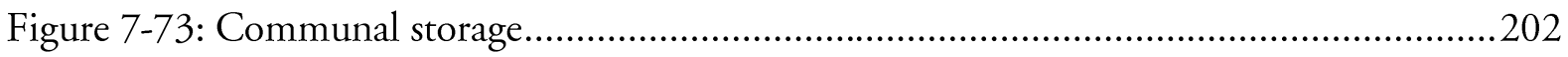

Figure 7-74: Changing sitting spaces according to the sun movement .................................206

Figure 7-75: Big lounge that created darkness in the inner area ............................................208

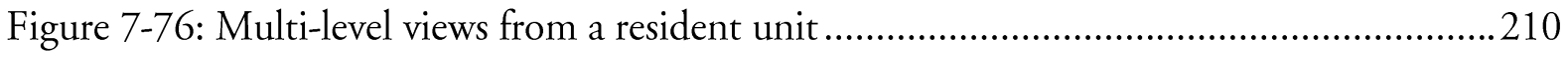

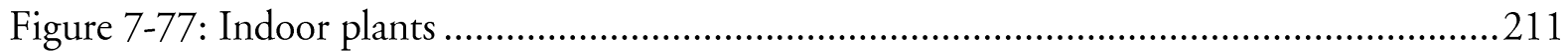

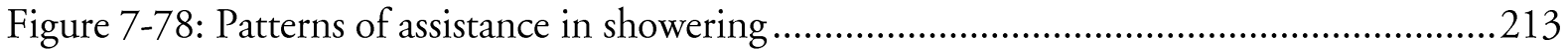

Figure 7-79: Example of insufficient space for caregiver assistance for washing.......................214

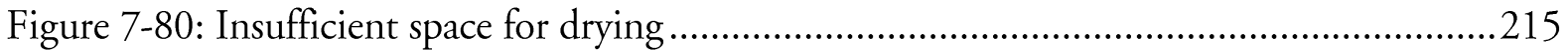

Figure 7-81: Example of space for clothes/shoes and caregiver movements...........................215

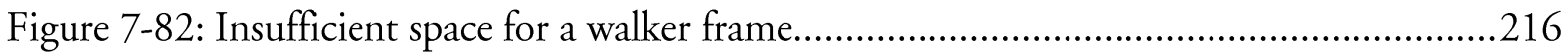

Figure 7-83: Moving a chair for bathroom set-up...............................................................217

Figure 7-84: Holding on things to hold onto with both hands while the caregiver washes the

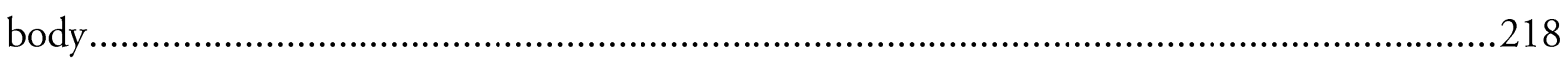

Figure 7-85: Caregiver's movements between the bathroom and the washing machine ...........220

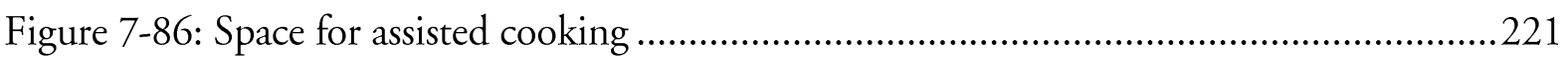

Figure 8-1: Relationships between themes for QoL and design elements ..............................230

Figure 8-2: Examples of thresholds that create a level difference (left) and no level difference

(right).

Figure 8-3: Comparison of trouble of a walker-frame user in opening hinged and sliding doors

Figure 8-4: Example of an external door with a small level difference (detailed section)...........236

Figure 8-5: Example of an external door with no level difference..........................................237

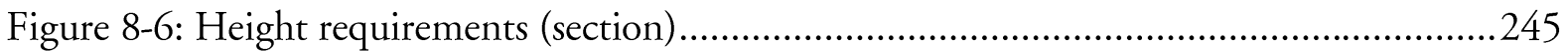

Figure 8-7: Ideas for interior layout that avoids turning around ..........................................249

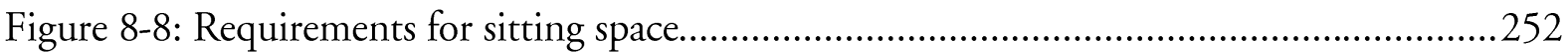

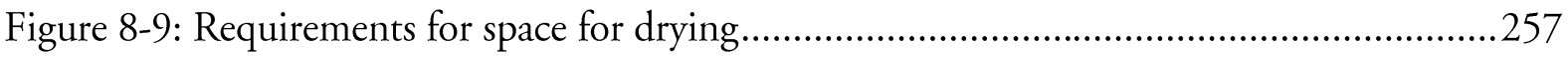




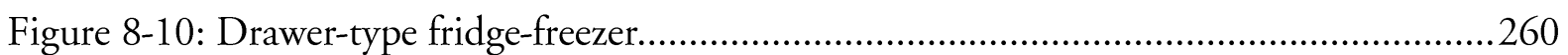

Figure 8-11: Examples of convenient storage under the bench corner...................................262

Figure 8-12: Considerations for the unit layout to provide a greater sense of homeliness and

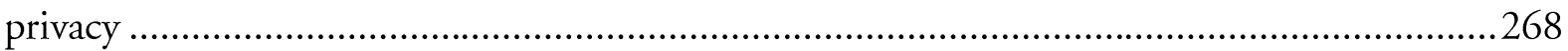

Figure 9-1: Layout of a passage that avoids needing to turn around......................................286

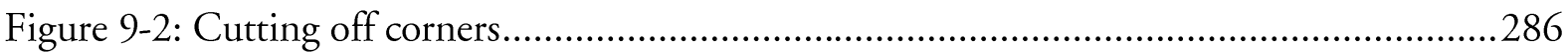

Figure 9-3: Provision of things to hold onto and light switches along the passage....................287

Figure 9-4: Creating multiple spaces along the passage .........................................................28

Figure 9-5: Layout of many spaces adjacent to the bathroom ..............................................28

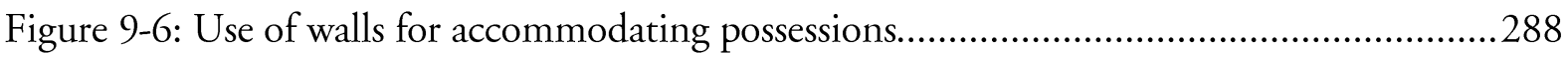

Figure 9-7: Removal of unnecessary doors and use of sliding doors .......................................289

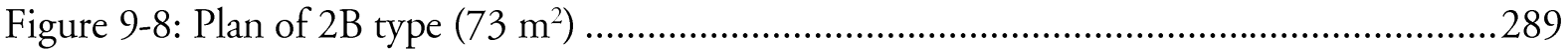

Figure 9-9: Plans of 1B-A type (left) and 1B-B (right) (both $50 \mathrm{~m}^{2}$ ) ....................................290

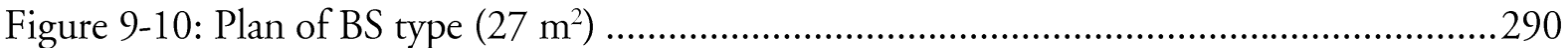

Figure 9-11: Perspective of the inner walls (1B-A) .................................................................291

Figure 9-12: Section of the lower part of the inner wall (1B-A) ...........................................292

Figure 9-13: Interior elevations of the inner walls (1B-A) ....................................................292

Figure 9-14: Plan of the inner walls at $1500 \mathrm{~mm}$ high (1B-A) ..............................................293

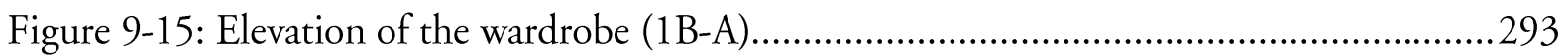

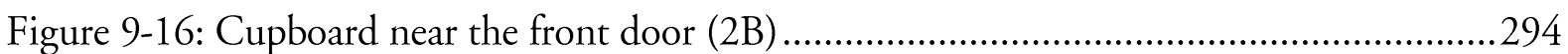

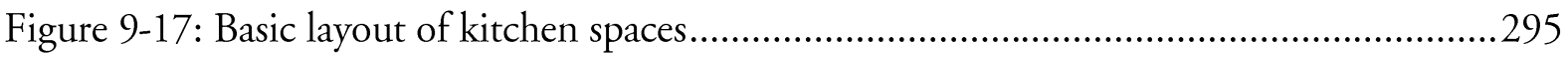

Figure 9-18: Overall floor plans of the kitchen (1B-A) ........................................................295

Figure 9-19: Bench space for food preparation (1B-A) ..........................................................296

Figure 9-20: Perspective of the bench space for food preparation (1B-A) ...............................296

Figure 9-21: Layout of storage in proximity to the space for food preparation (1B-A) ............296

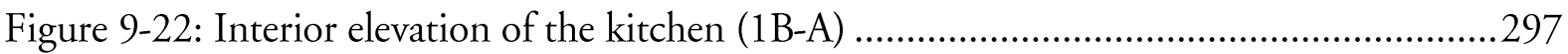

Figure 9-23: Options for the space for a microwave oven (1B-A) …….................................297

Figure 9-24: Options for having an oven and a dishwasher (1B-A) .....................................298

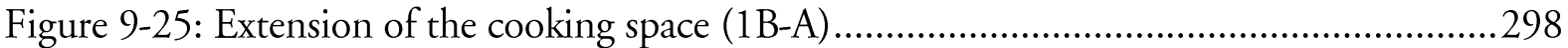

Figure 9-26: Section of the kitchen with a lowered bench (h=700 mm) (1B-A).....................299

Figure 9-27: Kitchen with a lowered bench (h=700 mm) (1B-A) ........................................299

Figure 9-28: Folding bench and under bench storage units (1B-A)......................................299

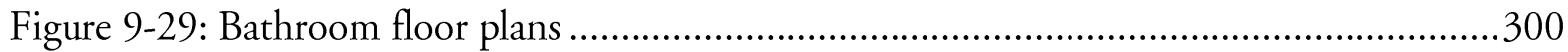

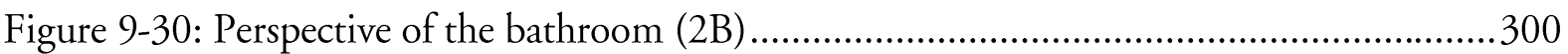




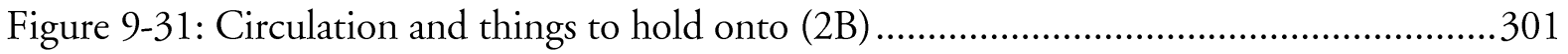

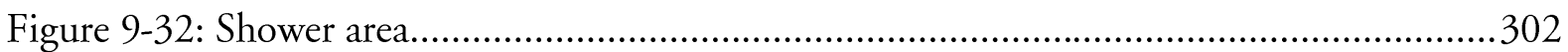

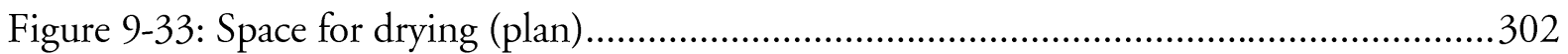

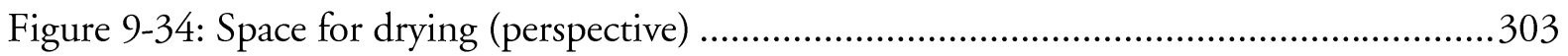

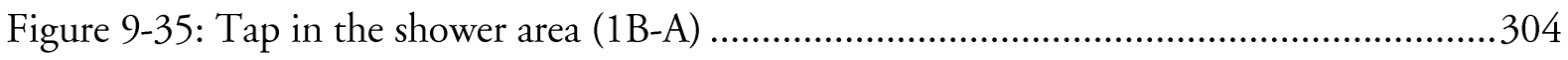

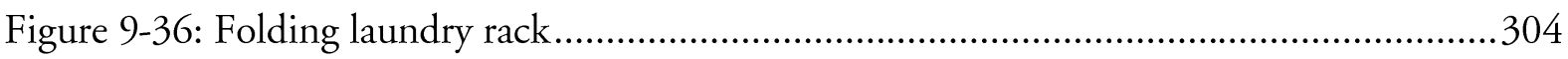

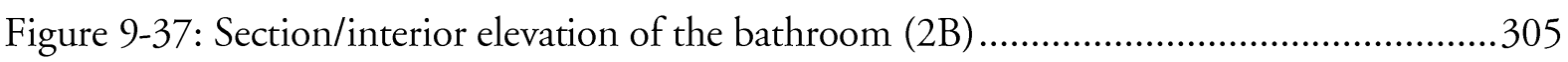

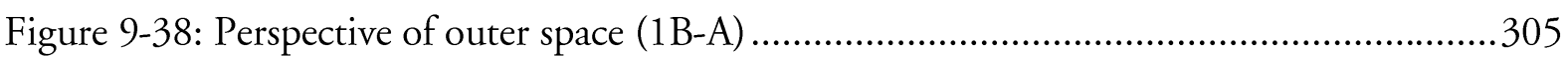

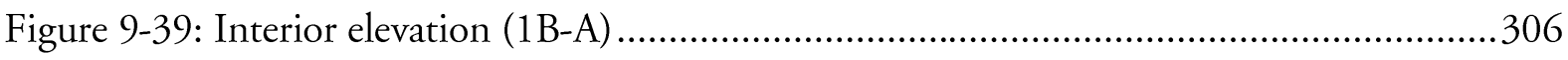

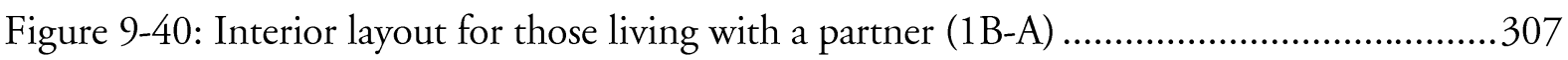

Figure 9-41: Interior layout of sitting space for those with limited mobility ...........................308

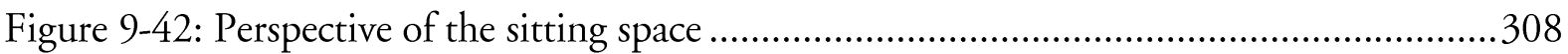

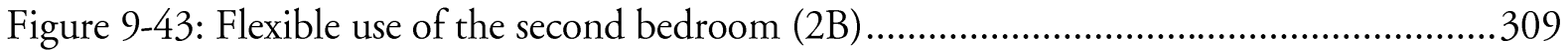

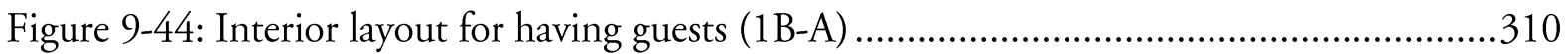

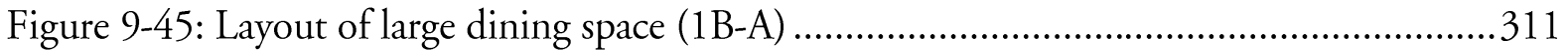

Figure 9-46: Creating an additional bedroom for an overnight guest (1B-A) .........................311

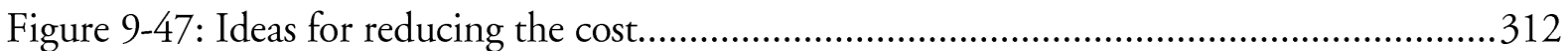

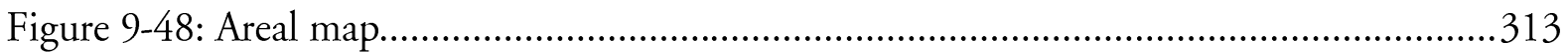

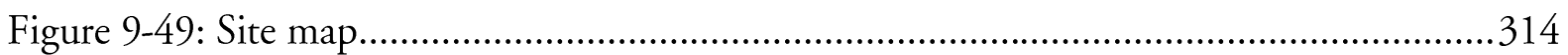

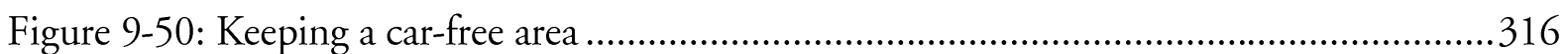

Figure 9-51: Layout of units with respect to the north and communal pathways.....................317

Figure 9-52: Deployment of three types of communal spaces...............................................317

Figure 9-53: Rental housing, self-contained model - ground floor plan (22/49 units)..............319

Figure 9-54: Rental housing, self-contained model — first and second floor plans (27/49 units)

Figure 9-55: Rental housing, communal-living model (28 units) .........................................321

Figure 9-56: Retirement-village, supported-living model (17 units (excluding care units)) ......322

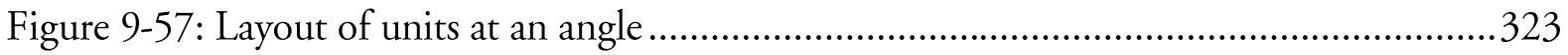

Figure 9-58: Provision of enough distance and the use of walls/glasses for maintaining resident

privacy .

Figure 9-59: Strategy for maintaining resident privacy and a sense of connection 1 - the use of a wall/glass

Figure 9-60: Strategy for maintaining resident privacy and a sense of connection 2 - the use of blind panels, level louvres and trees....... 
Figure 9-61: Section: relationships between units and communal pathways ...........................326

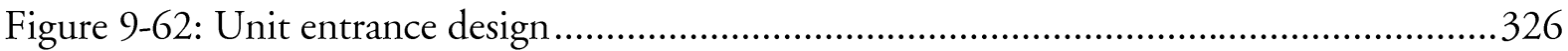

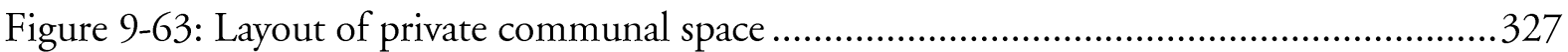

Figure 9-64: Space for displaying personal items at the entry of resident units........................327

Figure 9-65: Clustering of units with specific-use communal spaces.......................................328

Figure 9-66: Clustering of units with specific-use communal spaces.....................................328

Figure 9-67: Example of display space for art work ..............................................................329

Figure 9-68: Layout of the music room and the communal garden in the centre of the complex

Figure 9-69: Unit layout with respect to the staff space and the care centre ............................330

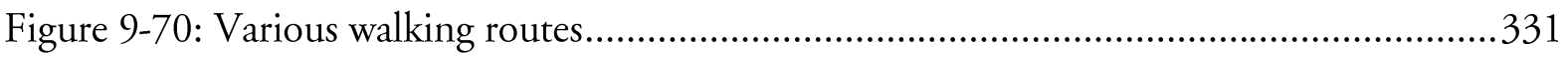

Figure 9-71: Layout of attracting features along communal pathways....................................331

Figure 9-72: Definition of public access area and the layout of communal space welcoming the

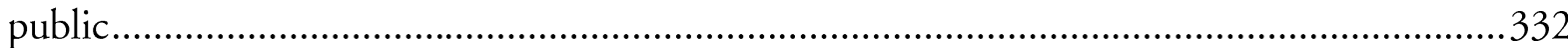

Figure 9-73: Connection to the wider community and children ...........................................333 


\section{LIST OF TABLES}

Table 2-1: Classification of housing for the elderly in the UK made by Robson et al................19

Table 2-2: Number of independent and supported-living units (2016) ...................................23

Table 3-1: Design considerations in the existing frameworks................................................... 49

Table 3-2: Themes of QoL, problems and scarcity of knowledge ..........................................67

Table 5-1: Participation in the Phase 1-1 survey ....................................................................76

Table 5-2: Participation of the Phase 1-2 survey (aggregated data) ..........................................78

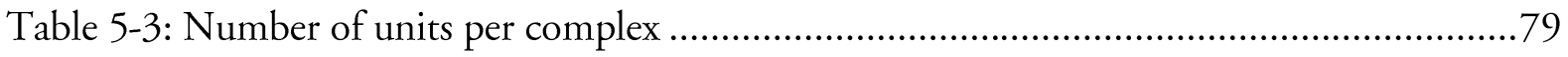

Table 5-4: Average percentage of units that have access and facilities for the disabled* ..............82

Table 5-5: Average Service Score by the housing-complex type ................................................ 84

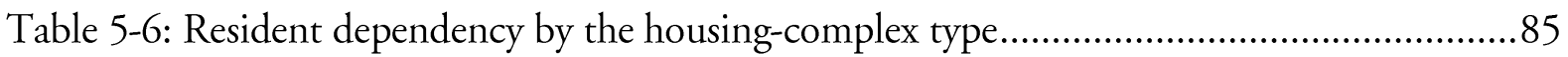

Table 5-7: Proportion of residents receiving care from professional caregivers or family/friends 87

Table 6-1: Interview questions for residents ..................................................................... 100

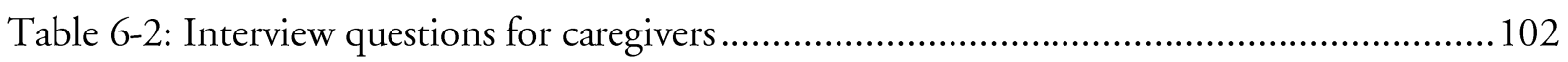

Table 7-1: Age, gender, living arrangements and marital status of residents............................ 108

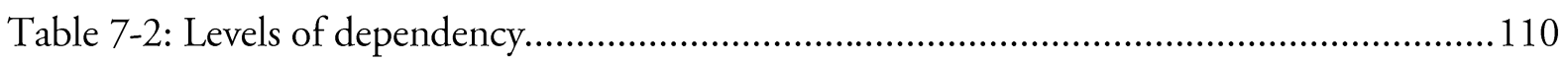

Table 7-3: Frequency of professional caregiver visits............................................................ 110

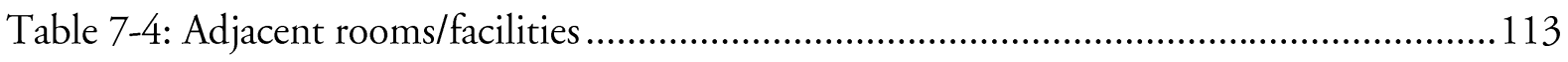

Table 7-5: Services and care that can provided/arranged by housing providers for residents .... 114

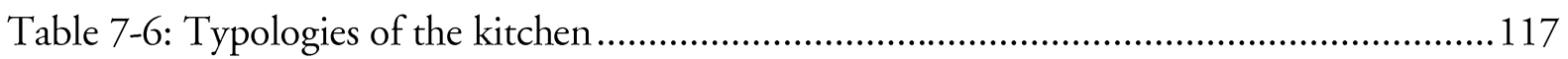

Table 8-1: Emergent QoL themes relating to design elements..............................................229

Table 8-2: Framework for the design of housing that improves the QoL of the high-needs elderly

Table 8-3: Themes for QoL (the legend for Table 8-2) ........................................................281

Table 9-1: Provided kitchen spaces and amenities .................................................................294

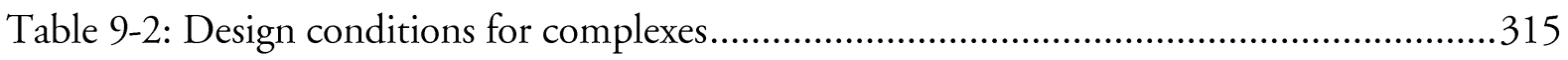




\section{LIST OF ACRONYMS}

QoL: Quality of life

ILE: Independent life expectancy

CCRC: Continuing Care Retirement Community

DHB: District Health Board

HNZ: Housing New Zealand

CHP: Community Housing Provider

Stats NZ: Statistics New Zealand

NZG: New Zealand Government

MOH: Ministry of Health

MSD: Ministry of Social Development

WHO: World Health Organization

ADL: Activity of daily living

IADL: Instrumental activity of daily living 


\section{GLOSSARY OF KEY TERMS}

Elderly people: Elderly people refer to people aged 65 years old and older.

High-needs elderly people: In this thesis, high-needs elderly refer to those elderly who require personal care from professional caregivers.

Personal care: Personal care refers to assistance in activities of daily living such as walking, bathing and putting on clothes, and medication management.

Residential care: Residential care is defined as accommodation combined with support and care, which include domestic services (laundry, cleaning), help with performing daily tasks (moving around, dressing, personal hygiene, eating) and medical care (various levels of nursing care and therapy services)(WHO, 2004). Residential care in New Zealand includes the following types of long-term care: rest home care, continuing care (hospital), dementia care and specialised hospital care (psychogeriatric care) (MOH, 2013b), and is classified in 'institution' in a 'non-private dwelling type' (Stats NZ, 2013).

Live independently: In this thesis, living independently refers to living in their own home, and not in residential care nor in their family's home. Therefore, it includes the situations in which elderly people live in their own home with some assistance in daily living.

Universal Design: "Universal Design" is 'the design and composition of an environment so that it can be accessed, understood and used to the greatest extent possible by all people regardless of their age, size, ability or disability. An environment (or any building, product, or service in that environment) should be designed to meet the needs of all people who wish to use it' (The Centre for Excellence in Universal Design, 2014). Universal Design in housing is both accessible and barrier-free, it is a concept distinguished from accessible or barrier-free housing; while accessible and barrier-free features in buildings (typically other than private housing) have been mandated by building codes or laws and defined by minimum standards, Universal Design in housing goes far beyond the minimum specifications (Mace, 1998). in New Zealand, Universal Design is represented by the Lifemark Design Standards (Lifetime Design Limited, 2012). 


\section{CHAPTER 1: INTRODUCTION}

This chapter aims to introduce this research, and includes the description of the research context, problem statement, aims and scope and overview.

\subsection{Research context and problem statement}

In New Zealand, people are living longer than previously. Life expectancy was recorded at an average 80.0 years for males and 83.4 years for females in 2015-17 (Stats NZ, 2018). Both these statistics indicate an increase of over 10 years in life expectancy during the past 60 years (Figure 1-1) (Stats NZ, n.d.). This increase in longevity, combined with a decline in fertility, has led to a rapid growth in both the size of New Zealand's population of elderly people and their proportion in the wider population. This is projected to accelerate further in the next 50 years (Figure 1-2) (Stats NZ, 2013, 2016b).

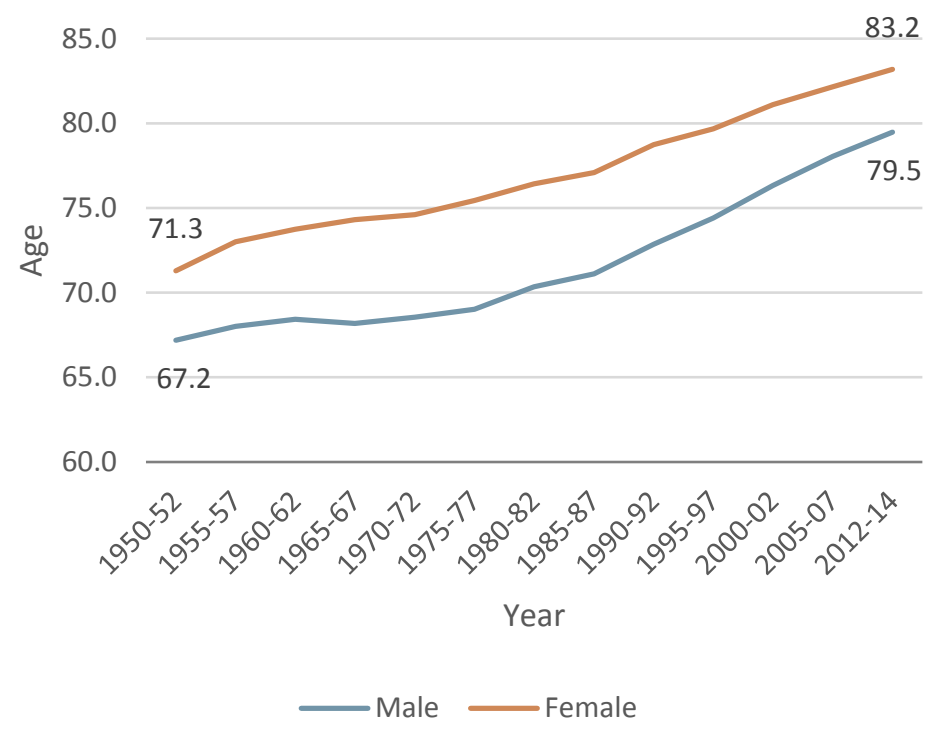

Figure 1-1: Life expectancy Data source: Stats NZ, n.d. 


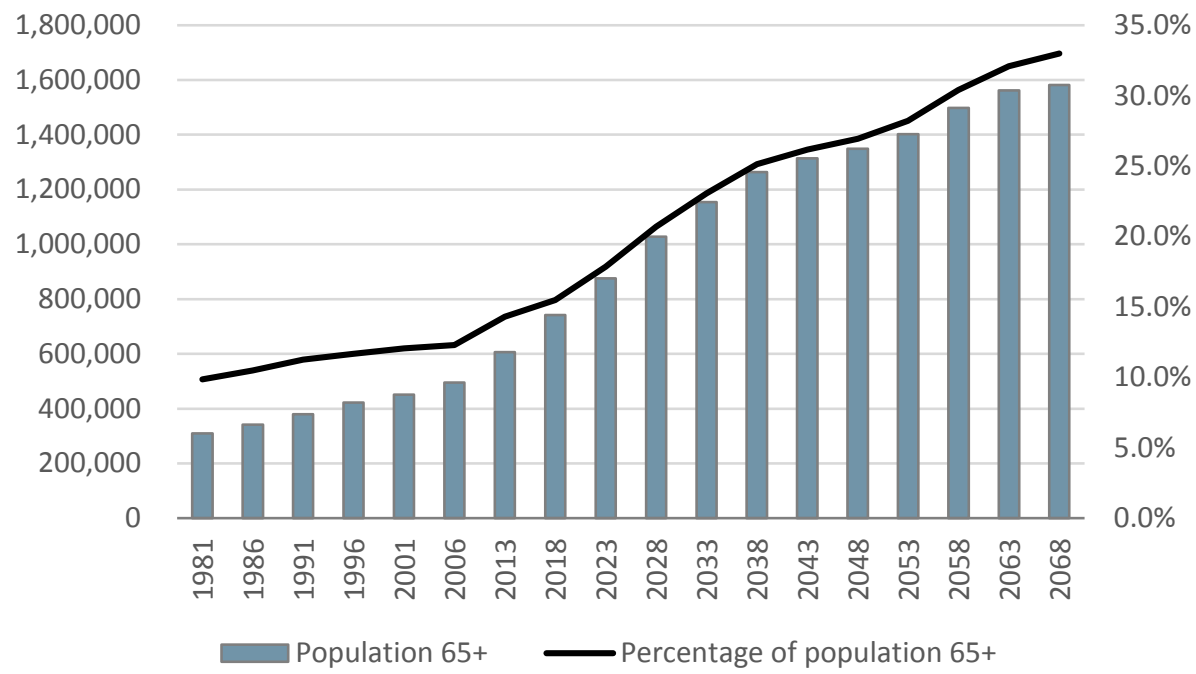

Figure 1-2: Population and the proportion of people aged 65+

(Data for 2018-2068 is projection)

Data source: Statistics New Zealand, 2013a, 2016b

\section{Increase in the elderly with high-care needs}

As people age, they tend to have greater health issues and impairments, which leads to an

increased need for care and support. Independent life expectancy (ILE) is the average number of years it is expected people will live independently, with no functional limitation that leads them to need assistance (MOH, 2013a). Figure 1-3 shows life expectancy and ILE at the age of 65 in 1996 and 2013. In 2013, the average life expectancy of people at age 65 was approximately 85, giving them an average 20 years of life remaining (18.9 years in males and 21.3 years in female), and the average ILE was approximately half of that (10.2 years in males and 10.6 years in females). This means that, on average, those who are 65 years old required assistance for about half of their remaining years before death (8.7 years in males and 10.7 years in females) $(\mathrm{MOH}$, 2015). The data of life expectancy and ILE between 1996 and 2006 showed that, while life expectancy had grown by 3.3 years in males and 2.2 years in females, the growth in ILE was only 1.1 years in males and 0.2 years in females. This indicates that as longevity increases, it tends to prolong the period in which the elderly require assistance, rather than the period they do not require assistance. 


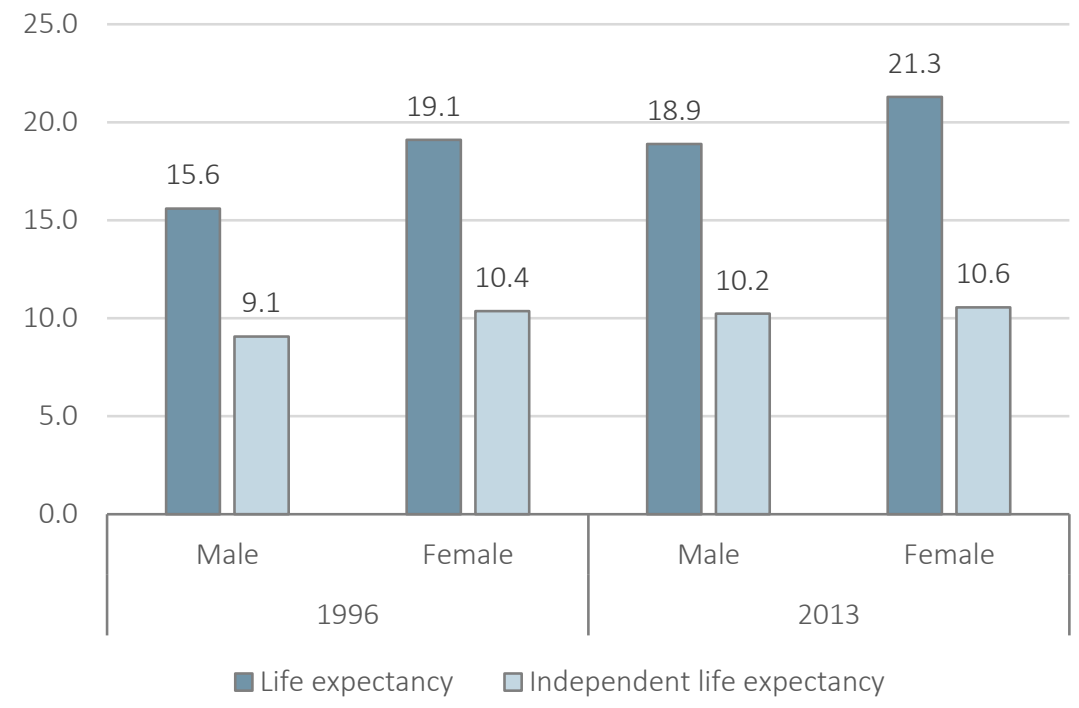

Figure 1-3: Life expectancy and ILE at age 65, 1996 and 2013

Data source: $\mathrm{MOH}, 2015$

In terms of the population, elderly people who require care and support are projected to increase, as shown in Figure 1-4 (Te Pou - The National Centre of Mental Health Research, 2011).

During the 20 years between 2016 and 2036, the high support needs will potentially increase by 1.9 times (from 77,000 to 146,000 ). This is a higher rate than the increase rate of the whole ageing population for the similar period of 2018 - 2038, which will be 1.7 times according to Figure 1-2.

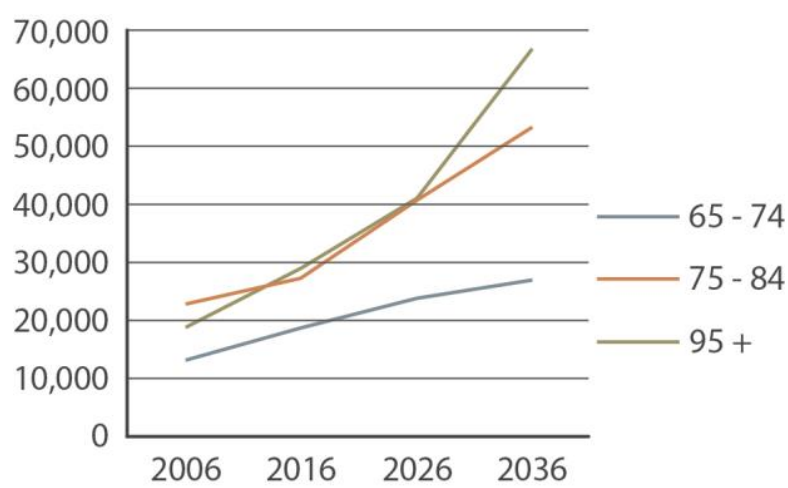

Figure 1-4: Projected number of older people with high-support needs

Data source: Te Pou - The National Centre of Mental Health Research, 2011

\section{Increasing demand for accommodating the elderly with care needs outside of residential care}

Residential care is a major accommodation option for the elderly with high-care needs in New Zealand. While the majority of elderly people live in their private dwellings, the proportion of people living in residential care increases as they age (Stats NZ, 2013). Recent trends in providing long-term care for elderly people show that residential care has focused on 
accommodating more people with higher levels of dependency. A longitudinal study between 1988 and $2008^{1}$ reported a substantial decline in the proportion of residents with lower dependency and an increase in residents with higher dependency in residential care (Figure 1-5) (M. Boyd et al., 2011). It also reported a significant decline in residents' length of stay in residential care over this 20-year period. These trends indicate that the level of dependency of the elderly at their entry into residential care has increased. If these trends continue, there will be more people needing care and living in their own home who would have resided in residential care homes in the past.

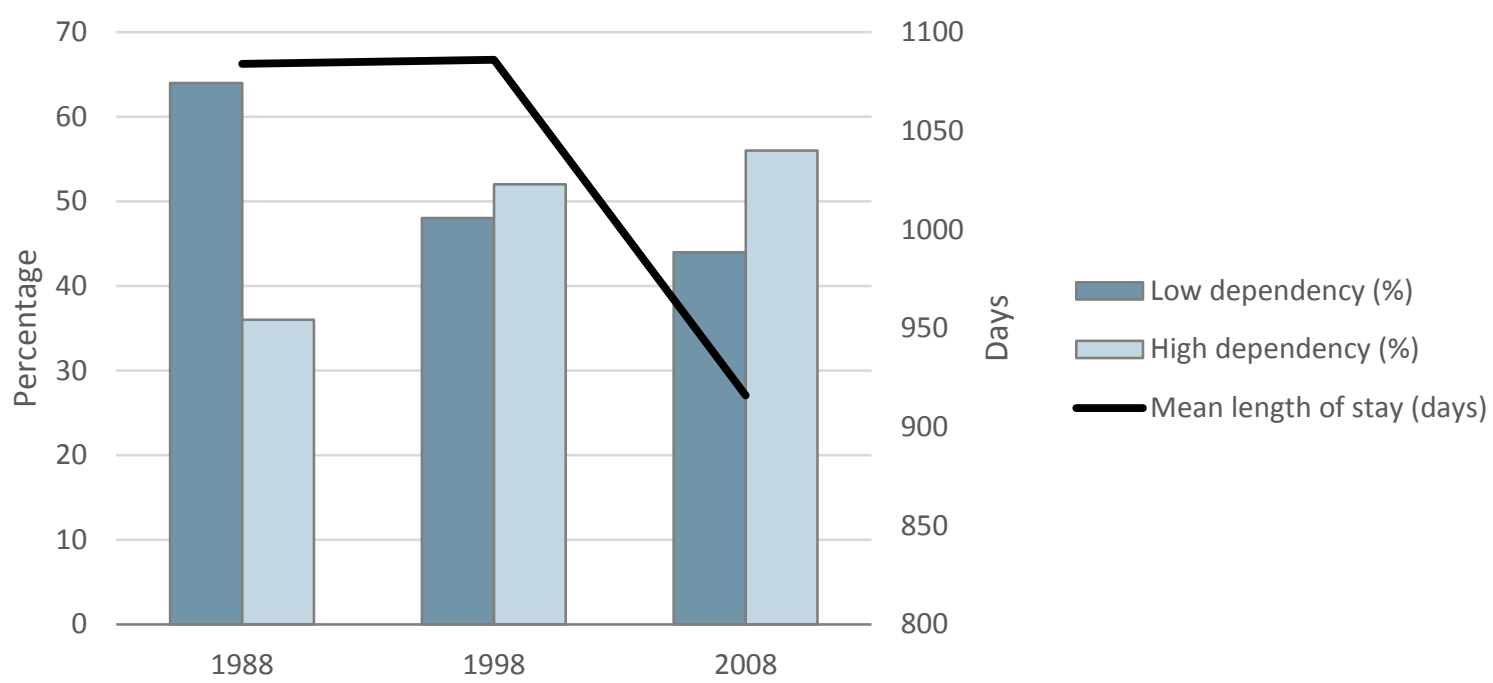

Figure 1-5: Proportion of residents by dependency levels and length of stay in residential care Data source: M. Boyd et al., 2011

Another factor that influences housing options for the high-needs elderly is the government policy for 'ageing-in-place' (MSD, 2001). This is a concept that has also been encouraged by international bodies (United Nations Population Fund and HelpAge International, 2012). It means that elderly people stay in their own home (including their family homes, in homes to which they have moved in mid or later life, or in supported accommodation of some type) and community that they have lived in as long as possible, rather than moving into residential care (Davey et al., 2004; Wiles, Leibing, Guberman, Reeve, \& Allen, 2012).

Combining two factors of the increase in the level of dependency of the elderly accommodated in residential care and the call for ageing-in-place by the government, there is a greater need for

\footnotetext{
${ }^{1}$ This study measured residents' dependency levels by the total scores for three categories of 'Self Care and Mobility,' 'Continence' and 'Memory Loss,' with each ranging between 1-5. 'Low dependency' and 'High dependency' had scores of 1-9 and 10-15 respectively.
} 
accommodation other than residential care, which can accommodate the elderly who require care.

\section{Option of staying put}

When impairments increase and care needs arise, the majority of New Zealand elderly people do not prefer to live with family as they do not want to be a burden on their children, and prefer to live their own homes (Davey, 2006a, p. 135; HART, 2014) (see also 2.1.2). However, the option of remaining in the home they have lived in up to that point often becomes unrealistic. One major reason is that older, frail people are likely to have trouble with inaccessibility in their house (Davey et al., 2004). For example, one study conducted on elderly people living in their own homes reported that the majority of respondents experienced difficulties, including difficulty with outside access (25\%), taking a bath or shower (20\%) and using indoor stairs or steps (8\%) (Povey \& Harris, 2006). These features sometimes caused residents to fall, and their fear of falls deterred them from moving around, which often led to lack of mobility (Howell, 2016).

Surveys of New Zealand's conventional housing report the need for modification to make houses suitable for them with increasing impairments, for at least the next 30 years (Saville-Smith, James, Warren, \& Coleman, 2009). However, housing modifications cost a substantial amount. For example, retrofitting a typical three-bedroom timber house with Universal Design features is estimated to cost approximately NZ $\$ 20,000$ on average, nearly four times more than adding Universal Design features at the stage of designing a new home (BRANZ, 2011). Given that the average annual income of a disabled person $65+$ in 2017 was NZ\$20,909 (Stats NZ, 2019), ${ }^{2}$ the expenditure of $\$ 20,000$ is unaffordable for many. Another study reports that the average expenditure for modifications that were actually made by elderly people over a 12 -month period was $\$ 16,345.63$, which was also a substantial expenditure (Saville-Smith, James, \& Fraser, 2008).

Even if housing is successfully modified, elderly people can still experience both physical and psychological problems. For example, the maintenance of a home and garden becomes more difficult (Davey et al., 2004; Povey \& Harris, 2006). Reported psychological problems include insecurity, loneliness, isolation and depression (Callaghan \& Towers, 2014; Davey et al., 2004;

\footnotetext{
${ }^{2}$ This cost is calculated based on the average weekly income of NZ\$401 in 2017.
} 
Greenbrook, 2005; Jaye et al., 2015). These issues relate to the changes in the spatial use of their home, which often occur when their health deteriorates, such as:

reorganizing of the downstairs part of the house into a [bedroom]/living area; closing off spare rooms or rooms not accessible or in use; parts of the house becoming chilly, musty and out of bounds ... Often parts of the house become shut off and much of the living begins to occur in only one or two rooms, such as the kitchen... (Hale, Barrett, \& Gauld, 2010, p. 33)

This spatial reorganisation results in adverse psychological conditions. In one study, Callaghan and Towers observed that ' $r$ ]estricted to living in only one or two rooms, many older people report[ed] being prisoners in their own homes, socially isolated and at risk from depression and anxiety'(2014).

High-needs elderly people tend to move to housing where residents can be provided with more suitable physical environments and greater care and support. This trend can be seen in the data for the percentage of people who moved house between 2001 and 2006, which dropped after the retirement age and rose at the age of 80 years and older (Stats NZ, 2009). This need is also reflected as a demand in suitable housing for this cohort in the policy context (see also 2.3.2).

There is a greater need for housing options where the high-needs elderly can live independently, other than the houses they have lived in previously, which are typically unable to accommodate their impairments and are detrimental to their independence and wellbeing.

\section{Housing options that supports independent life of the high-needs elderly}

Given that staying put is often not a realistic option due to the reasons mentioned earlier, other housing options that support the independent living of the elderly with care needs are required. There are three main types of housing that provide some level of care and assistance for the elderly: retirement villages, public-sector housing and private-sector rental housing for the elderly. Retirement villages, being viable options for current homeowners and the relatively welloff, offer company and security as well as home maintenance (Greenbrook, 2005). They often provide residents with support and care; among units supplied in retirement villages, supportedliving units provide residents with high-levels of care, which may include rest-home level care at the highest, while allowing residents' independence. By contrast, rental housing for the elderly provided by the public and private sector are generally affordable for those who cannot afford to live in retirement villages; however, most providers do not provide high levels of support and care 
for elderly people. It has been reported that options are scarce in New Zealand (Davey, 2006b; Reid, 2008) as with the rapid increase in the ageing population, there has been greater demand for such housing options (see also 2.3.2).

The quality of life (QoL) of high-needs elderly people has been studied by many researchers, and common themes include needs for independence, activities, relationships, identity, and quality of care (Murphy, Shea, \& Cooney, 2007; Tester, Hubbard, Downs, MacDonald, \& Murphy, 2004). QoL of this cohort is typically reduced in part due to increasing impairment but also due to unsuitable living environments (Hale et al., 2010; Nord, 2011) (see also Chapter 3). Given that retirement villages, particularly supported-living units, are options that have been designed to support independent life of the high-needs elderly, they are expected to result in greater QoL of residents. However, in recent times the QoL of residents in supported-living in retirement villages has been reported as low. They have reported a loss of privacy, independence and autonomy, poor social connections, and poor environments in private rooms; those in independent living arrangements were relatively satisfied (Hayward, 2012).

Without change to housing that offers support and care to facilitate independent living of the high-needs elderly, the reduced QoL of residents is likely to remain a problem. Yet, there is limited research addressing the role that each element of housing design can play to improve a wide range of aspects of QoL of elderly residents.

\subsection{Aim and scope}

This research aims to explore and clarify the requirements in housing design that can improve the QoL of high-needs elderly people living independently in New Zealand. As stated earlier, there are many factors that threaten residents' independent life when they stay put in the house they have lived in to date. These have resulted in a greater need for housing complexes designed for the elderly that support independent life by providing more suitable physical environments as well as accommodating levels of support and care. Responding to this need, the scope of this research excludes the option of staying put.

The elderly people addressed in this study are 'high-needs elderly', those who require personal care from professional caregivers. The rationale for this focus is that personal care is one of the higher levels of care for the elderly when compared to other types of care, such as assistance in household tasks and social support. Also, the majority of New Zealanders who require care, receive personal care from professional caregivers (see also 2.2.2). One of the limitations of this research is that it does not target those who receive personal care from their family, a tendency 
stronger in some ethnic groups (such as Māori and Pasifika) than others (particularly European New Zealanders); therefore, the findings do not represent the whole high-needs elderly population in New Zealand.

\subsection{Overview}

This thesis consists of ten chapters. Following this chapter, the context of this study is described in Chapter 2. Through the review of the circumstances for elderly people in New Zealand and housing options, it locates this study in a relevant context. In Chapter 3, a literature review focusing on the QoL of the elderly and the role of architecture in improving QoL is undertaken. This is a means to begin clarifying the requirements in housing design that improve the QoL of high-needs elderly people living independently in New Zealand. Through this review, the conceptual framework is developed and gaps in knowledge are identified, and the research questions are presented accordingly.

Chapter 4 describes the research strategy, which research establishes objectives and the four phases of the research procedures. Chapter 5 (Phase 1) presents the methods, findings and discussion of the preliminary investigation into the current situation of housing and elderly residents. It includes information that could not be obtained through literature; namely, the models of care and physical environments and the care and dependency of elderly residents. Chapter 6 and 7 (Phase 2) investigate the QoL of the high-needs elderly in relation to physical environments. In Chapter 6 , methods of investigation are discussed, followed by the presentation of findings in Chapter 7.

Chapter 8 establishes the design framework (Phase 3) through the discussion of the design considerations distilled in Phase 2. The design framework is then implemented in Chapter 9, and presents example models of housing design that improve the QoL of the high-needs elderly (Phase 4). Finally, in Chapter 10, outcomes of this research are critically viewed against the existing knowledge and frameworks. Contributions, limitations and expansion of research are discussed, followed by the conclusions of this thesis. 


\section{CHAPTER 2: BACKGROUND}

The design of housing for the high-needs elderly with a particular focus on the aspects of QoL that can influence design requires a fine-grained understanding of the conditions and needs of the high-needs elderly to establish performance criteria. This chapter reviews the current situation of the high-needs elderly and opportunities for independent living. First, it briefly reviews the socio-demographic features of elderly people such as age, gender, living arrangements, ethnicity, income and homeownership, which gives an overview of the elderly population generally in New Zealand. Next, the circumstances of the elderly requiring care are reviewed, starting with features of ageing and impairments and including situations of support and care offered in New Zealand. Finally, housing options for the high-needs elderly that enable them to live independently are reviewed. This includes a comparison of options in New Zealand with some international examples. Knowledge of these background contexts provides insight to the areas that require attention in the design of housing for the high-needs elderly in New Zealand contexts.

\subsection{Socio-demographic features of elderly people in New Zealand}

To provide a profile of elderly people in New Zealand, key characteristics such as age, gender, living arrangements, ethnicity, income and homeownership of elderly people are explored through a literature review.

\subsubsection{Age, gender and living arrangements}

A significant increase in the proportion of elderly people in New Zealand's population is projected in 30 years' time from 2013 to 2043 (Stats NZ, 2014d). The projected increase rate is higher as people age; the proportion of people aged $85+$ will increase at a higher rate than other groups (9\% increase in males and 5\% increase in females), while that of people aged 65-69 will decrease by $14 \%$ in males and $12 \%$ in females (Figure 2-1) (Stats NZ, 2014d). This means that people aged 85 + will account for a larger proportion among older people in 30 years. 


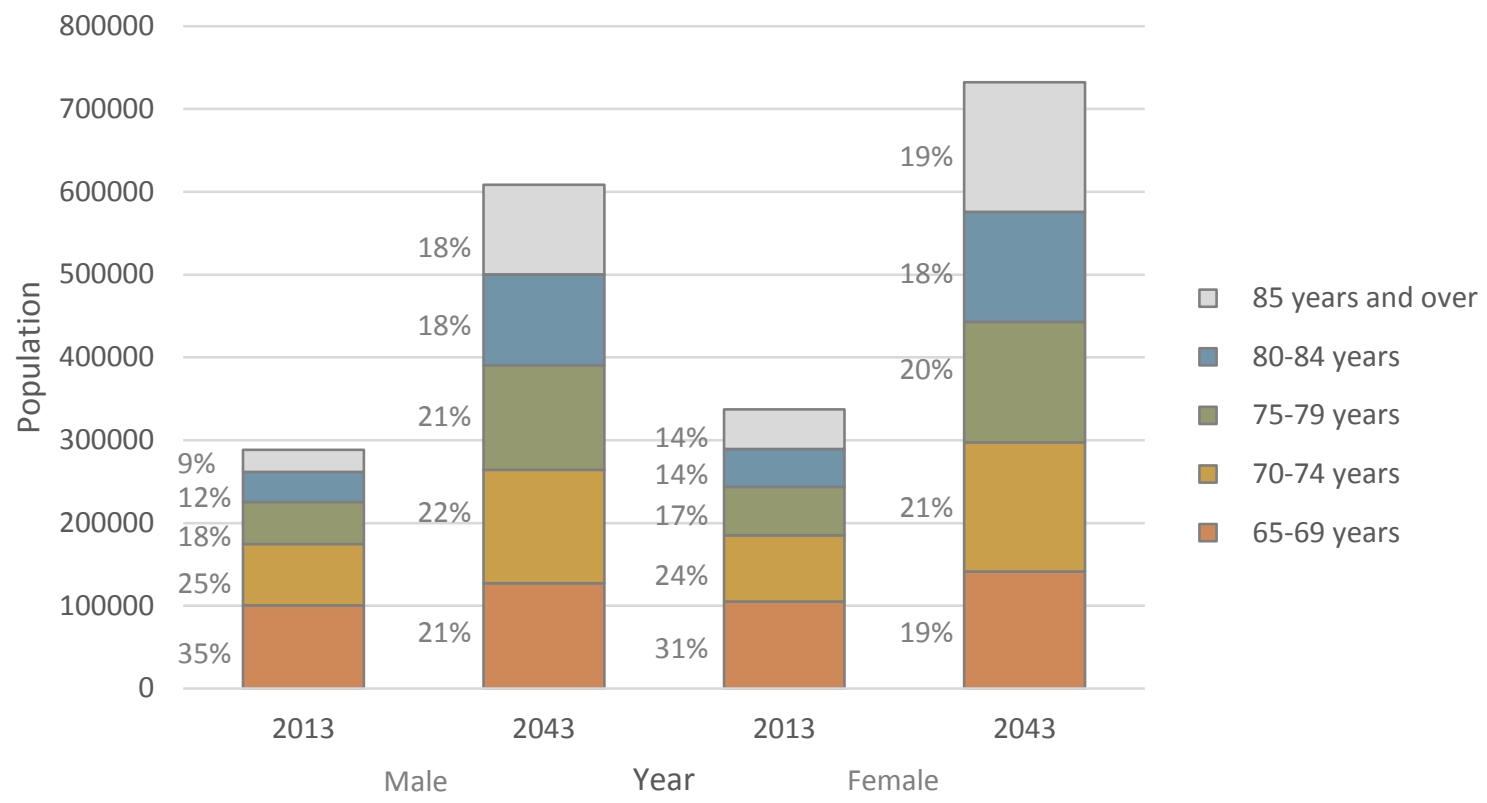

Figure 2-1: Population projection of people 65+

(The percentages beside columns represent those of each age group per the whole population)

Data source: Statistics New Zealand, 2014c

The majority (approximately 80\%) of people 65 and over either live alone or live only with a partner (Davey et al., 2004). Among those, the proportion of people living alone increases with age; at age 85+, a third of men and two-thirds of women live alone (Stats NZ, 2013, 2015).

This difference between genders reflects the difference in the life expectancy: the life expectancy is higher for women than for men, which results in 'more women being unpartnered in late life and more women living alone' (Davey et al., 2004).

\subsubsection{Ethnicity}

The older population will become more diverse in its ethnic composition (Davey et al., 2004). The proportion of non-European individuals, especially Māori and Asian, is expected to grow significantly in 25 years' time, though the population of $65+$ will remain predominantly European (over 70\%) (Figure 2-2) (Stats NZ, 2013). 


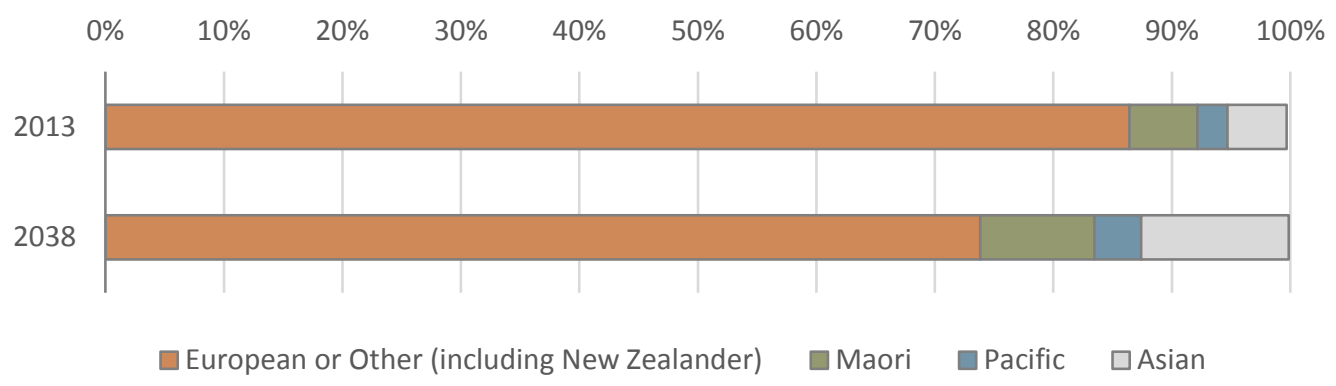

Figure 2-2: Projection of ethnic composition of people aged 65+

Data source: Statistics New Zealand, 2013a

This is significant because there are differences in living arrangements among ethnic groups.

According to a study on community-dwellings that focused on people aged 65 and over who had had InterRAI assessments for care needs ${ }^{3}$, those living with children or others accounted for $69 \%$ in Pasifika, 58\% in Asian and 42\% in Māori ethnic groups, which was much higher than the 14\% in the European/Other group (Figure 2-3) (Jamieson et al., 2018). In summary, living with children and others is a far more common living arrangement in non-Pakeha groups (Figure 23). This reflects differing cultural values; while Western cultures value individual independence, in Māori, Pasifika and Eastern cultures growing old is seen as positive and being cared for as a sign of love and respect (Ekelund, Dahlin-Ivanoff, \& Eklund, 2014).

\footnotetext{
${ }^{3}$ InterRAI New Zealand has conducted a universal standardised comprehensive geriatric assessment for both older people living in long-term care facilities and community-dwelling older people who are being considered for access to publicly-funded community services or residential care, aiming to standardise the assessments and care of older adults in New Zealand. Those assessed were mostly aged 65 and over, but included small proportion of people younger than 65 (interRAI New Zealand, 2016a, 2016b).
} 


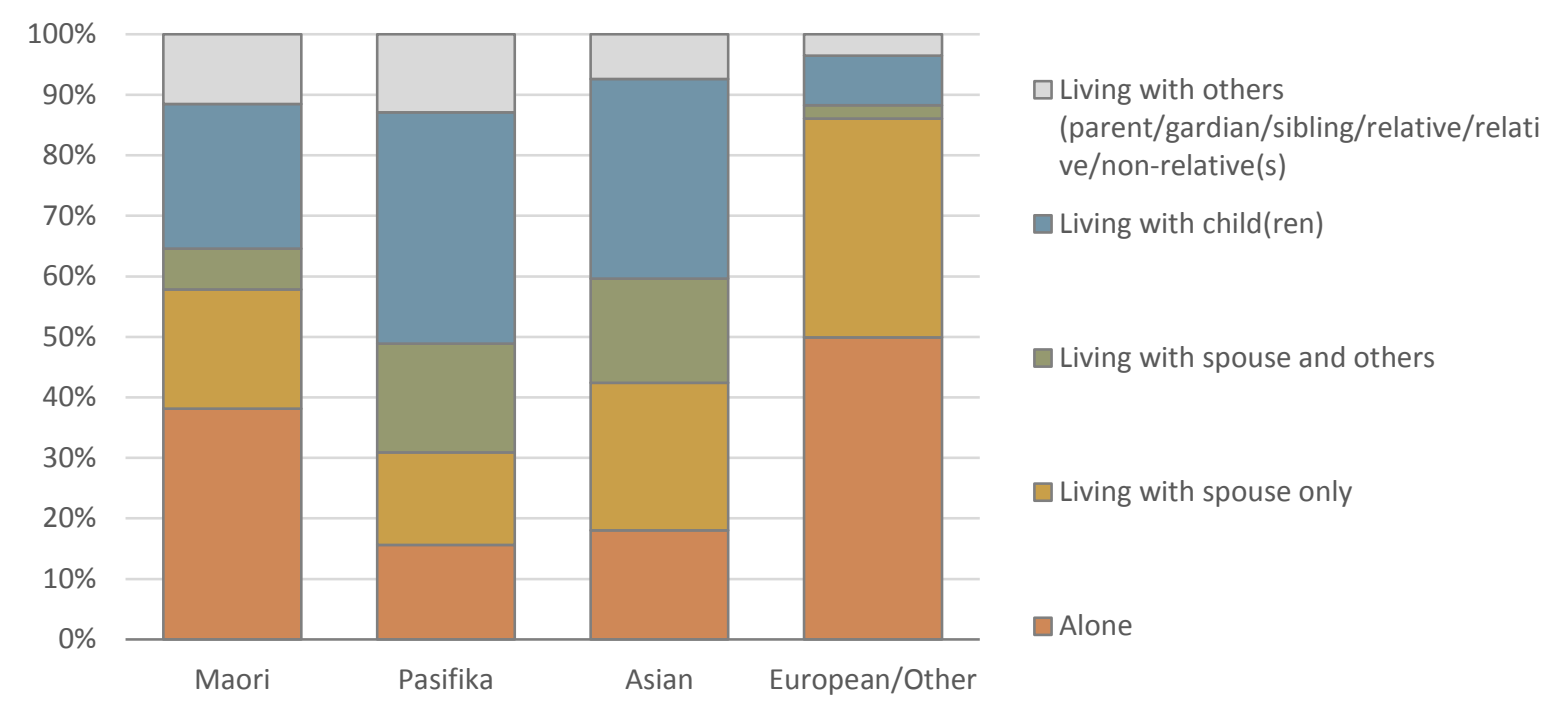

Figure 2-3: Living arrangements by ethnicity (Community-dwelling people aged 65 and over who had had InterRAI assessments)

Data source: Jamieson et al., 2018

However, there are changes to these trends. For example, despite the deep-rooted Māori values with respect to being cared for by their family, the proportion of Māori living in multi-family households is declining (Davey et al., 2004). This tendency is supported by a study of elderly Māori's perceptions, which reported that some of the older Māori consulted expressed a preference for living independently of their children and grandchildren. They reported wanting their whānau nearby, but did not necessarily want to live with them (Davey et al., 2004). Similarly, Pasifika people and Asians such as Chinese and Indians have traditionally lived in multi-family households where the elderly are cared for by their children, but research shows emerging preferences that the elderly do not want to be a burden on their children and prefer to live independently of their families (Davey, Keeling, \& Zodgekar, 2010; Li, 2011; Tamasese, Loudeen, \& Waldegrave, 2014).

\subsubsection{Income and homeownership}

Income begins to decline in later mid-life and reduces in retirement (Stats NZ, 2016a; Waldegrave, 2014). The source of income for people after retirement includes public provision from New Zealand Superannuation (NZS), veteran's pensions, private provision through KiwiSaver, occupational pensions, and private savings in bank deposits and shares. NZS is only just above the median income poverty threshold. This means that those with a low level of additional income are likely at risk of poverty, especially non-homeowners (Stephens, 2014). 
Historically, New Zealand has had a strong culture of homeownership (Davey, 2006a); however, the level of homeownership fell between 2001 and 2013, particularly in the middle-aged and younger age groups (Stats NZ, 2014a) (Figure 2-4). This trend will result in a decline in homeownership for future older populations and lead to a greater proportion of elderly people renting (New Zealand Property Investors' Federation, 2015). Along with an increasing ageing population, there has been a rise of nearly $30 \%$ in older renter households in both the privatesector and HNZ housing between 1996 and 2006 (Keeling, 2014).

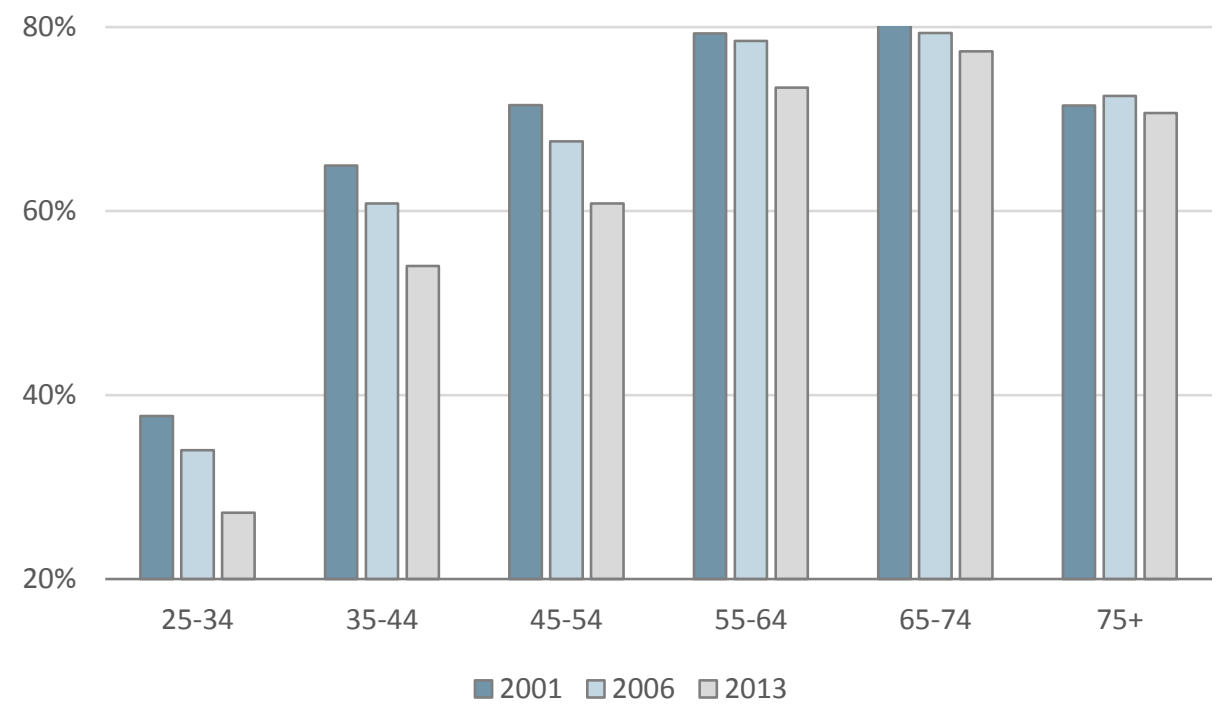

Figure 2-4: Homeownership rate by age group Data source: Statistics New Zealand, 2014a

\section{Summary}

In the next 30 years, there will be a significant increase in the proportion of older groups among people aged $65+$, with those living alone increasing at the highest rate. The vast majority of European New Zealanders live independently from their children, influenced by their individualistic culture. While European New Zealanders will remain the majority, there will be a greater proportion of non-European elderly people in the next 20 years. Older Māori, Pasifika and Asian people have traditionally lived with their families; however, the number of those who choose to live separately from families is increasing. This means that there will be greater demand for housing suitable for the elderly that can accommodate single persons in New Zealand. In addition, income typically declines after retirement, which may put some people into poverty; affordable housing options will be required for those financially in need. With the projected decline in the homeownership rate, the demand for affordable rental housing will increase. 


\subsection{Ageing and impairments of the elderly and support and care in New Zealand}

To capture the circumstances surrounding the high-needs elderly, features of biological ageing, impairments and the care and support provided for them in New Zealand are explored in this section.

\subsubsection{Biological ageing and impairments of elderly people}

Ageing has been defined as 'a progressive deterioration of physiological function, an intrinsic agerelated process of loss of viability and increase in vulnerability' (Magalhães, 2013). Interactive systems of the body that maintain its operation, such as mechanical and skeletal systems, control systems (affecting the digestive, respiratory and cardiovascular systems), detection senses and communicating systems (including the nervous system) deteriorate as people age (Eastman, 2013).

The muscular system loses strength as bodies age, and a decrease in movement can lead to muscular atrophy, which affects posture, endurance and joint positions (Eastman, 2013). The skeletal system loses its ability to bind appropriate minerals such as calcium within bone, making bone brittle and increasingly vulnerable to accidents (Arking, 2006; Eastman, 2013). The skeletal system also loses elasticity, affecting bending, kneeling, turning and rising. This impacts individual's gait, leading to shuffling or awkward steps and the increasing risk of falls. The decline in the efficiency of the respiratory and cardiovascular system increases the proneness to diseases that can reduce blood supply to the brain, causing dizziness, blackouts and blurred vision, which also increase the risk of falls.

Sight is also significantly affected by ageing. In addition to being less responsive to light, the diameter of the pupil decreases with age, which reduces the amount of light that can enter the eye. As a result, elderly people need a greater amount of light (as much as three times more than younger adults) to be able to see the shapes of objects and to perceive fine details (Haigh, 1993). Hearing impairment is another issue experienced by most individuals as they get older. The ability to hear higher frequencies is lost first (Eastman, 2013). Elderly people are less able to distinguish depth, quality and subtly of sounds, reducing the ability to hear voices clearly and to distinguish between speech and background noise (Goodman \& Smith, 1992).

The nervous system becomes less efficient at coordinating movement; reflexes degrade and reaction time slows, which can contribute to loss of balance (Eastman, 2013; Narayan, 2016). 
The mind's ability to store and recall information also diminishes with age, which causes memory loss (Eastman, 2013).

In New Zealand, as people age their propensity for impairment increases from $40 \%$ of the population aged $65-74$, to $60 \%$ of the population aged $75-84$ and $90 \%$ of the population aged 85+ (MOH, 2007; Stats NZ, 2013). The impairment types vary and include physical impairments such as mobility (46\%) and agility (28\%), sensory impairments such as hearing (28\%) and sight (11\%) and cognitive impairments such as remembering (10\%) and learning (7\%) (Stats NZ, 2014b) (Figure 2-5). Having multiple conditions is a feature of ageing ( $\mathrm{MOH}$, 2010).

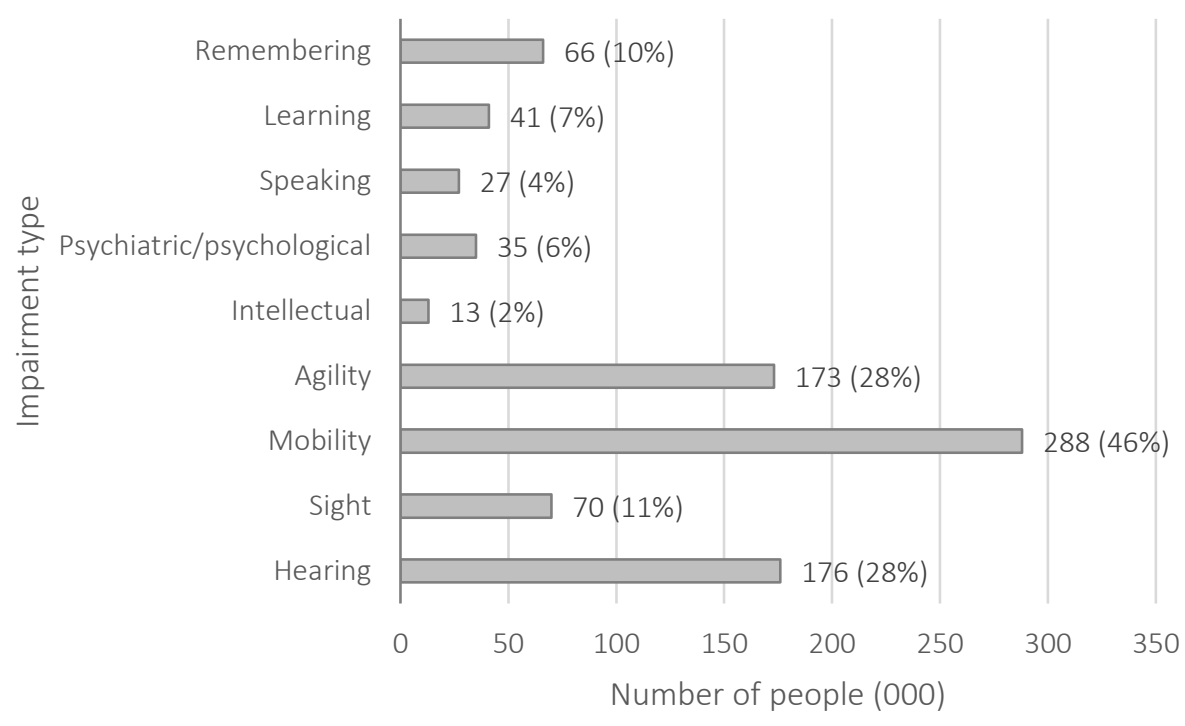

Figure 2-5: Impairment types and the population Data source: Statistics New Zealand "Disability Survey: 2013 -Tables"

\subsubsection{Care and support for elderly people living independently}

As impairments increase, elderly people require care and support from others to live independently. There are a wide range of types of care and support for the elderly in need, including personal care, assistance in household tasks, social and emotional support, meals and transportation services.

'Personal care' refers to assistance in activities of daily living such as walking, bathing and putting on clothes, and medication management. 'Assistance in household tasks' refers to help with shopping, laundry, preparing meals, gardening and household maintenance (Davey \& Keeling, 2004). 'Social and emotional support' refers to checking on the elderly person by phone, 
visiting, taking them out, and offering activities such as reading, writing letters and playing cards (Davey \& Keeling, 2004). Meal services such as Meals on Wheels are also available for those living independently (NZG, 2018). For emergency assistance, medical alarms worn on the neck or wrist are available, and are provided and monitored by St John or other private companies (Bupa New Zealand, 2018; St John, n.d.).

In New Zealand, the public health care system (District Health Boards (DHBs)) provides an individual assessment of needs for older people in order to provide care for individuals. Through this assessment the elderly may be provided with access to publicly-funded services, which are personalised depending on the levels of care needed and other personal circumstances. These services include home support services, such as help with household tasks and personal care in private homes, as well as residential care (MOH, 2011, 2012). Social support may also be publicly provided.

Types of care fall into two categories based on the type of caregivers: 'formal care' and 'informal care'. Formal care refers to care provided by paid caregivers, while informal care refers to that provided by unpaid caregivers such as family and friends. While the government health system entitles those in need to receive formal support for personal care and household tasks, the role of informal caregivers in supporting the elderly is significant, particularly for household tasks and for social and emotional support. It has been reported that $47 \%$ of disabled adults aged $85+$ receive informal support mainly with shopping and housework, while $10 \%$ received informal support with personal care (Te Pou - The National Centre of Mental Health Research, 2011). Another study conducted with elderly people in 150 households in Dunedin reported that over $80 \%$ had visits from family or friends once a week or more (Povey \& Harris, 2006). The demand for both types of caregivers (informal and formal) is expected to increase in New Zealand (Badkar \& Manning, 2009; Te Pou - The National Centre of Mental Health Research, 2011).

\subsubsection{Levels of dependency}

A high proportion of elderly people living in their own home require high levels of care; InterRAI New Zealand reported that over half of people who had home care assessments were in 
high to very high levels of need and might have a high risk of requiring hospital or aged residential care services ${ }^{4}$ (Figure 1-6) (interRAI New Zealand, 2016b).

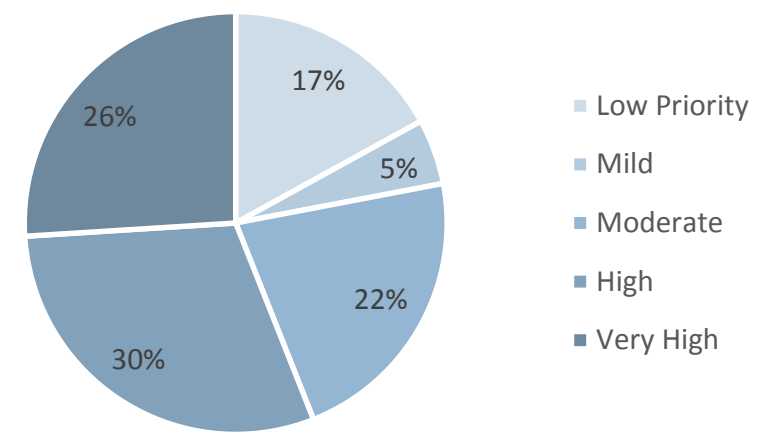

Figure 2-6: Proportion of older people living in their private homes with levels of priority for services Data source: interRAI New Zealand, 2016c

\section{Summary}

As age advances, elderly people experience a wide variety of physical changes, and impairments typically increase and become complex, which are relevant to the design of housing. In New Zealand, there are various types of care and support to assist the elderly who live independently. One of them is publicly-funded services for assistance in household tasks and personal care for the elderly in need. Other types of care, including social and emotional support, are provided by private agencies as well as family and friends. There are differences in the roles of formal caregivers and informal caregivers, with formal caregivers mainly providing help with household tasks and personal care, whereas informal caregivers provide assistance in household tasks as well as social and emotional support. There is an increasing demand for both types of caregiver in the future. Most elderly people who live independently and who had their care-needs assessed required high levels of care. This indicates there will be a higher demand for housing that can accommodate high levels of care and caregivers, as well as accommodate elderly residents' impairments in the future.

\footnotetext{
${ }^{4}$ Those assessed were mostly aged 65 and over, but included small proportion (5\%) of people younger than 65 (interRAI New Zealand, 2016b).
} 


\subsection{Housing options for high-needs elderly to live independently}

This section explores housing options for the high-needs elderly to live independently, which are relevant to those within the scope of this research and design changes recommended later. As noted earlier, these options exclude living in residential care and staying put. The exploration starts with review of international examples, followed by a review of housing options in New Zealand.

\subsubsection{Housing options for the high-needs elderly overseas}

Internationally, many types of housing for the elderly have developed over the past several decades. This section reviews the examples in the UK and North America. The UK examples are chosen because the New Zealand culture has been greatly influenced by the UK. Examples in North America are reviewed because they have developed Continuing Care Retirement Communities (CCRCs), which are similar to retirement villages in New Zealand. The review starts with an illustration of the typology of housing for the elderly in the UK as an example of the variety of housing types supporting different levels of care needs for elderly residents. After presenting this overview, housing types that accommodate high-needs elderly living independently in various other countries are examined, where the backgrounds of their development, the services provided for residents and the features of physical environments are briefly described.

\section{Typology for housing for the elderly in the UK}

Table 2-1 shows typologies of housing for the elderly in the UK, which are classified into seven levels depending on the levels of care and support provided for residents (Robson, Nicholson, \& Barker, 1997). 
Table 2-1: Classification of housing for the elderly in the UK made by Robson et al.

\begin{tabular}{|c|c|c|}
\hline Level & Description & Examples of housing types \\
\hline Level 1 & $\begin{array}{l}\text { Non-specialised and non-adapted dwellings ('staying } \\
\text { put' or living with relatives) }\end{array}$ & 'Staying put' or living with relatives \\
\hline Level 2 & $\begin{array}{l}\text { Independent dwellings which have been purpose-built } \\
\text { or adapted for fit and active elderly who may need } \\
\text { some support but can generally look after themselves }\end{array}$ & Retirement housing \\
\hline Level 3 & $\begin{array}{l}\text { Purpose-built, self-contained dwellings (to mobility } \\
\text { standards) in groups with warden attendance and } \\
\text { minimal communal facilities, for active elderly }\end{array}$ & Sheltered housing (Category 1) \\
\hline Level 4 & $\begin{array}{l}\text { Purpose-built, self-contained dwellings (to full mobility } \\
\text { and wheelchair standards) in groups with warden } \\
\text { attendance and access to communal facilities, for } \\
\text { physically frail elderly }\end{array}$ & Sheltered housing (Category 2) \\
\hline Level 5 & $\begin{array}{l}\text { Similar to Level } 4 \text { but with extra care support available } \\
\text { and the option to take communal meals }\end{array}$ & $\begin{array}{l}\text { Extra care housing (Category } 2.5 \\
\text { sheltered housing) }\end{array}$ \\
\hline Level 6 & $\begin{array}{l}\text { Residential care homes for elderly who may be } \\
\text { mentally and physically frail and in need of constant } \\
\text { personal care }\end{array}$ & Residential care homes \\
\hline Level 7 & $\begin{array}{l}\text { Nursing homes for elderly who are sick or very frail and } \\
\text { need qualified nursing care }\end{array}$ & Nursing homes and Geriatric care units \\
\hline
\end{tabular}

Level 1 is the accommodation option 'Staying put' or family houses, which are 'often ill-suited to the needs of frail elderly people, and extensive adaptations may be necessary' (Robson et al., 1997). Level 2 is 'Retirement Home', which is 'smaller and more convenient' but many '[fail] to meet mobility/wheelchair standards' and are 'unsuited for the needs of frail elderly people' (Robson et al., 1997). Housing types of Level 3-4 provide some levels of care and support for residents by warden attendance. However, Level 3 is not built to mobility/wheelchair standards, and although Level 4 is supposed to be built to these standards many are not and 'are unsuitable for frail elderly people' (Robson et al., 1997). Housing types of Level 6-7 are institutional residential care facilities, which are not private dwellings of the elderly, and are therefore excluded from the scope of this section. Level 5 housing type is situated between Level 4 and Level 6, and is described below.

\section{Extra care housing}

Extra care housing is the name for the Level 5 housing type in Table 2-1. This type emerged to fill in the gap between housing types of Level 4 and Level 6, with a focus on maintaining dignity and independence (often at stake in Level 6-7 residential care) while facilitating a variety of care delivery systems not facilitated by Level 4 housing (Department of Health, 2004; Robson et al., 1997). Some studies have reported overall positive outcomes in terms of residents' wellbeing in various aspects including independence and social life (Darton, Bäumker, Callaghan, \& Netten, 2011). Extra care housing meets full mobility/wheelchair standards and varies in the level of care 
provided for residents. Residents have self-contained units in the complexes that include a wider range of communal facilities, and are provided with personal care (Orrell et al., 2013). The number of extra care housing units has dramatically increased, from approximately 15,000 units in late the 1990s (Robson et al., 1997) to 49,000 units in 2014 (N. King, 2004; The Housing Learning and Improvement Network, n.d.). The size of complex varies from very small $(6-12$ properties) to very large retirement villages (300 or more properties) and puts great focus on the integration into the community (Robson et al., 1997; The Housing Learning and Improvement Network, 2011) (Robson et al., 1997).

\section{Assisted living}

Assisted living is a type of supported housing that was developed in the USA in the 1980s. It aims to create a homelike alternative to the institutional, medical model of the nursing home by providing private rooms or self-contained apartments with kitchen facilities and space for personal furniture and other belongings (Gusmano, 2004). One study has reported that assisted living residents had greater QoL compared to when they were in nursing homes; more specifically, they experienced a reduction in anxiety and depression and a significant improvement with their ability to make choices (Brandi, Kelley-Gillespie, Liese, \& Farley, 2003). The facilities often include supportive services and common spaces such as a dining room, a library and an activity room (Schwarz \& Brent, 1999). One of typical conceptual plan for assisted living is shown in Figure 2-7 (Eastman, 2013). 


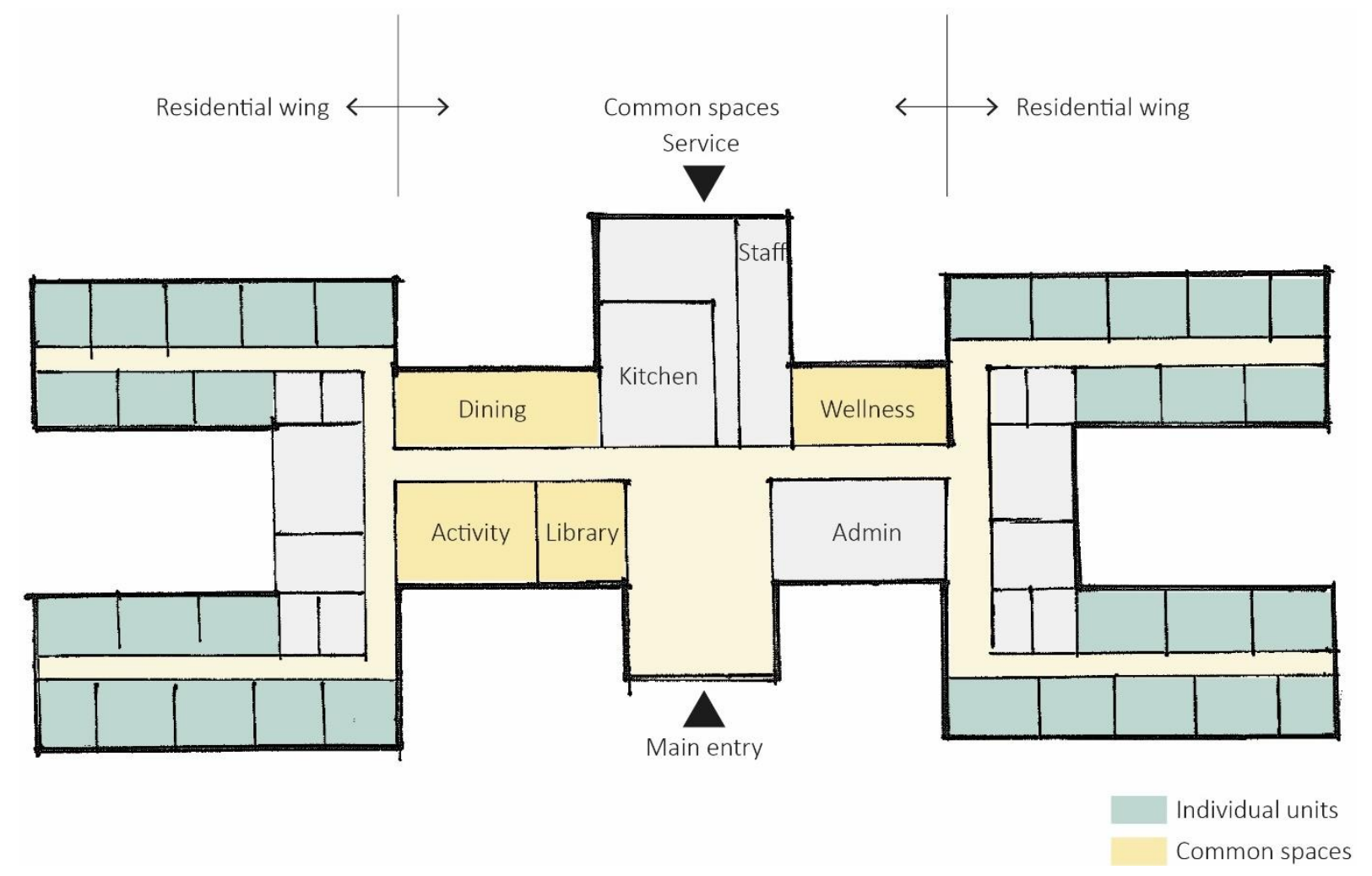

Figure 2-7: Concept plan for assisted living

Source: Drawing by author based on the image on Eastman, 2013

\section{Continuing Care Retirement Community (CCRC)}

Another housing type that can accommodate the elderly requiring care developed in North America is the CCRC. The CCRC offers a continuum of care for the elderly, combining housing options with different levels of care depending on residents' needs. There are typically three levels of housing and care: independent living, assisted living and skilled nursing care (Nelson, 2002). It offers this flexibility by maintaining an assortment of on-site medical and social services and facilities (Sanders, 1997). For the last 30-50 years, the majority of CCRCs were built in suburban and rural areas, which were typically composed of low-rise housing units and centrally located buildings near the major services and amenities (Eastman, 2013; B. Perkins, Hoglund, King, \& Cohen, 2004). In high-density urban areas, they may take the form of a high-rise building that is more likely 'integrated in the fabric of the city' (Eastman, 2013) . In accordance with the changes in attitudes of the elderly, new types of communities have emerged, such as those offering learning opportunities by locating the buildings adjacent to or within college and university campuses (Eastman, 2013).

\section{Public-sector affordable rental housing}

The public-sector has been the major provider of affordable housing for the elderly in many countries (Eastman, 2013). In the USA, the 'Section 202 Supportive Housing for the Elderly 
Program' operated by the Department of Housing and Urban Development (HUD) has provided housing for very low-income seniors, including the frail elderly, for over 50 years (Perl, 2010; U.S. Department of Housing and Urban Development, n.d.). It provides them with both housing and support services such as cleaning, cooking and transportation, and also offers rent subsidies (U.S. Department of Housing and Urban Development, n.d.). It was reported that 263,000 units were available in 2010, and a recommendation was made that 10,000 units be built annually for the next ten to fifteen years to provide the growing ageing population as a costeffective alternative to institutional residential care (Eastman, 2013; Perl, 2010).

However, problems with public-sector affordable housing have been reported regarding the limited ability to provide necessary services to allow residents to age in place, due to lack of funding. Solutions include inter-sectorial collaborations. One example is a partnership between the public-housing authority, a developer and a non-profit sponsor in Pittsburgh; jointly they converted two high-rises to provide assisted living (Eastman, 2013).

\subsubsection{Housing options for the high-needs elderly in New Zealand}

In this section, possible accommodation options enabling the high-needs elderly to live independently in New Zealand are explored. This section identifies three main types of housing designed for the elderly: public-sector housing, private-sector rental housing for the elderly and retirement villages. Inter-sectorial collaborations are also considered.

\section{Retirement villages}

Since 2003, retirement villages have been regulated and monitored under the Retirement Villages Act 2003, which requires formal registration as a retirement village. Under this act, residents and the operator enter into a contract called an 'Occupation Right Agreement,' which 'entitles a person to occupy a unit in the village and to use services and facilities' (NZG, 2003). The most common form of occupation right is a licence to occupy (James \& Saville-Smith, 2011). This contract normally requires capital contribution as well as weekly fees (normally ranging between NZ\$69 and \$150), so retirement villages tend to be an option for homeowners or those relatively well-off financially, but are often not options for low-income people or non-homeowners (Consumer NZ, 2013; Davey et al., 2004; Grant Thornton New Zealand, 2010; Greenbrook, 2005).

Residents have access to services and facilities under their occupation right agreements. As part of the services provided for residents, the operator is in charge of maintenance of all buildings, plants and equipment, as well as minor repairs and maintenance requested by residents (Ministry 
of Business, 2017). Other services may include nursing or medical services, the provision of meals, services for laundry, hairdressing, transport and recreation as well as security services (NZG, 2003).

Similarly to CCRCs in North America, retirement villages in New Zealand provide the elderly with housing options that have ranging levels of care (HJ SIMS, 2015). There are two main types of residential units, based on the type of services provided for occupants: independentliving units and supported-living units. Independent-living units include villas, townhouses, apartments or studios that are normally not combined with personal services. Supported-living units, often called 'serviced apartments', are designed for those who need regular help with dayto-day tasks such as cleaning and laundry or with medical care. They are generally one or two bedrooms. Meals may be provided in a communal dining room, although a kitchenette is usually included in the apartment (Retirement Village Association of New Zealand, 2014). Some retirement villages are also attached to residential care units (James \& Saville-Smith, 2011). Many villages assure a continuum of care, providing these different types of units with levels of care in one site.

According to the Retirement Villages Act 2003, a retirement village must contain two or more residential units as well as services and/or facilities (NZG, 2003). In practice, they range from small complexess with a few units to large complexes (James \& Saville-Smith, 2011). Based on the data for retirement villages supplied by six large New Zealand retirement village operators ${ }^{5}$ in 2016, the average number of units per village was 106, ranging between 42 and 175 depending on the operators (JLL, 2017). No comprehensive information is publicly available regarding the numbers of each type of units; however, Table 2-2 shows situations in some villages in the Wellington Region, which were publicly obtainable (Eldernet Ltd, 2018). Regarding accessibility, neither the Retirement Village Code of Practice nor Building Act 2004 specify the obligation for retirement villages to meet the access standards such as NZS 4121 (Ministry of Business, 2017).

Table 2-2: Number of independent and supported-living units (2016)

\begin{tabular}{|l|l|l|l|}
\hline Retirement villages & $\begin{array}{l}\text { Number of independent- } \\
\text { living units }\end{array}$ & $\begin{array}{l}\text { Number of supported- } \\
\text { living units }\end{array}$ & $\begin{array}{l}\text { Total number } \\
\text { of units }\end{array}$ \\
\hline Malvina Major retirement village & $116(69 \%)$ & $55(31 \%)$ & 171 \\
\hline Charles Fleming retirement village & $201(72 \%)$ & $79(28 \%)$ & 280 \\
\hline Summerset at the Course & $196(91 \%)$ & $20(9 \%)$ & 216 \\
\hline
\end{tabular}

\footnotetext{
${ }^{5}$ Six large RV operators are Ryman, Metlifecare, Summerset, Bupa, Oceania and Arvida.
} 
In 2017, there were 28,168 retirement village units in New Zealand (JLL, 2017). The proportion of those aged $65+$ living in retirement villages accounted for $5.2 \%$ and those aged 75 $+12.4 \%$ in 2014 (JLL, 2017). If this trend continues ( $12 \%$ in the age group $75+$ ), the future demand for retirement village units has been projected at an increase of 73,062 units between 2016 and 2068, which averages at 1,405 units per annum (JLL, 2017).

According to a study exploring baby boomers' expectations ${ }^{6}$ in older age, some people perceive retirement villages as a good model for those who could afford them, offering company and security, and reducing concerns about home maintenance (Glasgow, 2013).; however, other 'disliked the structured nature', or 'were concerned at the level of financial resources they used up' (Glasgow, 2013, p. 190). Moreover, the QoL of residents in supported-living in retirement villages has been reported as low. In one study exploring the perceptions of residents in retirement village independent-living units and supported-living units for their life in retirement villages, residents in supported-living units experienced a loss of privacy, independence and autonomy, poor social connections, and poor environments to accommodate their possessions in private rooms; those in independent-living arrangements were relatively satisfied (Hayward, 2012). There was no other research focusing on the physical environments and residents in supported-living units. Considering that elderly people with greater care needs are more likely to reside in supported-living units than in independent-living options, more research focusing on this type of accommodation is highly desirable.

\section{Public-sector housing}

Public-sector housing includes properties provided by central government (Housing New Zealand (HNZ)) and rental housing provided by local authorities. Elderly people account for approximately 20\% of all HNZ residents (NZG, 2015a) and most tenants of local authority housing are elderly people (Office for Senior Citizens, 2013). These properties are often supplied at subsidised rates (NZG, 2015b). However, HNZ does not usually provide support and care for residents. Assistance for the residents would be provided through the DHB. Some local authority housing is in a similar situation, while many local councils provide some assistance in household tasks and social support for elderly residents to live independently. For example, New Plymouth District Council housing provides regular visits by the Client Services Officers to check on maintenance issues and tenants' wellbeing (Davey et al., 2004). Another example is Christchurch City Council housing, which has traditionally accommodated many

\footnotetext{
${ }^{6}$ Baby boomers refer to the cohort who were born between 1946 and 1965 (Stats NZ, 2014c).
} 
elderly people. It has tenants' lounges within its complexes and recreation and outings are organised by activities coordinators (Davey et al., 2004).

Recently, housing provided by HNZ and local authorities was transferred to private-sector housing providers for a two year period (Alan Johnson, Philippa Howden-Chapman, \& Shamubeel Eaqub, 2018; NZG, 2015b). This movement has reduced the number of HNZ units from 66,127 units in 2011 to 60,301 units in 2017 (Alan Johnson et al., 2018). The Office for Senior Citizens reported in 2013 that 11,000 units were managed by local councils (Office for Senior Citizens, 2013), but many councils have sold their housing portfolios since and the number of units in 2017 has been reported as just under 8,000 (Alan Johnson et al., 2018). However, after the government change in 2017 , housing policies were refocussed on increasing the social housing portfolio (including HNZ units) to meet the growing demand (Alan Johnson et al., 2018; Hon Phil Twyford, 2017).

HNZ and local-authority housing, organisations that typically own old properties, are facing challenges in upgrading their stock to meet current desirable standards, such as the installation of ceiling and underfloor insulation. Some houses require major retrofitting to meet these standards due to issues such as insufficient space in roofs and under floors, or inadequate types of roof (Ian Page \& Fung, 2008). Despite these challenges, some HNZ homes are on their retrofit programme and will include upgrades like the installation of insulation and double-glazed windows, as well as improvements to air-tightness and ventilation and the installation of new heating (HNZ, 2018). Wellington City Council, who own over 2,000 units in 40 complexes, is also currently proceeding with a 20-year housing upgrade project, which includes the redevelopment of complexes with units that will meet the desirable standards (Wellington City Council, n.d.-a). Some of these units will be specially designed to accommodate elderly people or those with limited mobility, with features such as level-access showers, wide doors, power points and cupboards at suitable heights, and appropriate taps (Wellington City Council, n.d.b).

\section{Private-sector rental housing for the elderly}

This category of housing includes that provided by voluntary agencies and Community Housing Providers $(\mathrm{CHP})^{7}$. Voluntary agencies such as religious groups and charities play a significant role in supplying affordable rental housing for the elderly; however, they have been withdrawing

\footnotetext{
${ }^{7} \mathrm{CHPs}$ are sometimes categorised as providers of public-sector housing; however, they have been categorised in 'private-sector' in this project because it was often difficult to distinguish the voluntary agencies and CHPs, which may overlap.
} 
from the senior housing sector, largely as they are unable to afford to upgrade facilities (Povey \& Harris, 2006). Some offer elderly people independent-living units for rent in the community and others provide village-style accommodation. Some housing is located near existing rest homes.

Private-sector housing provides a range of services, such as communal areas, personal care, household assistance and staff visits. For example, Abbeyfield New Zealand provides home-like accommodation with individual resident rooms, common facilities such as a laundry, a dining room and a lounge, and employs a housekeeper who prepares two main meals a day (Abbeyfield New Zealand, 2016). The Salvation Army offers elderly people rented independent-living units, and has a referral officer to arrange letting and maintenance of units. Some complexes are located adjacent to rest homes, where tenants may purchase meals and laundry services (Davey et al., 2004; Salvation Army New Zealand, 2018). Upper Hutt City Council pensioner housing was sold off to Compassion Housing, a religious group that provides affordable rental housing for the elderly. This housing provides residents with staff visits at no extra charge, which includes visits from the Tenancy Manager for home maintenance, and from a registered nurse, who monitors residents' health needs (Sisters of Compassion Limited, n.d.).

CHPs are community-based housing service providers aiming to provide long-term, affordable and appropriate housing (Community Housing Aotearoa, 2018a). The Community Housing Regulations Authority authorises the registration of CHPs, which entitles elderly recipients to income-related rent subsidies (MSD, n.d.-a). Many of the voluntary agencies described above have CHP registration. The national Social Housing Reform Programme has encouraged CHPs to provide quality social housing for an increasing number of people in need (New Zealand Treasury, 2012; NZG, 2015b).

An increasing number of social housing options have been supplied by CHPs (MSD, n.d.-b). This sector currently owns or manages approximately 5,000 housing units in New Zealand (Office for Senior Citizens, 2013). However, if focusing on housing specially for the elderly population, the scale of development of housing provided by CHPs or voluntary agencies as described above is relatively small, and the total unit numbers offered by these groups accounts for only a small proportion of housing. For example, Seniorline (2016) listed 13 rental accommodations run by the voluntary sector in Auckland. Each site included an average of 16.3 units, ranging between 5 and 48. The total number of units was 212, which is a quite small number, compared to other types of housing for the elderly such as 1,300 pensioner housing 
units owned by Auckland City Council (Office for Senior Citizens, 2013). Shortages are currently reported in Auckland (Seniorline, 2016).

With the projected increase in older renters, there will be greater demand for private rental housing specially designed for the older age group in the future (New Zealand Property Investors' Federation, 2015). Through the Social Housing Reform Programme, there has been a great expectation put on CHPs for innovation and better combinations of housing and services that meet people's needs (NZG, 2015b). The role of private-sector rental housing will become more significant in the supply of housing for the high-needs elderly in the future.

\section{Inter-sectorial collaboration}

To provide not only housing but also care and support for elderly people in the New Zealand context, the need for inter-sectorial collaboration has been stressed by the government $(\mathrm{MOH}$, 2002; MSD, 2001; Reid, 2008). Several different types of partnerships have been reported, such as those between HNZ and the voluntary sector, and those between local authorities and the DHB or voluntary organisations (Davey et al., 2004). For example, the Canterbury DHB has worked with Christchurch City Council regarding subsidised housing for the elderly. They have been discussing partnerships with some private-sector agencies to develop housing that can accommodate the high-needs elderly (Davey et al., 2004). It has been reported that a partnership between a not-for-profit organisation and a rural District Council successfully made pensioner housing suitable for the high-needs elderly by adding communal facilities and employing support workers, thereby creating an alternative to the standard rest home (Reid, 2008).

\subsubsection{Policies for older people and housing}

This section reviews key New Zealand policies related to housing for the elderly in recent years. These policies are important in that they signal the areas of focus for housing for the elderly towards the future. Implications of these policies in housing for the elderly are identified, and finally are linked to the global context.

New Zealand Positive Ageing Strategy (MSD, 2001) states that the elderly are important members of society, who have skills, knowledge and experience and have the right to be afforded a life with dignity, and encourages their participation in the community, which would benefit the individual concerned, the community, and the country as a whole. For this objective, it identifies the barriers to their participation, and ten priority goals, with recommended actions to achieve these goals. Housing is set as one of the priority goals, particularly ensuring that they have adequate and affordable housing that meets their needs. This is important for them to 
maintain their independence and age-in-place successfully. As well as actions to keep housing cost affordable for the elderly general and with low income, it recommends strengthening legal protection for retirement village residents and increasing the supply of Universal Design and energy-efficient low-rental housing, including supported pensioner housing complexes. Another related priority goal of this strategy is supporting ageing-in-place, and it includes providing a wide range of services as one of the key actions.

'Older New Zealanders - Healthy, Independent, Connected and Respected (Office for Senior Citizens, 2013)' reviews the actions that had been taken for the past 12 years to demonstrate the commitment to the Positive Ageing Strategy vision and principles. This includes the review of affordable housing options such as state housing/HNZ and social housing (including localauthority housing and those provided by CHPs) (see also 2.3.2). It also outlines retirement villages as housing options with a range of care options so residents can remain living in a village for as long as they want. The Government supports living in retirement villages through advice, information and ongoing sector meetings. There has been greater attention to Universal Design in the design of housing provided by local authorities and CHPs. In the area of providing services to support ageing-in-place, there is an increase in the government spending for the provision of home support services as well as assistance for getting equipment (e.g. mobility aids, shower stools) and/or funding for housing/vehicle modifications (see also 2.2.2).

In 2019, the draft of Better Later Life - He Oranga Kaumātua 2019 to 2034 was released (MSD, 2019). This strategy also aims to embrace the opportunities that an ageing population and longevity brings and challenges the negative stereotypes of older people, but with a more focus on the diversity of the ageing population and consideration of the next generation of older people currently aged 50 to 64 . Housing is one of the key areas in this strategy as well. It defines the main functions of housing as protecting us from the elements and natural disasters, helping us to live decently on limited incomes, and being a place and base for us to enjoy life and stay connected (e.g. places for intimacy, hobbies, welcoming visitors and providing hospitality, caring for others and receiving care, and enjoying 'time-out') (Office for Seniors, 2019). In particular, 'providing housing choices and options so people can age in the community' has been identified as one of the five key areas for action by over $90 \%$ of submitters to the draft strategies (Office for Seniors, 2019). The draft points out the limited availability of accessible and affordable housing with good access to services, and the shortage of dwellings suitable for older people. Particularly, there is a growing demand for affordable rental and shared housing as well as for housing assistance, given the increase of renters and people paying mortgages among those $65+$, and the 
unaffordability of living in retirement villages for many people. Suggested actions include providing sufficient public and community housing suitable for older people with appropriate support, the central and local government working in partnership with CHPs. The draft also suggests the change in the size and mix of the housing stock to meet the needs of an ageing population. In relation to the housing design, the importance of Universal Design is stressed, and the importance of cultural diversity in the design and the provision of social services is also recognised.

Both strategies — New Zealand Positive Ageing Strategy and Better Later Life - He Oranga Kaumātua 2019 to 2034 — have determined that housing is one of the main focus in promoting positive ageing and the better life for the elderly. Both aim to help the elderly age-in-place or live in the home they choose to live in, and raise the priority area of providing affordable housing for the elderly (e.g. state housing, social housing). The provision of accessibility and Universal Design, as well as the provision of appropriate support service is also main areas of focus in both. In comparison, Better Later Life - He Oranga Kaumātua 2019 to 2034 focuses on the provision of more housing options for the ageing population, such as social housing and shared housing that are accessible and affordable. It introduces new perspectives on incorporating cultural diversity in the design of housing for the elderly.

Finally, these New Zealand policies are located within the global context, by referring to the World Health Organization (WHO) Global Strategy on Ageing and Health 2016 — 2020 (WHO, 2017). This global strategy values older people's participation in, and contribution to, society, and aims to promote healthy lives and well-being of all ages. In spite of the biological decline and losses the elderly are likely to experience, it emphasises the importance to challenge many of the stereotypes that currently define what it is to be "old". These descriptions are consistent with Better Later Life - He Oranga Kaumātua 2019 to 2034. The objectives it defines include developing age-friendly environments (which includes housing) and developing sustainable and equitable systems for providing long-term care (home, communities and institutions). The draft policy emphasises the importance of fostering autonomy, the rights to make choices and take control over a range of issues, including where they live. This part may correspond to the aim of providing greater housing options for the elderly and incorporating cultural diversity in the design, in Better Later Life - He Oranga Kaumātua 2019 to 2034.

The WHO strategy has a clear statement for the right of those with high-needs; 'in circumstances of significant loss of functioning, older people still... have the right and deserve the freedom to 
realize their continuing aspirations to well-being, meaning, and respect (WHO, 2017, p. 18).' It also refers to the role of age-friendly environments in supporting them; 'for people with significant declines in capacity, supportive environments can promote dignity, autonomy, functioning and continued personal growth (WHO, 2017, p. 4).' These aspects are included to some extent in the promotion of Universal Design in the housing design and the provision of support in New Zealand strategies, but there is no clear statement for the active and dignified living environments for those with high-needs. This is something that policies that emphasise active participation of older people are often criticised for; 'over-promotion of the positive image may result in insufficient attention being given to the needs of the frail old in policy and planning and increased denial of ageing amongst individuals. As a consequence, individuals may not prepare adequately for increasing dependency and may find it harder to adapt (Davey \& Glasgow, 2006, p. 25).’

\section{Discussion and summary}

Internationally, there has been a significant development over several decades in various types of housing for the elderly. There has been an increasing housing supply for those targeting the high-needs elderly, such as extra care housing, assisted living and CCRCs. Particularly extra care housing and assisted living have developed as an alternative to institutional residential care, providing residents with greater autonomy and independence. Some housing types have taken the form of a wide-spreading plans with low-rise housing units; however, the recent focus in planning has been focused on integration into the community. There is also increasing demand for housing suited for middle-income and low-income high-needs elderly people. Affordable housing for low-income individuals has been supplied mainly by the public sector; however, to meet the requirements for accommodating the high-needs, strategies such as inter-sectorial collaboration have been required.

Retirement villages in New Zealand are similar to CCRCs in some other countries and can provide ranging levels of care and support; however, they are not affordable for many New Zealanders. The demand for this housing type is expected to grow. On the other hand, publicsector housing typically offers affordable housing; however, the ability to provide support for elderly residents in this housing is limited, with some housing operators providing only social support. Some private-sector rental housing for the elderly provides higher levels of care than the public sector; however, the demand for these types of units far exceeds the supply. To meet the demand, the government expects CHPs or inter-sectorial collaboration to supply housing for low-income or non-homeowner elderly individuals. 
Comparing trends in New Zealand with those overseas, there are some similarities. One similarity is that there have been a growing number of housing units designed for the elderly requiring high levels of care; however, they are typically unaffordable for those with low levels of income or limited assets. There is also a similarity that affordable housing, provided by either the public or private sector, has been unable or is struggling to provide sufficient levels of care for the high-needs elderly, which has resulted in a shortage of supply against the demand. The common solutions include inter-sectorial collaboration. However, there are differences in the outcome of residents' QoL in the housing types that provide high levels of care; the levels of QoL of residents in extra care housing and assisted living have been reported as high, but those in supported-living units in retirement villages in New Zealand have been reported as low.

Key New Zealand policies related to housing for the elderly also state that more affordable and more housing options are required, with wider variety to suit individuals' needs. Housing should be suitable for the elderly, such as incorporating accessibility Universal Design features in the design as well as the provision of appropriate services for them. Most features are consistent with the WHO global policy, but the attention to housing for the high-needs elderly is lacking in those New Zealand policies reviewed, compared to the global policy.

The combined available information indicates that housing in New Zealand does not meet the needs of the high-needs elderly, due to both insufficient supply but also due to lack of affordability. This situation is similar to that seen in other countries. But, in New Zealand, even those residents in housing specially designed for high-needs elderly people to live independently perceived reduced QoL. There is a great need to clarify the improvements necessary to provide a greater QoL for the elderly requiring care. 


\section{CHAPTER 3:QOL AND ARCHITECTURE}

Chapter 1 identified the increasing demand for housing that facilitates independent living by the high-needs elderly and reported on their reduced QoL. To explore the best architectural means to improve QoL, the domestic and international literature on the QoL of the elderly and on the physical environment relevant to the QoL is reviewed in this chapter, broken into four sections. Prior to focusing on the relationships between the QoL of the high-needs elderly and physical environments, the first section provides an overview of the concept for QoL and the QoL of the high-needs elderly. This is followed by an exploration of the QoL of the high-needs elderly in relation to physical environments and establishes the conceptual framework for their QoL. The third section provides a review of existing design frameworks for housing for the elderly and examines their applicability to housing for the high-needs elderly in New Zealand. Finally, the outcomes of these three reviews are combined to identify the gaps in existing knowledge, and the research questions are developed.

\subsection{QoL of the high-needs elderly}

This section provides an overview of the QoL of the high-needs elderly, which starts with the exploration of the concept of QoL, followed by the review of the QoL of the high-needs elderly.

\subsubsection{QoL}

QoL is a difficult concept to define, but generally, it is considered 'a multidimensional concept emphasizing the self-perceptions of an individual's current state of mind' (Bonomi, Patrick, Bushnell, \& Martin, 2000, p. 1). The evaluations include individuals' 'emotional reactions to life occurrences, disposition, sense of life fulfilment and satisfaction, and satisfaction with work and personal relationships' (Theofilou, 2013, p. 151).' It is a subjective assessment, differing by individuals (Carr, Gibson, \& Robinson, 2001). For example, Calman has defined QoL as 'the gap between expectations and experience' (Calman, 1984); however, people have different expectations, which are influenced by social, psychological, socioeconomic, demographic, and other cultural factors. Moreover, expectations can vary between individuals even under similar conditions (Carr et al., 2001). The concept of QoL also changes with age (Eiser \& Jenney, 2007; Theofilou, 2013). Despite of this complexity, QoL is a relevant and important concept to consider in architecture, because physical environments are a factor influencing QoL (Mohit, 2013), and a high QoL of occupants is a component of the index for successful architectural 
design that enhances sustainability (Higher Education Funding Council for England, 2006; RIBA et al., 2017).

QoL is also difficult to measure (Theofilou, 2013). To date, more than 1000 instruments have been invented to measure QoL (Ubel et al, cited in Theofilou 2013). QoL measures typically consider a number of social, environmental, psychological and physical values (Theofilou, 2013). Some are generic, and others are for use of specific populations, such as patients of specific illnesses, specific age groups and those who require care ${ }^{8}$ (Eiser \& Jenney, 2007; Theofilou, 2013). Of the latter group, Health-Related-QoL (HRQoL) is concerned specifically with health aspects while accounting for general QoL 9 (Theofilou, 2013). While the measurement of QoL is useful to explore the impact of particular circumstances (e.g. diseases, physical conditions) on individuals' QoL (Theofilou, 2013), the use of standard tools has been criticized, due to the subjective nature of QoL as mentioned above. Predetermined measures contain items that may not be relevant for all individuals whose QoL is assessed (Eiser \& Jenney, 2007).

\subsubsection{QoL of the high-needs elderly}

Internationally, a lot of research has been specifically undertaken to clarify the QoL of the highneeds elderly (Cooney, Dowling, Gannon, Dempsey, \& Murphy, 2014; Dyck, Kontos, Angus, \& McKeever, 2005; Hale et al., 2010; Iwarsson \& Isacsson, 1997; E. C. King, Holliday, \& Andrews, 2016; Kontos, 1998; Morales, Rousseau, \& Passini, 2012; Murphy et al., 2007; Nord, 2011; Schillmeier, 2017; Tester et al., 2004). The QoL of the elderly is generally reduced as their care needs increase. This is typically seen in long-term care settings, where care is provided as efficiently as possible to accommodate large numbers of people. As such the care typically lacks certain core qualities of home, such as control, autonomy, choice, privacy and selfdetermination (Cooney, 2012; Custers, Westerhof, Kuin, Gerritsen, \& Riksen-Walraven, 2012; Granbom et al., 2014; Klaassens \& Meijering, 2015; Persson \& Wästerfors, 2009; Stabell, Eide, Solheim, Solberg, \& Rustøen, 2004). The individual's sense of self can be compromised as people are socialised into institutional routines (Wiersma \& Dupuis, 2010). These characteristics have been represented by the word 'institutional', defined by Goffman as a place of residence where a large number of like-situated individuals, are cut off from the wider society for an appreciable period of time and together lead an enclosed, formally administered life

\footnotetext{
${ }^{8}$ This group is called social care-related QoL (SCRoL) and include the ASCOT tool (University of Kent and the Personal Social Services Research Unit, 2018).

${ }^{9}$ HRQoL instruments include generic ones such as 36-Item Short Form Health Survey (SF-36) (John E. Ware \& Sherbourne, 1992) and WHOQOL (World Health Organisation, 1998), as well as disease-specific instruments.
} 
(Goffman, 1968). In particular, a lack of privacy was found in these settings (McColgan, 2005; Twigg, 2002). This effect has also been reported in New Zealand residential care, where admission into facilities providing full-care causes a decrease in autonomy and independence, which leads to an acceleration of mental and physical decline (Green Party of Aotearoa New Zealand and New Zealand Labour Party, 2010; Grimmer, Kay, Foot, \& Pastakia, 2015).

'Institutional' characteristics can also be found in home settings where residents receive care. When the home becomes a site for formal delivery of care, individuals' private space can be violated and their sense of home disrupted (Gillsjo, Schwartz-Barcott, \& von Post, 2011; Hale et al., 2010). Residents in assisted living and retirement communities generally reported better QoL than those in residential care (Biggs, Bernard, Kingston, \& Nettleton, 2000; Brandi et al., 2003); however, a lack of independence, control, privacy and autonomy of residents has also been reported in assisted living (Tompkins, Ihara, Cusick, \& Park, 2012; Young, 2005). Hayward's report on the reduced QoL of residents in New Zealand also indicated a lack of resident independence and autonomy and a lack of privacy in private rooms, which are typical characteristics of institutional living (Hayward, 2012). These outcomes indicate that housing combined with long-term care, irrespective of the type of accommodation, is prone to deteriorating resident QoL by 'institutionalisation.'

While the reduced QoL of the elderly requiring care is represented by the relatively general 'institutional' characteristic, some studies have refined the important components of QoL for them. For example, in the UK, Tester et al. identified four essential needs for QoL in frail elderly people in institutions: 1) retaining sense of self by keeping their preferred appearance, personal possessions and control over space, 2) the care environment that facilitates residents' dignity and individual needs as well as control in activities, 3) the ability to maintain connection to family and preferred relationships with other residents and staff, and 4) opportunities to engage with social and communal activities that interest them or give them stimulation (2004). Similarly, Murphy et al. in Ireland revealed four thematic domains of QoL: 1) care environment and ethos of care that facilitate resident autonomy, 2) maintaining personal identity through the provision of adequate personal space, 3) facilitating connectedness to family and community, and 4) provision of activities and therapies that are meaningful and purposeful (2007).

In conclusion, the QoL of the high-needs elderly is often reduced with their increasing care needs, which relates to 'institutionalisation,' regardless of the care type they receive (residential care or in-home care). In order to improve the QoL of the high-needs elderly, it is important to take account of a wide range of aspects of QoL, which include the maintenance of personal 
identity and important relationships as well as the provision of care that facilitates autonomy and individual needs and activities that are interesting and meaningful to the individuals.

\subsection{QoL of the high-needs elderly in relation to physical environments}

This section explores the QoL of the high-needs elderly in relation to the physical environments. First, the overall trend in the expectation of the elderly for their living environments is explored, which is followed by an extensive literature review on the QoL of the high-needs in relation to physical environments.

\subsubsection{Overview of the expectation of the elderly for their living environments in New}

\section{Zealand}

Prior to focusing on the high-needs, this section outlines the current and future trend in the expectation of the elderly in New Zealand. It largely focuses on baby boomers, who have started to enter their retirement, and will comprise a large part of the ageing population requiring care in the next 50 years. Baby boomers in New Zealand are characterised by individualism and liberalism (Martin, 2005; Sharon Buckland, 2009), which is 'centred on personal lifestyle choices'(Martin, 2005, p. 177). The diversity of this cohort has been also a notable characteristics (Glasgow, 2013; Koopman-Boyden \& Moosa, 2014). These attitudes have implications for their expectation for their living environments. Their main expectations for late life include the following;

- Avoidance of being a burden of family

There was a strong desire to ensure family were not burdened (Davey, 2006a; Glasgow, 2013).

- Avoidance of institutionalisation

Many desired not to enter residential care (Davey, 2006a) (see also Chapter 1). Boomers dislike of residential care may motivate them to explore alternative in-home care to a greater degree than current assumptions (Glasgow, 2013).

- Smaller housing for ageing in the community Many wish to age in their current dwellings (Glasgow, 2013; Povey \& Harris, 2006); however, they acknowledged that unsuitability of their home for those with impairments would require adaptations/modifications and maintenance services for the garden and property in the future (Davey, 2006a; Glasgow, 2013; Povey \& Harris, 2006). With these 
limitations, combined with financial concerns, many anticipated the need to move to a smaller house (Davey, 2006a; Glasgow, 2013; Povey \& Harris, 2006), but they strongly desired to live independently in the community (Davey, 2006a).

- A wider range of housing options, including communal housing options Some cohorts perceived retirement villages (one of the current major housing options for the elderly in New Zealand) as too structured and too costly, and desired a wider range of housing options. Through a focus group, a study revealed their expectations for various types of communal housing options such as multi-family dwellings, multi unrelated person houses with "flatting" style arrangements, cluster housing where residents were loosely linked by common values, such as an eco-village style and cooperative community (Glasgow, 2013). The motivations to co-reside were for social contact, costs, avoidance of institutionalisation and retaining a greater degree of control (Glasgow, 2013, pp. 266-270).

\subsubsection{QoL of the high-needs elderly in relation to physical environments}

This section explores the QoL of the high-needs elderly in relation to the physical environments, through an extensive literature review. The literature reviewed includes information on the relationships between their QoL and physical environments not only that in architecture but also in a wide range of disciplines such as social, medical and psychological areas. It excludes existing design frameworks (e.g. design standards, design guides), which will be reviewed in the following section. Through this review, the QoL of the high-needs elderly that relate to the physical environments is conceptualised with the categorisation of six themes of; independence and control, privacy, personal identity, activities, relationships, and quality of care. This review also leads to the identification of gaps in knowledge, which is combined with the outcome of the next section and presented in the final section of this chapter.

\section{Independence and control}

Independence is a core contributor to QoL. Studies have revealed a significant correlation between higher dependency and lower QoL (Callaghan \& Towers, 2014; Gabriel \& Bowling, 2004; Orrell et al., 2013). Needing assistance with the activities of daily living (ADLs) ${ }^{10}$ affects self-esteem, self-efficacy and sense of control (Boström et al., 2014).

\footnotetext{
${ }^{10}$ Activities of daily living (ADL) include bathing, dressing, going to toilet, transfer, continence and feeding, and have been are used for measure the functional dependency in clinical settings (Katz, Ford, Moskowitz, Jackson, \& Jaffe, 1963).
} 
An individual's dependency can be created or intensified by an unsuitable physical environment. This is well explained by the theory of person-environment $(\mathrm{P}-\mathrm{E})$ fit (Lawton, Altman, \& Wohlwill, 1984). P-E fit establishes that the degree to which individual and environmental characteristics match is influenced by environmental factors as well as by personal competence (Lawton et al., 1984). This P-E fit problem is more prominent in cases of lower competence and those who are more sensitive to the demands of the environment. For these reasons, it is applicable to the situation in housing for the elderly (Lawton et al., 1984). Research has focused specifically on the influence of the built environment on functional capacity; for example, abilities in $\mathrm{ADL}$ and $\mathrm{IADL}^{11}$ are affected by conditions in the physical housing environment (Iwarsson, 2005). The most common features in housing that adversely affect the elderly's independence include: stairs and level differences at doors and shower entry (Iwarsson, 2005); unsuitable heights of the work surface or storage in the kitchen (Iwarsson, 2005; Peace, 2017); a lack of assistive devices such as handrails beside slopes and in the bathroom (Burton, Reed, \& Chamberlain, 2011; Iwarsson, 2005); insufficient space for manoeuvring mobility aids (Iwarsson, 2005; Nordin et al., 2017); and hinged or heavy doors (Iwarsson, 2005; Nordin et al., 2017). The design of a bathroom also has a significant impact on the user's independence and ability to control. Key issues identified relate to residents' inconvenience and risks to their safety, with suggested improvements to the layout of bathing and showering spaces and the redesign of the shower entrance; for example, the spacious 'walk-in shower' with no curb at the entrance is suitable for wheelchair users. As for the issues relating to communal living settings, the size of the communal room and the length of corridors have been reported as affecting residents' independence (Hayward, 2012; Nordin et al., 2017). Support for cognitive frailties, such as sensory stimulation and ease of way-finding, has been identified as contributing to the positive emotion of residents (Parker et al., 2004).

However, in spite of the plethora of research in this area, there has been limited attention to the micro environments in which the high-needs elderly spend most of their time. It has been reported that in private dwellings a 'reorganisation of space', such as placing necessary items conveniently to hand (for example, on small tables on either side of the individual's chair) to ensure as little movement as possible (Hale et al., 2010) often occurs in order to afford as much

\footnotetext{
${ }^{11}$ IADL stands for Instrumental activities of daily living. It includes cleaning and maintaining the house, managing money, moving within the community, preparing meals, shopping for groceries and necessities, taking prescribed medications and using the telephone or other form of communication (Lawton \& Brody, 1970).
} 
control as possible. However, there is a lack of information on the important micro requirements for the design of living that can foster greater independence and control.

Privacy

Privacy is a key component of good QoL. If privacy is afforded, elderly people are able to safeguard their dignity, controlling and concealing their declining capacities in their daily life ( $\mathrm{R}$. Bland, 1999; Sarah Barnes \& the Design in Caring Environments Study Group, 2002). However, privacy is often at stake both in institutional settings and private homes for those receiving care (Dyck et al., 2005; Hale et al., 2010). Typical examples for loss of resident privacy in institutional settings are bathrooms shared with other residents and caregivers entering rooms without waiting for permission (Cooney et al., 2014; La Grow, Yeung, Towers, Alpass, \& Stephens, 2013). The privacy of the elderly who require care, even in their own home, is described as 'ambiguous (Nord, 2011)', which is the consequence of the caregiver's undesired presence in private spaces, of personal care being conducted in relatively public spaces, and of the lack of space where residents can be completely private (Hale et al., 2010; Klaassens \& Meijering, 2015; Nord, 2011, 2013; Young, 2005). To safeguard privacy, being able to lock doors is perceived as important (Klaassens \& Meijering, 2015). Recently, studies have increasingly focused on residents' individual private rooms (S. Hauge \& Heggen, 2008; Nord, 2011). Hauge and Heggen argue that residents should not be denied the right to pursue their individual life in their private rooms (2008).

In the design of housing and complexes, spaces are commonly laid out based on a hierarchy of privacy according to the levels of access allowed to those other than the residents. This refinement of space types has been categorised as private, semi-private, semi-public and public (The Royal Borough of Kensington and Chelsea, 2015). The concept is also known as 'progressive privacy' or 'graduation of space,' and is commonly used in the design of nursing homes and other housing and complexes for the elderly that include communal spaces that actively welcome visitors from the wider community (Altman, 1975; Sarah Barnes, 2006; The Royal Borough of Kensington and Chelsea, 2015; Tomah, Ismail, \& Abed, 2016; Torrington \& Bacon, 1997). While the provision of greater range of spaces, typically that of semi-public spaces in common areas, has a positive impact on resident QoL by affording residents more retreat options and places to socialise in smaller groups (Sarah Barnes, 2006), some literature has suggested that the rigid structure of space planned by progressive privacy does not improve the QoL of the high-needs elderly (Wojgani \& Hanson, 2007). For example, Wojgani and Hansen analysed the design of extra care housing using progressive privacy, and suggested that 
'institutional' feeling could be reduced by making the boundary between public and semi-public space less rigid (2007). In the private housing of those requiring care, Nord reported that the concept of progressive privacy is inapplicable because the caregiver's presence made residents' privacy ambiguous (2011).

\section{Personal identity}

The built environment has an influence on individuals' identity, which has been traditionally studied in psychology and supported by theories such as place-identity theory ( $\AA$. L. Hauge, 2007; Proshansky, Fabian, \& Kaminoff, 1983). For the elderly, having an environment consistent with personal history and identity is a key factor to maintaining a positive sense of self (M. M. Perkins, Ball, Whittington, \& Hollingsworth, 2012). The most visual way in which residents express their identity is through the personalisation of their space (Cooney, 2012). Creating personal space with their own belongings brought with them from their previous home, provides a connection between the person they are now and the person they had been prior to moving. Many cherished pictures of their family and friends (Nord, 2013), and space for their own furniture or plants is also important. However, commonly, the limited private spaces do not accommodate residents' belongings (Jaye et al., 2015; Murphy et al., 2007).

Personal identity may also be eroded at home through modification and layout changes to their house to accommodate difficulties due to ageing, such as remodelling of the kitchen or reorganising the downstairs area to create a bedroom or living space. Material possessions that mark social identity are often removed and individuals report that they feel 'invisible' as the person they used to be (Hale et al., 2010; Turner, 1969). Nord stressed the importance of residents' personal possessions in facilitating a dignified life in their private rooms (2013).

Having choices also facilitates personal identity (Tester et al., 2004). In New Zealand, the ageing population will become more diverse and individualistic in the future, and more housing options for the elderly that facilitate individuality are desired (Davey, 2006a). Koopman-Boyden and Moosa emphasised the heterogeneity of current cohorts and identified their different expectations of life. They found that meaningful life for elderly people is attained through the continuity of their chosen lifestyles, while providing care opportunities (2014). However, there has been criticism for the lack of individuality in the design of existing senior housing such as retirement villages (Glasgow, 2013; Peace, 2017). 


\section{Activities}

Activities contribute to elderly people's greater happiness, as well as providing stimulation and helping with the prevention of falls or physical and mental decline (Dias, Couceiro, Mendes, \& de Lurdes Almeida, 2017; Nordin et al., 2017; Sok Teng \& Thirumalaya, 2017; Tester et al., 2004). However, it has been said that 'elderly people fill their time with less action than younger people do' (Havighurst, 1961) and space used for activity becomes more restricted in old age, with activities more likely occurring within their private dwelling (Eakman, 2012; Gordon \& Gaitz, 1976). To facilitate greater participation in active activities, residential care facilities and various types of housing for the elderly provide activities for residents in communal space. However, some studies reported that the provision of activities in communal space was not successful; for example, residents felt some activities offered in residential care were boring and frustrating (Tester et al., 2004). Sikorska also revealed residents in assisted living facilities that offered more organised group activities tended to be less satisfied (1999). Recent studies have suggested greater need for improvements in the manner of providing activities and spaces for residents (Koehn, Mahmood, \& Stott-Eveneshen, 2016; Van Steenwinkel, Dierckx de Casterlé, \& Heylighen, 2017).

Recently, the importance of engaging in their meaningful activities has been receiving attention (Cooney et al., 2014; Eakman, Carlson, \& Clark, 2010; Kiata-Holland, 2010; KoopmanBoyden \& Moosa, 2014; Murphy et al., 2007; V. Wright-St Clair, 2011), which associates with great psychological well-being and health-related QoL (Eakman et al., 2010). Research has stressed the importance for activities and spaces to be designed to suit residents' particular interests and that residents are given choices for their participation (Koehn, Mahmood, \& StottEveneshen, 2016; Murphy et al., 2007; Van Steenwinkel, Dierckx de Casterlé, \& Heylighen, 2017). For example, National Care Standards in Britain recommend that communal spaces include rooms where various social, cultural, and religious activities can take place (the Department of Health, 2006). Barnes also reported that, in care homes with a greater variety of spaces, residents have higher levels of active behaviour (Sarah Barnes, 2006).

The private housing environment also has an impact on individual's meaningful activities. For example, providing an oven in the kitchen allows residents to make a snack, something very important to them; however, a full kitchen containing an oven was regarded as 'a waste of money and ... too dangerous for residents to use' by some providers and developers (Gusmano, 2004, p. 1230). The design of windows also mattered; large windows facilitated resident engagement in 
outdoor activities (Nordin et al., 2017). These are just two examples among many that illustrate the role of private housing in facilitating their meaningful activities.

In New Zealand, the focus has been on the diverse and individual preferences of the current and future cohorts (Glasgow, 2013; Peggy Koopman-Boyden, 2014). One factor for this diversity is cultural differences by ethnicity; a study reported differing valued activities for older Māori and older non-Māori. Māori frequently named extended-family related activities, such as supporting family and family gatherings, as being most important; whereas non-Māori named more recreation and leisure activities (V. A. Wright-St Clair et al., 2012). The desire for individuality also relates to the characteristics of baby-boomers. Kiata-Holland referred to this cohort as likely to embrace the concept of leisure (2010). Preferred leisure activities in New Zealand included those related to arts and culture (identified as most important) and reading newspapers, books or magazines (V. A. Wright-St Clair et al., 2012). Outdoor activities are another area that can enhance the wellbeing of New Zealand's elderly people (Kershaw, McIntosh, Marques, \& Cornwall, 2017; V. A. Wright-St Clair et al., 2012). One precedent demonstrating an architectural solution to facilitating individuality is De Hogeweyk in Netherlands, which is a dementia-care village consisting of seven clusters with physical environments facilitating different lifestyles (Anderzhon, Hughes, Judd, Kiyota, \& Wijnties, 2012). However, architectural means to facilitate particular genres of activities of the high-needs elderly have not been well developed in New Zealand.

\section{Relationships}

Relationships are a significant contributor for QoL of high-needs elderly (Yeun, Good, O'Donoghue, Spence, \& Ros, 2017). However, as mobility decreases, people typically spend more time in their personal space and the opportunity for social activities is likely to be restricted (Michal Boyd et al., 2009), leading to a loss of social connection (Kiwaki \& Arai, 2015; Toepoel, 2013). Loneliness and isolation are a growing social concern in the urban ageing population and are damaging to health and wellbeing, both mentally and physically (Hand et al., 2017; HoltLunstad, 2018). Loneliness has been a significant issue in New Zealand too (Jones, 2018; Sivignon, 2018). Physical environments have great potential to improve such issues relating to relationships of the high-needs elderly, through the provision of spaces for socialization (Tester et al., 2004).

In institutional settings, social interactions are commonly encouraged through communal activities provided for residents. However, there are differences in the preferences for socialising 
among elderly people. For example, Tester et al. reported that some prefer peace and quiet and valued the opportunity to keep themselves apart from the crowd, while others like to be among other people whether or not they are actually socialising (2004). Another study also reported that providing residents with multiple options whether they joined activities with other residents or retreated in their private rooms contributed to their greater sense of freedom (Van Steenwinkel et al., 2017). Attitudes towards other residents included hostility and indifference as well as sympathy and friendship (S. Hauge \& Heggen, 2008; Nord, 2013; Tester et al., 2004). Many residents have difficulty feeling a sense of 'belonging' or being a part of the group (Cooney, 2012; Van Steenwinkel et al., 2017). In retirement villages in New Zealand, residents, who are mostly not high-needs, generally place considerable importance on social networks, and most are satisfied with participating in communities of their own choice (Yeung, Good, O'Donoghue, Spence, \& Ros, 2017). In contrast, residents in supported living units in retirement villages, many of whom are high-needs, had difficulty in having a sense of belonging, because of the limited social space that they felt welcomed them (Hayward, 2012).

Personal relationships, such as visits from family and friends, are particularly valued by elderly residents of nursing homes and assisted living facilities (Cooney et al., 2014; Kontos, 1998; Tompkins et al., 2012). However, institutional settings often provided poor physical environments for entertaining guests; for example, residents experienced a lack of privacy for entertaining guests in their own room and had to entertain personal visitors in public spaces. Also in many places children were unwelcome (Murphy et al., 2007). The size of the dwelling matters; large private apartments have been found to better facilitate social activities than smaller ones (Nordin et al., 2017). In New Zealand, a loss of meaningful relationships was perceived by residents in supported-living units due to the reduced space for personal social activities (Hayward, 2012).

Architectural factors also play an important role in either encouraging or discouraging a connection to the wider community (Murphy et al., 2007; Tester et al., 2004). For example, a homely atmosphere in nursing homes was one of the main factors encouraging a sense of connection (Murphy et al., 2007). The size of the complexes also has influence on connection to the wider community (Orrell et al., 2013; Torrington, Barnes, McKee, Morgan, \& Tregenza, 2004). For example, one study on extra care housing reported that the village-type performed relatively poorly in facilitating a sense of connection with the wider community when compared to smaller living arrangements (Orrell et al., 2013). 


\section{Quality of care}

Assistance by caregivers is important for supporting the daily and recreational activities of the elderly. However, requiring assistance in ADLs affects residents' self-esteem and sense of control, particularly when they require personal care many times a day or need help with tasks normally considered private such as showering and bathing (Boström et al., 2014; Hale et al., 2010). However, this decline in QoL can be mitigated by the individualised manners and approach of caregivers (Nord, 2011; Robertson \& Fitzgerald, 2010); however, such models are often difficult due to a demanding workload (M. Bland, 2007; Dyck et al., 2005; Murphy et al., 2007; Tester et al., 2004). To facilitate individualised care, small-scale care settings have been suggested as preferable (Sarah Barnes \& the Design in Caring Environments Study Group, 2002; Van Steenwinkel et al., 2017); for example, in one study, caregivers reported that limited number of residents and compactness ( 8 resident units per care unit) contributed to greater ease of care for elderly individuals, increasing flexibility and control and eliminating strict rules and time schedules (Van Steenwinkel et al., 2017). While the problems described above can be fixed by the care models, the design of space has a great relevance to facilitating the ease of care, thereby supporting the individualised care.

While much literature focuses on the design of shower spaces for independent use by the elderly, there are few studies focusing on the design requirements for assisted bathing and showering. A recent Canadian study which ran focus groups to gather information about caregiver views on providing care for the elderly in the bathroom (E. C. King et al.) found that, in addition to other environmental factors, there was insufficient showering/bathing space to accommodate caregivers and care activities. Another Canadian study conducted semi-structured interviews with six residents and two caregivers to discuss their opinions on bathroom design (Morales et al., 2012). This study also found insufficient space and inaccessible features, as well as a diminished sense of comfort and privacy for elderly residents. While these studies support the need for improvements in bathing and showering spaces, there is limited information with respect to the design of space best suited for assisted showering, particularly regarding psychological comfort.

\subsubsection{Conclusion}

This section has explored the QoL of the high-needs elderly in relation to physical environments through two parts; first the overall trend in the expectation of the elderly in New Zealand was reviewed, second a review of extensive literature focusing on the high-needs was undertaken. Among current elderly people and baby boomers who are and will be joining the ageing population, there was a strong desire to live independently from family to avoid being a burden 
on them, other than in institutional settings. As for housing options, a house smaller than their family home in the community was the most common desire, and there was an expectation for a wider variety of options including various communal types of housing, where they could maintain preferred social connection while having an individual life as well as reduce the housing cost. In the second part, a literature review focusing on the high-needs elderly clarified their QoL connections to more detailed physical environments, categorising the QoL in the six emergent themes of; independence and control, privacy, personal identity, activities, relationships and quality of care. The matters relating to the relationships between their QoL and physical environments, such as problems with their QoL that could be addressed by the physical environments and potential influences of the physical environments on their QoL, are described by each theme. In the next section, existing frameworks for the design of housing for the elderly are reviewed to examine how they address the QoL of the high-needs elderly in the light of the outcomes of this section.

\subsection{Existing design frameworks for housing for the elderly that address their QoL}

This section reviews existing design frameworks for housing for the elderly in New Zealand and overseas. As there are no frameworks that specially focus on the high-needs, those dealing with the general elderly population are included for review. Therefore, this review aims to examine how the existing frameworks address the QoL of the high-needs elderly, and their applicability for the design of housing for the elderly. Two frameworks overseas, EVOLVE (Lewis et al., 2010) and Guidelines for the planning of housing for senior citizens (WEL_HOPS, 2007) were included for review. These frameworks were chosen, because they include numerous detailed design considerations that are linked to a range of the QoL of the elderly ${ }^{12}$. There are no frameworks that include design considerations for a range of aspects of QoL of the elderly in New Zealand, so those addressing some of the aspects of QoL were included, namely, New Zealand Standard 4121: Design for Access and Mobility — Buildings and Associated Facilities (Standards New Zealand, 2001) and Lifemark Design Standards (Lifetime Design Limited, 2012). Some relevant requirements are also outlined in a fragmentary way in Acceptable Solutions and Verification Methods For New Zealand Building Code Clause, particularly in D1 Access Routes (MBIE, 2016) and G1 Personal hygiene (Department of Building and Housing,

\footnotetext{
${ }^{12}$ For example, the ten design principles that the Housing our Ageing Population Panel for Innovation (HAPPI) (Innovation, 2009) were excluded from review, as they do not include sufficient details in the design considerations.
} 
2011a), but these are more comprehensively covered in NZS 4121; therefore, D1 and G1 were excluded from review. First, a brief overview of these frameworks is provided, which is followed by the in-depth review of the design considerations that they address including discussion in the light of the QoL issues of the high-needs elderly described in Section 3.3. The review concludes with discussion on the features of the existing frameworks, and the identification of gaps of knowledge in the existing frameworks.

\subsubsection{Overview of existing design frameworks}

This section provides an overview of the four significant design frameworks both overseas and in New Zealand as mentioned above, which will be closely reviewed in the next section. It includes consideration of the methods used in the development of the frameworks, their format and a summary of how they relate to a wide range of QoL of the elderly.

\section{EVOLVE}

EVOLVE (Evaluation of Older People's Living Environments) is a tool for evaluating the design of housing for the elderly, which was developed through a review of existing literature/standards, the involvement of focus groups with residents from four extra-care schemes in England and interviews with architects, as well as testing through surveys of 23 other extra-care housing schemes. It consists of a set of checklists for five areas of the housing scheme: living unit, communal facilities, circulation spaces, staff facilities and scheme site and location. It assesses how well a building contributes to physical support and personal well-being, which are shown by scores for 13 domains of user requirements ${ }^{13}$. Results are displayed in tables and in histograms, to show the strengths and weakness of the scheme or dwelling.

\section{Guidelines for the planning of housing for senior citizens (GPHSC)}

These guidelines have been developed in the course of a project involving five institutes in different countries in the European Union (EU) (Italy, Sweden, Spain, Hungary, the UK), and are aimed to be adaptable to the diverse contexts of the countries in the EU. They are recommendations for the planning and construction of new residences, the restructuring of existing ones and are also used for important urban transformations, that can sustain the elderly in their own homes for as long as possible. These were created by an interdisciplinary work group made up of sociologists, economists, architects, engineers and those responsible for study and

\footnotetext{
${ }^{13}$ The 13 domains consists of six domains in the 'Universal needs' category (Personal realisation and choice, Dignity and privacy, Comfort and control, Personal care, Social support inside building, Social contact outside) and seven domains in the 'Support for older age' category (Accessibility, Physical support, Sensory support, Dementia support, Health and safety, Security and Working care).
} 
research in welfare, and have been verified through numerous pilot experiences realised by the Public administration of the five project countries, planners as well as the elderly and their families, caregivers and the managers of residential structures.

The guidelines provide detailed architectonic and constructional requirements with the use of photos and explanatory images, and consist of four sections; A. the home, B. the building outside the home, C. spaces outside the building, and D. the urban context. Each section has $5-24$ themes which also consist of several (or more) detailed considerations that improves elderly people's QoL ${ }^{14}$.

\section{New Zealand Standard: Design for Access and Mobility - Buildings and Associated Facilities (NZS 4121)}

NZS 4121 provides information on the design requirements for built environments that are accessible to and usable by people with disabilities. The Building Act 1991 references this standard as a compliance document for the requirements in the Building Code ${ }^{15}$. This standard applies to all new/existing buildings, other than private residential buildings, when they are altered, or there is a change in use. It is applicable to some types of housing for the elderly such as pensioner flats which are classified in the 'accommodation for the public.'

\section{Lifemark Design Standards (Lifemark)}

Lifemark sets out voluntary design standards that meet the needs of people of different ages and abilities over time - which is the concept of Universal Design. They are standards for typical New Zealand residential housing 'based on similar underlying design principles to those reflected in NZS 4121 but with principles of safety and adaptability along with a healthy dose of common sense added to the mix' (Lifetime Design Limited, 2012, p. 3). They provide the 5-Star rating system, which is defined by the sum of the points assigned to each design requirement in seven

\footnotetext{
${ }^{14}$ For example, Section A includes nine themes for indispensable needs (Easy identification of the entrance to the home, Easy access to the home, Guaranteeing a stimulating external view, Creating the best conditions for rest and sleep, Taking care of one's own body, Preparing meals, Eating meals, Receiving treatment and care and Guaranteeing safety in surroundings) and 15 themes for complimentary needs (Acquiring familiarity with one's own home, Making one's own home comfortable, Increasing visibility between some rooms in the home, Receiving people/socializing, Looking after pets, Looking after plants and flowers, Doing recreational activities, Cleaning areas in the home, Devoting oneself to house hold chores, Guaranteeing personal safety, Guaranteeing safety in the home, Moving around easily in the home, Guaranteeing comfort in the home, Guaranteeing visual well being, Opening windows and blinds easily and Communicating with the outside)

15 The Building Act 2004 and NZBC require all buildings to which the public are admitted to have reasonable and adequate facilities for disabled people to visit, work, and carry out normal activities (Department of Building and Housing, 2011b). NZS 4121 is the code of practice for design regarding the access and use of buildings by persons with disabilities (Standards New Zealand, 2001).
} 
areas: Accessing the Dwelling, Getting Around, Fittings and Fixtures, Bedrooms, Dwelling Facilities, Bathrooms and Multi-Storey Access.

\subsubsection{Review of design frameworks by themes for the QoL of the high-needs elderly}

This section reviews the design considerations addressed by the four design frameworks for housing for the elderly in the light of the QoL for the high-needs elderly identified in Section 3.2 and examines how these frameworks address the QoL of the high-needs elderly.

Of these design frameworks, NZS 4121 and GPHSC deal with design requirements in wider areas than those relevant to residential settings, so the considerations other than residential settings are excluded from the review (e.g. considerations about moving walkways, public counters, places of assembly and entertainment, accessible outdoor public area, the urban context).

Design considerations included in the four frameworks are listed with respect to the QoL themes in Table 3-1. The summary of the considerations included, the features of frameworks in NZ and overseas, and how the QoL of the high-needs elderly is addressed are outlined by each them of QoL following the table. 


\section{Accessibility, safety}

Avoidance of level differences at entrances/doors. Elimination of thresholds where possible.

Requirements for ramps if there are differences (e.g. gradient, rails, landings).

Detailed requirements for stairs if unavoidable (e.g. stair types, number of risers in a flight, the rise height, width, handrail requirements, balustrade requirements, lighting, tactile warnings, nosing, a stair lift

and/or the reinforcement of the wall).
Detailed consideration for lifts (number, size, type (general/service) provision of seats, handrails, space out of the lift, door width, door opening time, lighting, emergency communication system with visual indication/ an induction coupler for hearing aids, call button/control requirements (tactile reading system, height).

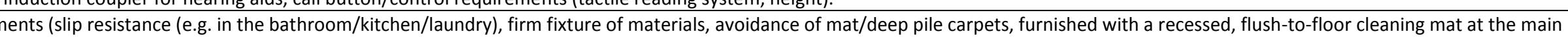

entrance).
Detailed requirements for handrails (location, height, firmness, diameter, the shape of the ends to avoid catching on clothing/handbags, being smooth to touch).

Doors/Windows that reque

Handle types that are easy to (eperate (e. D-shape, L-shape, lever handloor handle)

(n)

Electronic access (e.g. swipe-cards, key pads, door activating buttons) - detailed requirements for the size of the level area, the location/height of electronic access units

Windows that do not obstruct paths or walkways outside

Closers (e.g. building external doors swing closing by themselves, closers incorporating a delay and final damping)

A doorstop mechanism for a building entrance door

Front doors having a letter box and the requirements (e.g. the size, height)

Front door having a door bell

for wheelchairs

Door width (more than $760 / 800 / 810 \mathrm{~mm}$ ).

Width of coridor/pede.g. inside the entrance door/hallway, lounge entrance, kitchen, bathroom, around the bed.

Width of corridors/pedestrian access routes (e.g. more than $1100 / 1200 / 1800 \mathrm{~mm}$ or more, splayed/radius corners at corridor junctions, recess of the doors that open onto corridors).

Hand basin requirements (avoidance of the pedestal type, the height).

Distance between the opening edge of a hinged door and any adjacent wall (e.g. more than $300 \mathrm{~mm}$.

Spars

room, bathroom

Detailed requirements for car parking (e.g. location, number per home, size, provision of parking for disabled people/ staff/ visitors, parking for emergency vehicles in front of the building's main entrance, clearly visible signage, proximity to the unit/building (e.g. up to $100 \mathrm{~m}$ ))

Drop off bay (Long enough to allow 'tail loading' at the main entrance)

Routes from the car park to the building (e.g. weather protected, width, gradient)

Requirements for facilities accessible from a car (e.g. post boxes, ticket dispensers and other kerb-side machines)

\begin{tabular}{|l|l|l|l|}
\hline$x$ & $x$ & $x$ & $x$ \\
\hline$x$ & $x$ & $x$ & $x$ \\
\hline$x$ & $x$ & $x$ & $x$ \\
\hline$x$ & $x$ & $x$ & \\
\hline$x$ & $x$ & $x$ & $x$ \\
& & & \\
\hline$x$ & & $x$ & $x$ \\
\hline$x$ & $x$ & $x$ & \\
\hline$x$ & $x$ & & \\
\hline$x$ & $x$ & & \\
\hline$x$ & $x$ & & \\
\hline
\end{tabular}

Mobility scooter/electric wheelchair users

(e.g. more than 1200mm), a

widened corridor width adjacent to the electric scooter parks (e.g. more than $1800 \mathrm{~mm}$ ).

Motorcycle and bicycle parking (e.g. distance from the building)

Visibility/recognisability

Visible and recognisable position of entrance (unit, building). Avoidance of a monotonous succession of doors.

Colour contrast (floors/doors/handrals/swiches/(controls against walls, inside and outside of the unit, door handles against doors, garden paths against grass/ground cover).

Detaled requirements for artificill liektines requirements in every spa

(e.g. over kitchen counter, at

Natural lighting (e.g. public areas being more brightly lit with natural light than private areas.)

Finishes/materials to avoid dazzling (e.g. non-reflective satin/mat finish, concealing the light source from view, avoidance of large glass openings at the end of corridors)

Use of a glass with distinct markings (e.g. on main entrance doors)

Detailed requirements for signage (location, height, colour tone/contrast, lettering, use of written/tactile instructions, contents (e.g. the travel route from the main entrance to the reception point,

Ceiling height differences (e.g. public areas are indicated by their larger scale and higher ceilings)

Layout (e.g. visibility /from the site entrance to the main entrance, from the main entrance to the communal lounge/dining room, from the main entrance to the reception, from the reception to the

lift/staircase)

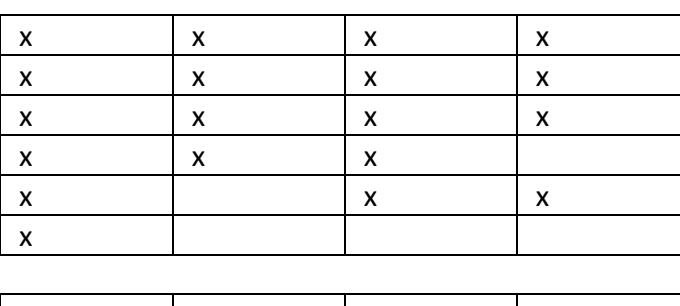




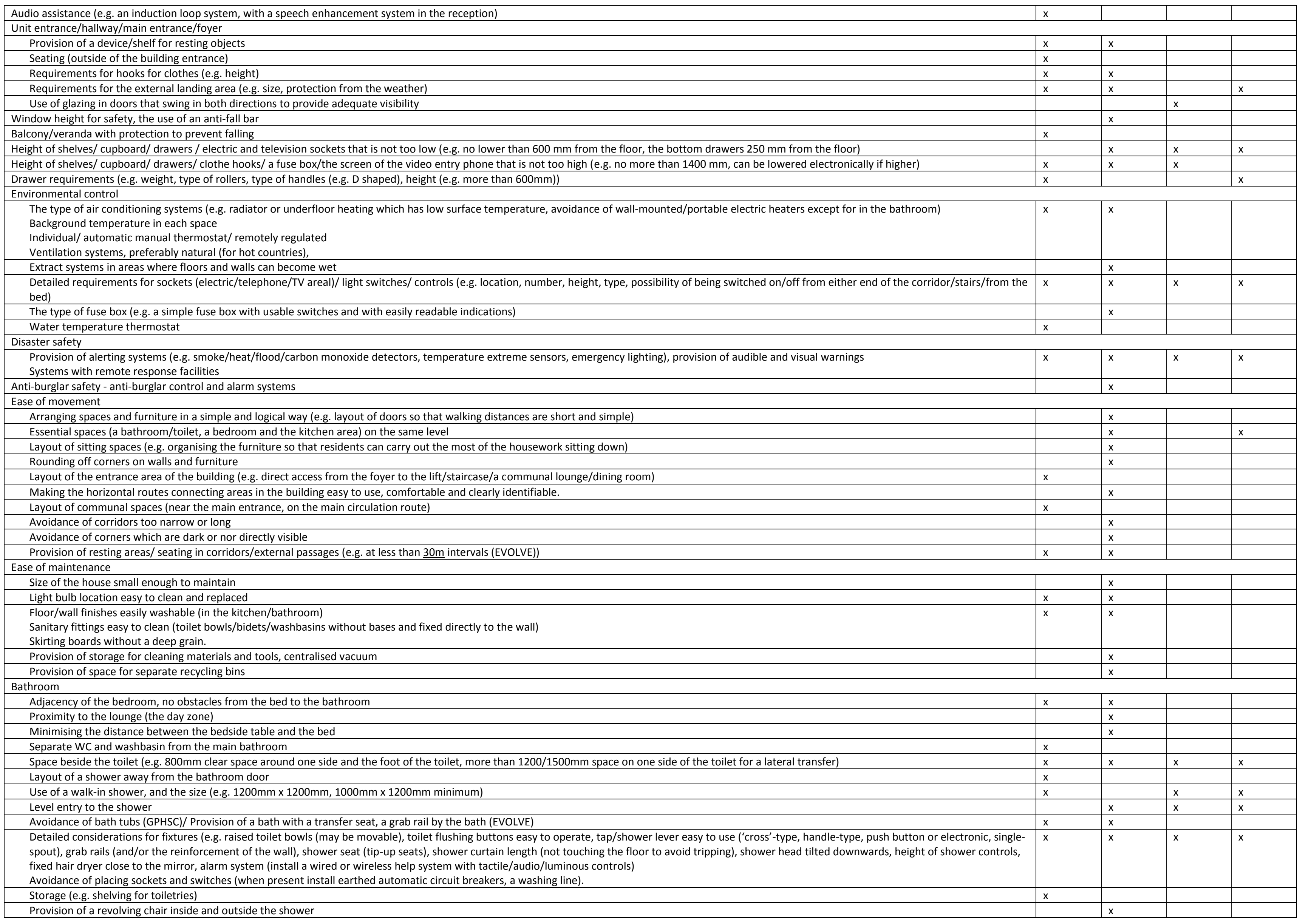


Usable taps

benches, major appliances and fittings (e.g. $1200 \mathrm{~mm}$, extending to a $1500 \mathrm{~mm}$ turning circle measured up to at least $250 \mathrm{~mm}$ above the floor)

Detalled requirements for kitchen facilities (e.g. space for a fridge with a freezer compartment, a sink, a cook

Washing machine, tumble dryer, dishwasher, a microwave oven, sink, an oven, range hood, draining board)

Requirements for working surfaces/sink/oven, (e.g. height, height-adjustable mechanism electronically controllable, rounded corners, heat-resistant)

Layout of appliances at least $300 \mathrm{~mm}$ from the inemal comers of bench units

(n) consisting of drawers, enclosed storage for food/utensils/cleaning equipment, storage for crockery, space for a waste bin, waste recycling containers)
Shelves (height, ope

Layout of spaces to reduce lateral movement (e.g. making kitchens with work areas in one incorporated block, possibility to slide goods along the surfaces)

Unit doors (e. . kitchen units fitted with sliding doors, doors that open through 180 degrees)

lighting under wall-mounted cabinet, over the table/worktop where food preparation occurs, over the hob/oven/stove area, sink

Illuminance requirements

Controls (e.g. height, illuminated indicators to oven/electro-domestic equipment when they are on, tactile markings)

Bedroom

Space around the bed (e.g. $800 / 900 \mathrm{~mm}$ clear space around one side and the foot of the bed, more than 1200/1500mm space on one side of the bed for a lateral transfer)

Access to facilities from the bed (e.g. light switches, curtain pull cords, telephone, television/radio controls)

Laundry - detailed space requirements for equipment (e.g. a washing machine easy to operate) and tasks (e.g. pilling dirty clothes, ace hanging and ironing clothes, drying clothes, the size of the clear space in

front of appliances, accommodating appliances at ground level)

Letter boxes requirements (e.g. Location (e.g. on the main circulation route), height, size, lock/opening system)

(Recyclable) refuse store requirements (e.g. accessibility)

Communal laundry requirements (washing machines, dryers and/or adequate space for hanging washed clothes, provide trolleys, ironing area))

Shop requirements

Communal WC requirements (e.g. proximity to main communal spaces)

Communal WC requir

Pedestrian routes clearly distinguishable from traffic routes, pedestrian crossings (width, the curb being dropped, tactile indicator paving), pedestrian access

Site entrance requirements (e.g. separate entrances for pedestrian/vehicle/delivery vehicles

Road design that restrict vehicle speeds.

Privacy

Provision of an independent bedroom (avoidance of bed-sits)

Sound insulation (bedroom)

Door design for greater privacy and security

Provision of partial opening, eye hole (and the height), avoidance of glazed front doors, burglar proof front doors

Layout of the door visible from the day zone

Insertion of glass in internal doors/walls for better visibility between the various areas of the day zone.

Provision of a system for observing who is calling at the doo

Privacy from neighbours - distance between neighbouring windows (more than 22m)

Privacy and security from the public/communal areas

Provision of clear delineation of public, community and private space
Provision of a security barrier between the public area and the living units/ lifts

Provision of a security barrier between the public area and the living units/ lifts

Privacy with reard to communal bathroms (e. the use of privacy sereens, access sing units without crossing communal lounges/dining areas

Privacy with regard to communal bathrooms (e.g. the use of privacy screens, access via private rather than communal thoroughfares)
Privacy from the outside of the complex - trees planted along the boundary

Privery friche

Accommodating residents' own furniture (

dressing table and a TV in the bedroom/

Design of space that provides multiple options for furniture layout

Avoidance of furniture with institutional appearance furniture (e.g. bed

Design process to incorporate residents' taste (e.g. provision of choices for the layout, finishing materials and decor)

Personalisation of space

Living unit entrance (e.g. set-back from the main travel route, provision of shelving, space for plants or flowers, avoidance of double-banked corridors (hotel-like))

Letter boxes (e.g. the names of each resident, not numbers).

Storage

General storage inside the hallway

Storage for personal objects not being used in Individual space and/or communal space (e.g. suitcases, tools, furniture, unseasonable clothin

Room shape for residents to furnish easily (as square as possible/rectangular, avoidance of rooms very narrow or curved)
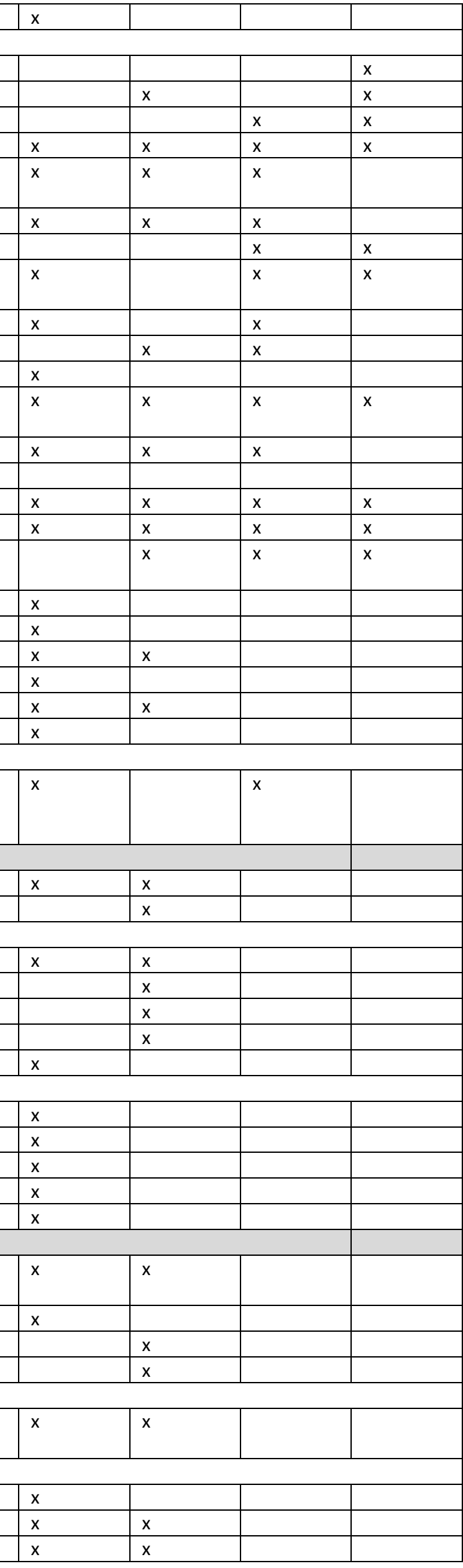


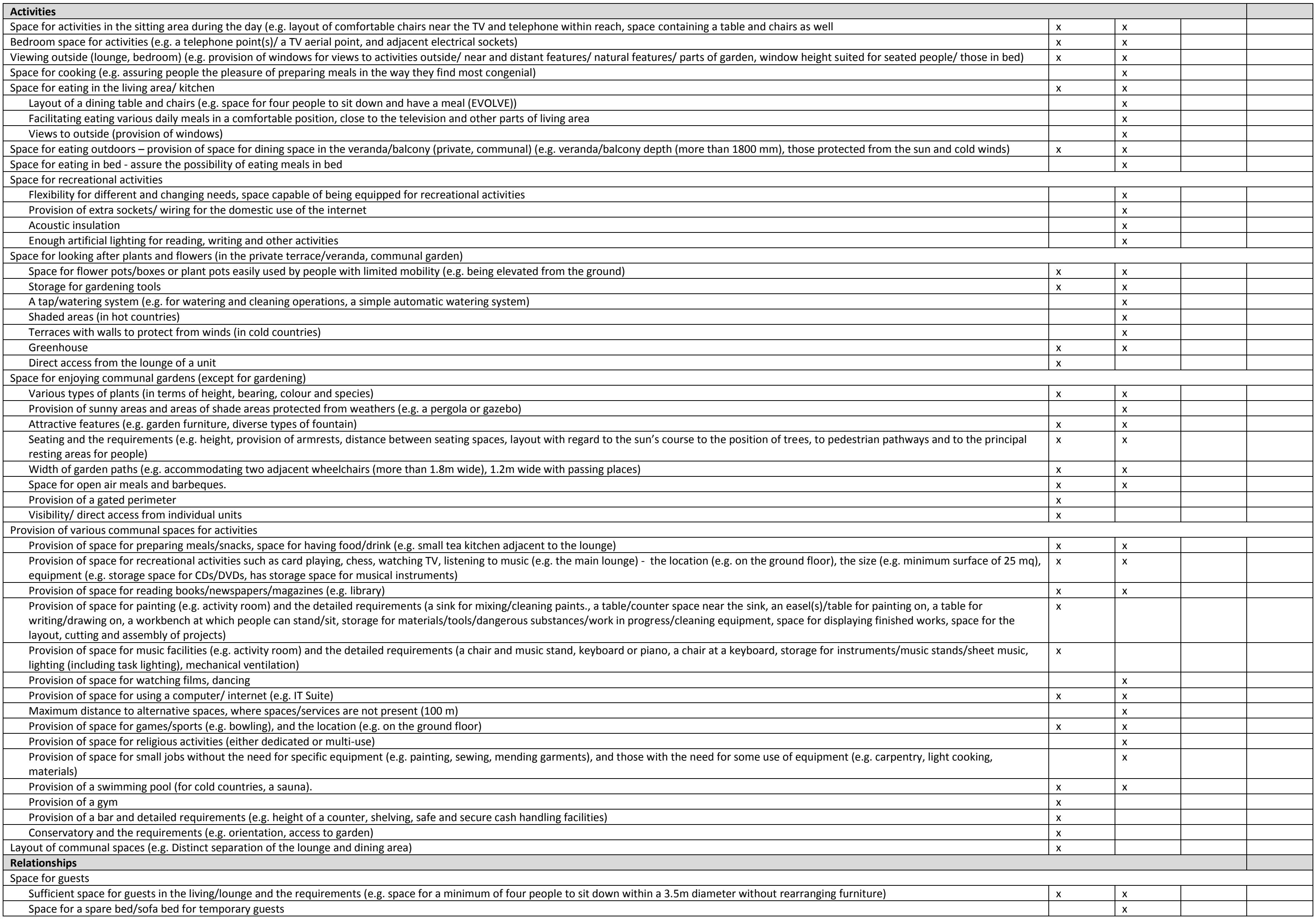


Space in the kitchen to involve guests in the preparation of meals

Space for sitting with guests in the balcony (e.g. space for a table and at least two chairs)

Welcoming guests

Layout of a sitting position allowing a person to see any callers from their sitting position

Entry phone system/CCTV system, provision of a video-entry phone on the bedside table

Opening system for the entrance door and vehicle/pedestrian gates which can be remotely operated from inside the home

Provision of space for pets (e.g. parrots, dogs, cats, fish and turtles) - provision of a small corner for feeding animals, small storage for food/equipment, a separate rubbish collection system for animal faces, a small space for cleaning the animals, space for looking after and walking pets in the communal garden, a dog ru

Provision of communal space for socialisation with other residents, the location (e.g. on each floor)

Security against the outside - scheme boundary (e.g. secure side/rear fencing, lockable full-height fencing side gates)

Space for an assistant in the kitchen

Space for an extra bed for a caregiver, and the layout of the bed so that the caregiver is aware of the resident moving around at night

Sufficient enclosure size

Provision of a low level shower enclosure to prevent the carer from getting wet

Sufficient space for a caregiver around a washbasin (e.g. $600 \mathrm{~mm}$ on the sides of a washbas in)

Sufficient space for a caregiver around the toilet (e.g. $600 \mathrm{~mm}$ on either side of the toilet)

Shared assisted bathroom

Location (e.g. on every floor), sufficient space around the bath (e.g. more than 700mm clear space either side of the bath for carers), detailed requirements for facilities (e.g. changing facility) and fittings (e.g. a shower fitting fixed to the bath, sufficient space for a hoist, a safety temperature thermostat, a privacy screen) Bathroom door type and opening direction which makes it easier for them to receive aid (e.g. opening outwards or sliding door). beacons)

Provision of a clinic/therapy room

etailed requirements for scheme/care manager's office (e.g. adjacency/views to the main entrance/the main route, acoustic privacy, space for specific furniture and equipment, tea making facilities, features

for greater accessibility and recognition to accommodate the elderly, lighting/thermal environments, CCTV monitoring system, communication system)

for the staff room (e.g. being located central to staff occupied areas/away from resident areas/with direct access to the main circulation route(s), access to the outside, staff

toilets/shower, changing area, staff kitchen and dining area, individual storage, accessibility, signage, lighting/thermal environments, an emergency call system, telephone)

Rect

Avoid institutional feeling - physically separating the kitchen/kitchenette from other area, provision of fixtures/furniture with non-clinical appearance (e.g. beds, grab rails)

Natural lighting, ventilation. (e.g. receiving sunlight in the morning and afternoon in every space, provision of living unit that is double aspect, provision of shading devices on

Environmental considerations (e.g. the avoidance of construction materials with high emission of VOC/formaldehyde or other chemical compositions dangerous to health, the use of construction materials which

have a good LCA, materials which have been ecologically certified and are easily recyclable

Wall colours (e.g. the use of light colours, colours linked to local tradition/the earth, avoidance of clinical colours)

Warning system for flooding, linked to the caretaker
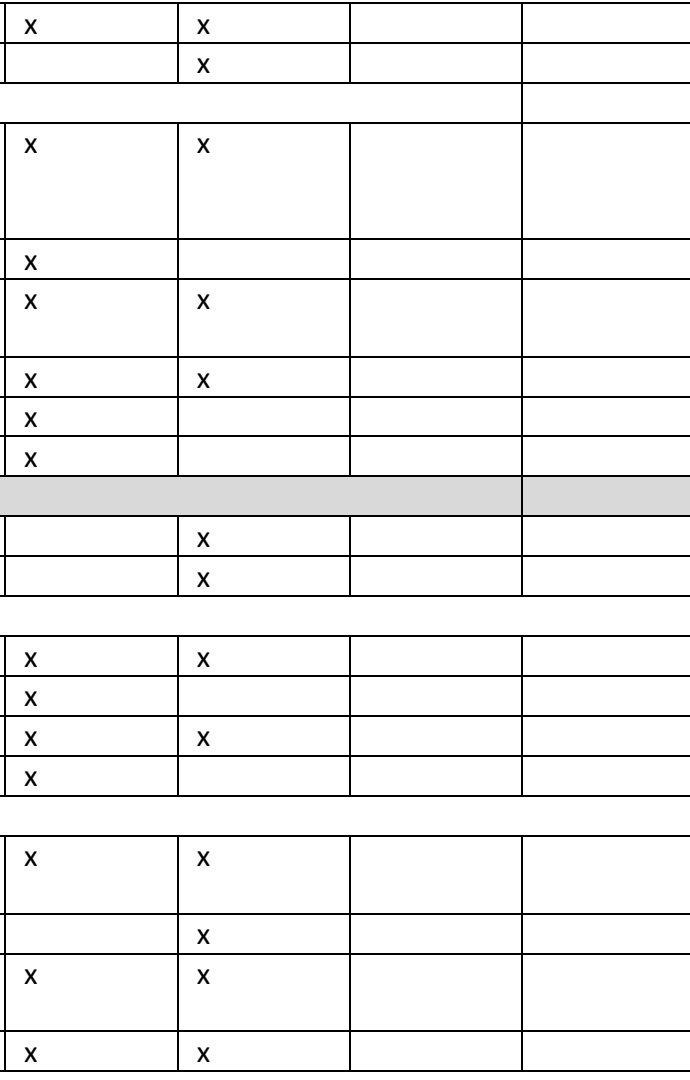

\begin{tabular}{l|l|l|l|l|}
\hline \multicolumn{1}{|l|}{$x$} & & & \\
\hline$x$ & & & \\
\hline & $x$ & & & \\
\hline & $x$ & $x$ & & \\
\hline$x$ & $x$ & & \\
\hline$x$ & $x$ & & \\
\hline & $x$ & & \\
\hline$x$ & $x$ & & \\
\hline$x$ & $x$ & & \\
\hline & $x$ & & \\
\hline
\end{tabular}




\section{Independence and control}

The considerations in this theme relate to accessibility (including that for mobility aids/special equipment users), visibility/recognisability, environmental control, ease of movement/maintenance and disaster/pedestrian safety, and encompass a wide range of design elements such as doors, windows, passages, the floor, the kitchen/bathroom/bedroom, lighting, finishes, fixtures such as door handles and taps, and safety systems. Many include detailed requirements such as dimensions of spaces and illumination levels.

All the frameworks reviewed include many considerations in this theme, and address detailed requirements for accessibility (with regard to floors, ramps, stairs, doors, fixtures such as handrails and taps, those for wheelchair users and car users), the height of shelves/cupboard/drawers etc, lighting, sockets/light switches/controls, alerting systems, and space and facilities in the bathroom/kitchen/bedroom/laundry. There are a few areas addressed only by NZ frameworks such as requirements for electronic access units (e.g. swipe-cards, key pads, door activating buttons), windows that do not obstruct paths or walkways outside, facilities accessible from a car (e.g. post boxes, ticket dispensers and other kerb-side machines), glazing in doors that swing in both directions, and the layout of a kitchen that is not a main thoroughfare in the home. In contrast, there are a number of requirements that are addressed by frameworks overseas but not by NZ frameworks such as requirements for building entrance doors/unit front doors (e.g. doorbells, letter boxes), those for parking for mobility scooters, motorcycles and bicycles, those for greater visibility/recognisability (e.g, the layout of front doors, finishes/materials to avoid dazzling, ceiling height and landmarks), environmental control systems, many requirements for greater ease of movement and maintenance, and those specific to communal facilities in housing complexes for the elderly (e.g. communal laundries, shops and hair salons).

Section 3.2 clarified that the independence of the high-needs elderly is affected by stairs and level differences at doors and shower entry, the wrong design of bathrooms (e.g. a lack of fixtures such as handrails), and kitchens (e.g. unsuitable heights of the work surface or storage). These problems are well addressed by the frameworks reviewed in this section. However, there has been less focus on the usual space of the high-needs elderly, which is important to them given their limited mobility. For example, it has been reported that they typically need to place necessary items conveniently to hand (for example, on small tables on either side of the individual's chair) from their chair to ensure as little movement as possible (Hale et al., 2010). 
However, design frameworks reviewed include limited spatial requirements with this respect (e.g. referring only to the layout of a telephone near the chair).

\section{Privacy}

Design considerations addressing the privacy of the high-needs elderly are included only in the frameworks overseas. Considerations include; the provision of an independent bedroom (avoidance of bed-sits), sound insulation, the door design for greater privacy and security (e.g. the provision of partial opening/an eye hole, the avoidance of glazed front doors, the layout of the door visible from the day zone, a system for observing who is calling at the door), privacy from neighbours (e.g. the minimum distance between neighbouring windows), privacy with regard to communal assisted bathrooms (e.g. the provision of privacy screens, access via private rather than communal thoroughfares), privacy and security from the public/communal areas (e.g. the provision of clear delineation of public, community and private space, the provision of a security barrier), and the privacy from the outside of the complex (e.g. the boundary design).

Common problems with the privacy of the high-needs elderly include bathrooms shared with other residents. For this issue, EVOLVE includes solutions in the design of communal assisted bathrooms as mentioned above. For the common issues of staff entering rooms without waiting for resident permission, the frameworks do not show effective solutions, though they include the requirements of the door design and spatial layout that enables residents to see who is calling, which may have some effect on improving resident privacy. EVOLVE includes the requirement for clear delineation of public, community and private space, applying the concept of 'progressive privacy' or 'graduation of space' (private, semi-private, semi-public and public), that is commonly used to provide adequate privacy for residents in housing and complexes that include communal spaces. The privacy of the elderly who require care at home is sometimes problematic, because of the undesired presence of a caregiver in private spaces (Nord, 2011). For this problem, there are no solutions identified by the frameworks reviewed.

\section{Personal identity}

Design considerations addressing this aspect of QoL of the elderly are included only in the frameworks overseas. The considerations with respect to this theme include; accommodating residents' own furniture, the design of space that provides multiple options for furniture layout, the design process to incorporate residents' taste, the design providing opportunities for personalisation (e.g. in the entrance, letter boxes), storage for personal items (including those not being used), and the room shape for residents to furnish easily. 
To provide greater sense of personal identity for the high-needs elderly, the personalisation of space has been suggested as useful, which is included in the requirements in the frameworks reviewed. For example, the personalisation of the area immediately adjoining the entrance to the home is encouraged, with the attention to maintaining the space of shared passageways (GPHSC). Regarding the spatial considerations accommodating important belongings indoors, the frameworks acknowledge the importance of assuring the use of residents' own furniture, and EVOLVE includes information on the kinds of furniture to be accommodated (e.g. a bookcase, $\mathrm{TV}, \mathrm{CD} /$ music player and a computer desk in the lounge). Considerations for storage in many parts of the dwelling are present in EVOLVE and GPHSC, such as in the entrance, kitchen, bathroom, as well as storage for objects and tools, furniture not currently used, which is important to keep residents' memories and attachments alive.

However, many of these considerations are too generic, and questions remain regarding what belongings are particularly important for the high-needs; for example, there is no requirement for providing space for pictures, that is important for the high-needs according to other literature. There is also little information regarding the spatial requirements for accommodating the belongings, other than that regarding the room shape (e.g, rectangular in plan (EVOLVE)). For example, information on the required floor area to accommodate objects/furniture would be highly useful for design.

\section{Activities}

Design considerations that facilitate the activities of the elderly are only included in the frameworks overseas. These considerations encompass those requirements for activities in individual and communal spaces. Regarding activities in individual space, the two frameworks include some considerations for activities during the day - mainly in the lounge, kitchen and terrace/balcony — as well as for activities in the bedroom. While the insights for individual space are limited, requirements for communal spaces in both frameworks are numerous and detailed, and encompass space for communal gardens, space for preparing meals/snacks (e.g. in the kitchen), space for having food/drinks (e.g. in the lounge, using a bar), as well as accommodation of various recreational activities (e.g. games, reading, painting, music activities, watching films, dancing, using computers/internet, swimming, exercise, bowling) and religious activities.

According to the literature review in Section 3.2, regarding the facilitation of the activities of the high-needs elderly, there has been great focus on providing communal spaces for various activities. This is well reflected in the frameworks overseas, both of which include many insights regarding the design of communal spaces for various activities. However, recently, facilitating 
individual meaningful activities has been receiving increasing attention — including, providing options in individual space (Koehn et al., 2016; Murphy et al., 2007; Van Steenwinkel et al., 2017). In individual space, there are detailed requirements for space for cooking and gardening; however, there is limited information on spaces for other activities. For example, both frameworks include requirements for the sitting space that facilitates watching TV and telephoning (e.g. a comfortable chair near a telephone and with respect to a TV). EVOLVE include requirements for space accommodating furniture such as a bookcase, TV, CD/music player and a computer desk, that implies possible resident activities. GPHSC includes a requirement for the day area large enough to contain a table and chairs, and a design consideration for greater 'flexibility for different and changing needs, space capable of being equipped for recreational activities (illustration showing painting — space with an easel and chair, an exercising machine)' (WEL_HOPS, 2007, p. 35). However, there are few specific requirements other than the provision of sufficient artificial lighting, extra sockets and sound insulation, and little information is given with regard to what kind of activities (and the spaces for them) should be taken into account in the design for great flexibility. In sum, information is lacking regarding the activities that the high-needs elderly would enjoy doing individually other than gardening and cooking, and the spatial requirements to facilitate them. These are important insights for providing the basis for design.

\section{Relationships}

Design considerations addressing this aspect of QoL of the elderly are included only in the frameworks overseas. The design considerations include those for accommodating guests in the lounge/kitchen/balcony, welcoming guests, accommodating a couple living together, accommodating pets, communal space for socialisation with other residents and welcome external visitors, and the relationships with the outside of the housing/complex.

According to Section 3.2 literature review, communal space is valued as a space for socialising, as the opportunities for the elderly to socialise typically decrease as their impairments increase. This is addressed by both overseas frameworks, encouraging the provision of communal space. Recently, it has been reported that there are differences in the preference of the high-needs elderly for socialising in communal space, and the importance of accommodating personal relationships has increasingly received attention (Cooney et al., 2014; Nordin et al., 2017). With regard to this trend, there are a few items relating to facilitating important relationships in individual space. One specific consideration included is the need for sufficient space to accommodate four people in the lounge and dining area (EVOLVE). However, questions remain 
as to if this requirement facilitates a variety of individual preferences in socialising; for example, it may not accommodate their children and grandchildren, which might be important.

Maintaining a connection to people outside of the housing/complex has also been reported as important. With this respect, there are some considerations included, regarding the maximum distance of the housing/complex from a public transport terminus (400m) (EVOLVE), and a boundary to provide greater security. However, this requirement regarding the distance to public transport is questionable, given the typical limitation in mobility. Finally, the literature suggests the size of the housing/complex has implications for the sense of connectedness; however, no requirements are included to address this.

\section{Quality of care}

This aspect of QoL is addressed only by the frameworks overseas. The main areas of considerations included are; space for a caregiver/assistant in the kitchen and bathroom/toilet (communal /private) as well as at night (the provision of an extra bed for them), the provision of safety systems (e.g. medical alarms), and spatial requirements for the staff (e.g. managers' offices, staff rooms, a catering kitchen).

Section 3.2 identified that the design of space that reduces the demanding workload of caregivers and the consideration in the size of care settings are relevant to facilitating the ease of care, thereby individualised care. The overseas frameworks include the necessary dimensions around the basin and toilet to accommodate a caregiver and the layout of a bed for the caregiver so that they are aware if the resident is moving around at night, both of which support their ease of care. The provision of safety systems can also reduce the caregiver workload by reducing their movement to supervise the elderly. However, there is insufficient considerations for reducing the caregiver workload. For example, there is no suggestion as to the layout of spaces that minimise caregiver movement, or requirements in the size of the housing/complex that facilitate individualised care. There is also insufficient requirements in the private bathroom suited for care practice in bathing/showering. For example, there is no detailed information on the size of a shower enclosure to accommodate them, or information on the spatial layout that eases caregivers' tasks, as well as modifications that can improve the psychological comfort for residents.

\subsubsection{Discussion}

Through the in-depth review of existing design frameworks in the light of the QoL of the highneeds elderly, five main features have been identified as follow. 


\section{Limited information on the spatial requirements for the high-needs}

All design frameworks lack sufficient design considerations for the high-needs elderly. Specifically, studies have reported the importance of private space for the high-needs, with respect to maintaining their control, keeping their important belongings and facilitating their individual recreational and social activities; however, there are limited design considerations for private space. Those design considerations identified/noted are ambiguously stated, particularly those that do not relate to the aspect of independence and control. Even with respect to the aspect of independence and control, there is insufficient information on the usual space (e.g. sitting space) for the high-needs elderly, which is especially important for them. This limitation in the considerations for private space is clear from the comparison with those considerations for communal/outdoor spaces, many of which are more numerous and detailed, particularly with regard to facilitating activities. For example, EVOLVE includes very detailed requirements for various communal recreational spaces - one example is the space for painting, which encompass the type of work surfaces/spaces and storage required for painting activities. Also some requirements included in the frameworks are too general and of little relevance to the high-needs; for example, the requirements for communal spaces addressed in EVOLVE and GPHSC include those for a gym and space for physical games and sports, the use of which by the high-needs is questionable. Other requirements not specific to the high-needs include the use of environmentally friendly materials (GPHSC). The inclusion of these requirements are deemed generic and not specific to the high-needs which blurs the priorities for them, posing questions as to what spaces are particularly necessary to facilitate the QoL for high-needs individuals. This knowledge is especially important in the design of housing for the elderly that do not provide many communal facilities, particularly given the increase of the demand of rental housing and limited resources as discussed in Chapter 2.

\section{Ambiguous design solutions}

In the frameworks reviewed, there are many design considerations that do not clearly show design solutions. The examples of the descriptions are shown in Table 3-2. 
Table 3-2: Ambiguous descriptions

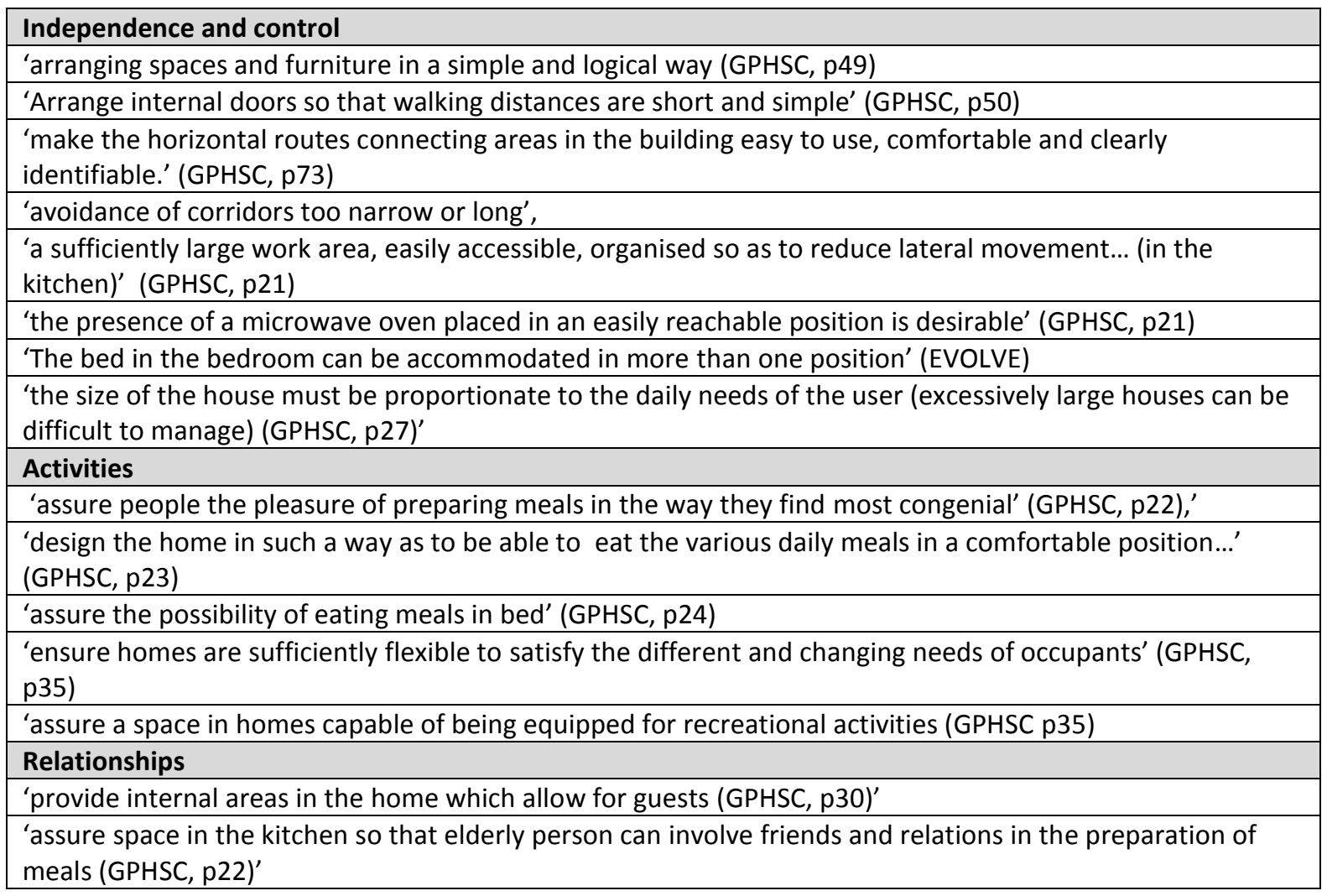

With regard to facilitating independence and control, no specific solutions are shown with the layout of spaces (in the unit and kitchen) and the length of corridor for greater ease of movement/recognition for the elderly and the size of the house small enough for ease of maintenance. EVOLVE recommends providing residents with multiple bed-layout options; however, limited insights regarding the bed-layout requirements are shown — so the optimal design of space is not clear. With regard to facilitating activities, the space for cooking, eating and recreational activities that meets the individual preferences and comfort are recommended. There is also limited description about detailed spatial requirements nor the description of their preferences that could inform the optimal design. GPHSC suggests that sufficient space for guests in the living and kitchen helps maintaining their personal relationships; however, there is no requirements for the size of the space nor the number of guests to be accommodated.

This ambiguity may be the results of the effort to keep the adaptability to various contexts (which was aimed in the development of GPHSC). However, it may also have resulted from a lack of knowledge on the unique spatial usage of high-needs elderly individuals and their perceptions/preferences. In order to design housing for the high-needs elderly, clearer insights into the spatial use, preferences and needs of the individuals. 


\section{Differences in the design requirements}

There are some differences in the requirements by the design frameworks, which are deemed to be consequences of the differences in the demographics and building types they aim to cater for as well as the social, cultural and climatic conditions.

For example, there are difference in the requirements for the minimum door width $(810 \mathrm{~mm}$ in NZS 4121, $800 \mathrm{~mm}$ in EVOLVE and GPHSC, $810 \mathrm{~mm}$ in Lifemark), for the minimum width of corridors/pedestrian access routes $(1800 \mathrm{~mm}$ to accommodate two wheelchairs side by side in EVOLVE, $1100 \mathrm{~mm}$ in GPHSC , $1200 \mathrm{~mm}$ in NZS 4121), and for the height of light switches from the floor (1000-1200 $\mathrm{mm}$ in EVOLVE, 900-1100 $\mathrm{mm}$ in GPHSC/Lifemark). This is likely because of the difference in the demographics that the framework is for (the level of impairments/care needed, the type of mobility aids). The building type that the framework aims to cater to is also relevant; GPHSC, designed mainly for apartment settings, includes considerations specific to apartment settings such as indoor parking areas, and anti-fall bars for windows in high-storey units. There are also differences in the requirements resulting from the cultural/social differences. For example, GPHSC only includes the avoidance of a bath tub, whereas EVOLVE include requirements in the case where a bath is provided (the provision of a transfer seat and a grab rail). Different climates also result in different design requirements; for example, GPHSC includes the installation of a system for refreshing the air, preferably natural, for hot countries, and the provision of protection from the sun and cold winds in balconies/verandas for cold countries. To develop an optimal design of housing, it is important to refer to the design framework that is designed for the right demographic and settings, and suitable for the right social/cultural contexts and climates. This is particularly important in the design for greater QoL, given the QoL is influenced by the social, demographic, and cultural factors as mentioned previously.

\section{Unevenness of addressing the key aspects for QoL}

All design frameworks include detailed considerations relating to various design elements with regard to facilitating the independence and control of the elderly in contrast to those relating to other aspects of QoL, and the number of considerations is also much greater (see also 'Independence and control' section in 3.3.2).

This trend coincides with the recent wider recognition of the importance of built environments facilitating this aspect, and great improvements in practice. Past decades have seen developments in 'accessible design,' 'inclusive design' and 'universal design' seeking the design of products and built environments that can be usable for everyone regardless of their age, ability and life 
situations, including pregnancy, childhood, injury, disability and old age (Clarkson, Keates, Coleman, \& Lebbon, 2003; Mace, 1998). These concepts have also been introduced into building codes and standards, as well as being embraced by Universal Design standards, such as Lifemark Design Standards in New Zealand. In contrast to this strong focus on the 'independence and control' aspects, there is less initiative to encourage incorporating considerations to address other aspects of QoL in the housing design in practice.

\section{Absence of design frameworks addressing a wide range of aspects of QoL of the elderly in New Zealand}

Both New Zealand frameworks are limited in the design requirements to facilitate the aspect of independence and control. Even in this aspect, considerations addressed are limited when compared with the frameworks overseas. For example, they do not include requirements specific to housing for the elderly that include communal areas.

\subsubsection{Conclusion}

This section has reviewed the existing frameworks for housing for the elderly, and examined how they address improving the QoL of the high-needs elderly. First, through the in-depth review of the frameworks in the light of their QoL discussed in the Section 3.2, gaps in knowledge were identified. Next, the features of the existing knowledge are identified, which included five main aspects of; 1) great focus on facilitating the aspect of independence and control of the elderly, 2) absence of design frameworks addressing a wide range of aspects of QoL of the elderly in New Zealand, 3) inclusion of limited information on the spatial requirements for the high-needs, 4) inclusion of ambiguous design solutions, and 5) differences in the design requirements.

The review in this section has clarified that none of these existing design frameworks are useful for the optimal design of housing for the high-needs elderly that improves a wide range of aspects of their QoL of in New Zealand. The creation of such a design framework in the New Zealand context would be beneficial given the increasing demand for housing the high-needs elderly in New Zealand.

\subsection{Conclusion, gaps in knowledge and research questions}

This chapter has explored the QoL of the high-needs elderly and the design considerations to improve it through a comprehensive literature review. Research has suggested that, the QoL of the elderly residents are typically reduced as the care needs increase, either receiving residential 
care or home-care. While the concept of QoL is hard to define, a review of a wide range of literature has identified the overall trend in the current and future ageing population in New Zealand, as well as conceptualised the QoL for elderly people requiring care that relates to physical environments, which are associated with six themes: independence and control, privacy, personal identity, activities, relationships and quality of care. This is followed by a review of existing design frameworks for housing for the elderly, to examine how they address the QoL of the high-needs elderly. In this section, the gaps in knowledge that have been identified through these reviews are combined, followed by the definition of the research questions.

\subsubsection{Gaps in knowledge}

Through literature review, main areas of gaps in the existing knowledge about housing design that can improve the QoL for the high-needs elderly have identified as below.

\section{Gap 1: Limited focus on a wider range of aspects of QoL other than independence and control}

In the course of conceptualising QoL, it has become apparent that, in the existing architectural research regarding QoL of the high-needs elderly, a significant focus has been put on improving elderly people's independence and control. Represented by the P-E fit theory, the influence of the physical environment on individuals' independence and control has well recognised and developed its application for the disabled in architecture discipline and its practice. However, other aspects, in particular, personal identity, activities, relationships and facilitating quality of care have been less developed in architecture. This trend has also been identified in the review of existing frameworks. In particular, the existing design frameworks in New Zealand focus mainly on the aspect of independence and control.

\section{Gap 2: A lack of knowledge for the spatial requirements for private space, which is important for the high-needs}

Another emerging trend in architectural research is about improving common spaces, particularly regarding residents' activities and relationships. However, more recently the significance of private space has been increasingly emphasised. There has been some literature referencing the relationships between built environments and each aspect of QoL. However, there has been a lack of knowledge being applied to actual design, particularly when it comes to the spatial requirements for residents' private space. Similarly, the importance of hosting personal guests and keeping personal possessions increases even though dwelling size often impairs residents' ability to do so, but there have been few studies closely examining spatial solutions that can be 
applied in actual design. The reduction of QoL closely relates to the presence of the caregiver; however, there is a lack of knowledge about architectural solutions that can accommodate residents in their private space without reducing the quality of care. This lack of knowledge in private space in contrast to that for common space has also been identified in the review of existing frameworks. There are limited insights with regard the spatial layout (in the unit/kitchen) to facilitate the ease of movement, as well as those mentioned above.

\section{Gap 3: Lack of knowledge for the design that facilitates the individuality of the high-needs elderly}

Finally, there has been significant attention to 'individuality' in QoL studies. There are differences in preferences for activities and manners in maintaining residents' relationships. In New Zealand in particular, the significance of the individuality and diversity is a notable characteristics of the elderly in New Zealand particularly the baby boomers (Glasgow, 2013; Koopman-Boyden \& Moosa, 2014), and intensified by the differing preferences by ethnicity (V. A. Wright-St Clair et al., 2012). However, older persons' dissatisfaction has been reported with regard to the environment that does not facilitate personal activities and preferred manners of socialising. This trend indicates an increasing need for considering the individuality of residents in the design of housing for the high-needs elderly. This trend has also been illuminated through the review of existing design frameworks, in which many design considerations to facilitate individual preferences and needs are described ambiguously and show limited design solutions. This implies the lack of existing knowledge as to the underlying information on the spatial use of the high-needs and their perceptions and preferences, that form the basis for design.

\section{Gap 4: No framework for the design of housing for the high-needs elderly that improves a wide range of their QoL in New Zealand}

The last gap in knowledge to mention is that there are no existing design frameworks that provide useful information for the optimal design of housing for the high-needs elderly that improves their wide-ranging QoL. There is no design framework that addresses the wide range of QoL of the elderly in New Zealand. Given that the design requirements, particularly with regard to the QoL, are subject to change by the social, cultural and demographic contexts, the application of frameworks overseas would be limited. Even with a review of existing frameworks overseas, there is limited knowledge in the requirements of private space as stated above, as many requirements are less relevant/ not specific to the high-needs, which blurs the priority of the spatial requirements specifically for them. 
More specifically with regard to Gaps 1 - 3, a lack of knowledge was found in a number of design requirements, which include;

- requirements for frequently occupied spaces (e.g. sitting space) in the private dwelling to facilitate independence,

- Requirements for the spatial layout (in the unit/kitchen) to facilitate the ease of movement

- Requirements with regard to the passage length/ distance to spaces

- requirements in the design of the private dwelling for greater privacy in the presence of the caregiver,

- requirements in the private dwelling that accommodate belongings important for the high-needs individuals,

- requirements in housing design that facilitate individual activities and the underlying information for individual preferences,

- requirements for space that can accommodate personal guests (including the number of guests) and facilitate other important relationships,

- requirements in housing design that facilitate individual preferences in socialising,

- requirements in the size of housing/complexes that enable a greater connection to the wider community

- requirements for improving resident privacy with regard to staff entering rooms without waiting for resident permission,

- requirements in the bathroom design to facilitate a great quality of care in assistance in showering, and

- requirements for space that reduces staff workload (e.g. the layout of spaces that minimise their movement).

In relation to each theme of QoL, the applicable theories and common problems regarding physical environments have been summarised along with gaps in knowledge for housing design in Table 3-3. 
Table 3-2: Themes of QoL, problems and scarcity of knowledge

\begin{tabular}{|c|c|c|c|}
\hline Themes of QoL & $\begin{array}{l}\text { Applicable } \\
\text { theory }\end{array}$ & $\begin{array}{l}\text { Common problems regarding physical } \\
\text { environments }\end{array}$ & $\begin{array}{l}\text { Gaps in knowledge in the } \\
\text { design solutions }\end{array}$ \\
\hline $\begin{array}{l}\text { Independence } \\
\text { and control }\end{array}$ & $\begin{array}{l}\text { Person- } \\
\text { environment } \\
(P-E) \text { fit }\end{array}$ & $\begin{array}{l}\text { - Unsuitable physical environments affect } \\
\text { daily activities } \\
\text { (stairs and level differences at doors and } \\
\text { shower entry, unsuitable heights of the work } \\
\text { surface or storages in the kitchen, a lack of } \\
\text { assistive devices such as handrails in the } \\
\text { slope and bathroom, too small space for } \\
\text { manoeuvring mobility aids, hinged and/or } \\
\text { heavy doors, communal settings, the size of } \\
\text { the communal room and the length of } \\
\text { corridors, sensory stimulation and ease of } \\
\text { way-finding) }\end{array}$ & $\begin{array}{l}\text { - Requirements for micro } \\
\text { space in private dwellings } \\
\text { to facilitate independence } \\
\text { - Requirements for the } \\
\text { spatial layout (in the } \\
\text { unit/kitchen) to facility the } \\
\text { ease of movement } \\
\text { - Requirements with regard } \\
\text { to the passage length/ } \\
\text { distance to spaces }\end{array}$ \\
\hline Privacy & $\begin{array}{l}\text { Hierarchy of } \\
\text { privacy (not } \\
\text { applicable to } \\
\text { the private } \\
\text { dwelling) }\end{array}$ & $\begin{array}{l}\text { - Loss/ambiguity of privacy in private space } \\
\text { (caregivers entering the room without } \\
\text { waiting for permission, their presence in } \\
\text { private space, personal care in relatively } \\
\text { public space, no place where residents } \\
\text { could be completely private) }\end{array}$ & $\begin{array}{l}\text { - Requirements in the design } \\
\text { of private dwellings for } \\
\text { greater privacy with the } \\
\text { presence of the caregiver } \\
\text { - Requirements for } \\
\text { improving resident privacy } \\
\text { with regard to staff } \\
\text { entering rooms without } \\
\text { waiting for resident } \\
\text { permission }\end{array}$ \\
\hline $\begin{array}{l}\text { Personal } \\
\text { identity }\end{array}$ & $\begin{array}{l}\text { Place-identity } \\
\text { Personalisatio } \\
\text { n of space }\end{array}$ & - Not enough space for personal belongings & $\begin{array}{l}\text { - Requirements in private } \\
\text { dwellings that } \\
\text { accommodates belongings } \\
\text { important for the high- } \\
\text { needs individuals }\end{array}$ \\
\hline Activities & N/A & $\begin{array}{l}\text { - Lack of activities in the non-communal } \\
\text { settings } \\
\text { - Unsuitable/lack of facilities/spaces for } \\
\text { individual meaningful activities }\end{array}$ & $\begin{array}{l}\text { - Requirements in housing } \\
\text { design that facilitates } \\
\text { individual activities }\end{array}$ \\
\hline Relationships & N/A & $\begin{array}{l}\text { - Lack of social connection, loneliness and } \\
\text { isolation in non-communal settings } \\
\text { - Insufficient space for accommodating } \\
\text { personal guests } \\
\text { - Lack of choices that facilitate individual } \\
\text { preferences in socialising } \\
\text { - Large facilities lack the connection to the } \\
\text { wider community }\end{array}$ & $\begin{array}{l}\text { - Requirements for the } \\
\text { space that can } \\
\text { accommodate personal } \\
\text { guests and other important } \\
\text { relationships } \\
\text { - Requirements in housing } \\
\text { design that facilitates } \\
\text { individual preferences in } \\
\text { socialising } \\
\text { - The size of } \\
\text { housing/complexes for a } \\
\text { greater connection to the } \\
\text { wider community }\end{array}$ \\
\hline Quality of care & N/A & $\begin{array}{l}\text { - Caregiver workload hindering the } \\
\text { individual care for residents } \\
\text { - Inappropriate/insufficient space for } \\
\text { accommodating the caregiver and care } \\
\text { activities }\end{array}$ & $\begin{array}{l}\text { - Requirements in bathroom } \\
\text { design to facilitate a great } \\
\text { quality of care in assistance } \\
\text { in showering } \\
\text { - Requirements for space } \\
\text { that reduces staff workload }\end{array}$ \\
\hline
\end{tabular}




\subsubsection{Research questions}

Given the significance and size of the gaps in our knowledge, it is necessary to explore the design of optimal housing that improves a wide range of QoL of the high-needs elderly in New Zealand. Given the absence of design frameworks that are useful for the design of such housing, developing an appropriate holistic design framework would be beneficial to the current New Zealand context. Therefore, this research aims to address the following questions:

'What is the optimal framework for the design of housing for the high-needs elderly in New Zealand that could improve a wide range of aspects of their QoL?' and

\section{'What are some desirable design solutions?'}

In order to explore the answer to these questions, the methodology of this research project is outlined in the next chapter. 


\section{CHAPTER 4: RESEARCH STRATEGY}

The literature review in Chapter 3 identified numerous gaps in the existing knowledge about housing design for the high-needs elderly in New Zealand that focuses on ways to improve QoL. Given the findings of the review, this research project aims to develop a more holistic framework for the design of housing for the high-needs elderly and then present some desirable designs. The aim of this chapter is to outline the various approaches to establishing a holistic design framework and the necessary investigations to inform its design. First, it identifies the scope, the selected research setting and the subjects, then it breaks the aims into a series of objectives. It then identifies the most suitable procedures to meet these objectives. The establishment of the methodological approach will be described in the subsequent chapters.

\subsection{Research scope, settings and subjects}

This study focuses on housing that could accommodate the high-needs elderly living independently. As reviewed in Chapter 2, the current types of housing for this cohort in New Zealand include retirement villages, public-sector housing, and private-sector rental housing for the elderly. These types of housing are the options for the high-needs elderly to move to when they have difficulty in maintaining their house and need greater support and care. Of the residents in these types of housing, the scope of this research focuses on those who receive personal care from professional caregivers. The study sites and participants are limited to those in the Wellington Region in New Zealand.

\subsection{Research objectives}

In order to develop a framework for requirements in the design of housing that improves the QoL of the high-needs elderly in New Zealand, it is essential to understand the current situations of housing and the elderly residents with respect to their care requirements as well as the models of care and physical environment that support them. This must be undertaken holistically to capture the wide range of aspects of QoL that relate to their physical environments. Once this primary data has been captured, analysis is necessary to develop a design framework. Finally, the data must be synthesised and application must be demonstrated. More specifically: 


\section{Objective 1: To establish the current situations with respect to models of care, physical}

environments and care requirements of elderly residents

The first objective is to clarify basic information relating to current situations of housing and elderly residents. Establishing the people and settings focused on is important, as it has an impact on the outcome of the optimal design framework this thesis aims to develop. The information to be clarified is that which could not be obtained through the literature review but is important basic information about the housing and the cohort focused in this study. In particular, while information on the models of care and physical environments is publicly available on retirement villages through literature review, information on public-sector housing and private-sector rental housing for the elderly is scarce. Similarly, care requirements for elderly residents living in the three types of housing are largely unknown. The first objective of this research is to establish the models of care and the physical environments of housing and the care requirements of residents, by housing type. This information will provide important insights with regard to the potential of housing to support the independent life of the high-needs elderly as well as provide basic information about the study settings and participants in the following investigations. The information obtained through this phase is also used for the site and participant selection in the Phase 2 survey.

\section{Objective 2: To investigate a wide range of aspects of QoL of high-needs elderly, that relate to physical environments}

The second objective is to holistically establish the architectural details of the living environments which impact on the QoL of the high-needs elderly. This investigation will provide the grounding for the design framework to be developed. This will address the gaps in knowledge identified in Chapter 3, such as information on the elderly's spatial use in their private dwelling. This, as well as their preferences in activities and socialisation, is particularly important for those with high needs, to provide insights into the design that facilitate individuality.

\section{Objective 3: To develop a design framework}

The third objective is to further analyse the outcome of the previous investigation to develop a framework for the requirements in the design of housing that improves the QoL of the highneeds elderly. 


\section{Objective 4: To apply this framework in the design of housing models}

Finally, example models of housing will be designed. This addresses the research question 'What are some desirable design solutions?' as well as tests the implementation potential for the design framework.

\subsection{Research procedures}

Following the defined objectives, the research has been split into four main phases and different investigative strategies have been adopted to best fit the data to be collected, resulting in a mixed method of data collection involving both quantitative and qualitative methods. First, questionnaires about the current situation of housing and care requirements of elderly residents are conducted. Second, investigation into residents' QoL and the built environment is undertaken. Third, a design framework is developed. Finally, models of housing are designed.

\section{Phase 1. Questionnaires on the current situations of housing and elderly residents}

In this phase, basic information on the current situation of three types of housing (retirement villages, public-sector housing and private-sector rental housing for the elderly) and elderly residents is gathered. More specifically, quantitative data for establishing the models of care and physical environments of housing and care requirements of the residents is gathered through two questionnaires; one for housing operators and the other for residents. To use the information obtained for the site and participant selection in the Phase 2 survey, the willingness to participate the survey is also asked. The methods of the Phase 1 survey as well as the findings are described in detail in Chapter 5.

\section{1-1. Models of care and physical environments of housing}

To establish the physical environments and models of care of three types of housing, data is collected from housing operators through an online questionnaire. Collected data about physical environments include: the type and number of units, the number of units suitable for the disabled, the adjacency of residential care facilities/units and communal spaces provided in the complex. Collected data about models of care provided for residents by the housing operators include various services encompassing social support, assistance in household tasks and personal care. These data will inform the potential of housing to support the independent life of the highneeds elderly. 


\section{1-2. Care requirements of elderly residents}

Information about care requirements of elderly residents is collected through paper-based questionnaires from all residents in three types of housing complexes where the housing operators give permission for conducting this survey. Collected information includes the degree of assistance residents need in daily activities as well as the type of caregiver who assists them.

\section{Phase 2. Qualitative investigation for the QoL of high-needs elderly with respect to the built environment}

This phase obtains holistic information on QoL of the high-needs elderly in relation to the built environment. The information is collected through the detailing of spatial use and the capture of user perceptions and preferences, The data collected is holistic to provide the grounding for the design framework to be developed and include information that addresses the gaps in knowledge. More specifically, data that relates to their private dwelling and their preferences in activities and socialisation and can provide insights into the design that facilitates individuality. To collect these data, a qualitative approach is selected, that can investigate QoL in relation to physical environments from a holistic perspective without hypothesising the components of physical environments or QoL. Among qualitative approaches, an ethnographical approach is selected, that can investigate the intimate space of the high-needs elderly while ensuring the consistency of data collected across participants. More specifically, it includes semi-structured interviews and daytime observation involving both elderly residents and their caregivers as well as data collection on physical environments. The exploration of optimal methodology and methods are described in detail in Chapter 6, and the collected data is presented in Chapter 7.

\section{Phase 3. Development of a design framework}

In this phase, based on the results of the Phase 2 investigation, a framework for the requirements in the design of housing that improves the QoL of the high-needs elderly is established. First, it establishes the relationships between design considerations and resident QoL, and next, design requirements are established through the consideration and synthesis of all relevant information by design elements and with reference to external sources. The design requirements are presented by design elements and with the relationships to the aspects of QoL identified (Chapter 8).

\section{Phase 4. Design of models of housing}

Based on the framework developed, models of housing are designed. The design includes both for individual units and complexes, given that the design framework includes design 
requirements for both areas. As for housing complexes, examples of three types of housing are designed incorporating the knowledge obtained through the Phase 1 investigation. Through the design, considerations and challenges in the implementation of the design framework are identified (Chapter 9).

The research procedure is summarised in Figure 4-1.

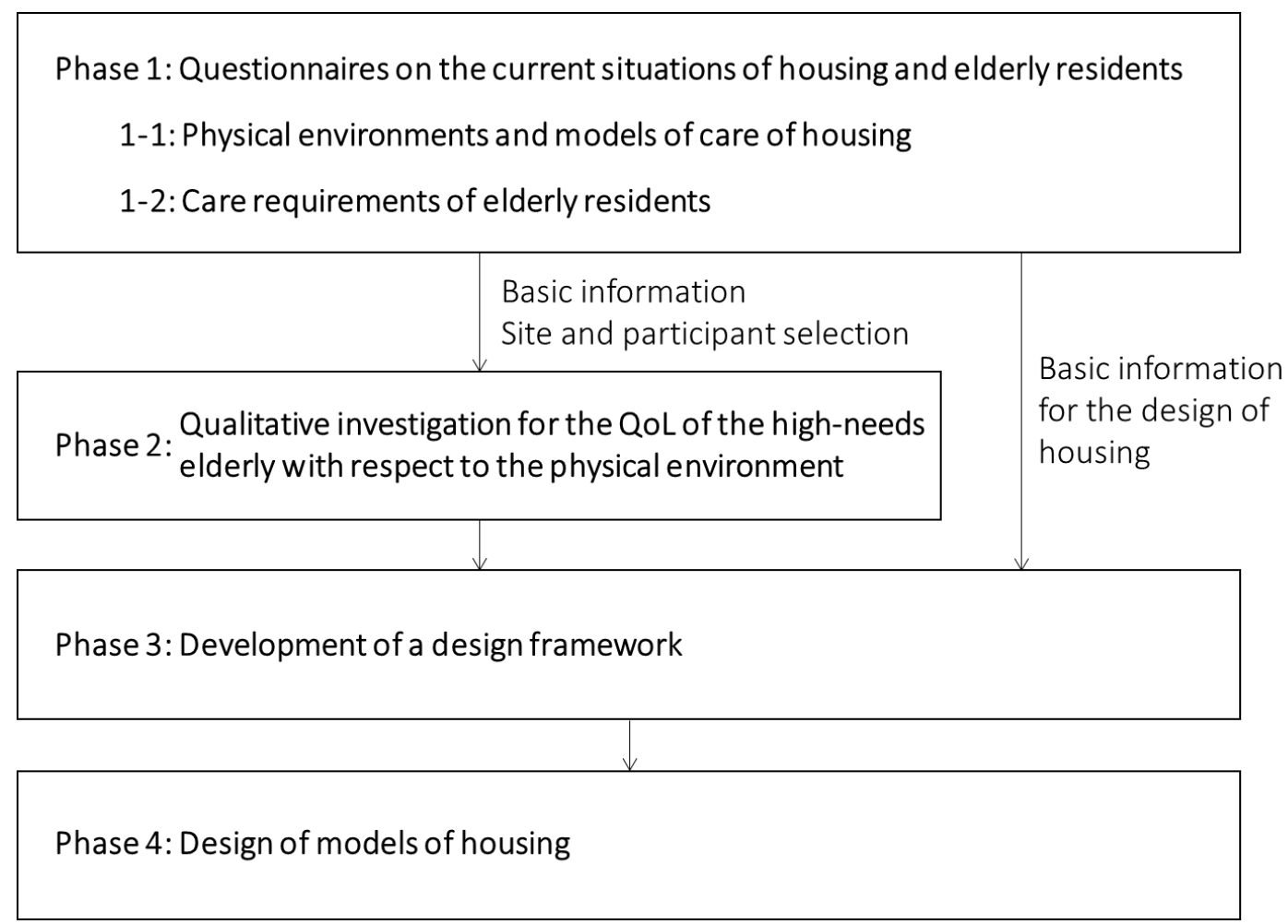

Figure 4-1: Research procedure

\subsection{Ethics approval}

For the Phases 1 and 2, ethics approval was obtained from the Victoria University Human Ethics Committee (approval number: 23243). Consent was obtained from the respondents in each phase. Details are described in the subsequent chapters. 


\section{CHAPTER 5: CURRENT SITUATIONS OF HOUSING FOR THE ELDERLY AND ELDERLY RESIDENTS (PHASE 1)}

This chapter establishes the current situations of housing for the elderly to live independently in New Zealand, which provides the basic knowledge for the following study investigating the QoL of the high-needs elderly in relation to physical environments. The information obtained provides the context for the design framework to be developed in the chapter ahead. This Phase 1 investigation explores models of care, physical environments and residents' care requirements in three types of housing: retirement villages, public-sector housing, and private-sector rental housing. These were previously identified as the main independent living options for the elderly.

The chapter starts with a description of the study methods used, followed by the presentation of the findings. It identifies the features of the three types of housing; leads into a discussion about combining services with housing provision; and, finally explores the possibility of providing housing that helps the elderly to live as independently as possible in New Zealand.

\subsection{Methods}

Data on models of care, physical environments and care of residents was collected through two surveys for housing operators and for residents. The focus of these surveys was on retirement villages and public-sector housing, and private-sector rental housing for the elderly in the Wellington Region of New Zealand.

\subsubsection{Phase 1-1: Data collection for models of care and physical environments}

Information on models of care and physical environments of housing was collected from the operators of retirement villages, public-sector housing and private-sector housing for the elderly.

Housing operators were identified in various ways, as there was no comprehensive database on all housing types studied. For retirement villages, the information was collected through Eldernet, an online directory of services related to elderly people, which includes retirement villages as well as aged care facilities (Eldernet Ltd, 2018). Information on public-sector housing was collected 
through web pages of local authorities ${ }^{16}$ and HNZ, as well as through email enquiries. Of eight local councils in the Wellington Region, two had sold their housing to private-sector agencies, and two had another council or a private company manage their housing; the remaining four councils managed their housing themselves. Information on private-sector housing for the elderly was collected through Eldernet (Eldernet Ltd, 2018), the online directory of CHPs (Community housing Aotearoa, 2018b) and other sources that referenced information about housing providers (Davey et al., 2004). The housing operators identified were sent an online questionnaire using Qualtrics (Qualtrics, 2018) as well as an information sheet, and asked for their response and consent to use the information.

A total of 47 housing operators were invited and 24 joined the survey (Table 5-1). From the public-sector housing groups, HNZ did not participate, so the results represent only the situations in local authority housing. One local authority housing organisation (Porirua City Housing) is managed by another local authority (Wellington City Council), who provided information for Porirua City Housing. In the analysis, retirement villages were divided into two types: independent-living units and supported-living units.

Table 5-1: Participation in the Phase 1-1 survey

\begin{tabular}{|l|l|l|l|}
\hline $\begin{array}{l}\text { Type of housing } \\
\text { complex }\end{array}$ & $\begin{array}{l}\text { Number of housing } \\
\text { operators invited }\end{array}$ & $\begin{array}{l}\text { Number of housing } \\
\text { operators responded }\end{array}$ & Response rate \\
\hline Retirement villages & 33 & 12 & $36 \%$ \\
\hline $\begin{array}{l}\text { Public-sector housing } \\
\text { (excluding HNZ) }\end{array}$ & 7 & 5 & $83 \%$ \\
\hline $\begin{array}{l}\text { Private-sector rental } \\
\text { housing for the elderly }\end{array}$ & 8 & 7 & $88 \%$ \\
\hline Total & 47 & 24 & $51 \%$ \\
\hline
\end{tabular}

Through the questionnaire, data on models of care and physical environments were collected.

Data collected about physical environments included: the type and number of units, the number of units suitable for the disabled (i.e., units that have access and facilities for the disabled), the adjacency of residential care facilities/units and communal spaces provided in the complex.

The services provided for residents by the housing operators focused on: regular staff visits, organisation of activities in communal areas, outings (including shopping trips), transportation services, emergency services on-call 24 hours a day, meals services, laundry services, assistance in

\footnotetext{
${ }^{16}$ Local authorities in Wellington Region are: Wellington City Council, Porirua City Council, Hutt City Council, Upper Hutt City Council, Kapiti Coast District Council, Masterton District Council, Carterton District Council and South Wairarapa District Council.
} 
household tasks, shopping on behalf of residents and personal care. Information regarding whether there was an associated extra cost was also collected.

\subsubsection{Phase 1-2: Data collection for the care of elderly residents}

Information on personal care was obtained through paper-based questionnaires responded to by residents. Those housing operators who participated in the Phase 1-1 survey and said that they were interested in this survey were contacted and asked for permission for their residents to take part in this survey. Ten housing operators gave permission. They were asked to distribute questionnaires and information sheets to all the residents. The completed questionnaires and consent forms were collected individually by the researcher, except in some housing complexes where the operators wished either to collect questionnaires themselves or to have them collected in an organised session.

To assure the validity of the study, it was essential to consider inclusion criteria of collected data for analysis, particularly the aspect of a response rate (M.B., K., M., \& Atatoa, 2012). For this reason, published research on survey participation rate was referred to. In response to the decline in research participation in recent decades, many studies have explored the influence of a low response rate on the validity of participant surveys (M.B. et al., 2012). One study examined the influence of low response rates on the validity by reviewing numerous studies and reported that there are few differences in bias in epidemiologic research between studies with low response rates (such as 30\%) and studies with response rates of 60\% to 70\% (Galea \& Tracy, 2007). Another study with a participation rate of $24.1 \%$ compared the distribution in gender, age and the district of residence of participants and the census data, and found that there were only small differences in the distribution (Bocquet et al., 2018). Considering these reports, in the analysis of this study data were limited to those from complexes where the response rates were more than $30 \%$ (33$82 \%)$.

The data on five retirement villages (of which two included supported-living units), three publicsector housing complexes, and four private-sector rental housing complexes were included. The situation on the participation is shown in Table 5-2. 
Table 5-2: Participation of the Phase 1-2 survey (aggregated data)

\begin{tabular}{|l|l|l|l|l|l|l|}
\hline $\begin{array}{l}\text { Type of housing } \\
\text { complexes }\end{array}$ & $\begin{array}{l}\text { Number of } \\
\text { housing } \\
\text { operators } \\
\text { who gave } \\
\text { permission }\end{array}$ & $\begin{array}{l}\text { Number of } \\
\text { housing } \\
\text { complexes } \\
\text { where the } \\
\text { questionnaire } \\
\text { was } \\
\text { conducted }\end{array}$ & $\begin{array}{l}\text { Number of } \\
\text { housing } \\
\text { complexes } \\
\text { with a } \\
\text { response } \\
\text { rate of 30\% } \\
+\end{array}$ & $\begin{array}{l}\text { Number of } \\
\text { residents } \\
\text { invited (in } \\
\text { housing } \\
\text { complexes } \\
\text { with a } \\
\text { response } \\
\text { rate of 30\% } \\
\text { ) }\end{array}$ & $\begin{array}{l}\text { Number of } \\
\text { respondents } \\
\text { (in housing } \\
\text { complexes } \\
\text { with a } \\
\text { response } \\
\text { rate of 30\% } \\
+ \text { ) }\end{array}$ & $\begin{array}{l}\text { Response } \\
\text { rate } \\
\text { (mean) }\end{array}$ \\
\cline { 1 - 5 } $\begin{array}{l}\text { RV independent- } \\
\text { living units }\end{array}$ & 6 & 6 & 5 & 573 & 250 & $43 \%$ \\
\cline { 1 - 6 } $\begin{array}{l}\text { RV supported- } \\
\text { living units }\end{array}$ & 2 & 2 & 50 & 22 & $44 \%$ \\
\hline $\begin{array}{l}\text { Public-sector } \\
\text { housing } \\
\text { (excluding HNZ) }\end{array}$ & 3 & 5 & 3 & 67 & 44 & $66 \%$ \\
\hline $\begin{array}{l}\text { Private-sector } \\
\text { rental housing for } \\
\text { the elderly }\end{array}$ & 4 & 9 & 4 & 172 & 90 & $62 \%$ \\
\hline Total & 13 & 17 & 14 & 862 & 406 & $47 \%$ \\
\hline
\end{tabular}

('RV' stands for 'retirement village')

The questionnaire was designed to collect data on the degree of assistance needed in six types of daily activities: bathing, dressing, personal hygiene, moving from bed to wheelchair/chair, walking indoors and eating. This questionnaire was created referring to the items in the Barthel Index (Mahoney \& Barthel, 1965), which is used in the clinical settings to measure patients' levels of dependency in ADL. Items were modified to suit the study settings; for example, the item of 'stairs' was excluded, as all residences were deemed single-story. Three choices were given for each activity based on the level of assistance needed: 1 . do not receive assistance, 2. sometimes receive assistance/supervision only, 3. receive assistance. The exception was for the activity of walking indoors, for which four choices were given: 1. walk independently (may use sticks, walker frames, etc.), 2. move in wheelchair independently, 3. walk with help of one person, 4. walk with help of more than one person/need assistance in moving in wheelchair. In addition to the assistance needed, information on the type of caregiver (informal/formal caregivers (see also 2.2.2)) was collected.

\subsubsection{Data analysis}

All the collected data were put into Excel spreadsheets for analysis. To enable a comparison of the results by the housing type, data is shown in tables or bar graphs organised by housing types.

To compare the levels of service, the services were given scores: provided without extra cost $=2$, provided with extra cost $=1$, not provided $=0$. Each housing complex was given a 'Service 
Score', which is the total score across 10 types of services. The levels of service were classified into three by the Service Score: low levels of service (Service Score $=0-3$ ), medium levels of service (Service Score $=4-9$ ), and high levels of service (Service Score $=10$ and over).

To compare the levels of dependency, the assistance in all activities was given scores of 0-2 or 0-3 depending on the degree of assistance provided. Each person was given a 'Dependency Score', the total score for assistance needed across six types of activities. The levels of dependency were classified into two categories by the Dependency Score: low levels of dependency (Dependency Score $=0-4$ ) and high levels of dependency (Dependency Score $=5$ and over).

\subsection{Findings}

This section reports the findings from two questionnaires; the Phase 1-1 questionnaire on physical environments and services and care of housing and the Phase 1-2 questionnaire on care requirements of elderly residents. These findings are synthesized by housing types and features of each type of housing are identified.

\subsubsection{Findings from the Phase 1-1 questionnaire}

\section{Number of units per complex and unit composition}

The data on the number of units per site were collected from the housing operators of 12 retirement villages, 61 public-sector housing complexes and 16 private-sector housing complexes for the elderly. Data on the number of units per complex is summarised in Table 5-3 and Figure $5-1$.

Table 5-3: Number of units per complex

\begin{tabular}{|l|l|l|}
\hline Type of housing complexes & Total number of units studied & $\begin{array}{l}\text { Average number of } \\
\text { units per complex }\end{array}$ \\
\hline RV ( $n=12)$ & 1467 units & 122 units \\
\hline $\begin{array}{l}\text { Public-sector housing complexes } \\
\text { (excluding HNZ) (n=61) }\end{array}$ & 2501 units* & 41 units \\
\hline $\begin{array}{l}\text { Private-sector rental housing } \\
\text { complexes ( } n=16)\end{array}$ & 223 units & 14 units \\
$\begin{array}{l}\text { ('RV' stands for 'retirement village') } \\
\text { *Of the public-sector housing, Wellington City Council housing units includes those supplied for younger } \\
\text { people. }\end{array}$
\end{tabular}




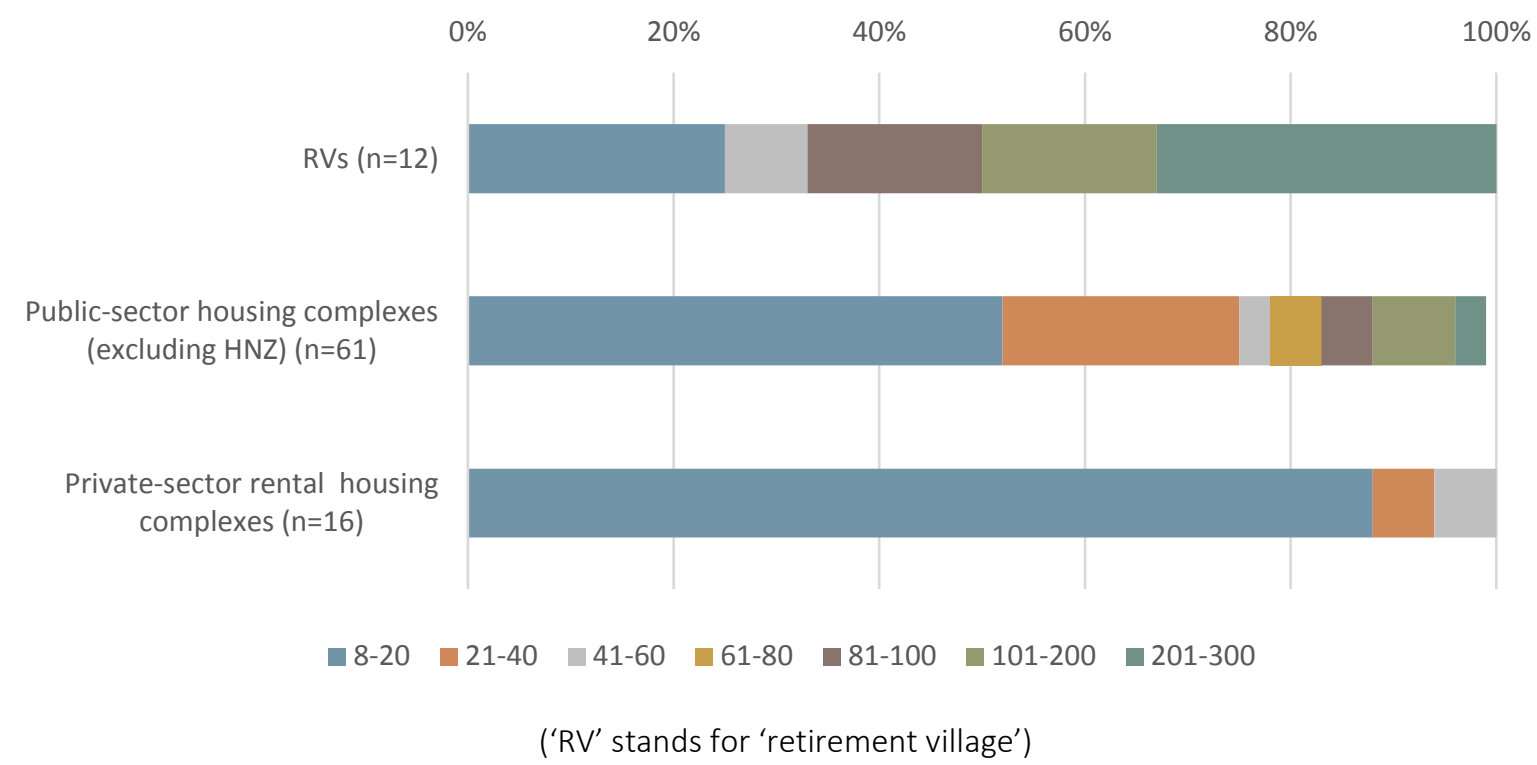

Figure 5-1: Distribution of number of units per complex**

**These percentages were calculated based on the number of complexes, not on the number of units.

The number of units per complex generally defines the size of the complex. The average number of units was the largest for retirement villages, which ranged widely in the size and contained from 20 units or less to over 200 units. The size of public-sector housing varied, with the majority being complexes of 20 units or less. The average size of a complex was the smallest in voluntary-sector housing, with $88 \%$ of the complexes containing 20 units or less.

\section{Unit composition and adjacency of residential care facilities/units}

The unit composition and adjacency of residential care facilities/units of the complexes are shown in Figure 5-2 and 5-3. The composition of independent-living units and supported-living units in retirement villages containing both types of units is shown in Figure 5-4.

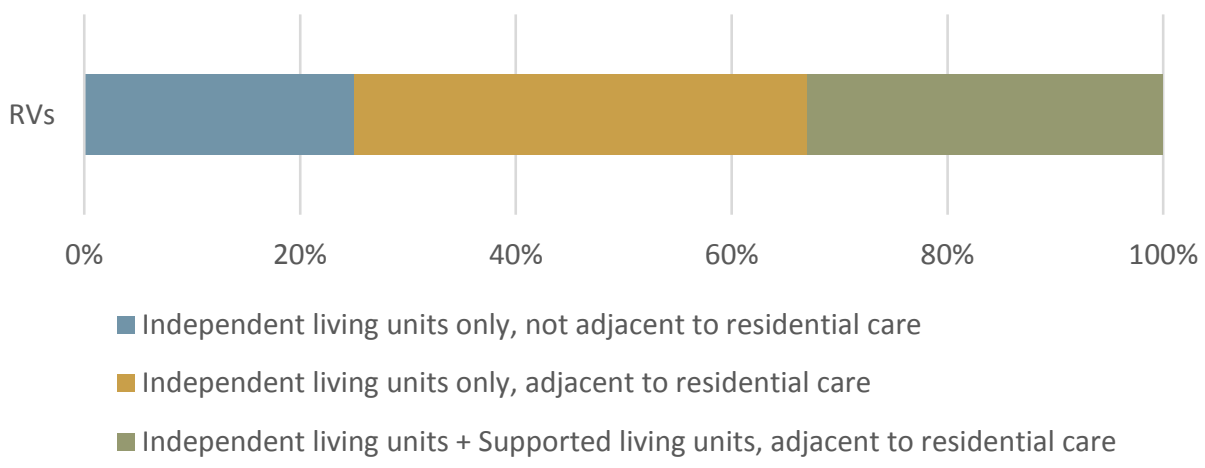

('RV' stands for 'retirement village')

Figure 5-2: Unit composition of retirement villages and the adjacency of residential care facilities 


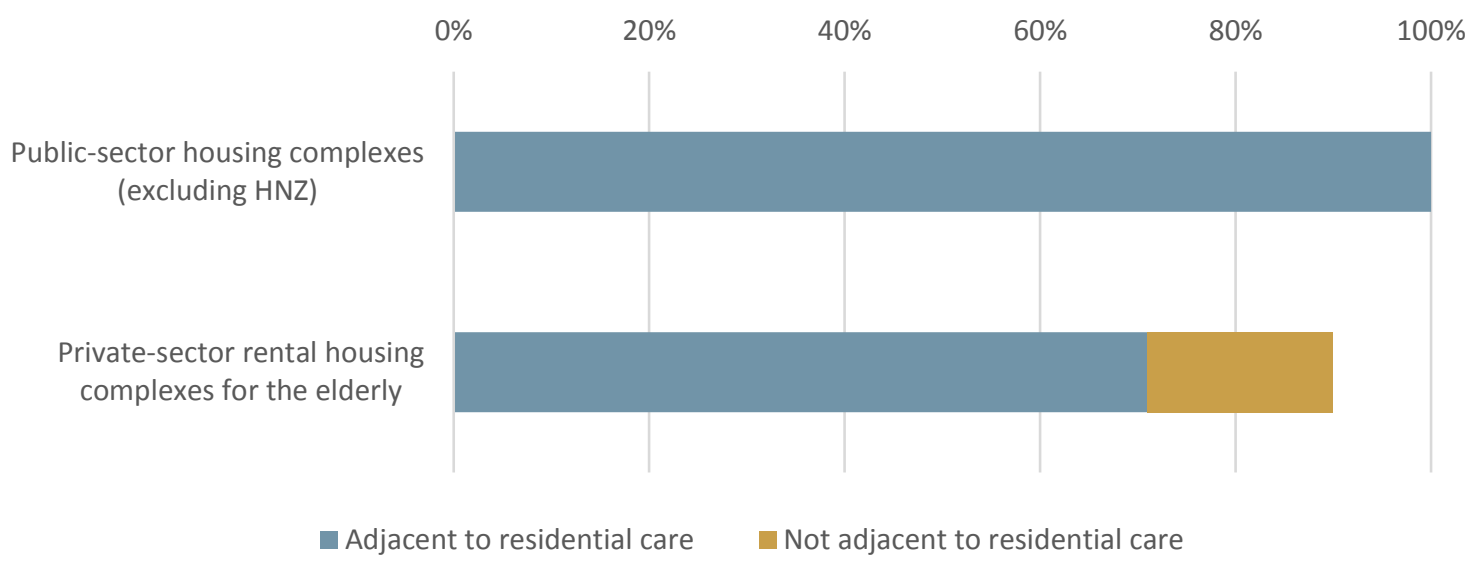

Figure 5-3: Adjacency of residential care facilities to rental housing complexes

RVs containing both independent living units and supported living units

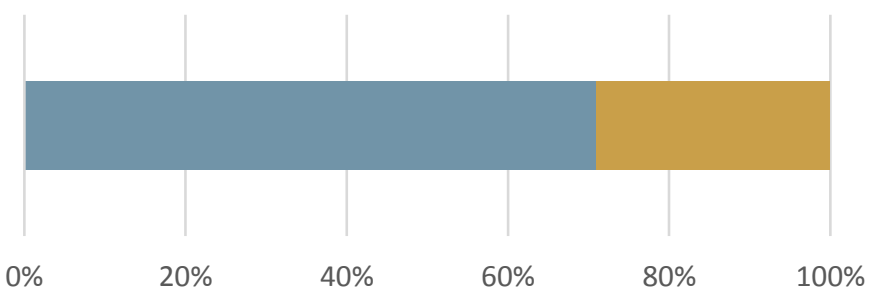

- Independent living units $\quad$ Supported living units

('RV' stands for 'retirement village')

Figure 5-4: Unit composition among retirement villages containing both independent and supported-living units

Retirement villages were composed of independent-living units only (25\%) or independentliving units and supported-living units (75\%). Residential care facilities/units were adjacent to many of the villages (75\%), particularly to villages with supported-living units. In those villages with both types of units, supported-living units accounted for $29 \%$ on average. All public-sector housing complexes (excluding HNZ) were rental housing units and no complex was adjacent to residential care facilities/units. Of the private-sector housing complexes, 19\% were adjacent to residential care facilities/units.

\section{Percentage of units suitable for the disabled}

The percentage of units that have access and facilities for the disabled ${ }^{17}$ is shown in Table 5-4 and Figure 5-5.

\footnotetext{
${ }^{17}$ This term refers to the term used in the Building Act 1991, which includes the requirements for access and facilities for people with disabilities (Standards New Zealand, 2001). People with disabilities are defined as 'people whose ability to be freely mobile or to access and use buildings is affected by mental, physical, hearing or sight impairment, such as: (a) An inability to walk; (b) Walking difficulties; (c) Reliance on walking aids; (d) Partial
} 
Table 5-4: Average percentage of units that have access and facilities for the disabled*

\begin{tabular}{|l|l|}
\hline Type of housing complexes & $\begin{array}{l}\text { Average percentage of units that have access and } \\
\text { facilities for the disabled }\end{array}$ \\
\hline RV independent-living units & $62 \%$ \\
\hline RV supported-living units & $62 \%$ \\
\hline Public-sector housing complexes (excluding HNZ) & $1 \%$ \\
\hline $\begin{array}{l}\text { Private-sector rental housing complexes for the } \\
\text { elderly }\end{array}$ & $14 \%$ \\
\hline
\end{tabular}

('RV' stands for 'retirement village')

*The unit number of housing sites where the data were not available was marked as 0 .

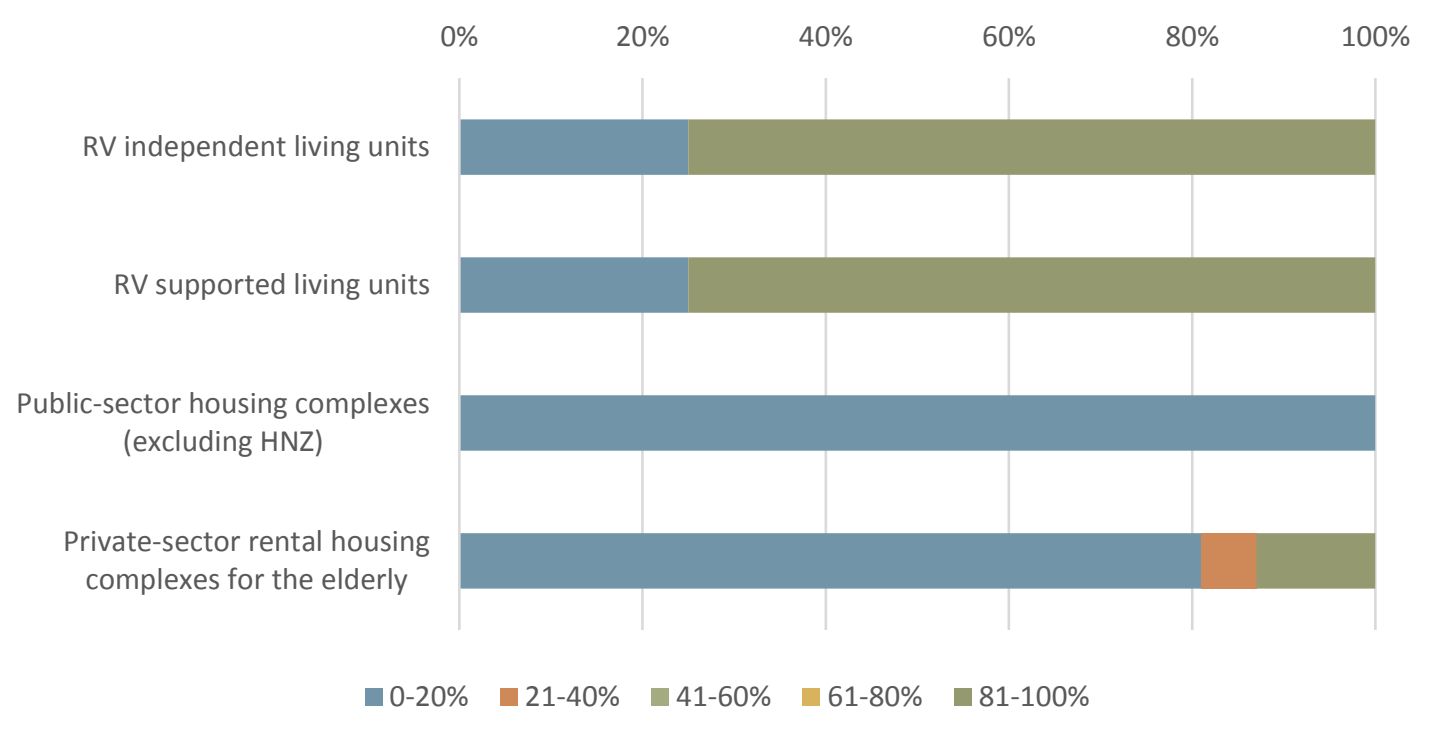

('RV' stands for 'retirement village')

Figure 5-5: Distribution of the percentage of units that have access and facilities for the disabled

Access and facilities for the disabled were provided for a high proportion of both types of units (independent and supported-living) in retirement villages. By contrast, public-sector rental housing rarely had units suitable for the elderly; only $20 \%$ or less of the units surveyed had access and facilities for the disabled. Most private-sector rental housing complexes had a small proportion of units suitable for the disabled, but there were a limited number of complexes that had a large proportion of units with access and facilities for the disabled.

\section{Communal areas}

Communal areas are common design features in housing for the elderly. The types of communal areas provided in the complexes is shown in Figure 5-6.

sightedness or total blindness; (e) Hearing disabilities; (f) Lack of co-ordination; (g) Reaching disabilities; (h) Manipulation disabilities; (i) Lack of stamina; (j) Difficulties in interpreting and reacting to sensory information; ( $\mathrm{k}$ ) Extremes of physical size; (l) Learning difficulties' (Standards New Zealand, 2001). 


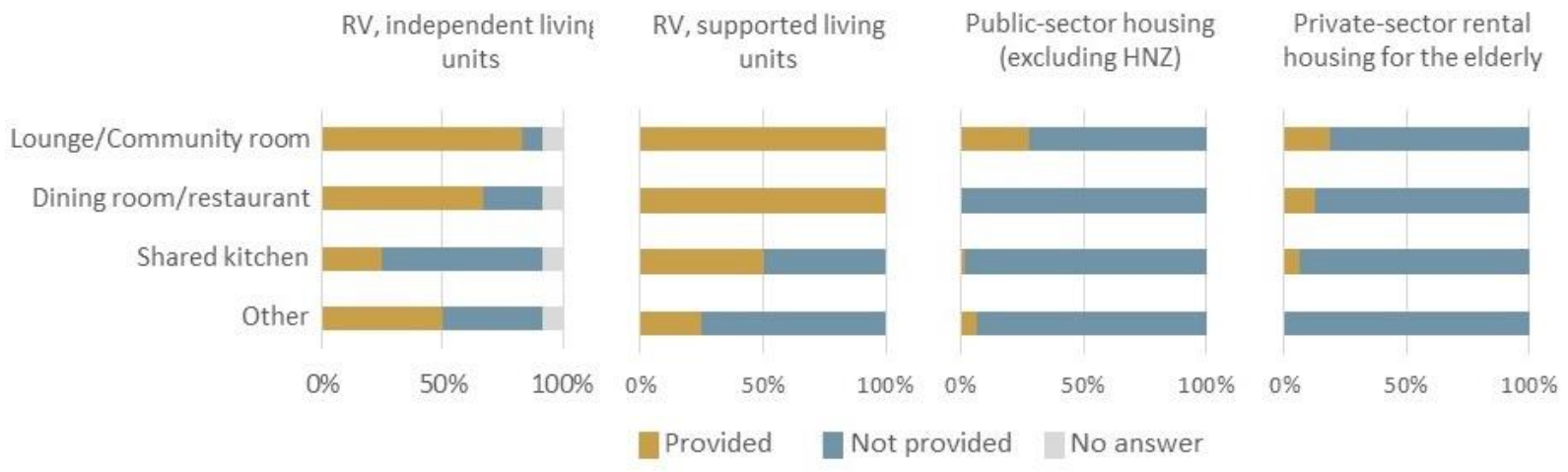

('RV' stands for 'retirement village')

Figure 5-6: Communal areas provided for residents

Retirement villages provided a wide range of communal facilities such as lounges, community rooms, dining rooms and restaurants. Residents in all supported-living units in retirement villages were provided with a lounge/community room and a dining room. By contrast, most public-sector housing complexes had either no communal space or only a community room for shared use. The number of communal spaces provided by the private sector was also limited.

\section{Services provided for residents}

Services and care provided for residents are a key element that supports the independent life of high-needs elderly people. The kinds of services provided for residents in each type of housing is shown in Figure 5-7. Service scores ${ }^{18}$ of housing complexes are shown in Table 5-5 and the level of service is shown in Figure 5-8.

${ }^{18}$ For the definition, see 5.1.3. 


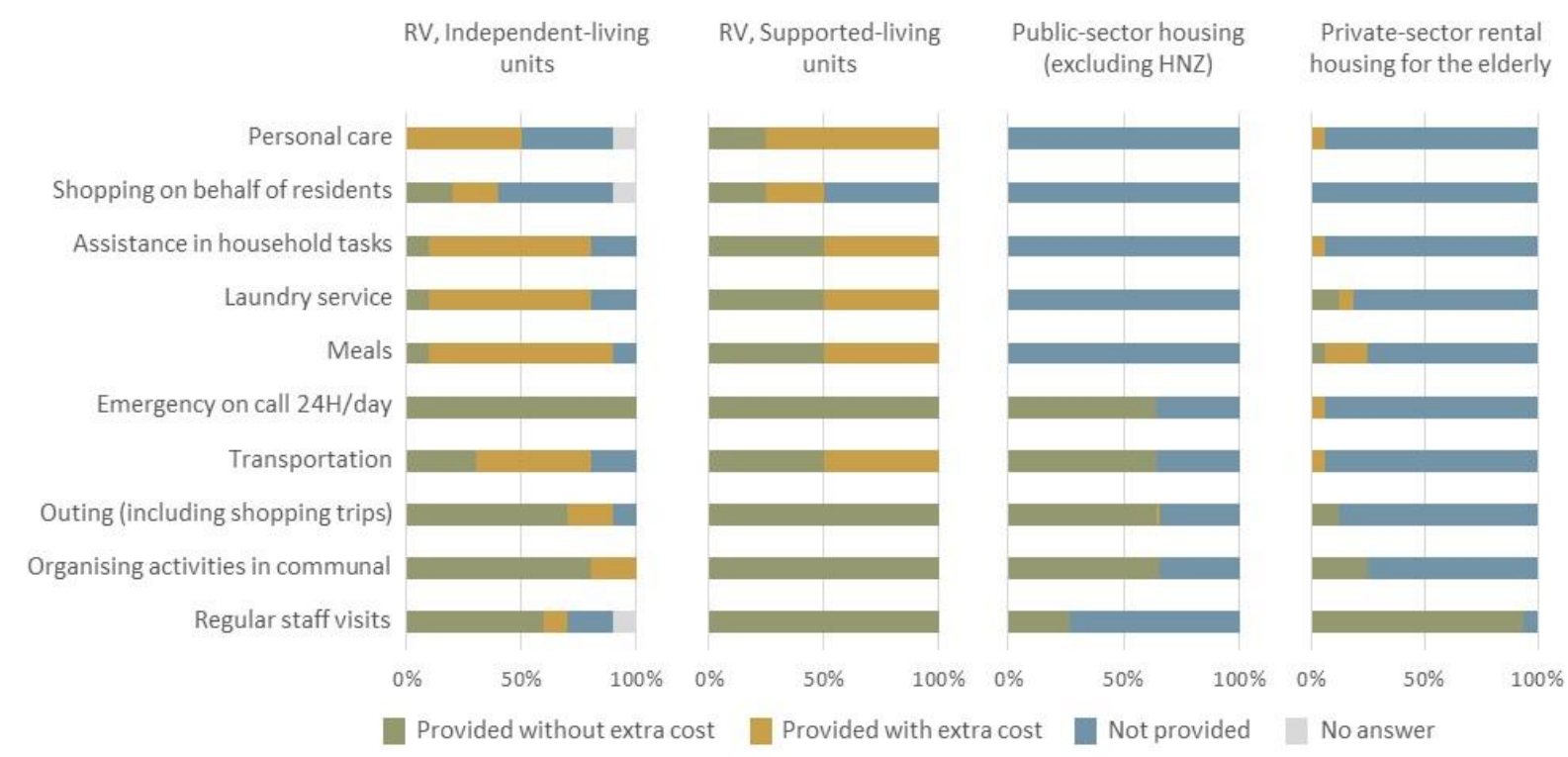

('RV' stands for 'retirement village')

Figure 5-7: Services provided for residents

Table 5-5: Average Service Score by the housing-complex type

\begin{tabular}{|l|l|}
\hline Type of housing complexes & Average Service Score \\
\hline RV independent-living units & 11.7 \\
\hline RV supported-living units & 16.0 \\
\hline Public-sector housing complexes (excluding HNZ) & 5.72 \\
\hline Private-sector rental housing for the elderly & 3.5 \\
\hline
\end{tabular}

('RV' stands for 'retirement village')

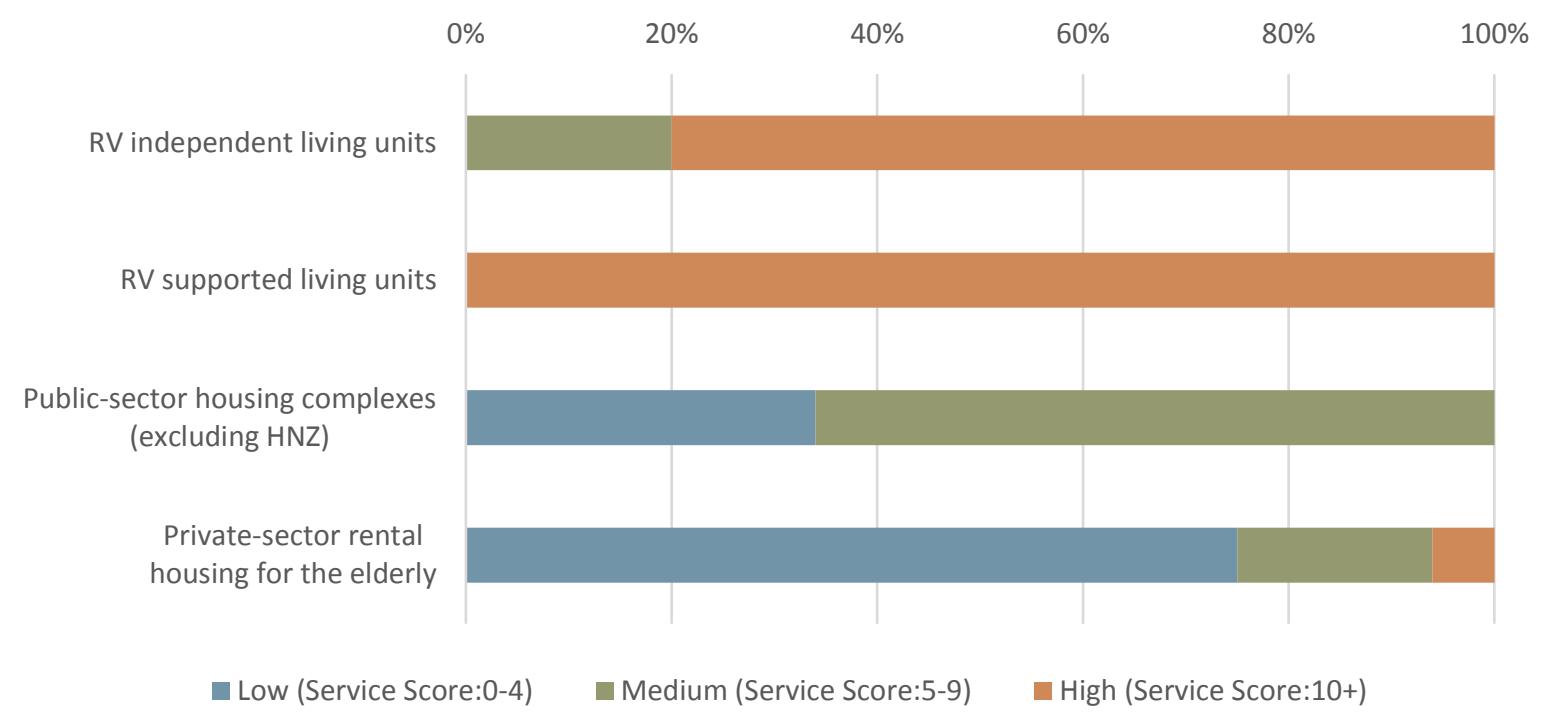

('RV' stands for 'retirement village')

Figure 5-8: Distribution of levels of service

retirement villages provided high levels and a wide range of services. For example, all villages provided emergency on-call 24 hours a day, and most arranged to visit residents regularly and 
organised activities and outings for no additional charge (the costs were often included as part of the 'Licence to Occupy', or in the 'Weekly Fees'). Assistance with transportation, meals, laundry and other support with household tasks was provided either inclusively or with extra cost.

The services for residents in supported-living units were greater than for those in independentliving units. In more than half the public-sector housing complexes, medium levels of services were provided, combining services such as emergency on-call with social support, and regular staff visits with organised activities and outings. The majority of private-sector housing provided low levels of service with regular staff visits, while some provided medium-high levels of services, with services offered varying by the site.

\subsubsection{Findings from the Phase 1-2 questionnaire}

\section{Resident dependency by the housing type}

The levels of dependency of residents are examined by housing types, using the Dependency Score ${ }^{19}$ and the percentage of those receiving care. In addition, the percentage of residents with low levels of dependency and those with high levels of dependency (defined by the Dependency Score) among those receiving personal care are also shown (Table 5-6, Figure 5-9).

Table 5-6: Resident dependency by the housing-complex type

\begin{tabular}{|l|l|l|}
\hline Type of housing complexes & Mean Dependency Score & $\begin{array}{l}\text { Percentage of those receiving } \\
\text { personal care }\end{array}$ \\
\hline RV independent-living units & 0.35 & $12 \%$ \\
\hline RV supported-living units & 2.14 & $46 \%$ \\
\hline $\begin{array}{l}\text { Public-sector housing complexes } \\
\text { (excluding HNZ) }\end{array}$ & 0.18 & $8 \%$ \\
\hline $\begin{array}{l}\text { Private-sector rental housing } \\
\text { complexes for the elderly }\end{array}$ & 0.58 & $21 \%$ \\
\hline 'RV' stands for'retirement village') &
\end{tabular}

('RV' stands for 'retirement village')

\footnotetext{
${ }^{19}$ For the definition, see 5.1.3.
} 


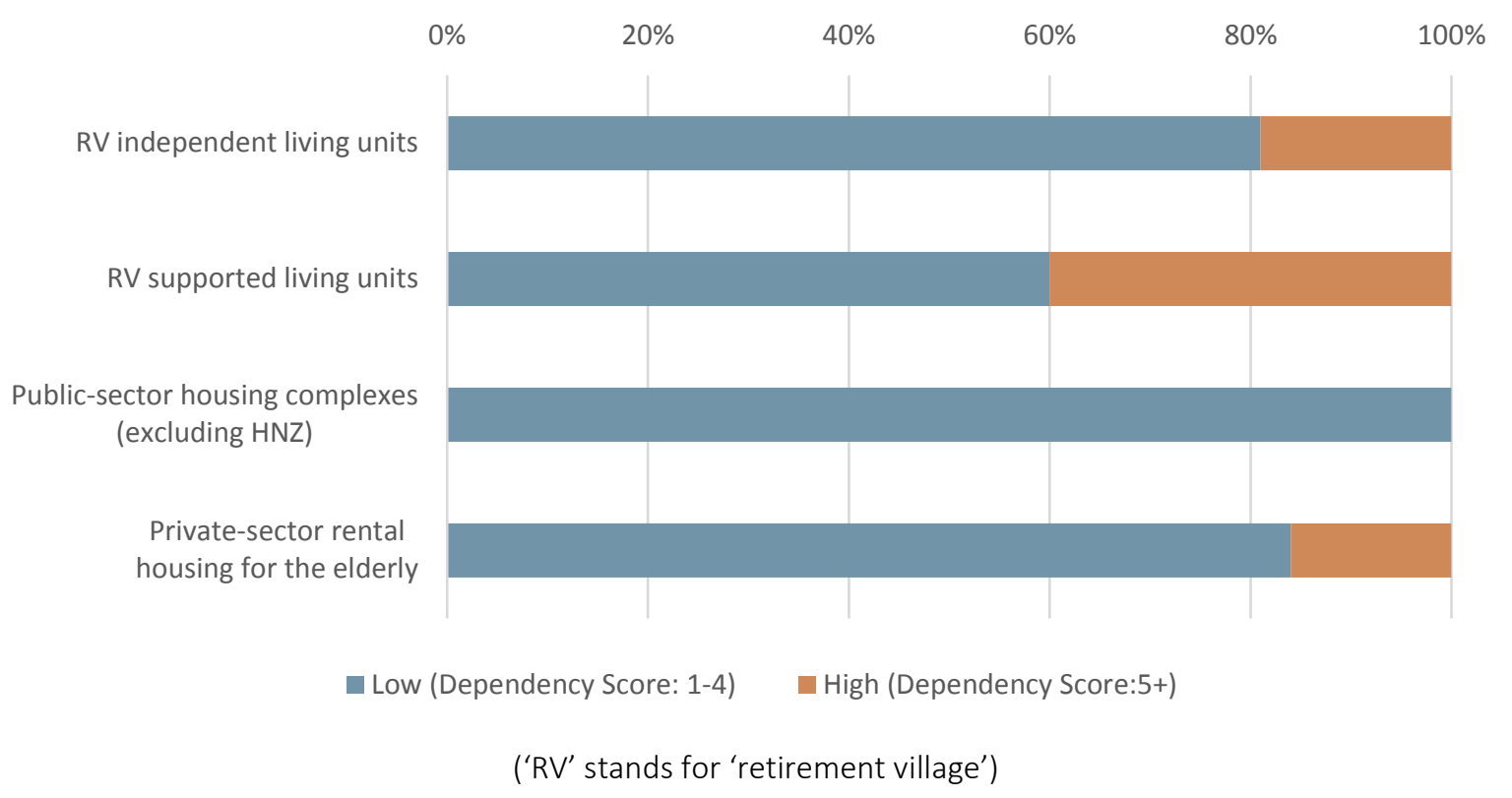

Figure 5-9: Levels of dependency among residents receiving personal care

Both the mean Dependency Score and the percentages of those who received personal care in six activities were highest in the retirement-village supported-living units $(2.14,46 \%)$, followed by the private-sector rental housing $(0.58,21 \%)$ and retirement-village independent-living units $(0.35,12 \%)$. These data were the lowest in public-sector housing $(0.18,8 \%)$. Regarding the proportion of residents with high levels of dependency, retirement-village supported-living units also accommodated the highest proportion of them (40\%). Retirement-village independentliving units accommodated the second largest proportion of residents with high levels of dependency (19\%), which was followed by private-sector rental housing for the elderly (16\%). Public-sector housing accommodated no residents with a high level of dependency.

\section{Assistance required by the levels of dependency}

The data on the status of personal care are compared between two groups with different levels of dependency: low levels (Dependency Score: 1-4) and high levels (Dependency Score: 5 and over) (Figure 5-10). 


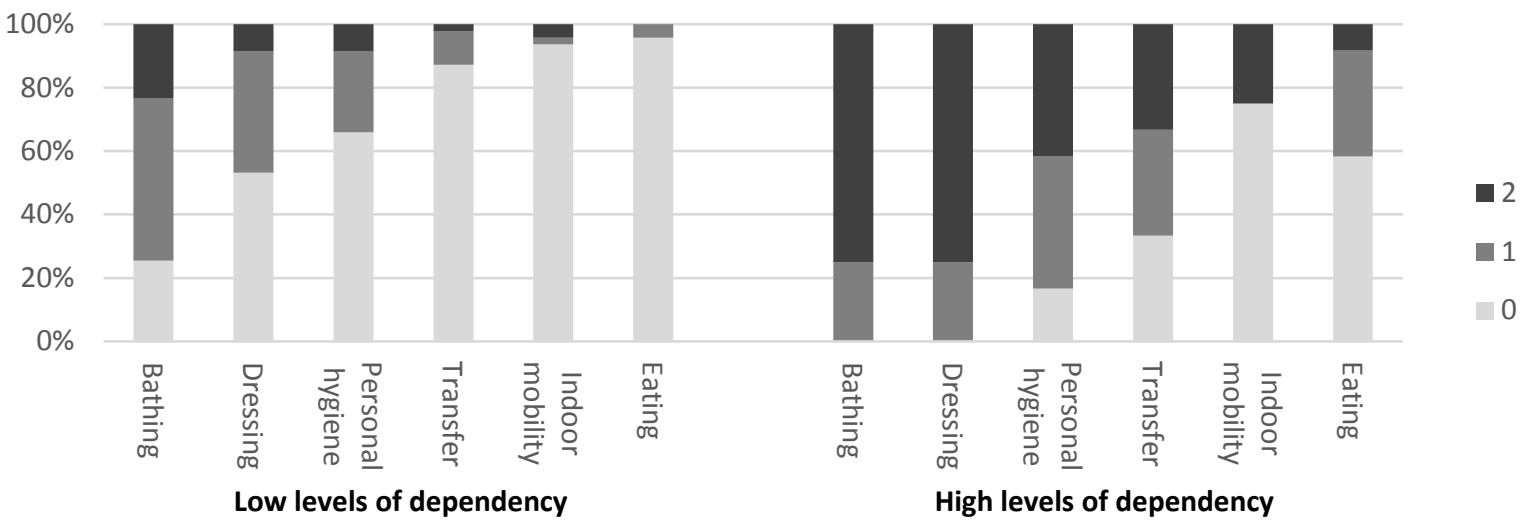

Figure 5-10: Type of assistance required

Where the dependency level was lower, elderly people often required assistance in bathing, dressing and grooming, with a small proportion of people requiring assistance with transferring from bed to wheelchair/chair, mobility and feeding. As the levels of dependency grew higher, the types of assistance required increased; residents needed assistance both in bathing and dressing, and the proportion of those who required assistance in transfer, indoor mobility and eating increased.

\section{Type of caregivers}

There are two types of caregivers; informal caregivers (family/friends) and formal caregivers (professional caregivers). The results for the type of caregivers offering assistance in any of the six activities is shown in Table 5-7.

Table 5-7: Proportion of residents receiving care from professional caregivers or family/friends

\begin{tabular}{|l|l|l|}
\hline \multirow{2}{*}{ Type of housing complexes } & \multicolumn{2}{|l|}{ Of those who require care in any of the six activities } \\
\cline { 2 - 3 } & $\begin{array}{l}\text { Receive care from } \\
\text { family/friends }\end{array}$ & $\begin{array}{l}\text { Receive care from } \\
\text { professional caregivers }\end{array}$ \\
\hline RV independent-living units & $45 \%$ & $81 \%$ \\
\hline RV supported-living units & $30 \%$ & $100 \%$ \\
\hline $\begin{array}{l}\text { Public-sector housing complexes } \\
\text { (excluding HNZ) }\end{array}$ & $33 \%$ & $67 \%$ \\
\hline $\begin{array}{l}\text { Private-sector rental housing } \\
\text { complexes for the elderly }\end{array}$ & $47 \%$ & $74 \%$ \\
\hline Average & $43 \%$ & $81 \%$ \\
\hline
\end{tabular}

('RV' stands for 'retirement village')

Of the elderly who received at least one type of personal care, over $80 \%$ were assisted by professional caregivers. This trend was more prominent in retirement-villages than any other housing types. 


\subsubsection{Summary: Feature of each type of housing}

Synthesising the data found through two questionnaires by the housing type, the features of each type of housing are summarised.

\section{Retirement villages}

The size of retirement-villages varied; villages with over 200 units comprised one-third of those surveyed, and those with less than 20 units one-quarter. All villages provided independent-living units and many also provided supported-living units. Supported-living units accounted for approximately $30 \%$ of the total number of units on average, among villages supplying supportedliving units. Many were adjacent to residential care facilities. The majority of units had access and facilities for the disabled and residents were provided with a greater variety of communal facilities, such as a lounge and dining rooms. Retirement villages provided the widest range of services of all housing types, such as emergency on-call 24 hours a day, regular resident visits and organised activities and outings for no extra cost (the costs are often included as part of the 'Licence to Occupy', or in the 'Weekly Fees'). Assistance with transportation, meals, laundry and other support with household tasks was provided either inclusively or with extra cost in these units.

Supported-living units were normally adjacent to residential care facilities and residents had access to a dining room as well as a lounge. The services for residents in supported-living units were greater than those provided in independent-living units; meals, household assistance and personal care were provided in all supported-living units, whereas some residents in independentliving units did not have access to support provided by the village. Supported-living units accommodated elderly residents with much higher levels of dependency than independent-living units did.

\section{Public-sector housing}

The majority of public-sector housing complexes were made up of less than 20 units, but the size varied and over $10 \%$ were large complexes with over 100 units. The units suitable for the disabled were few and most complexes had either no communal space or only a lounge or community room. At over half of the complexes, residents were provided with medium levels of services. Sometimes, this included emergency on-call service and social support such as regular staff visits and organised activities and outings. The proportion of residents receiving personal care was low, and this type of housing normally failed to accommodate those with high levels of dependency. 


\section{Private-sector rental housing for the elderly}

The size of most private-sector housing complexes for the elderly was small, with 20 units or less. The proportion of units that had access and facilities for the disabled was low. The communal spaces provided for residents were also limited and varied in the type of space. Most complexes provided regular staff visits, which were sometimes accompanied by other services varying between complexes; for example, some provided meals at a cost or included in the rent, and personal care at a cost. The proportion of residents receiving personal care was relatively high (one in five), which was higher than in independent-living units in retirement villages. However, the proportion of those with high levels of dependency was low (16\%) compared to independent-living units in retirement villages (19\%).

\subsection{Discussion and conclusion}

This Phase 1 study investigated the current situations of housing for the elderly and the elderly residents through two questionnaires, one on the models of care and physical environments of three types of housing (retirement villages, public-sector housing (excluding HNZ) and privatesector rental housing for the elderly), the second on the care requirements of the residents. This responded to the first objective of this research as stated in Chapter 4, which was to establish the models of care and the physical environment of housing and the care requirements of residents. The findings provided information that was not publicly available. Through comparison of data for the three housing types, it also identified the features of each.

Chapter 2 has shown that retirement villages are the most desirable option for those with sufficient capital, and the main housing option for the elderly who cannot afford to live in retirement villages is rental housing. The research in this chapter has demonstrated that, in retirement villages, the elderly who need assistance are aided to live independently with a wide range of services, which has resulted in attracting a high proportion of elderly residents with high levels of dependency. In contrast, rental housing provided by both the public- and private-sectors is less likely to provide high levels of services for the elderly, with some exceptions in privatesector rental housing. For those individuals who require rental housing, options for elderly people who need high levels of care are scarce; these individuals are more likely to have no option but to enter residential care. 
The objective of encouraging the high-needs elderly to live independently as long as possible cannot be achieved without suitable housing combined with service and care that supports the elderly person's independent life. This is also a key focus in the New Zealand policies related to housing for the elderly (MSD, 2001, 2019). However, the combination of service provision with housing creates difficulties for housing providers. In particular, the public and private-sector rental housing providers are limited in what they can afford with the limited resources made available from low-income rentals. Rental housing providers, particularly in the private-sector, operate with smaller numbers of units, making provision of additional in-house services uneconomic. Both public- and private-sector rental housing typically operates with an older housing stock that has not been designed for elderly people who have mobility issues or are not fully independent.

With limited resources comes the need for expanded ways of thinking and greater creativity. Strategies for housing with limited resources to improve the quality of space for the high-needs elderly include more flexible design and more efficient use of adjacent or community resources and facilities. For example:

a. In public and private-sector rental housing with medium levels of care, the communal space, often called the 'community room', could be easily adapted for various organised activities and other services.

b. Physically locating the housing units adjacent to care service providers, such as homecare service stations and residential care facilities, could be used as a strategy for providing high levels of care (as seen in the supported-living units in retirement villages and some private-sector rental housing complexes). By using the spaces and staff of the service providers, services and care such as organised activities, meals and home-care can be provided with greater ease and flexibility.

c. For rental-housing residents, activities and other services could be provided using public/private facilities in the community. The service provision could be accompanied either by its close proximity to housing or by providing transportation services for those with mobility difficulties.

Increasingly, providers of social housing for the elderly such as CHPs are expected to exhibit greater innovation and develop better combinations of housing and services to meet people's needs (NZG, 2015b). Partnerships between housing providers and service and care providers 
could also offer a more supportive service system for the elderly to live independently. There have been some local authorities that facilitate high levels of care for the elderly through partnerships between housing and/or services providers, such as DHBs and private care providers (Davey et al., 2004; Reid, 2008), though this study could identify no such example in publicsector housing in the Wellington Region. It has been expected that more innovative models of housing with care will be developed by $\mathrm{CHPs}$ and intersectoral collaboration. For the development of such housing, consideration for the design aspects is essential, as considered design can provide a more effective combination of care with housing. This study has identified some strategies such as the layout and flexible design of communal spaces. Consideration would also be necessary for accommodating care in private spaces. For example, given that assistance in bathing/showering and dressing are major requirements for those with care needs, the design of a bathroom should comfortably accommodate the resident and their caregiver. Greater knowledge is required to accommodate necessary services and care for the elderly in an effective way in every space of housing units and complexes.

In relation to the research questions of this thesis, the investigation in this chapter has illuminated the need for improvements in both the physical environments and in the models of care particularly in rental housing, as a context for developing a design framework for housing that enables the high-needs elderly to live independently. The research finds that agencies that provide both housing and care are most common in retirement villages of three types; however, in retirement village supported living units, that have designed to accommodate the high-needs, problems with regard to a low QoL of residents have been reported (Hayward, 2012). In all housing types in both the private- and public-sectors, further research is needed for specific housing models that could provide greater QoL along with optimal services to accommodate the elderly requiring care.

Finally, there are some limitations in this study. First, only limited information was collected with regard to the physical environments of housing that are suitable for the elderly; further investigation would be required with greater attention to the details of physical environments, which could be investigated in the following phase. Second, the research has analysed the degree of services provided in relation to the requirement for extra costs, but without the information on the costs included in the 'Licence to Occupy', or in the 'Weekly Fees' or the rent. Including this information would provide a more accurate understanding of the range of service provision. 


\section{CHAPTER 6: METHODOLOGY AND METHODS FOR RESEARCH ON QOL IN RELATION TO PHYSICAL ENVIRONMENTS (PHASE 2)}

The literature review in Chapter 3 identified significant gaps in the existing knowledge useful for the design of housing that improves the QoL of the high-needs elderly and led to the question 'What is the optimal framework for the design of housing for the high-needs elderly that will address the wide range of aspects of their QoL in New Zealand?'. Phase 1 of the methods section consisted of a quantitative investigation into the current situations of housing for the elderly and the elderly residents - more specifically, on the models of care and physical environments of housing as well as care requirements of the residents. This investigation illuminated the need for improvements in both the physical environments of housing along with the models of care, and that the design has a great potential to help the efficient combination of care with housing; however, it did not focus on the impact on the resident QoL. This chapter discusses the usefulness of methodology for the investigation of the QoL of elderly residents in relation to physical environments through examination of quantitative and qualitative approaches, which establishes the combination of qualitative methods suited for this study. This is followed by a detailed design of procedures for data collection, analysis and presentation. Implementation of these procedures will draw findings, which will be presented in Chapter 7.

\subsection{Exploration of methodology}

In this section, literature is re-examined to clarify the most effective methodology for the development of a framework for the design of housing for the high-needs elderly that addresses the wide range of their QoL. The reviewed literature includes the qualitative and quantitative research focusing on the relationships between their QoL and housing design or physical environments.

There are many pieces of quantitative research examining the relationships between the housing environment and resident QoL (Callaghan \& Towers, 2014; Iwarsson \& Isacsson, 1997; Nakhodaeezadeh, Jafarabadi, Allahverdipour, Matlabi, \& Dehkordi, 2017; Orrell et al., 2013; Sarah Barnes, 2006; Sikorska, 1999). However, studies provided only limited insights into housing design with respect to those factors that impacted on QoL through the conversion into quantifiable values. More specifically, they examined the influence of those design elements that could be represented by values, such as the size of private space and the number of communal 
spaces; however, many failed to provide core information that is difficult to represent by values but crucial to housing design, such as the organisation of spaces and the use of materials. For example, an UK study examined the relationships between 'graduation of space (the variety of spaces afforded in terms of the levels of privacy, such as private, semi-private and public spaces)' in care homes on resident QoL (Sarah Barnes, 2006). This study developed and used a scale to measure the extent of graduation of space provided by the building ${ }^{20}$, and showed a positive association between the graduation of space score and resident wellbeing; however, it did not provide information essential to design such as where and how each space should be laid out. Moreover, the critical weakness common to most quantitative methods is that they hypothesise the architectural elements influential to QoL which set the variables before the data collection. This strategy cannot capture the holistic nature and complexity of interrelationships including the potential influence of elements which have not been established.

In the sphere of qualitative research, many studies fragmentally focused on the relationships between QoL and physical environments, either focusing on a limited range of QoL with limited description of relevant physical environments (Cooney et al., 2014; Dyck et al., 2005; Hale et al., 2010; Kontos, 1998; Nord, 2011) or focusing on particular aspects of physical environments and their influences on the QoL (E. C. King et al., 2016; Morales et al., 2012; Schillmeier, 2017). There have been few studies focusing on a wide range of aspects of QoL and the influences of physical environments. One recent study, which applied a mixed method of qualitative and quantitative aspects, investigated the relationships between the QoL for elderly residents in residential care facilities and their physical environments (Nordin et al., 2017). Along with the use of quantitative measures for physical environments and resident activities and affective states, qualitative field notes were recorded through unstructured observations of the physical environment, and walk-along interviews with residents, a relative and staff members. The qualitative part of the investigation found rich and useful information for housing design that improves various aspects of resident QoL, such as independence, social interactions, activities and accommodating personal belongings. The design considerations that emerged from the qualitative part of the study included many aspects such as the interior layout, floor materials, types of doors, windows and fixtures and types of spaces, as well as the availability of assistive devices and the size of rooms. The number of the design considerations were small (17 in total) and included limited and segmented knowledge but did not holistically address their QoL.

\footnotetext{
${ }^{20}$ This scale consisted 12 items such as 'range of spaces for sitting in circulation space,' 'choice of locations for daily activities' 'space for family gatherings,' and 'kitchenette to make drinks' and the score is represented by the proportion the 12 items that are present or not.
} 
However, this research showed the potential of a qualitative method in clarifying design considerations to address a wide range of aspects of QoL — something that has not been established. This success is explained by the nature of qualitative research, in which the relationships between physical environments and QoL can be investigated through a naturalistic approach and can allow any theme to emerge freely in the analysis (Wang \& Groat, 2013).

\section{Ethnography}

While being rooted in anthropology, ethnography has been adopted by a number of other disciplines, including sociology, human geography and cultural studies (Wang \& Groat, 2013). It is suited to study on a particular group/culture, and requires researchers to spend considerable time in the field in order to find out how the members of the group/culture being studied understand things, and to holistically explain what they have seen (Juárez-Cedillo et al., 2015). In architecture, it has been particularly deemed useful for post-occupancy studies where the spatial usage of the occupants is examined (Lucas, 2016).

The primary mode of data collection is direct observation, often supplementing observation data with interview texts, documents or other artefacts for profound understanding and explanation of the reasons/contexts behind the behaviour/event (Crouch \& Pearce, 2012; Wang \& Groat, 2013). Observations and unstructured interviews are particularly complementary methods; conducting unstructured interviews during the flow of work is useful for deepening understanding (Crabtree, Rouncefield, \& Tolmie, 2012; Crouch \& Pearce, 2012).

The limitations of this approach are that it cannot collect information on what doesn't take place during the study period; for example, it cannot capture what an individual would like to do but actually cannot as it is not observable, therefore the physical environments that could address the aspects of QoL cannot be found. The additional use of semi-structured interviews with ethnographical methods can overcome this weakness. A semi-structured interview allows a researcher to prepare a list of questions to obtain information that cannot be collected during the observation and maintain the consistency of data collected across participants (Denscombe, 2014). The relaxation of structured interviews allow interviewees 'develop ideas and speak more widely on the issues raised by the researcher (Denscombe, 2014, p. 187)', which often enables the collection of their in-depth opinions, feelings, emotions and experiences (Denscombe, 2014). This mixing of methods, is commonplace in qualitative research (Crouch \& Pearce, 2012), and the advantages of the combination include providing the researcher with a fuller perspective (Crouch \& Pearce, 2012). In the context of this research, combining ethnography with semistructured interviews can clarify various aspects of QoL for participants, providing clear accounts 
of physical factors relating to such aspects of QoL. The combination of methods also has a benefit of providing opportunities for triangulation of data which leads to the greater credibility of the research (Crouch \& Pearce, 2012; Frances, 2000).

\section{Focus groups}

Another possible qualitative method to note would be the use of focus groups. A focus group is a discussion conducted by a group made up of people (typically five to ten) who have certain characteristics in common, to explore a specific set of issues (Kitzinger \& Barbour, 1999; Krueger $\&$ Casey, 2000) and is distinguishable from a group interview in that participants in focus groups are encouraged to talk to one another, ask questions, exchange anecdotes, and comment on each other's experiences and points of view — allowing a coproduction of knowledge and strategies for transcending their circumstances (Kitzinger \& Barbour, 1999). Originally established in social anthropology, media/cultural studies and health research (Kitzinger \& Barbour, 1999), it has been widely used in housing research to collect the perceptions of housing occupants (Hall, 2008; Howden-Chapman et al., 2000; Lewis et al., 2010). For example, the EVOLVE tools reviewed in Chapter 3 were developed using focus groups of the elderly and other related people as part of the methods (Lewis et al., 2010). However, the EVOLVE design framework included only limited information on the relationships between the physical environments and the QoL for the high-needs elderly, and the reasons for the requirements were often unclear. But more importantly, the gaps in existing knowledge were in the requirements for private space that would facilitate QoL. These require more sensitive and intimate investigation with regard to the usage of private space. An investigation through the use of focus groups would not delve into the close examination of spatial circumstances and the influences on occupants' QoL in the 'field,' and would be less likely address this gap. For these reasons, an ethnographical approach is deemed to be better suited for the objective of this research.

\section{Validity and bias}

In qualitative research, validity is a concept that cannot be dismissed, as it 'points to a question: Are these findings sufficiently authentic (isomorphic to some reality, trustworthy, related to the way others construct their social worlds)?' (Denzin \& Lincoln, 2000, p. 120). It is said that qualitative research is characterised by the robust internal validity, which can be enforced by the use of multiple tactics and the researchers asking a series of questions (Juárez-Cedillo et al., 2015; Wang \& Groat, 2013). On the other hand, its limitations include weakness in the external validity (Juárez-Cedillo et al., 2015). External validity refers to the extent to which the results of a study can be generalized to other settings (ecological validity), other people (population 
validity) and over time (historical validity), and it is increased by the more 'natural' settings (McLeod, 2013). Ethnography is said to have strong 'ecological validity' (Denscombe, 2014); however, the ecological validity of ethnography is challenged by the influence of the researcher's presence on the behaviour of those who are observed, which makes what is observed 'never be wholly natural setting' (Crouch \& Pearce, 2012, p. 94). In addition, there is the risk that the researcher's interpretation 'relies on what the researcher already knows and already believes' (Denscombe, 2014, p. 88)' and that this pre-existing knowledge or assumption may cause bias. This bias is shown in Crouch \& Pearce's argument regarding 'insider' and 'outsider' researchers in ethnography:

Observing a research setting as an insider reduces considerably the potential for fresh insights, as much of the setting that would seem new and different to an outsider will escape an insider's attention. Insider researchers need to be particularly aware of the need to problematize common sense perspectives and to try to see the familiar as if it were strange. The outside observer, while having focusing attention on what is novel or exotic to them and so losing sight of the everyday experiences. There are thus different challenges associated with making the familiar strange, and making the strange familiar (Crouch \& Pearce, 2012, p. 94).

For example, the researcher's age (half or less than the age of the elderly participants) would provide the researcher with completely outsider perspective in addition to her cultural background from Japan.

\subsection{Data collection}

The information was collected from both the elderly residents and their caregivers. Data collection from caregivers was essential to collecting the information on the quality-of-care, one of the important components of QoL, and related spatial factors. In addition, data collection from more than one party effectuates the triangulation by obtaining different sources of data, which enforces the validity (Crouch \& Pearce, 2012).

\subsubsection{Participant selection}

Resident participants were recruited from the Phase 1 questionnaires which explored the features of the current housing for the elderly and the residents. Using these questionnaires, the eligibility was assessed against the selection criteria of; being 70 years and older; receiving personal care 
from professional caregivers; and being willing to participate in this survey. The reason of limiting the age to 70 years and older was to exclude the younger group who are not likely to have the features of ageing such as 'loss of viability and increase in vulnerability (Magalhães, 2013).' Fifty-nine possible participants, who met the criteria mentioned above were identified. Of these, 30 residents actually participated. Exclusion criteria included those with severe cognitive impairments, as they were unable to complete the survey, those with serious deterioration in health, potential participants who moved out or passed away during the study, and those who declined to participate. The professional caregivers were contacted through the resident participants, and 21 caregivers agreed to participate.

\section{Sample size and bias in participant selection}

In qualitative studies, the adequacy of the sample size depends on the research methods. For example, Morse (2000) maintained that the necessary sample size depends on the amount and richness of the data obtained. A large number of participants (at least 30 to 60) is only needed if only relatively shallow data is required and fewer participants in the study (perhaps only 6 to 10) are suited if a large amount of data for each participant is obtained (Morse, 2000). The datacollection method in this research combines multiple tactics of a semi-structured interview and ethnographical observation for each participant, generating rich data. Therefore, the sample size of 30 is considered sufficient to draw meaningful results.

This study acknowledges there may be biases through the participant selection which include the volunteer bias and the bias in their health conditions (Kopec, Sinclair, \& Matthes, 2010). It is well known that there are groups of people who are more likely to volunteer their time for a research study than other people; for example, they might agree to participate because 'they are motivated and concerned about an issue at hand, or simply have more free time in their lives to commit to the surveys' (Kopec et al., 2010, p. 86). In this survey, because participants of this study were recruited from those who were willing to participate, they might be more aware of the issues related to this study, or do have more problems with their living environment than the average. It is important to acknowledge this bias, as the QoL of residents in this survey may be lower than the average of the cohorts.

\subsubsection{Procedures}

The data collection methods are described in three categories of; 1) the physical environment; 2) from residents; and 3) from their caregivers. The survey normally took two-three days per person. The procedures are outlined in Figure 6-1. 


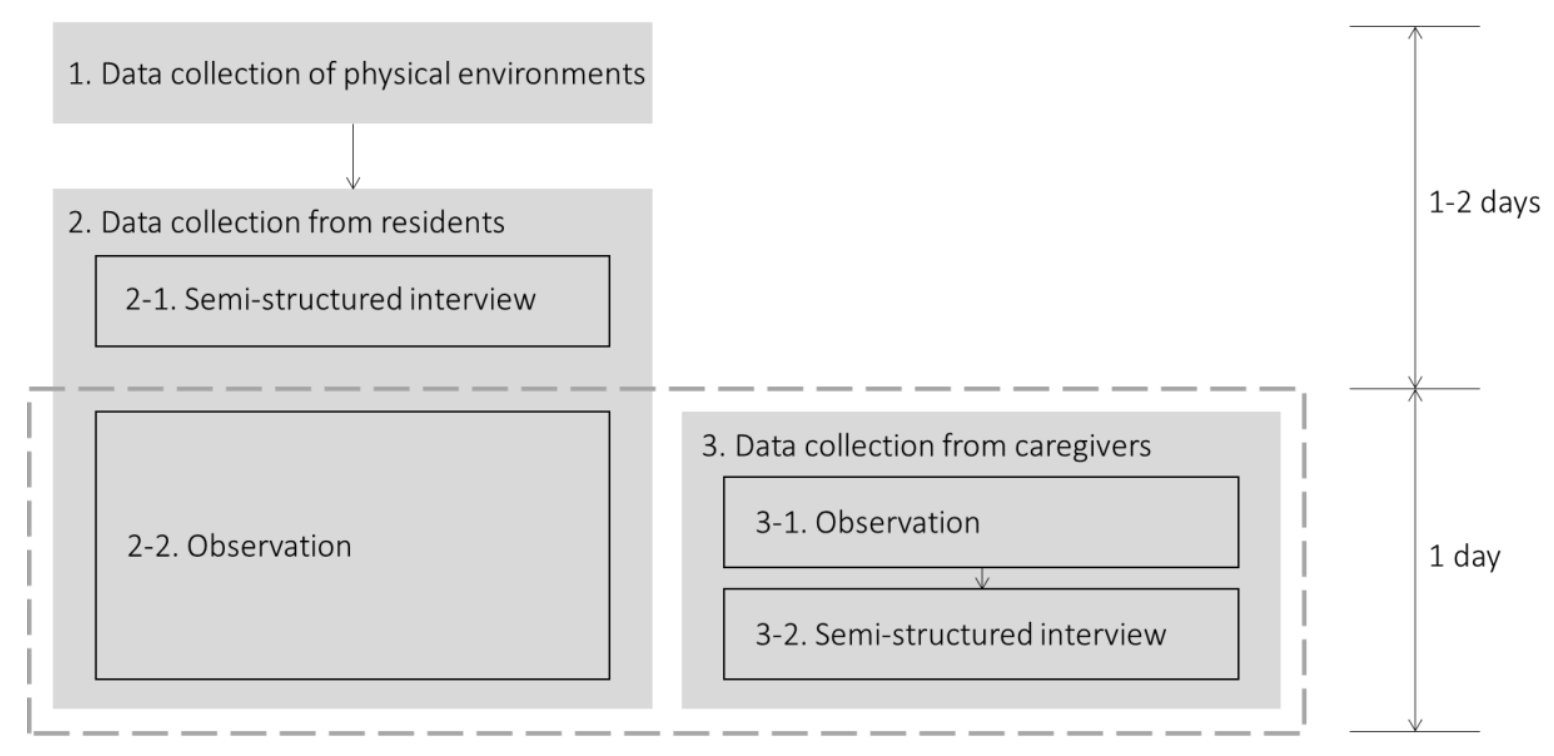

Figure 6-1: Data collection procedures

\section{Data collection of physical environments}

Data on physical environments of individual units, as well as public spaces in housing complexes, were collected through the documentation of physical environments. This included the measurement of the layout of the complex, buildings and rooms, furniture and fixtures, which were collected in plans, sketches and field notes, using laser and tape measures. Photographs were also taken. The drawings of units took between half an hour and two hours per unit depending on the size. Site and floor plans were provided by the housing operators, if available. Plan sketches were digitised in a CAD software for analyses of the spaces (Appendix 2).

\section{Data collection from residents}

Data were collected from residents regarding their perceptions, experiences and spatial use, through semi-structured interviews and day-time observation.

\section{2-1. Semi-structured interviews}

Semi-structured interviews were conducted to collect resident's perceptions and experiences. For creating the interview schedule, the themes for QoL conceptualised through the literature review in Chapter 3 were used, which included; 'independence,' 'privacy,' 'personal identity,' 'activities, 'relationships,' and 'quality of care.' To collect the in-depth data, 'it is important to choose questions which are open (rather than closed), and which do not make too many assumptions towards particular answers (Smith, Flowers, \& Larkin, 2009).' In addition to questions relating themes for QoL, questions for basic information of participants and those for their overall perceptions were prepared. All the questions were put in order so that more general/easy questions come first to quickly make participants become comfortable talking, and questions that 
are sensitive or need deliberation were put later (Table 6-1). In case they could not think of answers or in order to delve into the topic, some prompts and sub-questions were prepared for each question; particularly, it was deemed useful to use prompts such as 'Can you tell me a bit more about that? (Smith et al., 2009).'

Table 6-1: Interview questions for residents

\begin{tabular}{|l|l|}
\hline $\begin{array}{l}\text { Themes found in } \\
\text { literature review }\end{array}$ & Interview questions \\
\hline Basic information & 1. How long have you lived here? \\
\hline Activities & 2. Can you tell me about your most important activities? \\
\hline Relationships & 3. Can you tell me about your most important people? \\
\hline Basic information & 4. Can you tell me about your health conditions (diseases, disabilities, pains)? \\
\hline Independence & 5. Can you describe any difficulties that you experience when moving? \\
\hline Independence & 6. Can you tell me how convenient is the set-up of this house for you? \\
\hline Independence & 7. What do you think about the size of this house? \\
\hline Overall & 8. Can you describe your favourite space in this house, and why? \\
\hline Overall & 9. Can you describe the space you don't like in this house, and why? \\
\hline Identity & 10. Can you tell me the space that you customised after moving? \\
\hline Identity & 11. Can you tell me the most important possessions in this house, and why? \\
\hline Privacy & $\begin{array}{l}\text { 12. Can you describe any concern in privacy while living in this house or in this } \\
\text { village/complex? }\end{array}$ \\
\hline Identity & 13. Can you tell me about your previous house, that you moved from? \\
\hline Independence & 14. What is the reason that you moved house? \\
\hline Identity & 15. What kind of things of your previous house do you wish for this house? \\
\hline Overall & 16. How do you like this village/complex overall? \\
\hline Relationships & 17. How do you like the [communal space]? \\
\hline Relationships & 18. What kind of facilities do you use in this village/complex? \\
\hline Relationships & $\begin{array}{l}\text { 19. How do you like the way that this village/complex is connected or integrated } \\
\text { to the wider community? }\end{array}$ \\
\hline Basic information & 20. What was your past occupation? (If this has not been clarified) \\
\hline
\end{tabular}

Interviews were conducted in the participants' dwellings, to remind them more clearly of their experiences in their space. Interviews were audio-recorded supplemented with interview notes. Each interview took at least one hour, and the longest interview was 3-hours long. Most sessions were completed in a single session, but the interview for one participant was divided into three sessions due to her fatigue.

\section{2-2. Observation}

Observation of residents was conducted to witness the interaction between the user and physical environments first hand, so as to investigate the role of physical environments in influencing resident QoL. The researcher stayed for seven-eight hours in residents' house during the daytime on a typical day when their caregiver visited. The starting and ending times were arranged so that the whole caregiver visits could be observed, as well as meeting residents' preference. The duration of observation was established to holistically capture residents' spatial use in daily life, as 
well as short enough to avoid tiring them too much. With a 7-8-hour stay, it was deemed sufficient to observe how they spend their free time in their private space, as well as some other daily activities such as having a meal. During observation, unstructured interviews were conducted to clarify their behaviours, which is common and effective in ethnographical studies (Crouch \& Pearce, 2012). The resident was told that the researcher would like to observe their usual life; however, their conversation with the researcher was allowed if they wanted to do so.

The presence of the researcher was likely to affect residents' behaviours — by that their behaviours could not be completely natural. However, the interaction with residents could be regarded as part of a natural setting. Crouch \& Peace clarifies;

As the research progresses, you may find that the process continues beyond the formal data collection period as you bump into participants regularly and become drawn in to the natural interactions that take place in the setting where you are observing. Although such opportunistic interactions may not have formed part of your planned observations, they nevertheless will be invaluable (2012, p. 96).

The informal conversation was certainly invaluable in the observation sessions of this research, where residents 'pretended to' perform as usually as possible as if the researcher was not there; they could do so, at times, by providing an explanation for their behaviours which could have been seen just strange otherwise. Their explanation was definitely useful for deepening the researcher understanding of their behaviours. The resident's reaction to the researcher was sometimes that of guests, which helped reveal their sense of privacy.

In ethnography, it is important to develop 'coherent analytic accounts,' through 'thick description,' which is to report the sequence of the work with its rich context (Crabtree et al., 2012). For this aim, observation notes were taken continuously during the observation by the researcher. Floor plans of the unit were used, and information was noted on the location of the resident and their behaviours over time. When unstructured interviews or informal conversation occurred, they were noted as much as possible. The researcher comments and interpretations were noted as well. In order to fully record their comments, it was useful to write them down while the experience was fresh in memory (Crouch \& Pearce, 2012); therefore it was done during the observation sessions, and additional notes were written up in full as soon as the completion of the session. 


\section{Data collection from caregivers}

Data from caregivers were collected through observations and interviews.

\section{3-1. Observation}

Caregivers were observed during the observation sessions for residents. The data collection method was similar to the resident observation; the information on their location, behaviours, time and what they said was collected. Unstructured interviews were conducted if necessary. The data were recorded in the observation notes. The caregiver information was noted on the same form as the resident information, using different pen colours, so that the different spatial use of the two persons could be distinguished.

\section{3-2. Semi-structured interviews}

Using semi-structured interviews, caregivers' views were collected with respect to the care for residents in relation to the physical environment. Interview schedules were created in a similar manner for in the semi-structured interviews for residents (Table 6-2).

Table 6-2: Interview questions for caregivers

\begin{tabular}{|l|l|}
\hline $\begin{array}{l}\text { Themes found in } \\
\text { literature review }\end{array}$ & Interview questions \\
\hline Basic information & 1. What kind of assistance do you provide? \\
\hline Quality of care & 2. Can you describe any inconvenience that you find when assisting [Resident]? \\
\hline Quality of care & 3. How could it be improved? \\
\hline
\end{tabular}

The interview was conducted during the visit to the resident, after care activities, so that caregivers could better recall the care situation. This normally took 5-10 minutes. When participants did not want their care activities such as bathing and toileting to be observed, caregivers were questioned afterwards but in the same space as where the care was provided (e.g. in the bathroom), about the detailed spatial use as part of Question 1.

\subsubsection{Ethics considerations}

In ethnographical research, typically involving direct observation, ethical considerations are essential (Crouch \& Pearce, 2012; Denscombe, 2014). Ethical considerations 'rule out covert observation when individuals are going to be observed closely' (Crouch \& Pearce, 2012, p. 95). In this research, there were substantial considerations for ethical aspects, along with the process for obtaining ethics approval. The information sheets were prepared and explained separately for elderly residents and caregivers before their consent was given. The elderly participants could listen to the briefing of the survey with their family, if they wished. It was also important to gain approval from 'gatekeepers' (Denscombe, 2014, p. 85). In this research, the researcher contacted 
housing operators and explained the whole procedures with an information sheet before obtaining their consent.

\subsection{Data analysis and presentation}

The analysis of the data from semi-structured interviews and observation was conducted separately. In the course of the analysis, the data on physical environments (floor plans, sketches and photographs) were referred to and included in the analysis outcomes.

\section{Coding data from semi-structured interviews}

Semi-structured interviews were used to collect and organise data on resident QoL (perceptions and experiences) from residents and caregivers in relation with physical environments. Recorded data in this study were professionally transcribed, and cross-checked by the researcher while listening to the original recordings. In the analysis process, the interview notes were referred to when the recording was not clear, as well as used for triangulation. In qualitative research, interview transcripts, observation notes or other documentation must be coded, "reduced" to "data", in which some categorisation of the examined phenomena must be identified or screened out from the rest of the environment being studied (Wang \& Groat, 2013). When coding extensive raw data which the qualitative research generally produces, using a coding scheme is useful to 'retain mindfulness in coding' (Wang \& Groat, 2013, p. 246). In coding of data from semi-structured interviews, the coding scheme typically used in Interpretative Phenomenological Analysis was referred to as described by Smith, et al (2009). For this process, a table was created in an MS Word document with four columns, which showed transcript, explanatory comments, themes for QoL and relevant physical environments (Appendix 2). After reading and rereading the interview transcript, comprehensive explanatory comments were noted in the 'explanatory comments' column. This step aims to produce a comprehensive and detailed set of notes and comments on the data, and includes describing the meaning for participants, commenting on the contexts (including the description of physical environments) and identifying more abstract concepts that helps make sense of the data (Smith et al., 2009). The next step was developing emergent themes, where explanatory comments, along with original transcript, were reduced to produce a 'concise and pitchy' statement of what was important to the comments (Smith et al., 2009). The themes emerged were then put in the 'themes for QoL' column. This information was coded using different colours, by emergent themes for QoL. The related physical environments were noted in the 'relevant physical environments' column, which was thought to 
be useful in examining the relationships between themes for QoL and physical environments in the later phase.

\section{Coding data from observation}

Observation was conducted following an ethnographical approach. In ethnography, 'analysis is not something that happens after fieldwork, but runs through it' (Crabtree et al., 2012, p. 122). Therefore, some analytic accounts had been included in the field notes. Data were coded using a table in an MS Word document, similarly to the coding of data from semi-structured interviews. Data on time, participant activities, location and posture and resident remarks were filled in the table, based on observation notes. Researcher explanatory comments, themes for QoL and relevant design themes were also added, in the same way as in coding data from semi-structured interviews (Appendix 2).

\section{Organising the emergent themes into super-ordinate themes}

The final step in the analysis method used was to identify the patterns and connections between the emergent themes, which involved re-evaluation of some themes, and to emerge the superordinate themes at a higher level as a result of putting the themes together (Smith et al., 2009). In this process, themes for QoL were synthesized into six main themes;

1) Control in daily basic activities,

2) Meaningful leisure activities,

3) Meaningful relationships,

4) Maintenance of possessions,

5) Comfort, and

6) Quality of care.

For each theme of QoL, the influence of the physical environment was examined qualitatively. This examination involved an analysis for the elements of physical environments, which included existence of spatial elements and relationships between elements such as height, width, distances and directions.

\section{Data presentation}

Rich descriptions are generally valued in qualitative research (Denzin $\&$ Lincoln, 2000), and one main feature of ethnography is that the reporting of data typically takes up a substantial amount of text. Write-ups are the essence of ethnography, in which the thick description with a full context is generally presented (Crouch \& Pearce, 2012). The use of extracts is another feature of 
the report of findings in this method. They often form part of the final texts in ethnography, and should be labelled with participants' unique identifier to allow the reader to follow the story of individuals (Crouch \& Pearce, 2012; Smith et al., 2009). Participant identifiers have been used in the report of findings of this study, formed from the combination of an abbreviation for the housing type and a number. Four abbreviations of PU, PR, RVI and RVS were used to signify the housing types of public-sector rental housing units, private-sector rental housing for the elderly, retirement-village independent-living units, and retirement-village supported-living units, respectively. In addition to extracts, sketches and photographs are useful to enrich the description.

In the next chapter, findings of this study will be presented by emergent themes, which have been identified through the analysis described in this chapter. This report contains analytic accounts and a full description of contexts that support them, including extracts, sketches and photographs. This presentation is designed to effectively facilitate readers' understanding of participants' QoL. 


\section{CHAPTER 7: QOL OF THE HIGH-NEEDS ELDERLY IN RELATION TO PHYSICAL ENVIRONMENTS (PHASE 2)}

To understand the QoL of independently-living high-needs elderly individuals in relation to physical environments, it is important to understand their basic characteristics as people, as well as the other key stakeholders and physical elements that contribute to their QoL. Similarly, the settings that high-needs elderly people are occupying need careful consideration as these factors impact on individual's QoL. As QoL can be assessed with respect to six main themes, the analysis of these components can help to identify design features that should be considered to ensure a high QoL of the high-needs elderly.

\subsection{Understanding participants and settings}

In a high-needs care environment, two people interact as part of daily living: the resident and the person who assists in their daily care. The characteristics of the resident, such as age, gender, living arrangements, marital status, health conditions/impairments, types and degrees of care needs, and the use of mobility aids, have an important bearing on the resident's ability to use their space. Similarly, the characteristics of caregivers, such as gender, are relevant to particular elements for resident QoL. These data provide important basic information that has implications for the QoL of residents.

\subsubsection{Participants}

The basic information of participants is shown separately for residents and caregivers.

\section{Residents}

Resident participants' basic information, such as gender, age, ethnicity, living arrangements and marital status is listed in Table 7-1. Of the 30 residents, 13 were male and 17 were female. The majority $(n=19)$ were aged between 80 and 89 , and the remainder were either in their 70 s $(n=6)$ or 90s $(n=5)$. The participants' ethnicity was largely European and/or New Zealand, but included one Middle Eastern and one Asian individual. Most participants were living alone, while three were living with their partner. The majority were widowed, and the rest were either married $(n=4)$, divorced $(n=3)$ or unmarried $(n=2)$. 
Table 7-1: Age, gender, living arrangements and marital status of residents

\begin{tabular}{|c|c|c|c|c|c|c|c|c|c|}
\hline \multicolumn{2}{|l|}{ Gender } & \multicolumn{2}{|c|}{ Age group } & \multicolumn{2}{|l|}{ Ethnicity } & \multicolumn{2}{|c|}{ Living arrangements } & \multicolumn{2}{|c|}{ Marital Status } \\
\hline Male & 13 & $70-79$ & 6 & European/NZ & 28 & Alone & 27 & Widowed & 21 \\
\hline Female & 17 & $\begin{array}{l}80-89 \\
90-99\end{array}$ & $\begin{array}{r}19 \\
5\end{array}$ & $\begin{array}{l}\text { Middle Eastern } \\
\text { Asian }\end{array}$ & $\begin{array}{l}1 \\
1\end{array}$ & With partner & 3 & $\begin{array}{l}\text { Married } \\
\text { Divorced } \\
\text { Unmarried }\end{array}$ & $\begin{array}{l}4 \\
3 \\
2\end{array}$ \\
\hline
\end{tabular}

(the numbers in italics show the number of residents)

The number and type of conditions/impairments that were mentioned by residents are summarised in Figures 7-1 and 7-2. The majority mentioned more than one condition/impairment, and the type of conditions/impairments varied. The most common condition was pain or arthritis, which was experienced by half of the residents, followed by cardiac conditions and diabetes/high blood pressure $(n=8)$. Five residents had experienced one or more stroke. Urinary/bowel conditions, injuries by recent falls and sight impairments were mentioned by four residents.

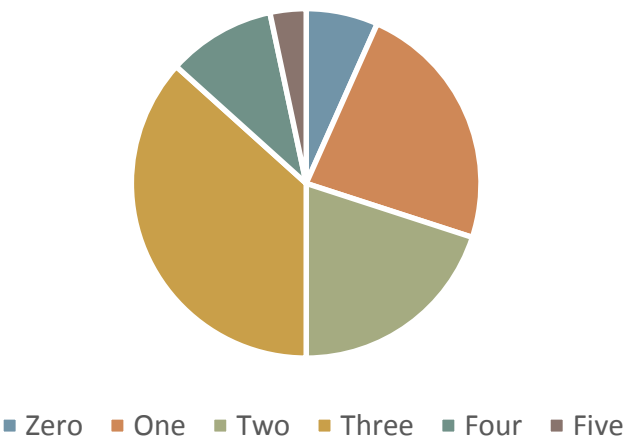

Figure 7-1: Number of conditions/impairments stated 


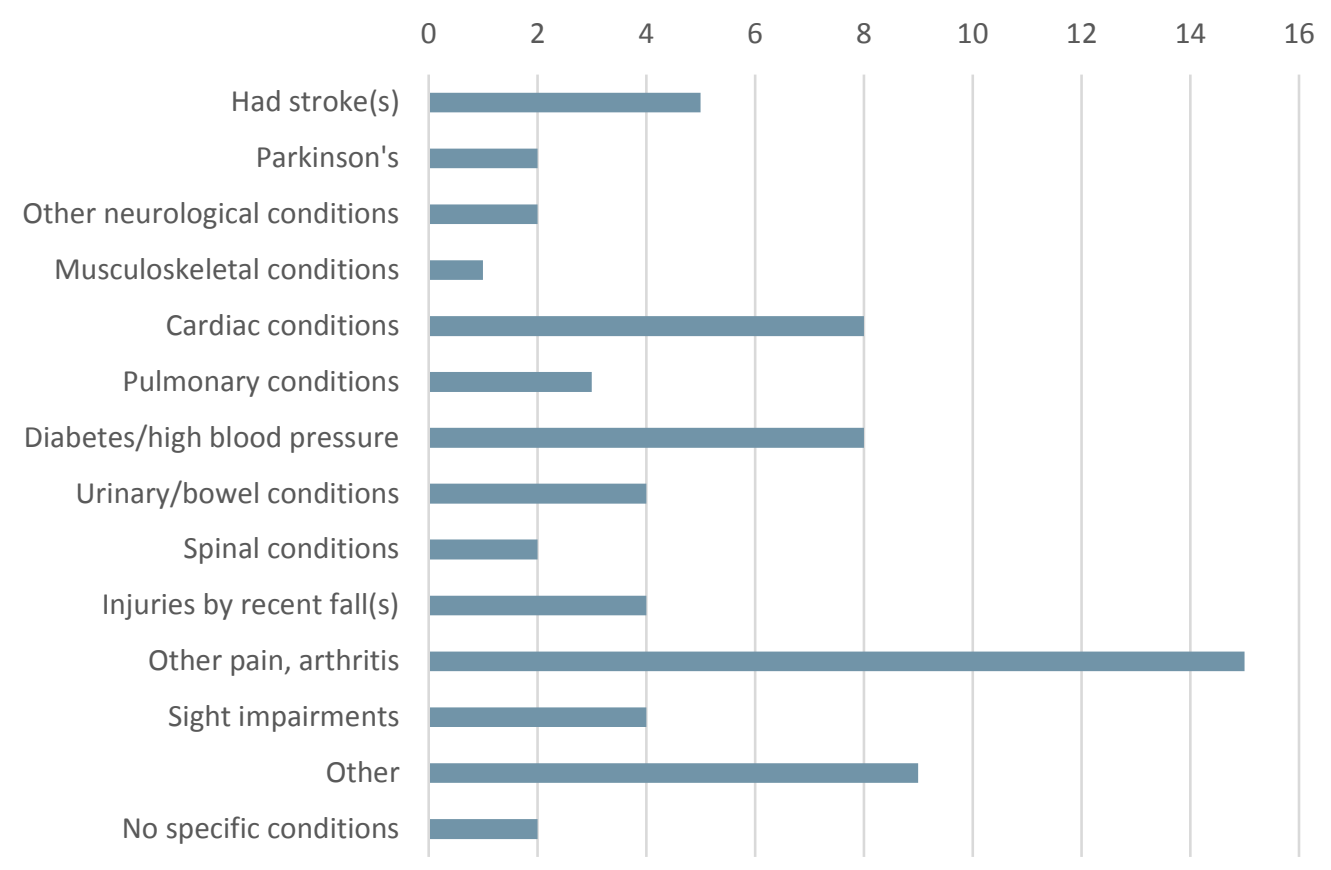

Figure 7-2: Type of conditions/impairments stated

Information on the assistance and the dependency levels of residents is summarised in Figure 73, Tables 7-2 and 7-3. The most common types of personal care required were showering $(n=23)$, dressing $(n=18)$ and personal hygiene $(n=10)$; five residents said they might require assistance in moving from bed to chair or wheelchair, and two in moving around indoors (Figure 7-3). 22 residents had lower levels of dependency (Dependency Score=1-4), and eight had high levels of dependency (Table 7-2). Most had household assistance once to twice a week or fortnightly, and three had assistance every day. The frequency of caregiver visits for personal care varied from one day to seven days a week; 13 were visited every day, and the rest were visited either for 1-2 days ( $\mathrm{n}=6)$, for 3-4 days ( $\mathrm{n}=6$ ) or for 5-6 days $(\mathrm{n}=5)$ (Table 7-3). 


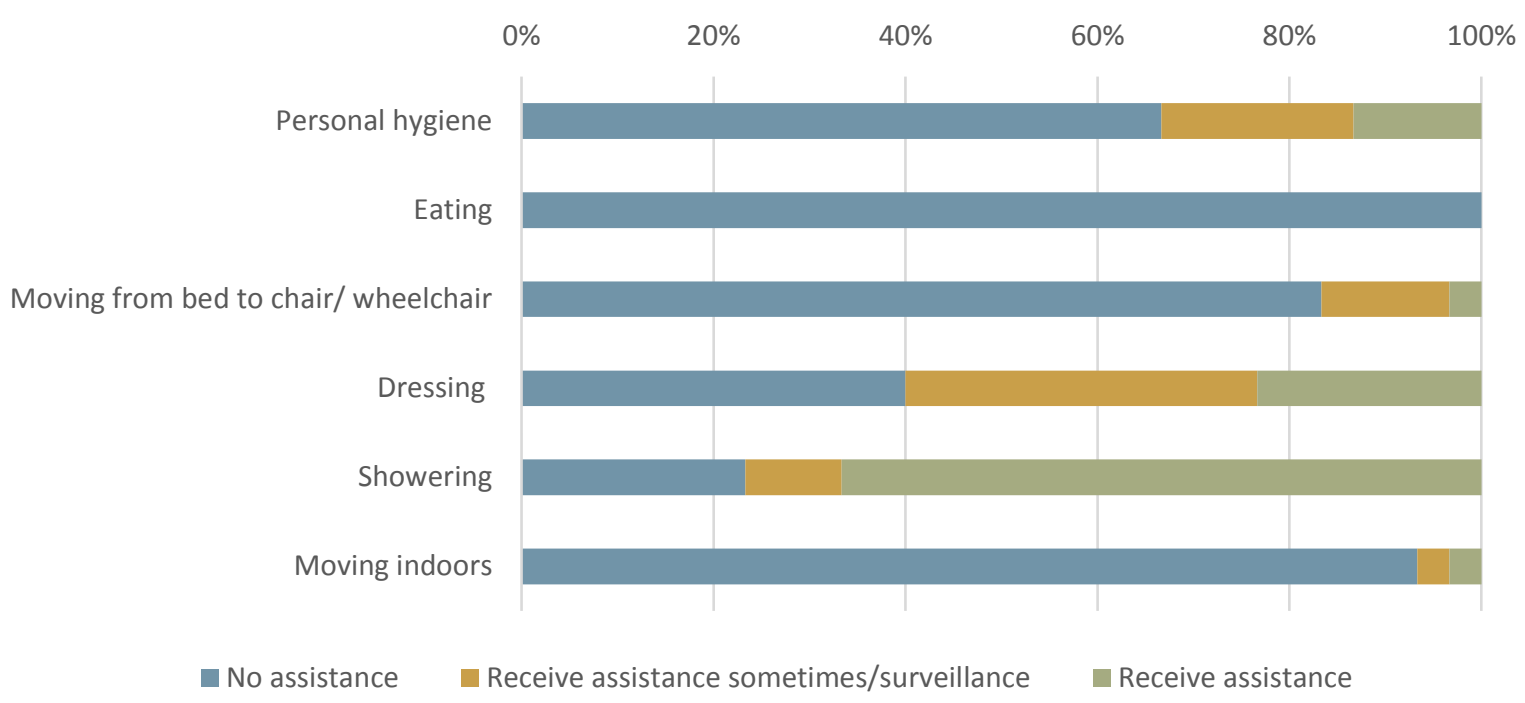

Figure 7-3: Requirements in assistance (personal care) by activities

(Moving indoors includes moving using walking aids, such as walking sticks, walker flames and wheelchairs)

Table 7-2: Levels of dependency

\begin{tabular}{|llr|}
\hline Higher levels of dependency & $\begin{array}{l}\text { (Dependency } \\
\text { Score=1-4) }\end{array}$ & 22 \\
Lower levels of dependency & $\begin{array}{l}\text { (Dependency } \\
\text { Score=5+) }\end{array}$ & 8 \\
\hline
\end{tabular}

(the numbers in italics show the number of residents)

Table 7-3: Frequency of professional caregiver visits

\begin{tabular}{|lr|lr|}
\hline $\begin{array}{l}\text { Visits for household assistance (per week) } \\
\text { (0.5: once a fortnight) }\end{array}$ & Visits for personal care (per week) \\
\hline None & 2 & $1-2$ days & 6 \\
$0.5-2$ days & 25 & $3-4$ days & 6 \\
7 days & 3 & $5-6$ days & 5 \\
& & 7 days & 13 \\
\hline
\end{tabular}

(the numbers in italics show the number of residents)

Most residents used mobility devices both indoors and/or outdoors. The type of mobility aids residents mainly used are shown in Figure 7-4. Outdoors, most residents used some mobility aids and only five (17\%) required no aids. The most common type of aid was a walker frame $(\mathrm{n}=13,43 \%)$, followed by a walking stick $(\mathrm{n}=5,17 \%)$, a wheelchair $(\mathrm{n}=4,13 \%)$ and a mobility scooter $(n=2,7 \%)$. Indoors, mobility aids were required less often; 10 residents (33\%) required no aids. Where they were used, the walker frame or a trolley were most common $(n=16,53 \%)$, and a walking stick and a wheelchair were also used by two residents (7\%). 


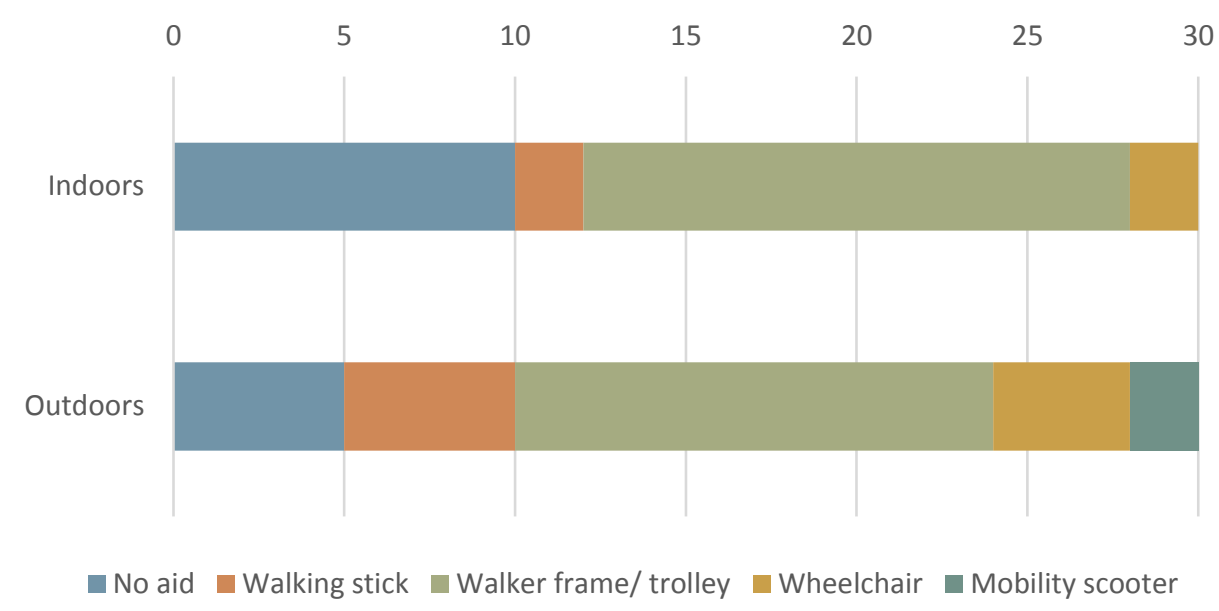

Figure 7-4: Type of mobility aids/vehicles mainly used

Some residents used more than one type of walking aid depending on the walking distance and their needs for carrying things (RVI1, RV16, RVS3). For example, one apartment resident used three aids indoors at different times: a walking stick for moving around her unit; a walker frame with two wheels, which gave her more support, for going to the toilet at night; and a walker frame with four wheels for carrying a cup of tea to the communal lounge (RVS3). Two residents used a trolley indoors which had capacity for carrying things while also providing support similar to walker frames (PU1, RVI9). One resident, who was a post-stroke individual with hemiplegia, could not control a walker frame with one hand but had control using a trolley.

\section{Caregivers}

Twenty-one caregivers participated in the survey. The majority were female ( $\mathrm{n}=19)$ and only two were male.

\subsubsection{Settings}

The basic information about the participants' settings is shown separately for housing complexes and housing units.

\section{Housing complexes}

The housing complexes studied include five public-sector rental housing complexes for the elderly, three private-sector housing complexes for the elderly and five RVs. All RVs had independent-living units, and two had supported-living units in addition.

The information on the number of housing units is shown in Figure 7-5. Of eight public- and private-sector rental housing complexes, the majority (five complexes) contained a small number of units (10-39 units), two contained between 40 and 69 units, and one was larger, containing 
between 70 and 99 units. The five retirement villages varied in their number of units, with the smallest containing less than 40 units and the largest containing more than 200 units. Between 10 and 39 supported-living units were supplied in the two retirement villages containing supported-living units.

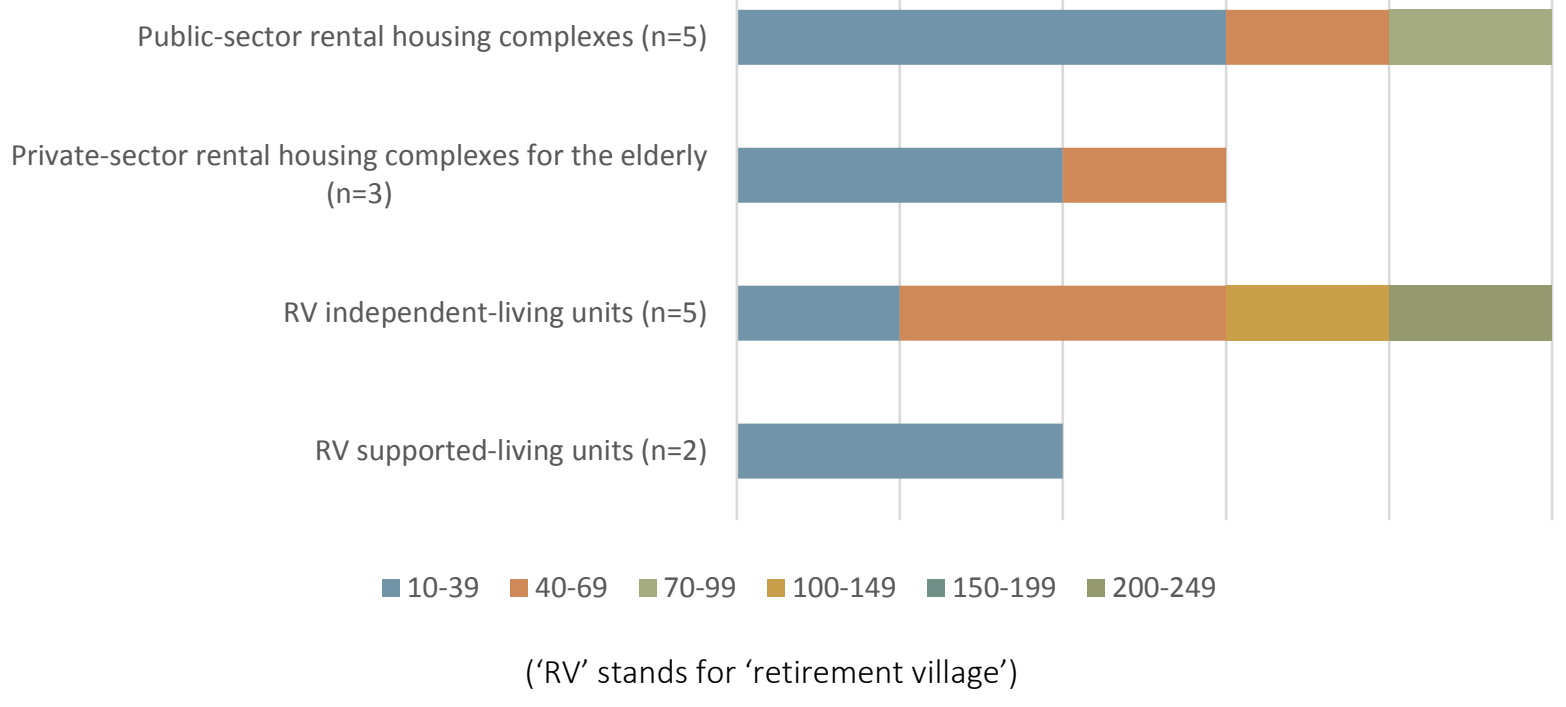

Figure 7-5: Number of units contained in a housing complex

The information on adjacent facilities to the complexes is described here and summarised in Table 7-4. One public-sector rental housing complex included a live-in custodian accommodation, and two private-sector rental housing complexes had rooms which were used by the staff during the daytime. All retirement villages had staff rooms. In the village where supported-living units were supplied, the medical/care staff rooms, as well as other staff rooms, were located near supported-living units. Multi-purpose communal spaces were supplied in some of the public- and private-sector rental housing complexes as well as in all retirement villages (both within supported-living unit buildings and outside). Communal dining rooms were placed near supported-living units and one private-sector rental housing complex, where meals were provided for residents.

Specific leisure rooms such as libraries, swimming pools/spa facilities and workshops/men's sheds were provided in some retirement villages. Communal laundries were provided in three rental housing complexes and in one retirement village with supported-living units. Some retirement villages and one private-sector rental housing complex were furnished with communal storage where storage spaces were allocated to individuals. One retirement village had a medical room that could be used for individual medical care, such as podiatrist visits. One private-sector rental 
housing complex was adjacent to a religious facility (a chapel) owned by the housing operator. Residential care facilities were adjacent to or located within all retirement villages, from which some services (e.g. meals, laundry services, recreational activities and hairdressers) were available to residents.

Table 7-4: Adjacent rooms/facilities

\begin{tabular}{|c|c|c|c|c|}
\hline \multirow{2}{*}{$\begin{array}{l}\text { Services and care for } \\
\text { residents }\end{array}$} & \multirow{2}{*}{$\begin{array}{l}\text { Public-sector } \\
\text { rental housing } \\
\text { complexes }(n=5)\end{array}$} & \multirow{2}{*}{$\begin{array}{l}\text { Private-sector } \\
\text { rental housing } \\
\text { complexes for the } \\
\text { elderly }(n=3)\end{array}$} & \multicolumn{2}{|c|}{ Retirement villages $(n=5)$} \\
\hline & & & $\begin{array}{l}\text { Outside supported- } \\
\text { living unit } \\
\text { building(s) }(n=5)\end{array}$ & $\begin{array}{l}\text { Within supported- } \\
\text { living unit } \\
\text { building(s) }(n=2)\end{array}$ \\
\hline $\begin{array}{l}\text { On-site staff } \\
\text { room/accommodation }\end{array}$ & 1 & 2 & 4 & 2 \\
\hline $\begin{array}{l}\text { Multi-purpose } \\
\text { communal space }\end{array}$ & 2 & 2 & 5 & 2 \\
\hline Dining room & - & 1 & - & 2 \\
\hline $\begin{array}{l}\text { Specific-leisure- } \\
\text { purpose room }{ }^{22}\end{array}$ & 1 & - & 4 & 1 \\
\hline $\begin{array}{l}\text { Communal laundry (for } \\
\text { resident use) }\end{array}$ & 1 & 2 & - & 1 \\
\hline $\begin{array}{l}\text { Communal storage (for } \\
\text { resident use) }\end{array}$ & - & 1 & 1 & 1 \\
\hline Medical room & - & - & 1 & - \\
\hline Religious facilities & - & 1 & - & - \\
\hline $\begin{array}{l}\text { Residential care } \\
\text { facilities }\end{array}$ & - & - & 3 & 2 \\
\hline
\end{tabular}

(The numbers in italics show the number of complexes)

\section{Services and care provided by the housing operators for residents}

The services and care provided for residents by the housing operators are shown in Table 7-5. Social support and emergency assistance are provided by some rental housing as well as by retirement villages. The on-site staff regularly visited residents in one public-sector housing complex and one private-sector housing complex (both every morning), as well as in retirement village supported-living units, where the staff visited residents once a day or more. In some other complexes, the on-site/off-site staff visited as the need arose. Residents had access to Emergency on call 24 hours a day in four public-sector and one private-sector housing complex, and in all retirement villages. However, the use of medical alarms provided by external services was encouraged in most complexes. Communal activities were organised by housing operators in

\footnotetext{
${ }^{21}$ Referred to as Lounge, Hall or Community room. These spaces may include kitchen/bar facilities, dining area, toilets, some bookshelves and storage spaces.

${ }^{22}$ Specific-purpose communal rooms include libraries, swimming pool/spa facilities and workshops/men's sheds.
} 
complexes containing communal areas, which included two public-sector and two private-sector rental housing complexes and all retirement villages. Activities for recreation, hobbies, exercises and socialising were organised every day in retirement villages, except for one village where a regular socialising activity was organised once a week. One public-sector housing complex provided residents with a weekly programme, in which they could bring in support agencies for education and advice. In private-sector rental housing complexes with a multi-purpose communal space, activities for hobbies, exercises and socialising were provided on weekdays. Outings and excursions were provided for residents in three public-sector and one private-sector rental housing complex, as well as in most retirement villages.

Assistance with daily living, such as meals, laundry and other household tasks were provided for residents in retirement villages, and not provided for those in rental housing, except for one private-sector rental housing that provided meal services for residents (the meal cost was included in the rent). Assistance in household tasks were also available to all residents in retirement villages, but they could also use external services such as publicly-funded home-based care. Personal care was available to supported-living unit residents in all retirement villages and in independent-living units in three retirement villages.

Table 7-5: Services and care that can provided/arranged by housing providers for residents

\begin{tabular}{|c|c|c|c|c|}
\hline \multirow{2}{*}{$\begin{array}{l}\text { Services and care for } \\
\text { residents }\end{array}$} & \multirow{2}{*}{$\begin{array}{l}\text { Public-sector } \\
\text { rental housing } \\
\text { complexes } \\
(n=5)\end{array}$} & \multirow{2}{*}{$\begin{array}{l}\text { Private-sector } \\
\text { rental housing } \\
\text { complexes for } \\
\text { the elderly }(n=3)\end{array}$} & \multicolumn{2}{|c|}{ Retirement villages $(n=5)$} \\
\hline & & & $\begin{array}{l}\text { For independent- } \\
\text { living unit residents } \\
(n=5)\end{array}$ & $\begin{array}{l}\text { For supported-living } \\
\text { unit residents }(n=2)\end{array}$ \\
\hline Regular staff visits & 1 & 1 & 0 & 2 \\
\hline $\begin{array}{l}\text { Emergency on call } 24 \\
\text { hours a day }\end{array}$ & 4 & 1 & 5 & 2 \\
\hline $\begin{array}{l}\text { Organising activities in } \\
\text { communal areas }\end{array}$ & 2 & 2 & 5 & 2 \\
\hline Outings & 3 & 1 & 4 & 1 \\
\hline Meals & - & 1 & 5 & 2 \\
\hline Laundry service & - & - & 5 & 2 \\
\hline $\begin{array}{l}\text { Assistance in household } \\
\text { tasks (excluding above) }\end{array}$ & - & - & 5 & 2 \\
\hline Personal care & - & - & 3 & 2 \\
\hline
\end{tabular}

\section{Housing units}

The information on housing units studied is shown in Figures 7-6 and 7-7. Regarding the unitlayout type, public- and private-sector rental housing units were either bedsit or one-bedroom 
type, whereas there were no bedsit types in retirement village units. All supported-living units in retirement villages contained one-bedroom types, whereas the independent-living units varied in the number of bedrooms and the presence of other rooms (such as an office and a garage). Most units were either semi-detached or apartments, and only two independent-living units in retirement villages were detached. All supported-living units in retirement villages, and some private-sector rental housing units and independent-living units in retirement villages, were the indoor-access apartment type.

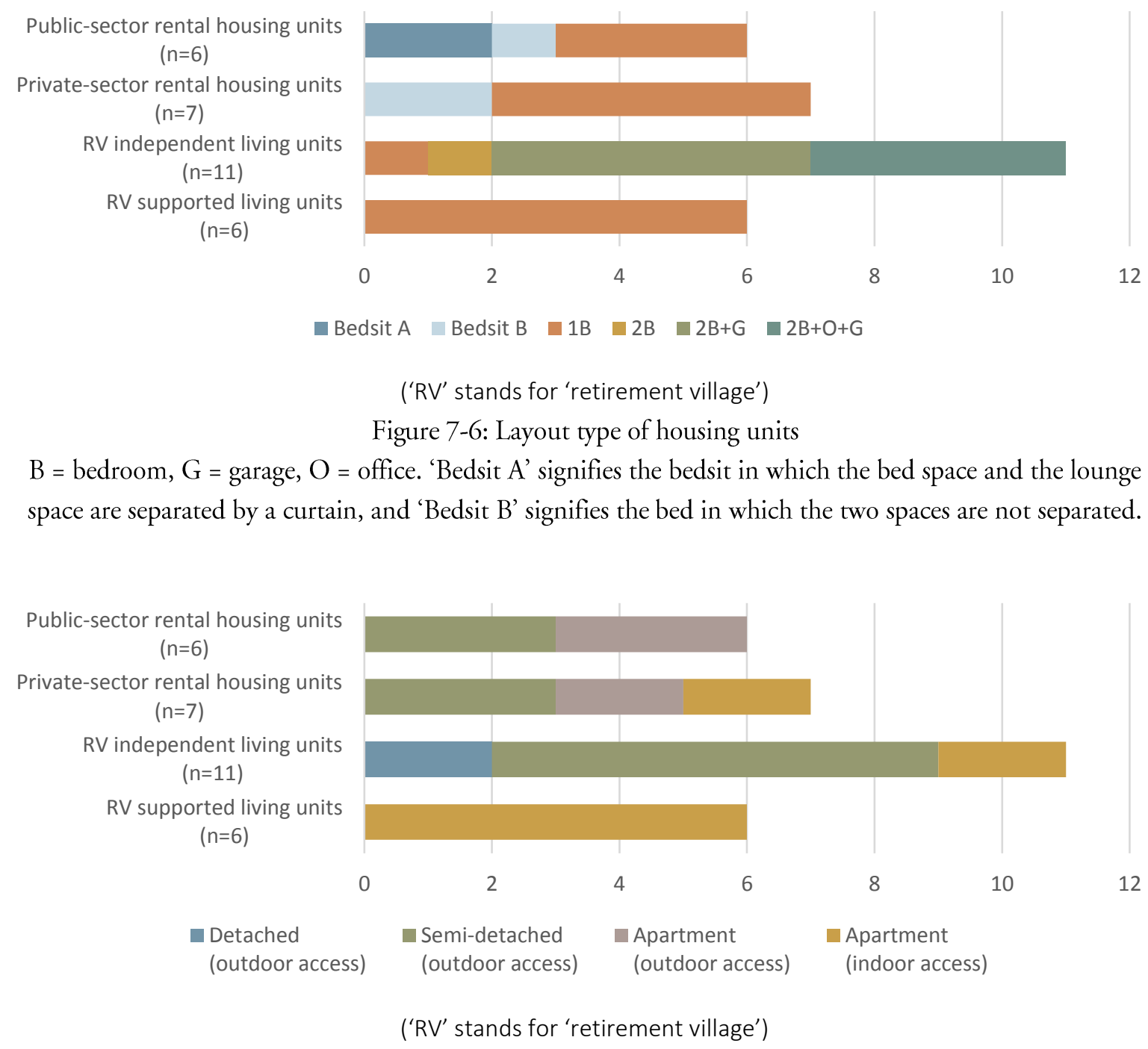

Figure 7-7: Building type of housing units

The relationships between the unit size, layout type and the type of housing complex are shown in Figure 7-8. The unit floor area of public-sector housing units varied between 31 and $45 \mathrm{~m}^{2}$, while private-sector rental housing units varied between $25 \mathrm{~m}^{2}$ and $57 \mathrm{~m}^{2}$. Retirement-village supported-living units were between $39-51 \mathrm{~m}^{2}$, whereas the size of independent-living units was larger than other types and varied between $59 \mathrm{~m}^{2}$ and $132 \mathrm{~m}^{2}$. The floor area was generally 
associated with the number of rooms. Bedsit units were between $25 \mathrm{~m}^{2}$ and $35 \mathrm{~m}^{2}$, one-bedroom units were between $39 \mathrm{~m}^{2}$ and $59 \mathrm{~m}^{2}$, a two-bedroom unit was $89 \mathrm{~m}^{2}$, two-bedroom units with a garage were between $96 \mathrm{~m}^{2}$ and $110 \mathrm{~m}^{2}$, and units containing two bedrooms, an office and a garage were between $118 \mathrm{~m}^{2}$ and $162 \mathrm{~m}^{2}$.

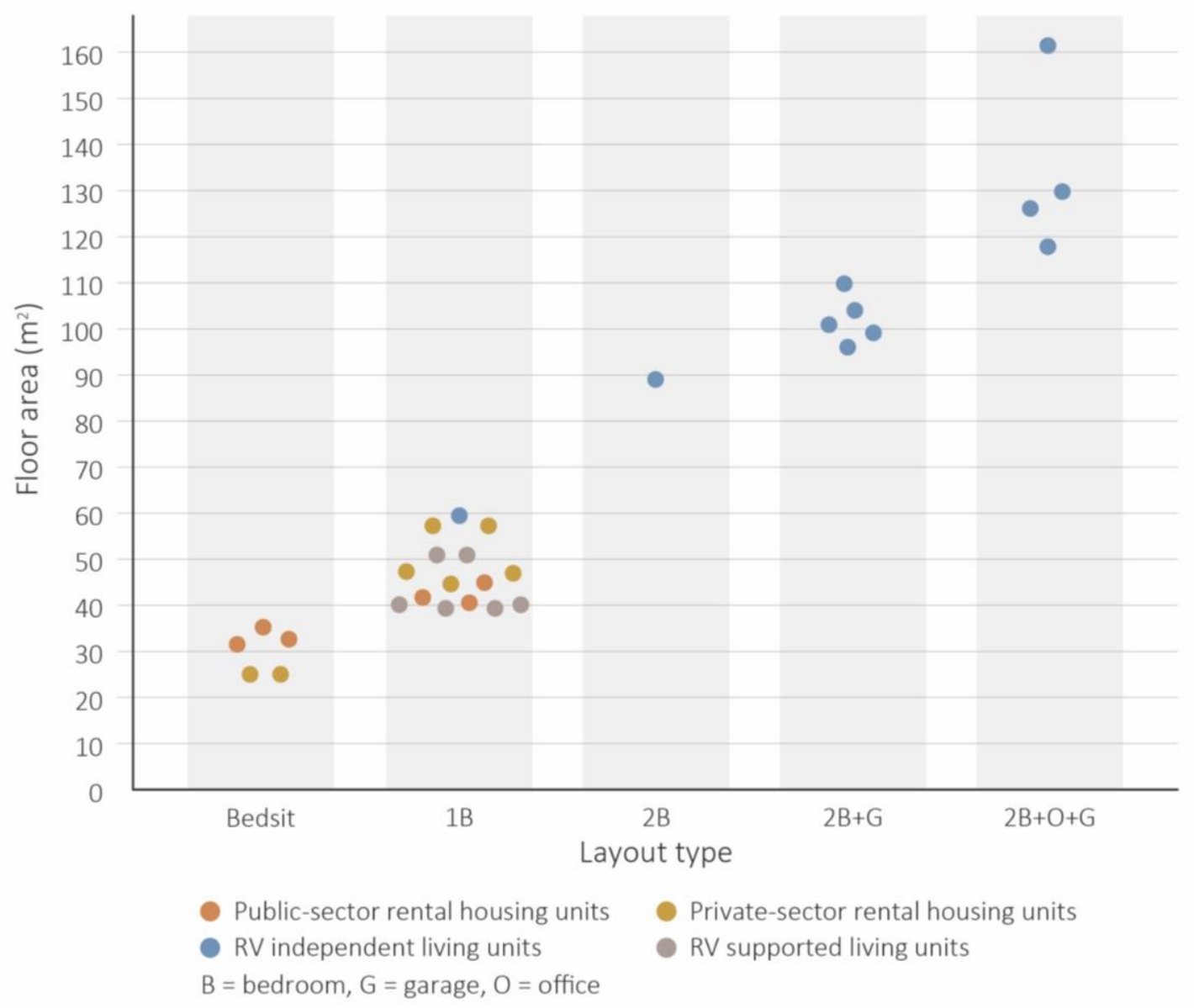

('RV' stands for 'retirement village')

Figure 7-8: Relationships between the unit size, layout type and type of housing complex

\section{Bathrooms and shower areas}

Of all units studied, three units contained two bathrooms (either two bathrooms with a shower or the combination of one bathroom with a shower area and a toilet room). The remaining 27 units contained only one bathroom. All the main bathrooms contained a toilet, a shower area and a vanity in the same room. Some also contained space for a washing machine. In all cases, a specific type of manufactured enclosure was used for the shower area. These shower areas have been classified into six typologies based on the type of enclosure and the presence of different levels at the shower entrance (Figure 7-9). 


\begin{tabular}{|l|c|c|c|}
\hline & Enclosed with shower curtain & $\begin{array}{c}\text { Enclosed with glass } \\
\text { screens }\end{array}$ & $\begin{array}{c}\text { Semi-enclosed with a } \\
\text { glass screen }\end{array}$ \\
\hline $\begin{array}{l}\text { Has a level } \\
\text { difference }\end{array}$ & & Type $\mathrm{A}(\mathrm{n}=2)$ & None \\
\hline $\begin{array}{l}\text { Has no } \\
\text { level } \\
\text { difference }\end{array}$ & &
\end{tabular}

Figure 7-9: Typologies of the shower area

\section{Kitchens}

The kitchens are classified into six typologies based on their level of amenities (Table 7-6).

Table 7-6: Typologies of the kitchen

\begin{tabular}{|c|c|c|c|c|c|c|}
\hline \multirow{2}{*}{$\begin{array}{l}\text { Kitchen } \\
\text { type }\end{array}$} & \multicolumn{5}{|c|}{ Kitchen amenities } & \multirow[t]{2}{*}{ Number of cases } \\
\hline & Sink & $\begin{array}{l}\text { Space for a } \\
\text { normal- } \\
\text { sized } \\
\text { fridge- } \\
\text { freezer }\end{array}$ & Hot plates & Oven & $\begin{array}{l}\text { Dish } \\
\text { washer }\end{array}$ & \\
\hline Type A & Yes & Yes & Yes & Yes & Yes & 10 (RVI1-5, 7-11) \\
\hline Type B & Yes & Yes & Yes & Yes & No & 9 (PU1-6, PR3-5) \\
\hline Type C & Yes & Yes & Yes & No & No & 5 (PR1-2, RVI6, RVS3-4) \\
\hline Type D & Yes & $\mathrm{No}^{* 1}$ & Yes & No & No & 2 (RVS1-2) \\
\hline Type E & Yes & Yes & No & No & No & 2 (RVS5-6) \\
\hline Type F & Yes & $\mathrm{No}^{* 1}$ & No & No & No & 2 (PR6-7) \\
\hline
\end{tabular}

*1 The kitchen has space for a small fridge under the bench.

Serviced apartments and one private-sector rental housing site provided all or some meals for residents, which were cooked by the on-site staff in the main kitchen (PR6, PR7, RVS1-6). The residents of serviced apartments were able to decide if they would like meals provided and where to have them (either in a communal dining room or in their own units). Of six serviced apartment residents, four only had a kitchenette without a stove or a space for a normal-size fridge-freezer. Another two had full kitchens. In one private-sector housing complex, residents were basically provided with all meals. They normally had lunch and dinner in the common 
dining room (but could choose to eat in their units on special occasions) and could choose where to eat breakfast (either in the communal dining room or bring it to their own units themselves). Resident units only contained a kitchenette without a stove.

Except for the housing units mentioned above, all housing units had a kitchen furnished with full amenities. Some participants used cooked meal delivery services, such as Meals on Wheels, for one meal a day (PR1, RVI2, RVI6, RVI7, RVI9, RVI10). Some residents purchased takeaway meals or pre-cooked food from the supermarket (PU1, PR4, RVI3, RVI4). Some had caregivers cook meals (RVI1, RVI7) and many had their caregivers wash dishes (PU1, PU5, PR5, RVI1, RVI2, RVI7, RVI11, RVS3).

\subsection{QoL of the high-needs elderly living independently}

Through data analysis, the themes for QoL emerged. They are categorised under six main headings: 1) Control in daily basic activities, 2) Meaningful leisure activities, 3) Meaningful relationships, 4) Maintenance of possessions, 5) Comfort, and 6) Quality of care.

\subsubsection{Theme 1: Control in daily basic activities}

Residents' impairments and other conditions affected their ability to accomplish their basic daily activities. Many residents were also at risk of falls or had experienced falls during activities in the past (PU4, PR5, PR6, RVI2, RVI5, RVI11) because of their conditions. As a result, having control in the activities was highly important for them. This section includes eight aspects of control: 1) basic movement, 2) going to the toilet, 3) showering, 4) cooking and dining, 5) laundering, 6) maintaining the home, 7) shopping, and 8) collecting mail.

\section{Theme 1-1: Control in basic movement}

Loss of mobility was a common experience for participants. While some experienced a gradual loss, others had experienced a sudden loss of mobility due to falls (PR5, PR6) or diseases such as strokes (PU1, PU, RVI3).

\section{Moving/walking}

One major difficulty residents mentioned was walking. They made comments such as, 'I have a wobble. I can't walk'(RVI2); and 'I don't walk very easily'(RVI4). Walking was often affected by a loss of balance due to arthritis and impairments in legs and knees, low blood pressure and other diseases (PU3, PU4, PR1, RVI10, RVI11). The pain also affected walking (PR2, RVI9). 
Walking aids such as a stick, a walker frame or a trolley assisted participants with balance to aid with walking. Some residents were completely reliant on their walker frame or trolley, making comments like, 'I use and quite depend on this little trolley(RVI9)', 'I couldn't live without it really'(PR1) 'I doubt whether I could move without it'(RVI1), and 'I can't walk without it. I would fall over'(RVI7). These residents kept their walker frames and trolleys within reach when sitting and used them to move around (PU1, PR1, PR6, PR7, RVI1, RVI2, RVI7, RVI9, RVI10, RVS1, RVS2, RVS3).

\section{Moving over level differences, slopes and unstable ground}

The elderly with limited mobility '[couldn't] lift their legs too high'(PR5 caregiver), which affected their ability to negotiate differences in levels. Many had difficulties in walking up or down steps (PU3, PU4, PU5, PU6, PR4, RVS2, RVS4) or could not do so at all and needed assistance (PU1, PR1, RVI1, RVS2, PRS5). Steps were a common feature of inaccessibility in the wider community, such as in family homes, cafes and transportation (e.g. cars, buses, trains) (RVI1, PU6, RVS4, RVS5). One resident had to give up going to a café he used to enjoy going to, because he was unable to get up the stairs to the café (PU6). Another resident in a wheelchair talked about the limiting opportunities of outings due to the steps in the car:

I always used to go to a 'mate's weekend' but I can't do that now... You know go away for a weekend with a group of mates from all over. That was lots of fun ... but you see, I can't... It's very limiting where I can go now. ... Most of my friends, they're about my age which is late 70s or 80s, and so I don't want them to be trying to get a wheelchair in and out of the back of their car. (RVS5)

Residents with a walker frame or a trolley also had difficulties with navigating the level differences (RVS2). Moving a walker frame over a few steps or a level difference at the door threshold was difficult and posed a risk to safety (PU5, PR5): 'You look at here [indicating a level difference]. How can the elderly clients stand on their legs and lift their walker? If they go down they'll bang their head over here'(PR5 caregiver). One resident wanted a small slope to fill a five-centimetre level difference at her back door, which she had to move over with her trolley when drying her washing (RVI9) (Figure 7-10). Wheelchair users also found it difficult to move over level differences. To move over the level difference onto the road, one resident used her feet while sitting on a wheelchair, a very technical process; she had to move her legs very fast to propel herself using the wheelchair's momentum (RVS5). 


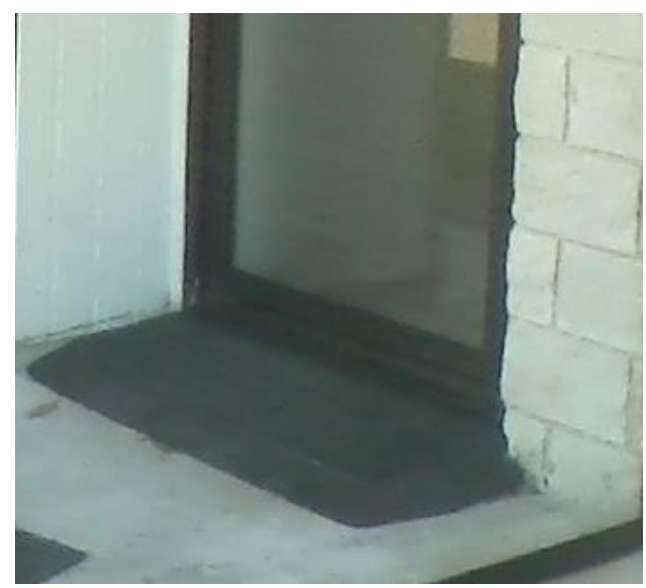

Figure 7-10: Filling the level difference with a small slope

A tiny level difference between steps was also a tripping hazard. One resident talked about their experience while walking without aids: 'I've got two sore legs... It's got no step, but it's not a step that caused a fall. It's the piece that sticks up ... That's what cut my legs'(PR5). Her caregiver also said, 'The small beading of the grout, she trips over that'(PR5 caregiver). The fear of falls deterred residents' active life. One caregiver said, 'Sometimes some of them are so scared to go out, because they are letting their fear that they will fall take over. Therefore, they are not moving'(PR5 caregiver). She maintained that 'entry doors should be like bedroom doors [with no small level difference]'. At the least, she said, level differences should be filled in with a small slope, which was also suggested by one resident using a walker frame (RVI9). Similar types of hazard for tripping over included a rug and a pet lead (PR5, RVS4).

Where the slope was too steep, residents also had difficulty walking without aid (RVS4). For many residents using a walker frame, the slope was easier to manipulate than a level difference (PR1, PR6, PR7, RVS2). Yet, some residents found it hard to walk across the slope between the outside ground level and the inside floor level with a gradient of 1 in 10.4 (490 mm in 5100 $\mathrm{mm}$ ), to get to the communal rubbish bins (PR1). Wheelchair users found a slope was easier than steps. One resident used a 500-mm long portable slope to fill the $70 \mathrm{~mm}$ difference between the unit level and the veranda level (the gradient was approximately 1 in 7 ), which was 'a little difficult, but $O K^{\prime}$ (RVS5). Another resident didn't use the communal room because it had a steep slope of a gradient of 1 in 5.8 at the entrance, which he could not go up even with his scooter (PU1). A long slope was particularly inconvenient for wheelchair users, because they had to use their upper body strength (which was often limited) to move up the slope. One resident who had a ramp of six metre (gradient was approximately 1 in 12) between the front door and the passage said, 'This is only a gradual climb as you can see. It's quite good but it's not 
convenient for people in wheelchairs'(PU3). One resident suggested '[the level access is] ideal; but for the level access, you can't do without a gutter [to run off rainwater]'(RVS5).

The grass lawn was not stable enough for participants to walk on. One resident fell over on the grass field: 'I just fell.... I was coming down the back, so it was just on the grass lawn here' (RVI2). One apartment resident also suggested there should be pavement instead of grass between her porch and the outdoor path (RVS4).

\section{Holding onto things}

Things to hold onto ensured residents' steadiness in walking. Handrails were desired for navigating steps, slippery floors (e.g. shower rooms, wooden decks) and paths. For example, the provision of 'handles around [steps]'(PR5 caregiver), and 'a railing [on the paths]'(PU2) was desired.

Some residents who relied on their walker frame outside their units didn't use it in their units, which were typically small (PU5, PU6, PR2, PR5, PR6, PR7). This was partly because the space was too small for them to use their walker frame, and partly because they could move holding onto furniture and fixtures. For example, one resident moved through her kitchen between her bedsit room and entrance by holding the walls, kitchen bench and fridge (PU5). Sofas and chairs also supported residents, who could hold on them in case they lost balance (PR2, PR6, RVI4). The provision of walls or fixtures that could be held on by both hands particularly ensured their safety. However, if support was not available continuously, their movement was unstable. For example, the opening of the shower door beside the toilet was a tripping hazard when one resident went to the toilet at night continuously holding onto walls and fixtures (the vanity counter and the shower enclosure) (RVI11) (Figure 7-11). Things used for holding onto could also be a hazard if they were not firmly installed; one resident had a fall when holding onto a drawer: 'The whole drawer fell over me, and I cut my right leg'(PR6). 


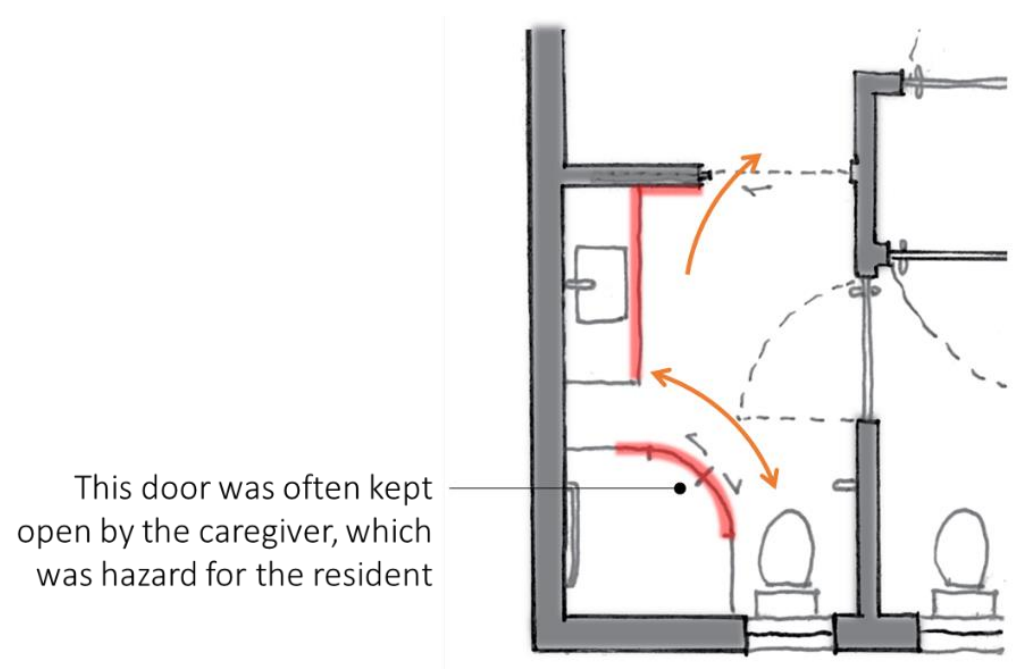

Figure 7-11: Opening of the shower door that was a tripping hazard

\section{Moving through doors}

Moving through doors was often challenging for those with limited mobility. Many residents had limited strength, and found some doors were very heavy (PR1, PR2, RVI7). One resident could not open it herself (RVI7): 'We do find the [main] doors really very hard for us [to open] when we have so little strength'(PR1). One resident with Parkinson's said she 'wouldn't be able to open the door and get through'(RVS1).

Some doors were unnecessary. A door between the kitchen and the lounge was never shut (PU1, PU4, PU6, PR3, PR4), except for one door which the resident shut when it was too draughty (PU5). One resident said, 'That one [door] I go through all the time... I think it's actually a silly place really to be perfectly honest. I don't think there's a need for it'(PU4). By contrast, one resident liked his open plan kitchen: 'You haven't got so many doors to go through'(PU3). The door between the bathroom and the laundry room was also often not used due to the difficulty of negotiating them and the separation into two rooms was not necessary for some residents (PU4, PR4). The bathroom door was mostly kept open unless there was a particular need to shut it (Figure 7-12). 


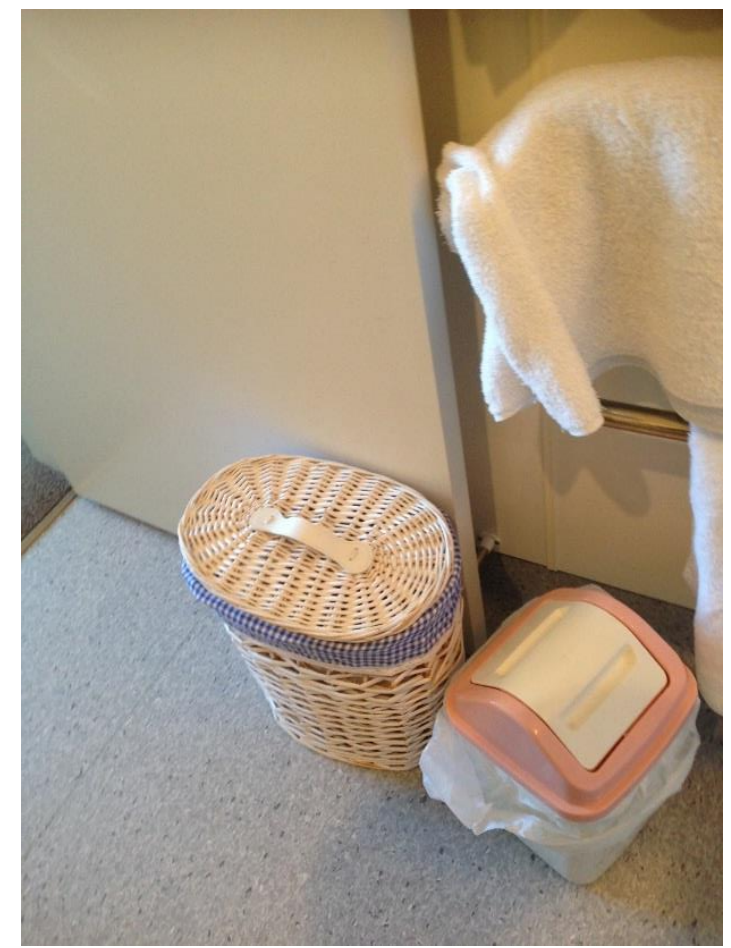

Figure 7-12: The bathroom door was normally kept open

Hinged doors were difficult to handle for those with a walker frame, because the walker frame position often interfered with the door swing when the door was pulled. Those who had difficulty with stretching their arms or limited balance also had trouble reaching over their walker frame to a door handle, which was particularly difficult when opening a hinged door. For example, one resident said, 'It opens that this way [pulled], so you have to pull the walker [frame] back. And trying [to] get the door open, [you have to] open in the distance. That is the same trouble as to open the other way'(RVI8) (Figure 7-13). Another resident also struggled to open and shut the bathroom door because the space between the door and the toilet was too small to allow her to approach with a walker frame (RVI7) (Figure 7-14). The door would not shut completely and as a result, she had to leave a little open during toileting. 
To open the hinged door inwards, the resident had to approach the door handle, then pull his walker frame back. He had to hold the door handle in the distance

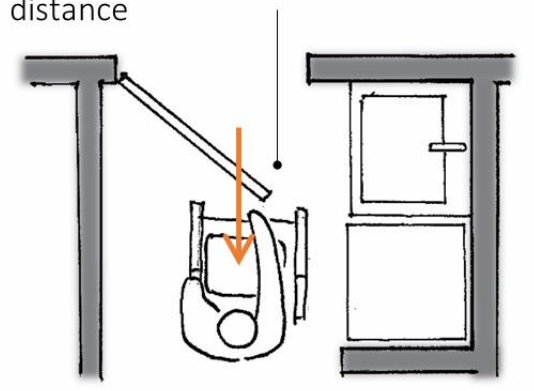

To open the hinged door outwards, the resident had to push the door in the distance while pushing his walker frame ahead.

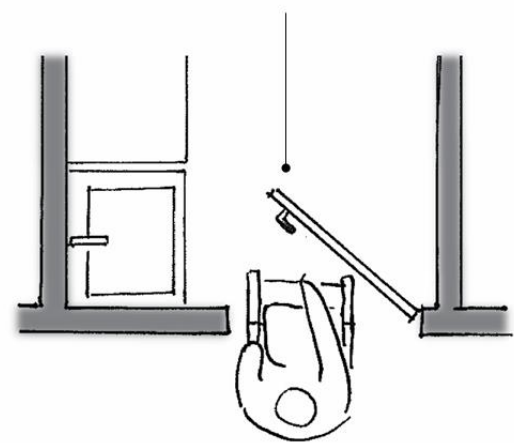

Figure 7-13: Trouble for a walker-frame user in opening a hinged door inwards (left) and outwards (right)

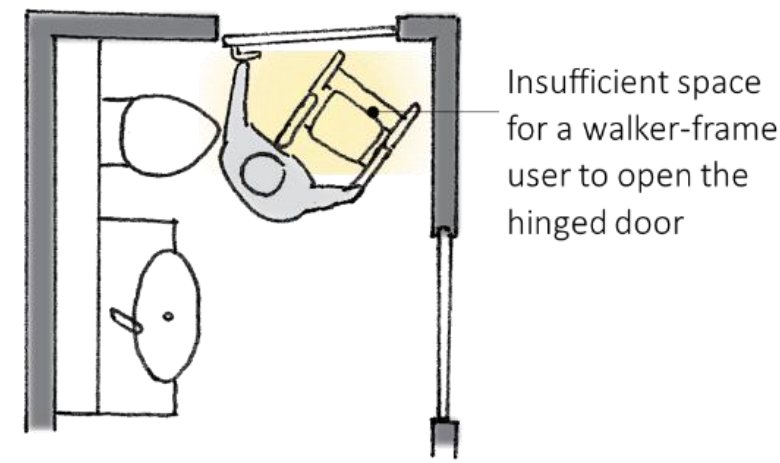

Figure 7-14: Insufficient space for a walker-frame user in front of a hinged door

A door swinging open in the wrong direction can affect wheelchair users as well as other aid users. In a narrow space, the swinging direction should have taken into account the direction of approach. One resident made the comment, 'The doors sometimes seem to open the wrong way. ... So if I had a wheelchair it would be quite difficult'(PR1) (Figure 7-15). A sliding door was preferred to a hinged door because it was easier to operate and took up less space (RVI9, RVI10). 


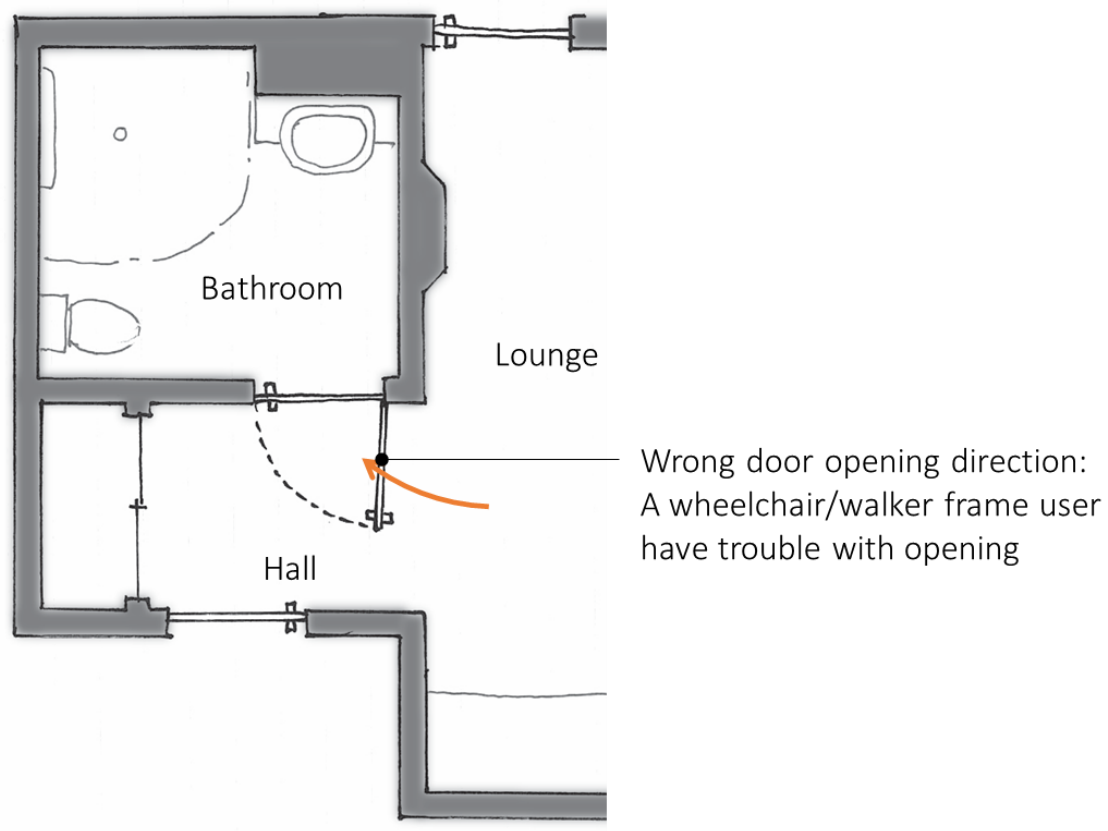

Figure 7-15: Hinged door with a wrong opening direction

Some hinged doors with door closers troubled those with a walker frame (PR2, PR6, PR7, RVS3), as it shut too fast and was 'hard to get in and out'(PR2). There was often a level difference at external hinged doors, which added to the difficulties. When one resident went through a hinged door with both a door closer and a level difference, first he held the door to keep it open, and then, counting 'one, two, three', let go of the door and moved his walker frame in or out as fast as he could (PR2). One resident preferred a sliding door to a hinged door for this reason (RVS3).

If the door had a hook-type hold-open, hooking the door back was useful for moving through the door slowly; however, it was hard to push the door by one hand and hook it by the other while holding a walker frame (PR2). While a magnetic hold-open could be handled by one hand and was easier (RVS1).

The location of the door handle was sometimes not appropriate. For one resident with a walker frame, the height of the handle of a door was too low, as it was blocked by the walker frame resting in front of the door (PU6).

Some residents appreciated having a door of $860 \mathrm{~mm}$ width, which was wide enough for a wheelchair: 'Even if I have to go into a wheelchair and I can't walk, I can still fit the wheelchairs through these doors'(RVI10). Another resident suggested the door width of $760 \mathrm{~mm}$ was too narrow for a neighbour when he was using a wheelchair (PR5). One wheelchair user had 
difficulty with passing through the doorway, which was narrowed to $750 \mathrm{~mm}$ wide by the layout of her furniture (RVS1).

\section{Turning around}

Although a small space could provide walls, fixtures and furniture for residents to hold onto and provide support for them as described earlier, having space that could comfortably accommodate a walker frame was generally appreciated — such as 'a wide passageway'(PR7), '[wide] doorways' (RVI7), and a spacious kitchen (PU3, PR2) which was 'easy to get around'(PR2). However, some residents didn't have enough space for a walker frame (particularly to turn around) in their bathroom (RVI2) and their narrow kitchen (PU6), and had to leave their walker frame outside the room. One apartment resident also left her walker frame in the corridor outside her apartment because the unit was too small to comfortably accommodate it (PR6). One resident '[didn't] need to turn around' in her bathroom when going to the toilet (RVI6), because she had two doors for her bathroom and she could enter through one door and exit through the other (Figure 7-16). Another resident had a fall when turning around with his walker frame in the bathroom, which was supposed to have enough space for turning around a wheelchair (the clear space of 1,500 mm diameter circle) (RVI8).

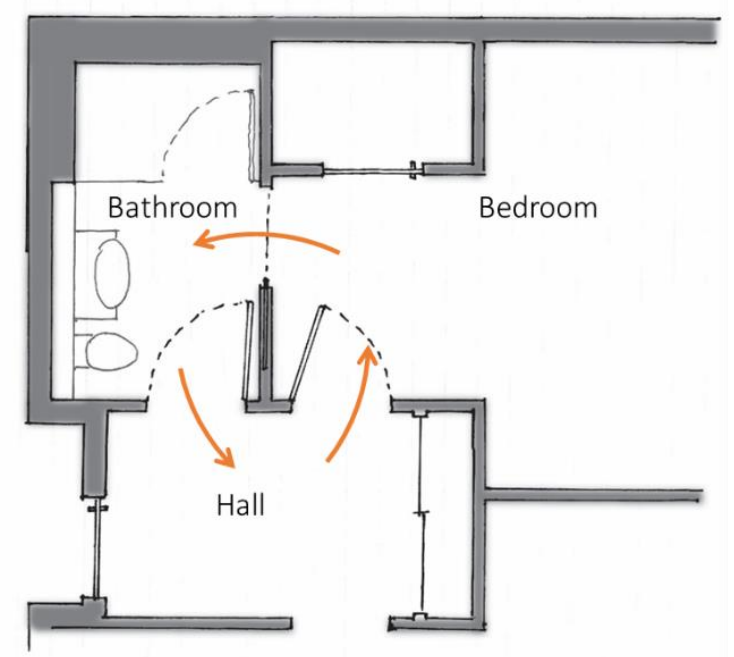

Figure 7-16: Having two bathroom doors facilitated a circulation that avoided turning around

Wall corners and objects that were sticking out from the wall were often hit by a walker frame. One resident reported regularly banging into the corner near the entry of his bedroom (RVI1) (Figure 7-17). Another resident actually hit his walker frame against the corners on the corridor (PU6). 


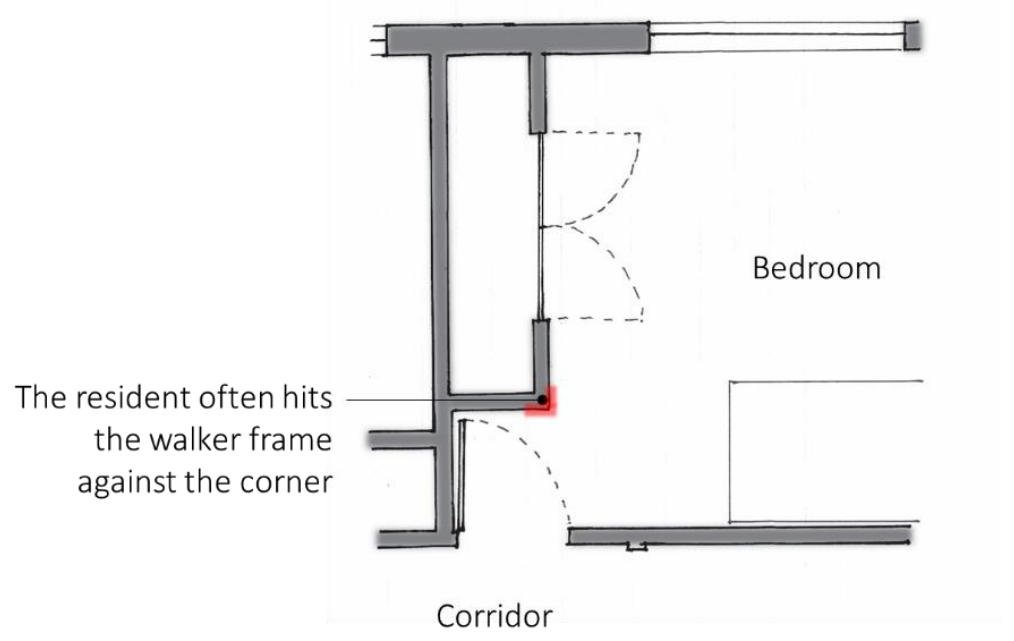

Figure 7-17: Wall corner often hit by a walker frame

\section{Floor material}

Floor materials were relevant to the movements with mobility aids. The slip resistance of the floor materials was important for walker-frame users. One resident was satisfied with her carpet, because 'it grip[ped] well'(RVS5). However, 'if the wheelchair [was] wet, it puts a black mark over the carpet'(RVS5). Dark-coloured carpet materials could mitigate the mark.

Unfixed rugs were found to be a hazard for tripping over, both for those not using aids (RVS4) and those using aids (RVS5). One resident said, 'The wrinkled carpet moves when the wheelchair moves. It could trip someone'(RVS5).

\section{Storage for mobility aids}

Appropriate design of storage for mobility aids was crucial for their independence in moving. Mobility scooters were sometimes stored in a garage (RVI8) or shared storage in a corridor (RVS2). One resident stored his scooter in his bedsit because he had no garage or accessible storage (Figure 7-18) and he couldn't store it on his porch as there was a one-centimetre lip at the entrance, hindering him from walking out of the room (PU1). 


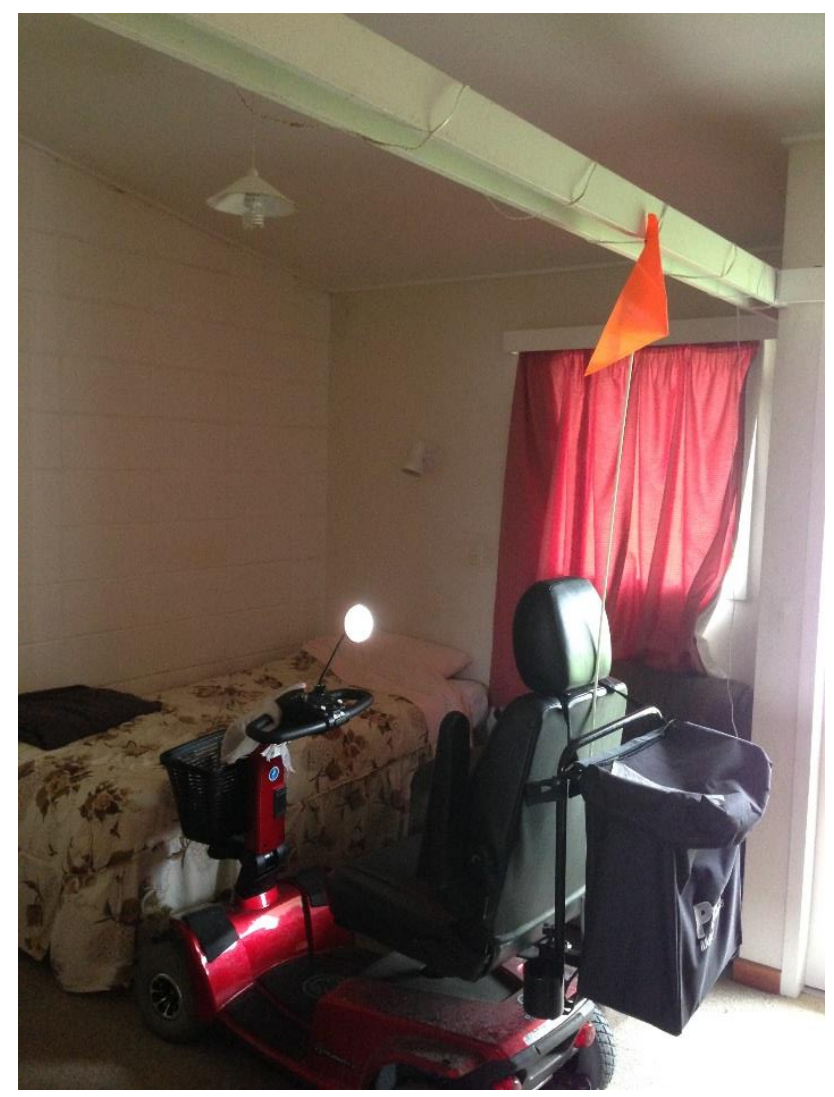

Figure 7-18: Mobility scooter kept in the bedsit unit

While in storage, enough space was necessary for mobility aids (his trolley or walker frame) to approach the scooter, and enough space was also required for the scooter to turn around. In order to go out with his mobility scooter, he walked with his trolley to the scooter. Next, he got onto the scooter, turned around in his bedsit (turning radius of approximately 2,050 mm was required), and went out of his unit (Figure 7-19).

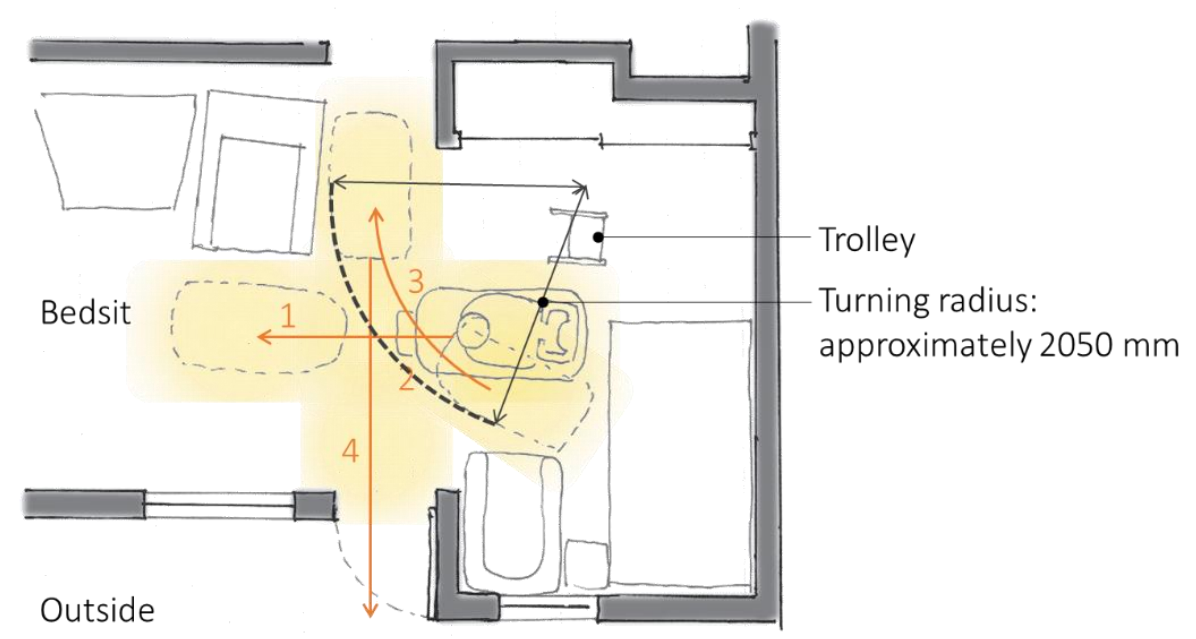

Figure 7-19: Procedure of turning around the mobility scooter and the required space 


\section{Sitting down, crouching/bending and getting up}

Some residents reported their difficulty sitting down on a low seat. Many used a toilet seat raiser to increase the height of the seat (the original seat height was approximately $400 \mathrm{~mm}$ high) (PU5, PU6, PR5, PR6, RVI8)' (Figure 7-20). A caregiver explained the difficulty in sitting on a low seat:

The toilet's too low ... so, they've got these big raised things and it helps people; otherwise, it's too low to sit down .... Just balance [is a problem], like a lot of older people fall and the body what, let's say gets stiff... the knees, hips, even a sore back... anything like that [can make it more difficult]. (PU5 caregiver)

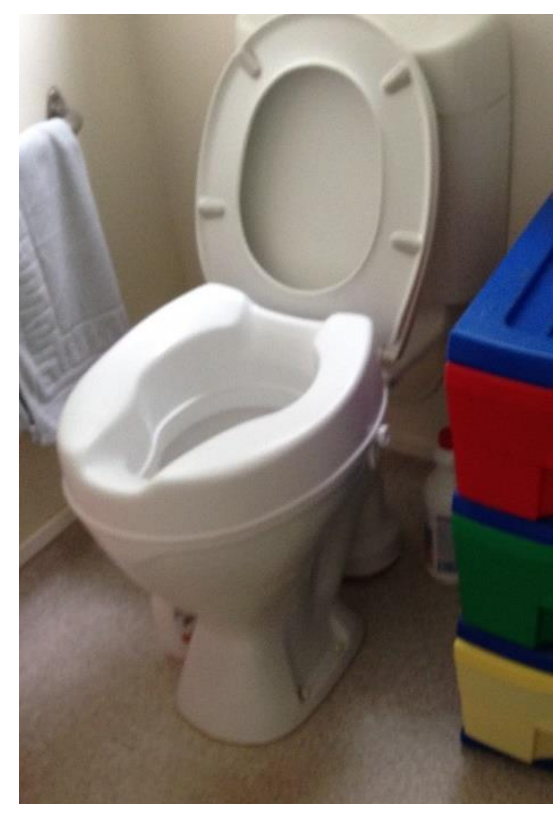

Figure 7-20: Toilet seat raiser

Many residents had difficulties in bending or crouching. Crouching to pick up something could cause a fall (PU1, PR6). One resident said, 'If anything drops on the floor it's got to stay there. I can't pick it up'(PR5). In observation sessions, some actually had difficulty crouching to reach things on the ground or in low areas, such as shelves (PR4), electric outlets (PU6, PR2) and door draught stoppers (PR7). Residents used tools to support the movement of picking up things, such as a 'picker uppers' (PU1, PR7) (Figure 7-21). This difficulty also affected gardening. One resident had to stand up frequently during kneeling to dig soil (PU4) and some had given it up because they couldn't kneel (PU5, RVI10). 


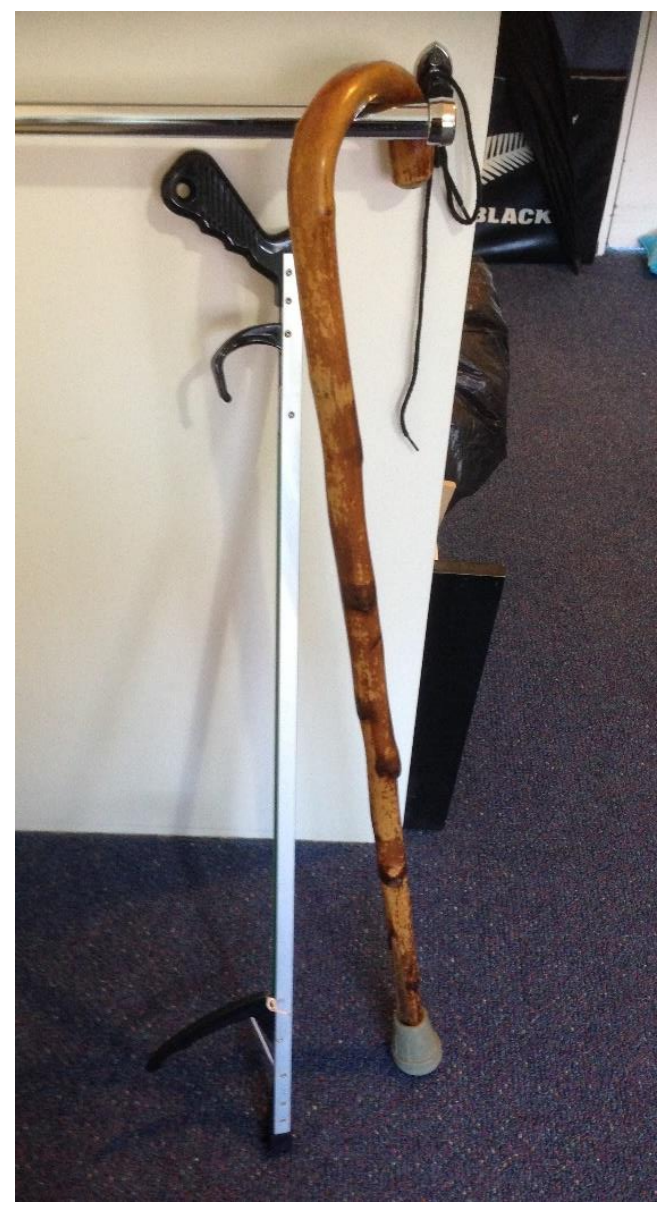

Figure 7-21: 'Picker upper' (and a walking stick)

Many residents experienced difficulty in getting up from chairs (PU5, PU4, RVI1, RVS3). For example, one resident who had low blood pressure observed, 'I can have fainting spells. If I stand up in a hurry I can fall down'(PU4). This difficulty was added in getting up from a low stool (RVS3). Having things to hold onto could provide support for residents in standing up, such as the armrests of an armchair or an adjacent table (RVS1, RVS3).

\section{Arm/hand movements}

Many residents had impairments in the hands or arms, which affected some movements. Some individuals had difficulty in stretching their arms. One resident said, 'Lifting things or putting my arms up, stretching up - that's when I have a bit of difficulty'(PU5). It affected reaching higher areas (such as shelves and cupboards).

The design of fixtures were often unsuitable for those with limited strength in their hands. One resident struggled with pulling drawers open, as the pulls were just small, tapered parts of the front boards that required power in the hands or fingers to open (RVS5). For those with shaky hands, it was difficult to turn a round-handle type tap (PU1). 


\section{Theme 1-2: Control in going to the toilet}

Many residents perceived the need for ease of access to the toilet. Some residents had severe issues of incontinence; even though using pads, one resident preferred to use the toilet to 'keep the pad dry'(PR6). Going to the toilet presented a particular challenge for those with severe mobility issue: 'Whilst it's only a step for you [to go to the toilet], it's a long step for me now' (RVS1).

One resident kept a 'piddle bottle' in the trolley at all times (PU1). When the researcher was situated near the sitting space during the observation session, the resident tried to hide and pass water in the kitchen (approximately $3 \mathrm{~m}$ away); however, he was unable to reach the privacy of the kitchen, passing water near the armchair because he had insufficient time to move (the researcher was asked to move to the kitchen).

There were greater needs for easy access to the toilet from the bedroom at night. 'If I go to toilet, I have to be quick, otherwise - wet pants' (RVI3), said one resident. Another mentioned his frequent toileting at night (every two hours). An en-suite bathroom adjacent to the bedroom was often preferred (PR1, RVS3). Those whose bathroom was far from the bedroom expressed a desire to have a bathroom in closer proximity (PU3, PR5). To make it easier to go to the toilet at night, residents often kept open doors on the way between the bedroom and the bathroom at night; such as the door of en-suite bathroom (PU2, RVI6, RVS1, RVS2) and a bedroom door (where the bathroom was not adjacent) (PU3, PR1, PR2, RVI2). One resident said, 'The bedroom door, it's a waste of time, (PU3). One couple had two toilets in their house, and they each used a different toilet (RVI3). The husband had limited mobility, and chose the one just across from the bedroom, which was ' handy' when going to the toilet at night. Just walking in a straight line was easier than having to change directions, which was necessary to use the wife's bathroom (Figure 7-22). 


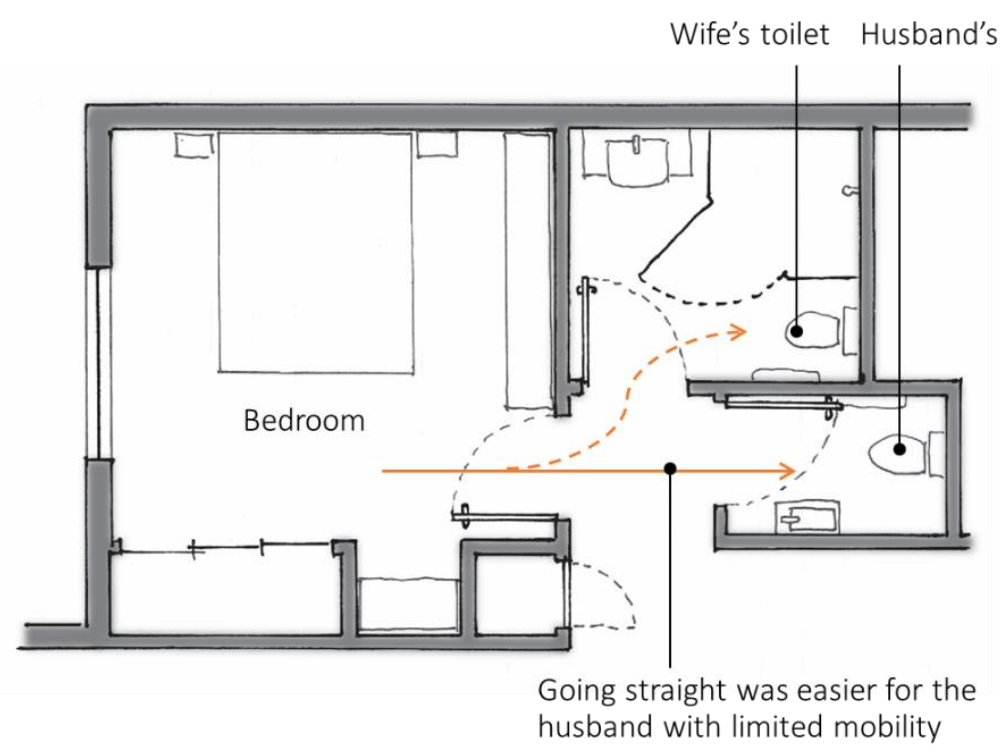

Figure 7-22: Easier route from the bedroom to the toilet

Ease of access to toilets was also important in the communal spaces, the absence of which could discourage residents with incontinence from participating in communal activities. One resident using a piddle bottle said, 'I've got this piddle bottle if I went down, but I'd have to disappear into some room with the piddle bottle. I cannot do it'(PU1).

\section{Theme 1-3: Control in showering}

Most residents who had a shower area with a level difference (shower type A-C) commented that they found it either dangerous or, at least, difficult to access, and that the area should be flat. A caregiver talked about the danger of the bathtub:

I feel that most elderly people shouldn't really have baths.... It's either better to have a low ... floor shower or one that you walk straight into, a walk-in shower... you have to think long-term as sometimes they start having falls, and they get arthritis and they can't really lift their legs up into the bath, and so I'm afraid [of residents] falling. (PU5 caregiver)

While the wet-area shower was desired by those who didn't have one, some people felt it was dangerous because of its sloping floor for drainage. A caregiver talked about a woman with limited eyesight who would not take a shower because of this fear:

She comments that the way the floor dips, she feels like she's going to lose her balance, so she gets a bit nervous about it. This woman over there won't actually shower, she'll only 
really have a body wash ... because she's so frightened she's going to lose balance and fall. (RVS2 caregiver)

In this wet-area shower, the resident/caregiver noted that, 'The water goes all over the floor. ... That shower was dreadful. Water all over the floor and, of course, that's quite dangerous. (RVS2 caregiver). After taking a shower in the wet-area shower, a mat was often put down to prevent falls; however, this too could be a hazard. A caregiver commented that the wet-area shower is not suited for those who walk in, although it is well suited for wheelchair users.

The shape and size of the bathroom affected the sense of safety when they walked in. A caregiver said,

One thing about a small space, a lot of people talked to me and said, "Oh I can hold on, I can hold on." They feel safe. Those modern ones [bathrooms] ... they're making them a big square and it's too big. ... There's nowhere to hang onto. The square is not good. ... In the shower, you just walk and pull a curtain ... and trying to get to the loo there's nowhere to hold on and they [the residents] are nervous ... (PU5 caregiver)

Things to hold onto for support were important. For the elderly with a fear of falling, objects to hold onto, such as grab-handles and handrails, provided them with a greater sense of safety.

Having the emergency alarm button in the appropriate location in the bathroom was very important, as individuals needed to remove their portable alarm buttons for showering. One man could not reach the pull of the alarm, which was $1200 \mathrm{~mm}$ high:

Someone falls in the middle of the floor, they can't get to the alarm. ... I fell here one morning at 8 o'clock. I had closed both doors ... and when I turned around, I fell. I was there about 40 minutes... The alarms... are in the wrong places. (RVI8)

The design of bathroom fixtures can have a significant effect on an individual's control. For example, in some cases the location of the shower mixer lever was poorly located. When a resident was showering herself in the bathtub in the absence of her caregiver, she could not stop the water if it became tepid because it was out of reach (PU5). In another bathroom, the shower hose often got hooked on the shower lever, creating problems during independent showering (RVI8). Both the placement of fixtures and the heights of fittings were critical. For example, a vanity height of $920 \mathrm{~mm}$ high was too high for a wheelchair user (RVS5). 
Objects to sit on allowed many residents to perform tasks with ease. A built-in bench and a folded chair attached to the wall in a confined bathroom were preferred by those who could shower themselves without assistance (PR5, PR2). However, even though a folding chair was installed, a resident used a movable chair with armrests in the shower area when requiring caregiver assistance. This was they could adjust the chair location so that the caregiver could easily wash the resident's body (PR1). Armrests were particularly necessary for residents when standing up and sitting down, which occurred when assisted by caregivers (PR1, RVI2, RVI9, RVS1). When drying, many residents sat on their bed or armchair, partly because it was 'nice and comfortable' (RVI9 caregiver).

In a small bathroom, a resident who was a wheelchair-user required two chairs, one for washing and one for drying (RVS5). These were moved before and after she showered by herself.

Bathroom storage space was highly valued. Built-in shelves and cupboards at practical heights were preferred (PU1, PU5, PR2, PR6). A resident who didn't have enough storage in her bathroom complained of not being able to store her personal effects (a pile of towels, toilet paper, hand wash, boxes of soap and bottles) (RVS5) while a resident who had ample space in the bathroom was able to put his own cupboard in it (PU3). The design of the storage significantly affected its usability.

A personal dressing table, which provided a mirror and storage, and was appropriate height for performing tasks in a sitting position, was put adjacent to the bathroom and used by some female residents for grooming activities such as brushing hair, putting on makeup and putting hearing aids (PR6, RVS2).

\section{Theme 1-4: Control in cooking and dining}

The manners in which residents engaged in kitchen activities and eating meals varied depending on the physical environment and any meal services provided by the housing, as well as personal preferences. While some individuals did not cook very much, others stressed the significance of cooking for them (PU5, RVS6, RVI10, RVS4, RVS5): 'I don't profess to be a top cook but I enjoy the cooking. Yeah, I do, yeah'(PU3).

\section{Preparing meals}

A microwave oven was used in every participant's kitchen for warming up meals or making tea or coffee. However, space for the microwave was problematic for some. When the space provided 
was a shelf higher than the kitchen bench, many participants found it difficult to use (RVI10, RVI11, RVS1, RVS2, RVS3) (Figure 7-23). One man with limited eyesight said,

The one thing I don't like - you see that alcove over on the top there above the oven, that's made for the microwave. ... If it's up here, and if you heat something up and you pull it out of the microwave, and it spills, it goes right down, and you burn your chest. (RVI11)

Another resident also shared similar difficulties: 'The microwave is too high. It's too heavy to put things in there'(RVS3). Those who had sufficient bench space kept the microwave on the bench rather than a shelf (RVI2, RVI10, RVI11), while others who did not have sufficient bench space had no choice but to put it on the shelf (RVS1, RVS2, RVS3).

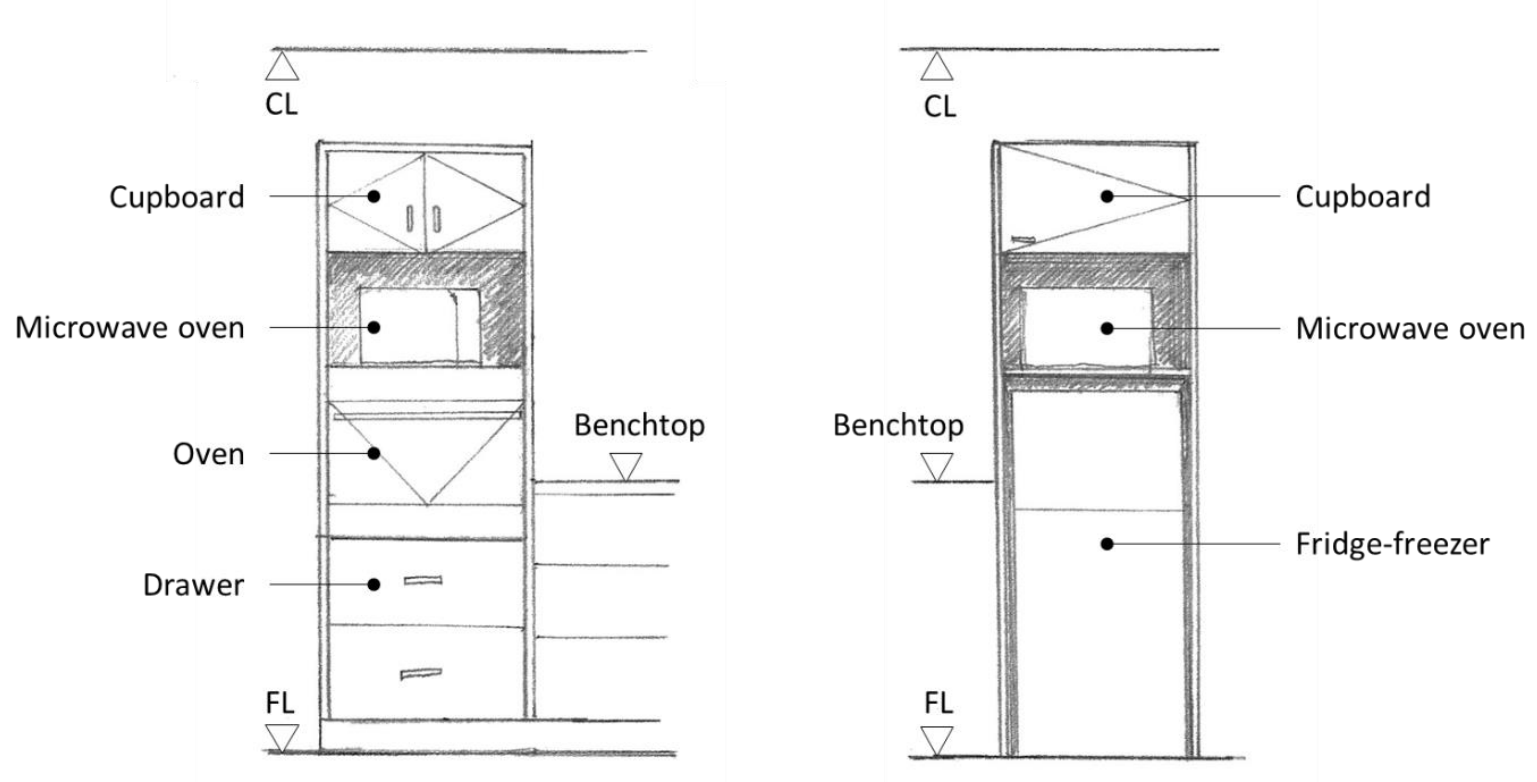

Figure 7-23: Space for a microwave that is too high

Hot plates were often used by residents for cooking various meals, such as boiling eggs, spaghetti, vegetables and sausages (PU3, PU4, RVI4, RVI10), making soup (PU5, RVI4), cooking stir fry (PU4, RVI4), making poached eggs (RVI10, RVS2), cooking bacon and eggs or scrambled egg (RVI2) and sautéing mushroom (PU4). However, one serviced apartment resident didn't have hot plates in her kitchen and said, 'It would be better if I had one'(RVS5).

Many residents had an oven installed in their kitchen. There were differences in preference about the use of an oven. Some residents hardly used it (PU3). However, some residents used their oven for baking (PU2, PU5), cooking roast meals (RVI10, RVS4) and cooking frozen food such as fish and chips (RVI4). One resident of a serviced apartment appreciated having the oven: 
'It's a very small stove here... It cooks alright. I can cook a meal, I can cook a roast, and I can cook chops... they have a good variety I can cook'(RVS4). Another resident had two ovens installed after moving in her house. One was for cooking and the other was used for warming plates (RVI10).

For breakfast and other light meals, residents often ate toast and sandwiches (PR2, PR4, PR7, RVI2, RVI3, RVI4, RVI9, RVI10, RVS2) as well as other simple food (e.g. cereals, porridge, fruits) (PU4, PR2, PR6, RVI2, RVI3, RVI10). To make toast or sandwiches, residents often took out frozen bread from the freezer and put it into the toaster (PU1, PU3, PU4, PU5, PU6, PR3, PR4, RVI9). The toaster was often kept on the bench, except for a few cases (PR4, RVI3 wife). Spreads such as butter and jam were taken out of the fridge or other storage space (PU1, PR2, PR3, PR7, RVI2, RVI4), and a knife was used for spreading. One resident used a sandwich maker to make a hot sandwich (RVI9).

Some participants liked cooking with a slow cooker (PR2, RVS5) and a rice cooker (PR3, RVS5). For example, a serviced apartment resident liked cooking with a slow cooker and a rice cooker for entertaining guests: 'A slow cooker, I have, I'm very keen on cooking. I like have people come over. I have a rice cooker, I still use the rice cooker.'(RVS5). One Asian resident kept her rice cooker on the bench (PR3).

Many residents had a cup of tea or coffee during the day (except for some, such as PR6, RVI1, RVI7). For many, making tea was an important routine (PU1, RVI2, RVS2), and enjoyed having a cup many times a day (RVI2, RVS4). Even in serviced apartments where tea was served by the staff at fixed times, residents made tea by themselves when they liked; for example, one resident liked to have tea at night (RVS4). Residents preferred having tea or coffee in the way they liked (such as a specific amount of milk or sugar added) (RVS1) and making the tea themselves was important to get this right. Making tea was also important as part of entertaining family and other visitors (PR2, RVI2, RVS2). For those with limited mobility to make tea, it was important that the bench, where a cup and an electric kettle are put, was in close proximity to: the sink and tap; the storage locations of a cup, tea bags, a bottle of instant coffee, sugar and a spoon; and the fridge where milk was kept. Where the fridge was on the opposite side of the bench, a resident who could not turn easily had difficulty in taking milk out of and returning it to the fridge (PU5).

Some participants had difficulty getting water from the tap into the kettle, and caregivers prepared it (PU5). Pouring boiled water into a cup was also difficult with a shaky hand or 
limited eyesight (PU1, RVI11); one resident with shaky hands kept his arm close to the body for support as he poured water, although the water still spilt (PU1). For a wheelchair user, a bench at $900 \mathrm{~mm}$ tall was too high for her to pour boiled water (RVS5).

Residents sometimes cut vegetables and other ingredients. However, those with limited strength in their hands found this challenging. One post-stroke elderly man '[couldn't] even cut a piece of cabbage'(PU1). Another man used a special cutting board, which didn't require much power or could be used with one hand (RVI4). One resident put a rubbish bin under bench in proximity to the space where she cut ingredients, which was convenient (PR3).

To be seated during cooking was important for some individuals. One man with hemiplegia put a table and a chair in his kitchen (PU1). The table depth of $700 \mathrm{~mm}$ allowed him to put dailyuse tools and foods on the table and still leave space where he could perform tasks such as spreading butter on bread (Figure 7-24).

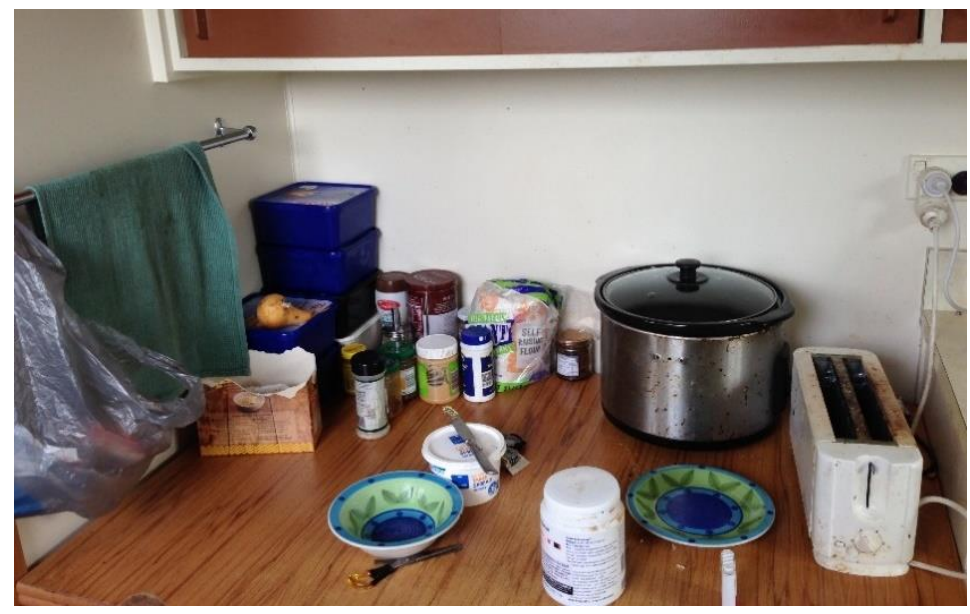

Figure 7-24: $700 \mathrm{~mm}$-deep table accommodating tools and foods while leaving space for tasks

A resident, who had difficulties in standing for long periods, put a high stool in her kitchen and had benches of $900 \mathrm{~mm}$ high on three sides. When she cooked lunch with a sandwich maker, she made sure all ingredients (such as bread and a tin) and tools (such as a sandwich maker and a tin opener) she would use were on the bench. She took these from shelves and the pantry as well as the fridge, and then sat on the stool to start cooking (RVI9) (Figure 7-25). She liked that her kitchen was compact; her storage spaces, fridge and bench were close and convenient. However, when she needed to take an additional tool from the drawer behind her, she had to stand up and turn around.

One wheelchair user had experienced difficulty in cooking in a kitchen unsuitable for wheelchair users, while in a previous dwelling: 'I used a stool because I couldn't do standing. But I had to 
move when I carried things from the microwave to stoves [because they were on opposite sides across the passage]. ... It was difficult'(RVS5). This was sufficiently inconvenient as to make her change dwellings. In her current dwelling, she had a kitchen bench of $730 \mathrm{~mm}$ high, which was convenient for her to perform most tasks for preparation and eating, but she found it still too high for cutting ingredients (RVS5). This height also allowed the resident to use a normalheight chair; particularly she liked to use a swivel chair, as well as her wheelchair, as it allowed her to easily change her direction and move between spaces.

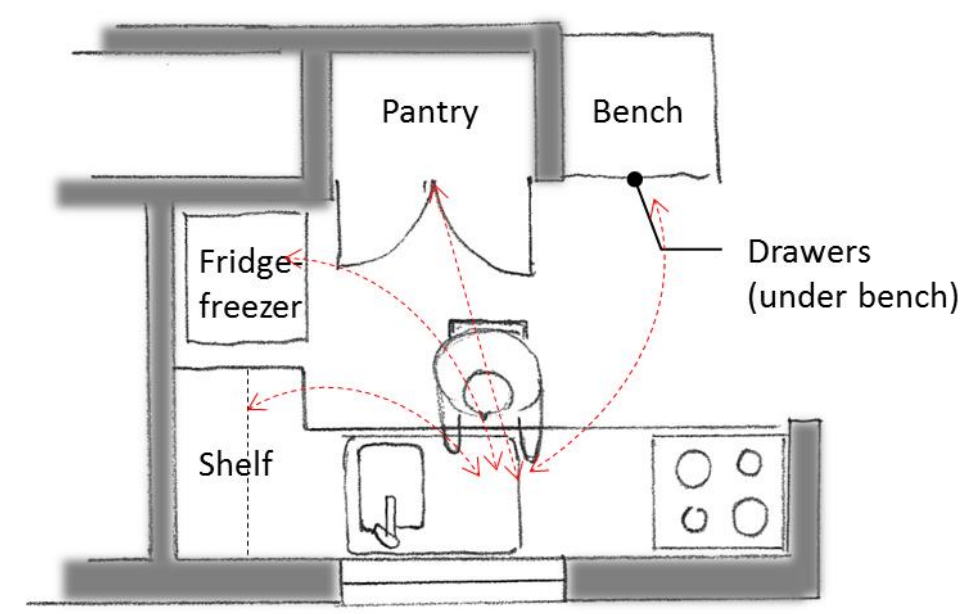

Figure 7-25: Gathering all foods and tools on the bench before cooking

\section{Carrying meals}

Some residents found carrying meals and drinks from the kitchen to the lounge a challenge. A walker frame or trolley was used for carrying meals and tea, particularly in case their eating space was far from the kitchen (RVS2, RVI2, RVI9, RVI10). However, one resident, who could not use her walker frame in her unit because of a lack of space, had trouble carrying meals; she could not use two hands to carry two plates at one time because she needed to hold onto a wall and furniture by one hand to keep her balance (PR6).

In a narrow kitchen without enough space for a walker frame or trolley, residents left their walking aid just out of the kitchen, and they either carried food unstably or took the walker frame into the kitchen just before carrying food (PU6, RVI9). One resident brought the walker frame to the end of the kitchen, which unfortunately blocked the fridge door from opening (RVS2). She then had to move out of the room backwards when she was using her walker frame. Carrying meals was also difficult for a wheelchair user (RVS5): when using an electronic wheelchair, she would have needed to operate the controls with one hand and could not hold 
things with both hands. So, she usually used a manual wheelchair, which allowed her to move her feet ahead and kept both her hands free, letting her carry meals with both hands.

One resident who had had a stroke and suffered from hemiplegia handled his trolley with one hand to carry tea or soup from the kitchen to his lounge. When he moved across the threshold between different floor materials, he had to be very careful not to spill the liquid he was carrying, even the one with less than a one-centimetre height difference (PU5).

Benches and tables provided residents with support in carrying meals as well as cooking. One resident said they held on 'everywhere [they could] hold on'(PR1). When the dining table was positioned close to the kitchen bench, residents had little trouble carrying food (PU3, PR1, PR2, RVI2), and they did not need to use their walker frame (PR2, RVI2).

\section{Dining}

Even in the complexes where meals were cooked in the main kitchen, residents had the choice of eating in their own room or in the communal dining room. Some preferred to eat in their own room (PR6, RVS3, RVS5): 'I have trouble swallowing and talking. As the day goes on my voice starts going. I'm backwards, because I just want to be able to eat and if I don't want to eat I don't'(RVS5). Those who used Meals on Wheels, or who chose to bring their meals from the main kitchen of their complex into their own space, often prepared their cutlery or added a drink or adjusted the meal's flavour by adding salt or seasoning themselves. It was important that there were adequate facilities in individual units to accommodate cutlery, a cup or glass, and containers of seasoning.

While some individuals used a dining table for meals (PU4, PU6, PR3, RVI1, RVI2, RVI4, RVI5, RVI9, RVI11, RVS1, RVS3, RVS4), many ate sitting in their armchair even if they had a dining table (PU1, PU2, PU3, PU5, PR2, PR4, PR5, RVI3, RVI4, RVI6, RVI7, RVI10, RVS2). A couple originally used their dining table for having meals, but they came to use their own armchairs for eating after one of them had a stroke and it became difficult to sit at the table (RVI3). Another resident got rid of her dining table because of insufficient space in her unit (PR4). Residents who ate in their armchair had a table (a low table or a portable table), a trolley or a kitchen bench beside their armchair, and used it to put plates, cups, and salt and pepper on, as well as using their knees or a tray. 
While having meals or tea in their units, residents often watched TV (PU1, PU2, PU3, PU4, PU5, PR1, PR3, PR5, RVI4, RVI6, RVI7, RVI10), while some read a book, magazine or their iPad (RVI9, RVS5).

\section{Washing dishes}

Washing dishes was a demanding task for those who had difficulty with standing and was often done by caregivers. Those who washed dishes themselves often stacked up used dishes throughout the day and washed them altogether once a day (PR4). A dish washer was appreciated by some (PR1, PR2, RVI3, RVI9). One resident said, 'I didn't use the dishwasher till I came here, but I must admit that it's nice'(PR1). Another resident also said, 'I don't know what to do if I didn't have it'(RVI10). Those who had a dishwasher used it daily or three to four times a week. Residents who didn't have a dishwasher had mixed opinions; some had concerns about the cost and space efficiency (PU1, RVS3), but others agreed that a small drawer type dishwasher would be beneficial despite these concerns (PU5, PR4, RVS4).

When residents used the dishwasher, they first sat on a stool in front of the sink, then ran water for prewashing dishes. Next, they put dishes into the dishwasher, then added detergent powder (often stored in the cupboard below the sink) and started the dishwasher (RVI4, RVI9). While sitting, another resident opened the cupboard under the bench to accommodate her legs (Figure 7-26).

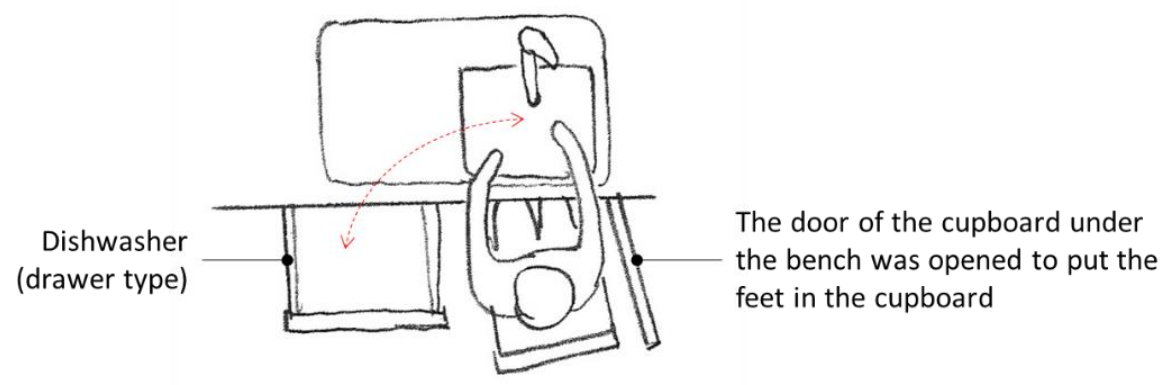

Figure 7-26: Sitting space for washing dishes with a dishwasher

The type of tap affected the task of washing dishes. One caregiver explained the need for a mixer tap: 'They have the [hot] water and cold water separate. It should not be like that, then people always have warm water [instead of hot water]. They can't use cold water, too, because they always feel cold'(PR5 caregiver). One man with shaky hands had difficulty in turning the round tap handle (PU1). 
Storage

Storage was important for participants, particularly for those who didn't go shopping often:

To have extra things, to get enough to last, I get enough each time to last me a fortnight. I buy extra if I can, and if I run out, I've just got to make do until I go and get my groceries next time. (PR5)

The fridge and freezer were important for storing food. They were often used for preserving food which had been previously cooked by residents, their caregivers or family (PU2, PU3, PU4, PU5, PU6, RVI3, RVI7, RVI10, RVI11), as well as for storage of other uncooked foods such as milk, yogurt and fruits. This is because, when cooking food, it was commonly too much for one meal for one person. One resident kept salad in the fridge: ' I can make a salad and it will keep for two weeks, in this Tupperware. I usually buy the packet lettuce(RVI10). A freezer enabled one woman to enjoy her favourite home-made meal at any time:

You can't beat a home-made soup. I had some old beef bones... So I put some in a big pot with barley and grated carrots and celery and onion, you name it - that's a proper soup. Yeah, like a big pot of everything and when it's cold I put it into containers and freeze it and just get it out when I need it. (PU5)

Generally, a big freezer was preferred (PR5, RVS4). Many residents stored daily foods such as bread, frozen pre-cooked or pre-cut food, such as frozen vegetables and meat or fish in the freezer (PU1, PU3, PR3, PR5, RVI4). One woman prepared a meal using mainly frozen ingredients, saying, 'Everything is in the freezer'(PU5). One male participant liked storing vegetables that had grown in his garden in his freezer: 'Broad beans, they grow during the winter - I must put some in... I got frozen veggies in the fridge[-freezer] here, you know'(PU3). A drawer-type freezer was valued because 'You could look into the inner part'(RVS4). However, some residents could not put the fridge-freezer with a drawer-type freezer because the fridge-freezer space was too narrow to accommodate the type of fridge-freezer, which was typically large (PR1, RVS4). One resident living in a serviced apartment with only a space for a mini-fridge below the kitchen bench brought in a normal-size fridge-freezer, which then protruded into the lounge area (RVS1).

There was also an issue in the design of some fridge-freezer spaces in a compact kitchen. One resident talked about her inconvenience in using the fridge-freezer: 'I can't open the door wide enough to get to get the shelves out'(Figure 7-27) (RVI9). 

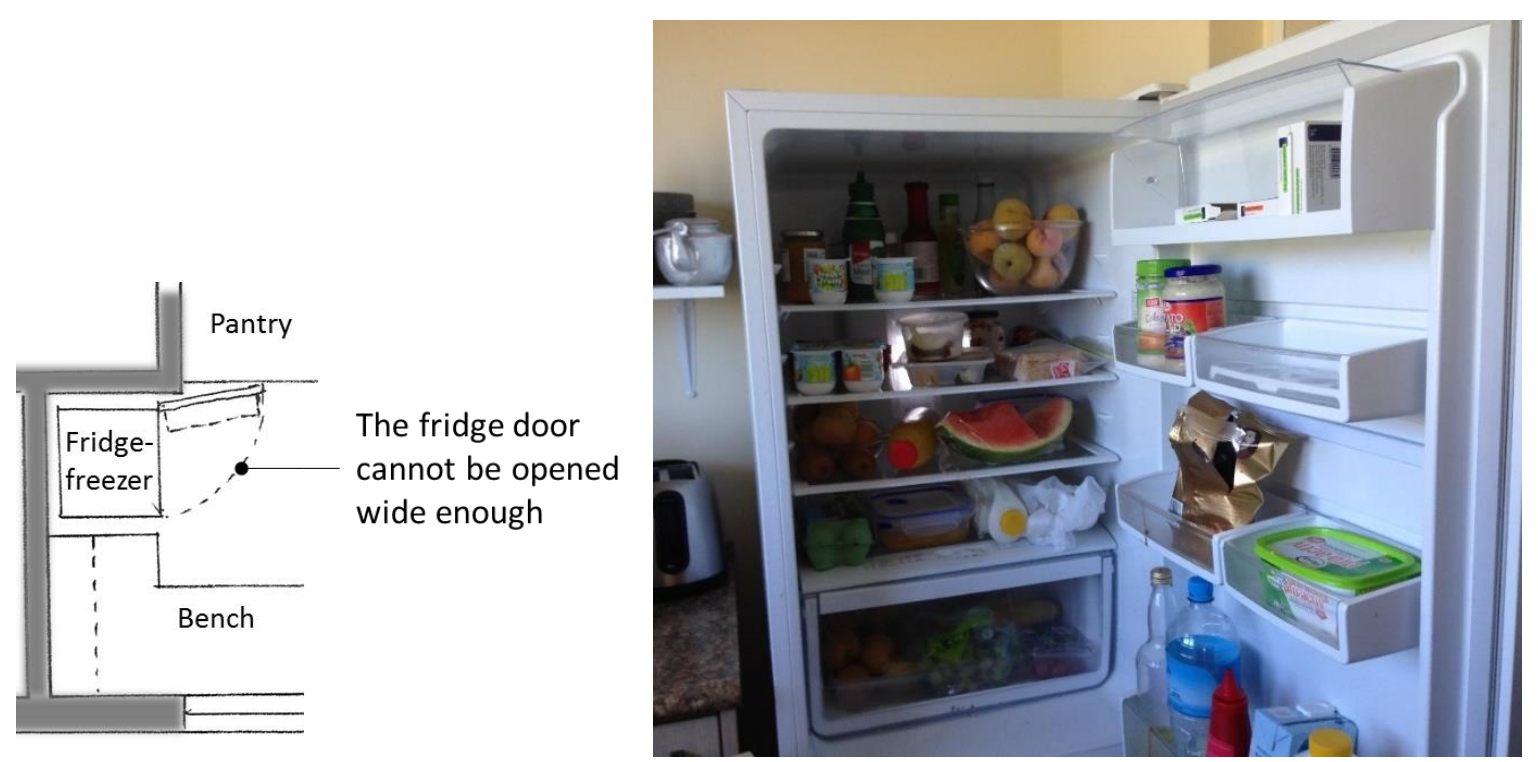

Figure 7-27: Inconvenient space for a fridge-freezer

Storage other than a fridge-freezer was also important. Not having not enough storage caused one resident trouble: 'I have to put something in the cupboard before I get something else out because I don't have enough room to put it otherwise'(PR5). A kitchen with limited storage could not meet the needs of those who liked cooking: 'I've got baking tins and things, they're [small kitchens] not designed to be accommodating to that sort of stuff (RVS5).

Having storage of appropriate height was important. Shelves and cupboards above the bench were often too high for participants (PU1, PU5, PR5, RVI2, RVI6, RVI9, RVS5) (see also Theme 4-3). Not having enough storage within reach meant they 'put a lot of things on the bench'(RVI6). Some residents filled the benchtop with pots, pans, tools and food, as there was not enough space for the resident or caregiver to put plates on the bench for food preparation (instead, the top of the range was sometimes used) (PU5, PR5) (Figure 7-28). 


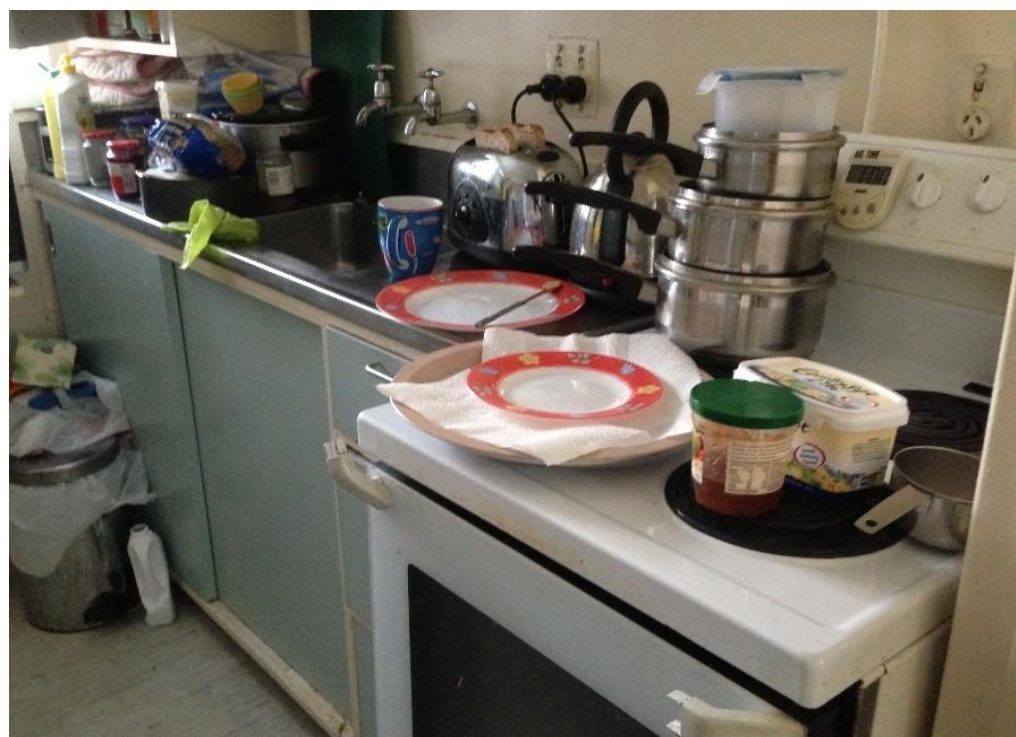

Figure 7-28: Insufficient storage resulted in insufficient bench space for cooking tasks

Low cupboards were problematic. One resident said, 'Because most of the things are down in the bottom cupboards ... I can't get at them if I want anything, so it's no use to put anything into the cupboards, it's no use'(PR5). However, drawers were perceived as relatively useful, even the lower draws (PR1, RVI10, RVS4). One resident said, 'a drawer underneath it [specify what] for pots and pans, that's all good'(PR1).

A pantry was useful for storing various things such as food and tableware. Those who didn't have a pantry wanted one (PU2, PR5). One resident of a serviced apartment put her own pantry cupboard near her kitchen beside the doorway, which made the space too narrow to pass through with a wheelchair (RVS1) (Figure 7-29).

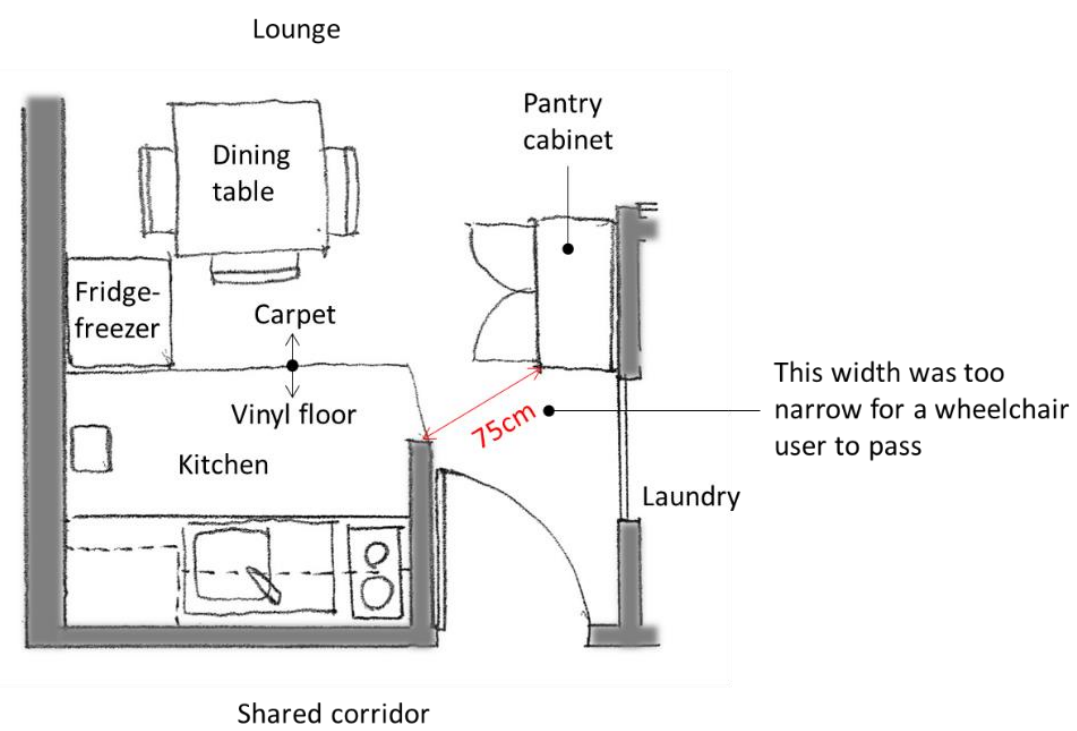

Figure 7-29: Insufficient kitchen space, which resulted in too narrow a passage for a wheelchair user 
Storage capable for tall things such as trays were preferred (PR4). One resident complained about a lack of storage for tall items: 'When I came here, oh, I've got these cupboards, but you can't put anything in. If you want to put something tall and you can't'(PR5). Another resident put a tray on the floor and had difficulty in picking it up as she had to crouch to do so (PR6).

The corner part of a cupboard under an L-shaped bench was often not efficiently utilised, as most of the space was difficult for residents to reach. This was problematic in a small kitchen. Some residents described as 'the waste of space'(PR4) or 'wasted space'(RVS4). In order to store various ingredients such as flour, sugar, and bottles with oil, vinegars and sauces in the space, one resident used four or five containers, which she had to 'take it out all the time' (RVS4). To use the space more easily and efficiently, some devices were required. For example, the resident explained one of the devices: 'I don't know whether you've seen them, they're corner shelves [designed so] that they can open the door, and the shelves come out'(RVS4). Another solution was installing a small turntable inside the corner part that assists in storing and taking out many items easily, which was actually appreciated by one participant (RVI9).

Open shelves were used a great deal. Tins, bins and containers of food such as coffee, tea and spices, as well as cups, were put on shallow open shelves or the raised part of the kitchen bench, which made them handy for use (PU3, PU4, PU5, PR3, RVS4). Deep, open shelves accommodated various things, including kitchen appliances (such as microwave ovens and cookers) and fresh vegetables and fruits (PU4, PR6). One resident used the shelf intended for a microwave oven for cook books (RVI2).

Hooks and railings on the wall were useful for hanging cups, teapots and utensils (PU2, PU5, PR3) (Figure 7-30). 


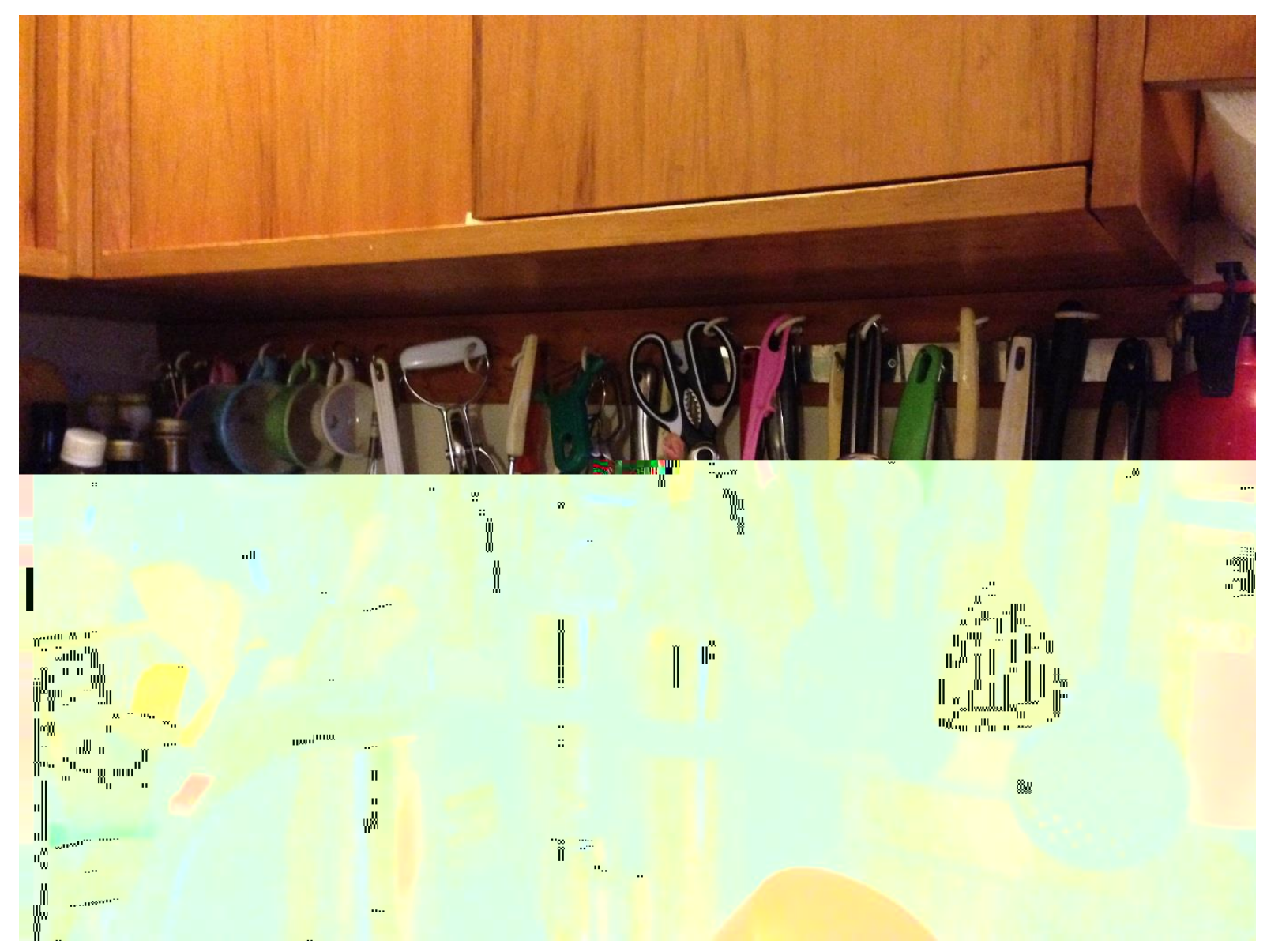

Figure 7-30: Use of railing and hooks

The bench space was of great use for storage, not only as a space for cooking. It was used to store kitchen appliances, food (fruits etc.) and medications, where there was no other adequate storage space for them. It was also used for storing daily items peripheral to cooking, such as telephones and eyeglasses (PU3, RVI11). Where space was abundant, decorative items such as flowers were displayed (RVI10). One resident who had sufficient bench space said, 'It's virtually cluttered with that and that and that, you know. The phone didn't have to go there because I've got that many phones - you've got a phone there, a phone around the corner. ... I've got plenty of bloody bits and pieces here, you know'(PU3). Another resident complained about his insufficient bench space, while praising the compactness of his kitchen (RVI11) (Figure 7-31). 


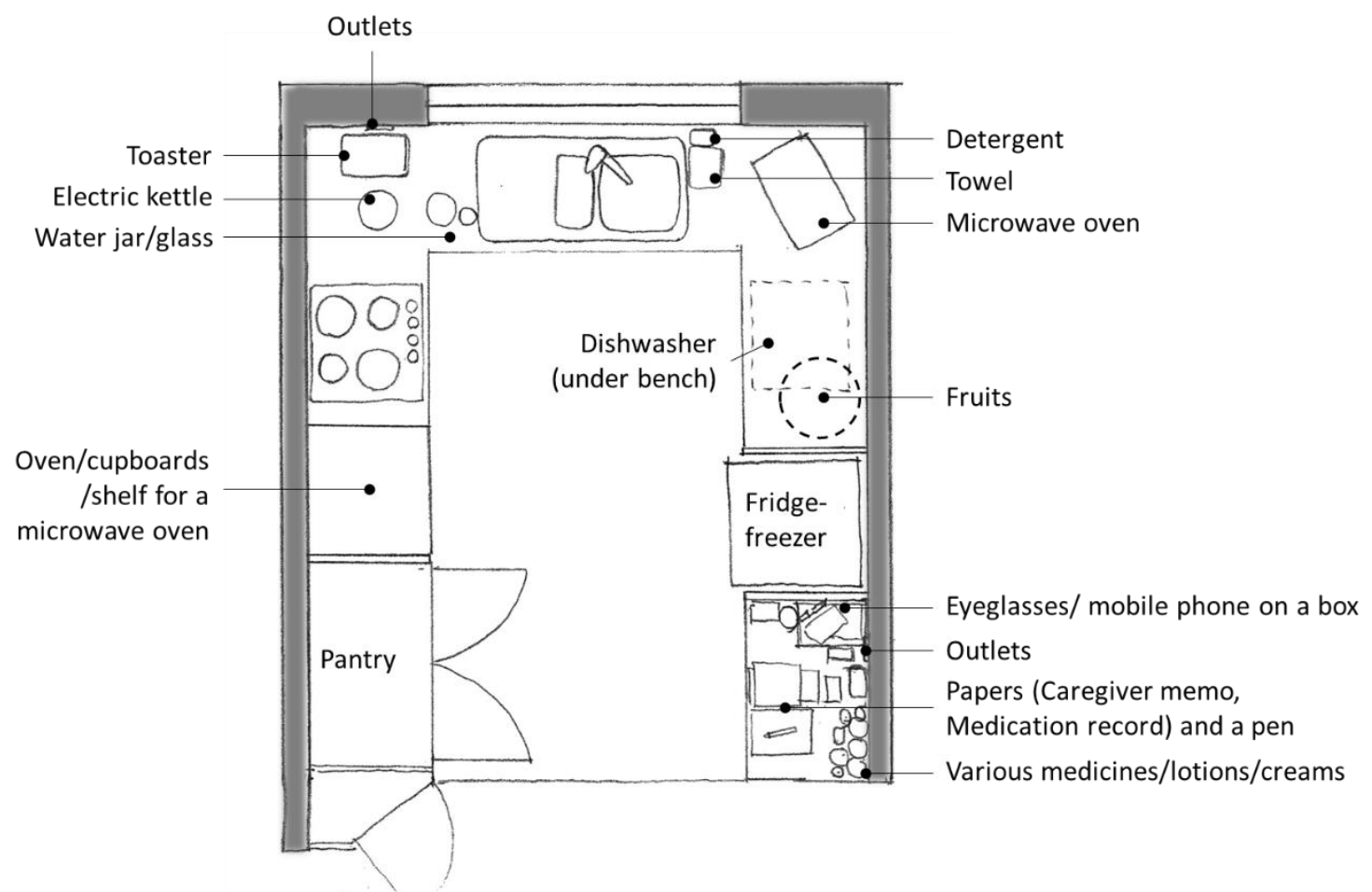

Figure 7-31: Bench space fully used for storing various items

\section{Theme 1-5: Control in laundering}

Some residents had their caregivers or friends do all or some of their laundry (PU5, PR5, RVS1). Serviced apartment residents could also use the laundry service provided by the housing. Those who used the service used it for limited washing, such as towels and bedding, but washed personal clothing items themselves (RVS1, RVS2). One caregiver said, 'We do offer a laundry service here. [But] she still likes to do some of her own stuff... And it's good independence, I think'(RVS1 caregiver). The daughter of one resident also said, 'Family could do the laundry, but Mum wants to be independent. And so, you know, if you've got clothes that you want to wash straight away, you can't leave them sitting to wait for somebody to come and take them' (RVS4 daughter).

\section{Washing}

The distance between the space where dirty items (such as clothes and towels) were stored and the washing machine affected the ease of tasks. One woman kept towels in the bathroom and dirty clothes in the closet near the bathroom then carried them on a trolley to the washing machine in the garage (RVI9) (Figure 7-32). This task tired her and she had to take a rest, sitting on a chair in the garage, before running the washing machine. In contrast, where a washing machine was in or near the bathroom, some residents took off clothes before showering and put them directly into the washing machine (PU3, PU4, PU6). 


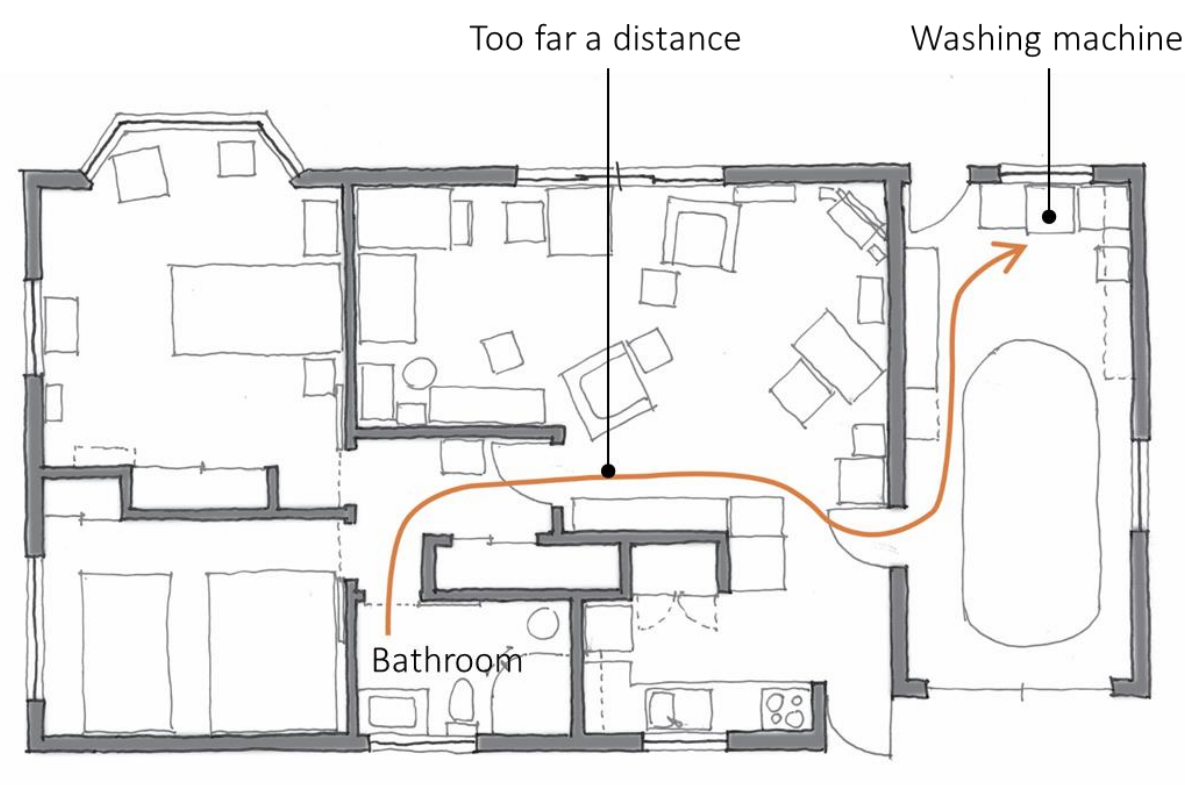

Figure 7-32: Too far a distance between the storage for dirty clothes and the washing machine

The laundry space was sometimes too small for residents with mobility aids. One resident who had a laundry room (not simply a cupboard) didn't have sufficient space to turn around with her walker frame, so she had to enter the laundry backwards (RVS2) (Figure 7-33). In contrast, a laundry cupboard was appreciated for being compact in a small apartment (RVI6, RVI7, RVS3, RVS4) (Figure 7-34).

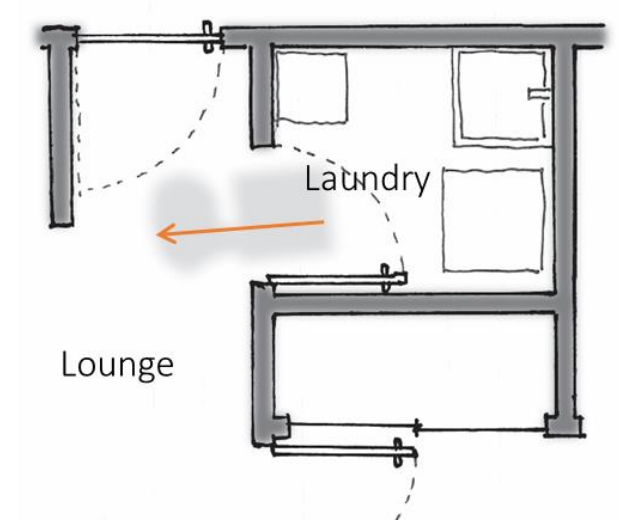

Figure 7-33: Insufficient space in the laundry room for turning around with a walker frame 


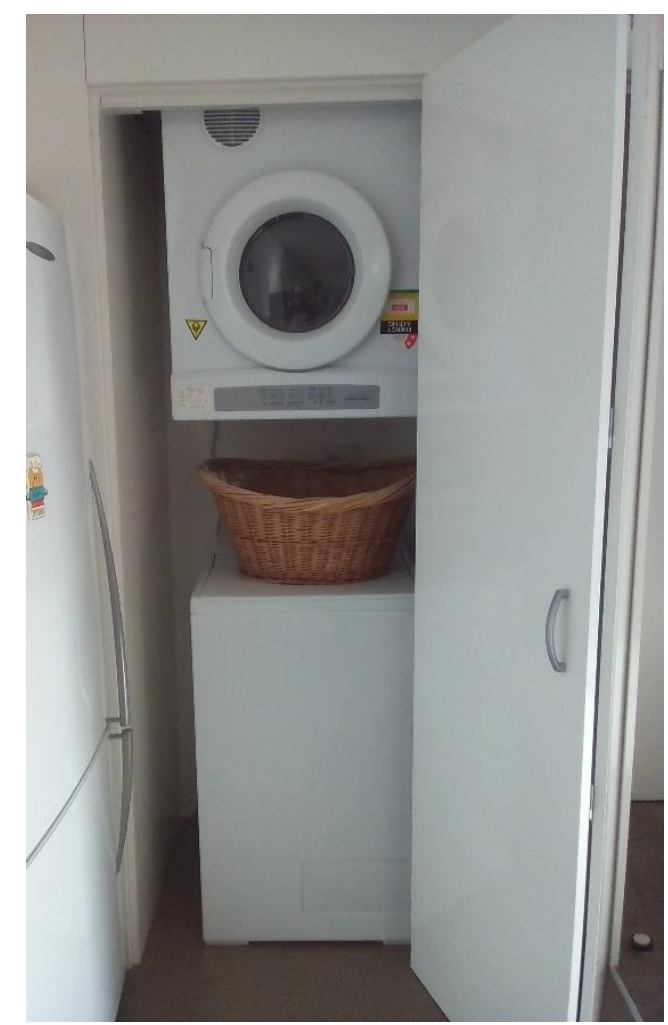

Figure 7-34: Laundry cupboard

\section{Drying}

Residents and caregivers used a range of strategies for drying items. One caregiver sometimes used a dryer and at other times used a clothesline outside. The resident herself used the communal airing room to dry clothes on the laundry rack 'to make sure you dry perfectly'(PR6). Another resident dried differently depending on the season; he dried clothes mainly in the bathroom in winter and used the clotheslines in front of his unit in summer (PU4).

Some residents liked to hang clothes on the clothesline outdoors (PU2, PU5): 'It's lovely drying it outside - nice and fresh'(PU5). Hanging laundry in the sun was preferred; one resident who only had south-facing porch dried laundry by bringing her rack onto the communal porch, which faced the north (RVS4). However, many residents had difficulty in using clotheslines and said clotheslines should be near their units. One resident said, 'I am frightened to fall. Walking with a basket and hanging [clothes]'(PR3). Another resident who had common clotheslines but didn't have a porch also said, 'It's almost like you need a wee porch, you could hang up a few things'(PU5). The height of clotheslines also mattered; the clothesline was too tall for one resident to use (RVI10). Even the height of a laundry rack was too high for one resident seated during trying her laundry, who suffered from her neck discomfort (RVI9). 
Clotheslines required privacy. One resident used a rack in her porch, mainly for underwear, while hanging other things on the communal clotheslines (PR5). A woman who lived in a firstfloor apartment didn't hang her clothes in her veranda, because 'it doesn't look good to have clothes out there'(RVS5). Another resident who didn't have a clothesline in her porch when she moved in installed her own clotheslines on her porch: 'The clothesline is important'(RVS4).

A laundry rack was used to dry clothes inside the dwelling when the weather was wet, when items had not completely dried, or in the case where residents were not able to use a clothesline. The drying rack was put in the bedroom (PU2, PR4, RVI2), bathroom (RVS4) and other places around the unit (PU5, PU6, RVI6, RVI1). Garages were also spaces used for drying clothes on ropes hung on the walls, as well as for putting a rack (RVI2, RVI3, RVI4, RVI10). A rail heater in the bathroom was also useful for drying (PR5, RVS4). Being able to dry indoors privately was important. One resident said, 'I put it here [in the laundry room] or I put it in the lounge or bedroom. And by the windows.... From the outside, it can't be seen'(PR4). However, one resident in a bedsit unit didn't have enough room for a rack. She put it in the bedsit room, which blocked the way to the bathroom and made it difficult for her to get around (Figure 7-35) (PU5).

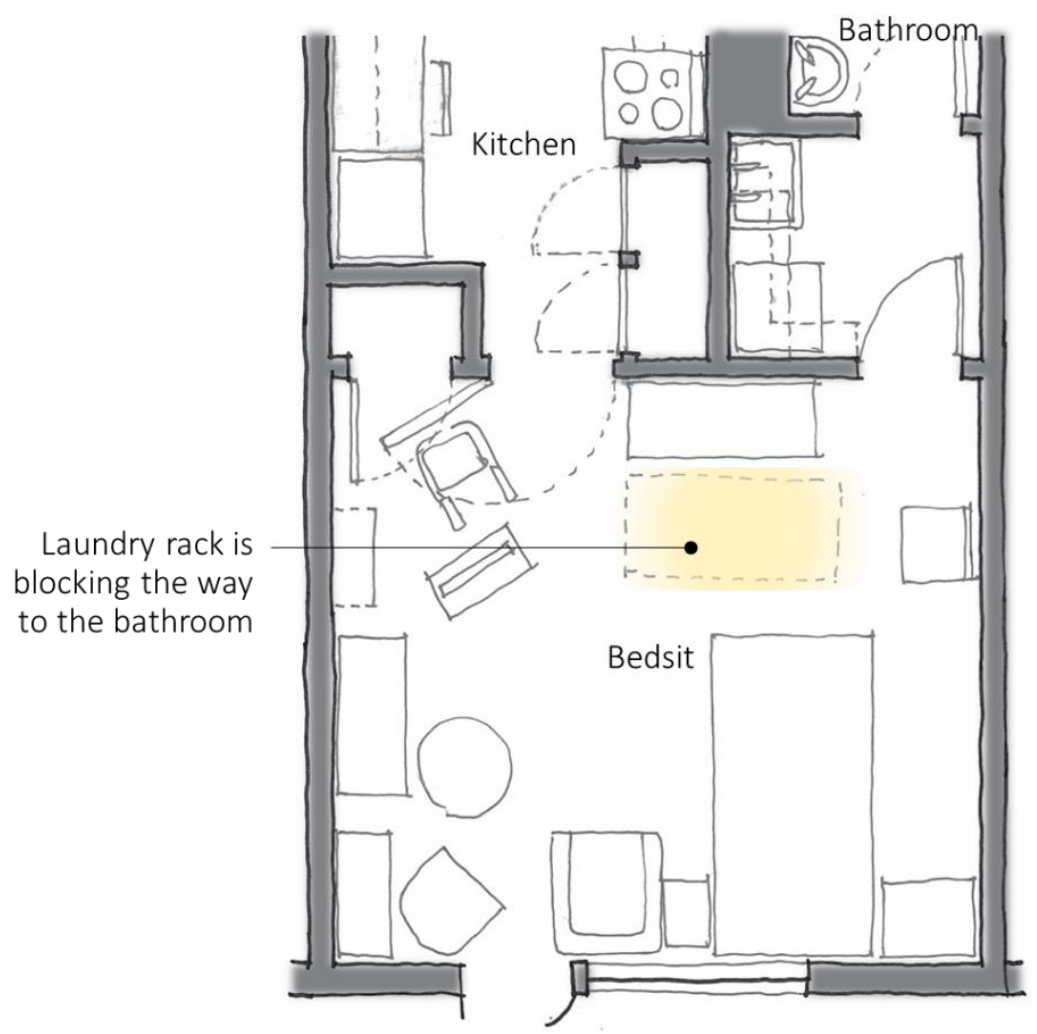

Figure 7-35: Lack of adequate space for a laundry rack 
Some people only used a clothes dryer in rainy weather or after drying in other manners, while others used it as the main way of drying clothes. A dryer was put in the porch in one serviced apartment because there was no space inside (RVS4). The height and placement of the clothes dryer required particular attention. Some residents put it upside down to make the controllers accessible when it was mounted on the wall, as shown in Figure 7-34 (RVI1, RVI7, RVS2).

Some residents had a concern about the cost for a clothes dryer: 'I wouldn't have a dryer, it's expensive'(PU5). One woman acknowledged the merits of sharing a dryer with other residents, while wishing for an individual washing machine (PR1).

\section{Theme 1-6: Control in maintaining the home}

Maintaining cleanliness was important for participants. All participants had a home helper who came once a week or fortnight. Two residents mentioned cleaning as one of their important activities (PR2, RVS6): 'I do my own cleaning, to the point where the cleaners come in and ask me what needs to be done because they can't see anything that needs being done', said one resident (RVS6).

\section{Ability to maintain the whole house}

It was important that elderly residents could manage to keep the whole house clean. One resident of a one-bedroom house said, 'It would be more difficult to manage [if my house was larger]. Now I can manage it because it's small with the lounge, the kitchen, the toilet and the bedroom'(PR3). If their house was bigger than its current size, the resident would have more 'maintenance and cleaning, everything, to take care of it'(PU6). One resident said the main reason for moving to their current house was 'because we couldn't clean out the gutters, couldn't clean the windows, you've gotta mow your lawn, you've gotta do your garden'(RVI5). Some residents didn't want an increase in house size because of the increased needs for maintenance (PU3, PR3, RVI2, RVI3, RVI5). These perceptions were shared by residents in houses of various size, from one-bedroom units of 40-60 $\mathrm{m}^{2}$ (PU3, PR3) to two-bedroom units or bigger, of $100-110 \mathrm{~m}^{2}$ (RVI2, RVI3).

\section{Keeping various elements of the house clean}

Residents often experienced difficulty with keeping various elements of the house clean, such as floors, doors, fixtures and high areas.

Floor materials that were easy to clean such as vinyl and linoleum were preferred for the kitchen and bathroom by many residents (PR1, PR2, PR3, PR4, RVI11): 'A vinyl floor is easy to clean, 
in case water spills'(PR2). One resident, who had shaky hands and often spilled liquid (such as tea and soup) didn't like his carpet. Pointing to a stain, he said, 'Look, if you see what's on this mat, you suppose something like that ever gets off, no matter what you do to clean it off?... What do you do if you do spill something like that again?'(PU1). The stain remained even after a professional cleaner cleaned the carpet. He wished for a non-slip tile floor to be laid all over the units.

The colour of floor materials also mattered. One resident liked her carpet colour because stains didn't show much (RVS2). One couple also acknowledged the current colour (light brown) didn't show stains, although they preferred their carpet to be brighter to match their furniture (RVI3).

One resident discussed the design of the bathroom floor for ease of cleaning. She explained that floors with small bumps, designed to prevent slips, are not always easy to clean depending on the shape of the bumps. Cylinder-shaped bumps were less slippery but not easy to clean, and hemisphere-shaped bumps were easier to clean but more slippery. She suggested raised bumps on the floor could be designed in a form that is both easy to clean and prevents slips (Figure 736).

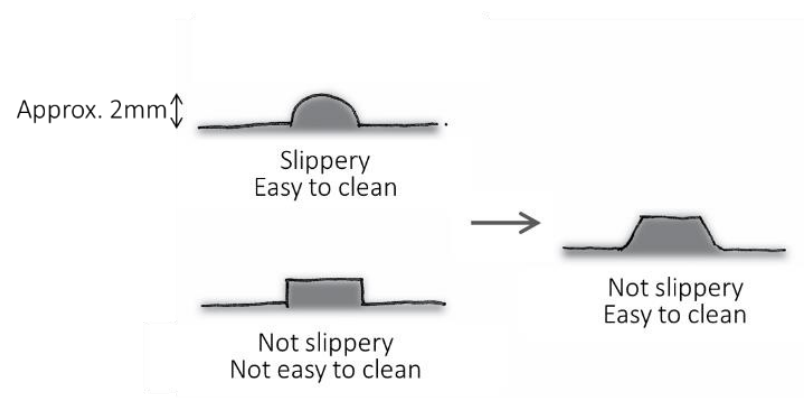

Figure 7-36: Consideration of non-slip floor design (floor sections)

One resident did not like having a shower curtain because it was prone to become 'full of mould' (RVI11). Another resident using a wheelchair pointed out the difficulty with cleaning a shower curtain: 'It would be a nuisance. To wash, you have to take it off and on'(RVS5).

\section{Taking rubbish out}

While some residents had their rubbish collected at their front door (RVI3, RVI4, RVI9, RVI10, RVI11), others needed to bring their individual rubbish and recycling bags or bins to the communal collection point (PU5, PR1-4, PR6,7, RVS1-4), which was often problematic due to inaccessibility such as a level difference and a slope (PR1, RVS2, RVS3). One resident, whose 
unit was too far from the collection point, had changed the way they managed rubbish collection: 'I used to bring the rubbish bags by car to the road, but now a private company comes to pick up the rubbish at the door once a week. I pay $\$ 5$ fortnightly’(PR5). However, she gave up some recycling: 'They [the rubbish company] don't recycle... I stopped putting the recycle things out because I can't put the bin out. I keep only papers in the storage and give them to my daughter'.

Rubbish and recycling storage was important where rubbish and recycling is collected individually. A garage was often used for storing rubbish (RVI3, RVI4, RVI9, RVI10, RVI11). Those who didn't have a garage stored their bins on the porch (PR5). One resident wanted storage space in the kitchen cupboard rather than in the garage:

Here to recycle, I've got to go out that door and put one [recycling bin] like there and the other in the paper [bin] and roll it around further, and it's a blooming nuisance. But there [in other units], they've got their recycling bins up in the cupboards, and it's so much easier. (RVI11)

\section{Theme 1-7: Control in shopping}

While some participants went shopping once or twice a week or fortnightly (PU1, PR2, PR5, RVS6), many didn't go shopping regularly. Reasons included: their limited mobility, the provision of all the meals (PR6, PR7); family or caregivers' support (PU6, PR1, RVI1, RVI7, RVI8, RVI11, RVS1, RVS2); and shopping online or by telephone (PU2, RVI9, RVI10).

Some people mentioned the difficulty in carrying heavy shopping items into their house, and often had family/friends or a taxi driver's assistance in doing so. The close proximity of the garage from the home was preferred (RVI2, RVI9). One woman appreciated the close proximity of her garage and kitchen (Figure 7-37): 'I like the garage right there, so when I do my shopping, I can drive my car into the garage, and then I can put my stuff on this trolley and the kitchen is right there. ... And you're not going through the house'(RVI9). However, she had a difficulty with the passage in the garage that was $2.9 \mathrm{~m}$ wide, because the garage was not wide enough for her to use a trolley. 


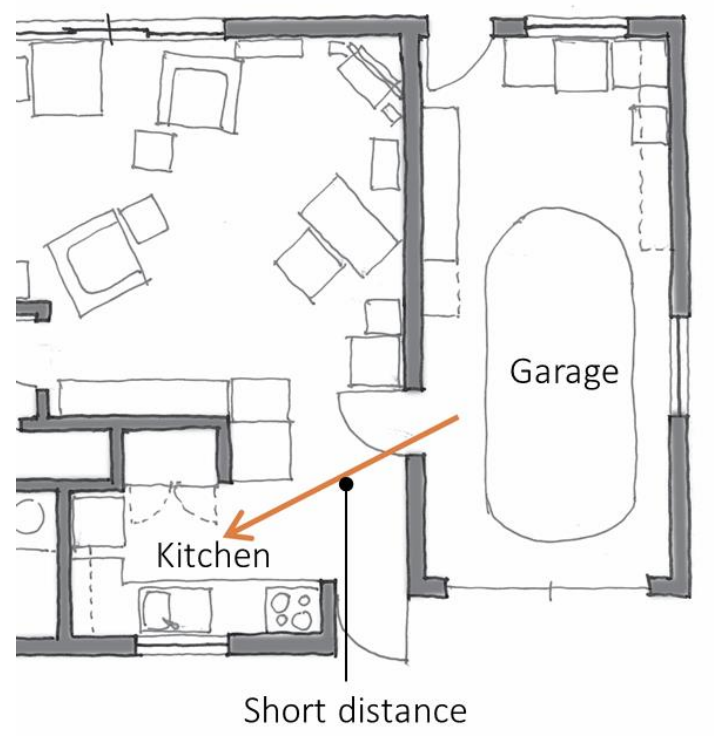

Figure 7-37: Convenient layout of the garage with respect to the kitchen

\section{On-site shop}

One RV had an on-site shop in the serviced apartments, which was convenient for some apartment residents to buy a few items they needed. An on-site café was desired for buying food or coffee (RVI2, RVI3, RVI4). One woman living in a public housing complex shared her views about on-site shops:

They used to have a vegetable cart coming in over by the community hall - that was years ago. Fresh veggies and eggs, but that doesn't come anymore. We used to have the fish man. He doesn't come anymore. And we used to have Mr Whippy, which is lovely, but he doesn't come anymore - that would be lovely! (PU2)

\section{Theme 1-8: Control in collecting mail}

Some residents used vehicles such as a scooter (PU1) or a bicycle (PU3) to get to their letterbox, while others had a neighbour or a caregiver collect their mail (PU5, RVI10, RVS1). Some people had difficulty with access to their letterbox, due to the hinged door with a level difference on the way to the letterbox (RVS3) and insufficient space in front of the letterbox to allow a walker-frame user to approach it (Figure 7-38) (RVI10). In yet another home, the letterbox was too high for the resident, who used a wheelchair, to open with the key and collect mail (RVS5). 


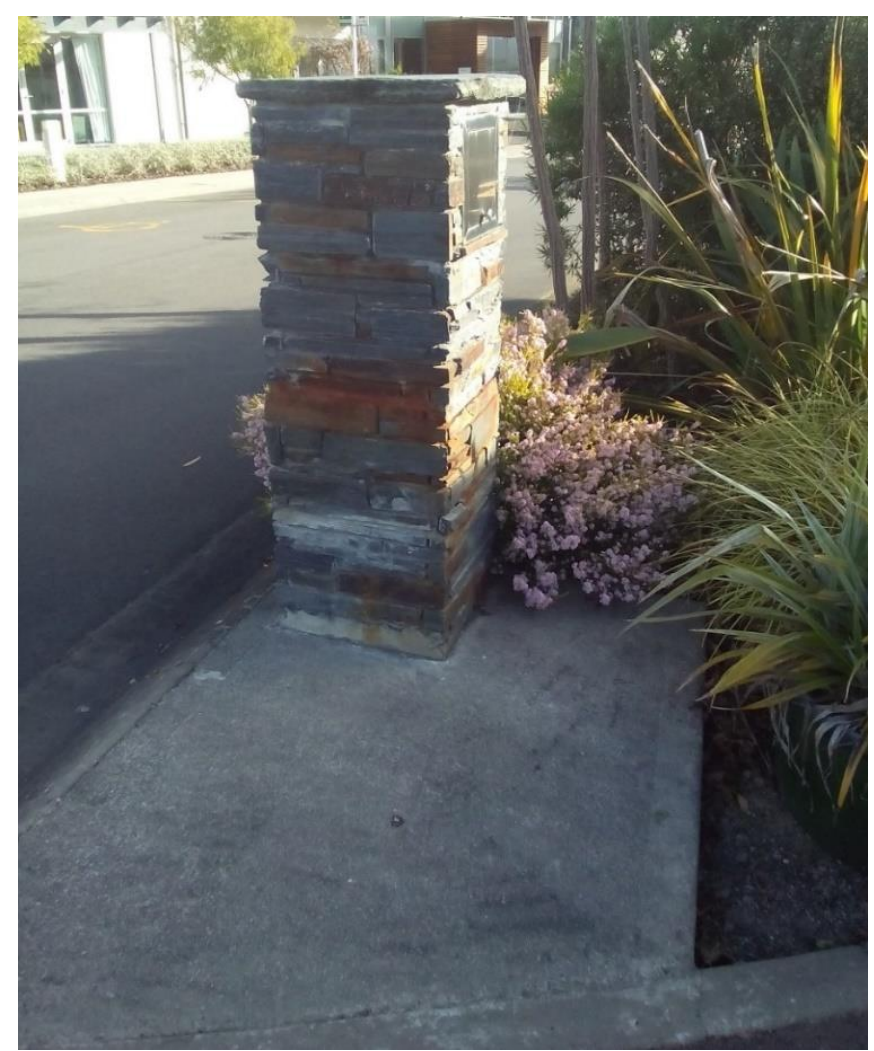

Figure 7-38: Letter box that was difficult for a walker-frame user to access

\subsubsection{Theme 2: Meaningful leisure activities}

Engagement in leisure activities of choice was important for residents. Five themes for activities and relationships emerged from the analysis: 1) continuing familiar activities, 2) staying active and able, 3) engagement in personal activities in private space, 4) enjoyment in activities in communal space, and 5) contribution. This theme included various preferences differing by individuals, which are summarised in Appendix 3.

\section{Theme 2-1: Continuing familiar activities}

Residents tried to continue participating in familiar activities. Key activities included religious activities, handcrafts, needlework, artwork, gardening, reading, building/DIY, sports and enjoying music.

\section{Religious activities}

For some people, church-related activities were important. Those who lived in a church-based complex with an adjacent chapel appreciated the ease of access to the chapel (PR1, PR3). Of those, a woman who lived under the same roof as the chapel moved into her current unit because 'Im]ainly I was getting less able to walk. I do like to go to the chapel every day' (PR1). Some RVs offered the communion or bible study in a communal lounge. However, a man who had 
experienced a deterioration in his mobility substituted attending church services with praying at home (PU6).

\section{Handcrafts/needlework/artwork}

Needlework such as knitting, patchwork, and embroidery was a familiar activity for many female participants (13 females and also one male). Though some had stopped because of problems with their hands, such as arthritis, others carried on. One woman discussed her motivation for the activity: 'I was a dressmaker for years. ... And I've got a half-finished patchwork quilt in there that I'm trying to get finished before my time comes to the end'(RVS2). Another woman continued making bobbin lace, even though '[she had] trouble doing anything with [her] hands' (RVS5).

For needlework, residents required a table for activities and space to store their tools. For example, one resident put a sewing table and a knitting table in her bedroom and stored tools such as needles, cloth and threads in the drawers (RVS2). However, another resident who only had space in her lounge $\left(13 \mathrm{~m}^{2}\right)$ for a table for needlework said,

It's a mishmash and having all this room taken up makes the room very cluttered ... I have got a lot of bits and pieces like these sorts of things; the room doesn't look tidy because of it.... This table and that chair and the lamp are there for a purpose for me. ... There's not enough room. (RVS5)

Ideally, a separate room or a separate space in a larger lounge would be used for the activity. She also wanted 'lot of drawer space, because having lace and embroidery takes a lot of room (RVS5).’

Another resident liked to press flowers. She used a folded card table, where she stuck flowers on cards and dried them for a couple days. She also needed drawers to store tools (PR4).

Art and craft activities such as painting and lost-wax casting were also important (PR1, PR2). These activities could have been better facilitated. 'It would be good if there was a room where we could leave our materials... [so that] if I was using pastels ... I wouldn't worry if I got pastel dust on the floor, cause it's very hard to clean up, it wouldn't matter', said one participant (PR1). A communal art room could be used for these activities. 


\section{Gardening}

The overwhelming majority of residents used to do gardening in their previous dwellings; some had owned a very large garden, including a quarter acre (RVS1), a plot of 100 roses (RVI9) and a one-hectare garden (RVS6). However, difficulty in maintaining their gardens was one of the major reasons residents had moved to their current dwellings. While some people didn't want to have a garden, many desired to continue gardening. Being able to have a garden was one of the main reasons some resident had chosen their current dwelling (RVS2, RVS3). One of them said, 'I felt I could live happily. The units in other villages had no room for a garden. That's something I miss - my garden’(RVS3).

Those who wanted or had a garden stressed the importance of it being suited to their ability to maintain it. This meant their desirable gardens varied in size and type. A woman who was relatively mobile wanted a vegetable garden of approximately $2.3 \mathrm{~m} \times 3 \mathrm{~m}$ (PR4). A man had various types of gardens including beds, pots and other enclosed sections, and enjoyed growing vegetables and fruit, saying,

Well I don't profess to be a brilliant gardener, but I enjoy doing it and I like to let things go to seed so I can get the seeds from the different veggies, you know. .... I enjoy my fruit and fresh vegetables. ... When it's a good day and there's some gardening to do, I'll only work out there for an hour. (PU3) (Figure 7-39)

Another male resident enjoyed looking after four bits of the garden for growing vegetables and fruit, which included one greenhouse (PU4). However, a woman with severe mobility issues had different ideas about what was a suitable garden for her: 'If I had a family I would have vegetables and fruits, but now... it's only flowers and bushes'(PR6). Those who didn't have garden beds enjoyed gardening with pots that didn't require as much maintenance as beds (RVS2, RVS3). One of them liked to 'fill all the space [in the porch and along the fence] with the pots'(RVS3) (Figure 7-40). 


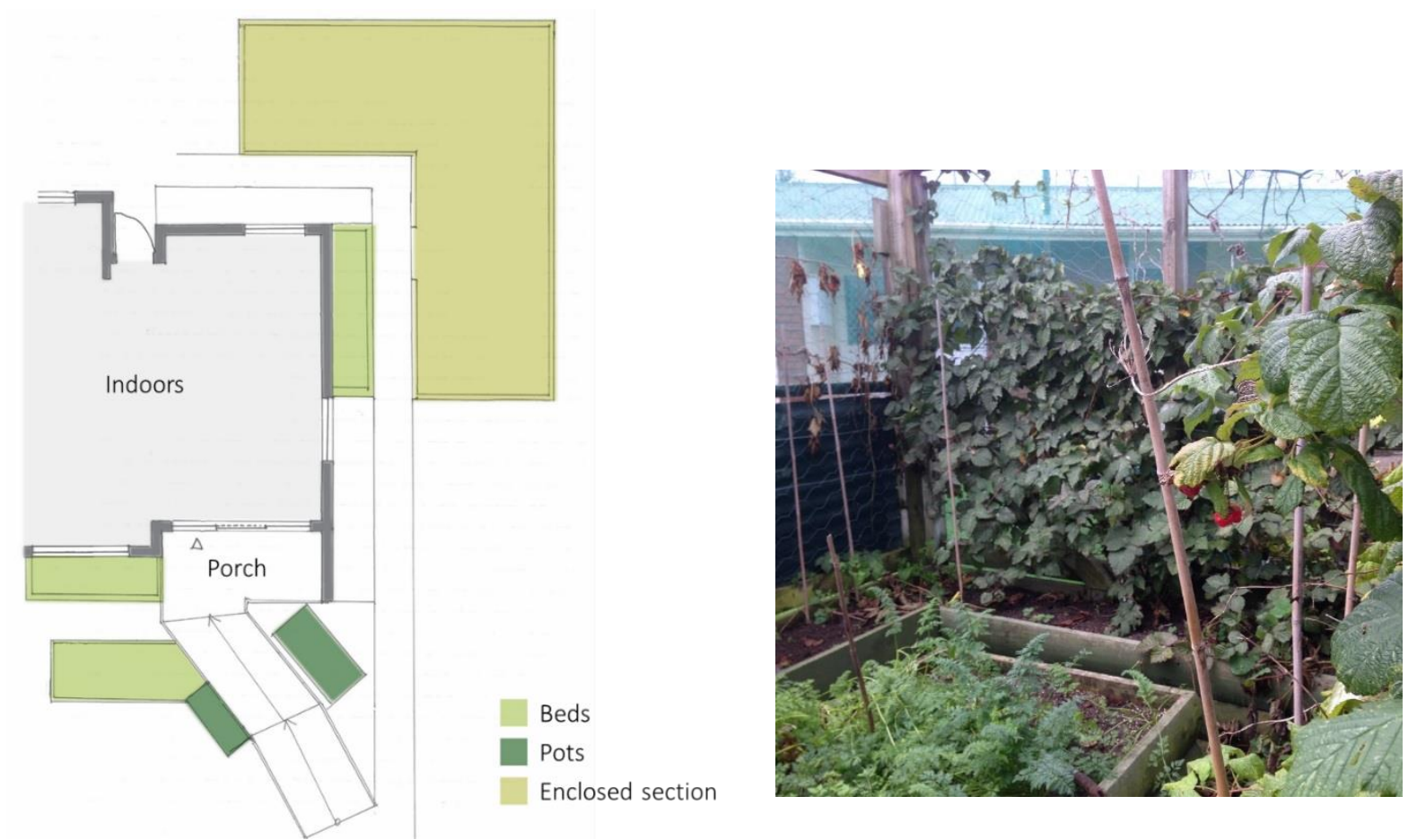

Figure 7-39: Plan of gardens (PU3) (left) and the photo of the enclosed section (right)
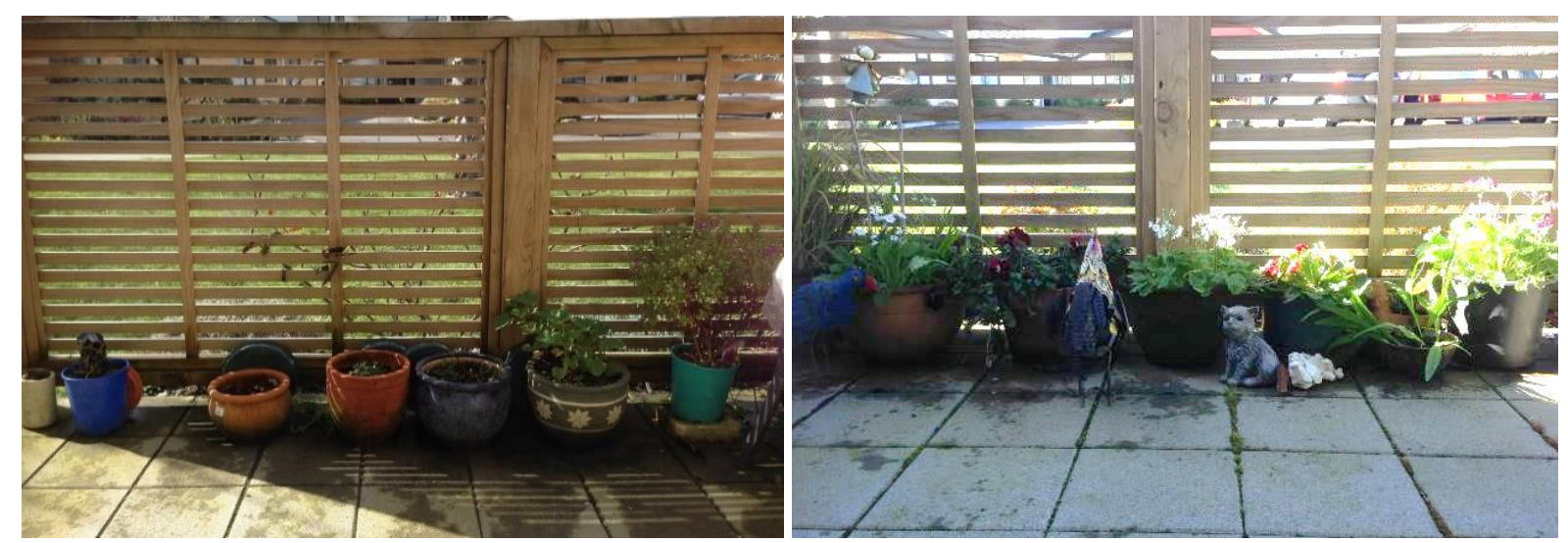

Figure 7-40: Putting pots along the porch fence

The difficulties residents had with gardening included postures such as kneeling for ploughing soil and weeding (PU4, RVS3) or just crouching for watering the pots (PR1).

Appropriate storage space for gardening tools was desired (PU2). A resident who stored gardening tools (e.g. spades, scissors, fertiliser) inside his house had to repeatedly move between the garden and the storage space (PU4). Another resident had a personal shed for gardening tools (e.g. shovels, spades, buckets, trollies), and suggested, 'There should be a big outside cupboard or something because, you know, we all have garden tools and things that you can't put inside — somewhere for storing stuff that you don't want in your house'(PU2). 
Reading was often an important activity, although in some cases issues in residents' eyes affected their ability to read (PU2, RVI10, RVS2). A man explained his preference for reading: 'I didn't have a hobby like making toys or carpentry or anything like that. ... I was more into books and things than I was into carpentry or doing anything active'(RVI1). Some kept their favourite books in their bookshelves (RVI1, PR2, PR3, RVS3). One of them also liked to display books showing the front covers: 'Yeah I like to ... put them out so they have the front page [showing] and sometimes people come in and they look through my books'(PR2) (Figure 7-41). One private-sector rental housing complex allowed residents to put personal books in the common shelves (PR7).

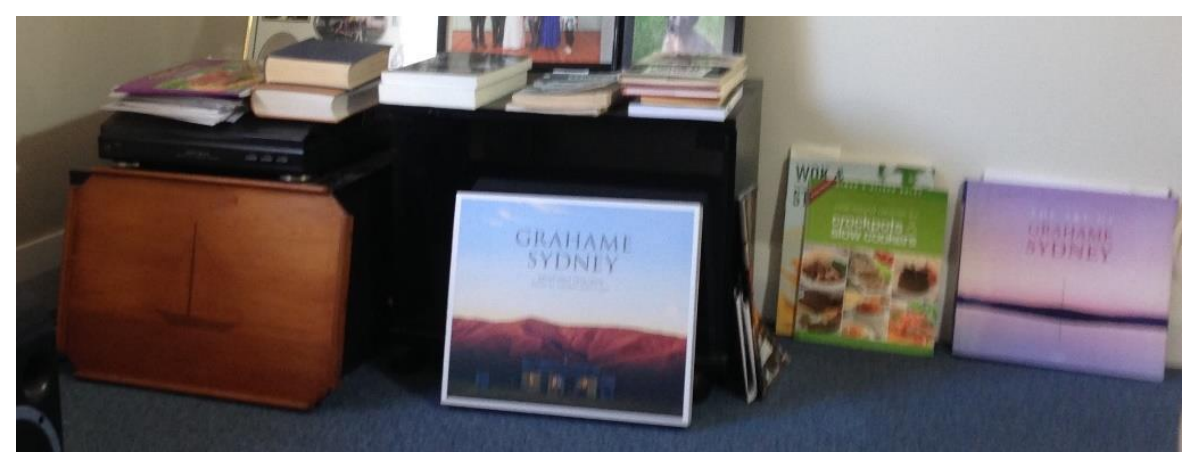

Figure 7-41: Displaying the front covers

Some liked to read books near the window, where natural light shone on their books (PU2, RVI1, RVI9); particularly reading in their armchair with their back on the north-facing window was preferred (RVI1, RVI9) (Figure 7-42). Others mainly used a lamp for reading (PR2, RVS1) (see also Theme 2-3).

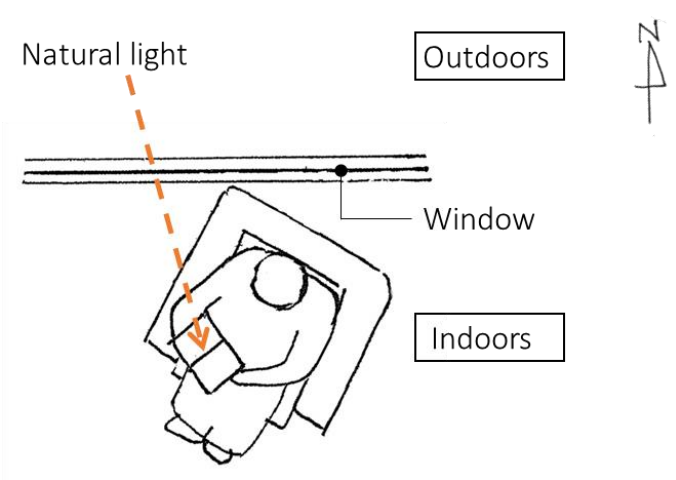

Figure 7-42: Reading in an armchair by the north-facing window

Choosing books at the library or from common bookshelves was an enjoyable activity for some people. Some RVs had libraries with a rich collection, described as 'marvellous' by one resident 
(RVI10). Even if they didn't have a specialised library space, bookshelves in the corridor or in the communal lounge that residents could take books away from freely were appreciated. One resident greatly appreciated the communal bookshelves in an alcove in the corridor, which was closer than the bigger library in her complex (RVS2). However, when another resident approached the bookshelves in her wheelchair, she found chairs for reading blocked the shelves and she could not reach books (RVS1).

Some complexes had mobile library visits. Either a mobile library bus with bookshelves would visit, or the library staff would bring books in and display them in the communal space. The mobile library bus had steps, which hindered access for users with mobility aids. They had to have the driver choose books for them (PR1).

\section{Building/DIY}

Some male participants used to do work related to building or DIY as a job or a hobby. A man who used to build houses said,

I'm still capable of doing some things. My enclosure around the corner, I built that while I was recovering from my hip operation, like a fool, but I did it. I've still got skills with, you know, electrical saws and things like that, you know, yeah. I'm very slow now but, you know, I just take my time. (PU3)

He stored his DIY tools on his porch. Another man who used to have a workshop in his garage missed it. He was storing the tools in an off-site rental storage. When asked if he would like to have a workshop now, he hesitantly answered, 'Yeah.... It's a bit selfish, I mean, I'm going to be the only one using it'(RVI3).

\section{Sports}

Many people had taken part in sports, such as rugby (PR7, RVI1), dancing (PR4, RVI7), golf (RVI5, RVI10), tramping (PR2, RVI2) and tennis (RVI6, RVI11). However, none of them continued these activities. One resident used to play rugby and enjoyed seeing rugby games in the park, but it was not possible for him to get there anymore. Watching weekly rugby games on TV substituted the activity, which he most cherished (PR7). Similarly, other elderly residents enjoyed watching sports on TV in their own room (RVI1, RVI10, RVI11). Those who used to do tramping liked to go for a walk in and out of their housing complex (PR2, RVI2) (see also Theme 2-2). 


\section{Enjoying music}

Singing and listening to familiar songs entertained several participants. One woman spoke about the joy of singing with others: 'Every Christmas, I think we have Christmas dinner, and then a sing-song. ... They're really all songs that we old people know, which is a lot of fun'(RVI10). Another resident went to the community day-care programme held in a church hall, where she and other elderly people enjoyed singing familiar songs accompanied by piano. When 'You Are My Sunshine' was played, she sang with tears, remembering her mother who used to sing this song (PR6). For these elderly people, communal areas played an important role of providing the space for this activity.

\section{Theme 2-2: Staying active and able}

The high-needs elderly interviewed wanted to be active and to maintain or improve their mobility and health and wellbeing.

\section{Walking}

One third of the respondents talked about walking as one important activity. A resident said,

I've always gone out for a walk. ... It keeps me moving. I don't want to become like some people, they spend too much time indoors and they don't get enough exercise, you know I mean they jump in the car and they go everywhere in a car ... They're becoming old before their time. (PU4)

A 93-year-old woman who used to enjoy tramping said, 'I love walking. I go for a short walk every day'(RVI2).

The ability to walk differed between individuals with different health conditions. Some people had difficulty walking because of breathlessness or pain in their legs or knees, which meant they had to take a rest during a walk. A woman who used to walk regularly and wished to continue spoke of her difficulty in walking the distance of $80 \mathrm{~m}$ : 'I would be get to, say, that hall quite comfortably ... but that means I would be sitting down half way, I'm sure. ..., I get too sore, and I have to stop, to let the pain go... I keep stopping more and more frequently'(RVI9). One man who had a stroke said, 'I can walk about 20 yards and then I've got to turn around and come back'(RVI8). One man had two walking routes of different distance (410 $\mathrm{m}$ and $640 \mathrm{~m}$ ), which he chose depending on how his conditions were (PU4). Providing walkways of different distance can accommodate residents' diverse conditions which differ both by the individual and by the day. 
Some complexes were poorly designed in terms of walkability. For example, residents of a big complex with little variety in walking routes walked to the gate of the village and then return to their houses. A resident expressed frustration about this: 'If you want to go for a walk, you do the same route all the time really'(RVS4).

Many people were prone to falls during a walk (see also Theme 1-1). A man who with limited eyesight preferred to walk on the road rather than on the footpath because 'it [gave him] more space to walk' (RVI11). A speed bump on the road was a hazard for him 'I forgot the hump in the road, you know? And I tripped over that hump, and I fell', he said (RVI11). Walking in darkness was also not preferred by those with vision impairments: appropriate provision of outdoor lights was desired (RVI10).

Car safety was a concern in large complexes with a long roadway. 'You get the occasional person who doesn't take any notice of the speed limit and they speed through the place.... So, you have to be more careful than you used to be crossing the road (RVI1).

Walking into town was a popular pastime for a man, who passed a lot of familiar venues, such as shops and cafés, each of which brought up associated memories which he discussed with the researcher (PR7) (Figure 7-43). During walks, well-maintained gardens were preferred (RVS4). Sometimes walking was motivated by having company: 'Sometimes [my daughters] will say, "Do you want to go for a walk?" And they'll come with me. I don't always go on my own'(RVS4). For a man who had a difficulty seeing, company was important for helping instruct on the direction to walk. On a narrow path he had to walk behind his companion, not side by side (RVI11). 


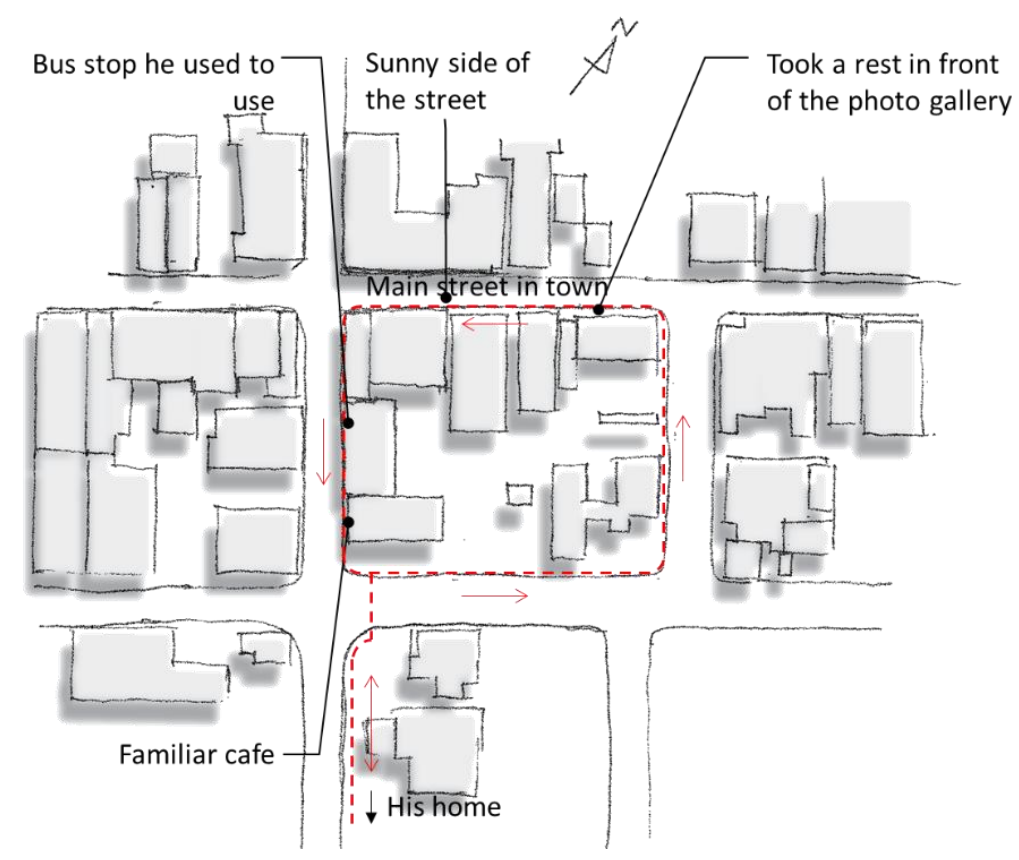

Figure 7-43: Walking route of a resident in the town

Indoor environments were also useful for walking exercise for some residents. One resident said, 'It's an exercise to walk this corridor'(PR2). In resident units, the space which was not too big improved walkers' sense of safety, because they had something to hold onto (such as wall and fixtures). Some residents separated a large lounge with armchairs and sofas, which created an indoor walking space with things to hold onto (the back of armchairs and sofas) (PR2, RVI4) (Figure 7-44).

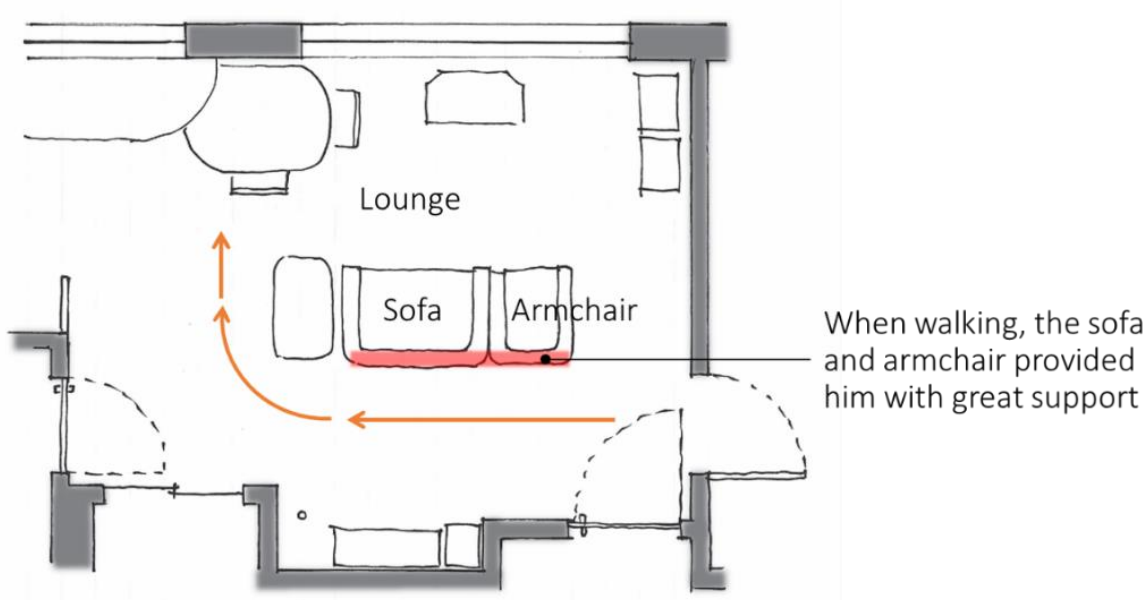

Figure 7-44: Using furniture as 'things to hold onto' 


\section{Other physical exercises}

In their own home, some residents did exercises on their own. Two men had an exercise bike at their unit and used it often. A post-stroke elderly man used his exercise bike quite regularly: ' $I I$ do exercise] at least once a day. ... Because I'm trying to get this leg okay, you know. (PR2)' The exercise bike was also used as an alternative for going out for a walk: 'I'm too light-headed, and I'm likely to have an angina attack or something, so when I can't walk outside or if it's too wet, I get on my exercycle' (RVI11). A man performed an exercise of raising his knees while sitting in his armchair: 'I do this. ... two, three, four, five, six, seven, eight, nine, ten. That's it. ... "Knees up Mother Brown” (RVI4). Another participant performed his routine exercises, moving his legs, ankles and hip lying on the bed (PU6). One lady who has arthritis in her hands did knitting to help her hands: 'I used to knit years ago. I'm starting up now to keep my hands, um, arthritis and that. If you don't work, you get all crippled, so I knit'(PU5). A man who had impairments following a stroke kept a ball on the side table by his armchair. He said, ' $M y$ left arm ... That's why I have a ball [for exercise] there'(RVI8). A Parkinson's patient was also keen to exercise his fingers by playing the piano, which could prevent the progress of his disease. His grand piano was put in communal space near his apartment (RVS6). This space, accommodating musical activities close to resident units, facilitated his exercise.

Communal exercise activities were popular with some participants, such as sitting exercises, Taichi and Yoga. One resident who enjoyed sitting exercises explained,

It's what they call "armchair dancing". You do exercises while sitting in an armchair with the music, you know. And another one, called "Use It or Lose It". .. You use your arms. They have a video that's played. You follow the actions of the person sitting in the chair. (RVS2)

A swimming pool was also preferred by some (RVI3, RVI4). A resident said he enjoyed 'walk[ing] up and down there, in the water'(RVI4).

\section{Moving between spaces}

Residents preferred to avoid a sedentary lifestyle. A male resident spent most of his time in his bedroom, except for the time he watched TV in the lounge. When asked whether he would like to have the TV in the bedroom, he said,

Oh yeah, it'd be quite nice... No, if I had the TV in here I would spend too much time sitting or lying down. I think having it in the lounge keeps me active... No, if it was in 
here I'd be lying on the bed for hours... In one sense it'd be nice, but it wouldn't do me any good. (RVI1)

One caregiver suggested the significance of space for moving around:

You have to have a small patio kind of area from their living room area they can go out on when it's a sunny day, where they can sit down and stay. So that will make them kind of move... They have to move around. (PR5)

\section{Theme 2-3: Engagement in personal activities in private space}

Some high-needs elderly expressed their boredom due to a limitation in activities. However, others succeeded in being engaged in private activities in their own dwellings. One resident said, 'I've got plenty to keep me occupied during the day... Oh I'm busy enough, you know. I'm not bored, put it that way. I love life, I love life'(PU3).

\section{Comfort and privacy}

High-needs elderly people often required rest in an easy posture between activities, which adds to the significance of their private space. All the respondents used certain types of chairs most of the time. Only one person stayed mostly in bed (PU6). An armchair was the most common type of the chair, which was often adjustable for leg and back positions (see also Theme 5-1).

Privacy was an important element for residents' space. One resident, who lived in an apartmenttype dwelling with shared spaces such as the dining room and the lounge, indicated her bed-sit room as her favourite space, 'because it's my own space'(PR6). Another woman who used an alcove within the lounge as sitting space (Figure 7-45) liked her space: 'It's a little ... "nook." It means a little space. "Nook"'(RVI7). Warmth was another important contributor to comfort in their space; some chose a location because of the proximity to a wall-mounted heater, such as a heat pump or an electric heater (RVI7, 10, RVI11). Others put a portable heater near their chair or bed when it was cold. One woman complained about a heater overhead on the ceiling, which was too hot (RVS1) (see also Theme 5-2). 


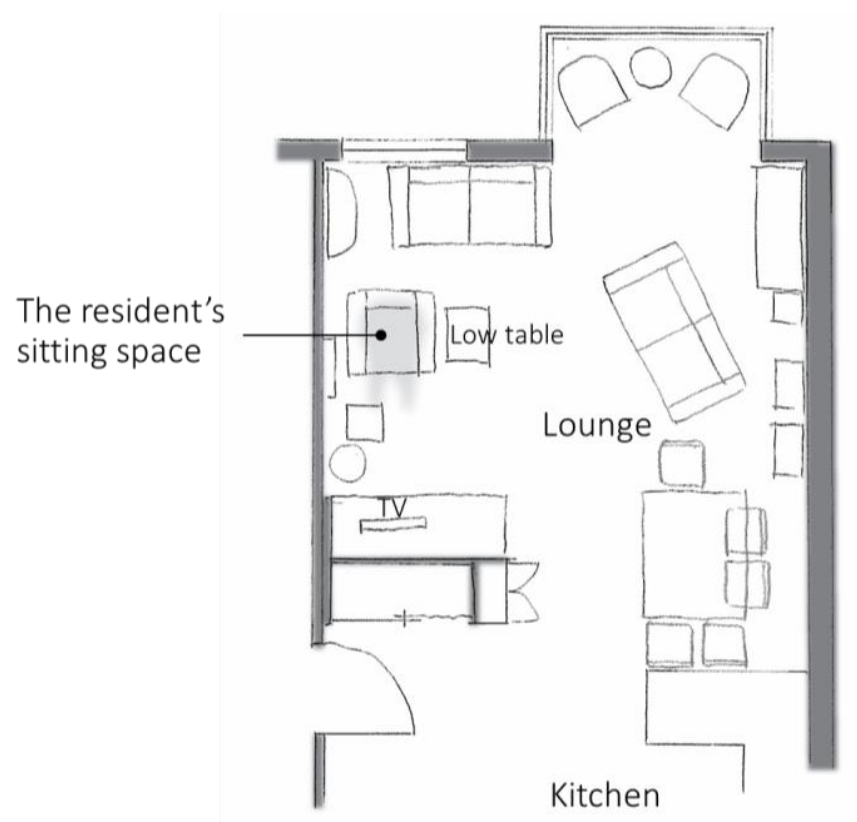

Figure 7-45: Layout of the sitting space in the alcove within the lounge

\section{Various activities to occupy residents at home}

Watching TV was the most common activity, and most seating was laid out in respect to the location and direction of the TV. In a small lounge, residents wanted to put the TV on the wall (PU5, RVS3). There were differences in preference and duration of this activity. A man who had very limited mobility watched TV all day, saying, 'That would be the only thing, I'd go nuts without the television. Gives me something to do'(PU1).

All sitting spaces or beds were laid out in respect to the location and direction of their TV, except for one person (RVI8). A participant with limited eyesight put his chair close to the TV (RVI11). Those who had sight impairments prefer to sit with their backs to the windows (PR4, RV7). One resident said, 'I don't think I could cope with that [the opposite layout]. Because you're looking into the light all the time'(PR4) (Figure 7-46). Residents had remote controls to turn on and change channels as well as adjust the screen brightness, and kept a program guide within their reach. 


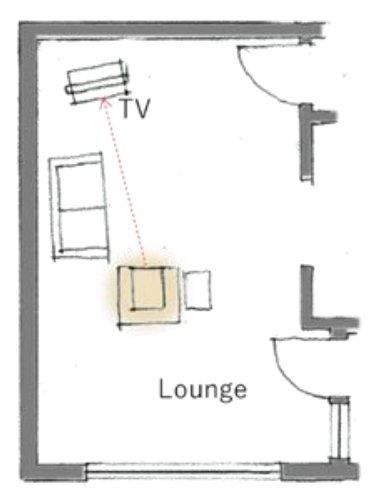

Suitable

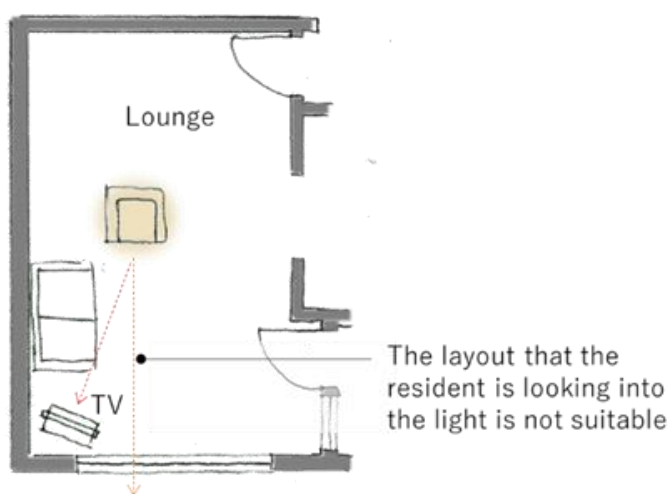

Not suitable

Figure 7-46: Layout of a chair and a TV for people with vision impairment

Reading was often an important activity during sitting for many residents (see also Theme 2-1). Reading a newspaper was problematic as the size of the newspaper was too large to easily manage. A woman with Parkinson's said, 'It's difficult to handle them without a table. It's too big' (RVS1). Necessary tools and furniture were put within reach of their sitting space, such as eyeglasses, a magnifier, a letter opener, a lamp, a level surface to put books on, and bookshelves.

Writing was a common preferred activity. Some often wrote letters or postcards (PR3, PR7, RVI6, RVI7, RVS3). Some wrote about their life (PR2, RVI6); one resident said, 'I do quite a bit of writing still. Now I just do diaries and things for my family really. ... A lot of questions [from her family]. And still writing the answers to her questions in this book'(PR2). A man whose wife had dementia and lived in a hospital said, 'I usually start writing two diaries. There's a diary for me as to what my activities have been during the day, and the other one is a diary about how I have found [my wife]'(RVS6). A dining table or a writing desk were often used for handwriting, and a PC table for writing with a computer.

Some people liked to listen to music, with CDs (PU1, PU2, PR2, RVI11, RVS1) and vinyl records (PU3), and some liked listening to the radio (PR2, RVI11, RVS4). A man with limited eyesight talked about the benefits of listening to music: 'I just like, sort of, listening to it. It passes, time goes quickly, and it helps get you relaxed, and feel all right'(RVI11). He also liked listening to audiobooks that he subscribed to, saying, 'I read a lot, I read, I listen to talking books. I have a lot of magazines and a lot of books'(RVI11). Another man who had also issues with eyesight used read-to-speech software to read the contents on the computer (PU4).

Crosswords were a common activity while sitting (PU2, PU5, RVS2, RVS3). Some people played games on a computer (PU2, PU3, PR1) or a tablet (PU5). A man explained the benefit 
of PC games: 'It keeps your brain ticking over, that's what it's all about, you know'(PU3). He played them sitting in his armchair, putting his laptop on the armrest, while a resident with hemiplegia needed to sit at a table to support his left arm (PU1) (Figure 7-47).

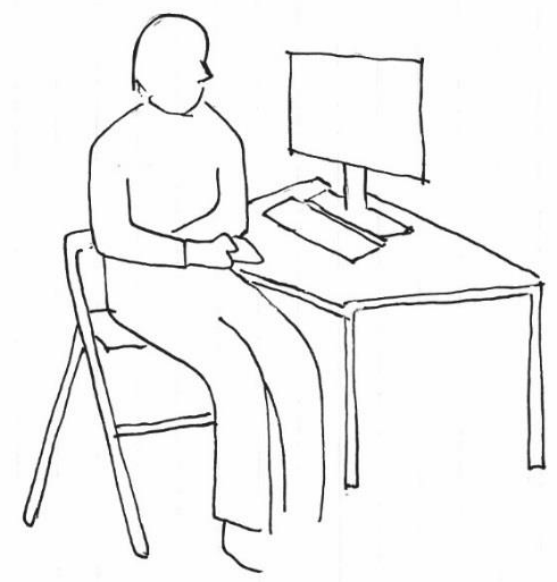

Figure 7-47: Posture of the resident with hemiplegia when sitting at the table

Having a cup of tea and meals often took place in this space (see also Theme 1-4). Many people used their knees, and adjacent level surfaces such as a side table, a portable table, a kitchen bench or their trolley to have meals.

Many people liked seeing outside, which was often the main reason for the choice of their sitting space, though some people had little concern about other people's movements outside (PR3, RVI3). Seeing moving things such as people and cars was preferred. A resident said, 'You can see out and see what's going on ... Reminds me I'm still alive'(RVI2). An elderly couple had their own preference different from each other regarding looking outside and arranged their armchairs differently (RVI3) (Figure 7-48).

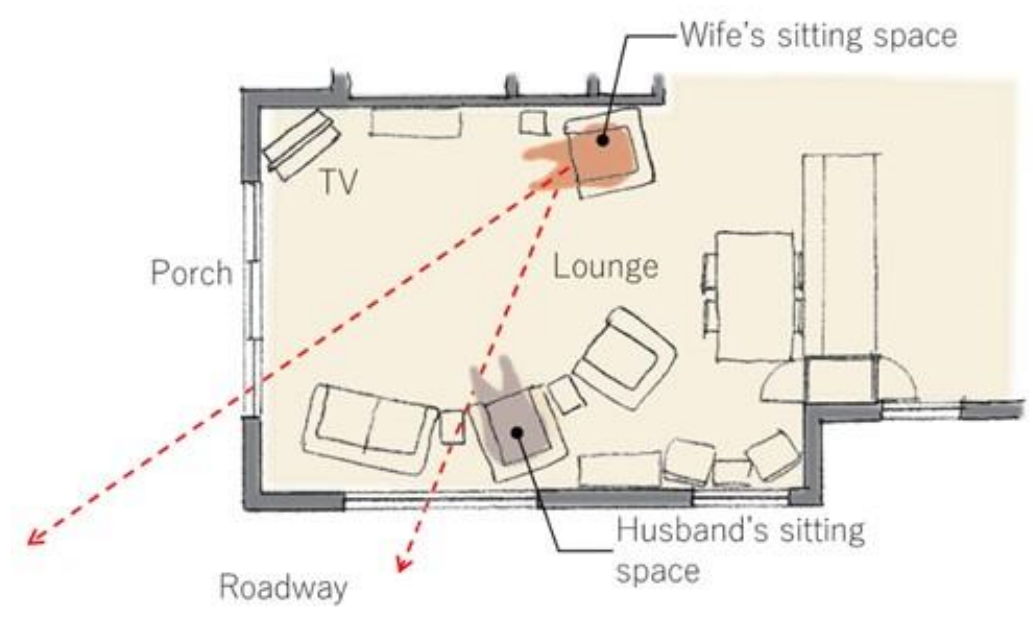

Figure 7-48: Difference in the sitting position depending on the preference for seeing outside 


\section{Control for doing everything from one space}

Some residents engaged in multiple activities and tasks, which could be fostered by the organisation of furnishings surrounding their sitting space. One resident sat at his chair facing his PC screen as well as his TV screen. While watching TV, the PC screen notified him of the arrival of messages from friends, at which point he walked to the PC and read the messages (PU4) (Figure 7-49 left). Another resident with a limited mobility could reach the mouse as well as see his PC screen, which allowed him to view emails from his armchair. He said, 'Yeah, I have to do everything, eat and everything here, from here and, um, do my computer over there'(PU1) (Figure 7-49 right). Another man said, 'I can do two things at once. The ear's that way [to the TV] but mostly the eyes are looking this way' [to his laptop] (PU3). He liked horse racing, which required him to watch TV, take notes on paper, and place bets through the computer that he rested on the armrest of his armchair (Figure 7-50). He said, 'I've got everything at my fingertips here'.
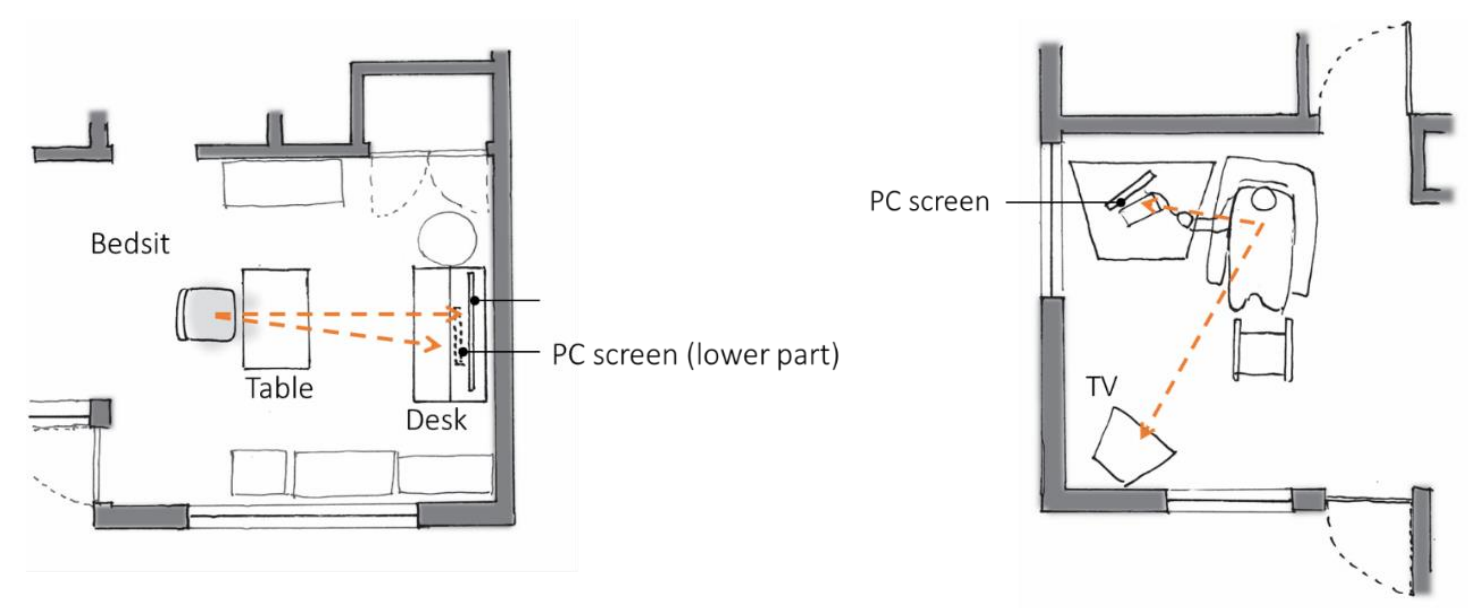

Figure 7-49: Layout of the PC screen that can be viewed from the sitting space 


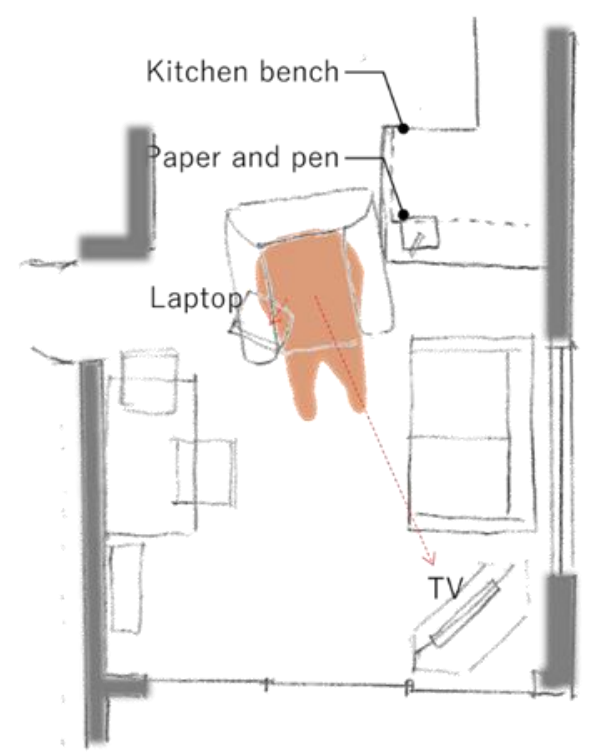

Figure 7-50: Sitting space that facilitated engagement in multiple activities

Various tools for activities and furniture and fixtures to accommodate them were placed within reach of residents' sitting space. Level surfaces such as shelves or cupboards with multiple drawers were particularly useful for keeping things tidy (PU3, PR1). A woman who was good at needlework made a handmade organizer for two remote controllers and stationary (scissors, a letter opener, two pens) that could be hung on the armrest of the armchair (RVS2) (Figure 751). Where there were not enough level surfaces, residents placed objects on an adjacent table or desk and put things on the floor (PU1, PU5, RVS3).

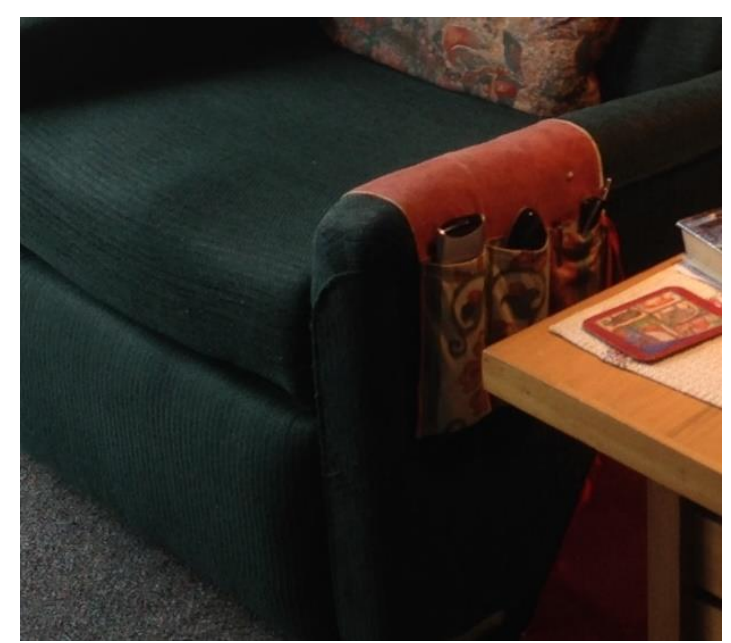

Figure 7-51: Hand-made storage for remote controls, pens and so on

\section{Using different spaces for different activities}

Some activities required a separate space with special furniture, such as a writing table, a PC desk or a table for handcrafts and needlework (see also Theme 2-1). One female resident who lived in a two-bedroom house had different spaces for different activities (Figure 7-52). She liked reading 
in an armchair by the window, with the sunlight falling on the book. She also had a card table where she liked to do jigsaw puzzles, which were kept on the table even if uncompleted. She operated her computer at her PC table, and she did writing at the writing desk in her bedroom, which she really liked because it was 'very private'(RVI9).

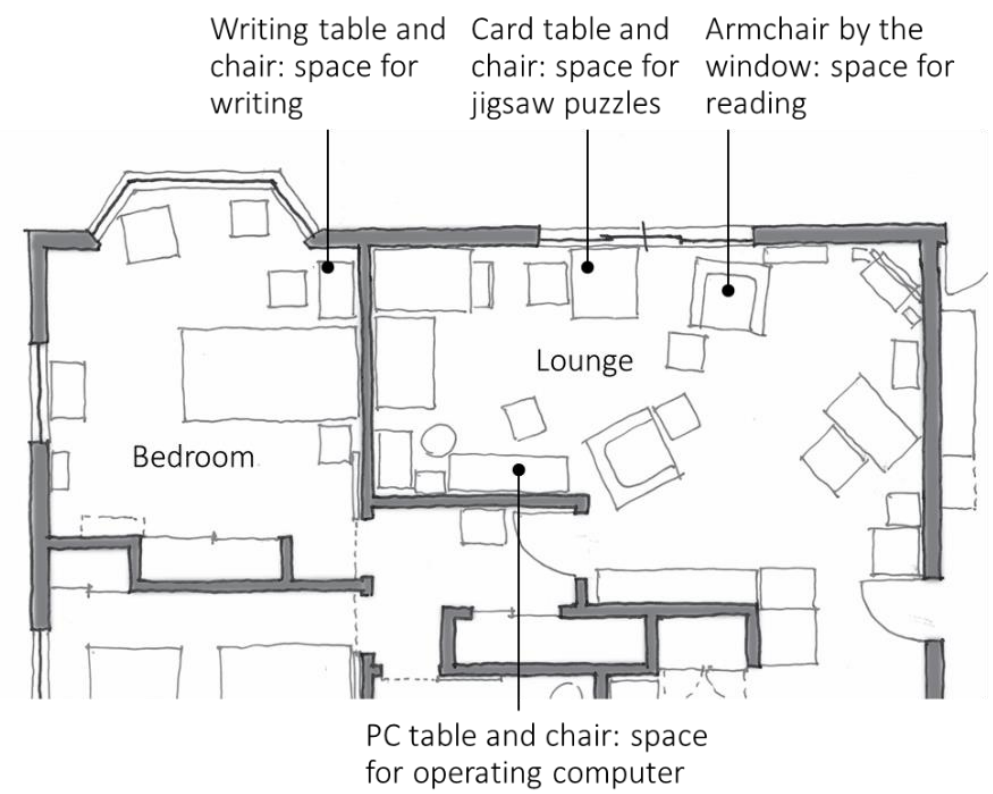

Figure 7-52: Using different spaces for various activities

\section{Theme 2-4: Contribution}

Many respondents had been involved with volunteer work related to their occupation, skills, religion and other interests, such as teaching (PR1), needlework (PR1, RVS2), playing a musical instrument (RVI8), building and machinery work (PU3, PU4), and helping at church and other organisations (RVI3, PR4, RVS4). While health conditions prevented continuing this work, some carried on contributing but scaled down their activities. For example, one woman, who used to teach needlework, assisted other women in her personal group, saying, 'I have [taught it] but not now really and we've got the new one so I've been helping her out'(RV5). Another woman wished to contribute by her piece of patchwork: 'If I could finish it, I'll give it to the Wellington Free Ambulance to raffle or to sell or whatever they wanted to do'(RVS2). Some residents were interested in teaching their skills to others. For example, one woman desired the opportunity to teach flower pressing (PR4).

As well as undertaking this work in their own dwellings, communal space was often the venue where residents took part in voluntary activities. One resident helped library services (RVS5). Some residents helped with setting the tables out for dinner (RVS6) or serving meals to everyone in their communal dining rooms (PR7). One resident competent in gardening looked after the 
garden bed in front of his neighbour's unit as well as his own (PU4). Another resident who played the piano actively arranged communal activities for residents: ' We, in conjunction with one of the local piano tutors, invited an Israeli concert pianist to come and play, and we held two concerts a year'(RVS6). Various communal spaces where residents can interact with other residents and people from the outside of the complex have great potential to facilitate elderly residents' desire for contribution.

\subsubsection{Theme 3: Meaningful relationships}

Relationships were one of the significant contributors to QoL for the high-needs elderly. The analysis has found four themes: 1) living with partner, 2) having personal guests, 3) relationships with other residents, and 4) connection to the wider community. This theme also found individual differences in meaningful relationships, which are shown in Appendix 3.

\section{Theme 3-1: Living with partner}

There were three residents who lived with their partners in RVs and five people who used to live with their partners in their current houses. One woman who chose to live in a serviced apartment spoke about her reason, saying,

My [late] husband thought he'd have to go to the hospital... With [this] apartment, we lived here together, that was the thing... We could be in here together. Rather than have a room each, you know, like in a hospital situation... That was really important, to be together. (RVS4)

The serviced apartment, which is typically one-bedroom, was too small for accommodating two people. In particular, participants often experienced a lack of wardrobe and other storage space. While residents in two-bedroom houses could have enough space by using the second-bedroom (RVI3, RVI8, RVI11), one-bedroom apartments typically did not contain enough wardrobe and storage areas for two people. One man said, 'It's sufficient for one person, not quite [sufficient] for two.'(RVS6).

In a serviced apartment, one resident explained the benefit of the sliding-door type partition between the bedroom and the lounge: 'You can seal off the bedroom, or you can... For instance, if I was unwell, and I'm in bed, my wife could sit here and still see me with the door open, or if I'm snoring a lot she could close it'(RVS6). He talked about greater privacy needs between the couple: 
For two people, it would be nice to have somewhere where the two people can be together but apart, if you like. A bit of privacy for one. ... For instance, supposing my wife was a writer. She would want somewhere to write, and she wouldn't want me and my activities distracting her, so it would be better to have two areas where each one of us can do our own thing.' (RVSG)

For example, having an office could provide separate spaces for two, and 'the office need[ed] not be very largé(RVS6).

\section{Theme 3-2: Having personal guests}

The family connection was incredibly important for most participants. Many had family visits once a week (PU1, PR2, PR3, RVI2, RVI8, RVS2) and some had them more frequently (PU2, PU6, PR1, RVI1, RVI3, RVS4). Family and friends visits were appreciated for both physical and mental support. A woman who had regular visits of a daughter living near her said,

She comes early evening and we have tea together and she'll see if there's anything I need that she can do. Or she'll sit and we just chat. Or I've got a piece of handcraft I couldn't do, cooking, other things. She'll fix that up and goes on with that for me. That sort of thing, just mother and daughter things. (PR1)

Another woman also enjoyed friends' visits:

I do have a lot of visitors, which I like. Most days there's somebody coming in... I'm really lucky, I am... They come and have a drink late afternoon before dinner for a wine or something, whatever they want, and we sit and have a chat and then they toddle off home. (PU2)

\section{Welcoming guests}

Being able to 'see who is coming'(PU1, PU3) before guests arrived was important for a feeling of control and in order to welcome the visitors. Unfortunately, residents in apartment-type accommodations typically did not have a view to see guests approaching (PR1, PR2, PR6, PR7, RVI6, RVI7). An apartment resident who could not see the door from her sitting space said, 'I can't go to the door easily, so I just wait. The door is unlocked (RVI7) (Figure 7-53). When residents called out, 'Come in!' while seated, the sound of their voice could not always be heard by guests outside the door because of the distance or blockages by walls (PU5, RVI7). An 
apartment resident also said, 'I cannot tell when they arrive because you cannot see nor hear them'(PR6).

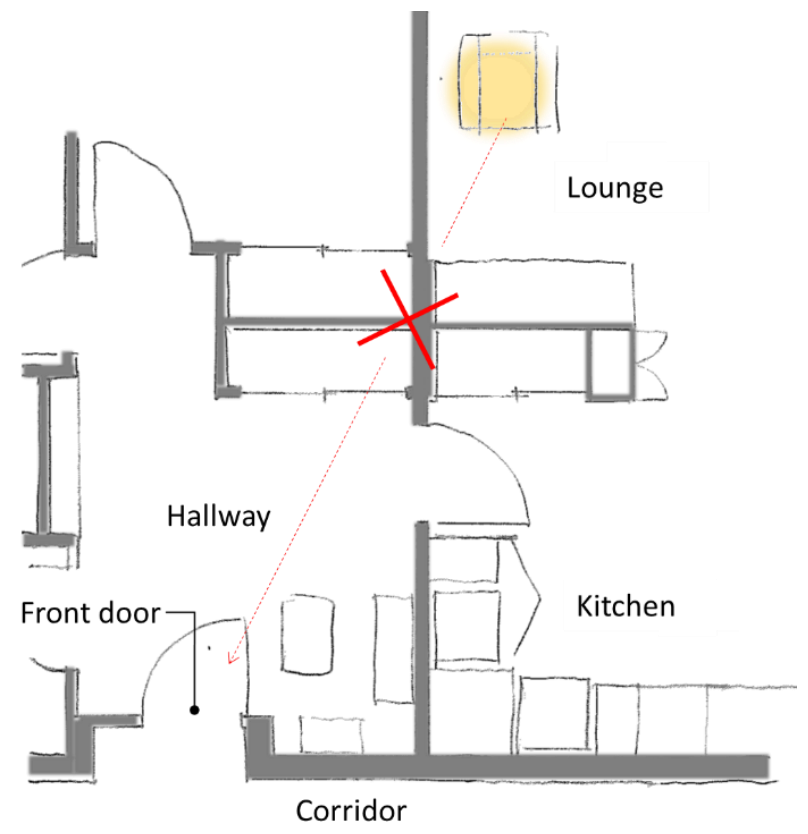

Figure 7-53: Interior layout that hinders the view from the sitting space to the door

Interphones were used in apartments with indoor access. An interphone notified a resident of a guest's visit, after which he could open the door of his unit before their arrival to the door and welcome them (PR2). A woman who lived in an apartment liked her individual external door more than a shared corridor and entrance; she said, 'I have that extra doorway. ... It goes to the outside, which I really like'. It was preferred by frequent visitors because of its homeliness as well as convenience:

My family loves to use the back entrance at the gate down at the end of the ramp. And even my helpers know they can just walk up that way without having to go ring a bell. ... Well, they like to feel, you know, it's more homely to them I think. And also, they don't have to get up steps and ring the bell, and I don't have to get up from wherever I am to answer the door. ... They can just come, and if I was not well [they can come in] also. It's good to know they don't have to get me up before they come. (PR1)

Another woman in an apartment with her own external door also had her family come through the porch door, rather than through a long corridor (approximately $35 \mathrm{~m}$ ), because it was easier (RVS4). 


\section{Entertaining guests}

Entertaining guests was an important activity. Most people had space for guests to sit, such as extra chairs and sofas. Activities for entertaining guests included serving tea (RVI2, RVS2), sharing a meal (RVS5) or a drink (RVI10, RVS1). For one woman, preparing a meal for a guest was important: 'It's something very simple, such as I've tried something in the crockpot ... a slow cooker. ... I'm very keen on cooking. I like have people come over'(RVS5). The dining table, which was not normally used for eating, was used for having meals with guests (PU3, PR4, RVI3). A folding table was used in a small lounge (PR4). The idea of having a café was preferred by one resident, who would use it when her niece visited her (RVI9).

Having a lounge separate from the bedroom was generally preferred for greater privacy when entertaining guests (PU1, PU2, PU3, PU5, PU6, PR1, PR2, PR5, PR6). One woman who enjoyed socialising did not have many guests because her bedsit room was 'more like a bedroom' (PU5). Another resident of a bedsit unit wanted a hard separation rather than his curtain (PU1). Another resident was also pleased to move from a bed-sit unit to a one-bedroom unit, where he used the lounge only when guests visited (PU6).

Some participants had a large number of guests at a time in their own home, such as extended family (PR6) or personal hobby or religious groups (PR1, RVS3, RVS5). A woman with limited mobility talked about the comfort of having a personal meeting with five people:

I have a group meeting here once a month. ... We share an intimate meeting in my house. ... That's just a very small part of the whole group but I can't get out the big meetings anymore so, we just have that small one here, very comfortable. (PR1)

A one-bedroom serviced apartment with a lounge of $13 \mathrm{~m}^{2}$ was too small to accommodate a group of people. The resident said, 'I do have sometimes eight or nine people, my little group of ten and that is very crushed, as you can imagine. ... I've had eleven I think, that was squashed (RVS5). In many houses, extra chairs were put in the lounge or stored in a bedroom when not in use. A woman who lived in a bedsit unit didn't have space to store chairs in her unit and so stacked two chairs in the corridor in front of her unit (PR6).

Common indoor and outdoor space could also be used to accommodate guests. One woman had held several soirées using the open lawn space in front of her unit as well as her lounge (PU2) (Figure 7-54). Some serviced apartment residents used communal indoor space in close proximity to their units: 'We use the table [in the communal dining room], because we have 
family meals and everyone brings something potluck, and we use that'(RVS4). However, when a woman tried to accommodate eight family members in the communal lounge, it was not possible because someone was watching TV (PR6).

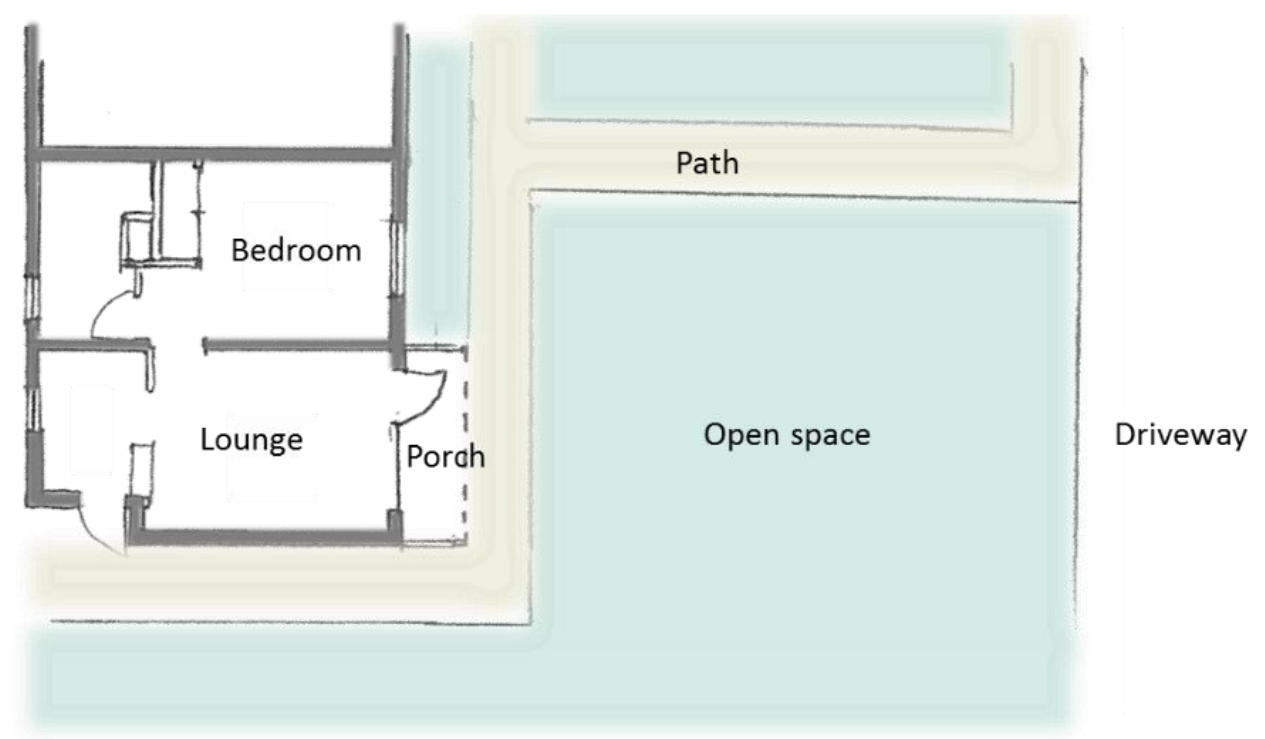

Figure 7-54: Open space in front of the unit, which was used for hosting large number of guests

Respondents particularly enjoyed having grandchildren and great-grandchildren to visit.

Watching the children was pleasing (PT1, PR5, RVI10); 'It's nice to see children running around'(PR1). One woman liked '[being able to see children] moving on the other side of the lake there, on their skateboards and bikes'(RVI10). Having a children's playground in the complex was suggested (RVI3).

However, special attention was needed for children's spatial usage. One resident's bedroom, where he normally stayed, was shut off to keep away from children. He said, 'The children will play around in the lounge. Their mother's very careful. She shuts my door so they can't get in here [his bedroom]. ... Yeah because they're very inquisitive'(RVI1). A man with sight impairments talked about the danger with children:

My biggest worry is little children, you know? About this high, running and scampering along. And I've got to be very careful that I don't run into them. ... But, no, I'm very careful. I keep watching down low rather than watching other people now. Watching for little children. (RVI11)

\section{Having overnight guests}

Most of the residents who had one bedroom or more used the second bedroom for overnight guests (8/10 cases), which was important to them. The second bedroom, if they had one, was 
used frequently (once a month or more) (RVI4, RVI1), and sometimes for periods as long as several weeks (RVI7, RVI1). One resident had had her son living overseas visit her, who stayed with her for three weeks (RVI7).

Most second bedrooms that were used for guests accommodated two beds (7/8 cases).

The garage was also used to accommodate guests (RVI9, RVI11). One woman parked her car out of the garage so that it could be used as a bedroom for two grandchildren. However, she said, 'It's not insulated, so it's OK in summer but not in winter'(RVI9). Another resident also said,

There's two bedrooms there... If [my daughter] brings her boys, they always stay out in the shed. They like it out there. It must be cold and drafty, but they say, "No, it's all right, "they read books, and they look at their computer all night. (RVI11)

This resident did not have a car and furnished his garage with chairs, a desk and a table, and he stored a mattress on the wall (Figure 7-55). Although he had two bedrooms he wished for a larger house:

Yeah, I wouldn't mind having an extra couple of bedrooms. I'm thinking if my children came and they can bring their grandchildren, they can bring their children with them, and it would be good. (RVI11)

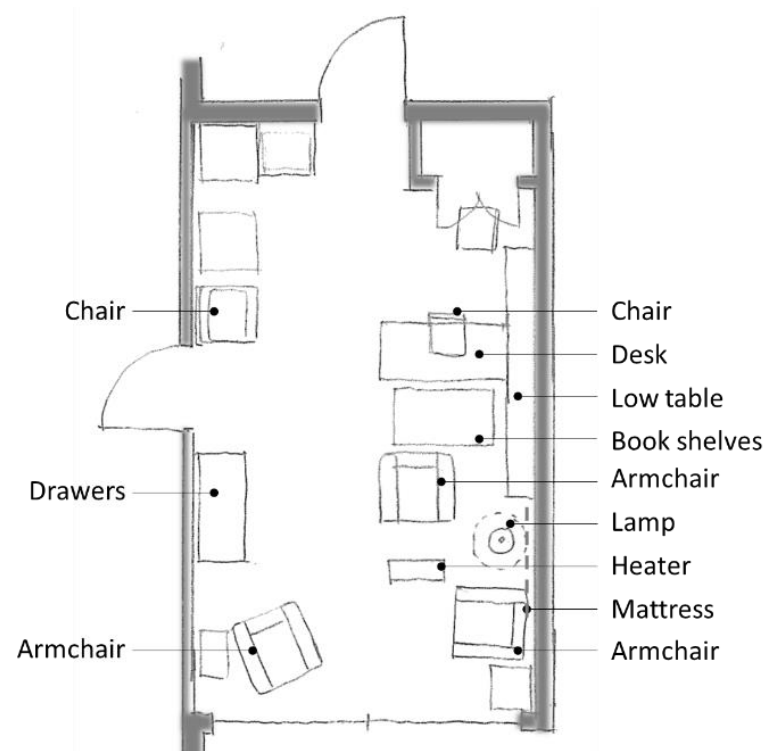

Figure 7-55: Accommodating overnight guests using the garage

In contrast, there were no bedsit residents who had guests stay overnight. One-bedroom unit could accommodate one or two family members or intimate friends. A woman who kept an 
extra bed explained, 'I was going to get rid of one of the beds, and they [my daughters] said, "No, just leave it there, because if you're sick, we can come and stay"'(RVS4). Those who had enough space were able to have guests stay in the lounge, using an extra bed or pulling out the sofa bed (PR4, RVS6). However, one woman wanted a second bedroom for her visiting son, who 'used to stay on his cushions ... on the floor [in the lounge]'(RVS1).

\section{Theme 3-3: Relationships with other residents}

Connection to other residents was important for many participants, as this contributed to a greater sense of security and safety, as well as a greater sense of homeliness.

Many residents supported each other. One woman said, 'It's comfort that there is someone who you can ask for help if needed'(RVS3). Some residents visited each other to check their safety (PU2, PR5). One couple had a neighbour who visited them every day: '[A neighbour] comes in. Every morning. And has a coffee, and something to eat usually and then he goes back. We encourage it to make sure that he's still up and alive'(RVI8). Complex settings where elderly residents live close to each other have a great potential in supporting each other's independent living.

\section{Familiarity and sense of homeliness}

Familiarity with other residents enhanced the sense of homeliness. A resident of a RV with over 200 units expressed a loss of sense of homeliness, which was related to the size of the village: ' $I$ liked the village as it was. The village has changed, and it's got bigger. ... They used to ... warmer. ... Yeah, you know? More friendly... Happier feeling... More homely. That's the word, homely'(RVI5). He attributed the change to the size of the village.

\section{Socialising in organised activities}

Many people enjoyed socialising in the communal tea and dinner (PR1, PR2, PR3, PR4, PR6, RVI4, RVI5, RVI7, RVI8, RVI10, RVS4, RVS6). One resident said, 'I go to the occasional monthly dinner. I went to the last one. It was Thai cooking. It was very nice'(RVS6). Another resident enjoyed Happy Hour at the bar; 'I have a wine or a beer, or something normally. ... I'm not a drinker, I mean ... I drink in moderation. Hahahaha ... '(RVI4).

The size of the communal space for the gatherings was discussed. A size that could accommodate all the residents was preferred. A resident who had a large communal space in her complex said, 'We can all fit in it'(PR1). A resident of a large RV said, 
It's a bit hard to get to know everybody when you've got too big of a village. ... Because sometimes when they want to have a function like a dinner, they gotta have it in three different sittings so you don't get to know everybody, you only get to know the people who go to the one sitting you would go to. (RVI3)

Another resident of a RV also talked about the inappropriate size of the communal space: 'It's not quite big enough for a village of this size. For instance, the two concerts that we held here, we fill the hall. It's fire-rated for 100 people. ... We had 120, and it was full'(RVS6). A dining room with a floor space of $18 \mathrm{~m}^{2}$ for 10 residents was too small for them to bring in their walker frames, and they left their frames outside the dining room (PR6, PR7) (Figure 7-56).

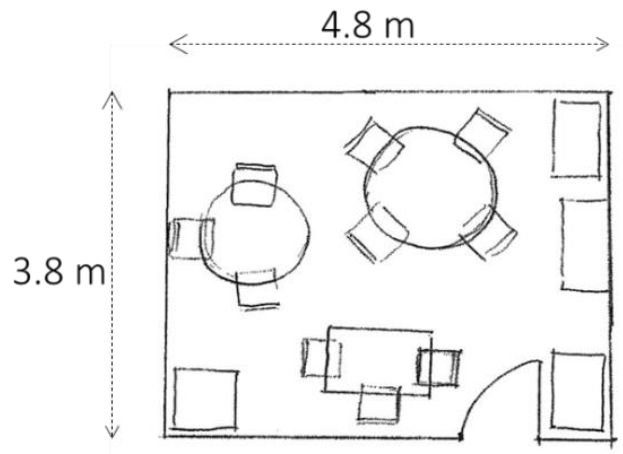

Figure 7-56: Insufficient dining space to accommodate ten residents and their walker frames

Communal activities were organised for the purpose of connecting residents in RVs and in some rental housing complexes. Socialising with other residents was sometimes more important than the activity itself: 'In the afternoons we have table games in the hall and I try and go to that to mix with the other residents. ... I just again, like to go even just have a cup of tea with them' (PR1). Residents enjoyed various social activities, such as card/board games and indoor bowls in the communal space (PR1, PR4, RVI5, RVI9, RVS3, RVS4, RVS5). A woman talked about an accidental encounter with an old friend: 'We went to the entertainment in the hall and she was there, so we had a little chat for a few minutes. It was nice'(RVS2). A resident of public-sector rental housing desired a communal space at her complex (PU5).

However, the preferences in communal activities varied, which was prominent among one couple: 'I go once a week. My wife goes several times a week... I don't mind being on my own. Right? My wife doesn't like being on her own'(RVI5). Some people did not join in communal activities because of their health conditions and various personal preferences. For example, some needed help with moving or had severe concerns with incontinence (PU5, RVI1). A resident with sight impairments said, 'It's difficult to mix with people. I have to ask their name when I see 
them'(RVI11). Having their own personal relationships reduced the desire for mixing with other residents through organised activities (RVI1, RVS4). One man said,

Personally, I don't use [the communal space] at all. Because I go out so often, I don't feel the social need that I would if I didn't go out. ... Because I've got family and church, there are plenty of people to look after me. I don't need anybody in the village, which sounds a bit selfish, but it's actually true. (RVI1)

The distance between housing units was a significant factor for residents' participation. Those with limited mobility generally preferred the communal space to be close by (PU5, RVI10, RVI2, RVI5, RVI7). One resident said, 'Its layout is quite good... it's a short distance, and it's fairly central for everybody'(PU4). In contrast, many residents had difficulties in reaching the communal area: one woman talked about the difficulty in reaching a communal space $22 \mathrm{~m}$ away: 'I get breathless'(RVI7). Another resident also talked about his difficulty:

I go to one, bible study in the village and ... it's a long walk. ... I go straight along here and then ... down to the main entrance. ... It is going to get worse. ... I could see me having to give it up because I couldn't walk that far. (RVI1)

A man with severe mobility limitations went to the hall at a $20 \mathrm{~m}$ distance in his mobility scooter (RVI8). There were also problems with accessibility to communal facilities, such as a slope in the indoor corridor (RVS2) and a heavy front door leading out to the indoor corridor (RVI7).

\section{Informal meetings with residents}

Encounters among residents often occurred near the unit entrance, such as in a corridor or on the porch. The view and proximity from the lounge to outside encouraged resident interaction. For example, one resident, who was sitting in his lounge saw a neighbour pass in front of his unit and talked to him on the porch (PU4) (Figure 7-57 left). One woman who lived in a complex where many people sat on porches said, 'I thought there's always somebody around, if you're lonely you could walk around and there's always somebody to talk to and it feels safe'(PU2).

In serviced apartments, a common lounge near resident units was used frequently by some residents for sitting in the sun and reading a book. However, one day a pool table was put in the space (temporarily during the refurbishment of the communal building). One resident said, 'We've got the pool table in the lounge, which is a nuisance really for us'(RV4). Another resident also missed the space before the pool table was moved there: 'Before the pool table came, there were more easy chairs and couches. This was a place to relax. We were close, because we 
felt "This is our room"'(RVS3). Even with the pool table there, she used the space often (10-12 times a month), sitting in the same chair (RVS3). The other two armchairs also had regular occupants (Figure 7-57 right).

In a small communal lounge in another block of serviced apartments, one resident who played the piano for his entertainment attracted other residents. He said,

When I do practice, within a matter of 60 seconds I have an audience. People come and sit around in that area... They're always saying, "When are you going to play the piano again?"(RVS6)
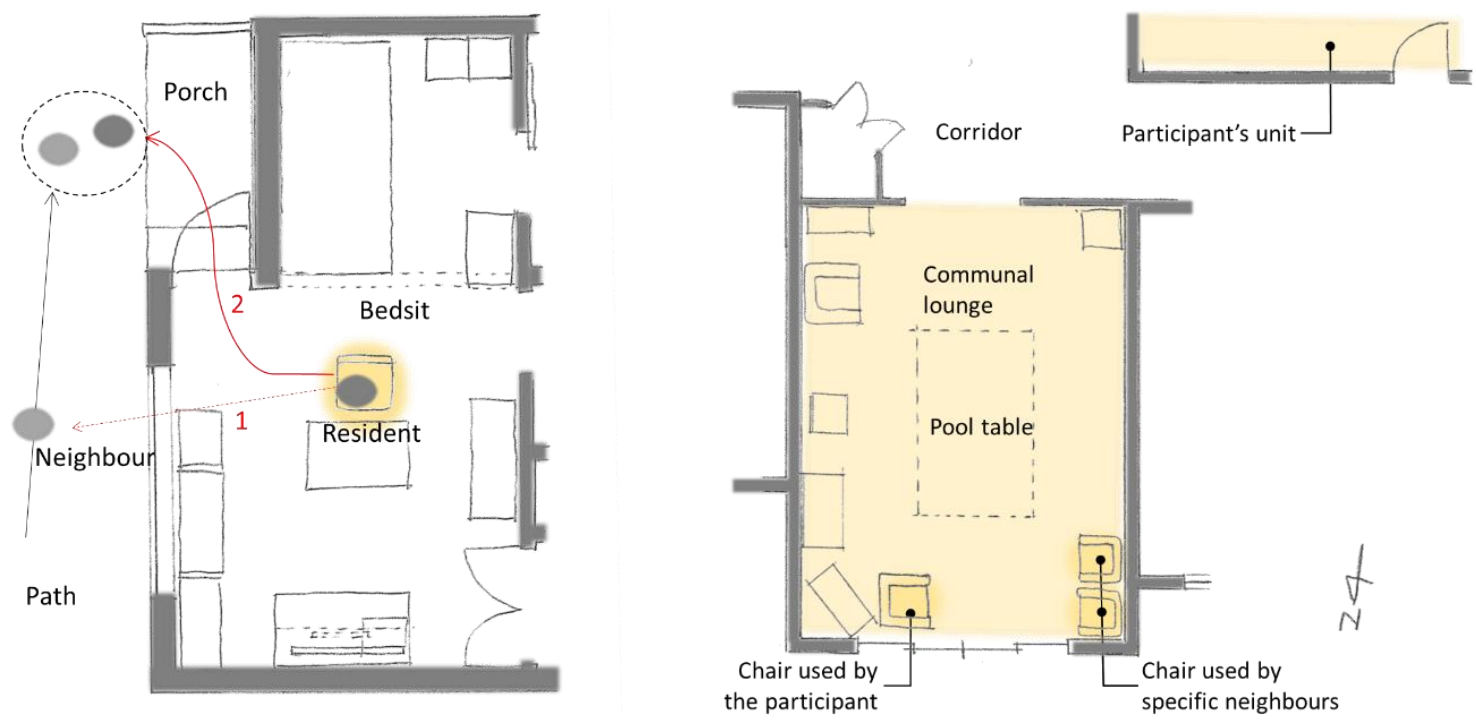

Figure 7-57: Spatial organisation that encouraged resident interaction (left) and their frequent use of communal space (right)

\section{Privacy and connection}

While most residents valued connection to other residents, they preferred to maintain their privacy. Residents commonly said, 'We're not living in each other's pocket'(PR1, PR2, PR3). Another resident also appreciated appropriate relationships between neighbours, saying, ' $M y$ neighbours are very friendly, but they don't interfere. But if you asked them for help, they would help you'(RVI7).

The layout of units and windows affected residents' privacy. The layout of houses with windows facing each other was not preferred (RVI1, RVI11, PU5). Many said they didn't want to look into other people's houses, and one woman didn't like that 'neighbours could think you're spying on them'(PU5). The short distance between units, as well as the parallel layout, was a problem. A woman talked about her impression of another village that she had visited: 'The 
buildings are looking at each other. To me, they are too close together'(RVS3). The proximity of other unit's window was an issue, particularly in regard to the bedroom (PR1). A woman praised the layout of the villas that had considered carefully the angle of and distance between houses (RVI9) (Figure 7-58).

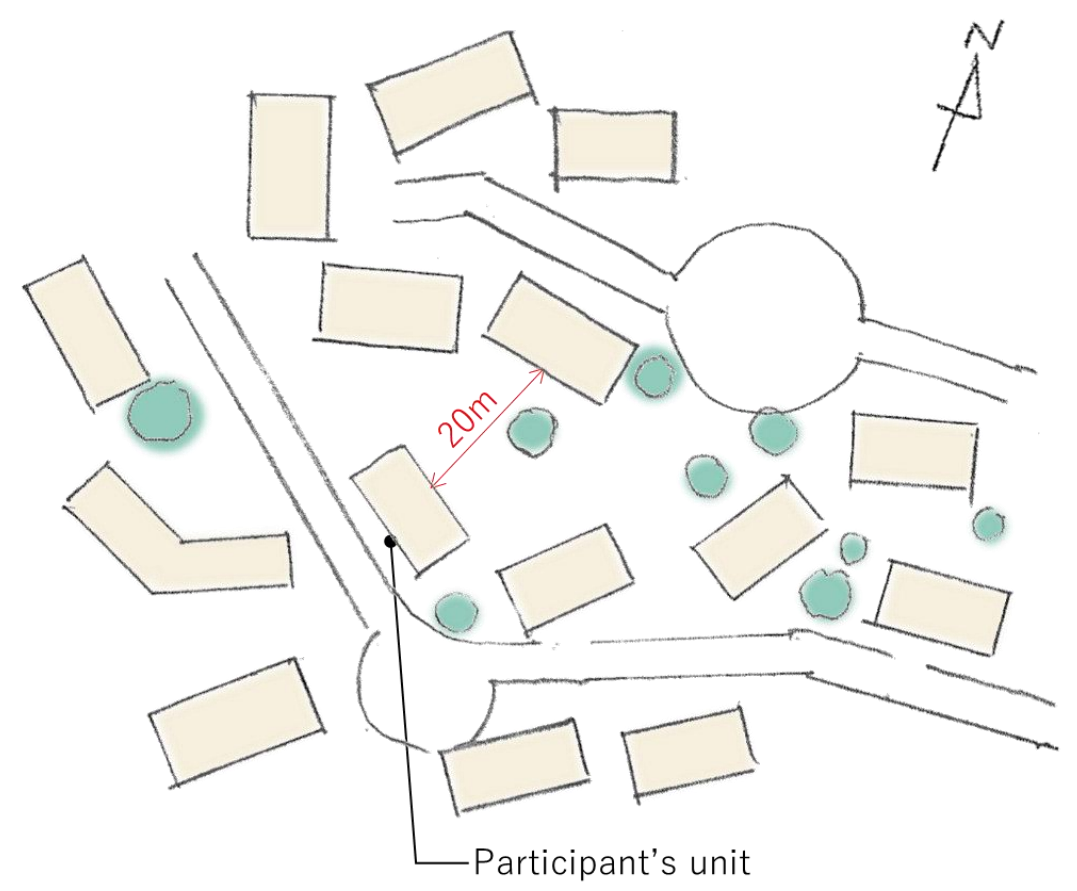

Figure 7-58: Layout of units with consideration to the distance and angles

The layout of the units, while affecting privacy, also had an impact on residents' sense of connection. One man whose window was facing towards an open space (a car park) appreciated his current situation:

It's certainly better than looking out on another row of houses like people further down in the village. They face each other. ... So in a sense, I'm a fair bit isolated, but at the same time I think it's a blessing that we're looking onto a car park and not another house. (RVI1)

Another resident, whose kitchen window faced her neighbour's window at a distance of $6 \mathrm{~m}$, appreciated the window's contribution to a greater sense of connection and safety, which outweighed her concerns about privacy:

This one, there's quite a few feet between us and it. It's not close enough to worry me. I quite like having neighbours because it's a bit of company around, isn't it? ... If my 
neighbours on my side don't see my blinds go up in the kitchen, they know there's something wrong. That works both ways, of course, I can see them. (RVI2) (Figure 759)

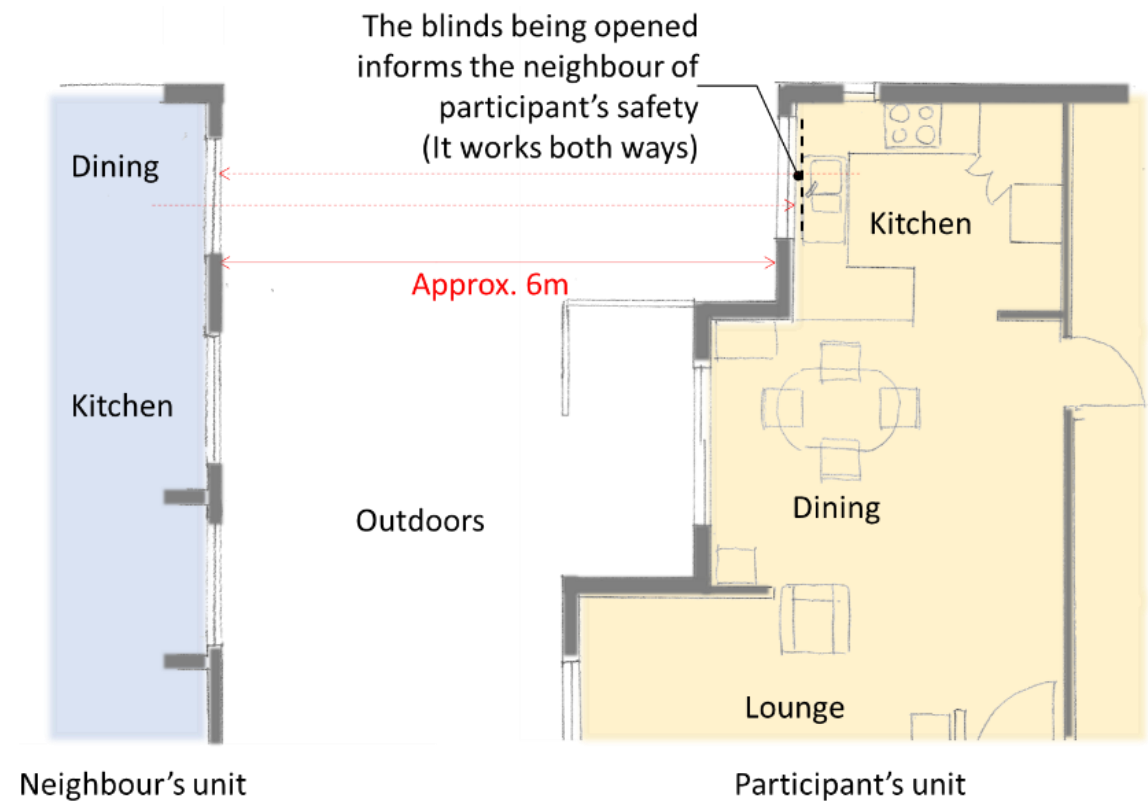

Figure 7-59: Unit layout that contributed to a greater sense of connection while maintaining privacy

By contrast, one apartment resident had a concern about privacy she had a window in close proximity to her neighbour's, which allowed views into her bedroom. She said, 'When they were looking through they could see me in that bed. ... I don't know if they really could or not, but I thought so, and then I used to close the door almost'(PR1) (Figure 7-60). 


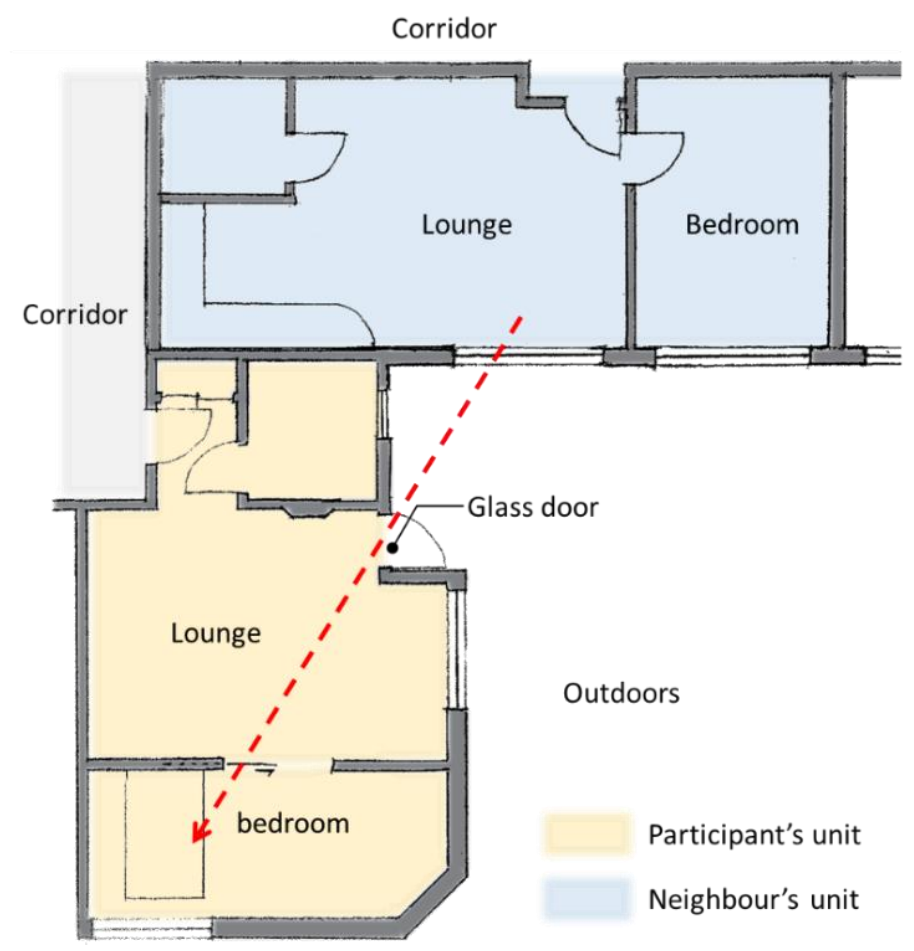

Figure 7-60: Layout of units and windows that affected privacy

A difference in levels was effective for maintaining privacy. A man who lived in a first-floor apartment said, 'I have stood down there and looked up here to see what people could see... You cannot see the settee... So, providing I'm not standing up against the window, I could stand here and nobody would see me'(RVS6) (Figure 7-61).

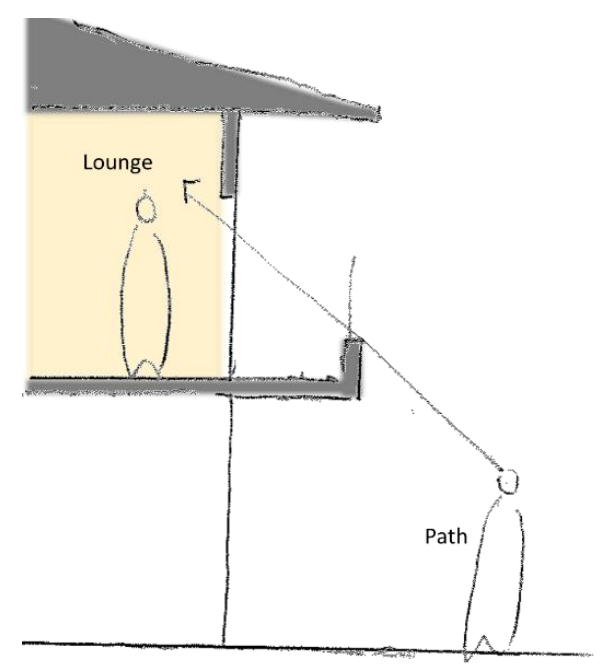

Figure 7-61: Level difference as a strategy for greater privacy

Using blinds is another solution that could shield views from outside and allowed residents views to the outside. One resident said, 'I think if you're building them [housing units] they should all 
come with blinds of some sort - Venetian or something, but they really can't afford to. And you have people here, they've got towels hanging up at the window or sheets, it's hideous'(PU2).

For most residents of apartment-type units with indoor access, the door that partitioned between the unit and the corridor was important for privacy. A female resident said, 'I like to shut the door. ... This is my little territory. Privacy'(RVS4). Another resident refused to move into the rest-home unit which had been encouraged by the staff for greater ease of care. She explained the difference between her current dwelling and a rest-home unit: 'I can shut these doors off. ... I wouldn't have a choice [to shut the door] there [in the rest home]'(RVS1). A translucent glass door was often used on the door to the corridor; however, it was often covered with a cloth or paper. A resident who put a cloth on it said that the light, with the reflection of his movement, went out to the corridor at night through the glass, which affected his privacy (PR7).

Some residents had concerns with neighbours' sounds. A resident of an apartment-type unit with outdoor access said, 'There's no insulation between the flats, so consequently noise can travel. If somebody has their radio too loud it could be heard in the next flat'(PU4). Another resident said, 'There used to be loud people, people play music loud'(PR6). She also said that she was sometimes worried about the noise she made. By contrast, some people had little concern about neighbours' sounds, particularly if there was enough soundproofing in their unit (RVI7, RVS6).

\section{Theme 3-4: Connection to the wider community}

While living in a housing complex, many participants left their complex for various personal activities and to maintain relationships.

While participants made new friends in the complex, their personal relationships such as family and friends were valuable, and many visited family and friend's house. Church also provided important connections (PU5, PU6, PR1, PR6, RVI1, RVI3, RVI4, RVS3). Some people belonged to personal social groups such as singing groups and bowls clubs (PU5, PR5). One resident said, 'I go to ten-pin bowls. But I don't bowl. I used to do a lot... I can't bowl, but I do raffle. I see old friends, it's something worthwhile'(PR5). Others joined in organised activities, such as events organised by local councils for residents (PU4, PU5, PU6) and a Community daycare programme (PR6).

Going into town was also a common enjoyable activity. One resident with limited mobility said, 'I go downtown once or twice a week, and this is what I look forward to'(PU1). Common 
activities in town included going to the café with families and friends (PU5, PU6, PR2, PR6, RVI1, RVI2, RVI9, RVI10, RVS2, RVS3, RVS4, RVS5), going to the club or RSA (PR2, PR7, RVI4, RVI9) and going to the public library (PR2, RVI4). One resident went to the club on Friday evening, saying, 'I've got a lot of friends who go to the club, so that's one of the main reasons. ... it's a just a change from here'(PR7).

Many people lived in complexes in their former neighbourhoods, and half of the participants expressed an attachment to the place (PU3, PU4, PU5, PR2, PR6, PR7, RVI2, RVI3, RVI5, RVI8, RVI11, RVS1, RVS3, RVS5, RVS6). One of them said, 'We lived in this area for all our married life. ... It's [their previous house] near here. It's just north by the light. When we go out, we see it every day'(RVI5). Another resident who had been in the same town all his life said, 'I do a lot of window shopping. ... And uh, I sorta take note of the shops, still there or closed down'(PR7).

\section{Accessibility and transportation}

On the way to and at their destination, inaccessibility hindered many resident's entry or use of buildings in the wider community. For some, getting out was demanding. An exterior hinged door with a door closer was often problematic, particularly when combined with a level difference on the floor (see also Theme 1-1).

While a few residents walked to the town (PU4, PR4, PR7), most used a car (13 drove, while others either had given up driving or had never been licenced to drive). For them, car access between the gate and the unit was an issue in large complexes. One resident complained about the narrow roadways: 'Everybody has to park up on the footpath'(RVI3). He also complained about the confusing road system with too many one-way streets: 'Once they developed right down to the end there, they changed all these streets here to one way. ... I mean, to try to direct someone through this to find this place [his complex] is very difficult'(RVI3) (Figure 7-62). 


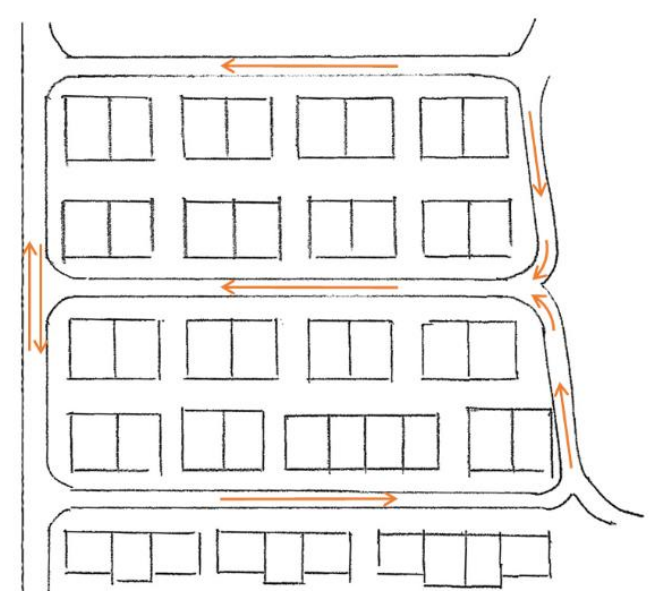

Figure 7-62: Confusing road system with many one-way roads

The access to public transportation was important. One resident said,

It will be great if, for instance, I can go out there and go out the front door and get a bus or something like that. ... Once I stopped driving the car, I've been dependent on other people. There was no way I could go out myself. Even if I could have walked or anything like that. (RVI1)

\section{Peacefulness and safety}

While the proximity to the town was convenient for many residents (PU5, PU6, PR1, PR2, PR3, PR4, PR7), 'peace and quiet,' and safety was also important (PU4, PR3, RVI9, PR6, RVI4, RVI11). A man talked about the importance of safety, referring to the situation in his previous house: 'I had a burglar. It frightened me. I started to think I want to live safe. I do feel safe here'(RVI4). The current housing was preferred by one resident 'because it's very peaceful and quiet and we have no interference, no problems from, you know, boys coming in... We are safe here'(PR3).

The enclosure of the site and the locking the gate or a common entrance increased the sense of safety (RVI3, RVI4, RVS2). One resident said, 'You lock that door at night. This is what I like about this place. I feel safe. ... There's no thought of any intruders or anything like that'(RVS2). A resident talked about public access, which was an issue in one council housing site which had pathways leading out to two streets:

School kids sometimes comes through and would look through your window... we have actually had sometimes kids come through and they've been on the boisterous side at night - about 10 o'clock, 11 o'clock at night. ... But I don't think the Council should 
allow that kind of thing. ... Although they've got signs up saying "No Access", nobody takes any notice of it (PU4).

\section{Visits by people outside the complex}

Inviting people from outside in the communal space was mentioned as a contributor to greater connection to the wider community (PU4, RVI7, RVI10). One person said, 'They bring along guest speakers and people like that to speak about different things. ... In the last 12 months, what, we've had the fire brigade ... we've had Age Concern, we've had Resolve'(PU4). People were also invited to entertainment such as concerts, exercises, handcrafts and speech-making in

RVs. Many residents had attended concerts, singing and dancing (10/17). A serviced apartment resident enjoyed 'entertainment like piano and ball-room dancing', adding, 'Sometimes little children come and sing' (RVS3). A resident with limited eyesight could enjoy musical entertainment: 'If there's anything interesting over at the landing like somebody playing the piano or somebody singing or a group singing, yeah, I go to that because that's something I can go to and listen to'(RVI11).

\section{Use of communication technology}

Most participants used a telephone daily. Telephone calls were important for many for mundane and occasional conversations with family and friends as well as other various purposes such as checking their medication (PU4, RVI5) and arranging visits (PU5, RVI4, RVS5, RVS6). In many cases, a landline telephone or its cordless handset was put within the reach of their sitting space (see also Theme 2-3). A woman, who used many spaces in her house, put a handset on her trolley and took it with her all the time (RVI9).

The internet was also used by many participants for corresponding with family and friends through emails and social networking websites such as Facebook (PU1, PU3, PU4, PR1, RVI1, RVI9, RVI10, RVS4, RVS5). It was particularly useful for contacting those who lived abroad or for receiving photos (RVI10). Social networking websites were used for actively building new relationships by one male participant, who had met a woman living overseas (PU4). While the computer was generally put on a desk (PU1, PU4, PR1, RVI1, RVI9), tablets such as an iPad didn't necessarily require dedicated space, and were used while sitting in their chair or wheelchair (PR5, RVS5) or put on a level surface such as a dining table (RVI10, RVS5). One resident missed her computer which she hadn't brought to her current home:

I would have liked to have brought the computer... I had a computer and a good computer table. A desk and a computer chair, which I enjoyed using. ... I missed it for 
emails and things. Sending to friends and that, overseas mainly. Keeping in touch. (RVS4)

She also used to use the internet for a wider range of information: 'I used to [use it for] fun, and [learning] a lot of information... I had a very good printer, and I used to print out articles and things. Look at different things, and that was always interesting'.

\subsubsection{Theme 4: Maintenance of possessions}

This section focuses on the maintenance of important possessions, that relates to residents' personal identity and control. Possessions also related to facilitating activities; however, this topic has been described in 7.2.2, and so has been omitted from this section.

Most residents had downsized from a previous larger house, and many of them had experienced getting rid of their possessions. Some had positive opinions for giving things away and adjusting to what they had in their units (PU2, PR4, RVI10, RVS2, RVS5), saying, 'I don't really miss anything'(PU2). However, some regretted what they did not bring: 'I didn't bring the things I should have...'(RVS1). Some residents said that everything they had in their home was important (PR3, PR4, RVI7): 'There's nothing that I don't want [in my house]. You can see it there - table and chairs in the lounge, the TV, the glass cabinet, the books'(PR3).

Inappropriate design or insufficient space for resident possessions at times resulted in a mess and created constraints on residents' activities. One caregiver talked about the situation of serviced apartments:

The apartments are very tiny, but she's also got a lot of stuff in there. ... That's probably half the problem, is the residents do like to have a lot of their stuff. So, it makes it difficult for, um, when you're transferring, and wheeling them about. (RVS1 caregiver)

\section{Theme 4-1: Maintenance of furniture}

Personal furniture was a significant contributor to residents' QoL. A dining table was important for some people (PU2, RVI9, RVS3, RVS5). For example, one resident talked about a unit with insufficient space for a dining table that she had visited: 'Residents were supposed to have meals at the kitchen bench. I wouldn't like that unit. When I saw this unit /with space for a dining table], I thought "I could live here quite comfortably" (RVS2). A wheelchair user who lived in a serviced apartment had a round table made of laminate that came furnished in her kitchen. The table was the appropriate height for her to sit at for cooking and eating meals. Not having 
enough space for her own dining table, she had no choice but to use it as her table, though '[she didn't] particularly like it, the look of it at all'(RVS5).

However, many didn't bring their dining table to their current home, or got rid of their dining table or were planning to do so (PU1, PU2, PU5, PR4, PR6, RVI6, RVS5, RVS6). This is because some felt there was no need for it, as well as there being insufficient space to accommodate it; the role of a dining table had been replaced by a smaller low or movable table, or by simply using their knees, when having meals.

The elderly often kept possessions they had owned for a long time such as desks, tables, chairs, drawers and cabinets (PU2, PU4, PU5, PR1, PR4, RVI7, RVI10) (Figure 7-63). One resident said, 'The TV, that's fairly new, the computer; otherwise I've made up most of the rest of what I've got from what I've sort of kept for many years, yeah so it's all pretty ancient really now' (PU4). Some kept their possessions they had brought from other countries (PR3, RVS2). One resident spoke about her furniture brought from England:

I rather treasure my table and chairs - those chairs are quite fabled, and they were handmade. ... When I was first married to my husband [in England]... we lived next door to a furniture shop - furniture man. And he made handmade furniture, and we liked it so much and we ordered four chairs, and they were $\$ 25$ each and that was quite expensive in those days. You know, I just treasure those. This is why I wanted to bring them. ... When we came from England to New Zealand in 1967, we brought every stick of furniture that we had with us. (RVS2) 


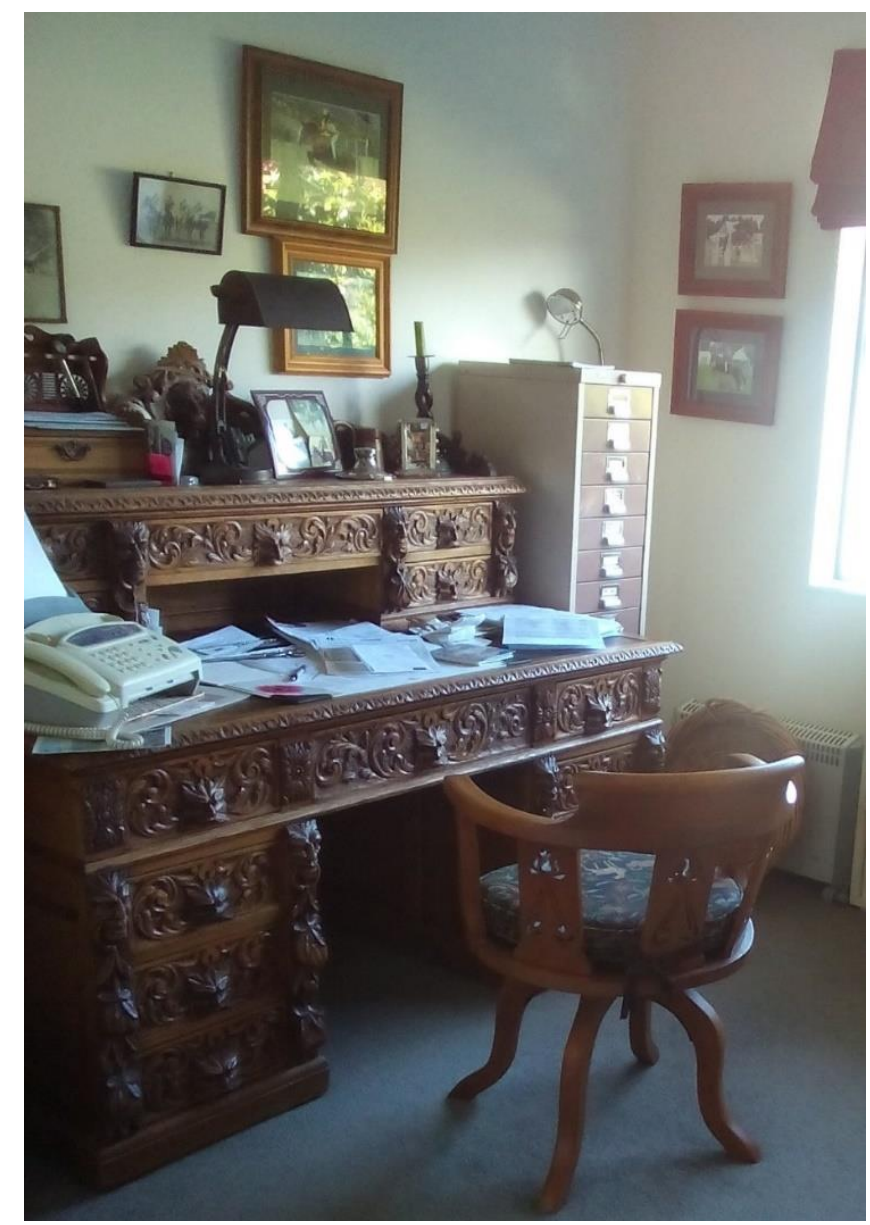

Figure 7-63: Desk that had been used for a long time

Some furniture had been given to resident from their family, which were also precious to many residents. One woman cherished a cabinet which had belonged to her father (PR4). Another resident kept a longcase clock that had been a gift, though she said, 'It doesn't go at the moment' (PU2). She also kept a low coffee table, saying 'my husband made [it] for me. No, I love it all actually, it all means something to me, it's part of my life.'

Attention was required in the layout of furniture. To prevent falls, the circulation space in a unit should have no furniture that could trigger tripping. For example, one resident said, 'That [low] table used to be there [in the middle of the room] but it's too dangerous if I staggered and fell, so my daughter moved it over there... If that table had been there I could have fallen over it'(PU2).

Walls were necessary for accommodating furniture. One caregiver suggested, 'They have all these empty walls [to accommodate furniture]'(PR5 caregiver). In a unit with ample empty wall space in the lounge, one resident said, 'When I first saw it I thought, it's so small. ... But I've been amazed at how much furniture fits in here without it being squashed'(PR1). One resident 
wanted more space for drawers in her bedroom (RVS5), while another had two display cabinets in her bedroom which would have been ideally placed in her lounge (RVS2) (Figure 7-64).

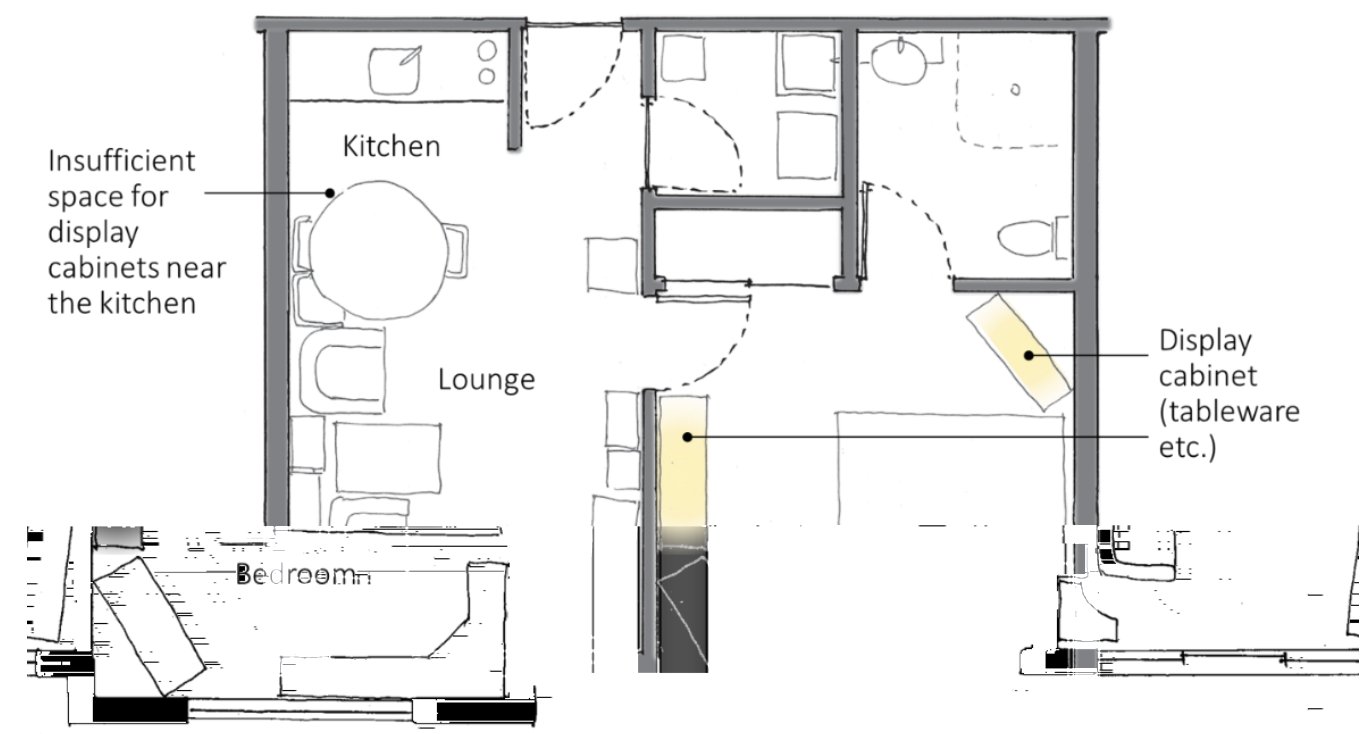

Figure 7-64: Unbalanced space for furniture in the lounge and the bedroom

One resident criticised a diagonal wall in her unit. She said,

I'm a terrible person for keeping on moving stuff around until I think I'm using the space best. But ... because of the unevenness of the walls there is some waste space in here. See, that second wardrobe has to go across and so [creates] the different corners, and of course I've filled them up with bags of wrapping paper and stuff like that to use the space, but that does bug me a little bit. (PR1) (Figure 7-65)

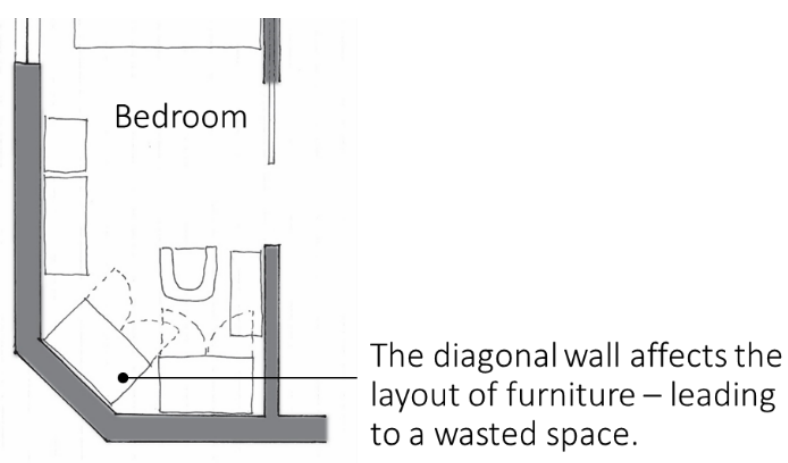

Figure 7-65: Inappropriate room shape for accommodating furniture

\section{Theme 4-2: Maintenance of nostalgic objects}

Keeping their nostalgic objects was important for elderly residents. In many cases, personal possessions marked their identities; one resident said, 'Books, records. Pictures are my life' (RVI6). Having her rugs and pictures also helped her with 'feel[ing] like [her] home.' 


\section{Needlework and paintings}

Some displayed the needlework they had done on the walls (RVI2, RVI7, RVI8). One resident talked about tapestries of her embroidery on the wall of her lounge: 'Well, I started at 16, embroidering a tablecloth. I haven't got it on today, but all my life I've done a bit of something... See those two pictures on the wall? I've done those. I've stitched them'(RVI2) (Figure 7-66). Another resident also cherished 'the few little pictures hanging up in the bedroom'which were 'just the sort of tapestries that she [his wife] did'(RVI11). One Asian resident hung a tapestry on the wall showing the traditional culture of her home country (PR3). One resident cherished her painting of the place she was previously living (RVI6).

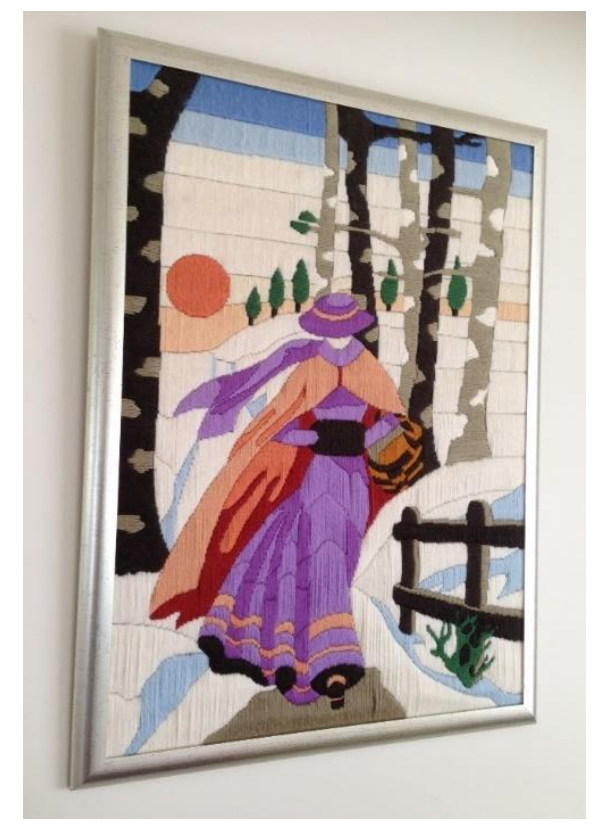

Figure 7-66: Needlework of one resident

\section{Artwork and ornaments}

Residents often displayed many items on shelves and other level surfaces. One resident kept a statue on the top of his bookshelves that he had made with lost-wax casting: 'I made that... in 1989. I've had that statue for 25, 30 years... To me it is [the most important], yes'(PR2). Another resident displayed an etching of her late husband's on the top of her writing table, saying, 'That is my biggest treasure. You see that round picture, that was given to me by my husband 69 years ago for my first wedding anniversary, and things like that to me are important. They are very important'(PU2). One resident who had lived with his wife before she entered a hospital displayed her ornaments in his bookshelf: 'I like my wife's ceramics. ... They're very delicate. The detail in them is quite remarkable'(RVS6). As well as open shelves and level surfaces, many residents kept their important items in a display cabinet/s (PU2, PU5, PR1, PR3, 
PR4, PR5, PR6, RVI1, RVI3, RVI5, RVI7, RVI10, RVI11, RVS1, RVS2, RVS4). They kept various ornaments such as tableware (PU5, RVI3, RVI7, RVS1, RVS2), ceramic ornaments (RVI1, RVI7, RVS4) and glasswork (PR3) and small religious items (RVI3). One resident living in a serviced apartment, who could not bring a lot of furniture, brought a lot of small items and displayed in a display cabinet: 'All my knick-knacks.... I've had lots of those plates, and ornaments and things. They're my treasures'(RVS4). Residents also displayed things that their family had made by hand, such as carvings made by his son when he was a schoolboy (RVI11).

One resident displayed a lot of candles on the table, which helped her with creating a religious space near her sitting space (PR3). A cross and religious pictures were also displayed on the walls.

\section{Photographs}

Photos were important for most people (PU2, PR1, PR4, PR5, PR6, PR7, RVI2, RVI5, RVI11, RVS2, RVS4). Photos were typically of their families, friends or past hobby groups. Some residents had a picture of familiar places from the past (RVI6, RVI8). Residents often displayed photographs on the walls, on shelves, on window sills, in a special display cabinet, or on furniture that provided a level surface/s such as drawers, a TV cabinet and a piano, as well as stored in albums (Figure 7-67). When asked what his favourite thing was, one resident said, 'Photographs of my wife, I suppose. ... Photographs of my wife and the few little pictures hanging up in the bedroom'(RVI11). In a rental house, the photos were displayed by putting them on the wall by tape which could be removed without making a mark (PR5).
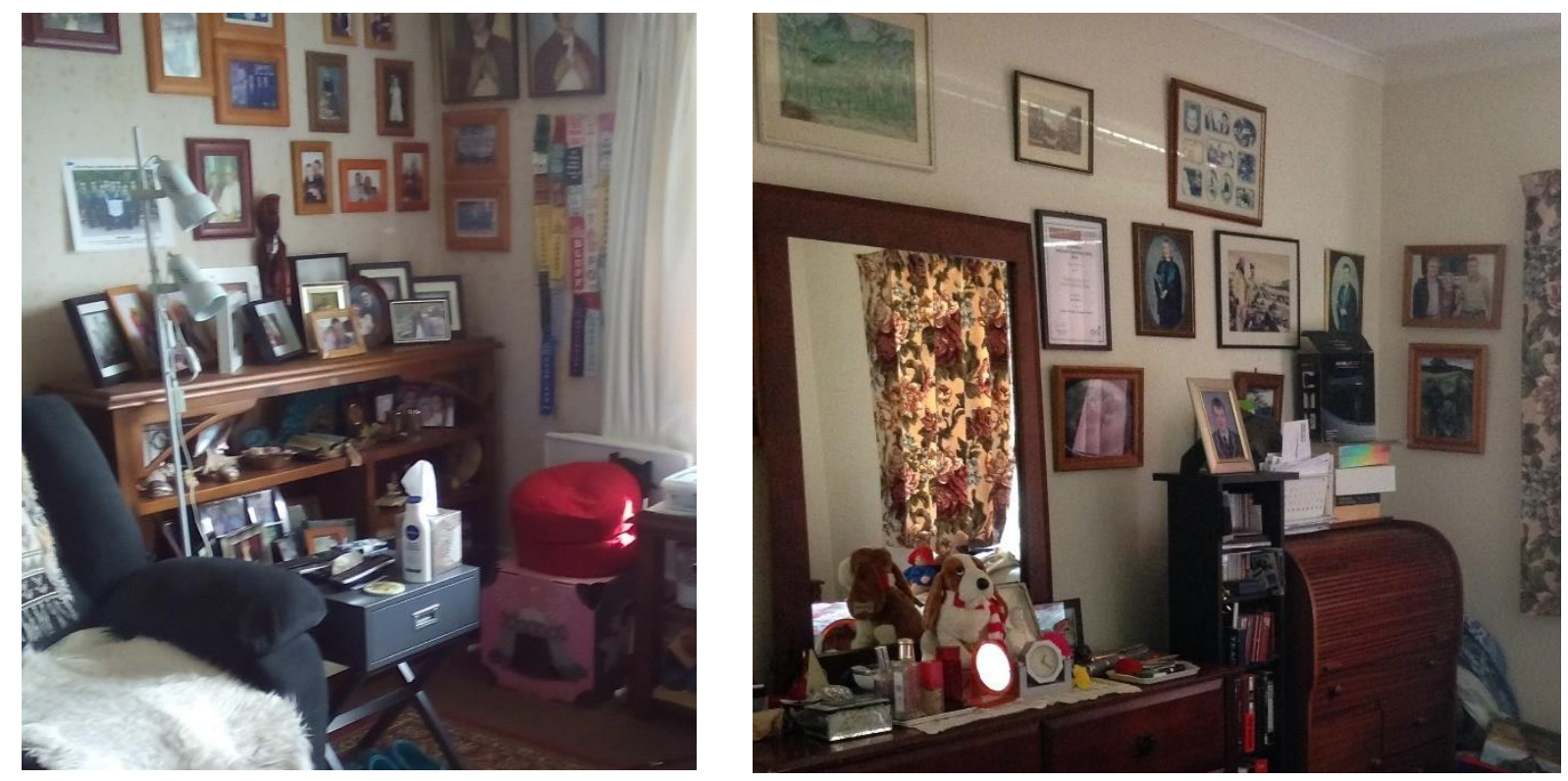

Figure 7-67: Photographs displayed on shelves and walls 
However, some photos were stored in less organised ways such as at the bottom of a bookshelf or in a cabinet or on the floor (PR4, PR5, RVS3). One resident, who put many photos all over the walls, complained,

There's nothing, you know, you can't have extra things around. This is why I have these sort of things to put in photos, because there's nowhere to put anything. And I'm a little bit crowded here, and I've got nowhere else to put them. (PR5)

Another resident also missed 'boxes of photographs'that were stored in her daughter's garage (RVS4).

\section{Tools for past hobbies and occupations}

Even though some people didn't do needlework anymore, they kept tools for needlework; for example, some kept a sewing machine and table or a knitting table in the spare bedroom (RVI2, RVI8). Another kept fishing rods in the wardrobe in a spare bedroom (RVI10).

One male resident who used to be a mechanic talked about what he didn't bring but had wanted to bring;

Well, all my tools - the tools of my trade. I mean I couldn't bring a lathe into a place like this; welding gear, welder, gas welding - I had a folder; I had about 30 grand's worth of tools, so I mean, yeah. And I've had to just get rid of it all. The things you have to do when you - you can't cart it all around, can you?' I mean I'd love to, but there wouldn't be any room for me if I put them in this flat. Yeah, there wouldn't be any room for me. (PU4)

\section{Books}

Many kept their cherished books and photo albums in their bookshelves (PU2, PU5, PR2, PR3, RVI1, RVS1, RVS2, RVS3), and some liked displaying the front page of their books (PR2) (see also Theme 2-1). One resident stored his bibles in his bookshelf near his armchair (RVI1). One resident had an oversized book, which was given by his late brother on his drawers in his bedroom (PU3). A built-in bookshelf was filled with the residents' own books (RVI7). One resident spoke of a precious book: 'Memoirs, that means the story of my life and my husband's. And one of my daughters put it all together and got it printed for me. It's very nicely done' (PR1). 


\section{Theme 4-3: Storing day-to-day items}

Open shelves and enclosed storage such as wardrobes and various cupboards were needed to accommodate various day-to-day items.

Shelves

Open shelves were useful to accommodate various items effectively, and the design had an impact on their ability to control their possessions. Many residents, particularly those living in one-bedroom or bed-sit units, added shelves after moving in (PU4, PU5, PR5). One resident put up open shelves for books, and said, 'There's two [shelves] in this house. ... There wasn't any room so friends of mine put them up' (PU5). She also had a lot of open shelves in the kitchen, which she appreciated (Figure 7-68). The alcove beneath the kitchen bench, designed for having meals with high stools, was often used for shelves or trolleys for storing things (PU3, RVI10) (Figure 7-69).
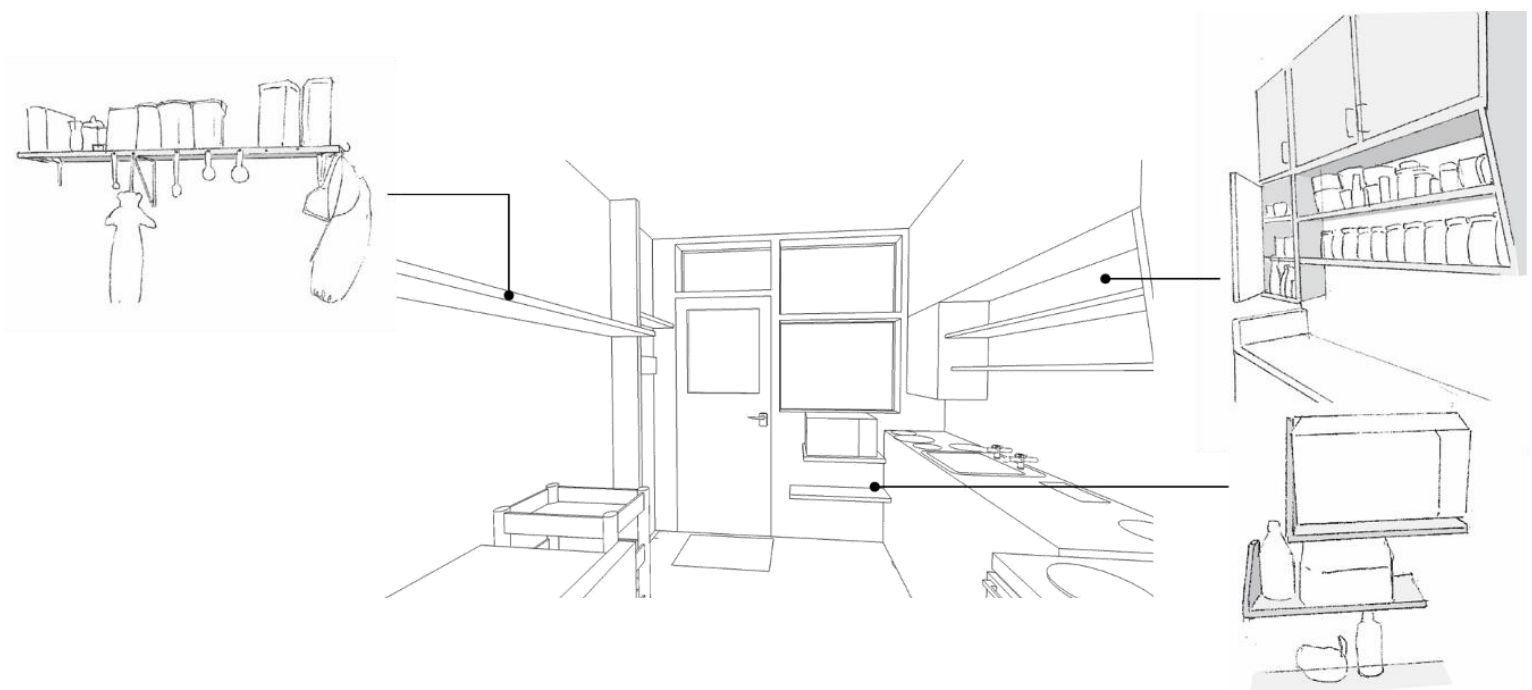

Figure 7-68: Shelves in the kitchen 


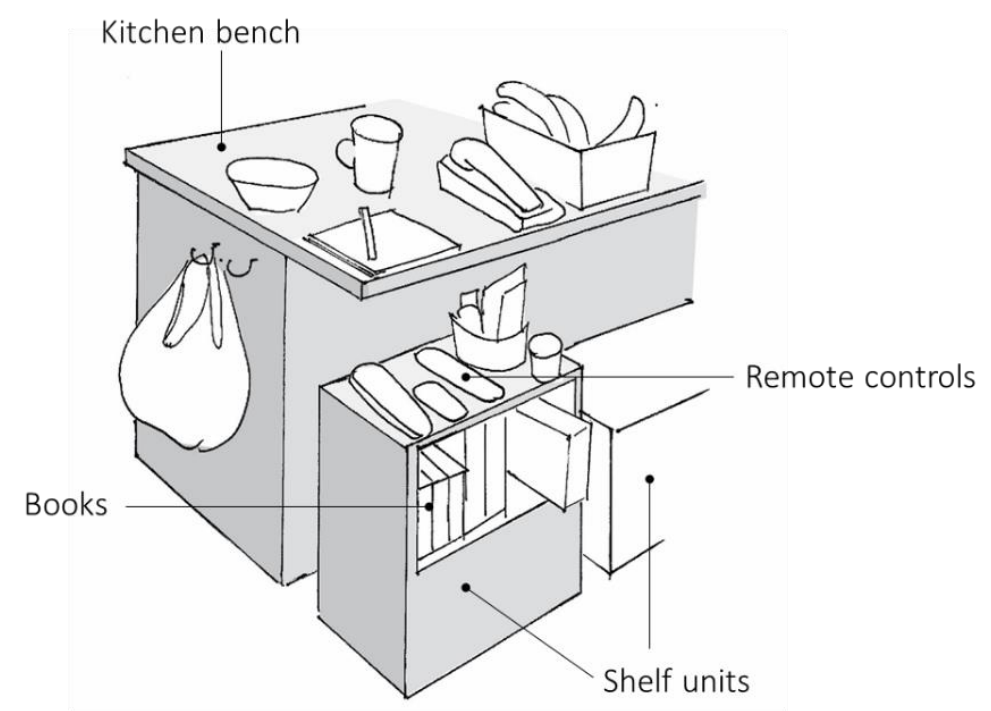

Figure 7-69: The recess under the kitchen bench that was used as storage

Shelving in cupboards was also important: 'I've got this cupboard here and one shelf. And my son put more shelves so, you know, storage space, there's nowhere to put anything' (PR5).

Another resident who felt there was a lack of storage had already put up one shelf but still wanted more. He said,

I mean the wardrobe's a good one, but it could do with somewhere where I can have shelves to put things. ... This one's got two doors to it but my previous one only had one door, so it was very tiny, but it had a lot of shelf space inside where I could put things on shelves, so I didn't need to have so many shelves up myself. (PU4)

Shelves being at a reachable height was important for elderly people, who tended to 'have shrunk' (PR5 caregiver). While some residents used a ladder, a step ladder or a stool to take something down from high shelves (PU3, PR4), it was impossible or dangerous for those with limited balance to climb on a chair or stool (PU1, PR5). One resident with hemiplegia said, 'I've got a little peg-chair over there, but the trouble is getting up on it. There is only one foot' (PU1). Many people had shelves or cupboards that were too high in the kitchen (PU5, PR5, RVI6, RVI7, RVI10).

In particular, upper shelves and cupboards above the bench that the kitchen came furnished with were unreachable for many, while lower shelves were generally reachable (PU1, PU5, PR5, RVI2, RVI6, RVI9, RVS5). One resident said, 'I can't climb up on a stool. The cupboards [at 1700 mm high] are too high for me. That's why I put my cups down on the bench'(PR5). Another resident could reach the $1650 \mathrm{~mm}$ high shelf (only the front part) but could not reach the 1850 $\mathrm{mm}$ shelf (PR6). Another resident was also able to reach the bottom shelf above the bench $(1550$ 
$\mathrm{mm}$ high) but not the second self (1730 $\mathrm{mm}$ high) (RVI6). One individual in a wheelchair had particular difficulty in reaching things and had to have someone else to help her (RVS5). Some residents used a stick to take things off high shelves (PR6, RVS4) (Figure 7-70). These efforts could also add risk of falling when they stretch, focussing on lifting something down.

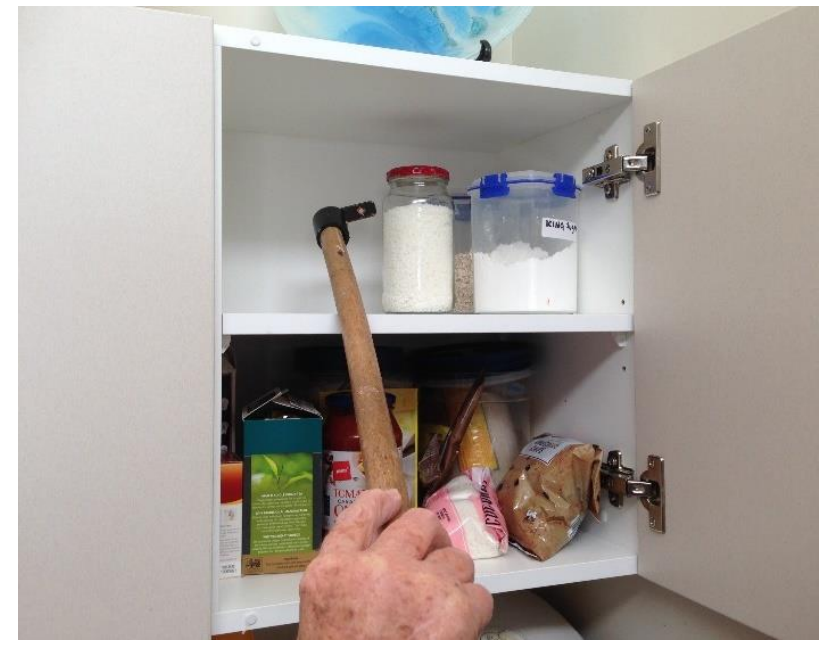

Figure 7-70: A hand-made stick used to take things down from high cupboards

Similarly, residents had difficulty in reaching lower shelves and spaces (PR5). 'They can't bend down. They can't go up, said a caregiver (PR5 caregiver), limiting the height of storage they could use. Acknowledging the limitation in space that she could use, one resident said, 'You have to use your space'(RVI10). Provision of inappropriate shelves and cupboards disregarding this limitation contributed to the inefficient use of space: 'More cupboards than I know what to do with. ... Plenty of cupboards'(RVI7). A caregiver suggested, 'It has to be a very reasonable size, a very reasonable height and reasonably long on the bottom... She [the resident] probably needs more, like things she can use'(PU5 caregiver).

The depth of the shelves added to difficulties: 'I can reach most of them. I think I can reach the top shelf, but I can't reach right ... to back of the shelf, I can't reach'(PU1).

\section{Wardrobes}

Wardrobes were important for storing clothes. For many participants living alone, a wardrobe of $0.8-1.7 \mathrm{~m}$ wide was not sufficient and extra storage was needed, such as a cupboard and many drawers of their own, or a wardrobe in their second bedroom (which was more than $1 \mathrm{~m}$ long) (PU4, PU5, PR5, PR6, PR7, RVI2, RVI4, RVI7, RVI8, RVS6). By contrast, 1.8-3 m wide wardrobes satisfied participants but were not too large for them (PU2, PR1, PR2, PR3, PR4). Most residents living with their partner used two wardrobes or more (RVI3, RVI8, RVS11). One couple used three wardrobes, with $5.4 \mathrm{~m}$ in total width (two built-in wardrobes and one 
cabinet of their own); the husband took three-quarters and the wife took one-quarter of the built-in wardrobe in the main bedroom. The wife took all the built-in wardrobe in the second bedroom and the husband took all the wardrobe in the office. One resident whose unit had two small wardrobes (each approximately $1 \mathrm{~m}$ wide) found it insufficient for two people (RVS6).

Having two wardrobe spaces was important for one resident to store his late wife's clothes as well as his own. One resident wanted to store his wife's clothes, which took up considerable space in the wardrobe (RVI11). Another brought with him 'those two [bedside drawers] there, one used to be mine, one my wife's'(PU4). A caregiver said,

Because sometimes they like, for example, their husband passed away. But for us, it is useless, but from the client's point of view, she likes to hold on [to] his blanket what they were using. Or maybe the pillow. ... For that client, for them [it has] so much memories so they want to keep those things. There should be a place that they can put their extra items [in], you know, storage, like a cupboard like this one or something. (PR5 caregiver)

A difference between genders in the use of the wardrobe was suggested: 'There is a big difference... [My wife] always took up three-quarters of the wardrobe and I had my quarter.... Women possibly do need to have more cupboard space and things like that'(PU4). However, this was not the case for other residents, as described earlier (RVI3).

Depending on the type of clothing, the requirements for space differed. One resident used the wardrobe for her 'hanging things' (RVS2), while things that could not be hung were put in her own drawers. More shelving 'instead of just a bar'(PR1) in the wardrobe was desired (PU2, PR1, RVI10). One resident put 'a set of bookshelves'(PR1) in the wardrobe and another also used shelves in her wardrobe, 'for scarves and cardigans and shawls and bags'(PU2). Another resident wanted 'shoe space in the wardrobe' (RVI9). In a spacious wardrobe, one resident was able to put in a shoe rack (PR4). For a couple living together, shoe space took half of the floor of both wardrobes in the main and second bedrooms (RVI3).

\section{Other storage}

A variety of cupboards were appreciated. Many houses contained no linen cupboard or one too small (PU5, RVI11, RVS2, RVS6); one resident had a linen cupboard with a projected area of $550 \mathrm{~mm}$ wide $\mathrm{x} 320 \mathrm{~mm}$ deep, which was insufficient (RVI11). In another house, the caregiver indicated a tiny cupboard and observed, 'That's the linen cupboard, where do you put all your 
linen?... If you had a lot of sheets and a lot of this and that, you know, you'd want another cupboard or another drawer' (PU5 caregiver). Another caregiver stressed the significance of storage that can accommodate the bedding, saying, 'Now, ornaments you can keep in a cupboard, but you know, there are so many other items. Their favourite shoes, their favourite pillow, their blanket, all sort of stuff. So many things they have'(PR5). A one-bedroom apartment resident stored his extra bedding for guests in a wooden box (RVS6). The importance of linen cupboards was significant, as potential space under the bed could become unusable when impairments increased. While one bedsit resident used the storage under the bed (two drawers) for bedding (PU5), another resident had replaced her previous bed with an electric adjustable bed, which no longer allowed for storage underneath it (RVI9).

Broom and bathroom cupboards were also desired (PU2, PU3). One resident said,

My broom's either in the hot water cupboard or I've got something behind the laundry door, or else on the back porch, but I think a nice little cupboard where you could put your brooms and your buckets, and everything would be nice. (PU2).

Another resident who had enough space to put his own cupboard into his bathroom said, ' $I$ think it's ample really, really good. ... The cupboard I've got in the bathroom there, well that's to keep all your bathroom stuff, you know, like your toilet paper and things'(PU3).

It was expected that a cupboard had room for a vacuum cleaner. A compact cordless vacuum cleaner was generally not too heavy and did not require residents to bend and was therefore usable (RVS4). Linen cupboards which had additional space to accommodate a vacuum cleaner were appreciated (PR3, RR4, RVI10), while those without it lamented their lack of storage (RVI11). In apartments with indoor access, a large vacuum cleaner, commonly used by professional cleaners, was stored in a communal space and shared with other residents (PR6, 7).

Hooks were effectively used for hanging outdoor clothes and bags near or at the front door (PU5, PR7) (Figure 7-71). However, one resident who experienced a lack of storage hung too many clothes at the front door (PR7) (Figure 7-71 right). 

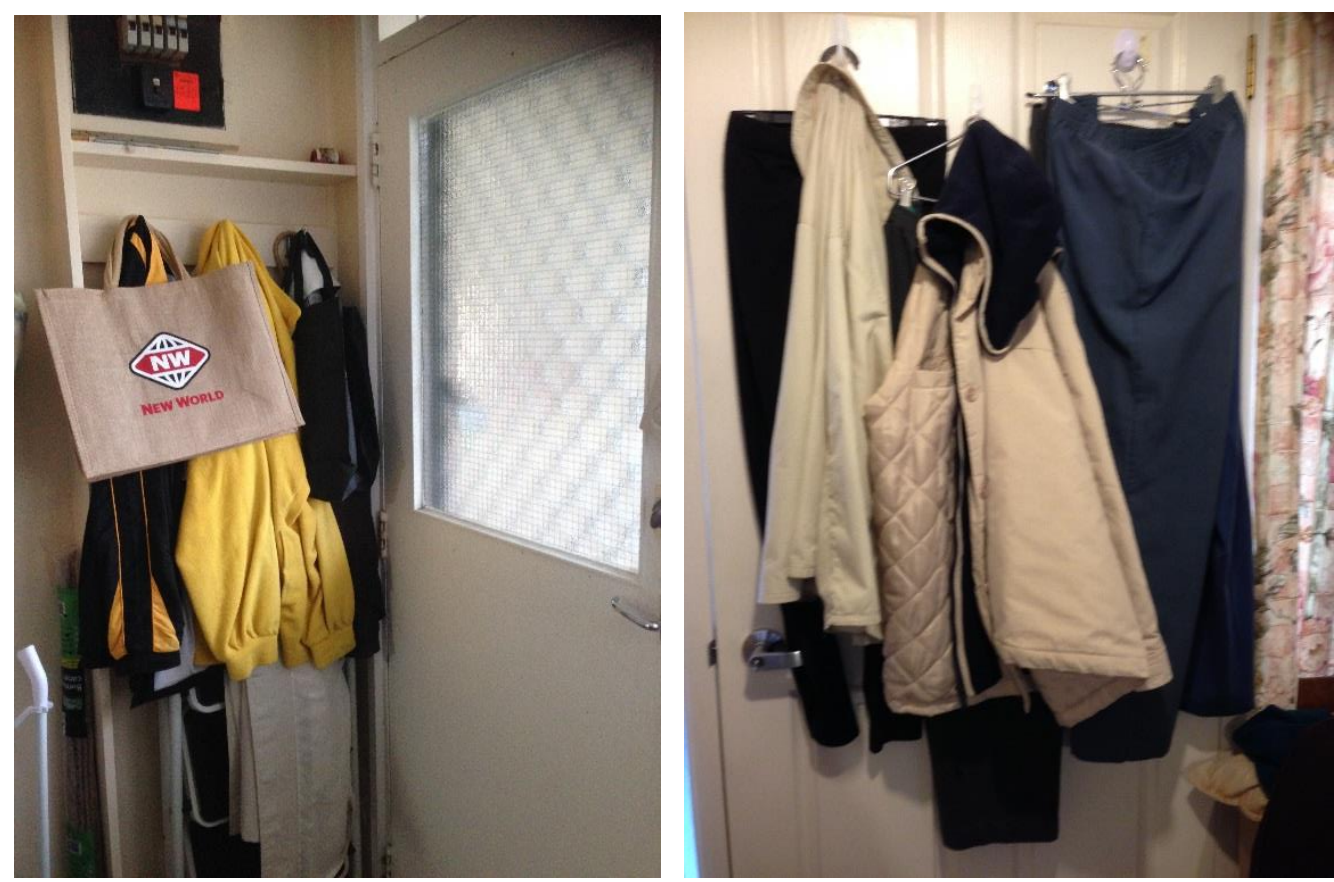

Figure 7-71: Hanging clothes and bags at the front door

\section{Theme 4-4: Storing items seldom used}

Storage for 'extra items' was important. One caregiver said,

Not their day to day items, but extra things that they don't like to throw away. They want to keep them with them.... So there should be a place... Some of the houses when we go there, we have to walk on a [set] path [through the house], because the rest of the area client has all the stuff. Too much stuff, but we can't ask them to throw it away. But you have to have some kind of a place for them to keep their extra items. (PR5 caregiver)

For those who had a garage or a spare room, it could be the space for their extra items. One couple said, 'We've got heaps of room, we can put stuff in the car shed. ... We put a bookcase in the shed'(RVI8). Many people had furnished their garages with shelves, cupboards and drawers (RVI1, RVI2, RVI3, RVI4, RVI5, RVI8, RVI9, RVI10, RVI11). One resident stored tableware, gardening pots and ornaments there, as well as various household items (RVI9) (Figure 7-72). A second bedroom was often used for storing extra items. One resident said, 'I just use the second bedroom as somewhere to put things, you know. I've got a wardrobe in there and a desk. I usually have my sewing machine in there'(RVI2). Another resident used her wardrobe in her spare bedroom as 'storage for fishing rods'(RVI10). 


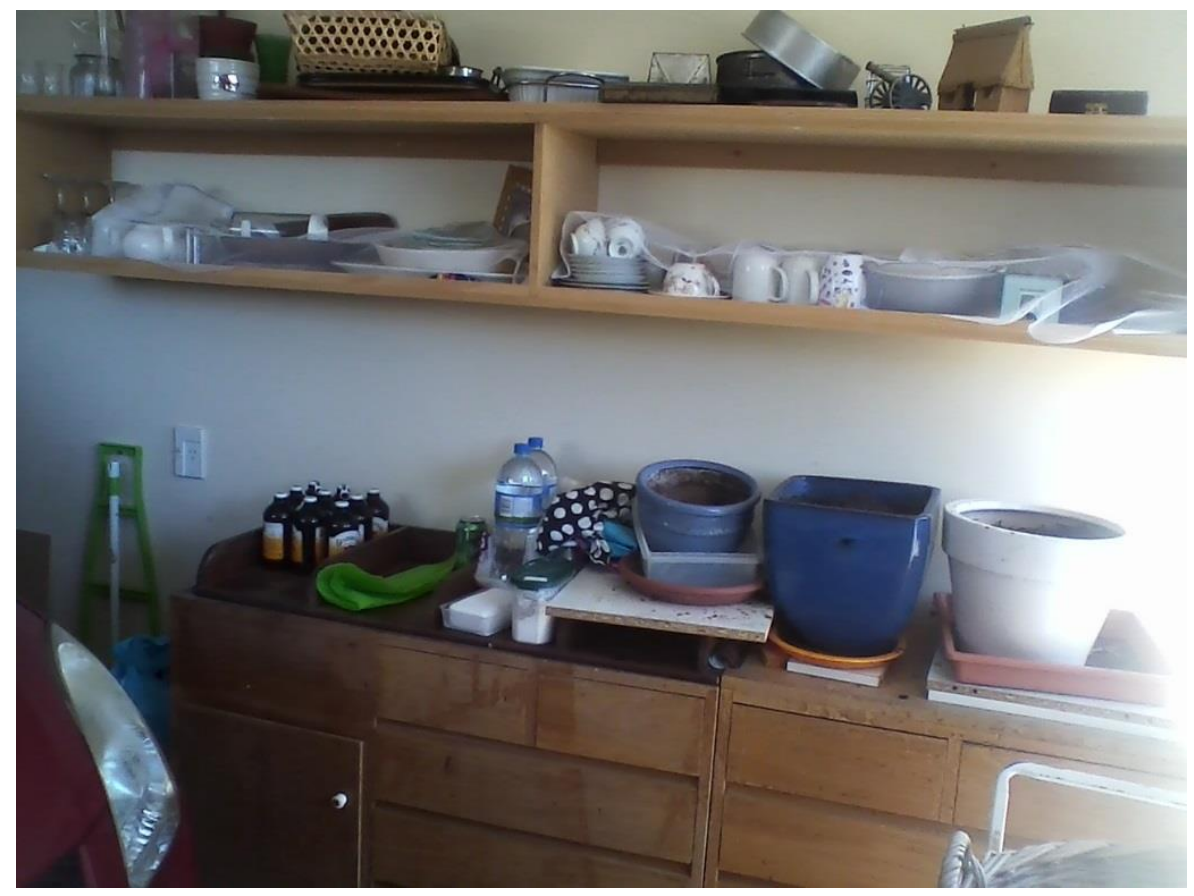

Figure 7-72: Using the garage as storage

In some RVs, residents could use the storage in the indoor shared garage allocated to individuals. One resident appreciated having one:

Because we have no family, we've got nowhere to store items that we would only occasionally need. A typical example really is winter jackets. We only need them for about four months of the year, so because of this insufficient room in here to keep all our clothes, I actually have some cupboards downstairs where I can keep that sort of thing. ... I have some tools that there would be nowhere in here to keep. Largely what's in the cupboards downstairs are things that there is insufficient storage within the apartment [to store them there]. You need an extra bit of storage somewhere else. (RVS6)'

Another resident stored their possessions in a self-storage area: 'The trouble is sometimes we've got a lot of stuff. We didn't want to get rid of it because we wanted to keep some of it, so it's in the storage area down there'(RVI3). One bedsit resident could use a communal storage room in her complex and put her three cases and several shelves there (Figure 7-73). However, she wanted to have more storage in her unit rather than using the common room (PR6). 


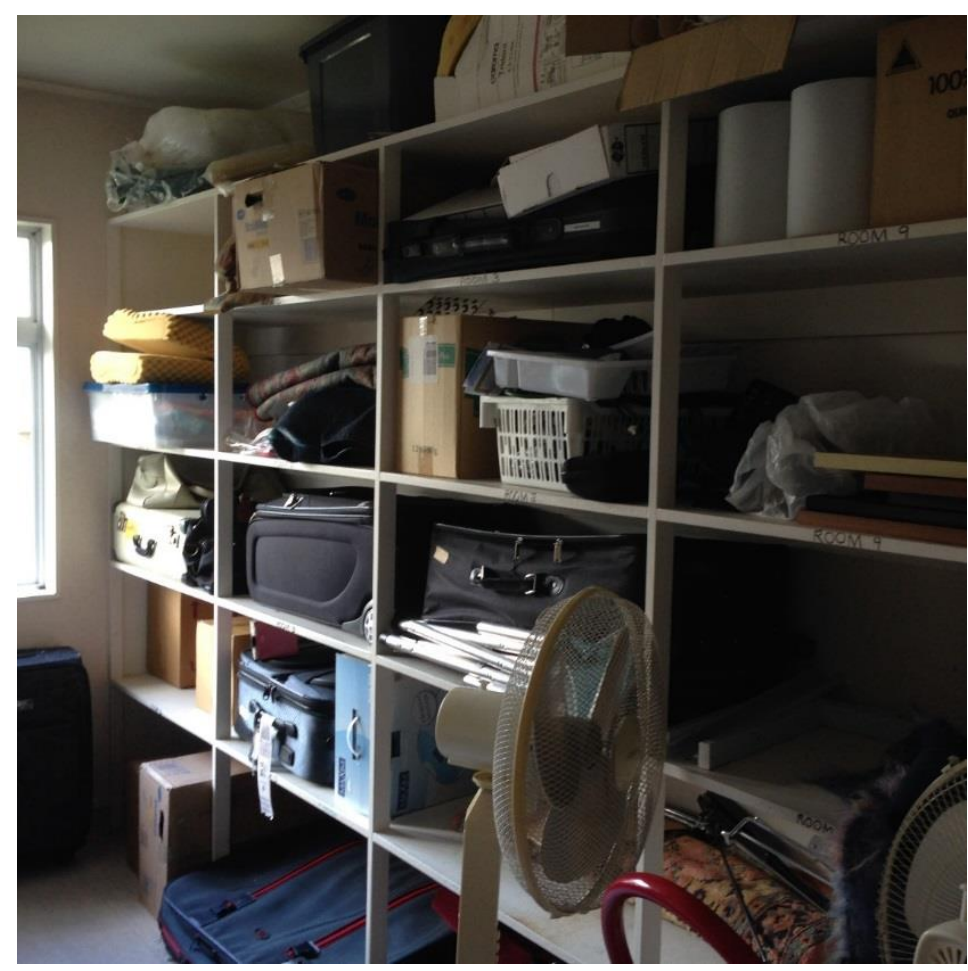

Figure 7-73: Communal storage

\subsubsection{Theme 5: Comfort}

This section focuses on four aspects of comfort for residents: 1) relief from fatigue and pains, 2) warmth and sunshine, 3) light, and 4) access to nature.

\section{Theme 5-1: Relief from fatigue and pains}

Many people felt fatigue and/or frequent pains. A woman in her 90s spoke about her fatigue: 'Since I've had this fall, I've been going to bed early, because I felt so tired. So tired during everything. Haven't seemed to get any energy, you know'(RVS2).

\section{Resting on bed}

Beds were valuable for many people (PR1, PR4, PR3, PR5, RVI7, RVI9). One resident said, 'I need a bed in which I can relax as much as possible, I'm with so many aches and pains and things... Sometimes I just need the bed'(PR1). Some residents used or preferred an adjustable bed for greater support in getting up and lifting their legs (PU1, PU3, PR5, RVI9). One resident who didn't have an adjustable bed needed another means to raise his legs, which was necessary due to his diabetic conditions: 'In my bed, I've got a pillow at the far end so that my legs are elevated'(PU3).

The darkness was important both for daytime naps and sleeping at night, and residents preferred being shielded from sunlight by doors and curtains (PR1, PU3): 'I've got dark drapes in there 
[the bedroom] so ... it was easier for me to go to sleep [during the day] when it's darker than what it is out there in the light'(PU3). Some residents kept the door almost shut to ensure their bedroom was dark enough, though they didn't shut it completely to avoid trouble to open it (PU5, RVI7, RVS1, RVS2, RVS3). While darkness was essential in a bedroom, light was also necessary when elderly individuals stayed in bed when feeling unwell. A resident pointed out the importance of windows that bring natural light in the bedroom: 'The bedroom ... it's just got a little narrow window [of $650 \mathrm{~mm}$ wide]. ... If you were spending a lot of time in bed, it would be pretty dark in there'(RVS5).

\section{Resting in a chair}

Sitting space was important for residents' rest: 'If I exert myself I've gotta come and sit down for a while, get my breath back'(RVI3). Many residents had specific sitting space in which they spend a lot of time (see also Theme 2-3). Many residents also needed to be seated while moving and performing daily basic tasks as well as leisure activities (see also Themes 2-1 and 2-3).

All the residents used specific chairs to spend most of their resting time, except for one person who stayed mostly in his bed. An armchair was the most common type of chair, which could allow an easy, relaxed posture (PU5, PR1, PR5, RVI1, RVI2). One resident said, 'because, I don't know where else to sit ... I can't sit up on the [normal] chair right now, I can't sit on that thing, because I can't get up properly'(PR5). The armrest was an important feature for many residents because it provided a support when they stood up (RVS3). Many armchairs could adjust back and feet positions. Raising the feet position was important for some (PU3, PR1, PR5, RVI4); one participant said, 'I've been told that, you know, because diabetics'... ankles are inclined to swell ... the more I can keep my legs elevated, the better I'm off (PU3). An adjustable chair also facilitated having a sleep in the early afternoon, which was a common routine for some (PU5, PR7, RVI8, RVS2). A resident wanted an electronically adjustable armchair instead of her armchair that was manually adjustable with a lever handle, 'because the lever handle is too hard for my shoulder'(RVS4).

While most elderly people used an armchair, one resident used another type of chair due to special requirements for his sitting posture. One resident used a side chair at a desk rather than an armchair like most residents used, because, he said, 'I have to sit up straight, I can't slouch. ... With the vertebrae, if I bend over like that, then those two are touching and that can cause pain, so I'm better sitting straight up so the weight is evenly distributed'(PU4). 


\section{Theme 5-2: Warmth and sunshine}

Warmth and sunshine were important for the elderly because, in one resident's words, 'as you get old, your skin becomes thinner, your blood becomes thinner, and you feel cold more'(RVI10).

Heating

Residents used various appliances for indoor heating such as an electric heater (PR4), an oil heater (PU6, PR3), a heat pump (PU3, RVI3, RVI4) and underfloor heating (RVS2, RVS3, RVS4). In one damp north-facing bathroom, the resident installed in a towel heater (PR5). Where a heat pump was used, residents arranged their sitting space near the heat pump mounted on the low part (RVI3, RVI7, RVI10). However, one resident was dissatisfied with her heat pump, which had been installed on the high part of the wall and didn't efficiently warm up her sitting space (RVI10). She would have preferred a heat pump on the floor.

Another resident had an infrared heater on the ceiling just above her sitting space, which she found 'too hot'(RVS1). In contrast, residents in serviced apartments who had underfloor heating were satisfied (RVS2, RVS3, RVS4). The cost for underfloor heating was included in the housing cost in serviced apartments, but residents of other housing types had a concern about the cost (PU1, RVI3). One resident said, 'Some of them [other units] have got underfloor heating but what they didn't know was ... their power bill in the winter would go up to $\$ 600$ a month. They're very expensive'(PU1). One couple didn't use their underfloor heating because it was 'far too expensive'(RVI3).

Warmth was also important when showering, and a heater was appreciated: 'I always turn the heater on about a quarter of an hour before I have a shower'(PU4). Coldness affected the motivation for showering if residents didn't have one: 'I go to one man and he won't have a shower, and what they need is, like, a wall heater in the bathroom to maybe turn that on. Get the room warm first and then they might consider going in the shower'(PR5 caregiver). Warmth was required particularly when drying. If the location of the heater was not close to the showering space, residents generally moved closer to the heater. However, those with particularly limited mobility had to stay in the same space even if it was far from the heater, and they didn't feel warm enough. One caregiver explained, 'This heater up here, gets turned on. But it's not very efficient being up there... [The resident] still does feel the cold in here'(RVI8 caregiver).

One individual had a strong attachment to the fireplace (PR7). He used to spend a lot of time in the communal lounge, because 'I used to like the fire down there. And I used to bring the wood and start the fire'. However, after the fireplace was packed up and replaced by a heat pump, he 
stopped using the lounge very often. He said; 'I do miss it'. Another resident enjoyed using the spa in the communal space, and said, 'On a nice, cold, wintry day, you can go down there and turn the fire on there. ... Hot facilities; I do use them'(RVS5).

One of the advantages of a small space was efficiency in heating (PR5, RVI11). One resident who lived in a one-bedroom unit said, 'As far as size goes, it's a small place to heat, you know, it's easier to heat'(PR5). She made the comment that, 'I've had a big place, I mean, [I've been] in a big place before, and you might ... stay in one room always. She kept the doors open between her lounge and bedroom. In contrast, one resident of a larger one-bedroom unit with a corridor between her lounge and bedroom always shut the corridor doors to 'keep warm'(PR4).

\section{Warmth in touch}

Carpets were preferred for warmth (PU2, PU5, PR3, PR6, RVI3, RVI5, RVS5) as well as softness (RVI11, RVS2). One resident said, 'Because, in New Zealand, carpet is warmer. Not like in Australia, [where] you have tiles... because they're cooler'(RVI5). Another resident had visited a place where the floor material of the living, dining, kitchen and bath rooms were all vinyl or lino, and she 'didn't like it'because it provided 'no feeling of warmth'(RVS5).

\section{Enjoying sunshine}

Accessibility to sun from the north significantly affected residents' satisfaction and their choice of the space they stayed in. One bedsit resident was fond of her sitting space with a north-facing window, because it got the sun. When she was offered to move to a newer and bigger apartment, she declined because of her attachment to the sunshine that came into her room: 'They said "you're a silly bugger." I said, "I know I am," but I like it here, I've got the sun, and I'm happy here. I'm happy here'(PU5). On the other hand, others were not satisfied with their access to the sun, especially those who did not have a north-facing window (PR1, RVI3, RVS3, RVS4). One resident said, 'I've got nothing facing north here, and it annoys me like mad'(RVI11). He moved his sitting spaces 'trying to find the sun all the time.' In the morning, he sat in a chair in the garage, looking out to the east; around noon he sat out in the deck where the sun shone from above; in the afternoon, he sat in the lounge with west-facing windows (Figure 7-74). 


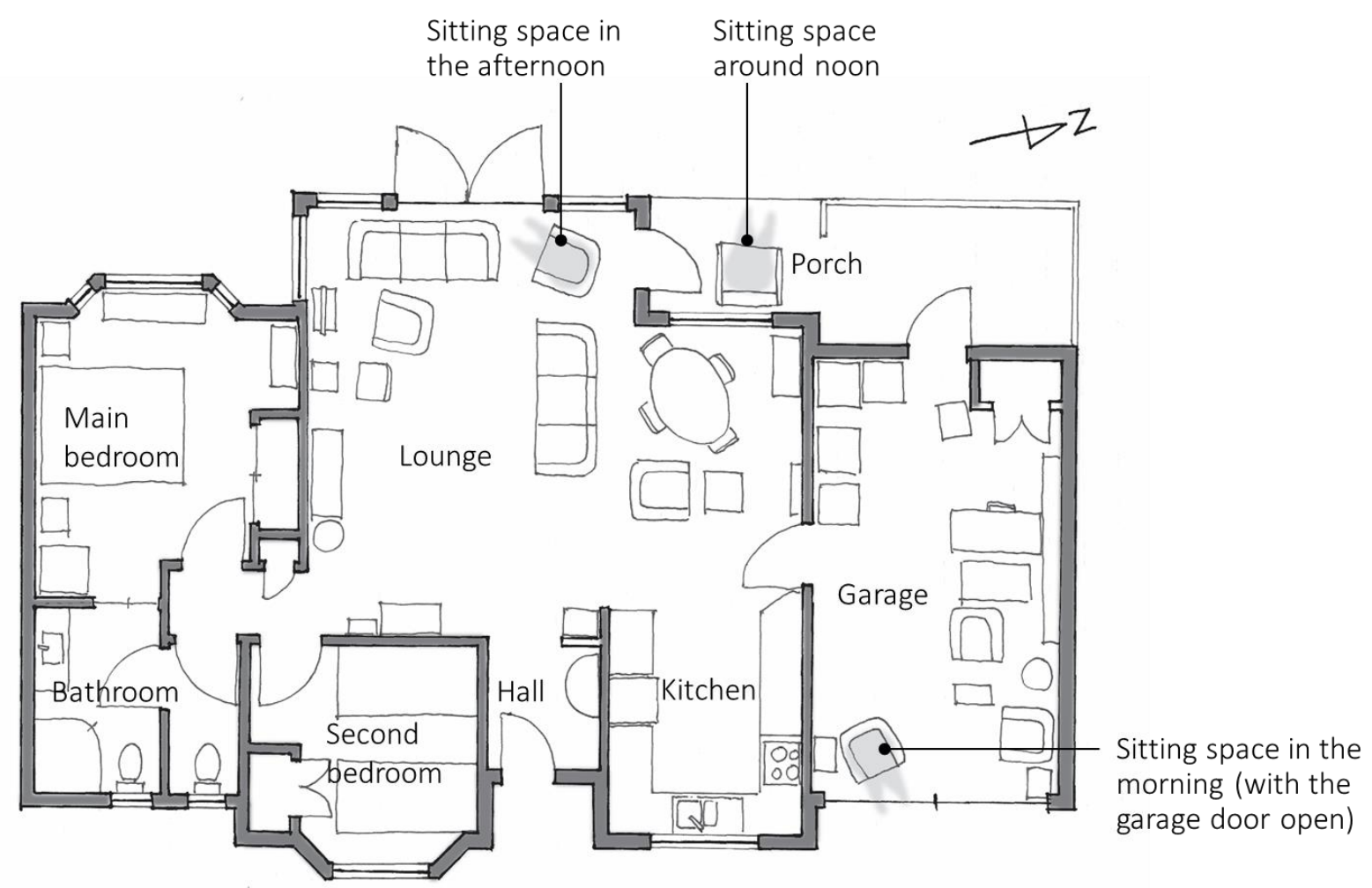

Figure 7-74: Changing sitting spaces according to the sun movement

In an apartment block with units on the north and south, residents on the southern side envied the northern side: 'I would like more sunshine. That's [the units on the northern side] the sunny side of the building'(RVS3). She and her next-door neighbour often sat in the communal lounge facing the north (RVS3, RVS4), and said 'I was freezing cold. ... This is warmer'(RVS3). Another participant who didn't have a north-facing window in his lounge mainly stayed in his north—facing bedroom, saying, 'It sounds silly to spend most of your time in the bedroom. ... It would be nicer, I suppose, if you sat out in the lounge and you got more sun'(RVI1). Apartment residents who had east-facing windows wished for more sunshine (RVS1, RVS2). One resident said,

It comes up early in the morning over the back of these, over these houses. Usually, it comes up over that highest point in the morning and then it makes its way over, and it's off of the terrace by about 2 o'clock - almost one or two. And then it goes over the house and it shines on that side for a little while, but on the whole, that's quite a cold side. Often people are saying, "Wish we had a bit more sun here". (RVS2)

The morning sun was preferred by some people (PU2, PR3, PR7, RVI10, RVI11). A resident who had a northeast-facing window said, 'In the mornings here it is absolutely beautiful and this 
flat's warmer, I think, because the sun in the morning warms it up'(PU2). Another resident also said, 'First thing in the morning the sun streams in. It's just lovely'(PR7). Getting the morning sun in the bedroom was particularly preferred. One resident talked about the ideal situation in his previous house: 'I liked the bedroom in the northeast corner, the main bedroom, so I got the sun in the morning. Wakes me up with the sun coming in'(RVI11). He suggested that all houses should face 10 degrees west of north, so that the bedroom in the northeast corner can get the morning sun through the east-facing window. Another resident also wished for the bedroom to be angled to get the morning sun: 'The bedroom of the next-door house is ideal, because it faces northeast and gets morning sun'(RVI10).

All-day sunshine was particularly pleasing. A first-floor-apartment resident could sit by the window without a concern about being watched. He enjoyed the sun all day through the northfacing window, and said, 'It [the sun] comes in here from sunrise to almost sunset. It's very warm in here'(RVS6).

Outdoor space, such as a porch, was used by many for sitting in the sunshine (PU2, PU5, PU6, PR2, PR5, RVI1, RVI2, RVI4, RVI6, RVI11, RVS1, RVS2). One resident referred to a direct health benefit: 'Vitamin D'(RVI4). Even those who could get plenty of sunshine indoors sometimes sat outdoors in the sun (PU5). However, direct sunshine that was too strong caused concerns for health. One resident went inside after staying outdoors for a while in the strong afternoon sunshine, saying, 'I can't stand it'(PR5). One woman suggested the health risk of the direct sunshine in New Zealand, and said 'It can be very dangerous, can't it?' We can end up with melanoma. ... I think we're in one of the worst countries in the world, aren't we ... for that?'(RVI10). Access to the sunshine was also affected by health conditions. Some residents couldn't sit in the sunshine because she would 'get a headache'(RVI2), and due to their diabetic conditions (RVI9). In contrast, one resident liked her outdoor sitting space with a roof: 'I spend quite a bit of time sitting there, because it gets the sun. It's sheltered. ... Yes, because it's sheltered and it's warm'(RVI2).

Having privacy when enjoying the sunshine was important for some individuals. One man could only sit in the morning sun facing a street. He said,

I'm looking out there in the sunshine. I don't look out to look at people. I look out because I like to get sun. ... But if I wanted to sit out on that terrace, you know, sunbathing with just my togs on or something, I can't do it because everybody's going to see me. (RVI11) 


\section{Theme 5-3 Light}

The requirements for light and brightness for the high-needs elderly related to their limited mobility, visual impairments and other conditions.

Brightness can be affected by the layout of space. One resident with limited mobility as well as an issue with incontinence needed high levels of privacy in his bedsit. This resulted in him keeping two curtains (of three) closed all the time during the daytime, which generated his desire for more light. He had a kitchen separated from his bedsit by the wall, but would have preferred an open kitchen separated from his bedsit only by the bench: 'That would let a bit little more light in' (PU1). In semi-detached houses, the open plan kitchen/lounge area provided greater access to the sun from multiple directions (PU3, RVI2) than that in closed-plan designs: 'I get the sun pretty well all day, because I get it in the side here in the morning, and I get it in that big window on the front for the rest of the day'(RVI2).

While installing a conservatory adjacent to their lounge allowed them to enjoy sunshine (but not in the lounge) as well as a larger interior space (RVI5), another resident who liked natural light didn't choose the option of installing a conservatory; they said, 'It makes the room darker' (RVI9). The distance from the window affected the illuminance; one woman felt it was too dark when sitting in a dining space that had no adjacent windows (EVI10) (Figure 7-75). Downlights with a low level of brightness were used there, which did not provide enough brightness.

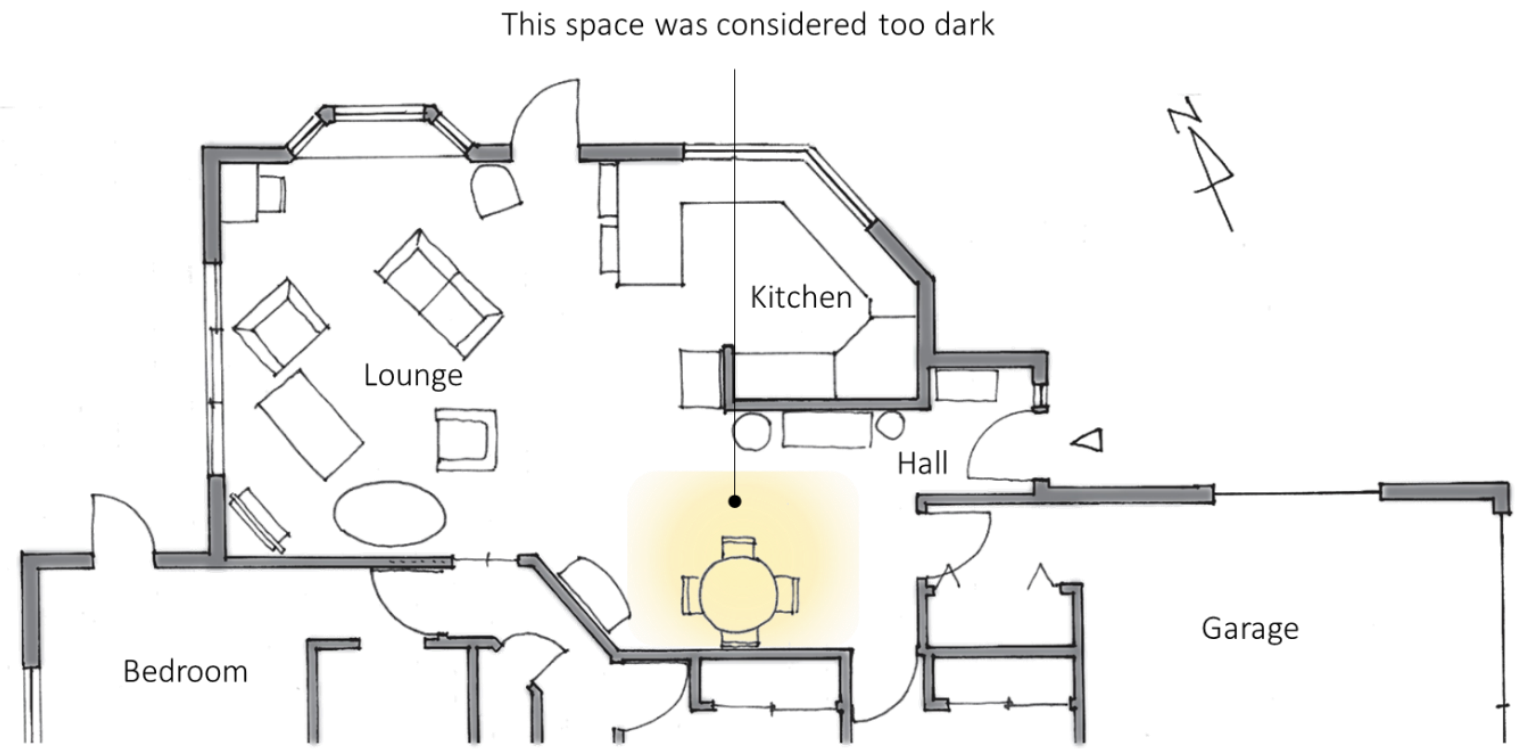

Figure 7-75: Big lounge that created darkness in the inner area 
Participants required extra illuminance in their space for activities. Several put a floor lamp or a table lamp beside their armchair for reading, writing and needlework (PR2, PR3, RVI1, RVS2, RVI5), while others instead sat beside windows and had enough natural light (PU2, PU5, RVI1, RVI9). One resident complained about the darkness of the bench space under a cupboard:

There's not enough light in that corner... With the light on and the big window there, on top there, it's still very gloomy working in that corner. Even washing dishes at night, it's not very good... Ideally, I would like a light under the cupboard, it would be quite handy. (RVS4)

Having a light in the pantry was appreciated by another resident (RVI10).

\section{Theme 5-4: Access to nature}

Access to nature was important for many residents. Important elements of the nature included mountains, the sea, plants. birds and just the outdoors.

\section{Views to mountains and the sea}

Views to nature were generally preferred by participants. Many liked views of the mountains, hills and bushes (PR1, PR2, PR3, RVI2, RVI3, RVI7, RVI10, RVS3, RVS4, RVS6). The reasons for the preference related to the situation of their previous houses (PR1, PR3, RVI7, RVI10, RVS4) and past activities of mountaineering and tramping (PR2, RVI2). For example, one woman said, 'Out the lounge window, I can just see the tops of the Tararua Range, which I used to walk across when I was tramping'(RVI2). However, another resident who used to enjoy mountaineering missed views to the mountains and said, 'I can't see any mountains here. You only see them when you're outside, going somewhere else, you know'(PR2).

Views to the sea were preferred by some (PR4, PR5), particularly with regards to Wellington Harbour, such as 'the sea in Petone'(PR5).

One woman who had multi-level views to the spacious grass area in front of her unit, to people moving on the road in the area, and across the country and the distant Tararua ranges (Figure 776) said, 'I love that. I often look out there on different days and wish I could paint. I wish I was an artist' (RVI10). Another resident also referred to the excellence of the unit layout for the views: 'How many [villages] are set such that every house has a view without impinging upon the neighbour? ... Even the hill [village topology] has been utilised to some extent, so ... everybody has a view. Brilliant'(RVS6). 


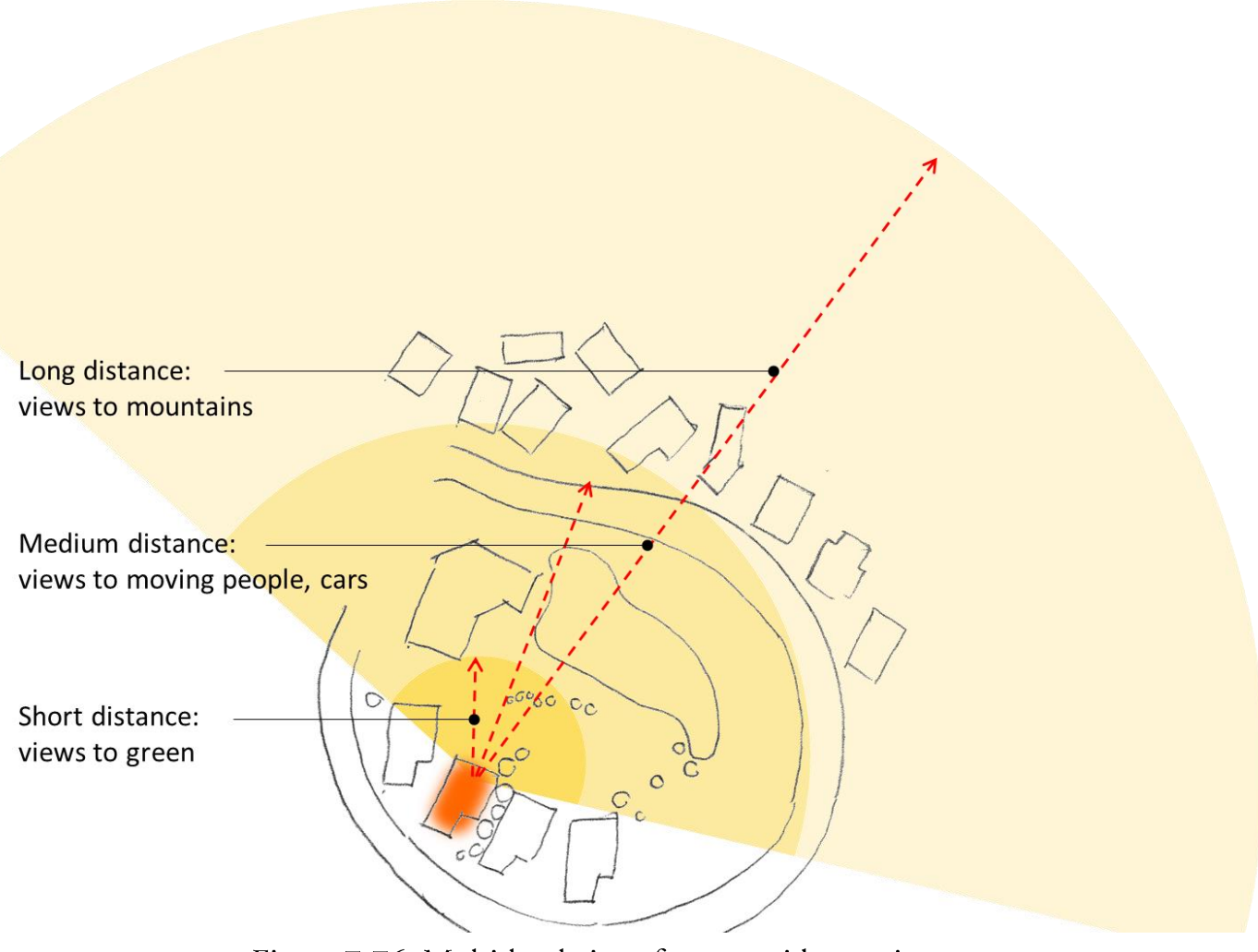

Figure 7-76: Multi-level views from a resident unit

\section{Access to plants and birds}

Trees and birds improved residents' QoL. Some residents liked to see trees, shrubs and birds from their sitting space (PR1, PRI5, RVI6, RVI10): 'On the couch here, ... I can look out at the cherry trees and Cyprus if I want to, and that's my favourite spot'(PR1). Another woman also praised the flowers around her unit: 'Those leaves, that bank [of a lake], they're all giant daffodils. Just had been a maze of yellow, beautiful'(RVI10). Native flora and fauna such as tui, wood pigeons, kowhai and flax were also preferred (PU2, PU5, PR5, RVI10). One resident pointed out the importance of trees, particularly in a confined situation: ' $I$ can see the oak tree and lots of birds... There is a building just over the fence, and if there were not trees, I wouldn't like it'(RVI6).

People often fed birds on their porch or a common deck (PR7, RVS2, RVS3). A woman said, 'The birds flock every morning. I feed them - give them a few crumbs outside there-and they sit away. As soon as I open my curtains, they're all sitting on the fence waiting for their breakfast'(RVS2). Some other residents also fed an apple (RVS3, PR7): 'I eat an apple ... on the [common] deck out there. ... birds come around, so they get an apple'(PR7). However, birds could cause trouble; one resident said, 'The birds make a mess of my windows' (RVS2). 
Trees and shrubs, while providing pleasant views and privacy for residents, sometimes caused residents some distress, such as limitations in the access to the sun (PU4, PU6). Another participant talked about the size of the tree that affected resident access to the sun: 'The trees block off sunlight and ... make the place rather cold. ... Smaller trees, bushes, they're more suited for this site rather than great big pohutukawas'(PU4).

Many female residents put their plants inside of their own dwellings, on a table or the kitchen bench (PU5, PR4, RVI2, RVI6, RVI7, RVI9, RVI10, RVS1, RVS2) (Figure 7-77).
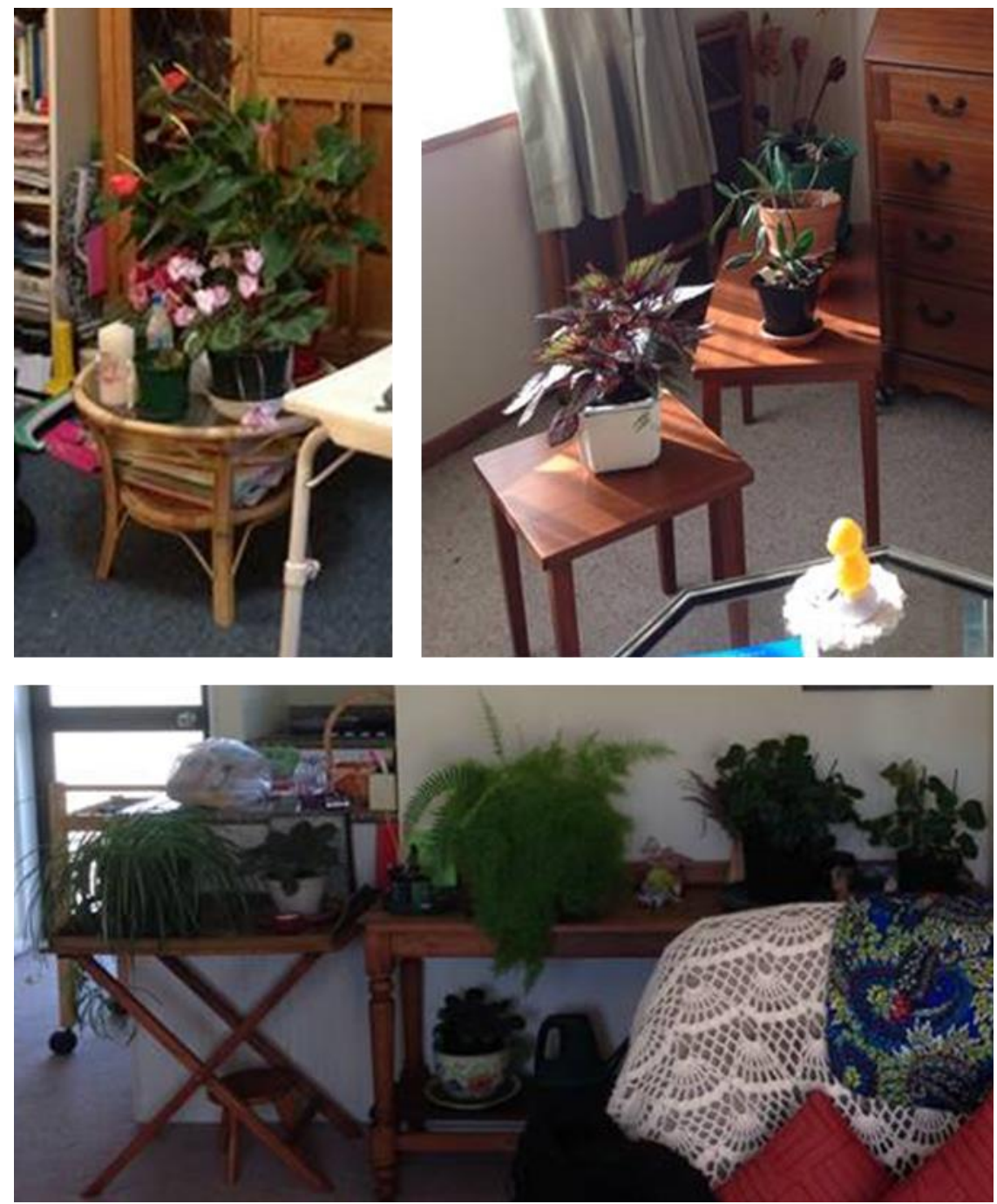

Figure 7-77: Indoor plants

\section{Access to the outdoors}

In small houses, having an outdoor space was highly valued. One caregiver observed, in relation to a client with a small bedsit unit, 
[There's] no little balcony - that's the thing, if you're in a high-rise it would be nice to be able to go outside, just a little spot where you can put a chair or hang a bit of string for your washing. That would be nice, wouldn't it? Because [his unit is] small it drives him crazy. He told me he'd go and walk down the street and sit in the bus shelter at night just to get out of here, just sort of, you know, in an open space. (PU5 caregiver)

Privacy was important in such an outdoor space. A woman said, 'If that front porch was closed in a bit - like Steve's next door is closed in - it's nice'(PU2). A resident who only had a common outdoor deck wished for her own outdoor space: 'A patio- you can go out around the garden. It would be quite nice'(PR6). Another man didn't have his own porch, but could enjoy sitting in a common porch adjacent to his unit that was little used by others: 'Usually when it's a nice day, I might go and sit out on the porch'(PR2). One couple installed additional fences around their porch to improve its privacy, which allowed them to enjoy 'a barbecue' and ' a few drinks out there'(RVI3).

\subsubsection{Theme 6: Quality of care}

This section focuses on the quality of care for residents, which has four aspects: 1) quality of care in assisted showering, 2) quality of care in household tasks, and 3) quality of care from on-site staff.

\section{Theme 6-1: Quality of care in assisted showering}

Many elderly people had health conditions that required assistance in showering. For example, one resident found she got 'very tired [from showering activities] because of [her] heart condition'(PR1). For a woman who had an intractable disease that affected her ability to undertake daily tasks, receiving assistance was an essential means to staying in her current home. She said,

I would like to stay here as long as possible [rather than going into the care centre]. And, when all's said and done, we can get plenty of help if we can't manage on our own. If I wished, I could have somebody here showering me, seven days a week. (RVI10)

The tasks required in assisted showering typically included five basic activities: undressing; washing the back, legs and/or hair; washing other parts of the body; drying; and dressing. One resident appreciated the assistance in washing and drying his toes: 'I find it difficult to clean and dry between my toes, and being a diabetic that's where gangrene starts - you know, dampness between your toes'(PU3). Another resident who had disabilities in arms said, 'I can't, sort of, 
dry myself properly'(PU1). Many residents were assisted with putting on his pants or socks, because of the difficulty in bending (PU6, PR6, PR7, RVI4). In addition to these main tasks, depending on the individual, other tasks occurred immediately between, before or after showering, such as toileting, brushing teeth and putting on moisture lotion or cream.

Required assistance in showering differed by individuals. The patterns of the combinations of the tasks which involved caregiver assistance are shown in Figure 7-78. There are six patterns of involvement of caregiver assistance. While some people were assisted with all tasks, some did not require any assistance with showering (though caregivers might provide surveillance). The tasks that most commonly required assistance were drying, washing the back and legs, and dressing. Assistance with washing parts of the body other than the back, legs and hair was the least common.

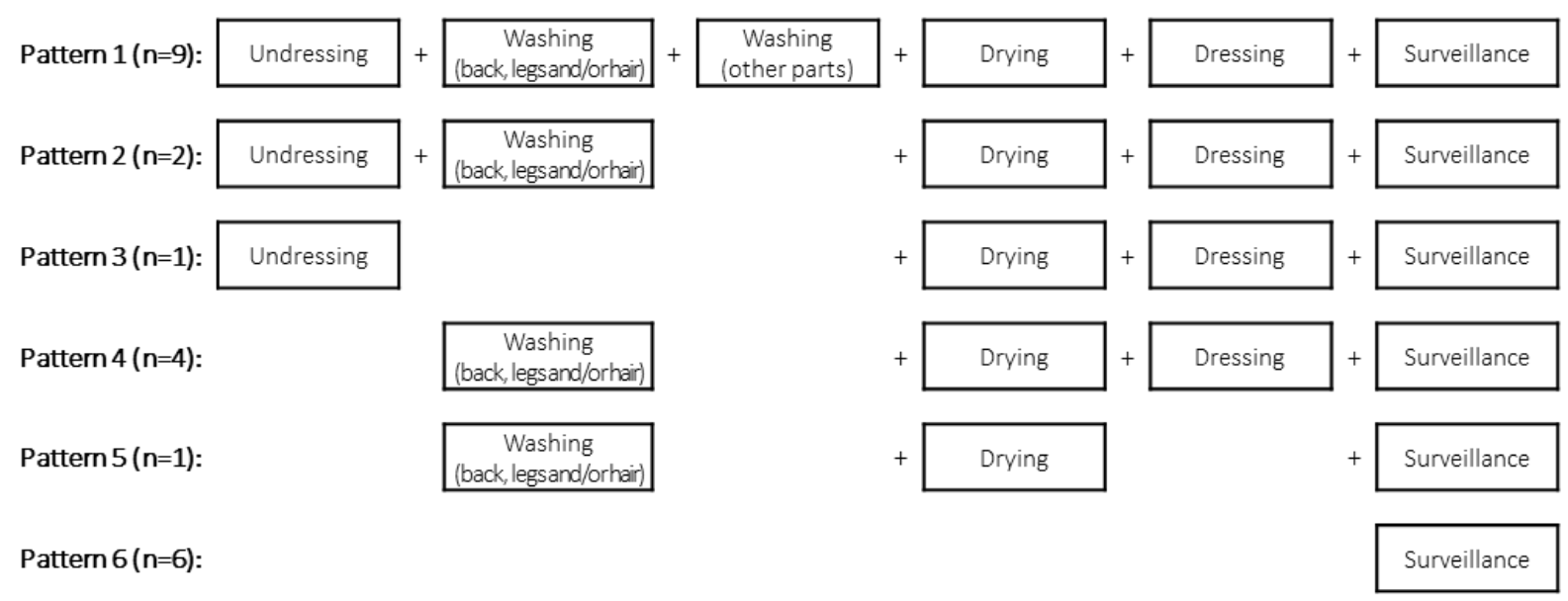

Figure 7-78: Patterns of assistance in showering 23

\section{Ease of care activities}

The square area of all shower stalls in the sample units containing a step or lip (Type B and C) were one-meter square or smaller. This was too small for the caregiver to come in to assist the resident (Figure 7-79). One caregiver described the situation: 'There is no room for me to get over there and wash his back, wash his legs. So, if ... without ever reaching around and try to get to him, I'd get soaked [because I'd be too close to him] probably'(PU3 caregiver). The bathtub/shower combination with a shower head fixed to the wall was the most problematic for users:

\footnotetext{
23 This chart doesn't include three cases in which information on the tasks requiring assistance was not obtained.
} 
This [shower head], I've got no control on where [it aims], you know, like I'm using this [bucket to draw water]. See what I mean? ... I've got to make sure the soap is away so that she [resident] doesn't slip ... and just to rinse her feet properly. Those showers with a long hose would be much better for the caregivers. (PU5 caregiver)

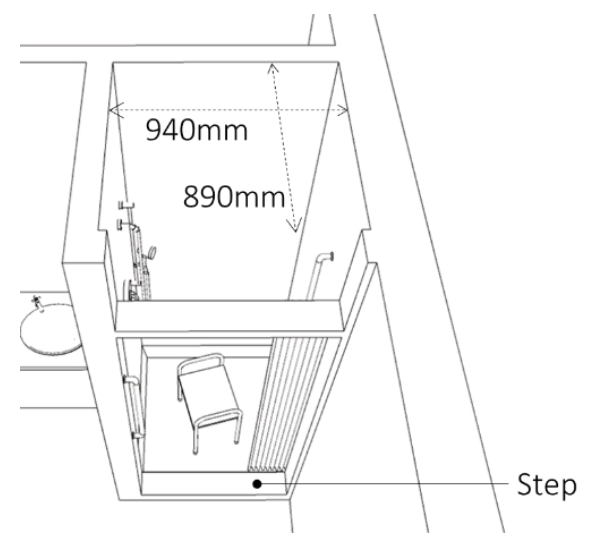

Figure 7-79: Example of insufficient space for caregiver assistance for washing

The door or curtains of the shower enclosure was normally kept open when caregivers assisted residents with washing in a small space. While this could be draughty, it allowed access for the caregiver. By contrast, a hinged door that opened in the wrong direction in shower areas disrupted caregivers' movement for assistance. Caregivers preferred other types of door, or a curtain: 'I keep banging into the door, she [the resident] jokes I'm trying to break it'(RVI2 caregiver).

Undressing, drying and dressing often took place in the bathroom; however, the space for undertaking those tasks was often not large enough (Figure 7-80). Space was lacking for a chair for the resident to sit on and for the caregiver to move around. In some cases, the toilet seat was used for the resident to sit on, and in other cases, residents kept standing: 'I'll have to dry her off, and if she's standing there [in front of the vanity]... there's not always a lot of room for me to get around'(RVI2 caregiver). 


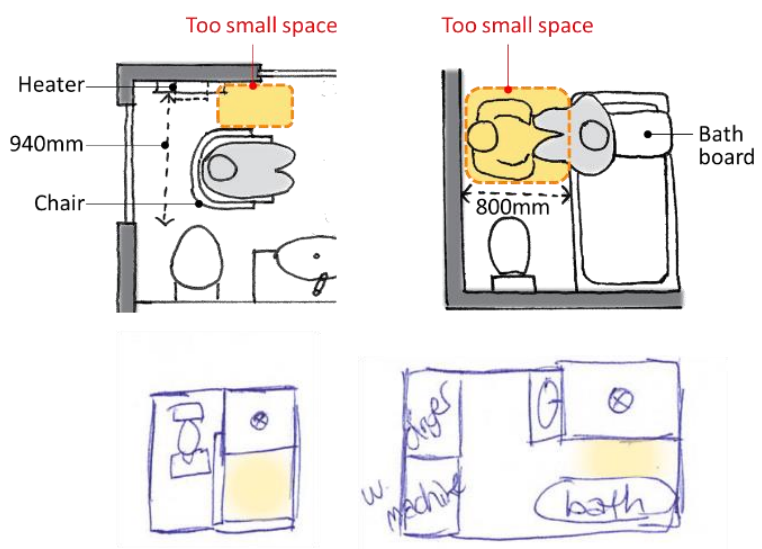

Figure 7-80: Insufficient space for drying

(The two sketches below were drawn by a caregiver)

Keeping wet and dry spaces separate within the bathroom was important. For those tasks which required a dry area (for example, to keep clothes and slippers or shoes dry), wet-area showers were at times problematic due to the spread of water over the floor. When the bathroom was not big enough or the design was inappropriate to accommodate keep clothes dry, the area immediately adjacent to the bathroom was frequently used (Figure 7-81). Clothes and shoes were often put on a chair or stool, on the walker frame or on the toilet seat to keep them dry.

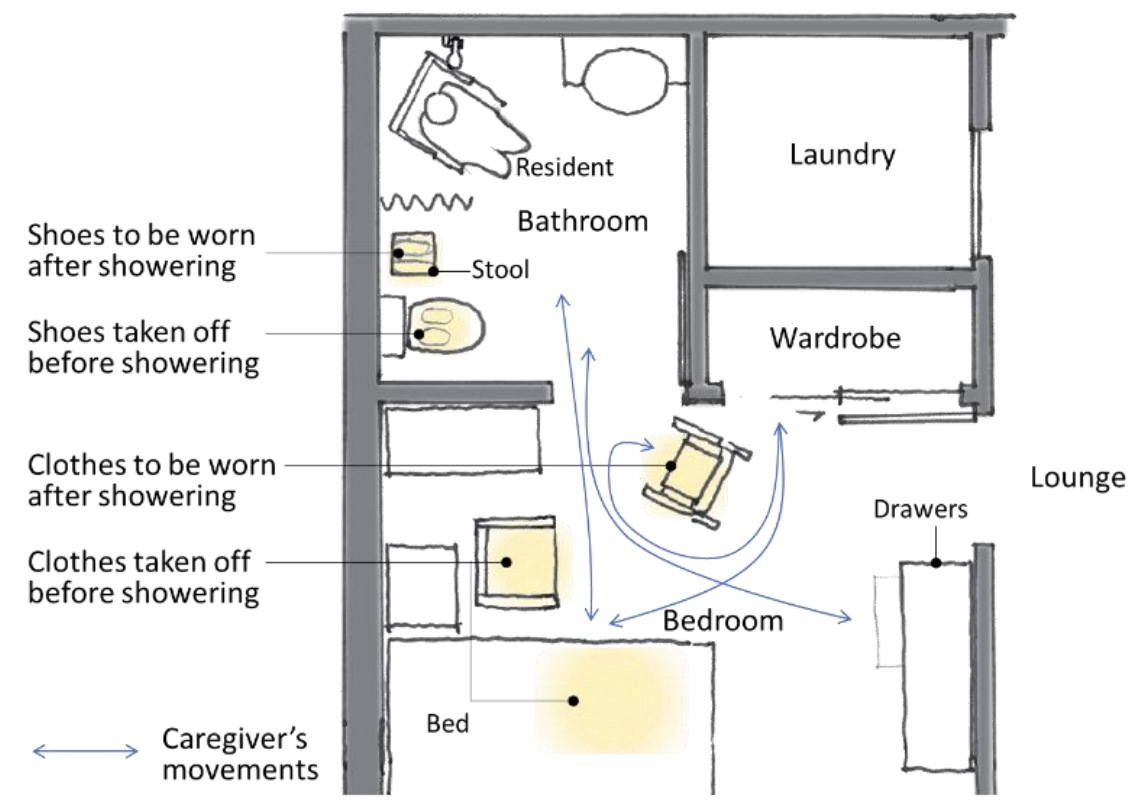

Figure 7-81: Example of space for clothes/shoes and caregiver movements

The accommodation of walker frames was mostly overlooked in the bathroom. When there was not enough or inappropriate space for the walker frame in the bathroom, the caregiver had to go in and out of the bathroom to move the walker frame: 
She leaves her walker out there [out of the bathroom], because there's not a lot of space. ... And once she's dry we'll bring her walker back through with her clothes, and squeeze in where we can and manoeuvre around that.... The clothes that we take off her or that we put back on her, she normally has them all on her walker. ... We leave that out there because there's just not really enough space, you know, with the water and everything. (PR1 caregiver)

The walker frame, in some cases, blocked the caregiver's way as they moved in and out of the bathroom (Figure 7-82).

Space for toileting before showering was also not large enough for those residents with a walker frame. The residents need to use the walker frame to have access the toilet. The caregiver was often keen to warm up the bathroom as the resident undressed and would typically turn on the shower during toileting to heat the room and the water. (Figure 7-82):

That [space in front of toilet] needs to be bigger... When he comes in with the walker, then he goes to sit down, he bends, hits his head on the handle of the walker, so I have to pull the walker around a bit ... before he's gets right down. (RVI8 caregiver)

Insufficient space also resulted in the walker frame getting wet.

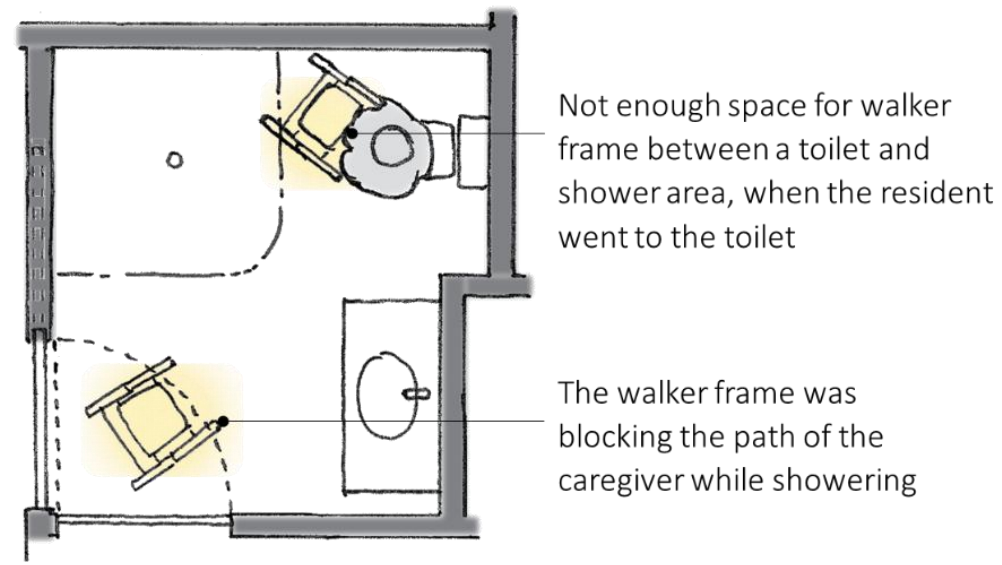

Figure 7-82: Insufficient space for a walker frame

One resident, who anticipated her deteriorating mobility, had requested a large glassed shower area: 'I said I would like it to be made bigger, so if the time did come, ... if I had to be put in a wheelchair, there'd be room for me to go in there with a wheelchair'(RVI11).

Sitting space was important. Some residents needed to sit during all the activities of undressing, washing, drying, dressing and other tasks in the bathroom (such as brushing teeth). The set-up 
of the bathroom was generally done by caregivers. Moving a chair for drying was a common setup task in a limited space (Figure 7-83). If there was enough space for two chairs, one for the dry area and one for the wet area, it saved the caregiver tasks and improved resident independence.

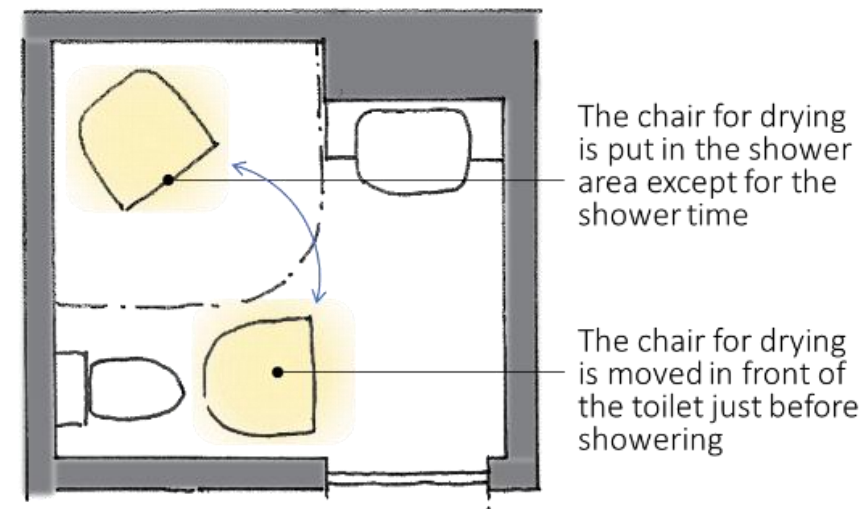

Figure 7-83: Moving a chair for bathroom set-up

\section{Safety in care}

In those bathrooms with a wet-area shower, caregiver safety was potentially at risk due to the slipperiness of the wet floor. One caregiver explained the safety concern

The water goes everywhere.... And it comes out from where the shower curtain is, it comes on to the main floor of the bathroom. And I think as a caregiver that's quite dangerous. Because I have to walk in the water to get to her to shower her. ... And then when she goes and she sits down, I mop the floor. And she always says to me "Don't worry about it," but I do it so that it saves me. (RVS2 caregiver)

While some caregivers tried to mitigate the risk by wearing gumboots or putting disposable gloves (for hands) on her shoes (RVS1 caregiver), others questioned the safety of this: 'Some caregivers wear gumboots, but I don't know whether that's actually any safer. That's to keep the feet dry. ... It's, cause I've got flat, non-slip soles on [my shoes]'(RVS2 caregiver). Some caregivers talked about the difficulties in drying the floor properly. One caregiver said, It can be quite hard on these winter mornings though, because things don't wanna dry as quickly as you want them to. ... After we mop up, we'll wipe it down with the towel as well'(PR1 caregiver).

One factor requiring consideration was the location of the drain: 'And I found it, like I said, I found it quite dangerous, cause when you're walking and there's water because the drain is in the middle of the room, the water will come on the other side of the drain'(RVS2 caregiver). This 
discussion extended to the type of shower enclosure as well as drainage. When asked if the enclosed shower was better, the same caregiver answered:

Ah, to some degree it does make it hard to access, but even if you like one side of the glass [closed] and one side open, it's just... oh, I don't, mainly it's just a floor slope. I don't know, but the water goes everywhere.

Grab handles were important not only when the resident moved around in the bathroom, but also when standing up and sitting down, which occurred recurrently with the tasks of washing and drying. A variety of objects provided support, such as the armrests of the shower chair or the walker frame and tables, as well as handrails. One resident who had Parkinson's disease held onto the handrail with one hand and onto the hand basin with the other, both of which were within reach while her caregiver washed her (Figure 7-84).

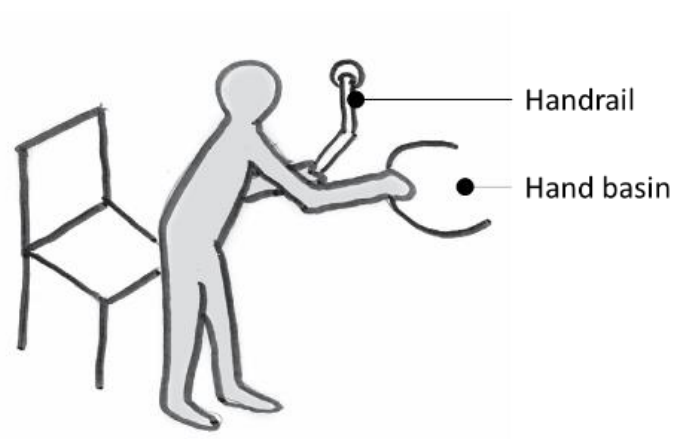

Figure 7-84: Holding on things to hold onto with both hands while the caregiver washes the body

\section{Independence, privacy and dignity}

Caregivers are trained to respect the independence of residents; however, the degree to which this happens is varied in practice. One caregiver said,

Each new person we go into is different. They have a support plan. ... It just depends on the person, how much help they want or need. ... Like, whatever they can do for themselves is great and we let them do that, and whatever they can't do we'll help them with. (PU5 caregiver).

For example, a resident who was 'semi-independent' had his caregiver wash his back, legs and feet, but did the rest of the washing himself. Some 'independent' residents did all tasks by themselves while their caregivers were out of the bathroom. Others had a strong wish for independence. A resident living in a supported-living unit in RV said, 'T have a shower [myself] 
... They will do anything for me because I'm part of the nursing home, but I like to be independent'(RVS5).

Some caregivers advised that the bathroom door and shower enclosure should be shut during showering to maintain resident's dignity. This is particularly the case in supported-living units in RVs, where the staff come in and out regularly. However, small wet-area-shower type bathrooms (such as the space $2 \mathrm{~m} \times 2 \mathrm{~m}$ ) made this impossible (RVI1, RVI3). One caregiver discussed their dilemma:

Researcher: 'Do you close the [shower] curtain?'

Caregiver: 'When she's showering, um, for dignity and stuff they should....'

Researcher: 'But if the space is not big, sometimes it's difficult?'

Caregiver: 'Yeah, it is... Yeah'. (RVS1)

Some caregivers had to leave the bathroom door open as they got breathless due to the small space and the insufficient ventilation capacity (RVS1 caregiver). In such a case, they relied on other means for privacy, such as shutting the curtain (RVS1) and the door of the adjacent bedroom (RVS3).

Many residents wished to be out of the sight of their caregivers when they didn't require assistance. One caregiver said, 'He 'never lets me in [the bathroom], he's a bit shy'(PR2 caregiver). Residents who had some degree of independence completed the set of activities from undressing to starting showering themselves. An elderly man had undressed and started to shower by himself, shutting the shower curtain, by the time his caregiver arrived. One woman did all the tasks by herself, which included using the toilet, undressing, putting a stool under the shower and starting her own shower, before needing assistance in washing her back. A caregiver said, 'When I've helped her, I'll leave her to enjoy the shower for a while. ... In case she's independent she might like privacy, so I might even be here or in there, just close by'(PU5 caregiver).

When a resident required assistance in most tasks, privacy was maintained while toileting by her caregiver leaving the bathroom after assisting her with sitting on the toilet. Residents were encouraged to wash genital areas by themselves using an extended shower hose, but the design with the shower head fixed to the wall often hindered this practice. 
The space requirements for the caregiver to be out of sight of the showering person differed by the type of the shower enclosure. In the case of a transparent glass-screen enclosure, the caregiver stayed out of the bathroom except for the time when assistance was required, doing other tasks such as laundry, bed making and washing dishes, or talking with the partner of the showering person. A caregiver went in and out of the bathroom from time to time, anticipating safety needs saying, 'Are you alright?' (RVI3). The wall between the bathroom and the main living area hindered communication between residents and caregivers, which was particularly difficult for those with poor hearing and who had no hearing aids while showering. A caregiver said, 'So when I come in and out I open the door. And sometimes I just come in and yell at her, like, she doesn't have any hearing aids while showering'(RVS2 caregiver).

\section{Theme 6-2: Quality of care in household tasks}

Each resident was allocated a certain time for their home-care. Some caregivers who had many tasks to perform emphasised their time constraints: 'I just think it'll take an hour, sometimes it's getting a bit stricter, the time ... Everyone is so different but if someone is a little bit prepared it's good with the timing'(PU5).

Another caregiver also said, 'It's always rush, rush, rush.... One job after another'(RVI11). In a dwelling where the bathroom was far away from the washing machine, one caregiver went back and forth in haste (RVI11 caregiver) (Figure 7-85).

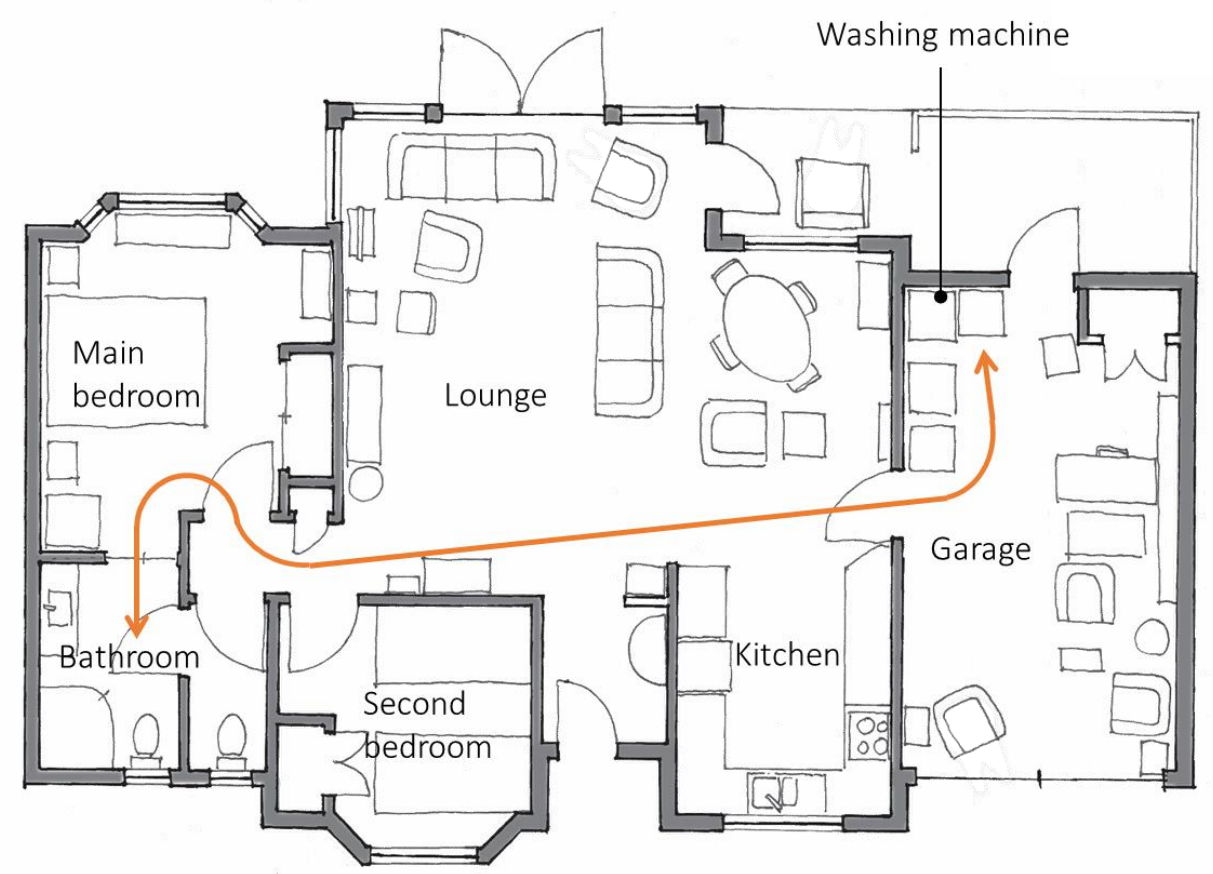

Figure 7-85: Caregiver's movements between the bathroom and the washing machine 
Often caregivers cooked meals for residents (PU5, RVI1, RVI7, RVI11, RVS3); some prepared three meals (RVI1, RVI7, RVI11), and others made only one light meal per day (PU5, RVS3). Washing dishes was often the caregivers' task as well (PU5, PR5, RVI1, RVI2, RVI7, RVI11, RVS3). One resident left used dishes on the kitchen bench, and her caregiver washed them the next morning (RVS3).

One caregiver, who didn't cook for the participant of this study but cooked for other elderly people, talked about the inconvenience of cooking in some houses (RVI9 caregiver). She said it was ideal that the caregiver and resident cook together, which would facilitate greater independence of the resident, but many kitchens didn't have enough space for two people as well as a walking aid, which often meant the caregiver cooked alone. A long, narrow kitchen was not good, because a person in a wheelchair couldn't turn around. If such a kitchen had a bench, the resident could see the caregiver cooking, which was better. A square kitchen that had enough space for a wheelchair user to turn around and could accommodate two people was best (Figure 7-86).
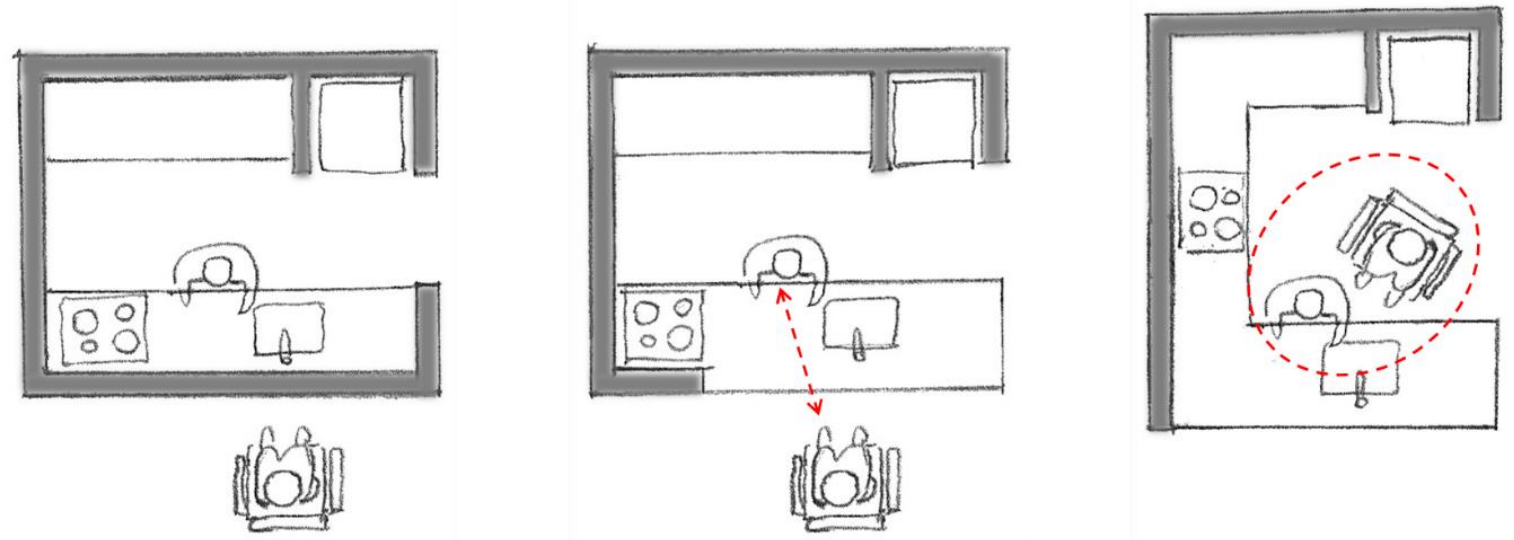

Figure 7-86: Space for assisted cooking

The long narrow kitchen is not good (left), but if it has a bench, the resident can see the caregiver cook (middle). The square kitchen is the best (right).

\section{Theme 6-3: Quality of care from on-site staff}

In complexes where the on-site staff provide support, residents generally appreciated the support, which also brought a sense of safety to them. This benefit was perceived by residents in a publicsector housing complex with a custodian, in a church-based rental housing complex and in RVs. One public-sector housing had an on-site custodian, but residents desired for attendance of medical and care staff: ' $I$ think in all these villages - of course we have to pay more if we had all these services - there should be a nurse. Like in the [RV nearby] there is a nurse full-time ... 
and she was very nice' (PU2). In serviced apartments, the flexible manner of care provision for individuals was appreciated: 'when I need care I get it and I can get full care... They'll just see whatever I need. ... I just want to have as much independence as much as I can. (RVS5)

\section{Efficient care provision}

One of the main benefits of the on-site staff was the quickness of their support when required. One resident said, 'You only need to ring [the custodian] if there's something wrong and he'd be here like a shot'(PU2). In serviced apartments, the medical and care staff who stayed near resident units provided greater care and support, which residents generally needed or appreciated. One resident of a serviced apartment appreciated the proximity of the staff, comparing this to a situation when she was living in an independent-living unit: 'If I have any problem they come to the door. ... If I do fall or get into any trouble, I've got instant help'(RVS5)

Serviced apartments are looked after by the staff (caregivers and other staff) based in the adjacent care facility. One caregiver said, 'Caregivers are very busy. And sometimes there are time constraints' or 'It's always busy. You can never stop'(RVS1 caregivers). Referring to one resident requiring a rest-home-level care, a caregiver said, 'Ideally [the resident] would be closer to this end of the facility... So we [could] can keep, um, easier visual, um, check on [the resident]' (RVS1 caregiver).

A staff member of another serviced apartment also noted the distance from their work station to resident units (RVS5 caregiver). She wanted multiple staff stations, where they could store care supply (medication etc.) and mobility aids (wheelchair etc.) to bring to residents, which would reduce their walking distance. She also commented on the layout that had resident units on one side along the corridor, which would have made their walking distance longer than the layout that had resident units on both sides of the corridor.

\section{Staff visits/surveillance and resident privacy}

While support from the staff was generally appreciated, the manner of the staff visits affected residents' privacy. Extra surveillance was not received well. One resident talked about her experience of this, saying a staff member came into her unit (and all residents' units) at night, twice a night, with a torch to check on her, and left all the doors open when they went out. She said, 'I thought that's invading my privacy... That's what they like, like to keep an eye on you of course'(RVS2). Another resident also disliked too much surveillance: 
I don't like them coming in in the middle of the night and making sure ... and so I've told them right from the beginning that I wouldn't have that. I don't care if I die and they don't find me 'til morning, you know. For me, that's not important. (RVS5)

In contrast, a resident who received lower levels of care wished for the staff visit just once a day:

Because we don't have any friends [or family]. ... The sad part is that four years ago my younger brother died, and it was five days before anybody knew. ... And I didn't want that to happen here, so I suggested that maybe the cleaner called each morning, or somebody did, just to make quite sure I'm still with it. (RVS6)

In some complexes, an on-site caretaker visited daily, which residents did not have complaints about (PU1, PU2, PR6): 'They make sure if you are all right in the morning, in case you can't say it in the night'(PU6). In another complex, for greater privacy of residents, the on-site staff avoided visiting them regularly; instead, they took another measure to ensure resident safety. One resident described the staff s considerate manner of care for residents, 'They leave us to be independent. But they always get around if somebody is not well. .... He's [the property manager] always around in the garden, doing jobs around the place'(PR1).

Loss of privacy was a particular issue for residents in serviced apartments, where many people received frequent staff visits for tea service as well as other support or medical activities. It was acknowledged that when staff waited for a resident to reply after ringing the doorbell or knocking before opening the door, it provided greater privacy for residents (RVS4). However, during the observation sessions, no staff member waited for residents to reply before opening the doors; one even entered without knocking (RVS1). In one case where a resident had a hearing impairment, a knock would not notify him of the staff arrival, particularly when not wearing hearing aids (e.g. in the early morning, at night) (RVS6). Front doors were also problematic in some cases; doors could not be shut properly unless pulled/pushed with extra strength. A light pull from a staff member didn't always make a hinged door shut properly, so the door was left half-open (RVS3, RVS4).

\section{Familiarity of the staff}

Familiarity with staff was preferred by residents, which also enhanced staff member's reliability. One resident complained about the frequent change of staff members: 'Staff come and go. Come and go, come and go, instead of ... having a set. You know. Staff that you can get to know 
and rely on'(RVS2). Presence of too many staff also decreased their familiarity: 'There are so many staff. I can't remember their names'(RVS2).

One resident referred to the size of the complex (her complex contained more than 200 units) as a factor to QoL: 'It's much nicer, I think, if it's smaller. ... They're [staff] always on the run, if you know what I mean. ... There's no sense of quietness, or community feeling'(RVS2).

\subsection{Discussion and conclusion}

Through a qualitative study for 30 high-needs elderly people and their caregivers as well as the physical environments, this chapter has reported in detail the QoL of elderly residents such as perceptions and experiences, and the influence of their housing environments on the QoL. These outcomes have been presented by six large emergent themes for QoL: 1) Control in daily basic activities, 2) Meaningful leisure activities, 3) Meaningful relationships, 4) Maintenance of possessions, 5) Comfort, and 6) Quality of care. This section summarises the findings of the chapter and provides an overview of the contribution to knowledge regarding the QoL for the high-needs elderly in relation to the physical environments. This reflection does not delve into detailed design considerations, which will be discussed in the next chapter.

In the first theme, Control in daily basic activities, numerous sub-themes were identified that link to various daily activities such as moving/walking, going to the toilet, showering, cooking, laundering, maintaining home, shopping and collecting mails. All of them were important for maintaining their independent life. Most of these sub-themes can be linked to the topics in the existing literature (Iwarsson, 2005; Burton, Reed, \& Chamberlain, 2011; Peace, 2017; Nordin et al., 2017). In the second theme, Meaningful leisure activities, the high-needs elderly's leisure activities and the relationships to space were clarified focusing on the meaningfulness for the individuals. This theme included diverse familiar activities important for the elderly such as religious activities and hobbies such as handcraft/needlework/artwork, gardening, reading, building/DIY, sports and enjoying music, which can be linked to the conventional knowledge as to important leisure activities for the elderly and spaces required for them (e.g. spaces in retirement villages). In addition, their meaningful activities included activities that helped them stay active, and the space for walking was of great importance. Facilitating meaningful activities is less focused in the existing literature regarding the QoL for the high-needs elderly. Also, the engagement in personal activities in private space was very important for the high-needs, who usually spent a long time in their home. Their spatial usage in private dwellings was analysed in great detail in this part, to address one of the gaps in knowledge identified in Chapter 3. The 
third theme was Meaningful relationships, which included facilitating various levels of relationships such as living with their partners, having personal guests, maintaining relationships with other residents and connection to the wider community. This section revealed the diversity in preferences and spatial usage, particularly in having personal guests and maintaining relationships with other residents and provided important knowledge regarding spatial requirements to facilitate individuality. The fourth theme, Maintenance of possessions, included sub-themes of maintaining furniture and objects including those seldom used, which related to facilitating their personal identity, as well as storage of day-to-day items. This section also added more detailed information to the existing knowledge (Lewis et al., 2010; WEL_HOPS, 2007) with regard to spatial requirements for keeping personal items. In the fifth theme, Comfort, four sub-themes have been found, which were; relief from fatigue and pain, warmth and sunshine, light, and access to nature. Among those, the space that has access to the north sun was so valuable for residents that it affected their occupancy of space. This illuminates the high level of importance of the access to the north sun (or the south sun in case of the northern hemisphere) for them, which has not been stressed in the existing literature. Finally, the sixth theme was Quality of care, which included three areas of quality of care; in assisted showering, in household tasks and from on-site staff. An important quality of care for residents related to privacy from caregivers/staff and their familiarity as well as easy and efficient manners of care, all of which had relationships to physical environments. These outcomes provide a wider knowledge with regard to housing design that can accommodate caregivers while allowing elderly people to live an independent life. This is particularly important, given the scarcity of knowledge relating to the architectural contribution.

The in-depth descriptions in this chapter — on the QoL of the high-needs elderly and the relationships to the physical environments - have provided a substantial volume of evidence for the need for better design. The next step is to conduct further analysis of design considerations indicated in this chapter, to establish a framework for the design of housing that provides greater QoL for residents and the synthesis of design considerations influential to various aspects of QoL. This examination will be conducted in the next chapter. 


\section{CHAPTER 8: DEVELOPMENT OF A DESIGN FRAMEWORK (PHASE 3)}

In Chapter 7, a wide variety of themes for QoL of the high-needs elderly that relate to physical environments were presented and categorised into six large themes. In Chapter 8, through examination of design considerations with respect to the QoL, a design framework has been developed.

The relationships between themes for QoL and design considerations have been established, by categorising design considerations into 24 design elements. These design elements were then aggregated into three larger design domains of; 1) general design requirements, 2) design of the individual unit, and 3) design of the complex. Next, design requirements have been distilled for each design element through the consideration and synthesis of all relevant information by design elements and with reference to external sources. The combination of the relationships between themes for QoL and design considerations with the design requirements results in a framework for the design of housing for the high-needs elderly.

\subsection{Establishing the relationships between themes for QoL and design considerations}

The relationships between themes for QoL and the design elements are summarised in Table 8-1 and Figure 8-1, where the number of design considerations is shown ${ }^{24}$. Within Figure 8-1, the number of design considerations is shown by the thickness of the lines connecting themes for QoL and design elements ${ }^{25}$. The analysis found complex relationships between various design elements and various aspects of QoL. The prominent features in the relationships between themes for QoL and design domains/themes can be grouped under needs for greater control, the importance of meaningful leisure activities, maintaining meaningful relationships, desires for comfort and needs for high quality of care.

Of the six themes for QoL, the largest number of considerations were related to 'control in daily basic activities' (200), followed by the desire for 'meaningful leisure activities' (75), maintenance of 'meaningful relationships' (58), and 'comfort' (56). Among all themes for QoL, the greatest

\footnotetext{
${ }^{24}$ In this calculation, the same design considerations relating to different themes for QoL were treated as different items.

${ }^{25}$ The number of design considerations is deemed to represent levels of relevance between design elements and themes for QoL to some extent, though it doesn't take account of the number of participants who experienced the significance of design considerations or the levels of QoL for individuals relating to each consideration.
} 
number of design considerations were found in the domain of the design of the individual unit. Within this design domain, the design of the kitchen and the dining space, the bathroom, and the planar relationships between spaces had the greatest influence on occupant control in daily basic activities. The bathroom design also strongly influenced to the quality of care. The design of storage and wall and elevation design largely related to the maintenance of possessions, as well as control in daily basic activities. The design of sitting spaces had a significant impact on facilitating meaningful leisure activities and maintaining relationships and comfort. The unit size and extra spaces also had implications for meaningful relationships. Somewhat surprisingly, individual outdoor space had a significant impact on residents' comfort.

In the domain of basic design considerations, which relate to both individual and communal space, the design of fixtures had a significant impact on elderly people's control and comfort as well as impacting on the quality of care. Design of floors/ground also had a great impact on control. The design of the complex also had great relevance to facilitating meaningful leisure activities and relationships, particularly indoor communal areas. Design of communal pathways in particular had great relevance to meaningful leisure activities. 
Table 8-1: Emergent QoL themes relating to design elements

\begin{tabular}{|c|c|c|c|c|c|c|c|}
\hline \multirow[t]{2}{*}{ Design domains } & \multirow[t]{2}{*}{ Design elements } & \multicolumn{6}{|l|}{ Themes for QoL } \\
\hline & & $\begin{array}{l}\text { Theme 1: } \\
\text { Control in daily basic } \\
\text { activities }\end{array}$ & $\begin{array}{l}\text { Theme 2: Meaningful } \\
\text { leisure activities }\end{array}$ & $\begin{array}{l}\text { Theme 3: Meaningful } \\
\text { relationships }\end{array}$ & $\begin{array}{l}\text { Theme 4: Maintenance } \\
\text { of possession }\end{array}$ & $\begin{array}{l}\text { Theme 5: } \\
\text { Comfort }\end{array}$ & $\begin{array}{l}\text { Theme 6: } \\
\text { Quality of care }\end{array}$ \\
\hline \multirow[t]{8}{*}{ General design requirements } & Floor & 15 & 4 & o & 1 & 1 & 2 \\
\hline & Door & 6 & p & 5 & 0 & 2 & 2 \\
\hline & Passage & 3 & p & 0 & 0 & p & p \\
\hline & Fixtures & 22 & 3 & 0 & 1 & 8 & 6 \\
\hline & Things to hold onto & 6 & 1 & o & 0 & o & 1 \\
\hline & Things to sit on & 5 & 3 & 1 & 0 & 0 & 1 \\
\hline & Space for mobility aids & 6 & 1 & 0 & 0 & 0 & 2 \\
\hline & Subtotal & 63 & 12 & 6 & 2 & 11 & 14 \\
\hline \multirow[t]{12}{*}{ Design of the individual unit } & Unit size & 5 & 3 & 5 & 1 & 2 & 3 \\
\hline & Wall and elevation design & 19 & 11 & 3 & 12 & 3 & p \\
\hline & Storage & 22 & 2 & 2 & 14 & 1 & 0 \\
\hline & Planar relationships between spaces & 10 & 1 & 4 & o & 4 & 1 \\
\hline & Sitting space & 3 & 18 & 6 & 0 & 8 & 1 \\
\hline & Bedroom & 2 & 0 & 0 & 3 & 4 & 0 \\
\hline & Extra rooms & 4 & 2 & 5 & 0 & 1 & 0 \\
\hline & Bathroom & 17 & p & o & 0 & 2 & 15 \\
\hline & Kitchen and dining space & 38 & 0 & 2 & 1 & 1 & 2 \\
\hline & Space for laundering and utility & 6 & 0 & 0 & 0 & 0 & 0 \\
\hline & Individual outdoor space & 7 & 4 & 2 & 0 & 7 & 0 \\
\hline & Subtotal & 132 & 41 & 29 & 31 & 33 & 22 \\
\hline \multirow[t]{7}{*}{ Design of the complex } & Complex size & 0 & 0 & 1 & o & 0 & 1 \\
\hline & Unit layout & p & 0 & 4 & 0 & 6 & 3 \\
\hline & Communal pathways & 2 & 10 & 4 & 0 & 0 & 0 \\
\hline & Roadways and carparks & p & 1 & 2 & 0 & 0 & p \\
\hline & Indoor communal areas & 3 & 10 & 10 & 2 & 2 & 0 \\
\hline & Open space & 0 & 1 & 2 & 0 & 4 & 0 \\
\hline & Subtotal & 5 & 22 & 23 & 2 & 12 & 4 \\
\hline Total & & 200 & 75 & 58 & 35 & 56 & 40 \\
\hline
\end{tabular}




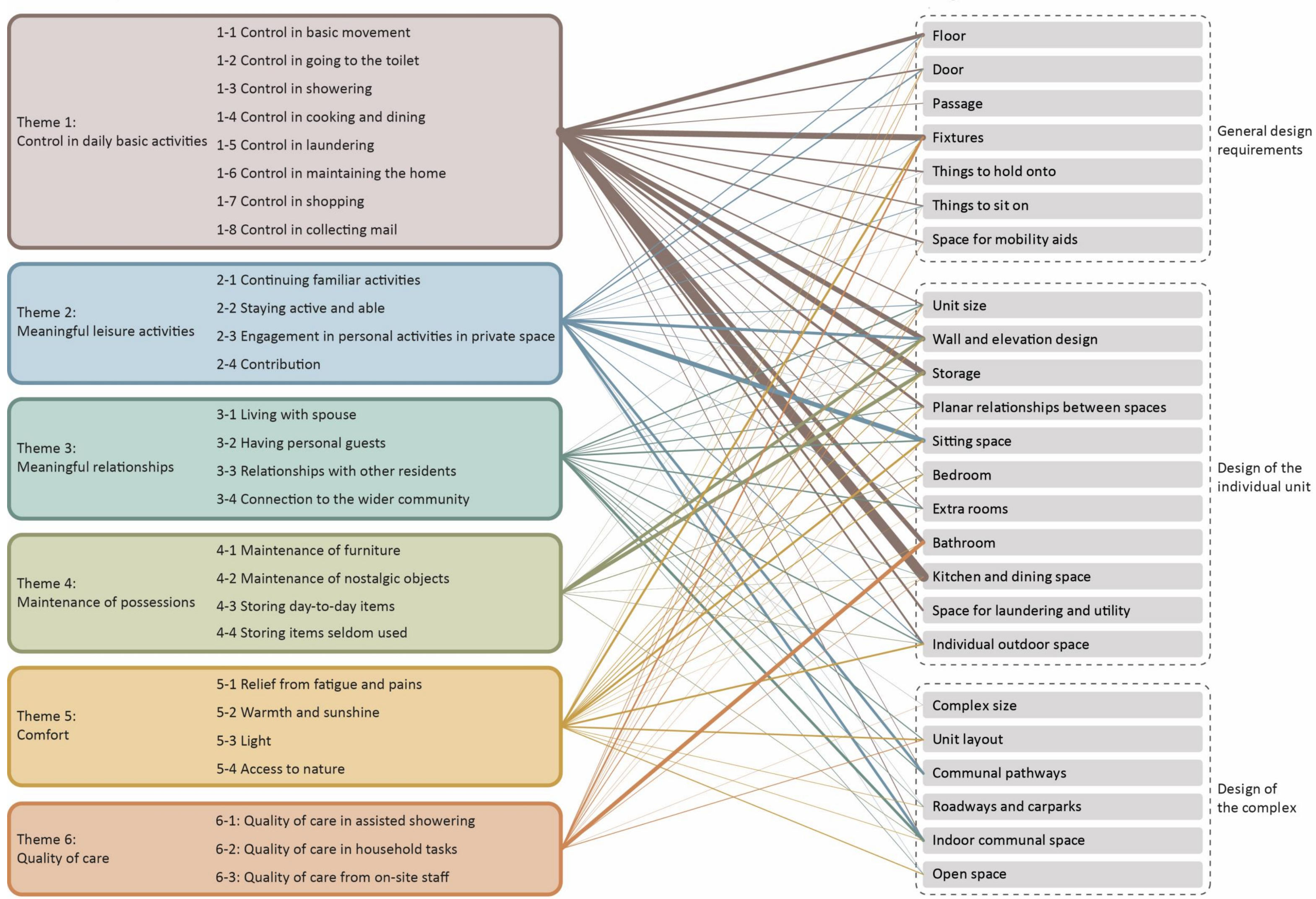

Legend: Line thickness and number of considerations 


\subsection{Establishing the design requirements}

To establish the design requirements to constitute a design framework, it is necessary to examine the design considerations that emerged in Chapter 7 and to synthesise those relating to each other. In synthesising information, it is important to consider how to deal with the outcomes that have resulted in variations and different requirements depending on the individuals. The factors for these variations include the type and level of impairments, changes in life situations as well as diverse preferences in personal and social activities, as well as in personal attachments to belongings.

Common impairments and related factors influencing spatial requirements included a decline in mobility in various body parts; visual and hearing impairments, as well as the use of different mobility aids. Given that the high-needs elderly generally have multiple impairments, taking account of all requirements covering their conditions and impairments is essential. Considering that their levels of dependency may also change, a Universal Design that meets different requirements could best support ageing-in-place and thereby enhance QoL.

Spatial requirements can change in the event of a loss of a partner, which is a common occurrence for the high-needs elderly. To design for this cohort, consideration for accommodating this change in life situation is essential. Similarly, it is important for a couple to continue to live in the same place even when the care requirements increasing for one person but not for the other. In this study, supported-living units in retirement villages could accommodate such couples; however, improvements are required to comfortably accommodate them. The strategies to accommodate the diverse preferences in activities and possessions are also considered through discussion in this section.

Design considerations shown in the study results are discussed by each design element to establish design requirements in this chapter. They are also discussed with regard to existing knowledge such as the New Zealand Building Codes, New Zealand Standard NZS 4121 (Standards New Zealand, 2001), the Lifemark Design Standards (Lifetime Design Limited, 2012) as well as EVOLVE (Lewis et al., 2010) and Guidelines for the planning of housing for senior citizens (WEL_HOPS, 2007). Drawing on these sources complements information obtained from this study and identifies the new knowledge that has been obtained. 


\subsubsection{General design requirements}

General design requirements that can apply to overall physical environments, either individual or communal spaces, are discussed in this section. Considerations encompass spatial elements such as the floors, doors, passages, fixtures, things to hold onto, things to sit on, and space for mobility aids.

\section{Floor}

To meet the needs of people who have difficulty raising their legs and people using mobility aids (other than walking sticks), ground level differences should be eliminated or minimised.

Considering that tiny level differences such as one centimetre can be difficult to move over with a walker frame or trolley and have great potential to cause the elderly to trip, there should be strategies for floor design that involve almost no level difference, both indoors and at external doors. Level floor surfaces are important for facilitating residents' active involvement in various activities as well as ensuring their safety.

In particular, a change of flooring materials that create a level difference at thresholds should be avoided (Figure 8-2 left). Examples of thresholds with no level difference are shown in Figure 82 right. An interior layout that reduces the changes in floor materials would also be effective; for example, using the same material for adjacent rooms, such as the kitchen and bathroom can eliminate a threshold between them. Having no level difference is particularly important at shower entries as this makes it easier for caregivers to provide care as well as offering greater safety for residents (for details, see also 8.2.2 'Bathroom'). Ideally, the principle of no level difference should extend to every space elderly residents can go, both in their private dwellings and communal indoor and outdoor spaces.
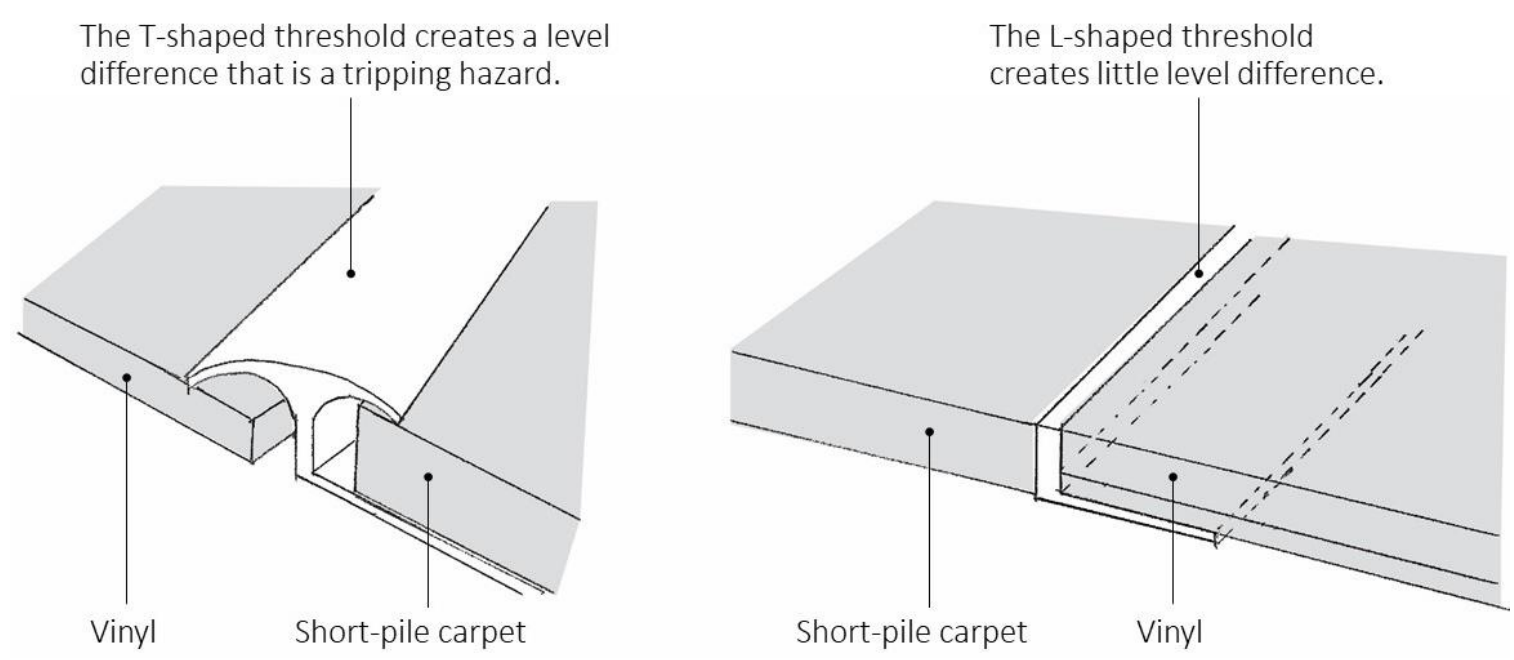

Figure 8-2: Examples of thresholds that create a level difference (left) and no level difference (right) 
Floor gradient is another element that requires consideration. NZS4121 states that a ramp should have a gradient of 1 in 12 or lower, and every effort should be made to construct a ramp as flat as possible (Standards New Zealand, 2001). In one complex studied, there was an external slope steeper than this requirement ( 1 in 10.4), where residents found it difficult to walk with a walker frame. Ramp length also requires attention. NZS4121 states that in the design of ramps (between 1 in 20 and 1 in 12) level surfaces should be provided at intervals and that ramps should be a maximum of nine meters in length (Standards New Zealand, 2001). However, given that many participants suggested a long slope of six metres (gradient: 1 in 12) as difficult for wheelchair users to climb up, the length of the slope should not exceed six metres.

Floor material choice should also take account of users with walker frames and wheelchairs. When choosing a carpet, material that can endure the weight of a wheelchair should be used, such as low pile carpets made of strong fibre such as nylon. Carpets should also provide sufficient grip for the wheels to move with ease. Floor materials should be firmly fixed to prevent being lifted by wheels.

There should also be consideration of flooring materials with respect to maintenance, as elderly people are more likely to spill things on the floor and a lower ability to clean it as their level of impairment increases. For those with difficulties in bending and crouching, the most suitable modes of cleaning are cordless vacuum cleaners or steam cleaners that are lightweight and can be used while standing. However, using only these tools is not effective for removing stains on carpets, including food stains and stains made by the wet wheels of a walker frame or wheelchair, which normally require wiping with a towel or paper while crouching on the floor. One participant in the study wanted a non-slip tile floor for their entire unit instead of carpets, which would make it easier to clean with a steam cleaner. However, there was a common preference for the warmth and softness of carpets in the spaces participants frequently used. This is particularly the case in New Zealand compared to warmer countries such as Australia.

There are a few options for floor materials with ease of maintenance, warmth and softness. First, choosing a stain-resistant carpet is effective; however, to have the greatest benefit, stains must be removed quickly with towels similarly to normal carpets, and this cannot be done by standing and using a vacuum cleaner or a normal steam cleaner. Another option is the use of tile carpets which would allow individual tiles to be replaced when stained. This is particularly effective because the areas in and around the sitting space are stained and damaged more easily than flooring in other areas. The ability to easily change carpet tiles also prevents residents from 
layering area rugs in their sitting space, which can be a tripping hazard. Selecting dark coloured carpets can also help residents with keeping a sense of cleanliness, which could hide stains until carpet tiles are replaced.

Stableness and slipperiness should also be considered in the choice of floor materials. NZBC D1 Access Routes requires that access routes 'have adequate slip-resistant walking surfaces'. ${ }^{26}$ This applies to public access, including routes to the main entrance of a house (MBIE, 2016). In addition, attention should be given to the layout of pathways to prevent residents from walking on a grass lawn or other unstable ground. Inside of dwellings, the Lifemark Standards require non-slip surfaces in areas other than access routes, such as kitchens, bathrooms and other wet areas. In this survey, participants stressed the importance of non-slip floors for the whole unit, particularly in wet areas such as the shower room (see also 8.2.2 'Bathroom').

\section{Door}

Interior doors serve to maintain privacy and to retain heat; however, they can be difficult to negotiate for those with limited mobility and the swing area can take up valuable space.

Therefore, the installation of interior doors should be kept to a minimum. For example, doors between the laundry and the bathroom, or the kitchen and the lounge, could be removed.

There should be sufficient door width to accommodate those using mobility aids. From the survey results, a width of $750 \mathrm{~mm}$ (the distance between furniture and the corner of a wing wall) was insufficient for wheelchair users to get through. Therefore, a door width of $760 \mathrm{~mm}$, which NZS 4121 defines as the minimum, is insufficient. An 810-mm width, which is the minimum requirement for a door in Lifemark Design Standards, is necessary to maintain impendence.

Hinged doors are difficult to manipulate for residents with mobility aids such as walker frames and wheelchairs because mobility aids often interfere with the space needed for the door to swing open. The difficulty is mitigated by providing enough space beside the door for mobility aids. For example, NZS 4121 states that a $300-\mathrm{mm}$ wide side panel is required for a hinged door. However, with a $300 \mathrm{~mm}$-wide side panel, it is difficult for walker-frame users to open inwards, because they cannot approach the door close enough to reach the door handle from the standing position in front of their walker frame (Figure 8-3 left). The side panel should be at least 600 $\mathrm{mm}$ wide to enable them to reach the door handle (Figure 8-3 centre). However, it still causes

\footnotetext{
${ }^{26}$ Acceptable Solution D1/AS1 requires a friction coefficient of 0.4 for level access routes used by the public.
} 
difficulty in moving their walker frame from and to their journey path. Sliding doors can eliminate this trouble, as well as causing no trouble with approaching the door (Figure 8-3 right).

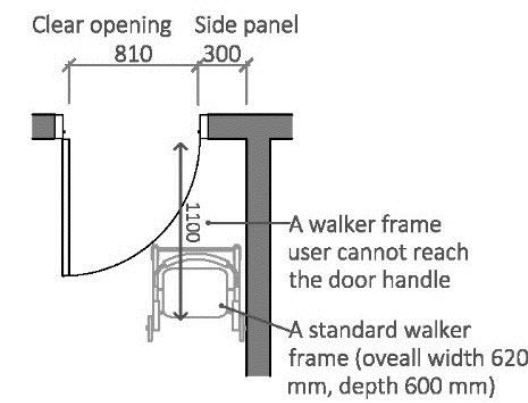

In the case of a hinged door

$+300-\mathrm{mm}$ wide side panel

(the minimum requirement in NZS4121)

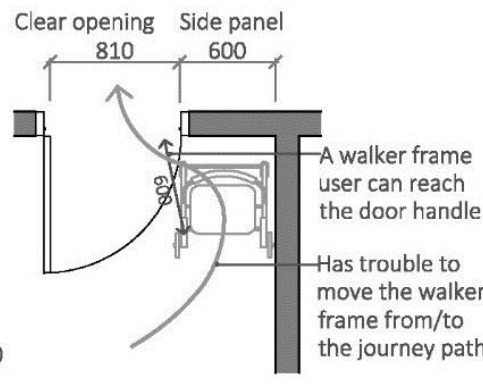

In the case of a hinged door + $600-\mathrm{mm}$ wide side panel

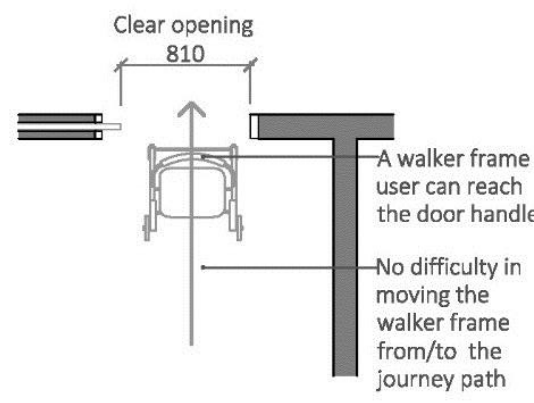

In the case of a sliding door

Figure 8-3: Comparison of trouble of a walker-frame user in opening hinged and sliding doors

The choice of sliding doors can also allow residents to keep the door either open or shut without taking up extra space. This contrasts with hinged doors that take space for the door swing when kept open. It can also enable a couple living together to have choices, either to have separate spaces or to have one space, depending on their needs and mood.

Sliding doors are recommended for all the reasons described; however, in case they cannot be installed, attention should be given to the direction of door swing and the provision of a side panel of at least $600 \mathrm{~mm}$ width as described earlier. Ideally, a door with a door closer should be avoided as it shuts faster than desirable for many and is difficult for those with mobility aids to go through. Where a door closer is unavoidable, an electromagnetic free-swing closer or electromagnetic hold-open, or at least, a magnetic hold-open should be provided. Doors should also not require strength to shut completely, and should be provided with some device to stop the door banging when closed.

External doors often have a level difference to ensure weather tightness; particularly, hinged doors with a threshold that has a level difference. However, to make it easier for those with mobility aids and difficulty in lifting their legs, level entry is an important consideration. The acceptable solutions for level access doors include those combining a channel with drainage 
provisions across the door opening, ${ }^{27}$ grating over the channel, ${ }^{28}$ and exterior paving ${ }^{29}$ (MBIE, 2014a). Another acceptable solution is to provide threshold weather stops projecting no more than $20 \mathrm{~mm}$ above the threshold's finished surface (Department of Building and Housing, 2011b). A threshold combining these two solutions is shown in Figure 8-4 and is an acceptable example (MBIE, 2014a). However, from this study's results, even a tiny level difference such as a one-timetre lip can be a hazard, particularly those that have a Icerprotruding vertical rise (Figure 8-4). A small sloped piece (e.g. a tapered reveal) to fill the difference eases the trouble; however, it still requires strength to manipulate. Therefore, providing completely level entry is an ideal solution. Combined with the recommendation for sliding doors, which are easier to open and close than hinged doors, an ideal solution would be a sliding door threshold with no raised lip, as shown in Figure 8-5.30

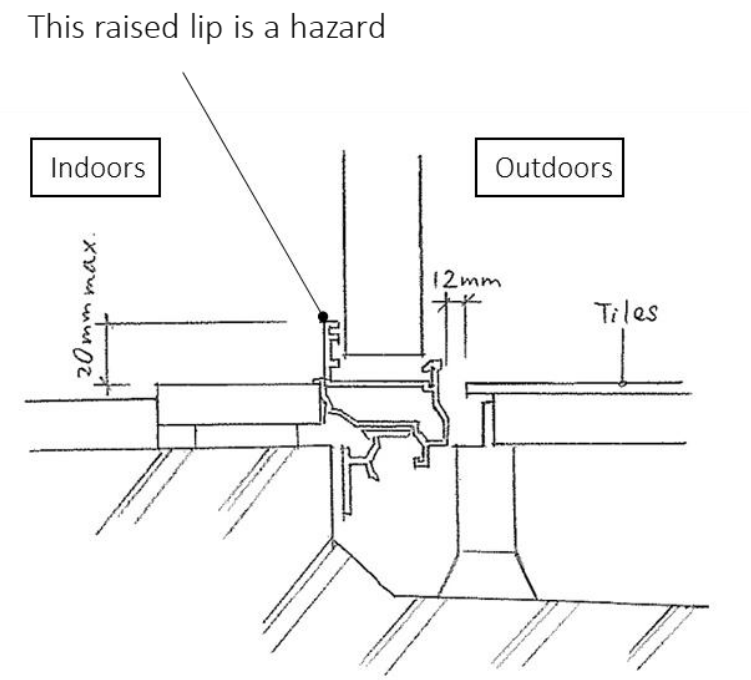

Figure 8-4: Example of an external door with a small level difference (detailed section)

Source: Drawing by author based on the image on NZBC E2/AS1 (MBIE, 2014a)

\footnotetext{
${ }^{27}$ The requirements for the channel include: i) the width to suit capacity in accordance with E1/AS1, ii) a minimum depth of $150 \mathrm{~mm}$, iii) a maximum length of $3700 \mathrm{~mm}$ and iv) 1:200 minimum fall along the length of channel towards a drainage outlet.

${ }^{28}$ The requirements for the grating include: i) being supported independently of the door frame, ii) being removable to allow access for cleaning, iii) being specifically designed to accommodate imposed loads, iv) having gaps sized to prevent the wheels of wheelchairs or mobility aids entering or being trapped and v) having a continuous gap of 12 $\mathrm{mm}$ minimum from door frame and wall cladding.

${ }^{29}$ The requirements for exterior paving include: i) a minimum fall of 1:40 away from the channel for a minimum distance of $1 \mathrm{~m}$ and ii) the surrounding paving and ground levels complies with drainage requirements of E1/AS1.

${ }^{3030}$ In using sliding doors for external doors, attention is required to the requirements for escape doors. For example, to comply with the safety requirements (MBIE, 2014b, 2017), it is necessary to allocate escape doors so that the number of occupants using one door is 20 or less.
} 

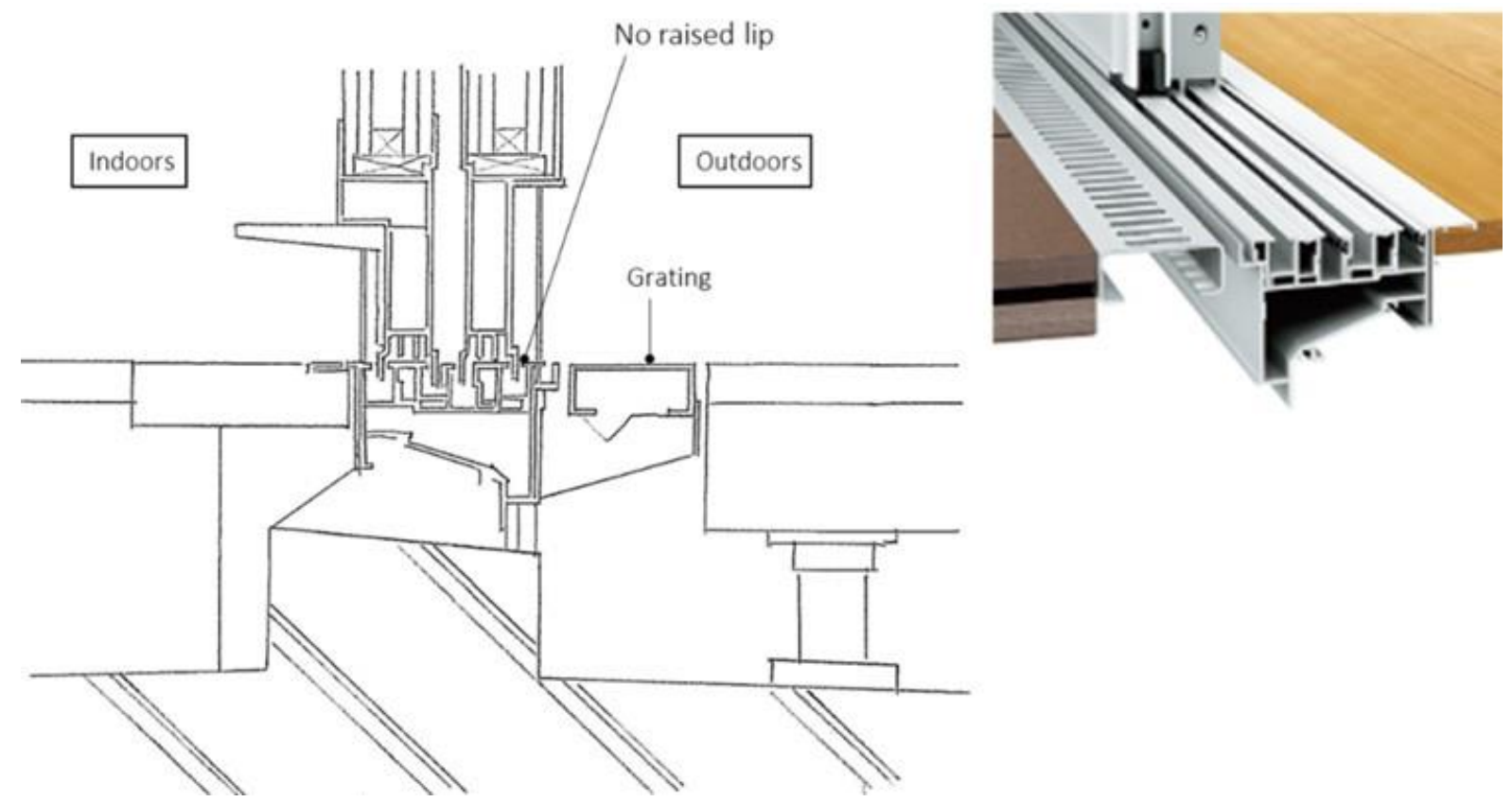

Figure 8-5: Example of an external door with no level difference

Source: https://www.ykkap.co.jp/search-b/elderly-sp/barrier-free.html (Drawing: by author based on this webpage)

Doors should also not be too heavy to open. Attention is required when using a door that faces the indoor corridor. It must be fire-rated, so it requires a door closer, which is normally too heavy for the high-needs to open. A solution for this is using a free swing closer or powered opening device, or alternative fire engineering solutions.

There should be attention given to the location of door handles. It is important to avoid interference with walker frame heights, which makes it difficult for the person to reach the handle. As a solution, handles can be installed slightly higher than the walker frame height. Considering that typical walker frames can be up to $970 \mathrm{~mm}$, the handle height should be at least 1,000 $\mathrm{mm}$ (see also 8.2.2 'Wall and elevation design'). The door handle height suitable for wheelchair users is between 900 - $1200 \mathrm{~mm}$ high (Standards New Zealand, 2001), door handles should be $1000-1200 \mathrm{~mm}$ high to meet both needs for walker frame users and wheelchair users is.

Other considerations in the door design include transparency. In apartments with indoor corridors, providing a vision panel (even frosted glass) should be avoided to maintain residents' privacy. The provision of individual doors that lead directly to the outdoors is preferred, so should be provided where possible. These are convenient for the occasions when family, friends and caregivers visit and contribute to a greater sense of homeliness. 
Passage

In considering passage width, it is essential to consider sufficient passage width for those using mobility aids. Given a wheelchair user in this study had difficulty passing the $760 \mathrm{~mm}$-wide door, the minimum requirement of NZS 4121 for the door width $(760 \mathrm{~mm})$ is not enough; the minimum width of $810 \mathrm{~mm}$ would be desirable, as recommended by Lifemark Design Standards. There should also be considerations given to a passage width that allow those with mobility aids to walk with another person side-by-side. a minimum requirement of $1200 \mathrm{~mm}$ width would be necessary, which NZS 4121 requires in public buildings to allow space for its use by people with disabilities and their aids (e.g. for a wheelchair and an ambulant person to pass, for a blind or aged person with a caregiver to walk beside them). In the case of footpaths that rise one step from roadways, the path should also be wide enough to eliminate the fear of falling off the footpath (see also 8.2.3 'Communal pathways').

In the design of passages, it is ideal to avoid routes that require users to change direction as those with limited mobility or those using mobility aids such as walker frames or wheelchairs find this difficult. At the very least, passages that residents use frequently and often in a hurry, such as those between the toilet and their sitting space or the bed, should be straight. Avoiding corners in passages can also reduce injury to themselves and damage to wall surfaces caused by bumping with mobility aids.

\section{Fixtures}

Design of various fixtures can affect residents' QoL. For greater control, fixtures that need to be operated frequently by residents such as handles, outlets and switches should be installed within easy reach of the residents: the typical height should be between 1,000 and 1,200 m high (see also 8.2.2 'Wall and elevation design'). The need for electrical outlets increases with additional medical equipment including electrically adjustable chairs and beds. Outlets should be appropriately installed with respect to the assumed or likely layout of electric appliances so that cords do not cross pathways, which may be a hazard. Design of handles, such as those of taps, doors and drawers, should allow easy use for individuals with limited strength in their hands. The lever type handle, particularly the type with a return, is easier to operate than the roundhandle type. Taps used for kitchen sinks, hand basins and the shower should be the mixed type, avoiding using the type with no mixer. The small, tapering handle design for drawers should be avoided.

Regarding the installation of heaters, it is essential to identify the space for which residents require heating; for example, residents' sitting space where they spend a lot of time, and the space 
they use when drying themselves in the bathroom. These considerations should be followed by the choice and location of heaters that efficiently heat up the space (see also 8.2.2 'Sitting space' and 'Bathroom'). One participant missed the experience of putting firewood on the fire; providing the option of a wood burner as a heater, or installing a fireplace in a communal space, enhances their QoL.

Lighting design is very important, when people age their sight often deteriorates and greater lighting levels are required. In particular, there should be adequate lighting in indoor workspaces (e.g. space for activities such as writing, reading, cooking, needlework, artwork and operating a computer) and storage areas. This study did not identify the required illuminance levels; however, appropriate lighting levels have been widely researched. For example, the required minimum illuminance levels for task lighting in the living and the kitchen have been specified as 750 lux and 500 lux respectively (Noell-Waggoner, 2017). The design should avoid creating dark spaces in the interior layout; for example, rooms that are too deep with respect to windows should be ideally avoided, and if they are unavoidable, there should be sufficient lighting in the areas far from windows (see also 8.2.2 'Storage', 'Unit size' and 'Kitchen and dining space').

Curtains and blinds serve to shield indoor spaces from sunshine and maintain privacy by blocking views from outside. The type of curtains and blinds should be chosen with respect to the level of darkness and privacy required. For example, a bedroom typically requires curtains that can block light for daytime sleep. These are the minimum requirements drawn from this research, but it is also important to follow the extensive research on lighting requirements for the elderly. ${ }^{31}$

Lighting is also required at night on outside paths as well as indoors, as this prevents accidents.

\section{Things to hold onto}

Things to hold onto help the elderly by providing greater balance. These include kitchen benches, vanity counters, firm furniture and walls, as well as fixtures such as grab bars and handrails. Elderly people may not always use mobility aids such as a walking stick or walker frame inside their dwelling, so the installation of things to hold onto is essential in the interior layout. The continuity of things to hold onto also supports them, particularly for those with limited eyesight. These considerations not only facilitate safe walking, but increase opportunities

\footnotetext{
${ }^{31}$ For example, some researchers clarified the optimal illuminance levels for the elderly which differ depending on the type of space in residential areas (Kunduraci, 2017; Noell-Waggoner, 2017). Shikder, Mourshed, \& Price investigated the therapeutic effect of lighting on the wellbeing of the elderly and found various attributes of lighting (e.g. illumination levels, luminance contrast, glare control) are influential to the psychological health (2012).
} 
to move around for those who want to exercise. Particular attention should be given to such installations in spaces where such mobility aids cannot always be used, such as a shower area when showering. In such spaces, the layout of walls, fixtures and furniture should incorporate objects that can be held onto with both hands. For example, an overly large shower area, in which residents do not have things to hold onto with both hands can prove dangerous (see also 8.2.2 'Bathroom').

For those with hemiplegia, often caused by a stroke, and other impairments such as arthritis, attention should be given to the position of things to hold so that residents can access them from their able side.

Things to hold onto also help the elderly with crouching or bending to pick up something on the floor, and simply getting up from their chair. Items useful for this purpose include armrests of armchairs, grab handles and level surfaces (e.g. tables, benches) that are adjacent to the sitting space and at a height that is easy to grasp or lean on.

Items for support should be designed or installed so that they can endure a heavy load. For example, when attaching grab handles it is essential that the wall's internal framing is in appropriate locations. Drawers can also be leant on. In the installation of drawers, particularly top drawers under kitchen benches and vanity counters, slides that can bear a heavy load should be used. Firm fixture of items for supports is essential in minimising injuries in the event of a stumble or fall.

\section{Things to sit on}

The elderly who walk (with or without an aid) may require spaces to sit on in numerous places, including the kitchen, bathroom and lounge. In the installation of seats (e.g. folding seats) the height should not be too low, minimising difficulties in sitting down and getting up. For example, a toilet seat of $400 \mathrm{~mm}$ high is too low. A height of $450-550 \mathrm{~mm}$, which is the height specified for a shower seat in NZS 4121, should be required. Things to hold onto adjacent to seating helps the elderly in getting up and sitting down. Armchairs are preferred for this reason, as well as for the comfortable posture they accommodate. Adjustable armchairs are appreciated by participants who needed to lift their legs to ease discomfort. Swivel chairs on casters are useful, allowing users to change directions and move along using other objects (such as a kitchen bench or vanity counter) while seated. This type of chair can be used in the kitchen and bathroom. 
Objects to sit on are required beside outdoor pathways too, providing the elderly with limited mobility with a place for taking a rest, thereby facilitating their movements and exercise activities.

\section{Space for mobility aids}

To accommodate those using mobility aids, spatial requirements arise in various aspects. Level differences such as steps and stairs are not suited for mobility aids; even a tiny level difference is difficult to move over with a walker frame and wheelchair (see also 'Floor'). Similarly, slopes are not suitable for mobility aids, while there are differences in the ability to move over them depending on the type of aid. As mentioned previously, attention should also be given to the door type, passage width and floor materials (see also 'Door' 'Passage' and 'Floor').

There should be sufficient space for turning around with a mobility aid, such as a walker frame and a wheelchair. This applies to any enclosed spaces and other spaces that residents need to approach, such as space in front of a letterbox, shelves in a library. Turning a walker frame or wheelchair in a small space requires extra strength. Turning a walker frame in a small room was actually avoided by many participants and in one incidence caused a fall (even in a room containing clear space of 1,500 m diameter turning circle). Considering these troubles and danger, providing spatial configurations that avoid the need to turn around is ideal (see also 8.2.2 'Planar relationships between spaces').

Storage for mobility scooters and electronic wheelchairs is necessary. Storage should be accessible from the residents' units, containing enough space for turning around, and indoors or at least half-enclosed, as electric outlets are needed to charge vehicles. This study found, in accommodating a scooter, a turning circle of 2,050 $\mathrm{mm}$ diameter was required This result is similar to the turning circle of 1,985 mm recommended in NZS 4121 (Standards New Zealand, 2001). Space is also required for a person with other mobility aids (e.g. a walker frame) to approach their scooter. Storage for mobility scooters should be located either in the individual's area or in a communal space. Preferably, this space should be located in residents' personal areas as they may leave behind other mobility aids such as a walker frame, a trolley or a walking stick after transferring to the vehicle. If communal storage for mobility scooters and wheelchairs is provided, there should be appropriate space that can accommodate these mobility aids.

\subsubsection{Requirements in the design of the individual unit}

In this section, design considerations relevant to individual units are discussed. These considerations include those relating to size, spatial elements such as walls and storage, and 
planar relationships between spaces, as well as spaces or rooms such as sitting spaces, bedrooms, toilets, kitchens, dining spaces and laundry spaces.

\section{Unit size}

Various factors to improve QoL have an impact on both an increase and decrease in housing size choices. This study found that the most important factor influencing a reduction in house size was the residents' ability to undertake home maintenance. In one-bedroom units, participants generally said that their house was small enough to maintain. Smaller sizes were also beneficial for heating efficiency and ventilation. Participants could keep doors open to let the heat circulate in their house, and the open doors facilitated ease of movement between spaces.

On the other hand, there were several factors that called for an increase in size, such as the need to accommodate mobility aids, caregivers and personal guests. For example, a bedsit unit was not desirable as most participants wanted a hard separation between the lounge and bedroom, rather than a curtain, especially when hosting guests. This generally required an increase in the floor area (bedsit units studied were 25-35 $\mathrm{m}^{2}$ and one-bedroom units were $39-59 \mathrm{~m}^{2}$ ).

Considerations for accommodating those living as a couple also necessitated an increased housing size. For example, wardrobe space that can accommodate clothing for two people is necessary, and having some separate spaces is preferred to maintain privacy from each other.

In the design of housing, it is important to meet both factors when establishing optimal size. The quality of the design has great implications for adequate size. Careful layout of space, combined with strategies for greater flexibility in use can minimise unit size, where less well considered design may necessitate a larger unit. The design of communal space can also be utilised for activities and storage of possessions that cannot be accommodated in individual dwellings. This will be discussed later.

\section{Wall and elevation design}

Furniture should typically be laid out along walls. The layout of low furniture, such as a low table, in the centre of the room should be avoided, as it is a tripping hazard. Walls should be designed so that they provide optimal space for furniture and fixtures. For example, walls with right angles rather than angled walls were preferred by participants to accommodate furniture. This outcome is consistent with the requirements in EVOLVE and Guidelines for the planning of housing for senior citizens which finds that the room shape should be as near to square as possible or rectangular for residents to furnish easily, avoiding very narrow or curved shapes (Lewis et al., 2010; WEL_HOPS, 2007). 
Allowing the layout of preferred furniture can enhance residents' sense of home and enable engagement in preferred personal and social leisure activities. It is important to provide space for furniture that facilitates activities of residents' choice in their personal space, while some spaces for activities can be located in a communal area.

To support watching TV, a TV can be installed on a wall, which was preferred particularly in a small room. Other common furniture for personal leisure activities included a writing table and a computer desk. Some people preferred tables for needlework (e.g. sewing, bobbin lace), or for artwork and for jigsaw puzzles, or a workshop table, with drawers to store tools, which could provide for 'works in progress'. Other items such as an exercise machine and musical instruments such as a piano were also commonplace. For those with severe mobility issues, work surfaces (e.g. tables, desks, benches) where they could perform tasks while seated facilitated their activities. The surfaces also played a role of supporting an impaired limb; for example, for those with hemiplegia.

There should also be consideration for space for furniture that is emotionally important for residents; this study has found that display cabinets, bookshelves or other cabinets were commonly used to accommodate residents' precious items. Other nostalgic and decorative furniture that depended on personal preferences included bedside drawers, longcase clocks, handmade furniture and low tables, tables for decorating candles to create a religious space, and tables/bases for indoor plants.

As well as furniture, walls can accommodate smaller decorative items and those for day-to-day use. Empty walls can be utilised for displaying photographs, needlework and artwork such as paintings and tapestries of memories. If provided with shelving, walls are also useful for displaying nostalgic objects such as artwork (e.g. a statue, an etching), photographs, various ornaments and books, as well as storing day-to-day items. Built-in shelves, other types of level surfaces such as seats and work surfaces can be provided for walls, which are used a great deal.

These insights provide a new understanding of the wide range of types of furniture and objects to accommodate to facilitate the preferences in activities and other desires of the high-needs elderly, compared to those of the existing design frameworks. For example, EVOLVE sets out a requirement of the lounge as being able to accommodate a bookcase, TV, CD/music player and a computer desk (Lewis et al., 2010) — it is doubtful if this requirement satisfies their desires based on the outcome of this research. 
In the wall design, it is essential to consider the height of fixtures so that they are easy to reach, sit on and enable elderly residents to perform tasks. Items that need particular consideration include shelves, cupboards, counters, outlets, window and door handles, wall-mounted clothes dryers and clotheslines. Attention should be given to ensuring a height that is usable both for those seated and those standing. Participants standing were typically unable to reach shelves of $1,800 \mathrm{~mm}$ or higher, and while they could reach the front of shelves at $1,650 \mathrm{~mm}$ and above they could not reach the space at the back. Participants had difficulty with bending to reach items under kitchen benches. The study did not extend to establishing the height requirements for wheelchair users; however, referring to NZS 4121, shelves should be installed at 230 - 1,350 $\mathrm{mm}$ height. Synthesising these requirements, the shelf height provided adjacent to sitting spaces should be $230-1,350 \mathrm{~mm}$ high. In other spaces, the height of shelves to accommodate highlyused items should be $1,000-1,350 \mathrm{~mm}$ high to ensure the usability both for wheelchair users and those who stand.

The height of electric outlets such as light switches and door handles also requires careful consideration (Standards New Zealand, 2001); though NZS 4121 defines the height as 900$1,200 \mathrm{~mm}$ above the floor level suited to meet the requirements for wheelchair users, to be usable for walker frame users, it should be at 1,000 mm or higher (see also 8.2.1 'Door'). Exceptions are the cases where outlets are provided adjacent to work surfaces and reached from a seated position, where they should be installed in the lower space just above the level surface.

Optimal height of work surfaces suited for a seated position can be applied in the design of many parts of housing for the high-needs elderly because many residents prefer to perform tasks while seated, and this design can also accommodate wheelchair users. According to NZS 4121, the height of the work surface should be less than $775 \mathrm{~mm}$ (Standards New Zealand, 2001). To accommodate legs under the surface, there needs to be clear under space of at least $675 \mathrm{~mm}$. Seating height must be considered as mentioned in 8.2.1 'Things to sit on', when providing a built-in or folding seat.

Figure 8-6 summarises the main height requirements. 

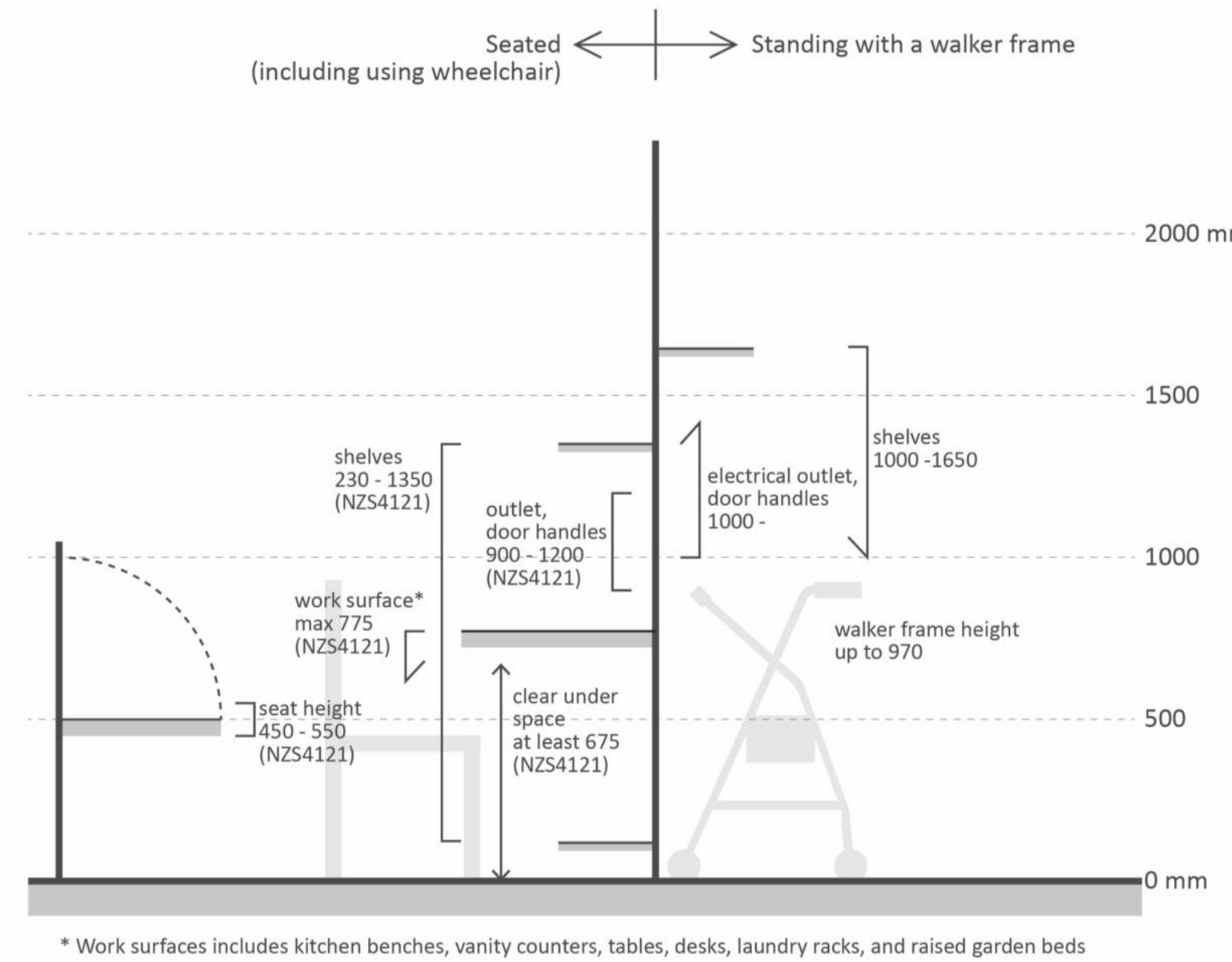

Figure 8-6: Height requirements (section)

While walls have significant roles in accommodating furniture and other items, it is also important to remove unnecessary walls to provide views and allow for natural light from various directions, which enhances residents' QoL. For example, an open plan kitchen/lounge area was preferred by many residents, as it eliminates the wall between the kitchen and lounge and provides residents with greater access to natural light.

In the design of external and intertenancy walls, additional sound insulation should be provided. This is particularly important for the high-needs elderly, who often use audio equipment such as a TV, a radio or audiobooks at higher than normal volume levels due to hearing loss.

\section{Storage}

Storage is important to maintain personal identity and provide residents with a greater sense of home, as well as maintaining control and facilitating a tidy living environment. The design of storage spaces should consider interior elevation and the necessary floor area required to accommodate personal objects and furniture. 
The wardrobe should be a sufficient size to accommodate clothing for all occupants, their accessories and other items such as those hobbies and general household linens. For those participants living alone, wardrobes of 0.8-1.7 $\mathrm{m}$ wide were not sufficient but those of 1.8-3 m wide were satisfactory. Ideally, there should be a wardrobe allowing expansion up to $3 \mathrm{~m}$ wide. Provision of enough wardrobe space for two people can also accommodate a resident's desire to keep the belongings of their partner.

In the design of the wardrobe, providing enough shelving that facilitates effectively storing bags, shawls or scarves, shoes and other small items is necessary. It is desirable to provide movable shelves so that elderly residents can customise them to suit their amount of clothes and other items as the amount differs by individuals. A walk-in closet is undesirable unless it contains sufficient space for turning around with a wheelchair or walker frame. To ensure an area is sufficiently illuminated for ageing eyes, there should be a light in any enclosed storage.

Shelves should be designed to be easily reachable (see also 'Wall and elevation design'). The appropriate height of shelves differs by the user posture, seated or standing. In the spaces where residents can both be standing or seated such as the kitchen, it is important to attain the height suited for both postures, which is $1000-1350 \mathrm{~mm}$ height. When determining the depth of shelves, the ability of residents to reach into the spaces should be considered, particularly for storage is higher areas. For example, the rear part of a $1800 \mathrm{~mm}$-high shelf was generally not reachable (see also 'Wall and elevation design'); therefore, the depth should be shallower. For example, assuming the use of high shelves in the kitchen for storing seldom used items such as containers and jars, the adequate depth of the shelves would be $200 \mathrm{~mm}$. In order to increase the storage efficiency, walls can be furnished with other items for storage, such as railing and hooks. Railings installed on walls facilitate the use of hooks according to the needs of individuals.

Drawer type storage eliminates the difficulty of reaching the deeper part of the space and is a good choice for storage. This is typically well suited under bench cupboards in the kitchen, but also be used in other low areas, instead of cupboards. When installing drawers, there should be a measure to ensure stability and prevent them from falling out in case users lean or hold onto them for support, such as the use of the slides bearing heavy loads (see also 8.2.1 'Things to hold onto'). A handle that is only operated with the fingertips, such as a tapered section in the front board, should also be avoided, as it is difficult for those with limited strength in their hands to operate. 
In the design of storage and shelves, the type of items that will be accommodated should be considered. This is especially important for items that need larger spaces. Space is necessary to accommodate long items, such as walking sticks or a 'picker upper' device. Provision of these kinds of storage spaces lead to greater safety as, if the tall items fall on the floor, they can be a tripping hazard. Other items such as compact vacuum cleaners with long handles are used by many elderly people frequently. Even though residents normally have regular visits from cleaners, keeping these items helps them keep their space clean in the event of spills without waiting for the next cleaner visit.

Storage for items should be located adjacent to the space where the related activities are performed. For example, a wardrobe should be located where residents wear or change clothes, commonly in a bedroom, where residents use their bed to sit on and put clothes to be worn on. It is convenient to have storage for outdoor clothes and bags (e.g. hooks on a wall) near a front door. Storage for garden tools should be in proximity to the door to the garden. Storage for rubbish is also important, particularly if rubbish and recycling items are collected individually. For example, storage under the kitchen bench for recycling items as well as rubbish was appreciated by participants.

Attention should be given to providing extra storage for items seldom used, such as seasonal items like heavy coats, suitcases, and nostalgic items such as the belongings of a late partner. These were often stored in the wardrobe of the second bedroom or in the garage by participants in this research. Where this space was insufficient the house tended to be untidy with those extra items. Therefore, it is important to provide enough storage for these items to keep their spaces tidy, and providing storage in communal space is an alternative solution where enough wardrobe space cannot be provided within the individual unit.

\section{Planar relationships between spaces}

Requirements were distilled regarding planar relationships between spaces. Such relationships relate to reducing the difficulty of elderly residents in moving around and ensuring specific views. and lines of sight. Given that the quality of care is often eroded by the demanding workloads and time constraints of caregivers, the layout of spaces that minimises the distance between spaces for care improves the quality of care.

\section{Proximity between a bathroom/toilet with respect to a bedroom, sitting space and a kitchen}

As incontinence is a common health issue experienced by the elderly, an environment to support residents' easy access to the toilet is important. For example, the proximity of the bedroom to 
the toilet is essential as the condition is worse in the night time when lighting and eyesight can also be worse. Attention should also be given to the path from the bedroom to the toilet; a straight route from the bed or sitting space is easier than a route involving a change in direction. This study has also shown that a proximity of less than $3 \mathrm{~m}$ is required between the sitting space and the toilet to meet the requirement of those with severe issues with incontinence and mobility. Solutions to meet both these requirements include having two toilets, having a toilet with two doors (on the bedroom side and the other side) or having a toilet in immediate proximity to the bedroom. The former solution is beneficial when residents have guests, as guests can use the bathroom without entering the resident's bedroom, thereby maintaining resident privacy.

The layout of a bathroom adjacent to the bedroom is also beneficial for residents' movements related to showering; for example, the bed is often used when removing and putting on clothes before and after showering as it provides residents with a comfortable place to sit and space for clothes. The proximity between the wardrobe and the bathroom also reduces the movements of residents or caregivers carrying a change of clothes.

Given that caregivers' care tasks often include bed-making, washing dishes, preparing meals, and laundry, the layout should ensure proximity of the bathroom to the kitchen, bedroom and washing machine. This has great potential to improve the quality of care, as caregivers can stay near residents even when they leave residents alone to give them privacy to shower themselves.

To reduce the distance for undertaking laundering, space for a washing machine should be located near the place where residents remove their clothes. Given that many clothes to be washed are taken off in bathrooms, space for a washing machine should be located near or within the bathroom. The space for washing (a washing machine) and access to drying areas (e.g. outdoor clotheslines, a laundry rack) should also be closely located (see also 'Space for laundering and utility').

\section{Separation of the bedroom from the lounge}

The separation of the bedroom from the lounge is highly recommended for greater privacy when welcoming guests. The door can be a sliding door, which can either be kept open or closed when the resident is alone. 
To provide greater control for guests, the front door should be close enough for a voice to reach the opposite side of the door.

\section{Proximity between a kitchen and a garage}

In dwellings with a garage, the kitchen should be laid out in proximity to this, which makes it easier for residents to carry their shopping to the kitchen.

\section{Layout of space that avoids turning around}

Given that turning around causes the elderly with a walker frame or wheelchair extra trouble, the layout of spaces that allow residents to avoid turning around mitigates their difficulty in moving (Figure 8-7).

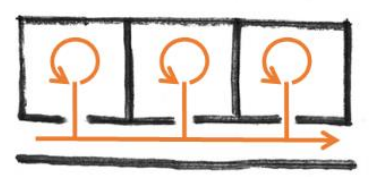

Turning around

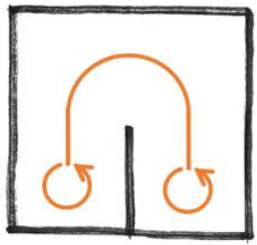

Turning around

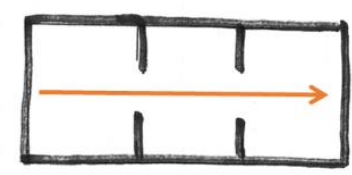

Avoid Turning around

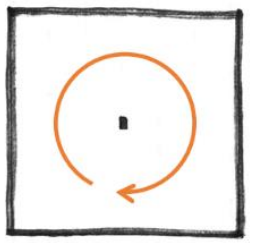

Avoid Turning around

Figure 8-7: Ideas for interior layout that avoids turning around

\section{Sitting space}

Most participants had a space where they sat for long periods of time, which became increasingly important as mobility declined. In the design of the sitting space, there should be careful consideration to facilitate residents' personal and social activities in a comfortable manner.

\section{Micro space to facilitate various activities while seated}

In residents' sitting space, an armchair is most commonly used as it allows for comfortable posture and offers support with armrests. Adjustable chairs are preferred. There should also be other seating options for sitting spaces, such as sitting on a side chair which is suitable for some people with spinal conditions. 
Careful consideration should be given to the micro environment surrounding a sitting area. A lot of activities can occur in this space, such as reading books and newspapers, knitting, sitting exercises, ball exercises, talking on the telephone, crosswords, cutting with scissors, writing letters and using the computer. The spatial organisation should allow for a layout including an adjustable armchair and level surfaces or storage to ensure necessary things are within reach, enhancing residents' control of their environment and facilitating activities. The adjacent level surfaces and storage areas include shelves, tables, desks, kitchen benches, drawers and trolleys that can accommodate various items such as a telephone/handset, remote controls, glasses, medicine, cups of tea, pens and paper. Attention should be given to the height of shelves adjacent to this space, which should be at $230-1350 \mathrm{~mm}$ high. Additional lighting should be provided for this space, where residents often enjoy reading.

\section{Views and privacy}

As watching TV was the most common activity, room layout should consider the location and proximity of the TV in relation to armchair location, which includes the option of hanging the TV on the wall. The layout of a TV in front of a window, which compels residents to stare at the window, should be avoided for those with eye impairments. Given that some elderly people like to view their computer screen to detect the arrival of new messages or updates on social media websites, similar attention should be given to views of a computer screen.

Attention should be given to the layout of doors and windows. First, the front door should be within sight of the sitting space to provide residents with a greater sense of control of visitors. The optimal layout should be considered regarding the sitting space and windows that provide views to outside. Views to the direction from which visitors approach improve residents' sense of control - this outcome is consistent with a requirement in Guidelines for the planning of housing for senior citizens that the front door should be visible from the day zone (WEL_HOPS, 2007). It is preferable to provide views to a personal garden in their porches, as well as distant nature such as mountains, hills and the sea. On the other hand, residents also liked the views of people and vehicles moving outside. Providing multi-level views of open space with trees in proximity, moving people, vehicles at a medium distance and nature at a long distance leads to a great QoL for residents (See also 8.2.3 'Unit layout' and 'Open space and outdoor communal areas').

Privacy is an important requirement for the sitting space. While windows should be positioned to provide a resident with views to the outside, visibility from the outside in should be limited. This is particularly important for those with severe incontinence and mobility concerns, who 
may use a commode or other devices in the lounge as well as in the bedroom. There should be enough consideration of the spatial organisation of exterior space and placement of windows to meet the conflicting needs for high levels of privacy and desires for views. Sheer or lace curtains can both let sunlight in and block views into rooms; however, they also block views to the outside and are not ideal for unobscured views. Using vertical blinds that can be angled is a better solution, which blocks most of views from the outside while ensuring views from the inside to the outside (though limited). Other strategies should also be considered, such as limiting the window height (e.g. avoiding full-height windows) and providing a level difference or enough distance between the unit and communal pathways (see also 8.2.3 'Open space and outdoor communal areas').

\section{Sunshine and warmth}

Warmth is essential in the sitting space. In New Zealand, the space should ideally have northfacing windows to get sunshine effectively and should avoid facing the south. Study participants in east-facing units felt cold and, only units facing between north and east were acceptable without complaints. A heat supply should be provided in proximity to the sitting space, and suited to warming lower areas where drafts are more prevalent. Floor heating or a heat pump mounted on the low part of the wall can address this concern. Infrared radiant heating on the ceiling is often too hot, so should be avoided. Considering that doors are often kept open between the lounge and adjacent rooms, the heating capacity of any heating device should take this into consideration.

Requirements for sitting space are summarised in Figure 8-8. 


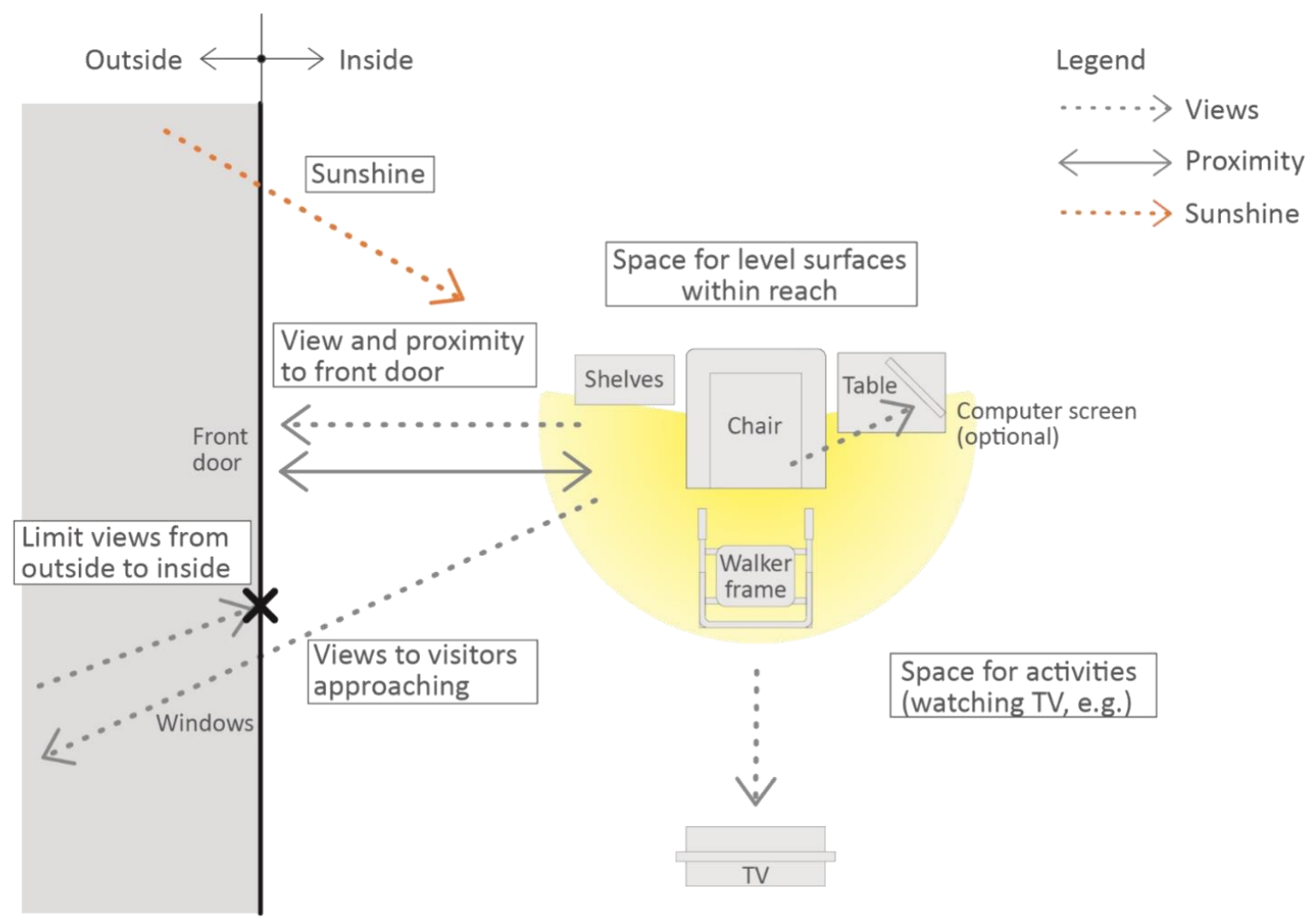

Figure 8-8: Requirements for sitting space

\section{Bedroom}

The design of a bedroom largely relates to residents' comfort, which is important as their discomfort increases due to health conditions. In this space, it is also important to accommodate sufficient space for possessions.

The design of the bedroom is important, given the high-needs elderly need to spend much time in bed. There should be door/s and curtains to provide enough darkness for sleep during the daytime but also sufficient windows for those who spend the daytime awake in bed to have access to natural light. Northeast facing windows let the morning sun in, which is pleasing for many elderly people.

Furniture in this space typically includes wardrobes, drawers and bedside tables as well as up to two beds. A dressing table is frequently desired mainly by female residents. Attention should be paid to providing sufficient wardrobe space (minimum $1.7 \mathrm{~m}$ wide per person as explained in 'Storage') and appropriate shelves inside as discussed in 'Storage'. This space should provide residents with a more private feeling than the sitting space. Therefore, photographs and artwork can also be kept here. A bedroom can also be a space for activities such as writing, which requires great concentration and privacy. Where a couple lives together, the provision of sufficient space 
for one member to spend their time, such as a table and a chair, in the bedroom can facilitate their separation within the dwelling, thereby increasing privacy from the other partner. This is particularly important in a small dwelling. There must also be sufficient room for turning around between furniture and the bed, potentially on both sides to accommodate couples. For example, NZS 4121 requires at least $900 \mathrm{~mm}$-wide passages on three sides of the bed, with turning circle space of $1500 \mathrm{~mm}$ diameter in one corner for a wheelchair user (Standards New Zealand, 2001).

\section{Extra spaces}

Extra spaces are required to accommodate guests and when living with a partner, as well as to ensure smooth movements with mobility aids and when engaging in daily activities. Attention should be given to the strategies that can mitigate an increase of space to meet these requirements, such as means to increase the flexibility in use.

\section{Space for guests}

As most participants had regular guests, sufficient room for visitors is necessary in the quasipublic areas of the unit. This space can typically be combined with the resident's sitting space. Attention should be given to accommodating a large number of guests such as up to 10 people at a time, as some residents in the study regularly had that many guests. For example, a lounge of $14 \mathrm{~m}^{2}$ was not enough to comfortably accommodate 10 guests. Space for extra chairs is also required within residents' units (including in other spaces such as the bedroom) or in communal space or corridors near the unit. Requirements increase when accommodating small children (e.g. grandchildren or great-grandchildren) which is commonplace and important for many elderly residents. It is desirable to have space for children to play away that is separate from residents' important things (e.g. books, hobby tools) to prevent children from damaging items.

Space for an extra bed is useful for having guests stay overnight. Even in a one-bedroom house, allowing sufficient space in a lounge for a sofa bed (being stretched out) or a portable bed (such as a trundle bed or inflatable bed) allows this.

\section{Garage}

In the design of a garage, there should be sufficient width for a walker frame or wheelchair to pass beside the car. The study results showed the width of $2.9 \mathrm{~m}$ is not enough for those using a walker frame or a trolley. This width is also too narrow for wheelchair users, who require the garage width of at least $3.5 \mathrm{~m}$ according to NZS 4121 (Standards New Zealand, 2001). In addition, space should be able to accommodate various personal possessions in addition to a car 
and passages. For example, in this study, a garage was used as storage for household items and for tools for hobbies such as DIY, as well as space for drying clothes and temporary accommodation for guests. Assuming an area of $250 \mathrm{~mm}$-depth storage on both long sides of the garage, a minimum garage width of at least $4 \mathrm{~m}$ would be required. As many elderly people typically give up driving as their ability to drive declines, the design of the garage should facilitate greater flexibility in its potential use. For example, the provision of sufficient insulation and layout of appropriate windows for ventilation and natural lighting can increase the opportunities to have overnight guests stay in this space.

\section{Space for greater privacy for those living with their partner}

For those living with their partner, there should be separate space that can shut off the sight of each other for greater privacy (e.g. when concentrating on writing). This is easily achievable where residents have extra rooms; however, just having a bedroom large enough for an activity space can also meet this requirement.

\section{Other}

Space for drying clothes is also important; particularly space for a laundry rack, which was often used both by participants and caregivers. For the indoor space for drying clothes, it is important to provide extra heating and ventilation as it creates excessive moisture. The option of having a conservatory can create additional indoor space, but there should be attention given to ensuring enough light in the inner room by providing windows on other walls.

\section{Bathroom}

The design of a bathroom and toilet greatly influence residents' QoL, particularly when their mobility declines and care needs increase. Consideration should be given not only to aiding their independence but to accommodating a caregiver and care activities.

\section{Floor}

The floor design is essential in improving the showering safety of the elderly who are prone to falls, as well as for facilitating the ease of caregiver activities and safety. The floor should have no level difference at the entry. The flooring should be designed for slip resistance and ease in cleaning, with consideration to the materials and form of the surface.

Design of shower drainage is very important because water on the floor increases the risk of falls. The slope should not be overly steep to mitigate the risk and fear of falling, particularly for someone with limited eyesight. There should be careful consideration of both the type and 
location of the drain and how their placement might affect the wetness of the area. Most bathrooms in this study had a round drain, while two cases had a trench drain. A trench drain typically provided a larger surface than the round drain, increasing the speed of drainage and reducing the floor slope gradient (both real and perceived).

\section{Shower enclosure}

Limiting the water flow over the floor is an important role of a shower enclosure. This matters not only for residents but also for caregivers and directly affects caregiver safety. The shower curtain or the glass screen door(s) is generally kept open when assistance is provided, so the degree of openness and direction the door opens should be well considered. Water collection on drying areas must be minimised and items that must be kept dry, such as clothes and towels, must not be impacted by spray or moisture. A shower enclosure also plays a role in limiting the spread of vapour, which can make a caregiver who is constantly moving breathless even with an extract fan, particularly in a small bathroom. The enclosure size should allow the caregiver to come in to assist the resident. A shower enclosure of $1 \mathrm{~m} \mathrm{x} 1 \mathrm{~m}$ is too small to accommodate assistance.

The preference for the type of shower enclosure material changes with the level of independence in showering. For those walk into the shower area, it is important to provide a solid enclosure, which can provide the necessary support for them and increase their sense of safety, and limits the water flow on the floor which increases safety for caregivers in assisting residents with washing. This requirement of a solid enclosure is contradictory to the recommendation for the open walk-in shower in many existing design frameworks for housing for the elderly (Lewis et al., 2010; Lifetime Design Limited, 2012; Standards New Zealand, 2001; WEL_HOPS, 2007). This is likely because the open walk-in shower is more suitable for wheelchair users or those who use a large chair or equipment, while most of the study participants were those who walked (with or without walking aids) (93\%) in contrast to a small proportion of wheelchair users (7\%). More importantly, the majority were walker frame/trolley users (53\%), who usually left behind their aids when going into the shower area and needed extra support in walking. Therefore, the requirement of a solid enclosure is particularly important for the design for those who are walker frame users and require assistance in showering. In the installation of doors to a shower enclosure, the opening direction should be considered as a door may need to be kept open when assistance is required and may get in the way of care activities. The design should also enable easy cleaning; for example, a shower curtain is prone to mould and is difficult for the elderly to take on and off to wash. Flexibility regarding enclosure modification, such as removal of a glass 
screen and installation of a shower curtain, is desirable to address any deterioration of mobility and needs for use of a larger chair and equipment.

There should also be special consideration for physical environments that can provide residents with greater privacy while supervised by the caregiver for safety. Ideally, the caregiver should be out of sight but still in the bathroom while providing supervision. The material of the enclosure should block views to provide a degree of privacy, rather than be fully transparent. This also prevents the caregiver from having to leave the bathroom, as leaving can mean an accident is not easily detected.

\section{Space for undressing/drying/dressing}

Tasks of undressing, drying and dressing typically take place in the bathroom and often with caregiver assistance. In most cases in this study, a chair was placed out of the shower area for the resident to sit on while the caregiver moved around them. Given this, there should be enough space for the caregiver to crouch in front of the resident to dry their legs and apply lotions or cream and to move beside the resident between their back and front sides. This typically requires an oval-shaped space of at least $1.2 \mathrm{~m} \mathrm{x} 1.7 \mathrm{~m}$ (Figure 8-9). For those with severe limitations in mobility, drying generally takes place in the same space as showering. The size and the opening of the shower enclosure should take into account the space for drying.

A dry area within the bathroom is necessary for keeping towels, clothes, shoes or slippers and a chair out of the water, which requires spatial solutions regarding the design of the shower enclosure. The adjacency of spaces can ease care tasks, such as close proximity of the rubbish bin to dispose of diapers or pads, of the chair to the storage of lotions or cream, and of a washing machine for dirty clothes, and the wardrobe to bring clothes from. Considering that the bed is often used for undressing and dressing, an adjacent bedroom helps. 


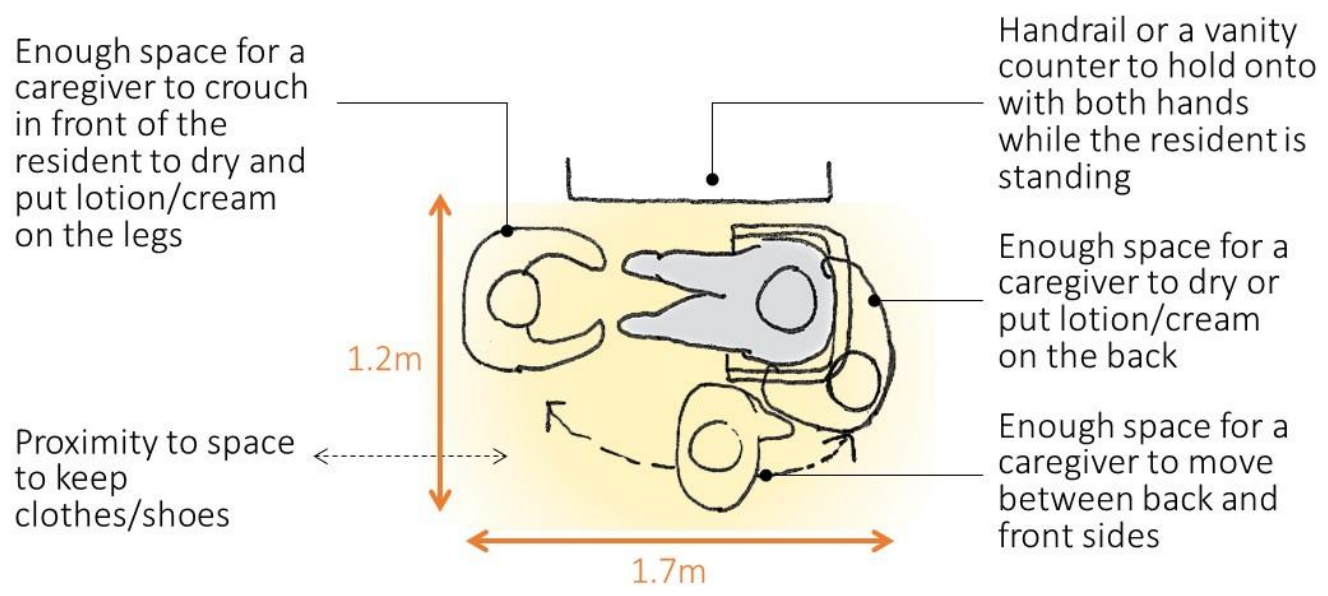

Figure 8-9: Requirements for space for drying

\section{Things to sit on}

Shower chairs are important for greater comfort of residents. Chair size can vary depending on the degree of support required for residents, and a larger space is required to accommodate a big chair with arms and back that provide greater support. A built-in bench or a folding seat attached to the wall was less used, because they lack armrests to support residents and often the location was not convenient for the caregiver. When attaching a folding/fixed chair in the shower area, it should be designed to allow space both in front and back of the seated person for their caregiver to assist washing them. There should also be things to hold onto while seated and when standing up/sitting down (ideally armrests). The height of the seat (e.g. shower chair, a toilet seat) should be appropriate and not too low for balance. The location of the seat should be considered in relation to handrails or grab handles to support the movements of sitting and standing, and that of other fixtures such as shower mixer levers and hoses that enable resident independence.

Two types of chair or seat (one 'wet' and one 'dry') are required for those who need to sit both for washing and for undressing/drying/dressing. A small bathroom often requires spatial rearrangement by moving the chairs before and after showering; however, the provision of appropriate space for chairs can reduce the effort and enhance the independence of those with limited mobility.

\section{Things to hold onto}

Things to hold onto (e.g. handrails, grab handles) are required when moving in the bathroom, and when standing up and sitting down not only when washing, but also when drying, dressing and undressing. When standing, those who have a great deal of difficulty in maintaining their 
balance prefer to lean on something with both hands. These latter activities typically take place out of the shower enclosure. Things to hold onto are particularly important in spaces where elderly residents cannot use their mobility aids (e.g. they can't bring a walker frame in the shower). In such spaces, it is also important to provide objects to hold onto that can be held by those with limited hand strength using both hands.

The shape and size of the bathroom and the layout of spaces such as a shower area, a vanity and a toilet should provide residents with support when moving in the bathroom and should enhance their sense of safety. The layout of solid walls and other elements, such as a vanity counter, with an appropriate distance between them can allow residents to hold support objects with both hands. The continuity of solid walls and other elements improves safety, particularly for those with limited eyesight, and a hole in the continuity of things to hold onto (such as the opening of the shower enclosure) can be a hazard.

\section{Space for mobility aids}

There should be enough space in the bathroom to accommodate a walker frame. A walker frame is commonly used for putting clothes on as well as for support in walking. This is particularly important in situations when the caregiver goes in and out of the bathroom while the resident is showering. An appropriate storage space out of the way of the caregiver reduces difficulty for both the resident and the caregiver.

For the walker frame user to use the toilet, the space in front of the toilet should be big enough for them to manoeuvre. Particularly in a wet-area shower, there should be enough space between the shower enclosure and the toilet, as the available dry space to keep a walker frame dry is often limited when the caregiver turns on hot water to warm up the room before showering.

\section{Fixtures}

The shower head should have a hose long enough for the resident to use it easily, enabling greater independence and privacy as well as greater ease of care activities. A fixed shower head attached to the wall should not be used. While both the shower hose and the mixer lever should be within reach of the resident, the shower hose should not be too close to the lever to avoid it being caught by the lever. Bathroom heaters enhance thermal comfort. The location of a heater should be in proximity to the space for drying. The location of the emergency alarm button can affect safety; the button or the pull cord for it should be located close enough to the floor that it can be easily reached in case of a fall, as well as reachable from the sitting position. 
Storage space is required for a variety of items including towels, toilet paper, bottles of hand or body wash and shampoo, boxes of soap, lotion or cream and diapers or pads. Built-in shelves and vanity counters of appropriate height for wheelchair users are necessary.

\section{Kitchen and dining space}

The design of a kitchen should consider control of the environment. Attention should be given to the layout to ensure kitchen appliances, utensils, tools and ingredients are within reach and to keep the moving distances short. Providing enough accessible storage is also essential. However, spatial requirements may vary depending on individual preferences for cooking and the meal service of the housing complex.

\section{Space for a microwave oven}

The microwave was commonly used by study participants. In the design of space for a microwave oven, the height should be carefully considered because many elderly residents have difficulty in taking out hot food and spills can lead to burns. Space should be provided at the height that is easy to reach. This requirement is consistent with those suggested in Guidelines for the planning of housing for senior citizens (WEL_HOPS, 2007). In addition, there should be a level surface to put food that has been taken out immediately in front of the microwave oven. Bench space was preferred by participants rather than a separate shelf.

\section{Space for a fridge-freezer}

In determining the space for a fridge-freezer, it is important to accommodate one that is large enough, as the high-needs elderly tend to store a lot of food in the fridge-freezer. For those who prepare at least one meal per a day, space for a normal-sized fridge-freezer (typically $600 \mathrm{~m}$ wide or wider, preferably with a drawer-type freezer) should be provided; the design should avoid only space for a mini fridge. Where three meals are provided, the space for a mini-fridge under the bench is likely to meet residents' needs. However, residents have difficulty taking things out unless they are of a drawer type (Figure 8-10). Therefore, a mini-fridge can be placed in a raised position, or a drawer-type fridge under the bench, would be optimal solutions. 


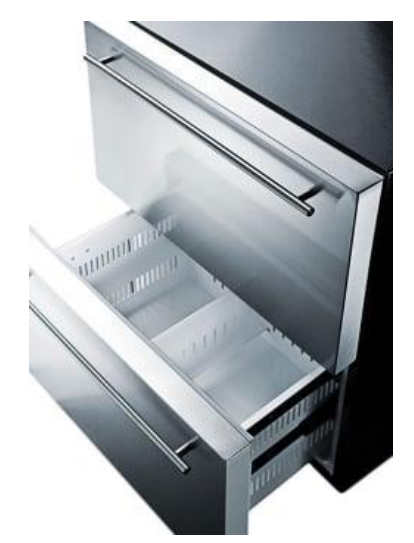

Figure 8-10: Drawer-type fridge-freezer

In the layout of space for a fridge-freezer, attention should also be given to the layout in relation to walls so that the fridge-freezer door can open more than 90 degrees.

\section{Kitchen facilities}

There were differences in participants' need for cooking facilities according to individual preference. Hot plates were often used even by participants who cook one meal a day, so should be furnished in residents' kitchens. Those who used an oven used it for simple cooking, such as cooking frozen food, as well as baking, and a compact oven could meet most residents' needs. Only one participant wanted two ovens, one for cooking and the other for warming dishes. It would be good to provide options for having one or two ovens, or not having one at all by providing space for components rather than built-in appliances.

A dishwasher can be of great help for the high-needs elderly. A small drawer type is preferred and should be installed close to the sink at a height is suited for those sitting on a chair. Space under the sink should have some open space so that residents can sit and put their legs under the sink.

\section{Bench space for meal preparation}

Bench space is centrally used for meal preparation, which includes tasks such as cutting and using appliances on the bench such as a slow cooker or rice cooker, a toaster, a sandwich maker and an electric kettle. As those with less strength and mobility have difficulties taking out appliances or putting them in place, the bench space should be big enough to comfortably accommodate dailyuse tools and appliances within reach, as well as provide enough open space for tasks. For example, a bench of $700 \mathrm{~mm}$ depth can accommodate appliances such as a toaster and a sandwich maker in the rear part and still leave space for tasks in the front. 
Cooking tasks often include carrying ingredients, water and tools. In the design of benches, attention should be given to the layout of spaces such as storage, a fridge-freezer, a sink and hot plates in close proximity to each other. This proximity is particularly important for those performing tasks while sitting on a chair. A continual bench between these spaces makes it easier to move items. An 'island kitchen' with two parallel benches can cause difficulty with carrying food or pans between the benches if spaces for preparation and cooking are separated. To keep the moving distance between spaces (e.g. space for tasks, storage, a fridge-freezer) in proximity to each other, an L-shaped bench is more effective than a rectangular bench. The space between benches and walls should be large enough to accommodate both a caregiver and a resident, allowing the caregiver to help the resident with cooking, and allowing for a wheelchair user to turn around. This also allows for taking a walker frame or trolley into the kitchen, which is necessary particularly when carrying meals to dining space.

The bench height should suit wheelchairs and those who want to be seated while cooking. This is particularly important for safety in tasks such as pouring boiled water from the electric kettle into a cup or taking heated food out from the microwave oven. For cutting food while seated, a height of $730 \mathrm{~mm}$ is slightly too high. There should be space beneath the bench for putting the legs and feet where they mainly perform the tasks, as well as under the sink and hot plates. The bench height should be carefully determined so that it is suited for various tasks, particularly cutting food, while accommodating legs underneath (see also 'Wall and elevation design').

The bench space adjacent to the sink is also used to store used dishes until caregivers visit and wash them. Under the shelves and cupboards above the bench, there should be a light to ensure enough brightness for tasks.

\section{Storage}

Storage is required for storing items such as pots, pans, tools, utensils, tableware, ingredients and food. In the design of storage, usability for the high-needs elderly should be closely considered, such as requirements for the shelf height and depth that they can reach as well as the type of storage.

Participants with limited mobility were not able to reach high cupboards or shelves, as the elderly typically can't reach shelves of $1800 \mathrm{~mm}$ or higher, and they can reach shelves of $1650 \mathrm{~mm}$ high but cannot easily reach the rear part (see also 'Wall and elevation design'). Open shelves at a reachable height above the bench are useful; shallow shelves (such as $150-250 \mathrm{~mm}$ deep) are ideal for storing cups and foods such as bottles or containers of coffee, tea, sugar, snacks and 
spices, and deeper shelves are useful for storing, kitchen appliances or cook books. Open shelves (or benches) are also useful for storing some fruit and vegetables that require fresh air at room temperature (often combined with the use of a basket). The use of hooks and railings also effectively increases storage capacity. Having a pantry was preferred by study participants because it could store a great amount at a reachable height and contained various heights of shelves. Having a light in the pantry made it easier to use.

Space under the bench is also useful as storage; however, here again it should be given to a type of storage that is easy for the elderly to reach. For example, a cupboard with just shelves is difficult to reach, particularly the rear section. Drawers are easier than a cupboard. Attention should be given to the height of drawers to accommodate various items, such as cutlery, ingredients with tall containers such as sauces and vinegars, kitchen appliances that are not often used (e.g. baking tools, a sandwich maker), pots and pans and trays. Bins for rubbish and recycling items can be installed in a drawer. In the design of drawers under the bench, mechanisms in the device should prevent the drawer from falling off when residents lean onto it, as this can lead to an injury (see also 8.2.1 'Things to hold onto'). There should be strategies for storage under the bench corner for ease in taking out food and tools, particularly in a small kitchen; solutions include using a 'Lazy Susan' and drawers in the corner (Figure 8-11).
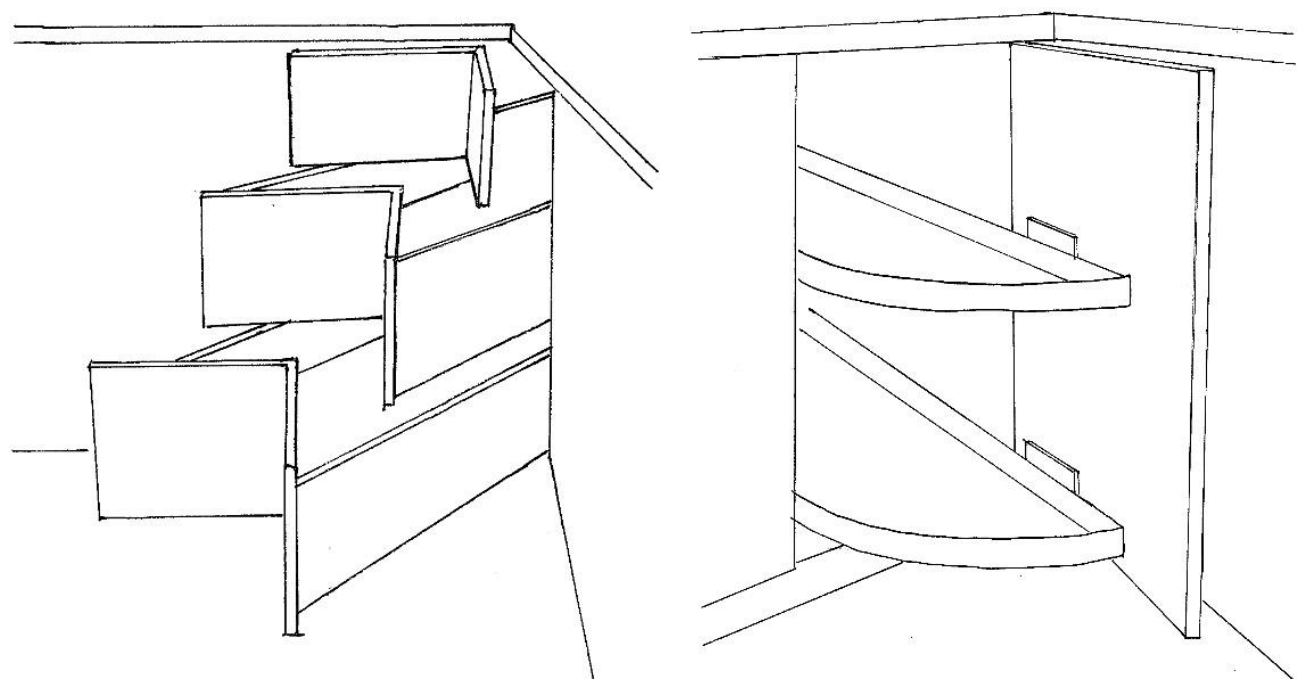

Figure 8-11: Examples of convenient storage under the bench corner

(Corner drawers (left) and Lazy Susan on the door (right))

Even for residents who are provided with three meals a day basically in a communal dining room, it is important to provide storage for some tableware (e.g. cutlery, cups, glasses, dishes) and seasoning, to provide an option of having meals in their private space. 


\section{Dining space}

Bench space for eating in an open kitchen saves those with limited mobility the trouble of carrying a meal to a separate dining space, and also facilitates conversation when having guests over and serving tea or food. The proximity of the dining space to the kitchen contributes greater ease for carrying food. This was particularly important for a wheelchair user in this study, who experienced difficulty operating the wheelchair with one hand if they were holding a dish with the other. Therefore, the layout of a bench between a kitchen and a lounge, as well as the proximity of the kitchen and the dining space, facilitates residents' control and independence.

Some participants had meals at a dining table, while others ate while sitting on the armchair that they used often. This difference in use was influenced by their preferences, as well as the size of the room. For example, those who needed great support in sitting liked to take meals in their armchair. On the other hand, space that could accommodate a dining table was particularly important for those who liked to have meals with guests such as family and friends. Having their own dining table also contributed greater homeliness. There should be flexibility in the design of dining spaces to accommodate preferences for both using a dining table and using an armchair.

Being able to watch TV from the dining space was preferred by most participants and being able to watch TV from the kitchen was also preferred by those who cooked for a long time in the kitchen. The layout of the dining space and kitchen should be able to provide views to the TV while having meals and cooking.

\section{Space for laundering and utility}

The design of space for laundering should provide residents with control in washing and drying. The layout should keep the moving distance short, as well as be user-friendly for those who can't reach high areas and those with mobility aids.

The washing machine should be put close to storage for dirty clothes to be washed to reduce resident movement. Considering dirty clothes are often kept in the bathroom, the space for the washing machine should be laid out in or near the bathroom (see also 'Planar relationships between spaces').

If space for washing is provided in an enclosed room, there should be enough space for turning around with a wheelchair or walker frame. By contrast, cupboards do not require space for turning around inside, which therefore saves space. 
A dryer can be mounted on a wall above the washing machine; however, attention should be given to ensure the height of the controls is within reach. If the dryer is mounted upside down (the lowest part of the dryer door can be $1500 \mathrm{~mm}$ high) they are within reach for elderly residents who can stand. However, for wheelchair users this height is unreachable. Where enough space for drying next to the washing machine cannot be provided, communal dryers can be used. This is also appreciated as an economical solution.

Hanging their laundry indoors and/or outdoors was preferred by some participants, even those who had a clothes dryer. Space for drying laundry should be provided in proximity to the washing machine. A clothesline or a laundry rack should be low enough for residents to reach while seated during the task of hanging up clothes. For example, there should be enough space to stretch out a folding laundry rack in a sunny room. A folding rack attached to a wall can be useful for those who have difficulty with carrying a rack. Privacy is preferred for space for hanging some clothes either indoors or outdoors.

\section{Individual outdoor space}

Design of individual outdoor space has great potential to provide residents with connections, comfort, and opportunities for preferred activities.

It is important for residents to have space to breathe outdoor air, particularly if their unit is small. This space should also facilitate moving around, increasing the chance of exercise. There should be a sunny porch so that residents can enjoy the sunshine. Having a roof or covering can extend the time spent outdoors as they can avoid sunshine that is too strong. Many participants preferred privacy for this space, where they could enjoy sunbathing and personal events such as a barbeque. Clotheslines can also be installed in the private space (See also 'Space for laundering and utility'). Views to neighbours passing by facilitate communication between residents, which leads to a greater sense of safety and can evoke mutual support, so options for spaces that facilitate such views and allow neighbours to approach are desirable. To provide both privacy and views to/from passers-by, ideally two kinds of spaces should be provided, that residents can choose from depending on their mood. Another solution is to use the type of enclosure both providing views to outside while having some effect on preventing passers-by to look inside, such as a screen or trellis.

Having a garden that is easy to maintain was desired and appreciated by several participants. A porch can accommodate a small space for garden beds or pots. To facilitate ease of gardening, attention should be given to a height that supports ease of tasks in gardening such as watering 
and weeding while seated. Provision of a tap can remove the need to carry water from inside of their dwelling. The layout of gardens that can be viewed from the sitting space increases residents' enjoyment. There should be storage for gardening tools, such as a spade, scissors, a watering can and fertiliser, in proximity to gardens.

Some participants liked to have larger gardens for growing fruit and vegetables, such as several sections and a greenhouse. There should be an option of providing such a larger garden depending on preferences in gardening and mobility levels. Community gardens can also be used for this purpose.

\subsubsection{Requirements in the design of the complex}

Through the analysis in Chapter 7, some considerations for the design of the complex emerged. In this section, these considerations are discussed by design elements and design requirements are established. Focussed design elements include; the complex size, unit layout, communal pathways, roadways and carparks, indoor communal areas, and open space.

\section{Complex size}

Attention should be given to the size of a complex in which residents can all know each other, therefore enhancing their sense of homeliness. The survey results showed that residents felt a loss of sense of homeliness as complexes became overly large. To specify the ideal complex size that would provide residents with the best QoL, a more precise survey focusing on this aspect should be conducted; however, based on this study results, the number of units should be no more than 200. In a larger complex, grouping units in some clusters, with common space provided for each, can be an alternative solution.

\section{Unit layout}

In the layout of units, attention should be given to maximising benefits from the sun and surrounding nature, as well as providing optimal privacy from neighbours. Attention should also be given to the layout of units facilitating the quality of care, by enhancing both care efficiency and resident privacy from the staff.

\section{Layout of units for sunshine}

Units should be laid out so that residents' sitting spaces can have north-facing windows (see also 8.2.2 'Sitting space'). East or west-facing windows cannot let in sufficient sunshine for residents, though windows facing northeast or northwest are acceptable. Bedrooms with northeast windows are preferable. 


\section{Layout of units for views and privacy}

The units should be laid out with respect to nature at a distance such as mountains and the sea. Providing a window for just a part of it can lead to great satisfaction. Views of passing people and even cars were also pleasing for many survey participants, if enough distance was assured. Views of activities such as children playing were particularly valued. Ideally, space for activities, such as communal spaces and children's playing area, should be laid out so that residents can view it from their units. In the layout of spaces for activities, communal pathways and roadways, it is important to provide sufficient privacy for residents. For ground-floor units, enough distance between units and paths (for example, using open space) is necessary. Greater privacy is provided on the upper floor if a balcony or long eaves is provided. The space in proximity to units can also be utilised to provide greenery and flowers that provide residents with pleasant views (see also 'Open space').

In the layout of units with respect to other units, units should avoid directly facing each other. The layout of units at an angle is an effective strategy. Distance also matters, and the requirements differ by the type of rooms facing each other, as rooms require different degrees of privacy (e.g. bathrooms and bedrooms requiring greater privacy). For example, resident lounges facing each other at a longer distance of $20 \mathrm{~m}$, can satisfy participants' sense of privacy. The requirements are different in case of kitchens; a kitchen window facing towards a neighbour's window at $6 \mathrm{~m}$ distance did not affect participants' privacy and contributed a greater sense of connection for one resident in the survey. With regard to the distance between units, EVOLVE specifies the distance between neighbouring windows that assures resident safety as more than $22 \mathrm{~m}$ (Lewis et al., 2010), which is a similar outcome to this research.

\section{Layout of units with respect to space for the staff and a care facility}

In complexes with on-site staff offices, while residents generally appreciated the support and sense of safety, the physical environments and staffing models are important to improve resident privacy and familiarity. In the layout of units with respect to space for staff or a care facility, there should be action taken to create physical environments and staffing models that increase the sense of residents' familiarity with the staff, which also increases their sense that staff are reliable. In the survey, in complexes where on-site staff visited residents and provided services and care for them, the presence of numerous staff members generated a feeling of unfamiliarity for residents. In contrast, in a complex where a housekeeper provided three meals a day and visited participants once a day (a different staff member in charge on weekends), residents did not have such feelings. In supported-living units adjacent to a care facility with 43 care units 
(offering rest-home and hospital level care) participants experienced a loss of familiarity. The scale of complexes and staffing models in both care facilities and serviced apartments should be considered, as well as the connection between the two. For example, The Green House concept, developed in the early 2000s, encourages person-directed nursing care in small-scale households with 10 residents, with each household staffed with two staff members performing all household tasks and providing nursing care (Anderzhon et al., 2012; The Green House Project, 2017). ${ }^{32}$ This strategy of clustering combined with the staffing model reduced the number of different staff members caring for one resident. Residential units provided adjacent to the care facility (e.g. retirement village serviced apartments) can be located extending from these care units.

In the layout of the on-site care staff space, it is also essential to consider efficient care provision. For example, the layout of an on-site staff station in proximity to residential units increases efficiency. Even simply providing storage to store medical supplies and other necessities reduces walking distance for staff when dealing with residents' requests. The layout should avoid the feeling of surveillance for residents. Given that one participant didn't like being watched going in and out of their room, the staff space should not be located where staff can see residents going in and out to help remove their feeling of surveillance.

\section{Communal pathways}

Communal pathways are a key to maintain residents' preferred activities and important connections, by facilitating their mobility to communal areas and in and out of the complexes. The safety from the public is also relevant to this theme. Topics of this section include space leading out from individual front doors, outdoor walkways and limiting public access.

\section{Space leading out from individual front doors}

Attention should be given to accessibility between individual space and communal pathways, particularly in the case of units with outdoor-access. It is important to note that concrete slab floors must be at least $150 \mathrm{~mm}$ above permanent paving and $225 \mathrm{~mm}$ above unpaved ground, while suspended timber floors are likely to be at least $400 \mathrm{~mm}$ above ground (MBIE, 2014a). Therefore, to avoid steps, there must be a slope between exterior grounds and resident unit, the gradient of which varies depending on the type of floor. The gradient should be made as small as possible, while the length of one ramp should be within $6 \mathrm{~m}$ (see also 8.2.1 'Floor').

\footnotetext{
${ }^{32}$ In addition, a registered nurse serves two or more households, depending on the shift. Specialists such as therapists, social workers, or doctors come to the households only when needed (Anderzhon et al., 2012).
} 
In the design of an entrance, attention should be given to providing greater homeliness and privacy for residents. For example, individual doors leading directly to the outdoors were preferred over those leading to an indoor corridor, providing participants with a greater sense of homeliness. Particularly indoor corridors where the staff passed frequently reduced the sense of homeliness, and shutting the door leading out to such a corridor was important for participants' privacy. Therefore, the provision of a door leading directly to the outside is recommended where possible; if it is not possible, alternative strategies are necessary to provide greater privacy and homeliness, such as keeping the door at a distance from the corridor that the staff pass frequently (Figure 8-12).

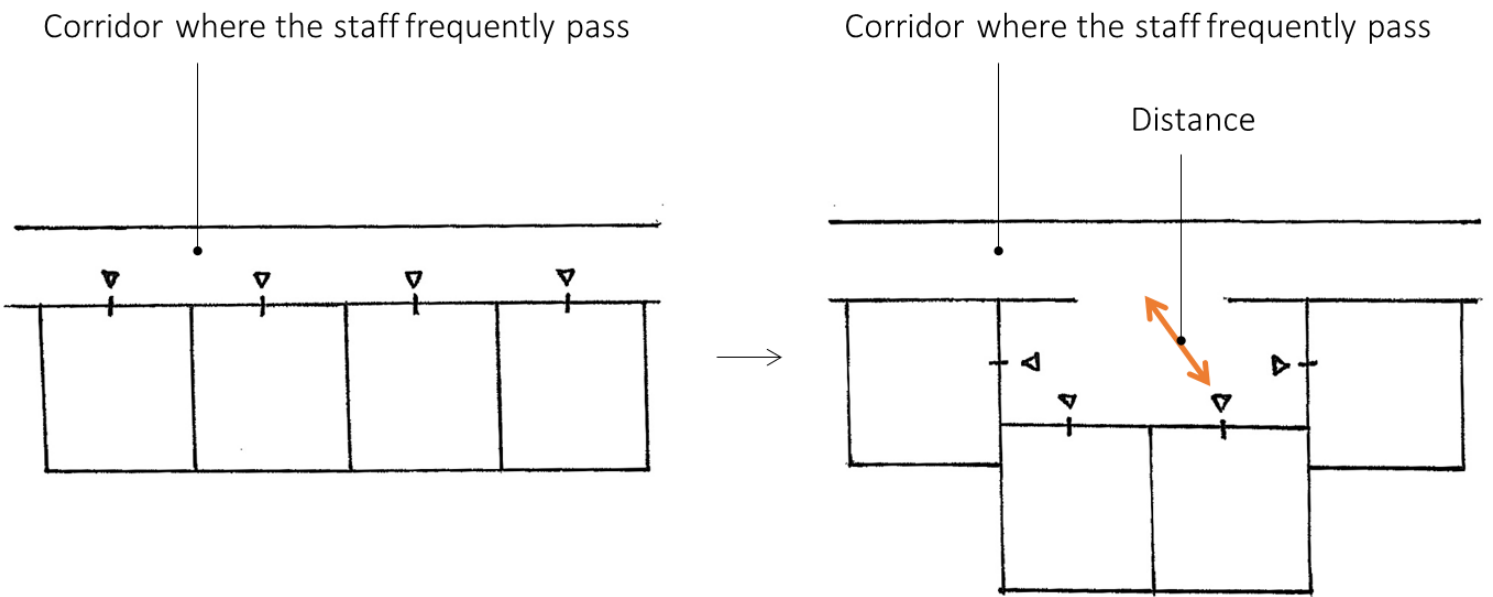

Figure 8-12: Considerations for the unit layout to provide a greater sense of homeliness and privacy

\section{Outdoor walkways}

Walking was an important activity for keeping fit and able, which most participants wanted. Therefore, in the design of walkways attention should be given to environments that facilitate residents' walking activity. Spaces to sit and rest should be placed at least $20 \mathrm{~m}$ intervals for those with severe mobility impairments and those who have pains or get breathless easily. This is a shorter interval for seating required in EVOLVE (Lewis et al., 2010), which is deemed a consequence of the focus of this study only on the high-needs. In the design of walking routes, there should be a variety of paths to meet the needs of the individual's mobility, as well as to avoid monotonous walking experiences. Providing loop routes is preferable, as this can provide more options than just going back and forth on the same paths. Walking routes should be designed so that they extend to the entrance or outside of the complex, which can provide long walking routes for those who are competent, as well as meeting their common desire to go out for a change. Walking is also encouraged by attractive features such as gardens and communal spaces that provide a purpose for visiting. 
For safe walking, there should be no level difference. Attention should be also given to ground finishes; unstable grounds such as grass lawns and slippery materials should be avoided. Outdoor lighting is also important for walking safety, particularly for those with eye impairments who need extra brightness. Footpaths should be wide enough to prevent residents with mobility aids or with limited eyesight from falling over the level difference between the footpath and the roadway. Wide walkways also facilitate walking side-by-side with their family, friend and caregiver. The survey results showed that a footpath of $1.1 \mathrm{~m}$ wide was too narrow for a wheelchair user to walk in a row with another person, or for those with limited eyesight to walk even by themselves. A width of $1.2 \mathrm{~m}$ is recommended by NZS 4121 (Standards New Zealand, 2001); however, it is doubtful if this width is sufficient for residents to walk with a sense of safety. The sufficient width was not made clear from the survey, but one solution is to eliminate any level differences. Without level differences, the footpaths could be indicated by other means, such as using different colours for footpaths and roadways. This is also a solution for eliminating residents' fear of cars when crossing roads (see also 'Roadways and carparks').

\section{Public access}

While a greater sense of connection to the wider community is aspired, it is important to limit unwanted public access to provide a greater sense of security for residents, something they become more sensitive to as they experience the loss of partner and decline in health. Recently, it has been suggested that many conventional retirement villages have been gated with 'one way in and one way out;' which is not desired by the current and future ageing population (Stock, 2018). The strategies for improving resident security without 'gating' includes limiting and clearly defining the space and pathways that allow public access. Given that just prohibiting the public access through the site was not effective in preventing the public to pass (internal pathways were used as shortcuts), the careful layout of pathways is needed so that it avoids creating a shortcut through the site. If the short cut is unavoidable, it would be a good way to make use of the short cut as the pathway allowing the public access. In the case of buildings with indoor-accessed units, it is also effective to limit the number of entrances, while residents are provided with some options for entrances to use (which should be accompanied by the use of security cards or facial recognition system).

\section{Roadways and carparks}

As long roadways allow cars to pick up speed, roads should be kept short. Ideally, there should be no road crossing (see also 'Communal pathways'). In the design of roadways, assuming that residents will walk on them is essential as roads are wider than walkways and easier to walk on for 
those with limited mobility and those with limited eyesight. Speedbumps are a falling hazard, so should not be used. Attention should also be given to roadway width. If roadways are too narrow, visitors (including caregivers) need to park their cars on footpaths, which causes residents trouble with walking. Roadways should be wide enough so that there is space for parking a car on the side while still leaving enough space for other cars to pass. In the layout of a large complex, a confusing road system should be avoided to remove trouble in wayfinding for residents and visitors.

Car parks that could be accessed from participant units through indoor areas or under roofs were preferred, as those with mobility aids had extra trouble in holding an umbrella. In car parks, there should be space for mobility aids where residents can leave wheelchairs or walker frames that they use to approach cars but do not take in the vehicle.

\section{Indoor communal areas}

In this section, indoor communal spaces where residents spend time are discussed. The design of communal space requires a wide range of considerations, such as requirements for accommodating health and mobility issues and for facilitating individual preferences in activities.

Access should be easy for those with limited mobility as they move between communal spaces and residential units. The ability to drive a car generally declines with age and half of the residents who participated in this research did not drive. Communal spaces should be accessible by walking (with or without mobility aids) and by wheelchair or mobility scooter. Therefore, the close proximity between residential units and communal space is advised. In communal spaces, there should be parking space for mobility scooters near the entrance so that residents can transfer from mobility scooters to aids (e.g. wheelchairs). Toilets should also be provided in communal spaces to accommodate those with continence issues.

\section{Large communal space}

One of the main uses of communal space is socialising. For example, many retirement villages and some public- and private-sector rental housing complexes in the survey provided communal tea and/or meals, which were very popular. Given that these meals have great potential to enhance residents' familiarity with other residents and to lead to a greater sense of homeliness, providing a communal space that can accommodate all residents is ideal.

In the determination of size, attention should be given to space for mobility aids. For example, space of approximately $18 \mathrm{~m}^{2}$ was not enough for accommodating 10 people and their walker 
frames, as shown in 7.2.3, even though it meets the requirement calculated with occupant densities defined in NZBC (MBIE, 2014b). ${ }^{33}$ It is important to assure the passage width and space for mobility aids are sufficient when the space also accommodates furniture, not only relying on the calculation based on occupant densities.

Other communal activities requiring relatively large space include: religious activities such as communion; communal exercises such as Tai-chi, yoga and sitting exercises; indoor bowls (which can be played while seated); watching sports on TV; concerts; guest speeches; and accommodating library visits. Required facilities and spaces include a kitchen facility, a TV monitor and storage for furniture and various tools. The activities can involve people from outside of the complex. Providing this space in a location that is easy for the public to use can also facilitate public use. This is recommended, as providing activities for people not only living in the complex but also from outside can foster a sense of connection to the wider community. This space can also provide space for volunteering activities of elderly residents and facilitate their desire for contribution. The integration of the complex into the wider community by opening the communal space to the outside is also an international trend in senior housing, such as extra care housing as referred to in Chapter 3. Spaces for some of these activities can also take place in smaller communal areas described below.

\section{Small communal space near resident units}

Communal space near resident units has great potential to accommodate residents' social activities and possessions that cannot be accommodated in residents' own units. This space can also facilitate various modes of socialising other than simply participating in communal activities. Three types of communal space can be provided near resident units; the communal lounge for residents, the space for guests and gathering and communal storage.

The communal lounge for residents is particularly important in the complex with small units. For example, a communal space for a small number of residents, rather than one large communal space for a large group of residents, can enhance their sense of community, as was seen in the case of providing a communal lounge of $33 \mathrm{~m}^{2}$ for residents in 10 units (RVS3, RVS4). The proximity to resident units greatly encourages visits of those with limited mobility: for example,

\footnotetext{
${ }^{33}$ The minimum floor area is determined using occupant densities defined in NZBC documents. For example, 'Space with fixed seating' is given the occupant density of $1.1\left(\mathrm{~m}^{2} /\right.$ person) for risk group CA (buildings, or parts of buildings where people congregate, participate in group activities, or where personal services are provided, such as halls, recreation centres and public libraries). Using this occupant density, the minimum floor area to accommodate 100 people is calculated as $1.1 \times 100=110 \mathrm{~m}^{2}$ (MBIE, 2014b).
} 
in the case of RVS3, RVS4, the distance between the communal lounge and the front door of residents who frequently used it was $9 \mathrm{~m}$ or less. The spontaneous use of spaces is motivated by a sunny area.

As well as communal lounge for residents, space is required for activities that cannot be accommodated in residents' individual units; examples include hosting personal guests and gatherings of personal hobby groups (e.g. 10 people) to take part in activities such as playing cards, bible studies and handcraft or needlework, where some residents can teach or help others with their skills. In such areas, it is important to provide optimal storage for tools and a kitchen facility for having tea and pot-luck meals. This space should preferably be separate from a communal lounge for residents, as space may be occupied by other residents, and social activities can be disrupted by the TV sound. Small communal spaces, or even an alcove in a corridor, can also accommodate some amenities such as bookshelves where residents can borrow books. In the layout of bookshelves and chairs, sufficient space for those with mobility aids to approach should be ensured. These amenities can also lead to facilitating residents' movement.

In space near resident units, it is important to provide storage for mobility scooters and wheelchairs. It is also important to provide collective rubbish and recycling bins in proximity to units, and these should be easily accessible (no level difference, avoidance of a hinged door). Depending on the model of care, dining space, kitchen facilities, laundry space (containing dryers and tubs) and storage for cleaning tools can be located near resident units.

\section{Communal space for specific use}

Some activities require special rooms and spaces. For example, in the survey, one participant wanted an art room, which allowed them to create some mess from their activities and to leave their work. A sink and storage for tools are necessary for this space. A soundproof music room can be also useful; one participant played the piano for hand exercises to delay the progress of his disease. Given that live music entertains other residents, the door of a music room could be designed so that is can be kept either wide open or closed.

Other spaces for special use include a café for buying food or coffee and visiting with a friend, a swimming pool for walking exercises and a spa to enjoy the warmth. Communal storage rooms are preferred to store items that are not often used (see also 8.2.2 'Storage'). 


\section{Open space}

Open space, such as that between individual units and between units and external pathways, plays an important role to provide residents with optimal privacy and preferred views. It can provide pleasant views for pedestrians as well.

Trees and plants that bloom or attract birds are preferred, such as cherry, cyprus, kowhai, flax and daffodils, to name a few. It is desirable to place them where they can be viewed from windows of resident units and residents' sitting spaces. In the choice of species, attention should be paid to the size and leaf cover that will not shield the sunshine from resident units, as well as orientation to the sun. Planting trees that attract birds can provide residents with a chance to feed birds. However, birds also dirty external walls and grounds with droppings, which may cause distress for neighbours. There should be some distance between the space for feeding birds and resident units.

Given that visits from children are enjoyed by many elderly people, space for children playing is of great use. There was also a preference for viewing children. For such people, having a play area for children on the site is suggested (see also 'Unit layout').

\subsection{Design framework}

Through the analysis of the relationships between the QoL and the physical environments and the discussion for the design requirements, a framework that can provide a holistic picture of the design of housing that provides the greater QoL for the high-needs elderly has been developed. The framework shows themes and requirements for design and related themes for QoL (Tables $8-2,8-3)$.

Table 8-2: Framework for the design of housing that improves the QoL of the high-needs elderly (Asterisks $\left(^{*}\right.$ ) show optional requirements to be applied depending on resident needs and preferences and care models)

\begin{tabular}{|c|c|}
\hline Design elements and requirements & $\begin{array}{l}\text { Relevant } \\
\text { themes } \\
\text { for QoL }\end{array}$ \\
\hline \multicolumn{2}{|l|}{ Basic requirements } \\
\hline \multicolumn{2}{|l|}{ Floor/ground } \\
\hline \multicolumn{2}{|l|}{ Level differences } \\
\hline Avoid level differences (e.g. steps, a tiny level difference, those created by thresholds) & $\begin{array}{l}1-1,1-4, \\
2-1,2-2, \\
3-4\end{array}$ \\
\hline \multicolumn{2}{|l|}{ Slope and stableness } \\
\hline Avoid slopes with a gradient of more than 1 in 12 & $1-1$ \\
\hline
\end{tabular}




\begin{tabular}{|c|c|}
\hline Avoid slopes longer than $6 \mathrm{~m}$ & $1-1$ \\
\hline Avoid unstable ground (grass lawn etc.) & $1-1$ \\
\hline \multicolumn{2}{|l|}{ Floor materials } \\
\hline $\begin{array}{l}\text { Choose floor materials that are easy to clean and warm to touch, as well as providing grip for mobility } \\
\text { aids }\end{array}$ & $\begin{array}{l}1-1,1-6, \\
5-2\end{array}$ \\
\hline Choose dark floor colours, that don't show stains and dirt by wheels & $1-6$ \\
\hline Choose materials that are not slippery in the kitchen and bathroom & $1-1$ \\
\hline Firmly fix carpets to substrate materials & $1-1$ \\
\hline \multicolumn{2}{|l|}{ Doors } \\
\hline Avoid unnecessary doors (e.g. between kitchen and lounge, between the bathroom and washroom) & $1-1$ \\
\hline Ensure sufficient door width for wheelchair users (e.g. minimum 810 mm is recommended) & $1-1$ \\
\hline Avoid hinged doors, provide sliding doors & $1-1,3-1$ \\
\hline \multicolumn{2}{|l|}{ In providing a hinged door, } \\
\hline Consider the door swing direction that doesn't interfere with user movement & $6-1$ \\
\hline Provide sufficient side panel, for walker-frame users: minimum 600 mm & 1-1 \\
\hline Avoid door closers & $1-1$ \\
\hline \multicolumn{2}{|l|}{$\begin{array}{l}\text { If a door closer is unavoidable, provide an electromagnetic free-swing closer or an } \\
\text { electromagnetic hold-open, or at least, a magnetic hold-open }\end{array}$} \\
\hline Avoid doors that require extra strength to shut & $6-3$ \\
\hline \multicolumn{2}{|l|}{ Provide level entry } \\
\hline Adopt strategies for water-tightness at external doors (e.g. the use of a gutter) & $1-1$ \\
\hline Eliminate any raised lip (e.g. $10 \mathrm{~mm}$ high) & $1-1$ \\
\hline If it cannot be avoided, fill the tiny level difference with a slope & $1-1$ \\
\hline Avoid heavy doors & $3-3,2-1$ \\
\hline Provide door handles at a height of 1000 - 1200 mm high & $1-1$ \\
\hline Avoid vision panels on the door leading out to the indoor corridor for greater privacy at night & $3-3$ \\
\hline Provide individual external doors & $3-2$ \\
\hline \multicolumn{2}{|l|}{ Passage } \\
\hline Provide sufficient passage width for wheelchair users (e.g. $810 \mathrm{~mm}$ wide) & $1-1$ \\
\hline Avoid wall corners that impede resident moving & $1-1$ \\
\hline Avoid a passage to the toilet that requires a change in direction & $1-2$ \\
\hline \multicolumn{2}{|l|}{ Fixtures } \\
\hline \multicolumn{2}{|l|}{ Electrical outlets } \\
\hline $\begin{array}{l}\text { Ensure the height of electrical outlets (e.g. light switches) are easy to use: the typical height should be } \\
1000-1200 \mathrm{~mm} \text { high. }\end{array}$ & $1-1$ \\
\hline $\begin{array}{l}\text { Ensure the appropriate layout of outlets for appliances typically required by the elderly (e.g. electrically } \\
\text { adjustable armchairs, beds) }\end{array}$ & $5-1$ \\
\hline \multicolumn{2}{|l|}{ Tap } \\
\hline Choose lever-type tap handles, avoid the round types & $1-1$ \\
\hline Avoid taps without a mixer & $1-4$ \\
\hline \multicolumn{2}{|l|}{ Heating } \\
\hline Consider the location of a heater with respect to the sitting spaces & $2-3$ \\
\hline $\begin{array}{l}\text { Choose a heating type that is suitable for sitting spaces (e.g. a heat pump mounted in the low area, } \\
\text { underfloor heating) }\end{array}$ & $2-3,5-2$ \\
\hline Provide a heater in the bathroom & $5-2$ \\
\hline \multicolumn{2}{|l|}{ Lighting } \\
\hline Provide light in workspaces and storage & $5-3$ \\
\hline Provide outdoor lights for walkways & $2-2$ \\
\hline \multicolumn{2}{|l|}{ Curtains and blinds } \\
\hline Provide curtains and blinds depending on the level of privacy required & $5-1$ \\
\hline \multicolumn{2}{|l|}{ Things to hold onto } \\
\hline Provide things to hold onto in the passage & $1-3$ \\
\hline Ensure firmness of walls, fixtures and furniture (heavy chairs sofas) for use as things to hold onto & $1-3$ \\
\hline Provide things to hold onto continuously & $1-1$ \\
\hline Provide things to hold onto by both hands in the bathroom (walls and fixtures) & $6-1$ \\
\hline $\begin{array}{l}\text { Provide fixtures to support residents in sitting down and standing up in sitting spaces (e.g. grab bars, } \\
\text { handrails, armrests, level surfaces) }\end{array}$ & $1-1,1-3$ \\
\hline
\end{tabular}


Ensure firm fixture of things to hold onto (e.g. install backing inside the wall for attaching grab handles)

For top drawers under kitchen benches and vanity counters, use slides that can bear a heavy load

Things to sit on

Avoid seating that is too low. Provide seats at least $450 \mathrm{~mm}$ high $1-1$

Choose seating with armrests

Provide spaces for a rest on outdoor pathways

Provide spaces for a rest on outdoor pathways

Consider chair types (e.g. swivel chair helps to change the direction and to move between spaces)

\section{Space for mobility aids}

Space for a wheelchair and a walker frame

Adopt a spatial configuration that avoids the need to turn around in a small space

(If providing enclosed spaces) Sufficient space for turning around with a wheelchair and a walker frame

(e.g. minimum a $1500 \mathrm{~mm}$ diameter turning circle)

$1-5,2-1$,

6-2

Storage for mobility scooters and wheelchairs

Provide accessibility in the storage for mobility aids (e.g. avoid level difference)

$1-1$

Provide indoor or at least half-enclosed space with electrical outlets

$1-1$

Provide space for a mobility scooter to turn around: $2050 \mathrm{~mm}$ - radius circle

Provide space for approaching with other aids (e.g. a walker frame)

$1-1$

In providing storage in communal areas, provide appropriate space for leaving other aids used to approach the storage (e.g. a walker frame, walking sticks)

\section{Requirements in the design of the individual unit}

\section{Unit size}

Keep housing units small enough to ensure ease of maintenance

$1-6,5-2$

Meet the needs that typically require an increase in the floor area, to name a few;

$1-1,6-1$,

- Accommodating mobility aids (e.g. a wheelchair, a walker frame)

$3-2,3-1$

- Accommodating caregivers

- Accommodating personal guests (e.g. separation of the lounge and the bedroom)

\section{Wall and elevation design}

Avoid the layout of low furniture (e.g. low tables) in the centre of the room

Avoid a corner angle that is not a right angle for walls that accommodate furniture

Provide sufficient empty walls for furniture

Provide an option of wall space for accommodating TV (e.g. mount a TV on the wall)

$1-1$

*Provide space for desks/tables for specific uses (a table for needlework and storage for tools) depending on individual preferences

*Provide space for an exercise machine depending on individual preferences

*Provide space for a piano in the individual unit or communal space

*Provide space for extra chairs

*Provide space for a PC desk (often with a printer) /writing desk

Provide space for display cabinets and bookshelves

*Provide extra empty walls for nostalgic furniture (e.g. bedside drawers, longcase clock, hand-made

4-1

4-1

table) and for displaying tapestries, paintings and photographs, depending on individual preferences

*Provide space for tables/bases for indoor plants

Provide shelves accommodating highly-used items at 230 - $1350 \mathrm{~mm}$ high adjacent to the sitting space,

at $1000-1350 \mathrm{~mm}$ high in other spaces

Provide electrical outlets often operated by residents (e.g. light switches), and door handles at 1000 -

1-1, 1-4,

$1200 \mathrm{~mm}$ high

Provide an emergency alarm within reach following a fall (e.g. $500 \mathrm{~mm}$ above the floor)

Provide work surfaces (e.g. benches, counters, tables, desks) to use while seated (less than $775 \mathrm{~mm}$ )

Provide open space beneath the bench/sink to accommodate legs when seated

Avoid too low seats, provide seats at 450-550 $\mathrm{mm}$ high

Provide sufficient sound insulation for external and intertenancy walls

\section{Storage}

Enclosed storage/wardrobe

Provide sufficient size of wardrobes (e.g. min $1.7 \mathrm{~m}$ wide per person)

Provide appropriate shelves in enclosed storage and wardrobes (e.g. for shoes, bags, shawls/scarves)

Provide enclosures for storage of clothing adjacent to a passage 


\begin{tabular}{|c|c|}
\hline Provide storage for linen and bedding & $4-3$ \\
\hline Provide light in enclosed storage and wardrobes & $5-3$ \\
\hline \multicolumn{2}{|l|}{ Shelves } \\
\hline Provide shelves at a height that is within reach & $\begin{array}{l}1-1,1-4 \\
4-3,4-4\end{array}$ \\
\hline \multicolumn{2}{|l|}{ Provide shelves at the height of no more than $1650 \mathrm{~mm}$ for those standing } \\
\hline Provide shelves at the height of $230-1350 \mathrm{~mm}$ for seated persons & $1-4$ \\
\hline Provide shelves with appropriate depth (reduce the depth of high shelves $1650 \mathrm{~mm}$ high and above) & $4-3$ \\
\hline \multicolumn{2}{|l|}{ Railings } \\
\hline Provide railings on walls to facilitate the use of hooks for greater efficiency in storing & $1-4$ \\
\hline \multicolumn{2}{|l|}{ Drawers } \\
\hline Use drawers in low areas, rather than cupboards where possible & $1-4$ \\
\hline Design drawer handles for those with limited hand strength & $1-1$ \\
\hline For top drawers under kitchen benches and vanity counters, use slides that can bear a heavy load & $3-1$ \\
\hline Provide drawers for storing household items, and tools for hobbies (e.g. needlework) & $2-1,4-3$ \\
\hline \multicolumn{2}{|l|}{ Accommodating items needing larger space } \\
\hline Provide storage for long items (e.g. walking sticks, picker uppers) within reach & $1-1$ \\
\hline Provide storage for a vacuum cleaner & $4-3$ \\
\hline \multicolumn{2}{|l|}{ Other } \\
\hline Provide storage for outdoor wear (e.g. coats, hats) near the entrance, which includes hooks & $4-3$ \\
\hline Provide storage for gardening tools in proximity to gardens & $2-1$ \\
\hline $\begin{array}{l}\text { Provide sufficient storage for items seldom used (e.g. extra storage, use of spare rooms, communal } \\
\text { storage) }\end{array}$ & $4-4$ \\
\hline Provide storage for rubbish/recycling items (e.g. under kitchen bench, garage) & $1-4,1-6$ \\
\hline \multicolumn{2}{|l|}{ Planar relationships between spaces } \\
\hline Lay out a bathroom/toilet in proximity to a bedroom, sitting space, a kitchen and space for laundering & $\begin{array}{l}1-2,1-5, \\
6-2\end{array}$ \\
\hline Lay out the sitting space in proximity to the front door & $3-2$ \\
\hline Create separation of the bedroom from the lounge & $3-2$ \\
\hline Layout of the garage in proximity to the kitchen where the garage is provided adjacent to/in the unit & $1-7$ \\
\hline Layout of spaces to avoid turning around in small areas & $1-1$ \\
\hline \multicolumn{2}{|l|}{ Sitting space } \\
\hline \multicolumn{2}{|l|}{ Micro space planning to facilitate various activities while seated } \\
\hline Assume the layout of an armchair for the space where the resident spends most time during the day & $2-3,5-1$ \\
\hline $\begin{array}{l}\text { Ensure space that can accommodate some variations in the type of chair depending on residents' } \\
\text { preferences (e.g. sitting on a side chair at a desk) }\end{array}$ & $5-1$ \\
\hline Provide an option of an armchair layout by the north-facing window & $2-3$ \\
\hline $\begin{array}{l}\text { Provide level surfaces and storage areas (e.g. shelves, tables, desks, trolleys) to accommodate various } \\
\text { items (e.g. telephone/handset, remote controls, glasses, medicine, cups of tea, pens and paper) within } \\
\text { reach from sitting space }\end{array}$ & $\begin{array}{l}2-1,2-3, \\
3-4\end{array}$ \\
\hline *Provide light for reading & $2-1$ \\
\hline \multicolumn{2}{|l|}{ Views and privacy } \\
\hline Lay out the sitting space with respect to the TV & $\begin{array}{l}2-1,2-3, \\
3-3\end{array}$ \\
\hline Lay out the sitting space with respect to PC screen & $2-3$ \\
\hline Lay out the front door within the sight from the sitting space & $3-2$ \\
\hline Lay out the sitting space with respect to windows for outside views & $2-3$ \\
\hline Provide views to the directions from which visitors (guests, staff) approach & $3-2,6-3$ \\
\hline *Provide views to personal gardens (e.g. beds and pots in the porch) & $5-4$ \\
\hline * Lay out windows with respect to the direction of mountains/vegetation or seas & $5-4$ \\
\hline Provide views to people and moving objects & $2-3,5-4$ \\
\hline Limit the window height to shield views from outside & $2-3$ \\
\hline \multicolumn{2}{|l|}{ Sunshine and warmth } \\
\hline $\begin{array}{l}\text { Provide north-facing windows, avoid windows facing south or east and west (those facing northeast and } \\
\text { northwest are acceptable) }\end{array}$ & $2-1$ \\
\hline Provide a heater in proximity to the sitting space & $2-3,5-2$ \\
\hline
\end{tabular}


Choose the heater type that is suitable to warm lower areas (e.g. floor heating, a heat pump mounted 2-3, 5-2 on the low part of the wall)

Space for seats for guests

Provide space for seats for guests, which include immovable furniture (e.g. sofas) and movable chairs

Bedroom

Provide doors and curtains to shield light

5-1

Provide windows that bring sufficient natural light in a bedroom for residents to stay during the day

Provide northeast windows that let in morning sun

Provide sufficient space for furniture and storage (up to two beds, wardrobe/s, drawer/s, bedside

table/s)

*Where a couple living together, provide sufficient space for one member to spend their time in for

their greater privacy from each other

Provide sufficient size of wardrobes (at least $1.7 \mathrm{~m}$ wide for one person)

Provide appropriate shelves in wardrobes (e.g. for shoes, bags, shawls/scarves)

*Provide a dressing table adjacent to the bathroom (typically for female residents)

\section{Extra rooms}

Space for guests

Provide an option of space that can accommodate up to ten people

$3-2$

Provide space for extra chairs

*Provide an option of space for children, separate from the residents' space

$3-2$

Provide space for an extra bed or sofa bed for guests (e.g. in the lounge)

$3-2$

Garage

Provide sufficient garage width for a walker frame when moving beside the car and putting storage and

$1-6,1-7$,

other items (e.g. $4 \mathrm{~m}$ wide)

Assume greater flexibility in use

$3-2$

Provide insulation and appropriate windows for an indoor garage adjacent to resident units

$3-2$

Accommodating those living as a couple

Provide separate space for greater privacy between a couple

Provide appropriate space for drying laundry indoors, such as sufficient space for a laundry rack or space $1-5$

for hanging ropes (e.g. in a garage, bedroom, spare room)

*Provide an option of attaching a conservatory

Ensure that the inner room has windows on other wall/s when providing a conservatory

\section{Bathroom}

Floor

Avoid level differences at the entry of shower space

$1-3,6-1$

Choose the floor material for slip resistance and ease of cleaning (e.g. design of the form of the surface)

$1-1,1-6$

Avoid creating a steep slope for drainage that causes user fears for safety

Consider the type and location of a drain for rapid drainage (e.g. choice of a trench drain)

$1-3,6-1$

Shower enclosure

Choose the type of enclosure that limits water flow as well as moisture (using a curtain is not

$1-3,6-1$

appropriate)

Choose a solid enclosure that can provide support for residents

$1-3$

Choose the enclosure material that is easy to clean (avoid a curtain, that is typically prone to mould)

$1-6$

Provide the door so that the opening direction doesn't affect caregiver movements while care is

provided with the door open

Provide the door so that water dispersion doesn't affect dry items (e.g. towels, clothes, shoes)

Choose the enclosure material to obscure caregiver views for greater privacy and safety of residents

Install an enclosure so that it can be removed when needs arise in the future

Provide sufficient space to accommodate both the resident and the caregiver ( $1 \mathrm{~m} \times 1 \mathrm{~m}$ space is not enough)

Space for undressing/drying/dressing

Provide sufficient space for a caregiver to dry a resident (an oval-shaped space of at least a $1.2 \mathrm{~m} \times 1.7$ m)

Provide space for dry items such as towels, clothes and shoes adjacent to the drying space 
In providing a folding/fixed chair, design it so that it allows the space both in front and behind the seated 1-3 person for their caregiver to assist washing them, and provide things to hold onto (e.g. armrests)

Things to hold onto

Provide fixtures to support residents in moving, sitting down and standing up (e.g. grab bars, handrails) 1-3

Provide things to hold onto when moving by both hands where residents can't use their mobility aids $6-1$

Consider the appropriate size and shape of rooms that provide walls and fixtures to hold onto with $1-3$

both hands

Provide things to hold onto continuously

Space for mobility aids

Provide sufficient space for a walker frame that doesn't block caregiver movements (e.g. avoid blocking 6-1

the doorway)

Provide sufficient space in front of the toilet that can be kept dry

Fixtures

Provide a hose to the shower head

6-1

Provide a shower mixer lever and shower hose in proximity to the seated person

$1-3$

Locate a shower mixer lever and shower hose at distance to avoid the hose being caught by the lever

$1-3$

Provide a heater with respect to the space for drying

$5-2$

Provide an emergency alarm within reach following a fall

$1-3$

Storage

Provide sufficient storage for items (e.g. towels, toilet paper, bottles of hand or body wash and

shampoo, boxes of soap, lotion or cream and diapers or pads)

Provide shelves and vanity counter at a height suited for wheelchair users/ seated persons

Kitchen/dining space (for those preparing at least one meal per day, unless otherwise noted)

Space for a microwave oven

Provide space for a microwave oven at the height that is easy to reach

1-4

Provide a level surface immediately in front of the microwave oven

Space for a fridge-freezer

Provide space that can accommodate a normal-sized fridge-freezer (600 $\mathrm{mm}$ wide or wider)

1-4

* Provide space for mini-fridge in a raised position or drawer-type fridge under the bench for those who

are provided with three meals a day

Lay out adjacent walls so that the fridge door opens more than 90 degrees

Kitchen facilities

Install hot plates

$1-4$

*Provide optional space for installing a mini-oven, normal oven, or double oven

$1-4$

*Provide optional space for installing a small drawer-type dishwasher in proximity to the sink

$1-4$

Bench space for meal preparation

Provide a bench with a sufficient space to comfortably accommodate daily-use tools (e.g. an electric 1-4

kettle, a toaster), as well as provide enough open space for tasks

Provide a bench of at least $700 \mathrm{~mm}$ deep

*Provide space for a slow cooker/rice cooker depending on preferences

$1-4$

*Provide space for a special cutting board

$1-4$

Lay out spaces such as storage, a fridge-freezer, a sink and hot plates in proximity to each other

$1-4$

$1-4$

Consider the bench shape that keeps the distance between spaces short (e.g. an L-shaped bench is better than a rectangular bench)

Provide a bench that provides continuity and does not require turning around (avoid an 'island kitchen' 1-4

with two parallel benches)

Provide open space in front of the bench, with sufficient size for a wheelchair user to turn around as well $6-2$

as accommodating a caregiver

Provide a bench with the height suited for cooking while seated: lower than $730 \mathrm{~mm}$

Provide open space beneath the bench/sink to accommodate legs when seated 1-4

Provide sufficient bench space adjacent to the sink for storing dirty dishes $6-2$

Provide a light with respect to the space for meal preparation $5-3$

Storage

Provide storage at the height that is within reach

$1-4$

Provide shelves within reach from the sitting position

$1-4$ 
Provide open shelves: shallow shelves (e.g. 150-200 mm deep) for storing cups and foods such as bottles 1-4 or containers of coffee, tea, sugar, snacks and spices, deeper shelves for kitchen appliances, cook books and food

\begin{tabular}{ll}
\hline Use hooks and railings for greater efficiency in storing & $1-4$
\end{tabular}

Mainly use drawers for storage under the bench $1-4$

Provide drawers of various height to store items such as cutlery, ingredients with tall containers (e.g. $\quad$ 1-4 bottles of sauces and vinegar), kitchen appliances, pots and pans and trays

*Provide storage for some tableware and seasoning even for residents who are provided with three $1-4$ meals a day

Consider strategies to efficiently use the space under the bench corner (e.g. corner drawers, Lazy Susan 1-4 on the door)

Provide bins for rubbish and recycling items under the bench, near the space for meal preparation 1-4, 1-6

Dining space

Provide options for the layout of dining space in proximity to the kitchen 1-4

*Provide an option of putting a personal dining table and chairs $\quad 1-4,3-2$

*Provide an option of taking meals in an armchair

Provide a table or bench between the kitchen and the lounge, which mitigates the trouble of carrying

$1-4,2-3$ meals

Provide views to the TV

Space for laundering and utility

Space for washing and utility

Provide a washing machine in proximity to the storage for items to be washed

$1-5$

Provide a tub/sink in proximity to a washing machine

$1-5$

Install a tub either in private dwellings or in communal areas

$1-6$

Provide storage for cleaning tools

Provide sufficient space for a walker-frame user to turn around

$1-6$

Choose the type of door/enclosure for a washing machine that is easy to use (e.g. cupboard etc.)

$1-5$

pace for drying

Provide space for a clothes dryer either in individual houses or in communal areas

$1-5$

Lay out clotheslines/a laundry rack in proximity to the washing machine 1-5

*Provide a wall-mounted folding rack

Individual outdoor space

Porch and outdoor sitting space

Provide sunny outdoor space (e.g. a north-facing porch)

2-2, 5-2,

$5-4$

Provide a roof for covering to provide shade

$5-2$

Consider providing both privacy and views to pathways: provide privacy for private activities (e.g.

1-5, 3-3,

sunbathing) and drying clothes, and provide views for greater sense of connection

$5-2,5-4$

Provide two spaces; one providing privacy (e.g. not facing a street), and the other allowing views

Provide the type of enclosure providing both privacy and views (e.g. trellises)

*Provide a pathway and space that allow for others approaching for those who prefer

Provide porches for feeding birds depending on individual preferences, not close to houses to avoid the 5-4 mess caused by birds

Personal garden

Provide the size and type of gardens suitable for their ability (e.g. garden beds/pots, several

sections/only in the porch)

Provide raised garden beds or space for pots that are suitable for a seated person

2-1

Provide a tap in the porch

Provide storage for gardening tools in proximity to gardens

Provide an option of having a larger garden depending on individual preferences

Requirements in the design of the complex

Complex size

Limit complexes to maximum 200 units for maintaining a sense of homeliness

Unit layout

Layout of units with respect to the sunshine

Lay out the sitting space facing to the north

*Provide northeast-facing windows in the bedroom if preferred 
Layout of units for views and privacy

* Lay out units with respect to surrounding nature (mountains, sea)

Lay out units that provides views to communal areas and pathways

Provide sufficient distance between units and communal pathways for greater privacy

Use level differences for greater privacy

Utilise the space in proximity to the unit for providing pleasant views for residents (e.g. greenery)

Avoid directly facing each other for greater privacy, lay out units at an angle

Provide sufficient distance between units

Ideally $20 \mathrm{~m}$ for units where lounges are facing each other

$6 \mathrm{~m}$ or more, where only a kitchen window is facing to other house windows

Avoid layout of rooms that require greater privacy near windows and outdoor pathways

Layout of units with respect to space for the staff and a care facility

Provide strategies in the unit layout to keep the staff number caring for one person small (e.g. clustering of 6-3

care units, combined with an optimal staff model)

Lay out units to ensure the proximity between individual units and the staff station

Lay out storage for medical/other supplies near resident units

Lay out units that are out of sight from the staff station

\section{Communal pathways}

Space leading out from individual front doors

Ensure accessibility of units with outdoor-access (level differences, the gradient and the length of ramps)

Provide an individual door leading directly to the outdoors, where possible

Provide some strategies to improve resident privacy and homeliness for resident units leading out to a

corridor where the staff frequently pass

Outdoor walkways

Provide spaces for a rest at short intervals (at least every $20 \mathrm{~m}$ )

Eliminate a level difference between footpaths and roadways

Use other means to indicate footpaths (e.g. distinction by using different colours)

Use ground materials that are stable and not slippery, avoid grass lawn $2-2$

Provide a variety of walking routes that can extend to the entrance or outside of the complex $2-2$

Avoid road crossing

Provide outdoor lights

Provide attracting features (e.g. beautiful gardens)

Limiting public access

Clearly define the public-accessed area and pathways

Avoid creating pathways that can be used as shortcuts

If a shortcut is unavoidable, make use of the shortcut as the pathway allowing the public-access

Limit the number of entrances, yet provide some options

Internal roadways and car parks

Avoid long roadways to reduce the car speed

Avoid using speedbumps

Provide roads wide enough to allow parking cars beside where applicable

Avoid a confusing road system (e.g. using too many one-way roads)

Indoor communal areas

Lay out communal space in proximity to resident units

Provide space for mobility aids and wheelchairs

Provide toilets in communal space

Large communal areas

Provide communal space that can accommodate all residents and their mobility aids for communal

meals/tea

Provide space for various communal activities (communion; communal exercises; indoor bowls; watching

Provide a kitchen facility, a TV monitor and storage for furniture and various tools

Lay out communal space in a location that is easy for the public to use

Small communal spaces near resident units

Provide communal lounges for residents in close proximity to resident units 


\begin{tabular}{|c|c|c|}
\hline \multicolumn{3}{|l|}{ Provide sunny space } \\
\hline \multicolumn{2}{|c|}{$\begin{array}{l}\text { Provide small space for hosting personal guests and for gatherings of personal hobbies separately from the } \\
\text { communal lounge for residents, which is particularly important where the unit is small }\end{array}$} & $2-4,3-2$ \\
\hline \multicolumn{2}{|c|}{ Provide bookshelves in small lounges and alcoves } & $2-1$ \\
\hline \multicolumn{2}{|c|}{ Provide shelves within reach from residents } & $2-1$ \\
\hline \multicolumn{2}{|c|}{ Provide communal rubbish/recycling bins in accessible areas, where necessary } & $1-6$ \\
\hline \multicolumn{2}{|c|}{ Provide communal laundry (dryers and tubs) and storage for cleaning tools, where necessary } & $4-3$ \\
\hline \multicolumn{3}{|c|}{ Communal space for specific use } \\
\hline \multicolumn{2}{|c|}{ *Provide an art room that allows dirt from art activities, depending on individual preferences } & $2-1$ \\
\hline \multicolumn{2}{|c|}{ *Provide a music room, depending on individual preferences } & $\begin{array}{l}2-1,2-2 \\
3-3\end{array}$ \\
\hline \multicolumn{3}{|c|}{ Provide soundproof walls and a door that can be either closed or wide open } \\
\hline \multicolumn{2}{|c|}{ *Provide an on-site shop and a café } & $1-7,3-2$ \\
\hline \multicolumn{2}{|c|}{ *Provide a pool and a spa, depending on individual preferences } & $2-2,5-2$ \\
\hline \multicolumn{2}{|c|}{ Provide communal storage rooms for resident items seldom used } & $4-4$ \\
\hline \multicolumn{3}{|c|}{ Open space } \\
\hline \multicolumn{3}{|l|}{ Trees and plants } \\
\hline \multicolumn{2}{|c|}{ Lay out trees and flowers with respect to resident windows } & $5-4$ \\
\hline \multicolumn{2}{|c|}{ Choose trees that bloom and attract birds } & $5-4$ \\
\hline \multicolumn{2}{|c|}{ Choose trees by the size and leaf coverage that will not affect resident access to the sun } & $5-4$ \\
\hline \multicolumn{3}{|c|}{ Space for children } \\
\hline \multicolumn{2}{|c|}{ Provide children playing area, depending on individual preferences } & $3-2$ \\
\hline \multicolumn{3}{|c|}{ Table 8-3: Themes for QoL (the legend for Table 8-2) } \\
\hline Theme1: Control in daily basic activities & $\begin{array}{l}\text { 1-1 Control in basic movement } \\
1-2 \text { Control in going to the toilet } \\
1-3 \text { Control in showering } \\
1-4 \text { Control in cooking and dining } \\
1-5 \text { Control in laundering } \\
1-6 \text { Control in maintaining the home } \\
1-7 \text { Control in shopping } \\
1-8 \text { Control in collecting mail }\end{array}$ & \\
\hline Theme 2: Meaningful leisure activities & $\begin{array}{l}\text { 2-1 Continuing familiar activities } \\
\text { 2-2 Staying active and able } \\
\text { 2-3 Engagement in personal activities in private space } \\
\text { 2-4 Contribution }\end{array}$ & \\
\hline Theme 3: Meaningful relationships & $\begin{array}{l}\text { 3-1 Living with partner } \\
\text { 3-2 Having personal guests } \\
\text { 3-3 Relationships with other residents } \\
\text { 3-4 Connection to the wider community }\end{array}$ & \\
\hline Theme 4: Maintenance of possessions & $\begin{array}{l}\text { 4-1 Maintenance of furniture } \\
\text { 4-2 Maintenance of nostalgic objects } \\
\text { 4-3 Storing day-to-day items } \\
\text { 4-4 Storing items seldom used }\end{array}$ & \\
\hline Theme 5: Comfort & $\begin{array}{l}\text { 5-1 Relief from fatigue and pains } \\
\text { 5-2 Warmth and sunshine } \\
\text { 5-3 Light } \\
\text { 5-4 Access to nature }\end{array}$ & \\
\hline Theme 6: Quality of care & $\begin{array}{l}\text { 6-1: Quality of care in assisted showering } \\
\text { 6-2: Quality of care in household tasks } \\
\text { 6-3: Quality of care from on-site staff }\end{array}$ & \\
\hline
\end{tabular}




\subsection{Discussion and conclusion}

Following the presentation of the QoL of the high-needs elderly in Chapter 7, this chapter has developed a framework for the design of housing that improves their QoL. First, through the analysis of relationships between themes for QoL and design considerations, the basic design framework was established, where 24 main design elements and three superordinate design domains, as well as their overall relationships to QoL themes, were identified. Next, synthesising design considerations and discussion in reference to existing design standards, design requirements are established for each design element. Combining the outcomes of these two procedures, the design framework is established.

This framework shows what design elements contribute to which aspects of QoL and how, as well as being useful as a guideline in the design. This is beneficial because understanding the relevance of the design to the occupants' QoL is expected to lead to a better design, which is not just what is compliant with the minimum standards. To summarize the relevance, numeral design requirements were formed in 24 designs and three design domains, that had complex relationships to themes for QoL. Of all design domains, the design of the individual unit contained the greatest numbers of design considerations in all themes for QoL. This signifies the design of the individual unit is a significant contributor to the QoL of the high-needs elderly.

Within individual units, the design of wall and elevation design, and storage design had a considerable impact on resident QoL, particularly on their control in daily and leisure activities and maintenance of possessions. This is largely because spaces elderly residents can reach becomes limited in the vertical direction when impairments and needs for mobility aids increase. Design of sitting space was also significantly influential to resident QoL, particularly in facilitating their meaningful leisure activities and comfort. This relates to the fact that the highneeds elderly typically spent their most of their time in their armchair, that allowed them to be seated in a comfortable posture, where they enjoyed various leisure activities. This space also contributed to facilitating residents' meaningful relationships, as the layout of this space with respect to their front door and windows was a key to their greater control of visitors. The design of a bathroom, a kitchen and dining space had also a great impact on their QoL, largely on their control in daily activities such as cooking and showering. Quality of care was influenced by the bathroom design, which related to the fact that the majority of the study participants received care in showering. The optimal design of individual outdoor space greatly contributed to resident comfort, as this space provides opportunities to enjoy the sunshine as well as space to breathe. This space was also relevant to facilitating connection to other residents. Planar 
relationships between spaces were largely related to maintaining their control, by keeping the moving distance short. The size of the room and the design of extra spaces had implications for resident meaningful relationships, mainly relating to the ability to accommodate personal guests.

The number of design considerations distilled in the domain of complex design was small (less than one-fourth of those distilled in the design of the individual unit). Among those, the design of indoor communal areas had a great impact on resident QoL, particularly on their meaningful leisure activities and relationships. Depending on the design and layout, communal spaces could facilitate various connections between residents, which also lead to a greater sense of safety and homeliness. The optimal design of communal pathways also facilitates resident walking, which was a meaningful leisure activity for many participants that could keep them active.

In the examination of design requirements, factors leading to diverse requirements differing by the individual should be taken into account. Among those, it was required to accommodate the diversity in the type and level of impairments, the type of mobility aids used and life situations, to support ageing-in-place, providing greater QoL. For example, this consideration was implemented in the development of requirements for the elevation design, where requirements for seated people (including wheelchairs) and for those standing with a walker frame were combined to create the requirements suited for both groups. In order to accommodate the deterioration in mobility, the adaptability is required, for example, in the design of a shower area. Incontinence is another condition common to the elderly. To accommodate this, a toilet should be in proximity to a bedroom and a sitting space. There should also be consideration for providing high-levels of privacy for those with severe mobility concerns, where toileting can occur in the lounge as well as in the bedroom through the use of a commode or other devices.

While this Universal Design concept is essential to design for the ageing population, current standards such as NZS 4121 and the Lifemark Design Standards, commonly used in practice, fail to meet all the requirements of the high-needs wishing to remain independent. For example, the space containing 1,500 mm-diameter turning circle cannot ensure the ease/safety in turning around with mobility aids, particularly for those with limited strength in their arms. The requirements in NZS 4121 and the Lifemark Design Standards for the height of door handles and electrical outlets do not take account of the requirements for walker-frame users. In contrast, there were also excessive regulations, such as the requirement to attach a folding seat to the wall in a shower area, which was not suited for assisted showering. To design housing for the high-needs elderly, careful considerations are required so that it meets every requirement for impairments and conditions related to ageing. 
There was also diversity in the personal preferences in leisure activities and manners of maintaining relationships with others, as well as in their belongings to keep and other physical environments. These insights are new knowledge obtained in this study, considering that these are not shown in domestic existing frameworks, and knowledge included in international existing frameworks relating to these areas is limited and ambiguous, and requires examination for the applicability to the New Zealand contexts as these areas greatly relate to culture. w. In the design of housing, one of the strategies is to facilitate flexibility to accommodate various preferred activities. For example, attention should be given to the optimal design of residents' sitting space, which can facilitate many activities in a comfortable posture. To accommodate resident activities that require some furniture and fixtures, as well as to enable the maintenance of their valuable furniture, there should be sufficient wall space. Those activities that cannot be accommodated in individual dwellings and communal activities can be accommodated in communal spaces. In the design of communal space, flexibility is also required to facilitate various activities. However, there are some activities that require specific spaces, such as art and music and those requiring facilities such as swimming pools and spas. Many large retirement villages provide various facilities including these specific-purpose facilities; however, considering the difficulty of residents with limited mobility in traveling to the communal area, clustering residential units into small groups by the preferences can better facilitate their activities. Strategies are also required for smaller complexes that have less ability to provide many spaces. Diversifying the design of dwellings and communities based on these preferences would be a solution to best facilitate individual preferences.

The framework constructed through this chapter will serve as a guideline in the design of housing for the high-needs elderly. Meeting all design requirements in this framework is desirable for designing housing that provides them with the greatest QoL; however, there can be potential conflicts between requirements. For example, the dwelling size should be large enough to accommodate various activities, relationships and possessions, but it needs to be small enough for greater ease of maintenance. There may be challenges in the design to accommodate diverse preferences of the elderly. In the next chapter, some example models of housing that improves the QoL of the high-needs elderly will be designed, which shows how the design framework, with these potential conflicts and challenges, can be implemented. 


\section{CHAPTER 9: DESIGN OF HOUSING (PHASE 4)}

Chapter 8 presented a framework for the design of housing that improves the QoL for the highneeds elderly that enable them to live independently. In this chapter, based on the requirements in this framework, models of individual dwellings and complexes are designed. The essential aim of this process is to examine the implementation potential for the framework — for example, given the concern about the potential conflicts and challenges identified in some of the requirements, how the design can meet the requirements are examined. The outputs of this chapter are not generalisable, as the designs include solutions for specific contexts as well as those that can be applied to a range of contexts. The design is presented through two main parts: first, the design of individual dwellings is outlined, followed by the design of complexes.

\subsection{Design of individual dwellings}

The design of individual dwellings starts with the definition of the concepts for basic interior layout, using key design requirements shown in Chapter 8. With these concepts, four models of dwellings are designed:

1) two-bedroom unit (2B);

2) one-bedroom unit, type $A(1 \mathrm{~B}-\mathrm{A})$;

3) one-bedroom unit, type $B(1 B-B)$; and

4) bedsit unit (BS).

Along with a visual presentation of the design, the design solutions are explained regarding every element of a dwelling, such as a bathroom, kitchen, sitting space and spaces in order to accommodate various life situations and individual preferences.

\section{Concepts for the basic interior layout}

From the design requirements relating to the interior layout, seven design concepts are defined to develop seven models for basic interior layout.

\section{Layout of the main passage that avoids needing to turn around}

People who use a mobility aid, such as a walker frame or wheelchair, have difficulty turning around with it in small spaces. As a solution, a main passage that allows residents to avoid turning around is set as a circle (Figure 9-1). 


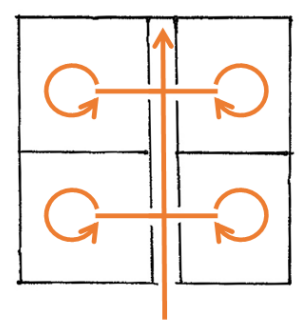

Turning around

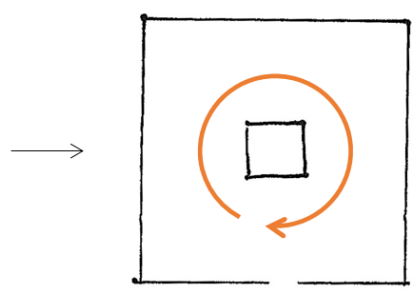

Avoid Turning around

Figure 9-1: Layout of a passage that avoids needing to turn around

\section{Cutting off corners in walls}

Corners in walls are often hit by a walker frame or wheelchair, so corners in these models have been cut off to make individuals' movement smoother, as well as prevent damage to walls. This also helps to improve views from residents' sitting space. Views to the front door, for example, provide residents with greater control over visitors. Important views to the outside include nature, moving people and objects and approaching visitors (Figure 9-2).
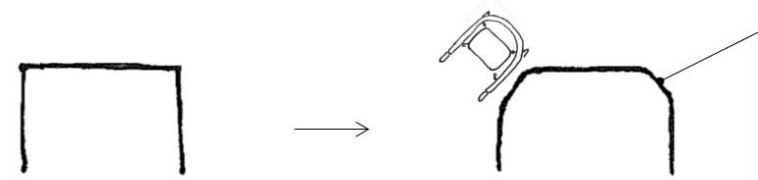

Cutting off the corners contributes to smoother movement with mobility aids

Figure 9-2: Cutting off corners

\section{Providing things to hold onto along the main passage + ease of access to light switches}

It is important to provide things for residents to hold onto continuously along the main passage, giving residents support in moving (Figure 9-3). These objects include handrails and shelves as well as walls. Light switches are provided adjacent to this passage as well. 


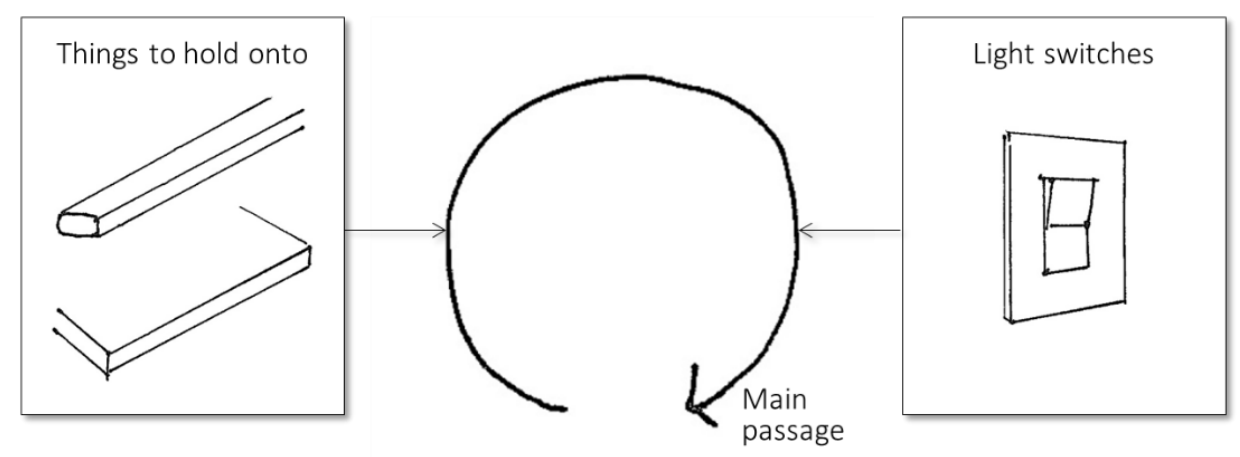

Figure 9-3: Provision of things to hold onto and light switches along the passage

\section{Creating multiple spaces along the main passage}

Spaces for different purposes are laid out along the passage to avoiding creating a space that is used only as a corridor. This allows a more efficient use of space for a range of activities and for possessions, as well as helping keep the distance between different spaces to a minimum (Figure $9-4)$.

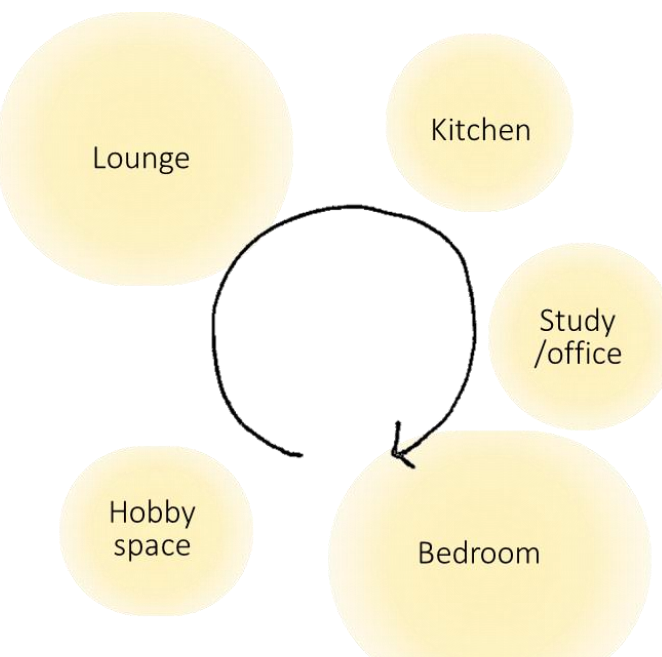

Figure 9-4: Creating multiple spaces along the passage

\section{Layout of the bathroom adjacent to many spaces}

Many requirements have been found for planar relationships relating to the layout of the bathroom. First, the bathroom is laid out in proximity to the bedroom and the sitting space; a proximity of less than $3 \mathrm{~m}$ is required for those that have conditions of both incontinence and limited mobility. The passage between the bed and the toilet is straight with no change in direction. Next, a washing machine adjacent to or in the bathroom is desirable as this reduces the trouble of carrying clothes or towels to be washed. Proximity between the kitchen and the bathroom also helps caregivers move smoothly between spaces and increases resident safety, allowing caregivers to provide surveillance over residents showering while they perform tasks in 
the kitchen. Finally, proximity between the washing machine and the space for drying clothes also reduces the distance washed laundry items need to be carried (Figure 9-5).

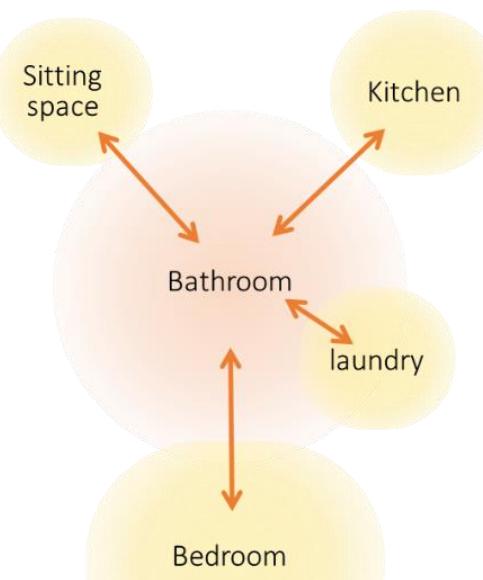

Figure 9-5: Layout of many spaces adjacent to the bathroom

\section{Provision of space for possessions}

Elderly people need space that can accommodate many personal possessions, including items that facilitate their favourite activities and those that mark their life history. Personal possessions also increase residents' sense of homeliness. Items include furniture, clothing, and small objects, as well as pictures and photographs. In consideration of this, inner walls are designed to mainly accommodate enclosed storage and shelves, while outer walls are left as simple walls for accommodating furniture and pictures or photographs (Figure 9-6).

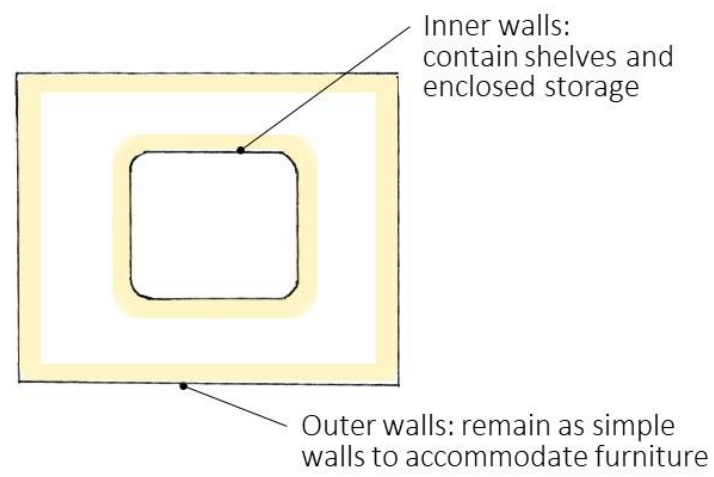

Figure 9-6: Use of walls for accommodating possessions

\section{Removal of unnecessary doors and use of sliding doors}

Opening and closing doors causes those with limited mobility some difficulty. Therefore, unnecessary doors have been removed, such as that between a kitchen and a lounge. Only doors that are necessary for resident privacy when having guests are included, such as those for a 
bathroom and bedroom. All doors are sliding doors, which are not only easier to manoeuvre than hinged doors but can be kept open without taking up extra space (Figure 9-7).
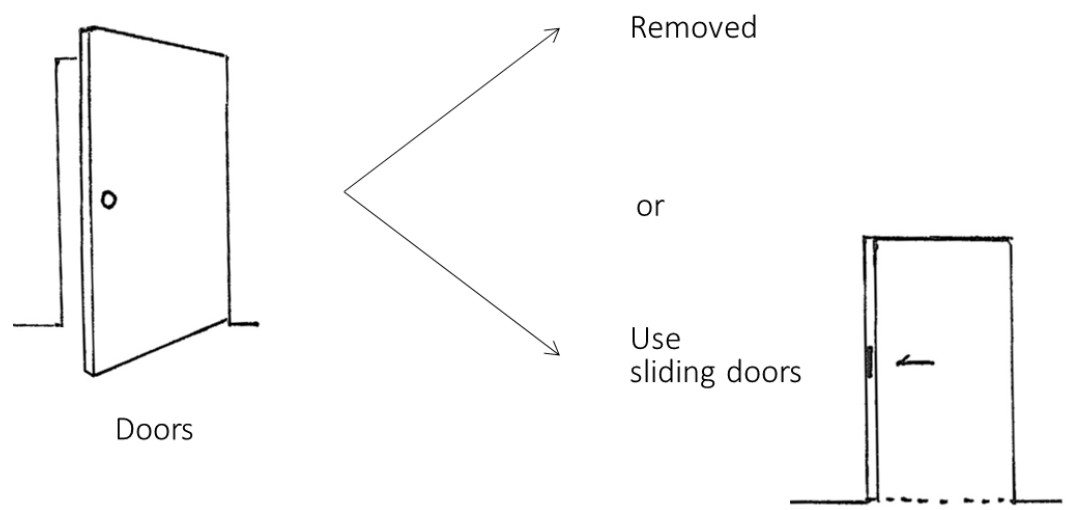

Figure 9-7: Removal of unnecessary doors and use of sliding doors

\section{Design}

Based on these concepts, four models of individual units have been designed (Figure 9-8 - 910). The floor area of one-bedroom and two-bedroom units is defined as $50 \mathrm{~m}^{2}$ and $73 \mathrm{~m}^{2}$ respectively, to meet various inner requirements as well as to keep it small. The unit floor area is also decided referring to the units studied in this research (one-bedroom units studied are 39-59 $\mathrm{m}^{2}$ and two-bedroom units are $89 \mathrm{~m}^{2}$ ). Other information on the floor area of local-authority housing units has also been referred to; according to Povey \& Harris, there was an increase in the floor area of one-bedroom units in a Dunedin City Council housing from $40 \mathrm{~m}^{2}$ in 1966 to 53 $55 \mathrm{~m}^{2}$ in 2006, and in two-bedroom units from $60 \mathrm{~m}^{2}$ in 1966 to $73 \mathrm{~m}^{2}$ in 2006 (2006). The units can be adjoined (semi-detached type or apartment type), as shown in Appendix 5.

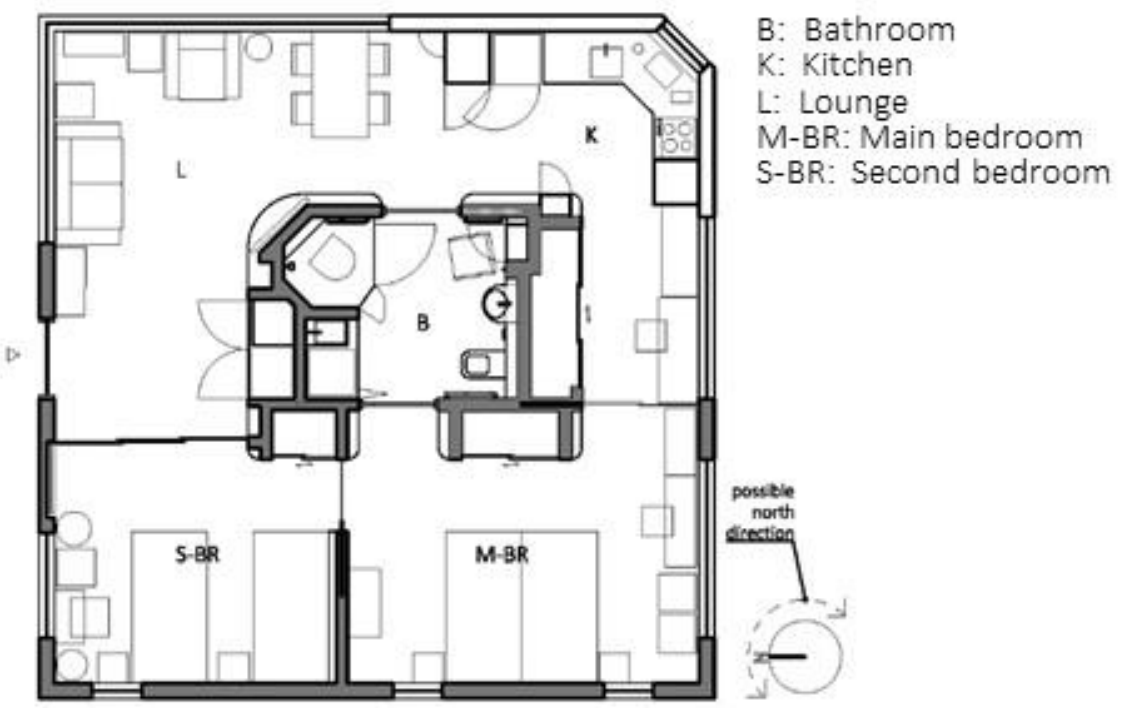

Figure 9-8: Plan of $2 \mathrm{~B}$ type $\left(73 \mathrm{~m}^{2}\right)$ 

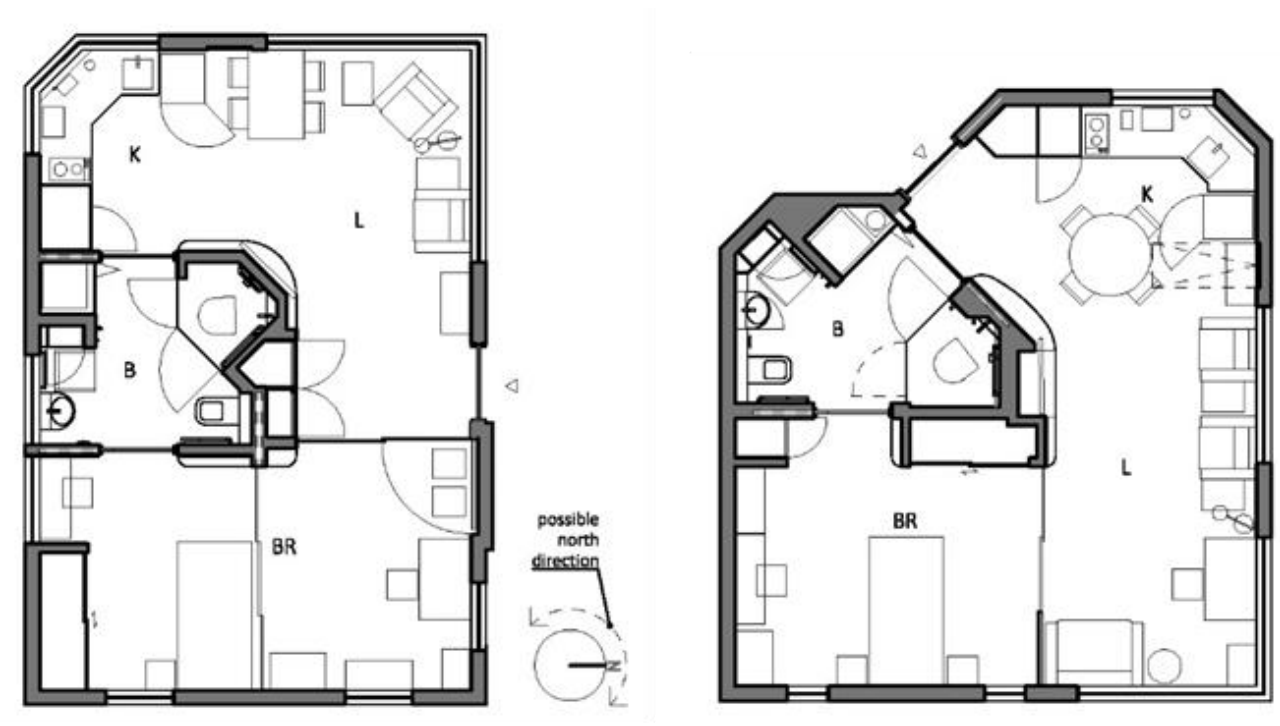

B: Bathroom

K: Kitchen

L: Lounge

BR: Bedroom

Figure 9-9: Plans of 1B-A type (left) and 1B-B (right) (both $50 \mathrm{~m}^{2}$ )

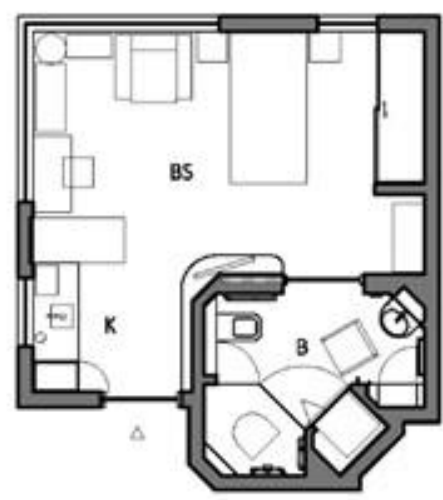

B: Bathroom

$\mathrm{K}:$ Kitchenette

BS: Bedsit

Figure 9-10: Plan of BS type $\left(27 \mathrm{~m}^{2}\right)$

\section{Inner walls}

Inner walls contain enclosed storage (cupboards, drawers and glass cabinets) and open shelves, as well as things to hold onto. 


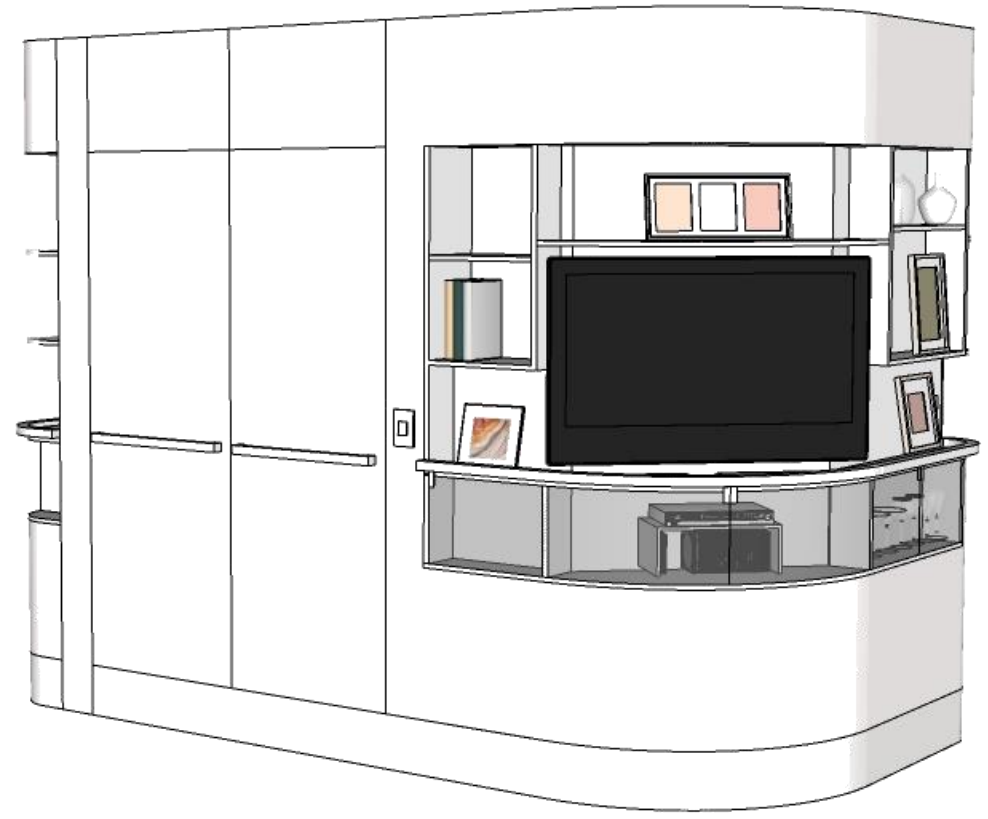

Figure 9-11: Perspective of the inner walls (1B-A)

Objects to hold onto (such as handrails incorporated into shelves and handles of cupboards or wardrobes) are provided at $1010 \mathrm{~mm}$ high (Figure 9-12). Except for cupboards and wardrobes, the wall area above the handrails contains open shelves at $1330 \mathrm{~mm}$ high and $1650 \mathrm{~mm}$ high, allowing those standing to easily reach the storage space. Some shelves above $1330 \mathrm{~mm}$ high are designed as movable shelves, allowing wheelchair users who don't use them to remove unnecessary shelving and providing greater flexibility in accommodating objects of various sizes. The area below the handrails contains enclosed storage such as glass cabinets and drawers, which helps residents avoid bumping objects when moving along the walls. To mitigate any difficulties for residents standing to reach stored items, drawers are used in lower areas. Light switches are located at $1100 \mathrm{~mm}$ high (Figure 9-13). 

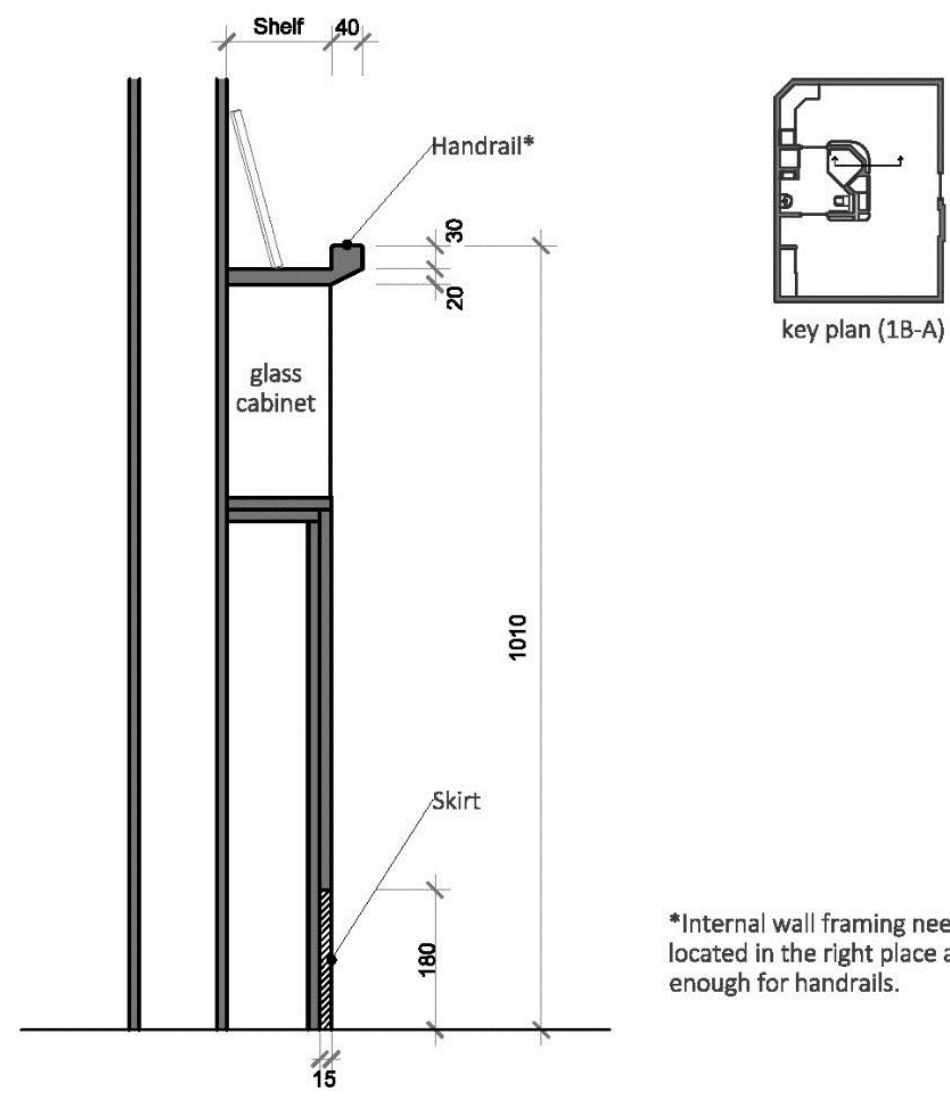

*Internal wall framing needs to be located in the right place and strong enough for handrails.

Figure 9-12: Section of the lower part of the inner wall (1B-A)

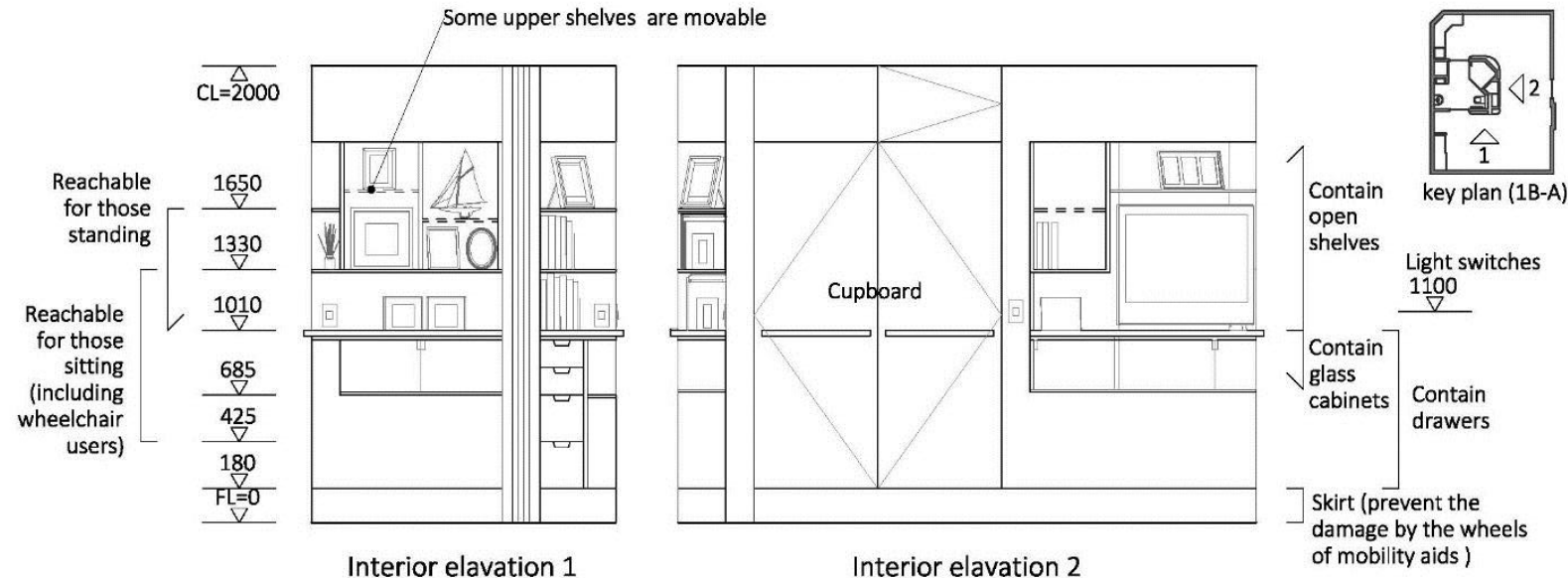

Figure 9-13: Interior elevations of the inner walls (1B-A)

Shelves of three different depths are provided to accommodate objects: a) $150 \mathrm{~mm}$ for photo and picture frames, small objects, and so on; b) 230-250 mm for books, etc.; and c) $300 \mathrm{~mm}$ for a TV and audio-visual devices (Figure 9-14). 


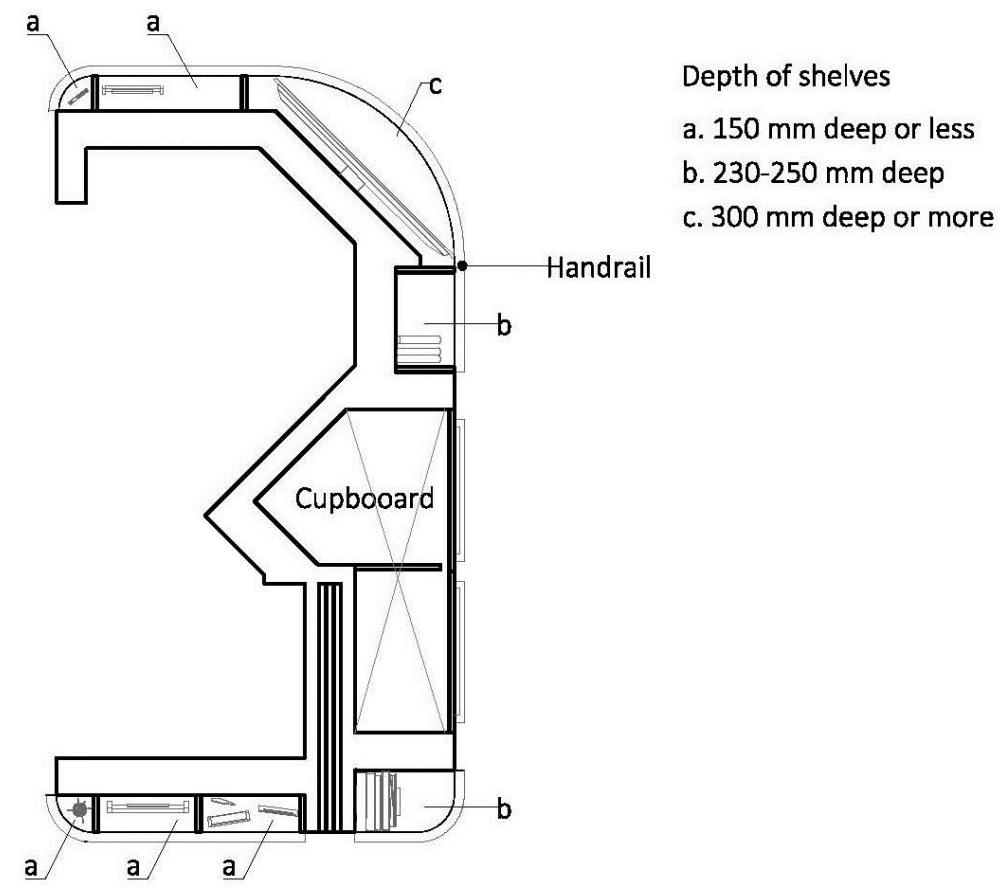

Figure 9-14: Plan of the inner walls at $1500 \mathrm{~mm}$ high (1B-A)

\section{Enclosed storage}

A wardrobe of at least a $1.7 \mathrm{~m}$ width is provided for one person. Wardrobes contain shelves, drawers and hooks, as well as railing, to accommodate various types of clothing including shawls or scarves, bags and shoes. Rail supports are provided at $1350 \mathrm{~mm}$ high and $1650 \mathrm{~mm}$ to suit both wheelchair users and those who walk. Drawer-type storage is provided in the lower areas (lower than $760 \mathrm{~mm}$ ) to mitigate difficulty reaching items (Figure 9-15).

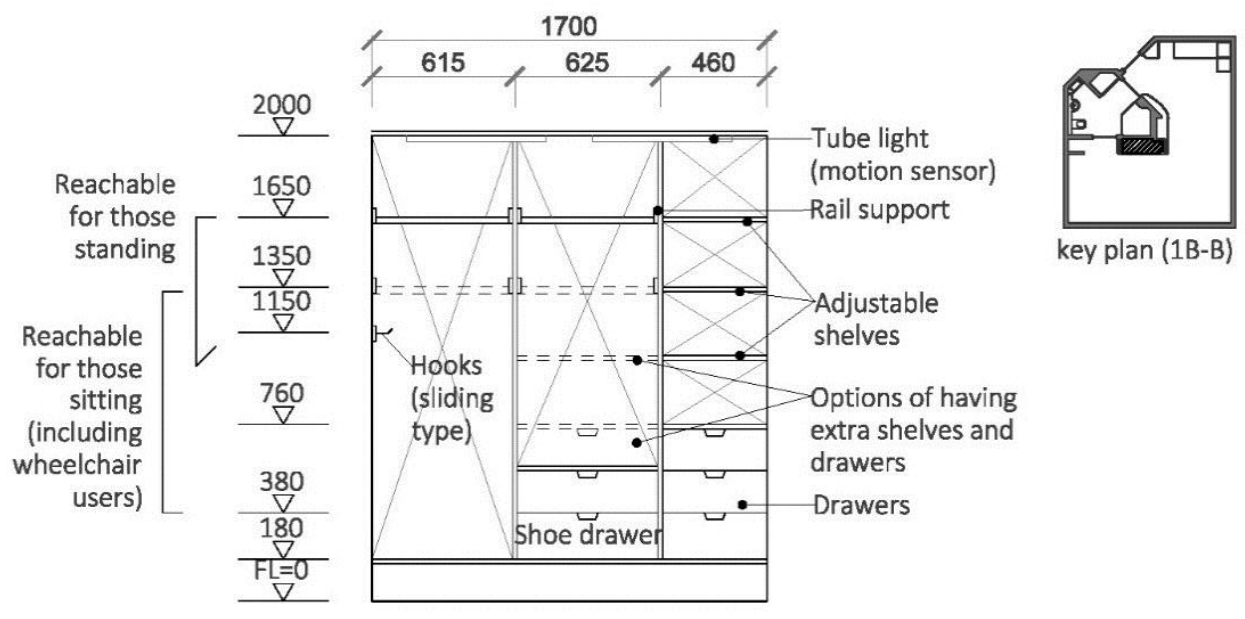

Figure 9-15: Elevation of the wardrobe (1B-A)

Storage is also provided near the front door for each type of housing unit, which includes shelves and drawers to store household items and gardening tools, as well as railing and hooks for hanging up outdoor wear (Figure 9-16). 


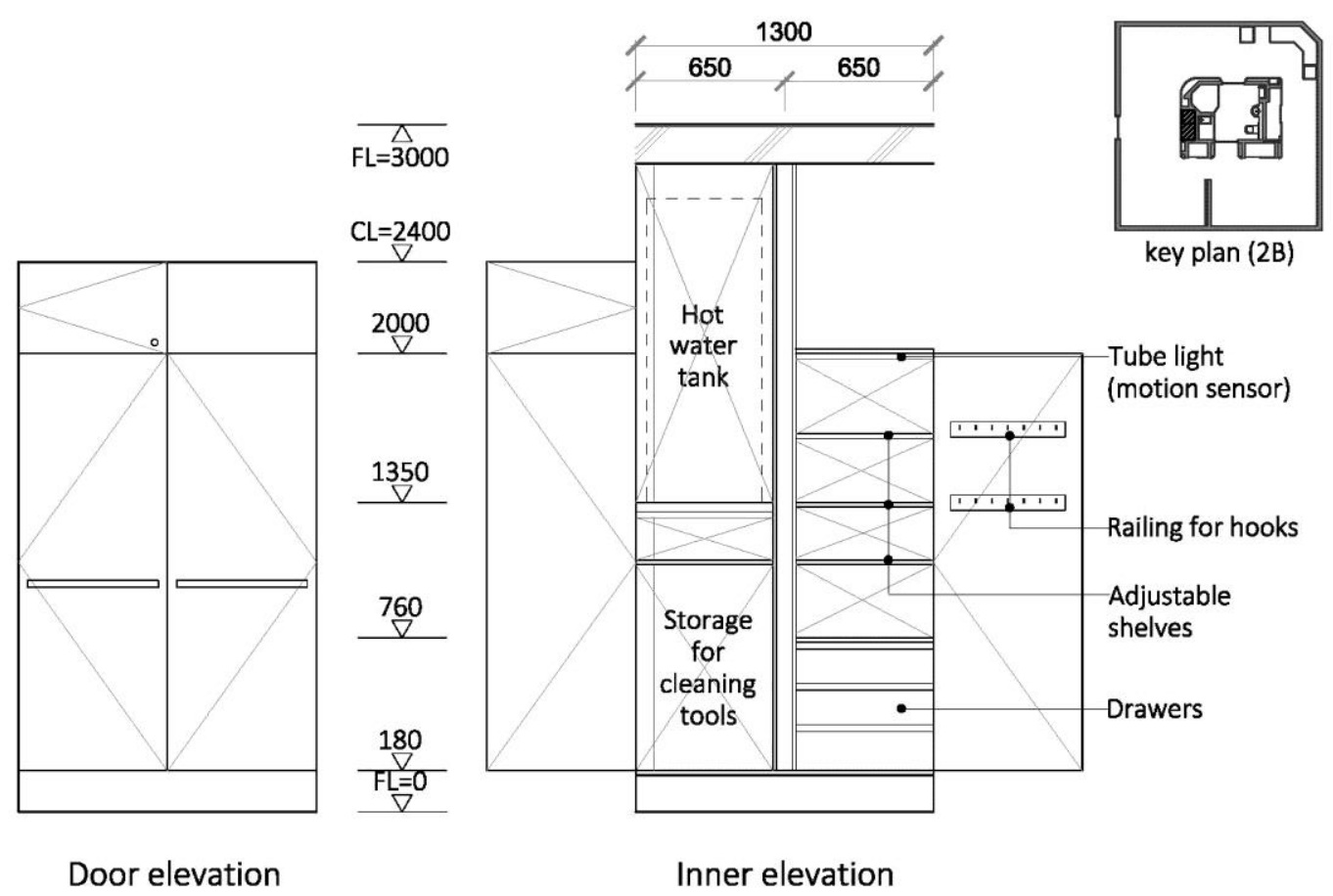

Figure 9-16: Cupboard near the front door (2B)

\section{Kitchen}

The kitchen is designed to accommodate kitchen spaces and amenities shown in Table 9-1, depending on the unit type. Bench space, a sink, space for a fridge-freezer and full-height storage are provided for all unit types. Hot plates are provided for all unit types except for the BS-type, in which residents are provided with three meals as part of their standard services. An oven (or double oven) and a dishwasher is provided in the $2 \mathrm{~B}$ type, and the option to have a compact oven in the full-height storage space and to have a dishwasher is provided in the $1 \mathrm{~B}$ types.

Table 9-1: Provided kitchen spaces and amenities

\begin{tabular}{|l|l|l|l|l|}
\hline & 2B & 1B-A & 1B-B & BS \\
\hline a. Bench space for food preparation & Yes & Yes & Yes & Yes \\
\hline b. Sink & Yes & Yes & Yes & Yes \\
\hline c. Hot plates & Yes & Yes & Yes & No \\
\hline d. Space for fridge-freezer & Yes & Yes & Yes & Yes * \\
\hline e. Full-height storage & Yes & Yes & Yes & Yes \\
\hline f. Oven & Yes & Optional & Optional & No \\
\hline g. Dish washer (under bench) & Yes & Optional & Optional & No \\
\hline h. Folding bench & No & Yes & Yes & Yes \\
\hline
\end{tabular}

* A drawer-type fridge-freezer is provided under the kitchen bench

An L-shape kitchen bench is used to minimise the moving distance between spaces as well reduce the difficulty of turning around 180 degrees. This use of space allows both the resident and their caregiver to approach the bench, as well as ensuring enough space to turn around with a mobility aid. The basic pattern of the spatial layout has the bench space for food preparation located in 
the centre. This is adjoined to the sink and hot plates, with storage (full-height storage and a fridge-freezer) placed in the outer area (Figure 9-17). A dishwasher is provided in proximity to the sink. The overall floor plans of the kitchen are shown in Figure 9-18.

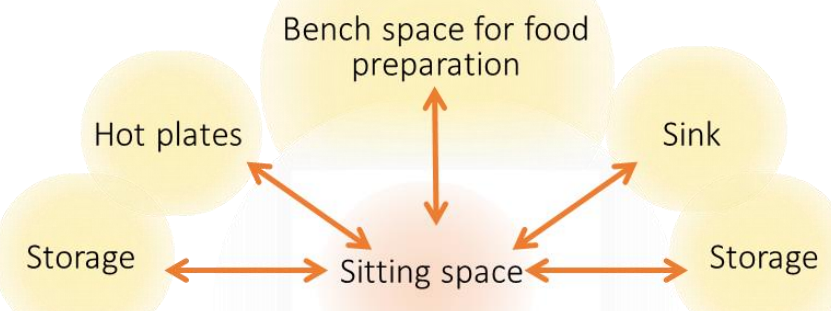

Figure 9-17: Basic layout of kitchen spaces

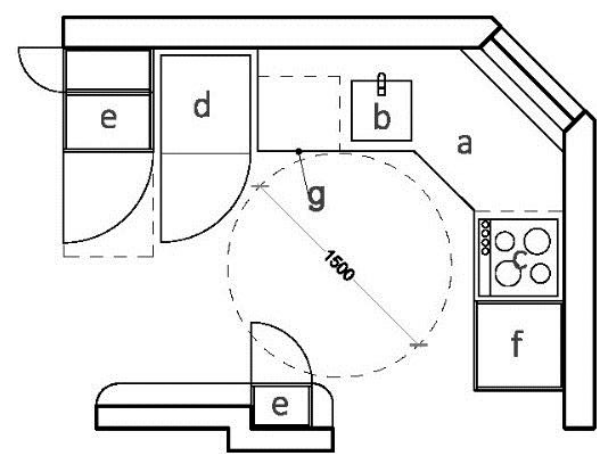

2B

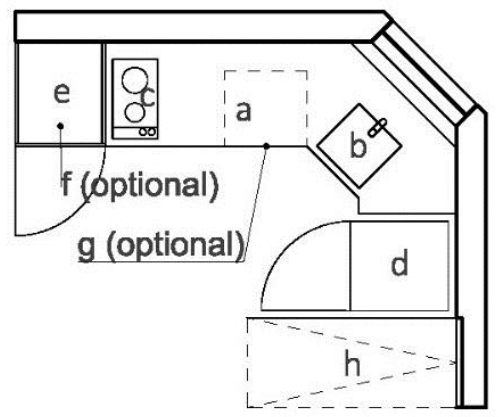

1B-B

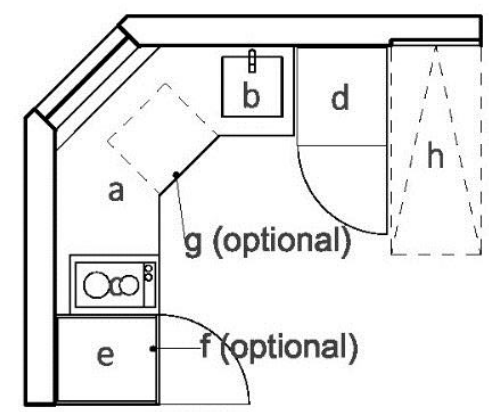

1B-A

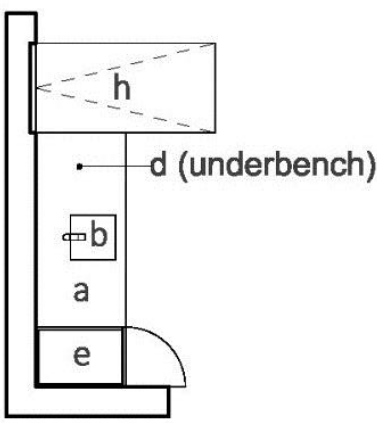

BS a. Bench space for food preparation

b. Sink

c. Hot plates

d. Space for fridge-freezer

e. Full-height storage

f. Oven

(at waist high for wheelchair users)

g. Dish washer (underbench)

h. Folding bench

Figure 9-18: Overall floor plans of the kitchen (1B-A)

Bench space for food preparation is $700 \mathrm{~mm}$ deep, providing space for a microwave oven, a toaster and an electric kettle on the bench, while still leaving enough space for food preparation (Figure 9-19). Storage (shelves, cupboards and drawers) is laid out to accommodate various items that are within reach from the front of this space (Figure 9-20, 9-21). Light is provided at the bottom of the hanging wall. 


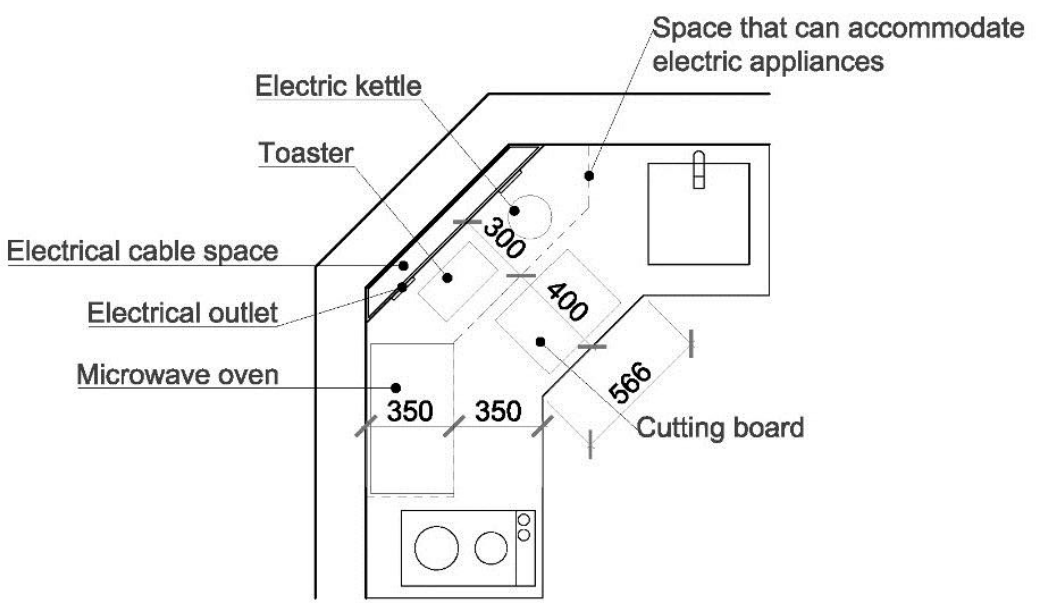

Figure 9-19: Bench space for food preparation (1B-A)

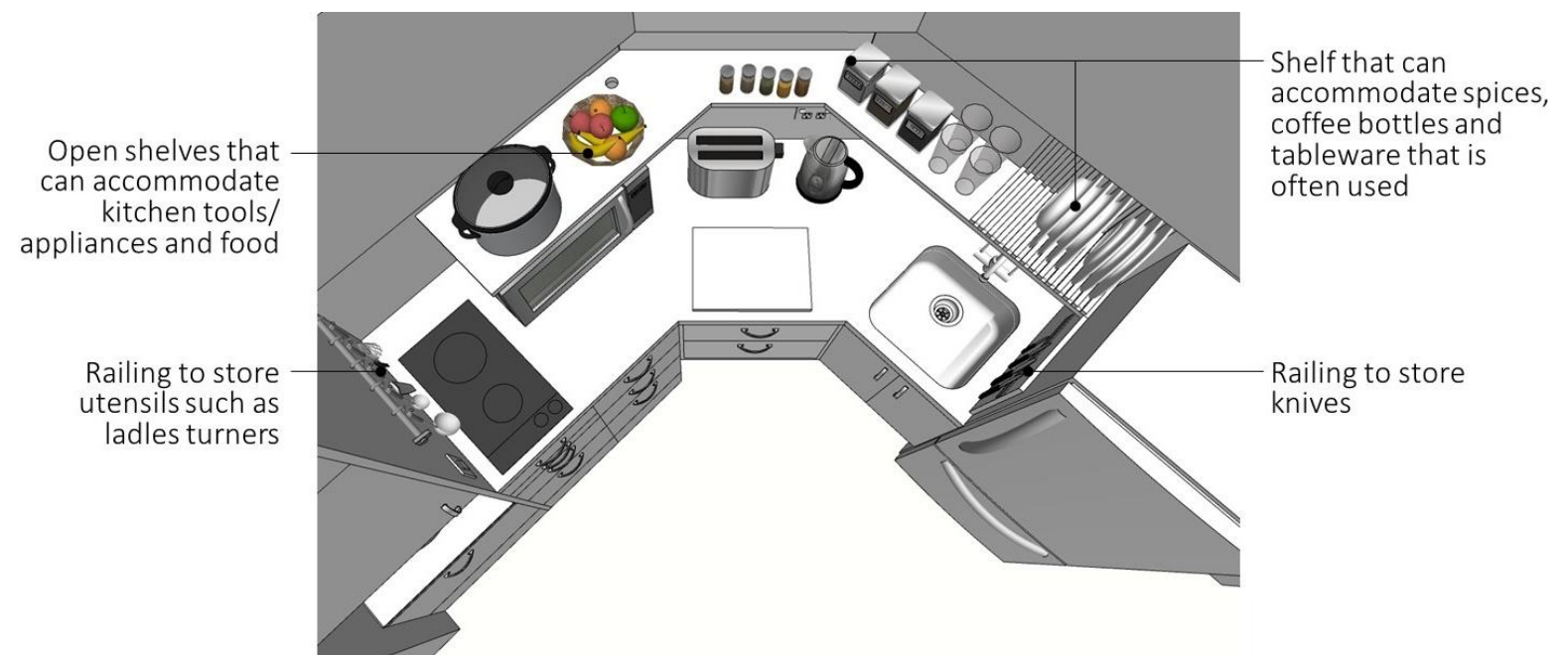

Figure 9-20: Perspective of the bench space for food preparation (1B-A)

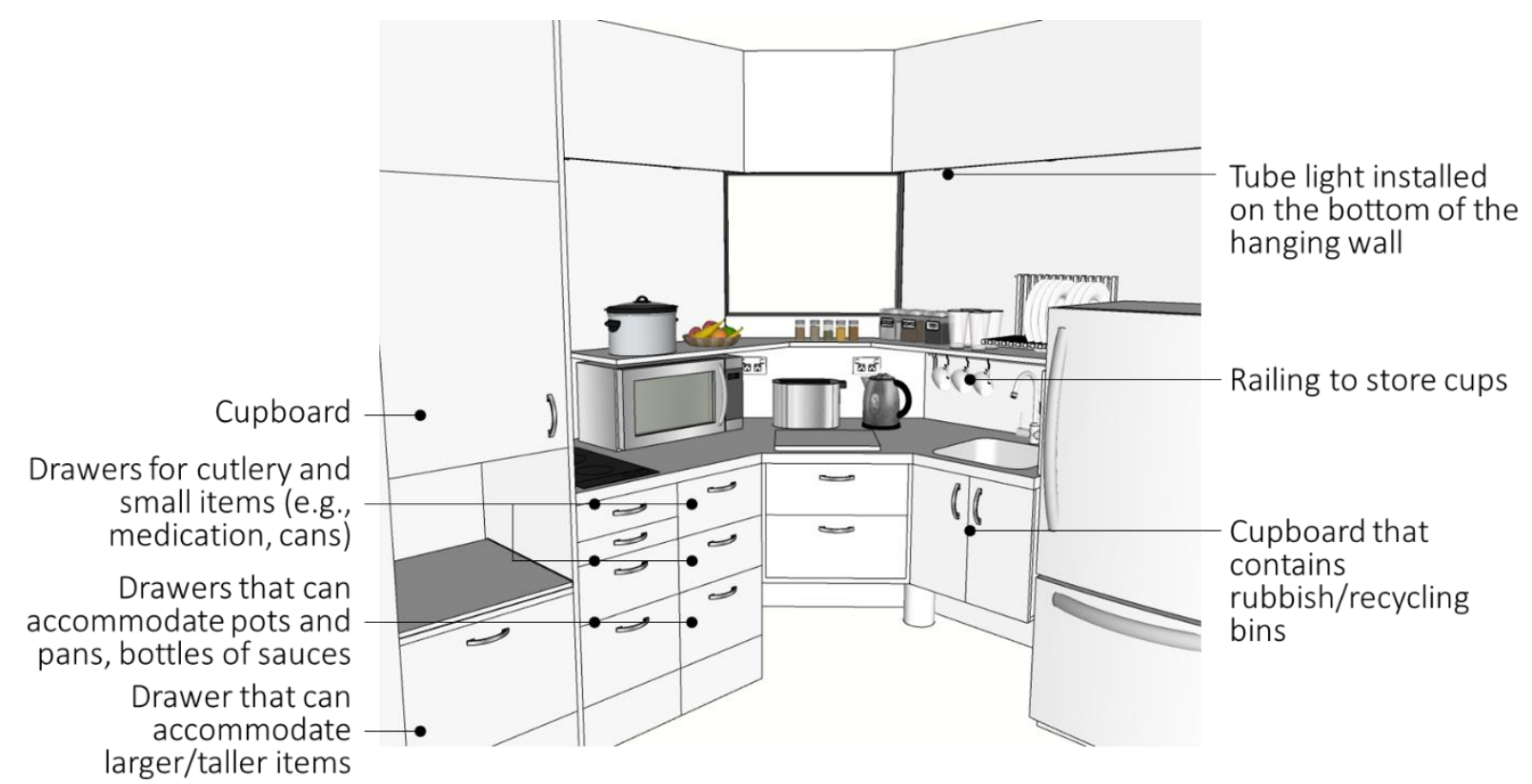

Figure 9-21: Layout of storage in proximity to the space for food preparation (1B-A) 
Storage (cupboards, drawers, shelves and railings) and electric outlets are provided within reach of the resident (Figure 9-22).
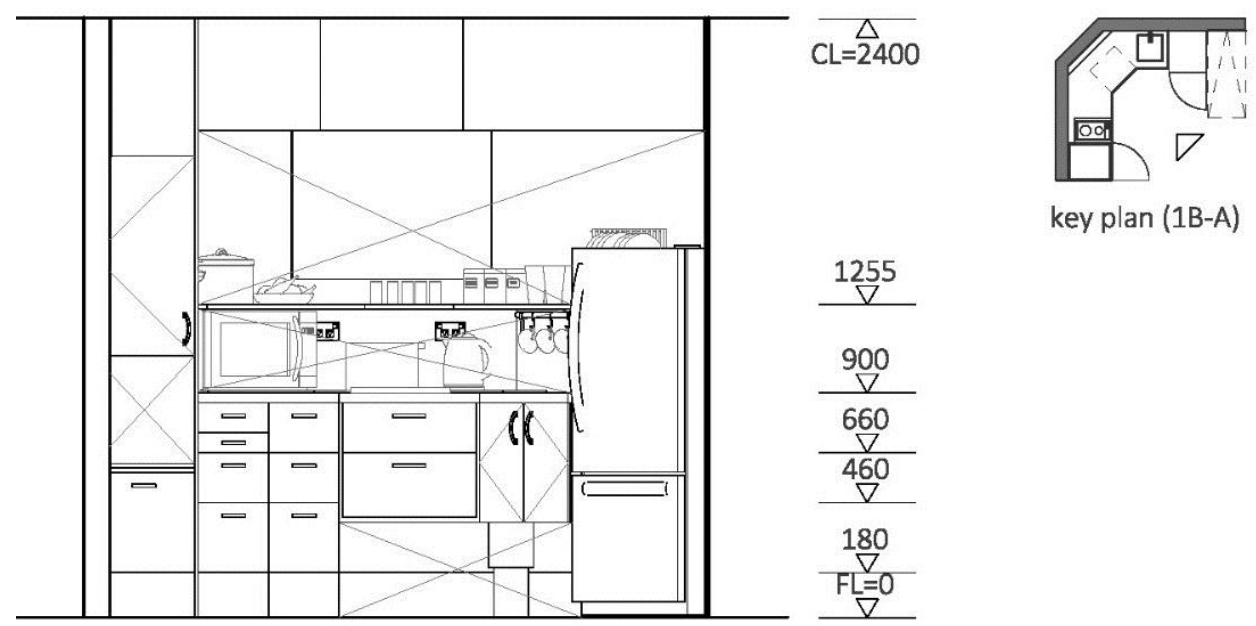

key plan (1B-A)

Figure 9-22: Interior elevation of the kitchen (1B-A)

Two options are provided for space for a microwave oven — in the rear part of the bench and on the shelf above the bench - so that residents can take out meals from the microwave oven in a safe manner (Figure 9-23).
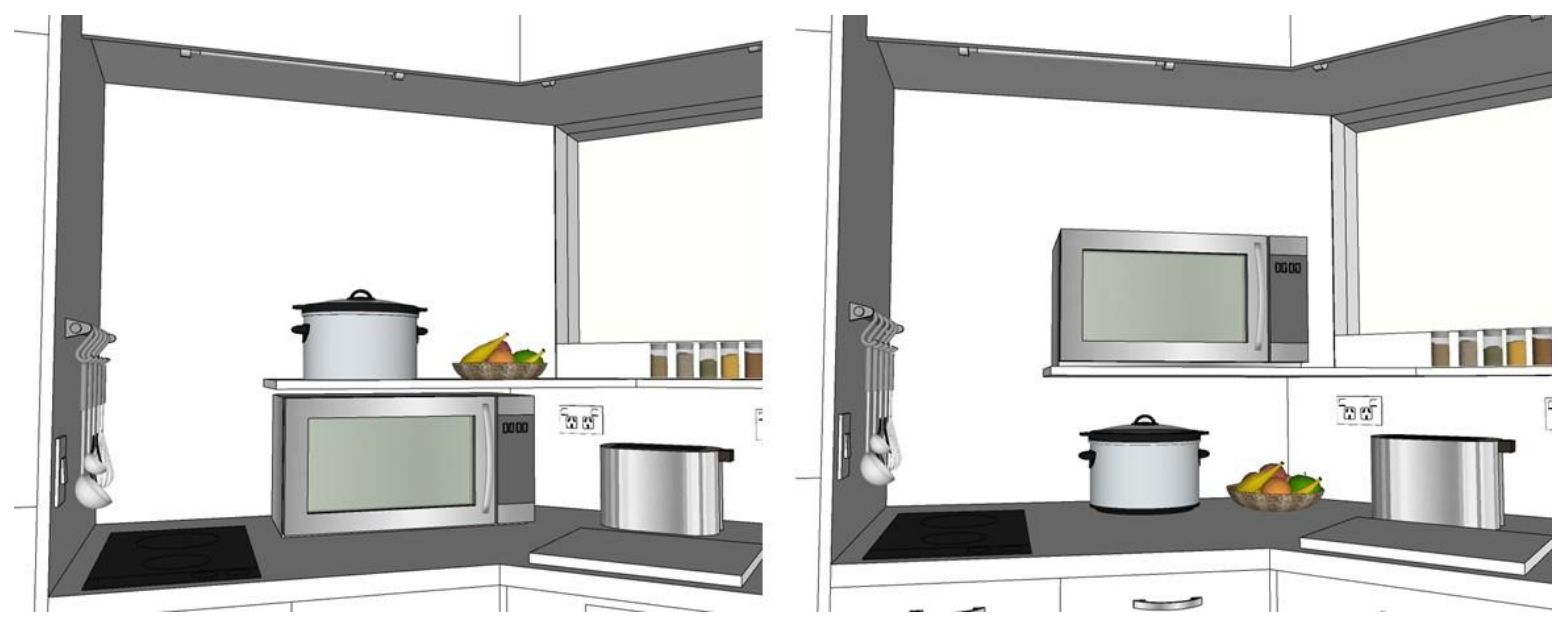

Figure 9-23: Options for the space for a microwave oven (1B-A)

To accommodate preferences for cooking, some flexibility is afforded to the kitchen. In the 1Btype units, spaces that can accommodate a small oven and a small drawer-type dishwasher are provided (Figure 9-24). 


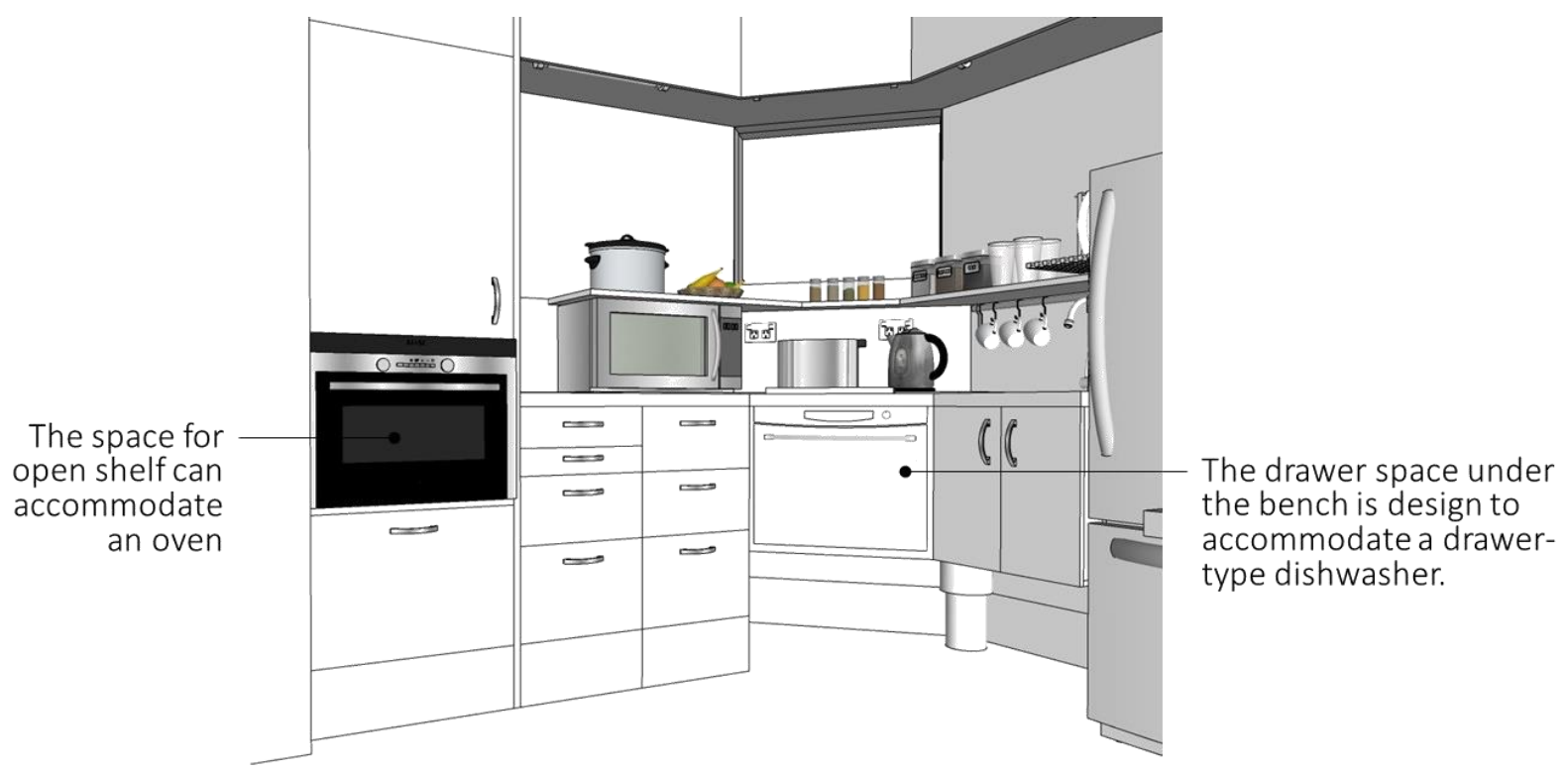

Figure 9-24: Options for having an oven and a dishwasher (1B-A)

To provide larger space for cooking, a sliding shelf and a folding bench are provided (Figure 925).

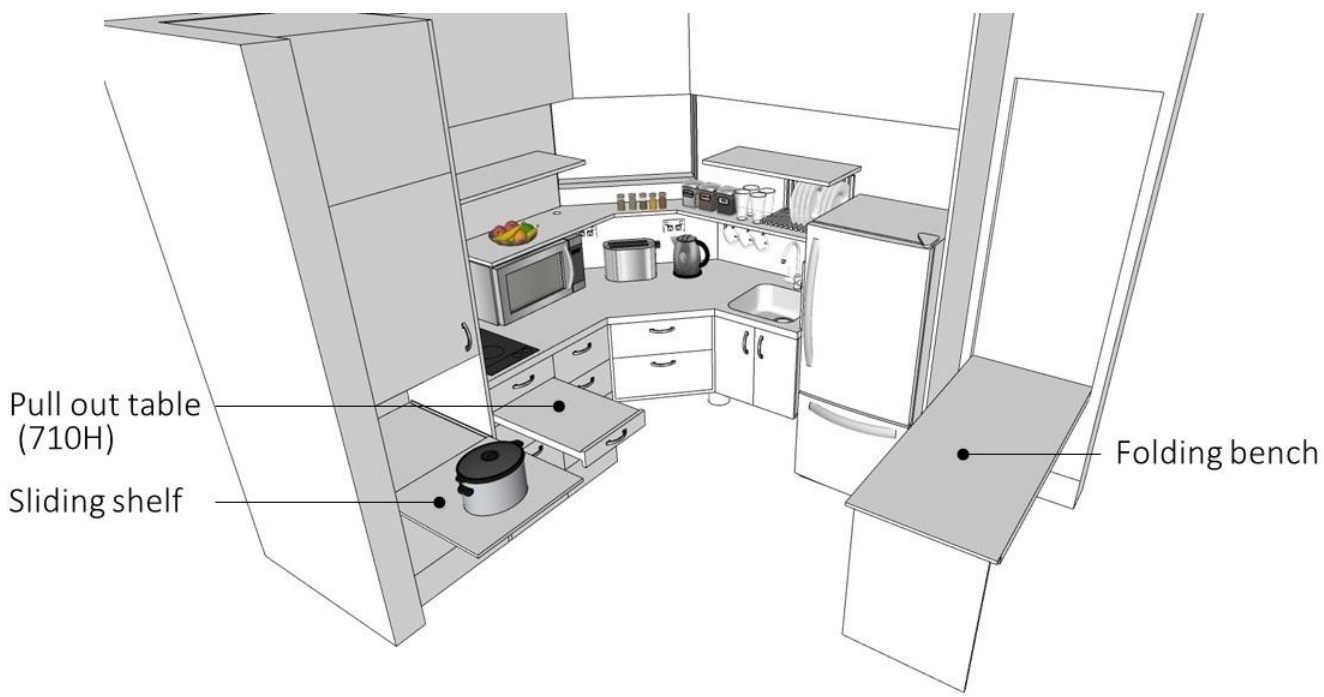

Figure 9-25: Extension of the cooking space (1B-A)

The bench height can be adjusted between $700 \mathrm{~mm}$ and $900 \mathrm{~mm}$ high to suit residents both standing and seated, including wheelchair users (Figure 9-26, 9-27). Some drawer units can be taken out when used by seated residents. The units taken out can fit under the folding bench (Figure 9-28). 

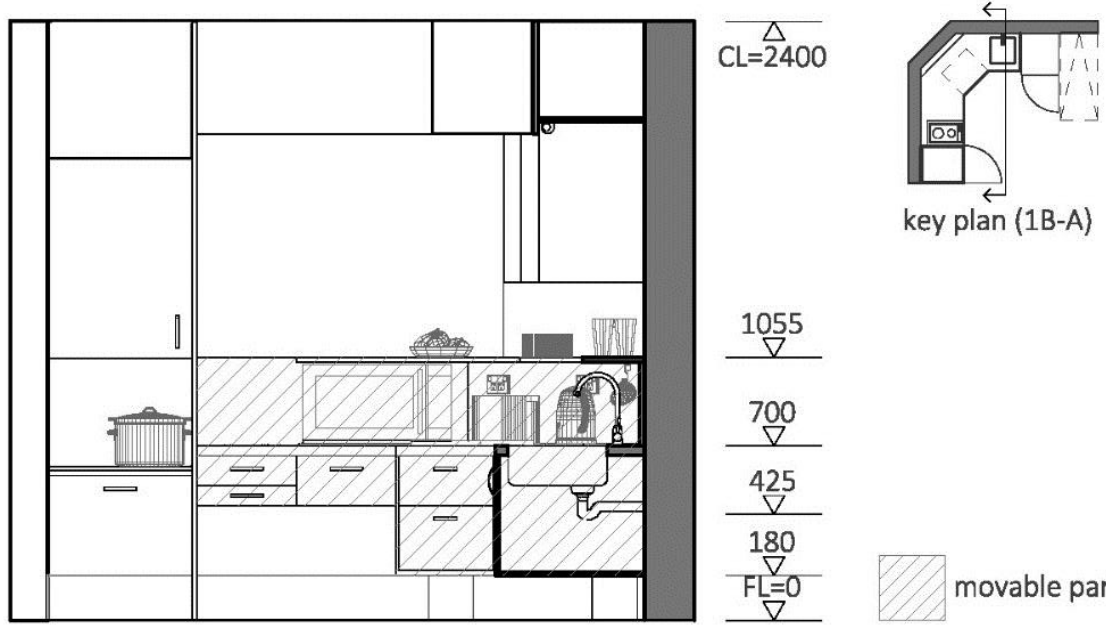

Figure 9-26: Section of the kitchen with a lowered bench $(\mathrm{h}=700 \mathrm{~mm})(1 \mathrm{~B}-\mathrm{A})$

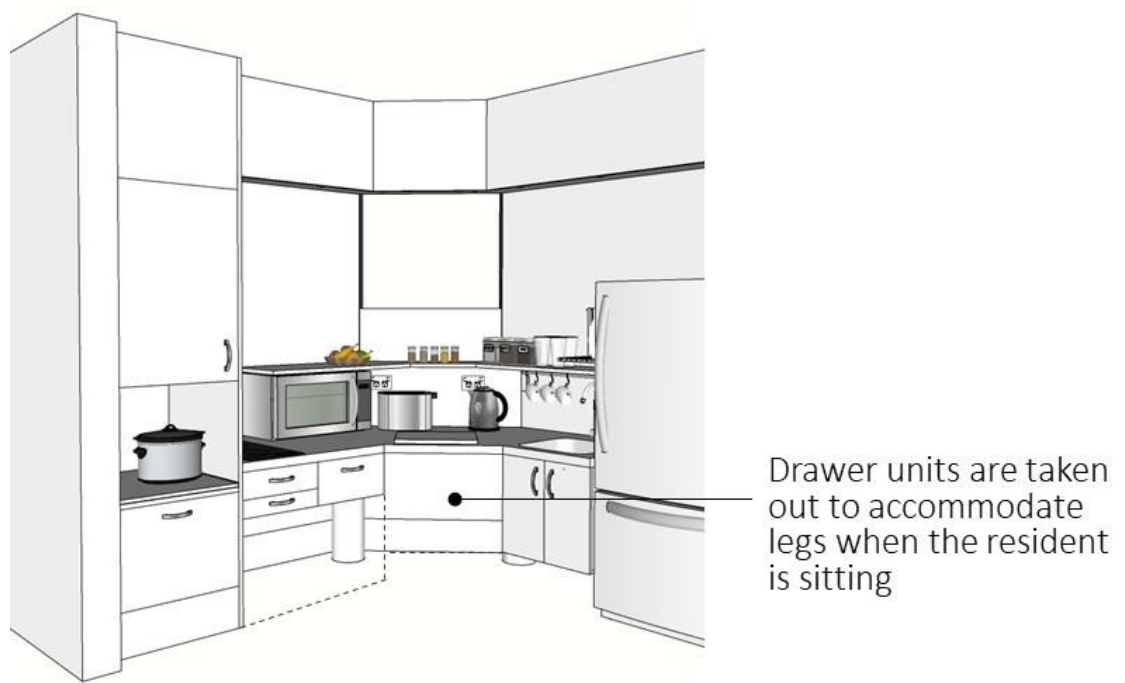

Figure 9-27: Kitchen with a lowered bench (h=700 mm) (1B-A)

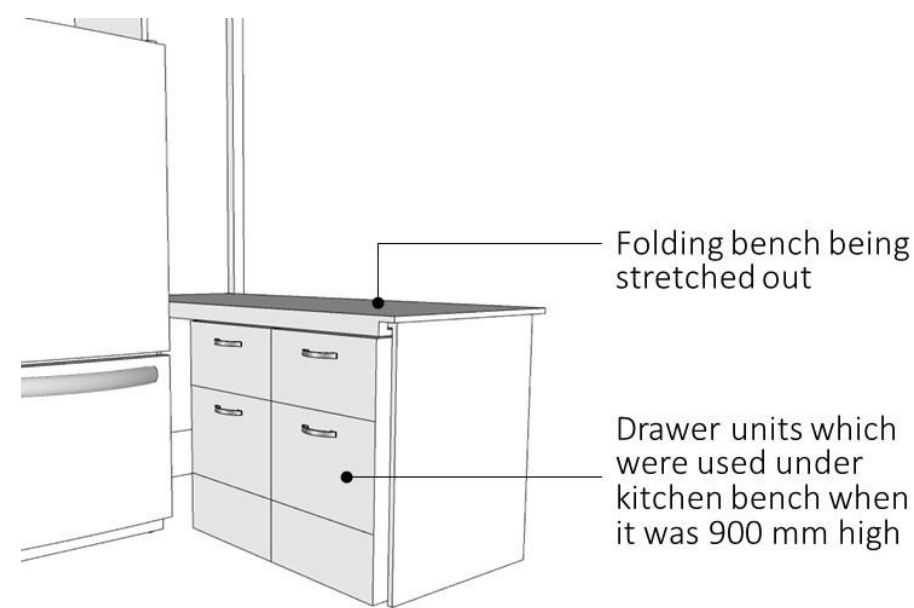

Figure 9-28: Folding bench and under bench storage units (1B-A) 


\section{Bathroom}

The bathroom is designed to contain a toilet, a hand basin, a shower area, a laundry and storage (Figure 9-29, 9-30). It is designed to have enough space for undressing, drying and dressing for assisted showering, as well as turning around with a wheelchair. The shower area has a clear moving space of $1200 \mathrm{~mm}$ in diameter to ensure room for a caregiver assisting in washing a seated resident.

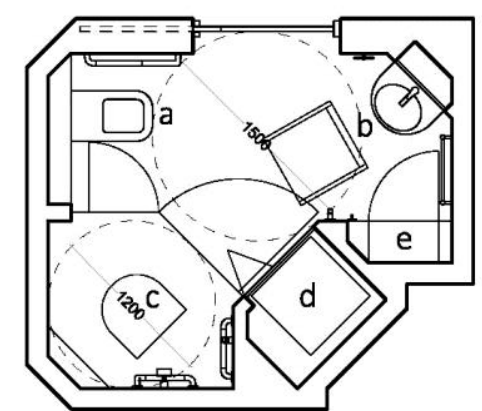

BS

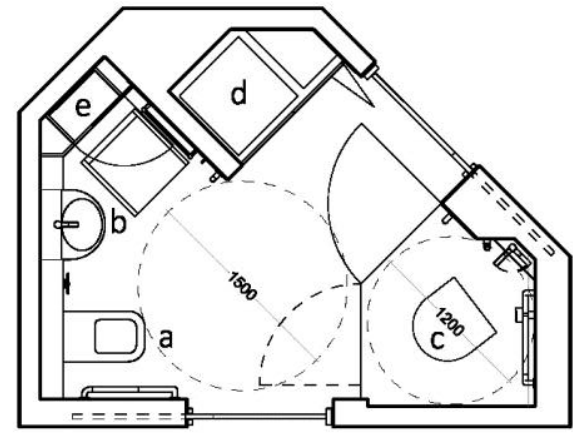

1B-B

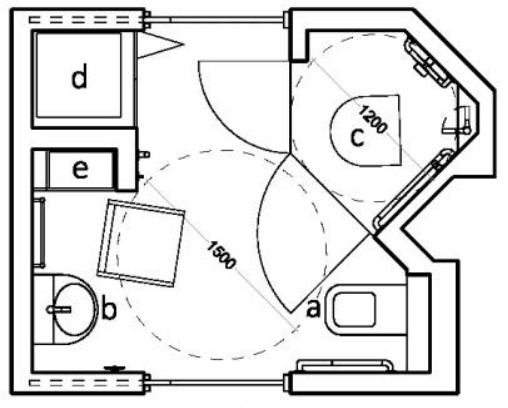

1B-A

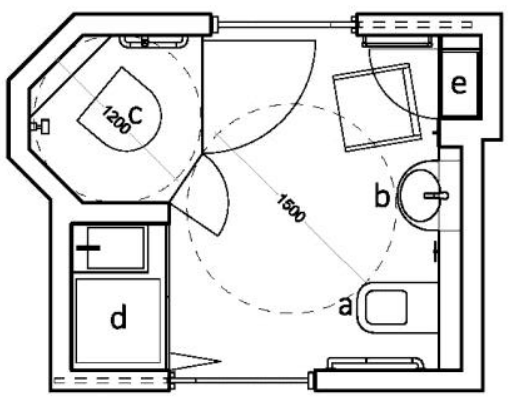

2B a. Toilet

b. Hand basin

c. Shower area

d. Laundry

e. Storage

Figure 9-29: Bathroom floor plans

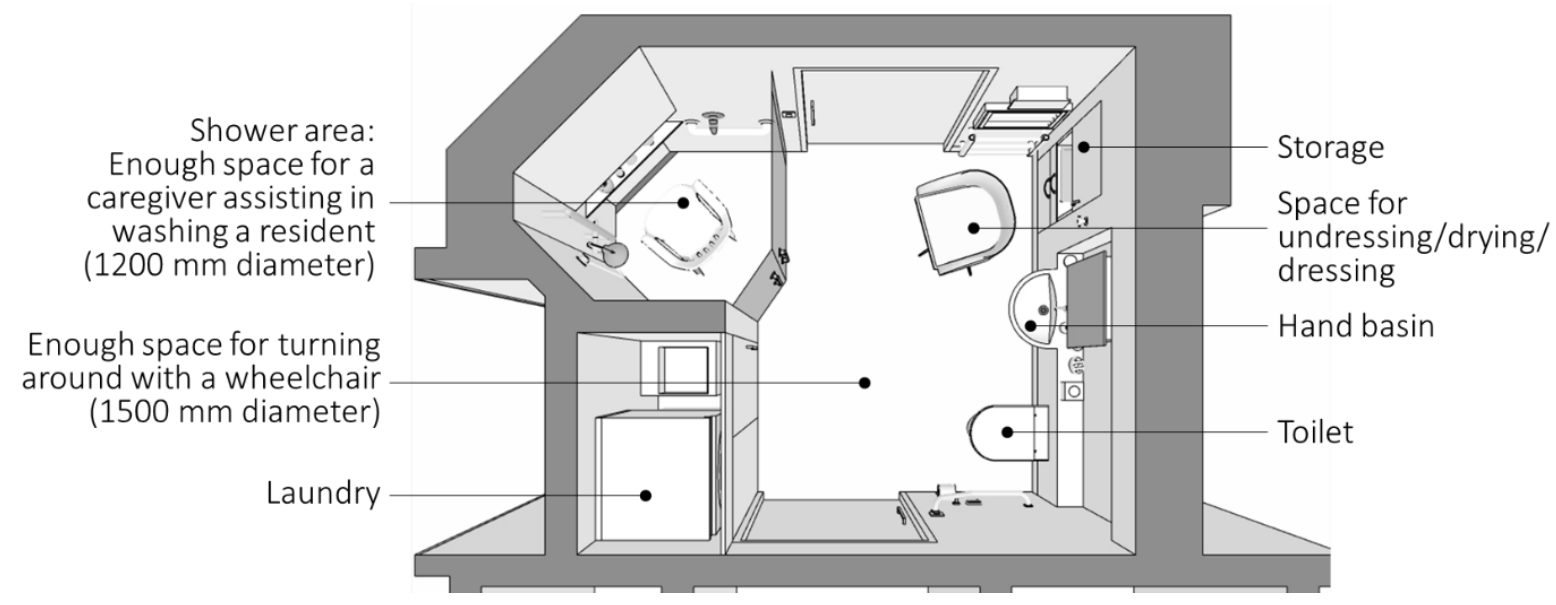

Figure 9-30: Perspective of the bathroom (2B)

The spaces in the bathroom are laid out to minimise the moving distance of the resident and the caregiver, and things to hold onto are provided for the resident's easy movement around the 
room (Figure 9-31). The toilet is located in the closest space to the bedroom for those with severe needs in toileting at night. Two doors are provided in the $1 \mathrm{~B}$ and $2 \mathrm{~B}$-types to reduce the moving distance of caregivers between the bathroom and the kitchen, which increases the efficiency of care tasks. Having two doors also increases privacy in accommodating guests, as visitors can use the toilet without passing through the bedroom.

Resident circulation

Spaces are laid out along the resident circulation

The sequence of things to hold onto (vanity counter and handrails) enhances a sense of security

\section{Caregiver circulation}

The layout of the

bathroom with respect to

the laundry, the bedroom and the kitchen in

proximity to the shower

space reduces caregiver workload and increases resident safety

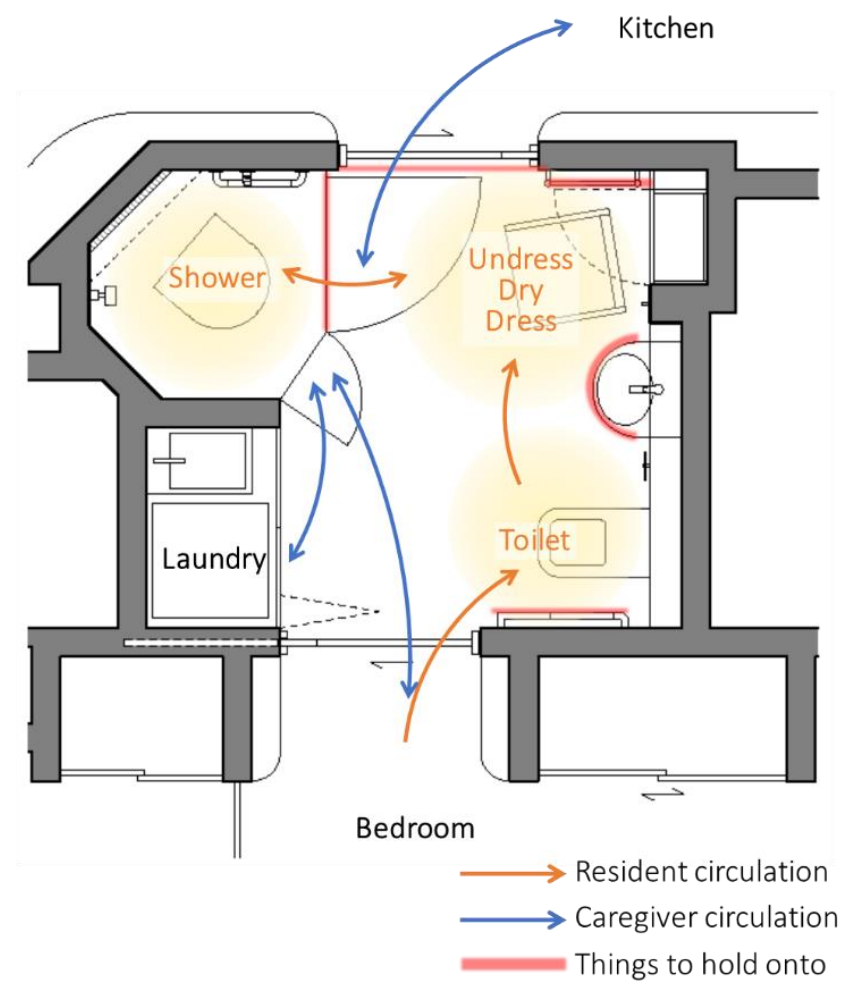

Figure 9-31: Circulation and things to hold onto (2B)

For the shower area, an enclosure is used to limit water flow for greater safety of caregivers when assisting residents with washing. A trench drain is used rather than a round drain, which typically increases the capacity in the water catchment and lessens the floor gradient, thereby reducing resident fear of falling. A semi-translucent glass screen is used to provide the resident with greater privacy from caregivers while caregivers provide supervision outside the shower area. This also allows caregivers to remain in the bathroom while residents maintain their privacy, thereby increasing resident safety (Figure 9-32). 


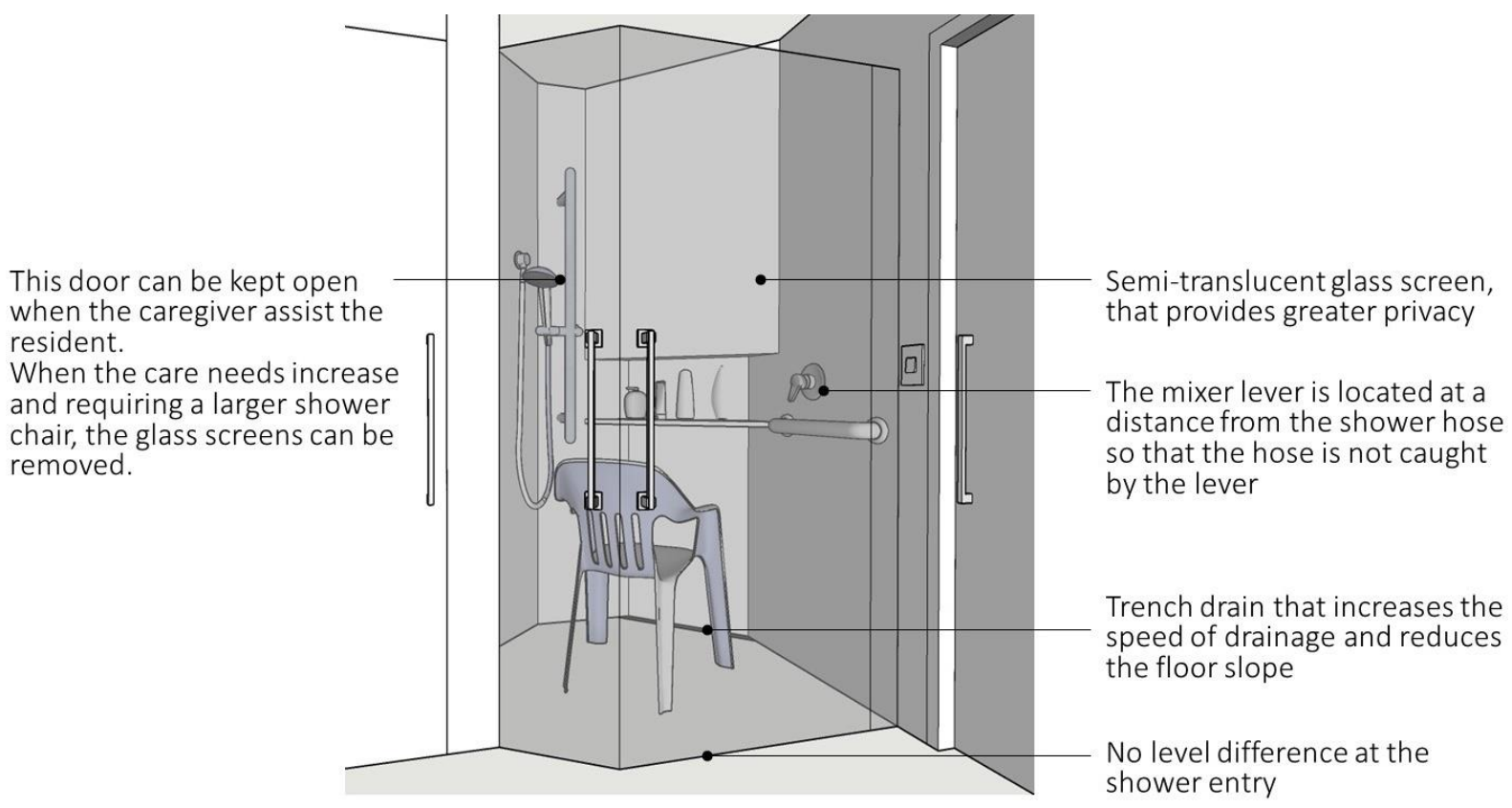

Figure 9-32: Shower area

The space for undressing, drying and dressing contains enough room for a caregiver to dry a resident and to provide things for residents to hold onto when standing up for assisted drying, as well as storage for dry items (such as towels and clothes) and a heater (Figure 9-33, 9-34).

Enough space for a caregiver to move between back and front sides

Enough space for a caregiver to crouch in front of the resident to dry and put lotion/cream on the legs

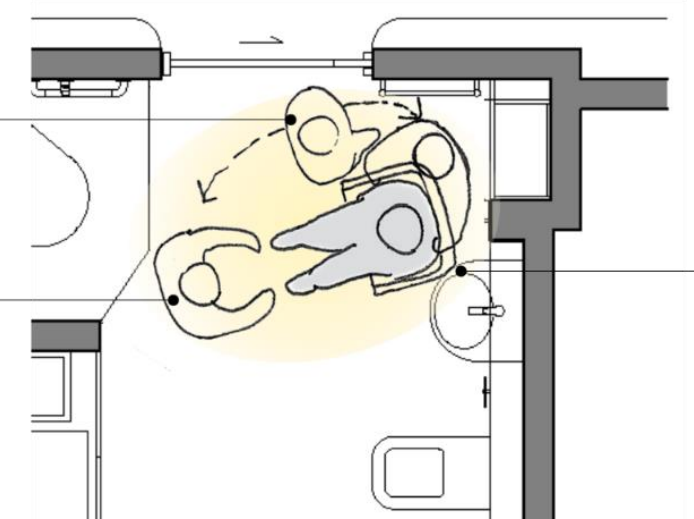

Figure 9-33: Space for drying (plan)
A vanity counter to hold onto while the resident is standing 


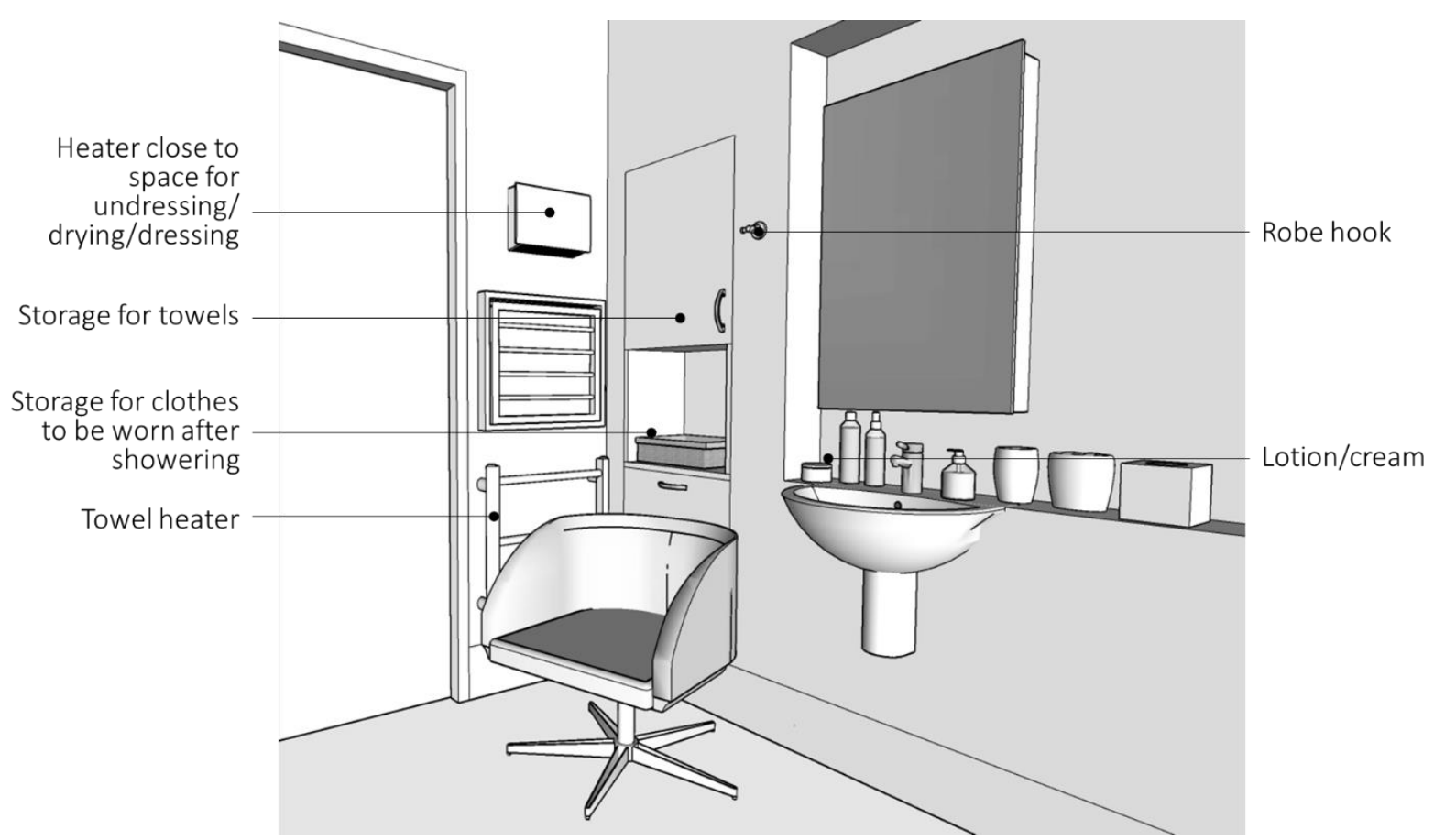

Figure 9-34: Space for drying (perspective)

The laundry is designed with space for a washing machine as well as a clothes dryer wallmounted above the washing machine, except for the BS-type where space for a clothes dryer is not provided (the one in the communal laundry can be used). A tub, which is often used by a professional cleaner, is provided for the 2B-type, while it is not provided for other unit types. Instead, for the 1B-types an additional tap is provided in the shower area for putting water in a bucket (Figure 9-35). For the BS-type, a communal laundry space including a tub is assumed to be used instead. A folding laundry rack is provided in the bathroom (Figure 9-36). 


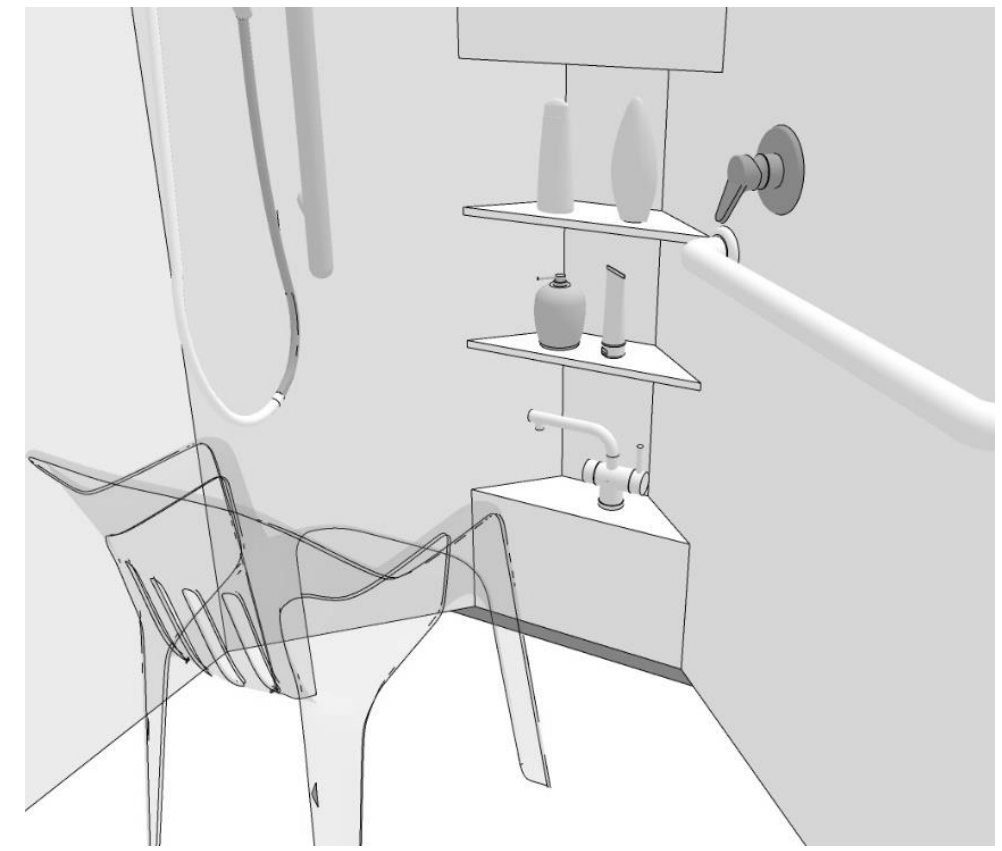

Figure 9-35: Tap in the shower area (1B-A)

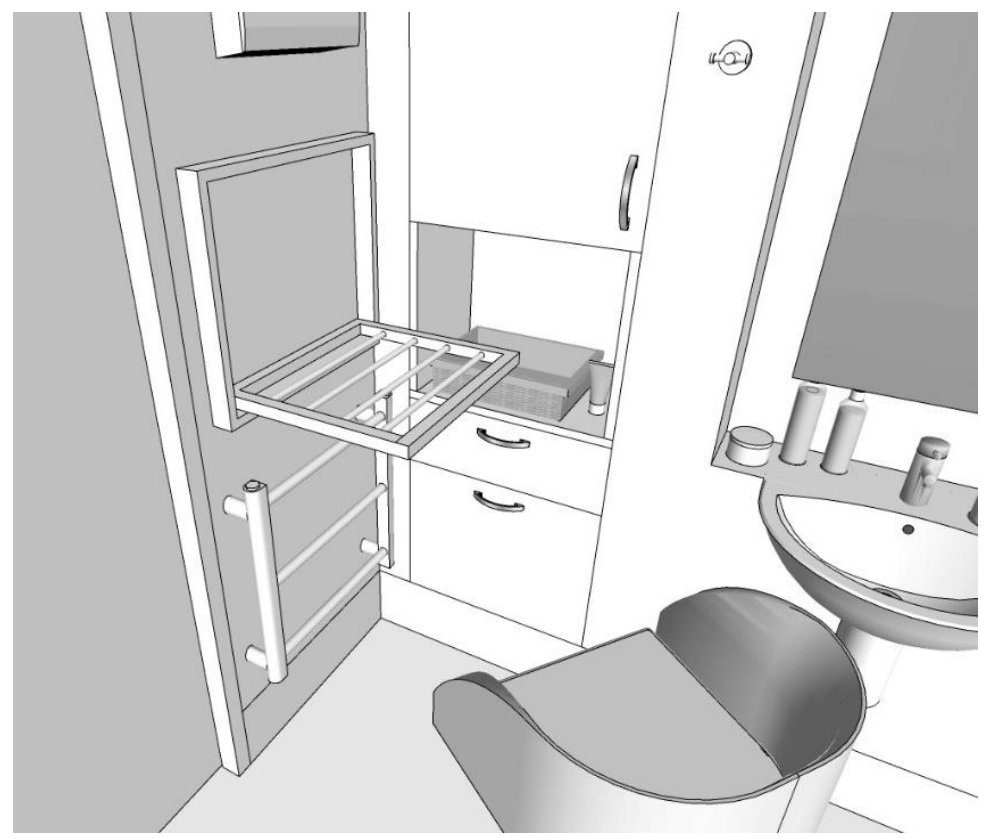

Figure 9-36: Folding laundry rack

Shelves and shower fixtures are provided within reach of a seated resident in the shower area. An emergency button is provided in the area which can be reached following a fall. Bathroom storage (shelves, cupboard and drawers) is also provided at a height within reach (Figure 9-37). 


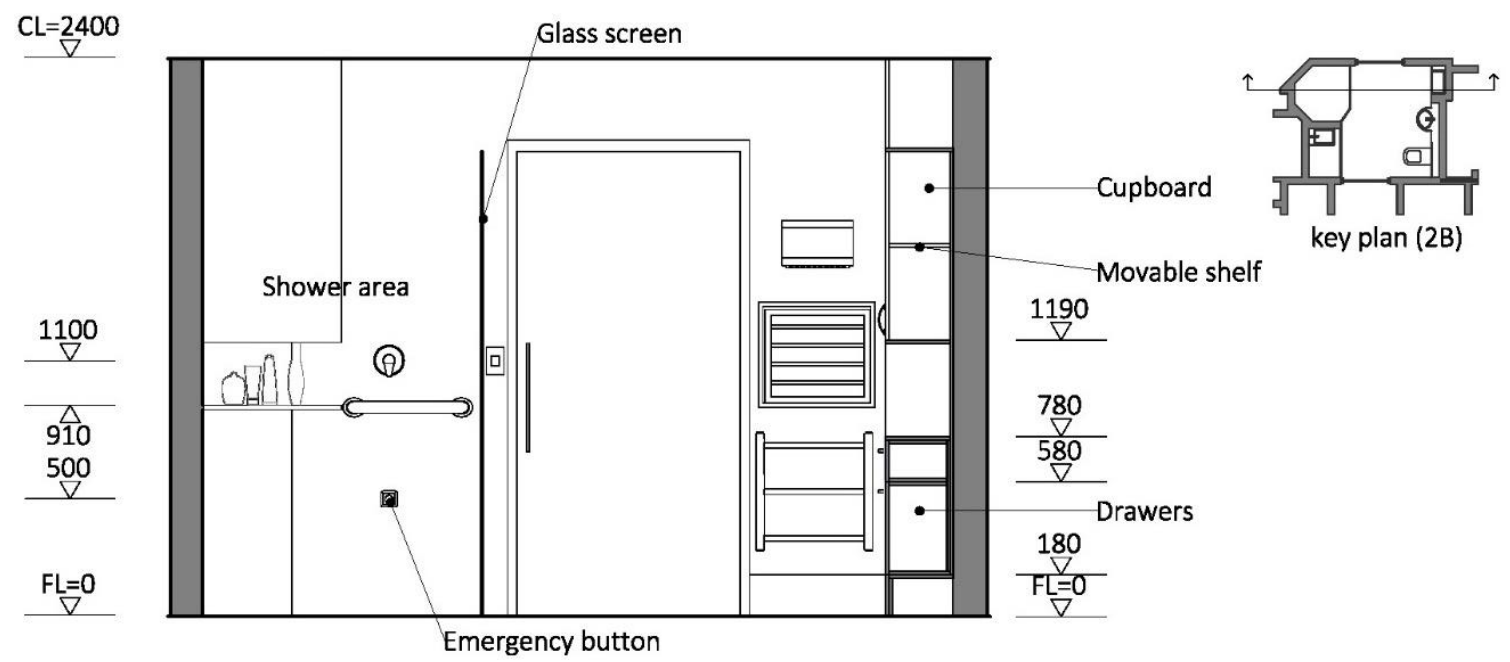

Figure 9-37: Section/interior elevation of the bathroom (2B)

\section{Outer space}

The outer space (excluding the kitchen and bathroom) is designed to accommodate furniture along the wall, as well as decorative items such as paintings (Figure 9-38). The sitting space is designed to accommodate an armchair with enough space for adjacent tables. It is also possible to accommodate special furniture for preferred activities or items that the resident treasures using the space between the bed and sitting space. For example, residents can put items such as a sewing table, a workshop bench, an exercise machine or a piano here.

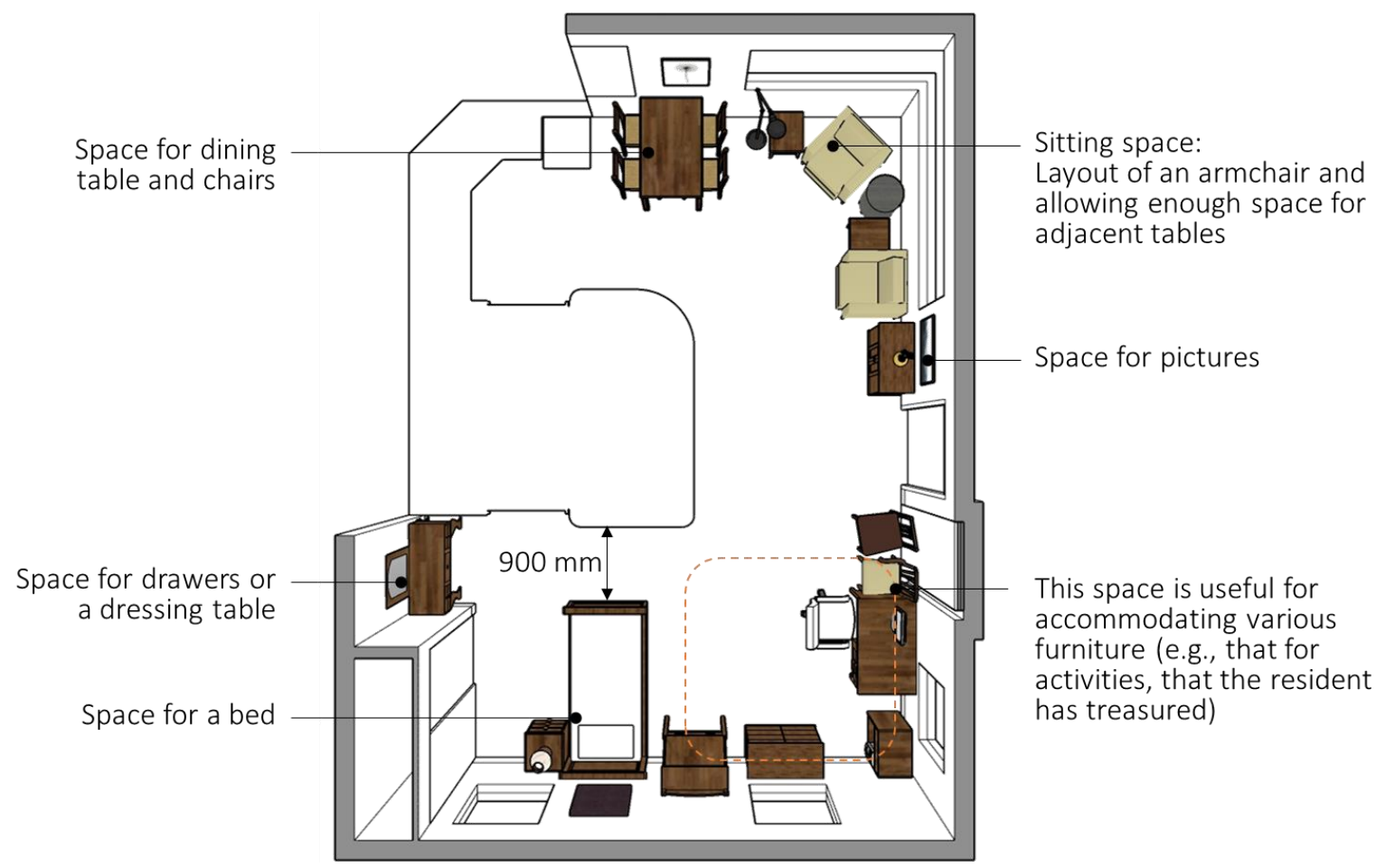

Figure 9-38: Perspective of outer space (1B-A) 
The bottom height of the windows is set at $900 \mathrm{~mm}$ high, which can provide sitting residents with views to outside, as well as accommodate many types of furniture underneath the windowsill (Figure 9-39).

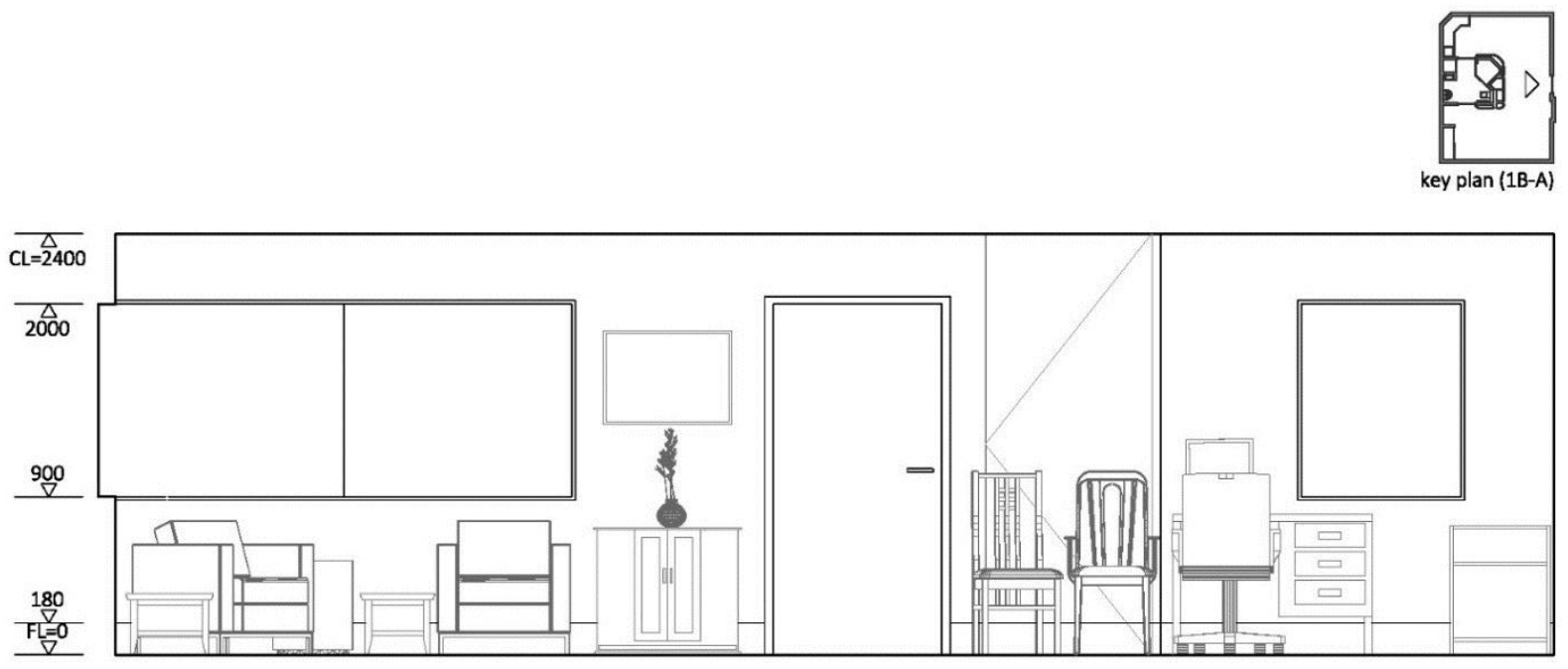

Figure 9-39: Interior elevation (1B-A)

\section{Considerations for flexibility}

The design should accommodate changes in residents' mobility and type of mobility aids used, as well as life changes and individual preferences. In the design of individual dwellings, some level of flexibility is afforded to accommodate these variations. (Some solutions have already been shown in the design of both the bathroom and the kitchen.)

To accommodate the change in life situation typified by the death of a partner, two-bedroom and one-bedroom units have been designed to accommodate either one or two people (Figure 940). For example, there should be space for two beds and wardrobes for two people in the bedroom. Separate spaces for both members are required for privacy from each other, particularly when doing activities requiring concentration, such as writing. When one resident is unwell and in bed, a sliding door can be closed when quietness is required for sleeping but can be open at other times. 


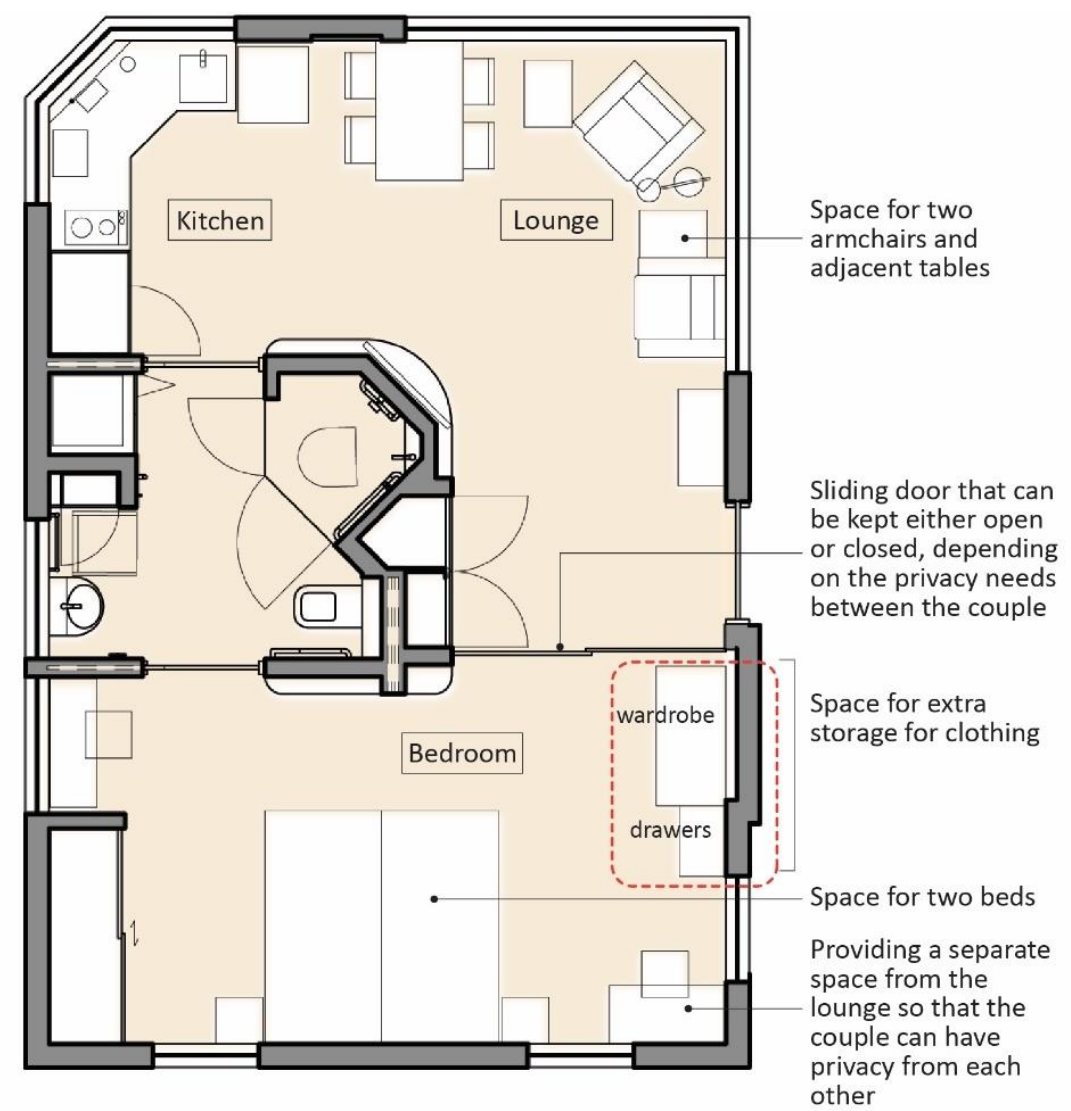

Figure 9-40: Interior layout for those living with a partner (1B-A)

There are some options for the sitting space in the lounge of the 1B-A type. The sitting position, as shown in Figure 9-41, is the option suitable for residents' whose mobility is decreasing. In this layout, the dining table is not used and the folding bench attached to the wall is stretched out, which can provide residents with greater control in activities. For example, the folding bench provides a large, level surface for the resident to put things on. Putting the kitchen storage units under the bench provides many drawers that are within reach of the resident. A computer, a laptop or a tablet can be put on the bench, which enables the resident to use it (e.g. checking messages, playing games) while they are watching TV. There is also enough space to put another desk adjacent to the armchair, which provides support for those with hemiplegia when operating a computer (Figure 9-42). 


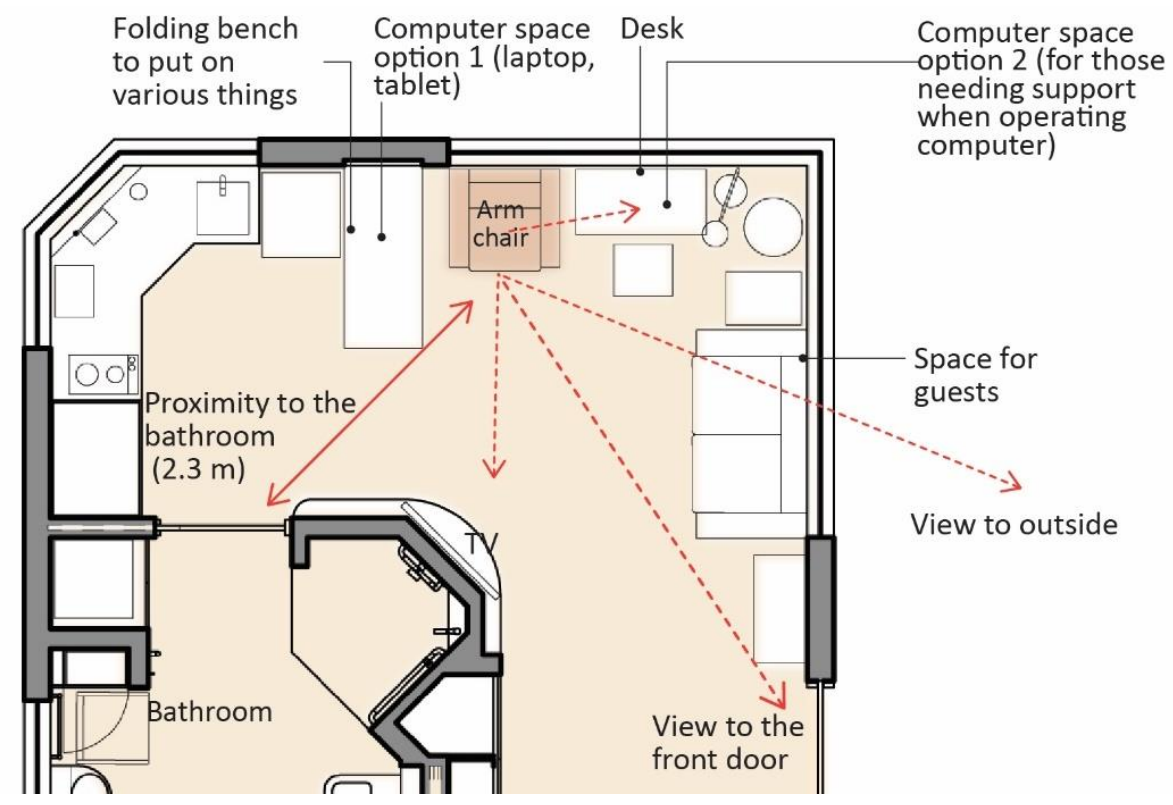

Figure 9-41: Interior layout of sitting space for those with limited mobility

The folding bench can accommodate a lot of items such as a telephone, remote controls, a pen and a paper, a cup of tea, a plate, eye glasses and books.

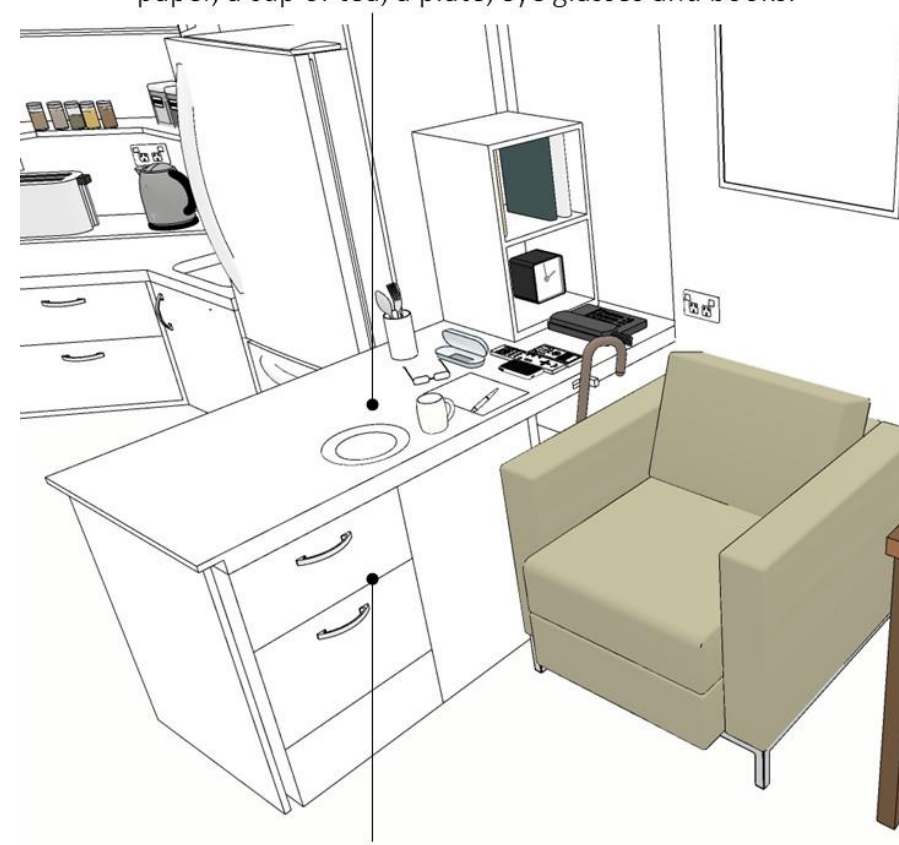

A computer screen can be placed on the adjacent desk, so that the resident can check messages and so on.

Drawer units (which are designed to be put under the kitchen bench) can be used here, to accommodate daily tools such as scissors and letter pads.

Figure 9-42: Perspective of the sitting space

Accommodating guests in individual dwellings is valuable for many elderly people. The use of sliding doors allows two rooms to be converted into one large room. In the two-bedroom unit, the second bedroom is useful for having guests stay overnight. Providing two doors for the bathroom also allows the guest to use the bathroom without passing through the main bedroom 
and affecting residents' privacy (Figure 9-43). When not having overnight guests, the lounge and the second bedroom can be used efficiently as one large lounge by folding the bed into the wall, which is useful for accommodating many guests or having a children's playing area.

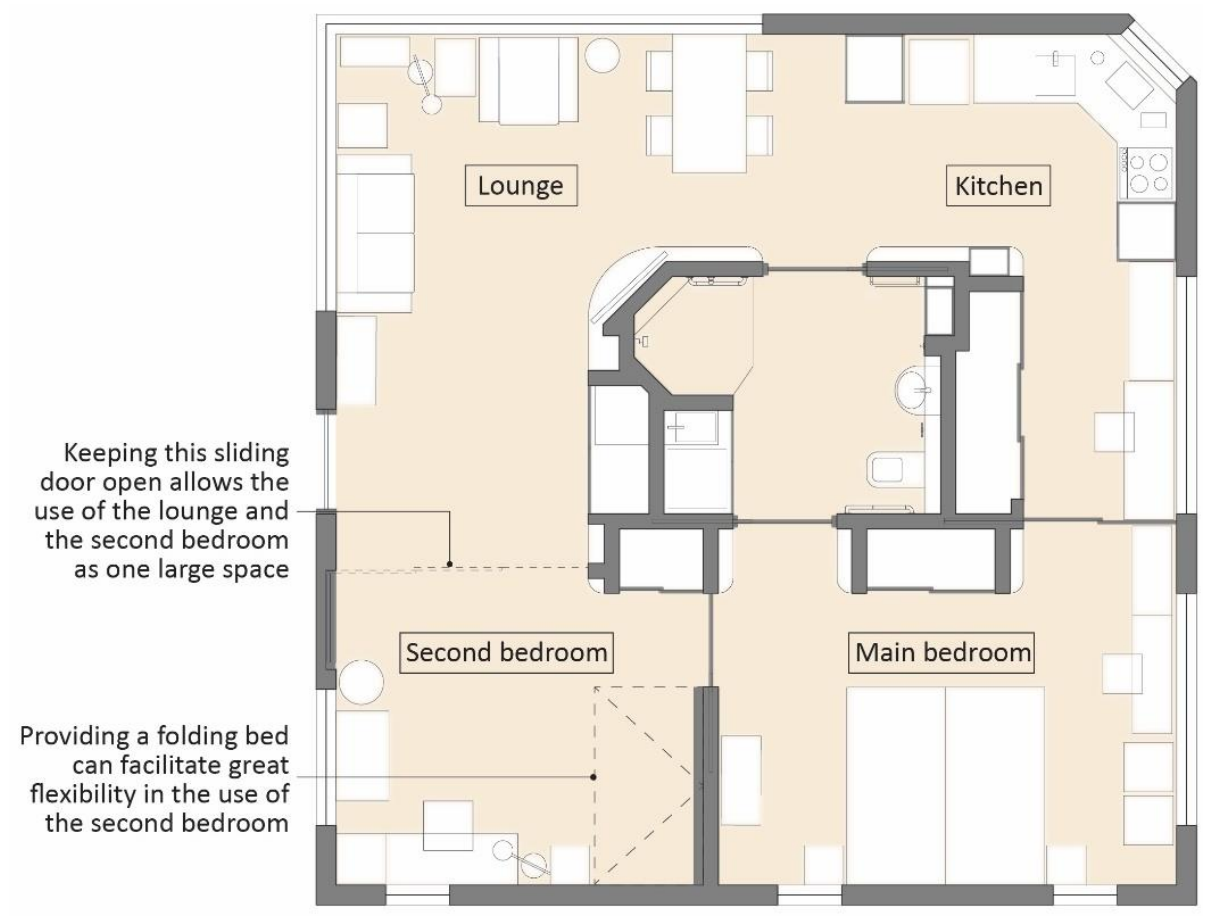

Figure 9-43: Flexible use of the second bedroom (2B)

Even in the one-bedroom units, the provision of extra space and two doors between the lounge and the bed enables residents to have space for many guests or children (Figure 9-44). If they don't need a large bedroom, residents can have extended dining space for entertaining guests with meals (Figure 9-45). The provision of an additional sliding door, combined with the use of a sofa bed, also allows for the creation of an additional bedroom (Figure 9-46). This flexibility also allows for units to be set up differently by residents on a semi-permanent basis to meet their own needs and wants. 


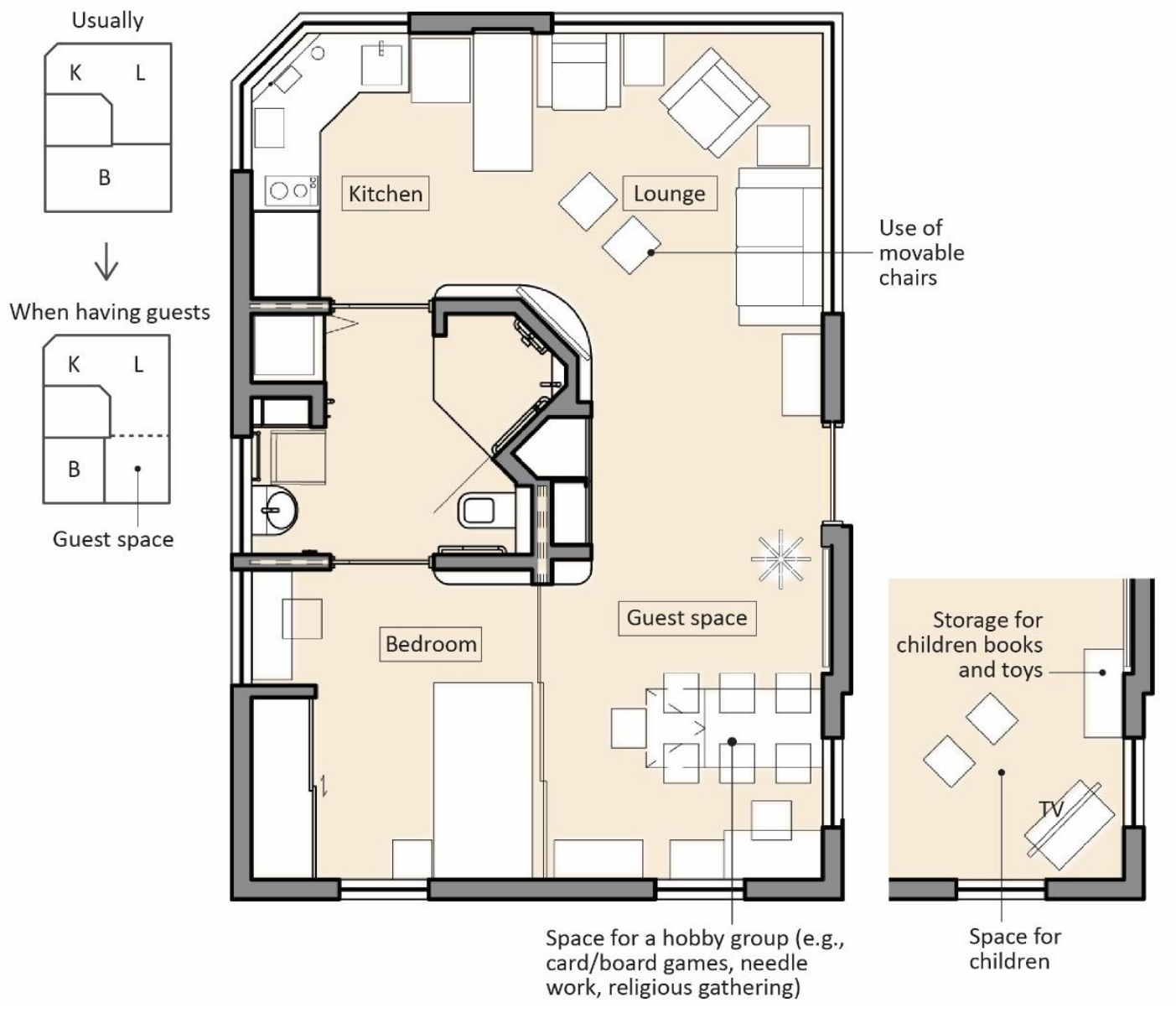

Figure 9-44: Interior layout for having guests (1B-A) 

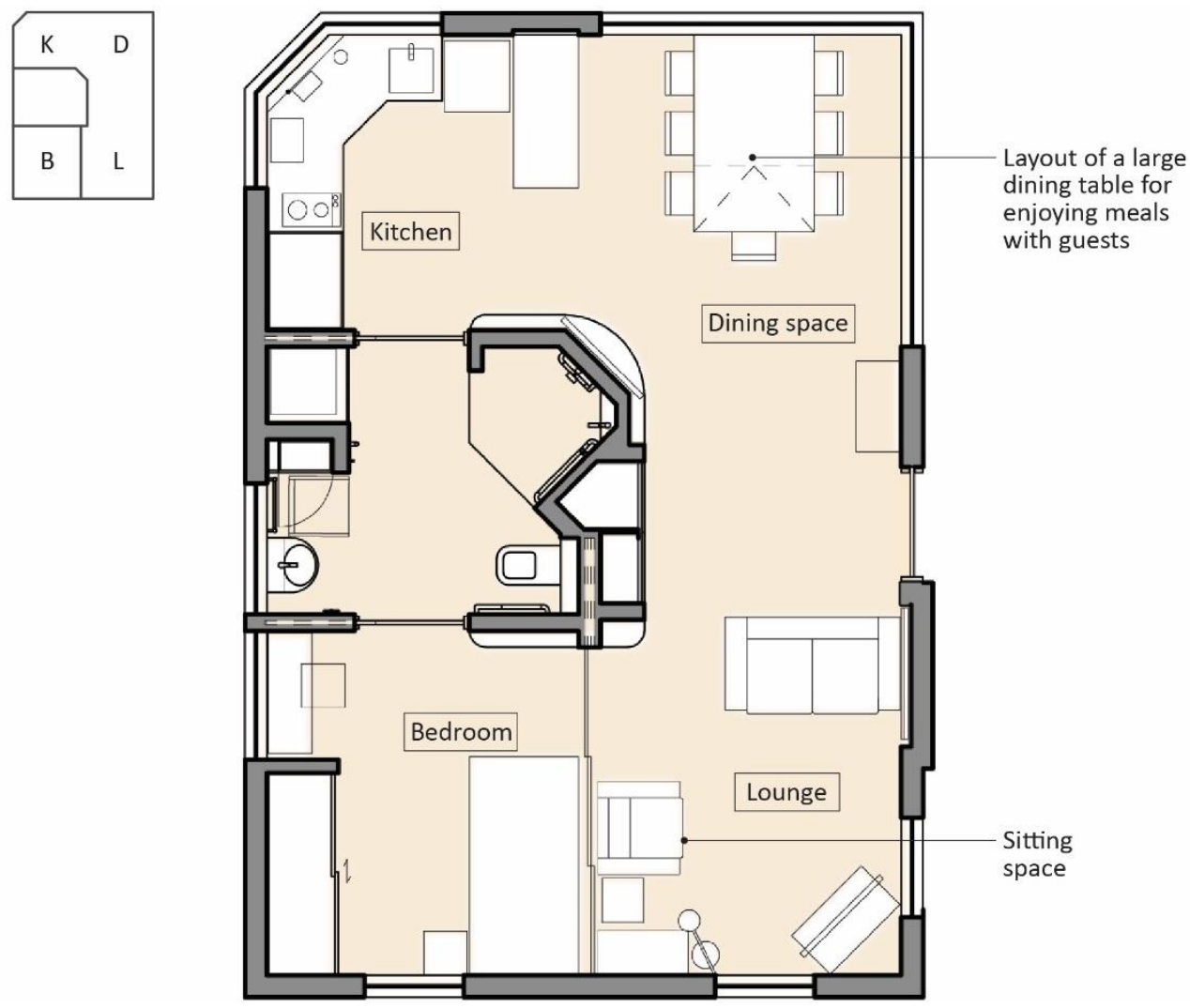

Figure 9-45: Layout of large dining space (1B-A)
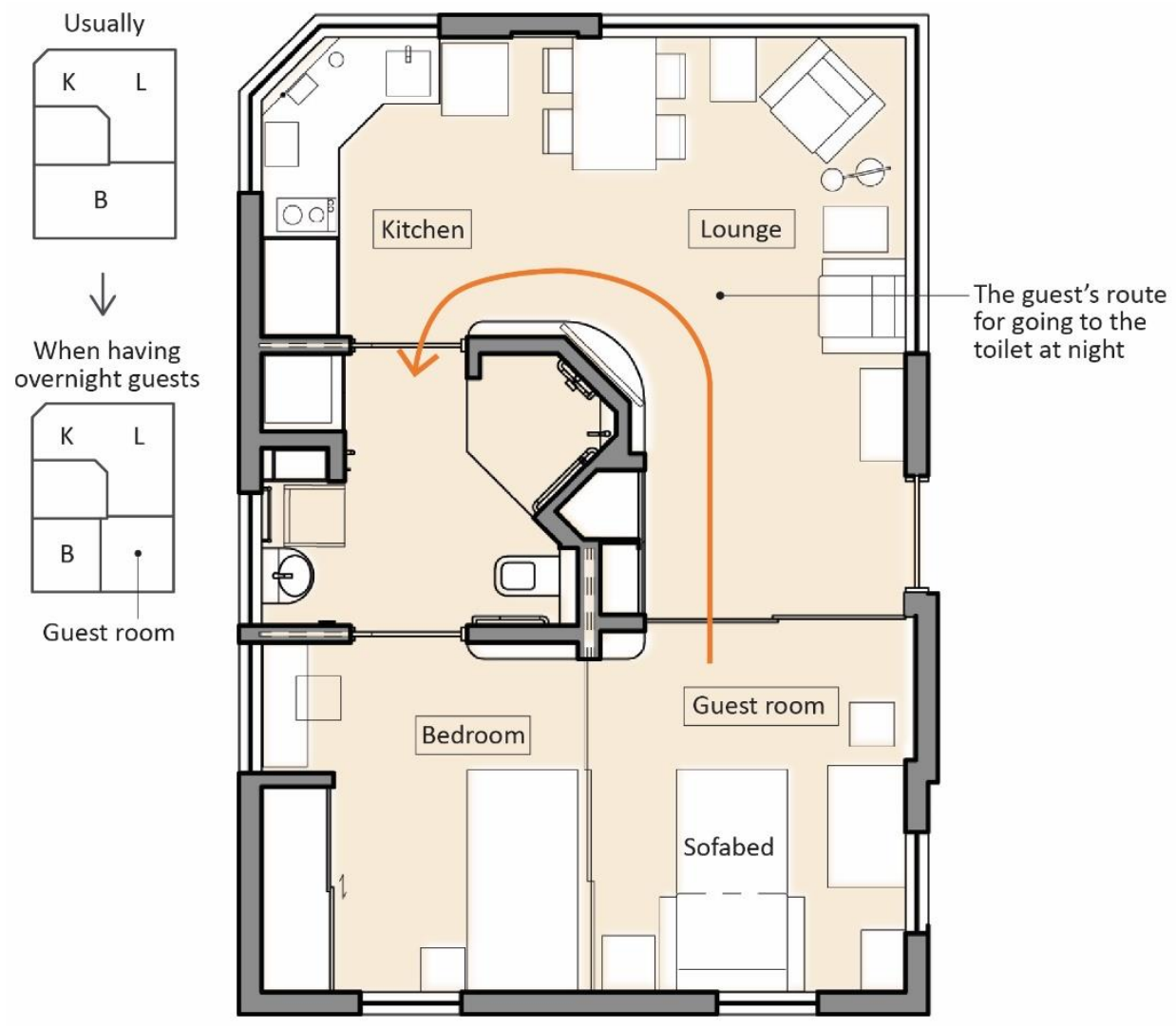

Figure 9-46: Creating an additional bedroom for an overnight guest (1B-A) 
The BS type is not designed to accommodate guests in the unit; the strategies to accommodate guests are considered with use of communal areas.

\section{Consideration of the cost}

Desirable models for the individual units, designed to facilitate the greatest QoL and maximum flexibility, have been shown. Figure 9-47 shows a model of the 1B-A unit with a reduced cost, compromising some of the QoL. For example, sliding doors stored in walls can be replaced with a roll screen and a door with the frame outside the wall, reducing some level of privacy when accommodating guests and the degree of flexibility in use of space. A kitchen bench can be fixed at either $700 \mathrm{~mm}$ or $900 \mathrm{~mm}$ high, which limits the suitability for different users and therefore the length of time that an individual can stay in the unit. These negotiations would be necessary depending on the budget and what kind of QoL is valued.

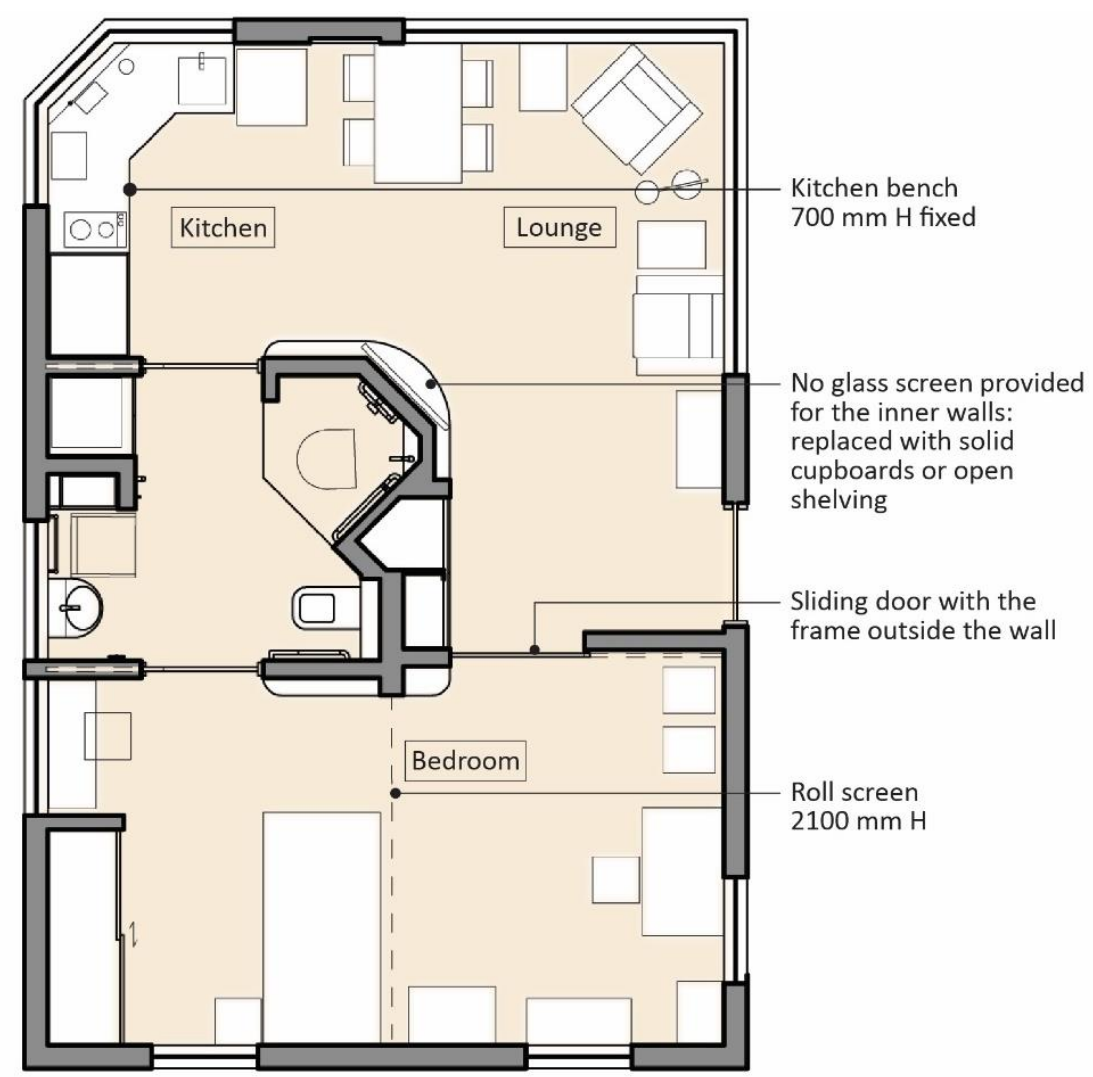

Figure 9-47: Ideas for reducing the cost

\subsection{Design of complexes}

Using the housing units designed in the previous section, three models of housing complexes are designed: 
1) rental housing, self-contained model;

2) rental housing, communal-living model; and

3) Retirement-village supported-living model.

\section{Design conditions}

Design of complexes requires consideration of conditions such as the geographical context and surrounding environments. In addition, in the design of housing for the high-needs elderly it is essential to define the service models, which include services for residents, staffing and communal areas.

\section{Site selection}

For the three complex models, one site has been selected in the central area of Karori, a Wellington suburb. One of the features of Karori is that it is surrounded by hills and forests, which can be utilised in the design to enhance resident QoL (Figure 9-48). A $4720 \mathrm{~m}^{2}$ residential area in central Karori was selected for the housing site. A community centre is located adjacent to the site, and other facilities such as the city library are located in the same block (Figure 9-49).

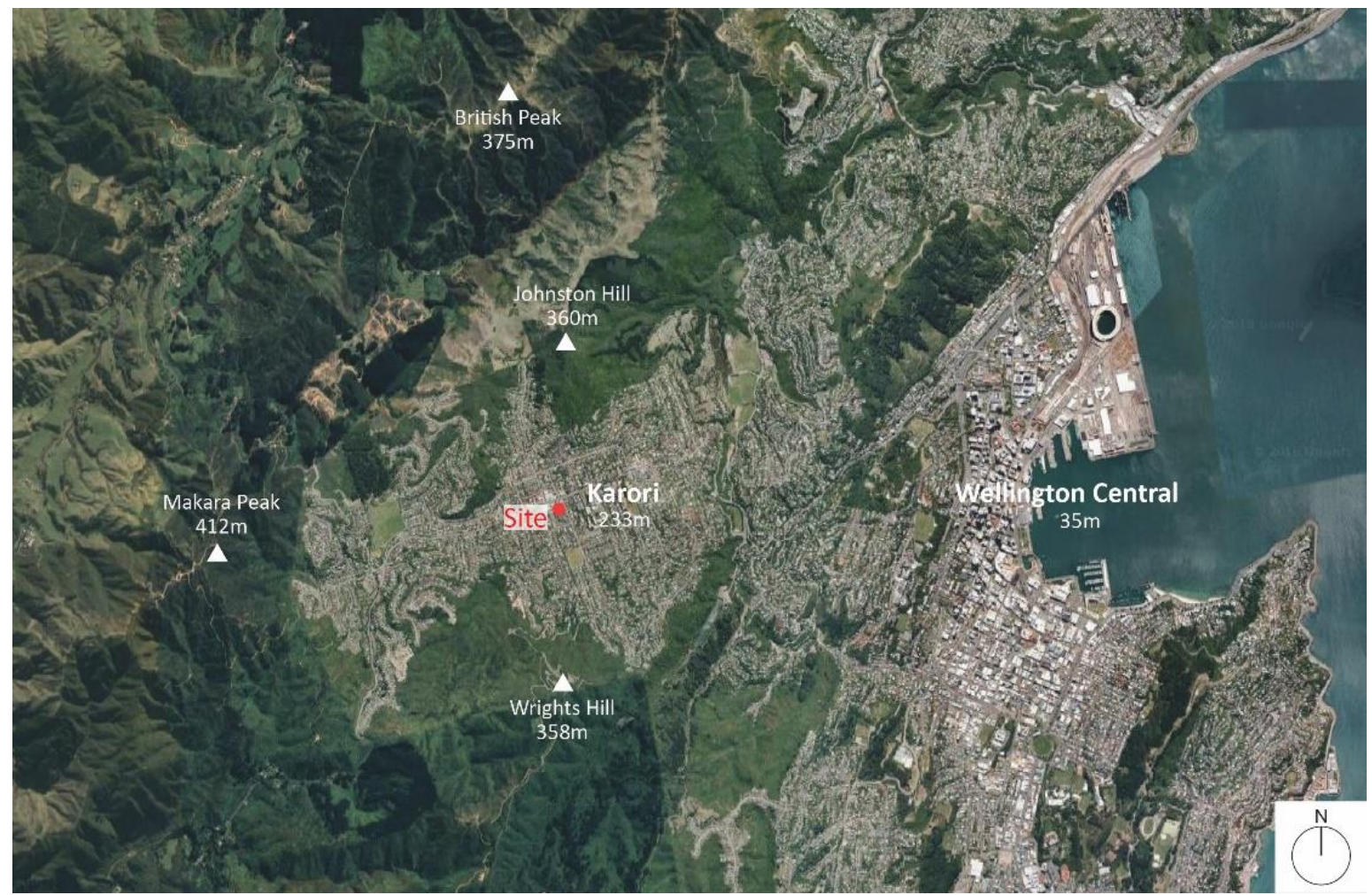

Figure 9-48: Areal map 


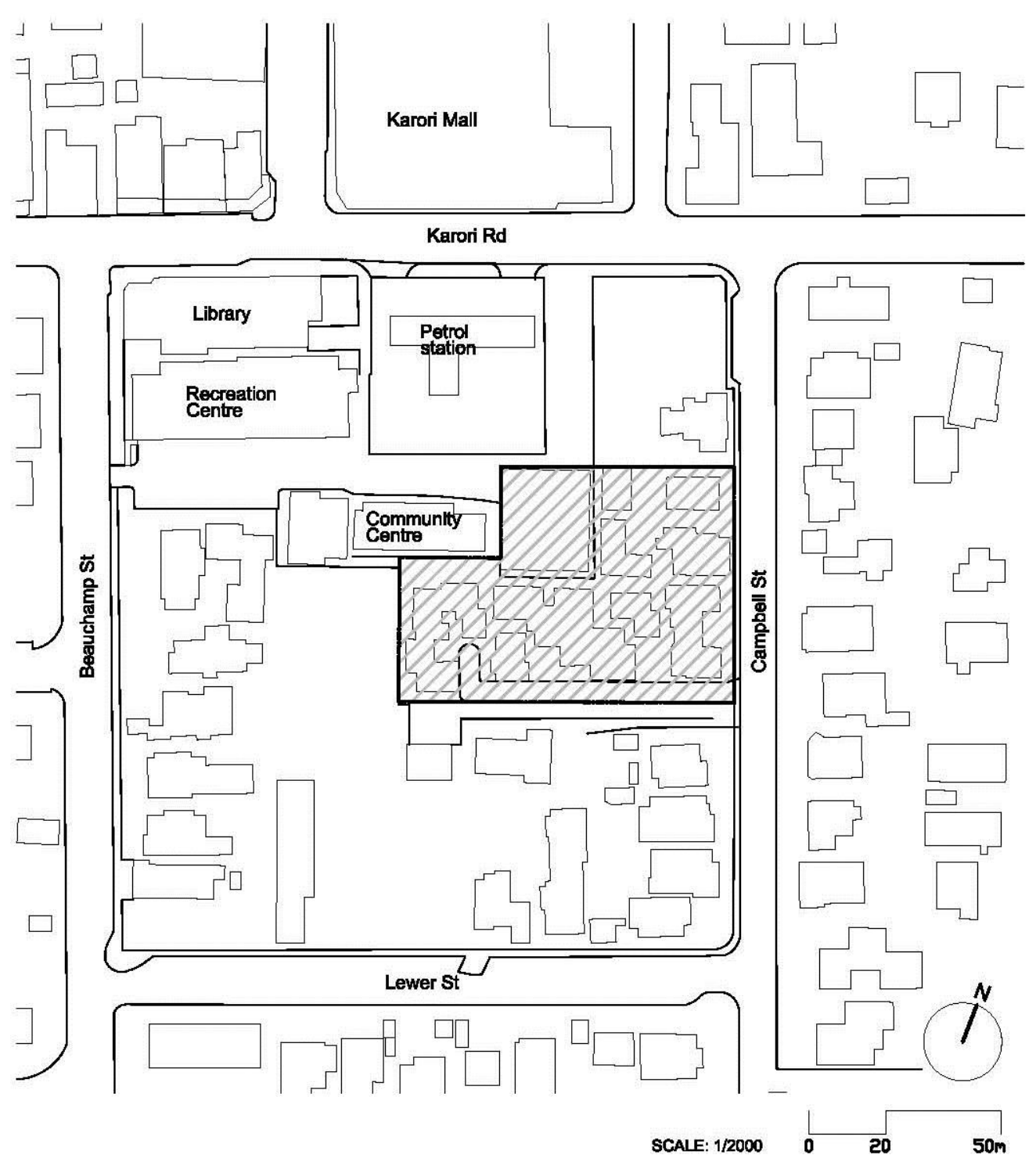

Figure 9-49: Site map

Service models and building/unit types

Based on current situations of housing as clarified in Phase 1 and 2 of this research, services combined with housing, staff spaces and communal spaces for residents, as well as the type of building and individual units, are shown in Table 9-2. 
Table 9-2: Design conditions for complexes

\begin{tabular}{|c|c|c|c|c|c|c|}
\hline & Features & $\begin{array}{l}\text { Services combined } \\
\text { with housing }\end{array}$ & $\begin{array}{l}\text { On-site staff } \\
\text { space }\end{array}$ & $\begin{array}{l}\text { Indoor communal } \\
\text { spaces }\end{array}$ & $\begin{array}{l}\text { Building } \\
\text { type }\end{array}$ & $\begin{array}{l}\text { Individual } \\
\text { unit } \\
\text { types }\end{array}$ \\
\hline $\begin{array}{l}\text { Rental } \\
\text { housing, } \\
\text { self- } \\
\text { contained } \\
\text { model }\end{array}$ & $\begin{array}{l}\text { The complex is } \\
\text { developed in } \\
\text { collaboration } \\
\text { with the local } \\
\text { council and } \\
\text { DHB, to share } \\
\text { the resources. } \\
\text { This complex } \\
\text { facilitates } \\
\text { gardening and } \\
\text { musical } \\
\text { activities. }\end{array}$ & $\begin{array}{l}\text { - Staff visits on } \\
\text { request } \\
\text { - Emergency on } \\
\text { call } 24 \mathrm{~h} \\
\text { - Weekly } \\
\text { organised } \\
\text { activities } \\
\text { - Personal care }\end{array}$ & $\begin{array}{l}\text { Yes (an office } \\
\text { is provided } \\
\text { for a DHB- } \\
\text { contracted } \\
\text { caregiver } \\
\text { who also } \\
\text { looks after } \\
\text { the } \\
\text { community) }\end{array}$ & $\begin{array}{l}\text { - Small } \\
\text { communal } \\
\text { spaces } \\
\text { (outdoors) } \\
\text { - A music room } \\
\text { (communally } \\
\text { used with } \\
\text { Community } \\
\text { Centre) } \\
\text { - For } \\
\text { annual/monthly } \\
\text { gatherings, the } \\
\text { room in the } \\
\text { adjacent facility } \\
\text { (community } \\
\text { centre) is used. }\end{array}$ & $\begin{array}{l}\text { Apartment } \\
\text { type with } \\
\text { outdoor } \\
\text { access, } \\
\text { multi- } \\
\text { story }\end{array}$ & $\begin{array}{l}\text { 1B-A, 1B- } \\
B, 2 B\end{array}$ \\
\hline $\begin{array}{l}\text { Rental } \\
\text { housing, } \\
\text { communal } \\
\text { living model }\end{array}$ & $\begin{array}{l}\text { This complex } \\
\text { provides the } \\
\text { meal service for } \\
\text { residents. Units } \\
\text { are grouped } \\
\text { into two } \\
\text { themed } \\
\text { clusters, which } \\
\text { facilitate } \\
\text { specific } \\
\text { activities. }\end{array}$ & $\begin{array}{l}\text { - Staff visits on } \\
\text { request } \\
\text { - Emergency on } \\
\text { call } 24 \mathrm{~h} \\
\text { - Weekly } \\
\text { organised } \\
\text { activities } \\
\text { - Three meals a } \\
\text { day }\end{array}$ & $\begin{array}{l}\text { Yes (a } \\
\text { housekeeper } \\
\text { is available } \\
\text { daytime) }\end{array}$ & $\begin{array}{l}\text { - Communal } \\
\text { kitchen and } \\
\text { dining room } \\
\text { - Multi-purpose } \\
\text { room } \\
\text { - Mini lounges } \\
\text { - Activity lounges } \\
\text { (e.g. art room, } \\
\text { reading lounge) } \\
\text { - Communal } \\
\text { storage for } \\
\text { residents } \\
\text { - Communal } \\
\text { laundry and } \\
\text { utility room }\end{array}$ & $\begin{array}{l}\text { Apartment } \\
\text { type with } \\
\text { indoor } \\
\text { access } \\
\text { single- } \\
\text { story }\end{array}$ & BS \\
\hline $\begin{array}{l}\text { Retirement- } \\
\text { village, } \\
\text { supported- } \\
\text { living model }\end{array}$ & $\begin{array}{l}\text { The complex is } \\
\text { provided } \\
\text { adjacent to a } \\
\text { residential care } \\
\text { facility, and the } \\
\text { facility staff } \\
\text { provides } \\
\text { services for } \\
\text { residents. }\end{array}$ & $\begin{array}{l}\text { - Staff visits on } \\
\text { request } \\
\text { - Emergency on } \\
\text { call } 24 \mathrm{~h} \\
\text { - Daily organised } \\
\text { activities } \\
\text { - One or two } \\
\text { meals a day } \\
\text { - Laundry service } \\
\text { - Daily assistance } \\
\text { in household } \\
\text { tasks } \\
\text { - Personal care, } \\
\text { rest-home level } \\
\text { care }\end{array}$ & $\begin{array}{l}\text { Yes (in the } \\
\text { adjacent care } \\
\text { facility, the } \\
\text { medical staff } \\
\text { are available } \\
\text { all day) }\end{array}$ & $\begin{array}{l}\text { - Main } \\
\text { communal } \\
\text { room } \\
\text { - Small } \\
\text { communal } \\
\text { spaces } \\
\text { - Dining room } \\
\text { - Guest rooms } \\
\text { - Art room } \\
\text { - Music room }\end{array}$ & $\begin{array}{l}\text { Apartment } \\
\text { type with } \\
\text { indoor } \\
\text { access; } \\
\text { single- } \\
\text { story }\end{array}$ & $\begin{array}{l}\text { 1B-A, 1B- } \\
B\end{array}$ \\
\hline
\end{tabular}

\section{Concepts for basic layout}

Based on three concepts for basic layout shown here, the models of complexes are designed as follows. 
For greater walkability, car parks are limited to the outer area of the housing complex, making the space inside the property car-free (Figure 9-50).

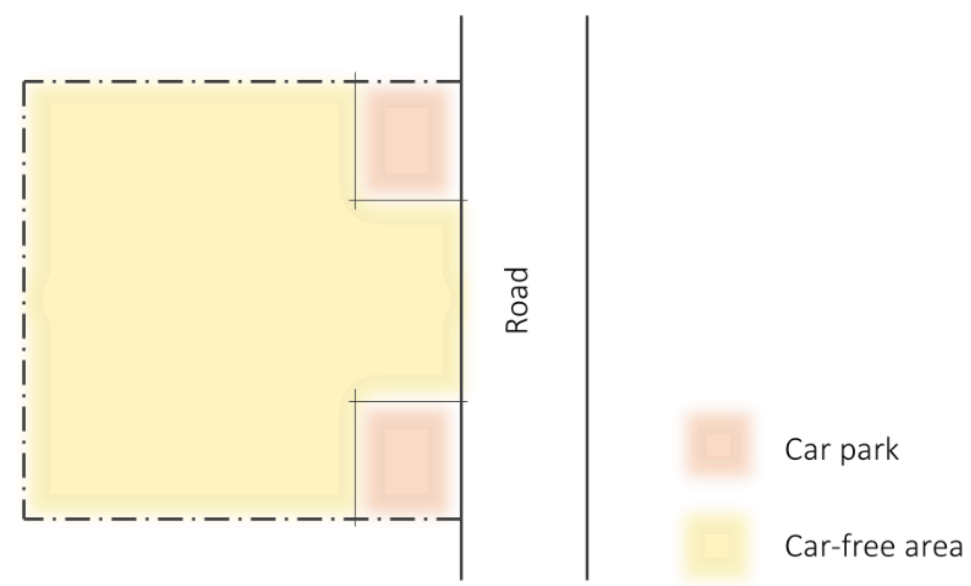

Figure 9-50: Keeping a car-free area

\section{Layout of all units with respect to north and to communal pathways}

Housing units are laid out so that the lounge can have north-facing windows (including northeast and northwest windows). Depending on the relationship between the entry and the lounge (on the same or opposite side) as well as the orientation of the communal pathway, three layout patterns are used (1B-B, BS), and those with the lounge on the same side as the entry ( $2 \mathrm{~B}$. 1B-A). The patterns of layout regarding orientation to the communal pathway are shown in Figure 9-51. 


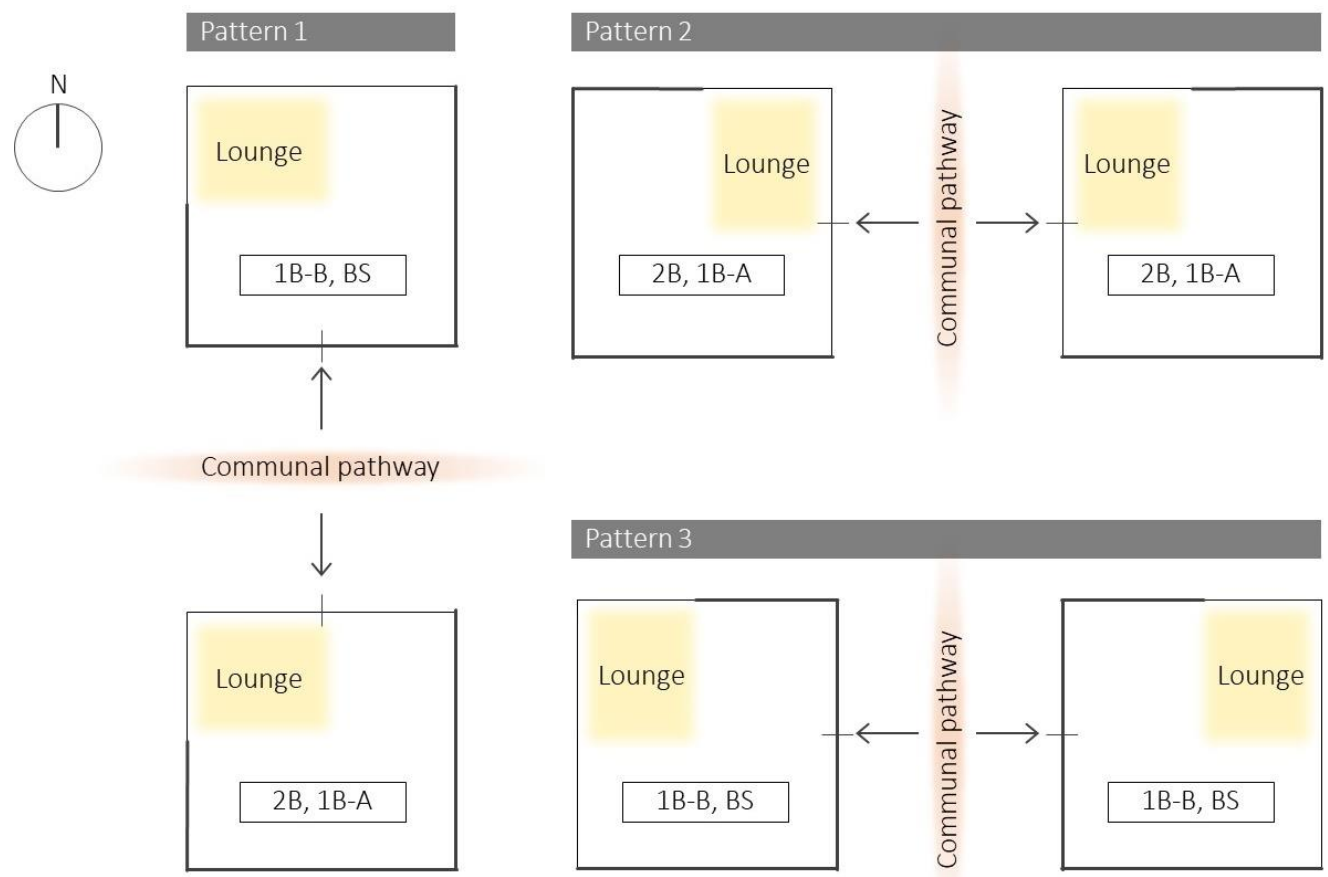

Figure 9-51: Layout of units with respect to the north and communal pathways

\section{Placement of communal spaces for facilitating specific preferred activities}

Communal spaces are provided depending on the service model of the housing complex. Three types of recreational communal space are laid out: the main multi-purpose communal space, small communal space near individual units, and space for specific use (Figure 9-52). Other necessary spaces, such as communal storage, letterboxes and rubbish collection points, are also provided.

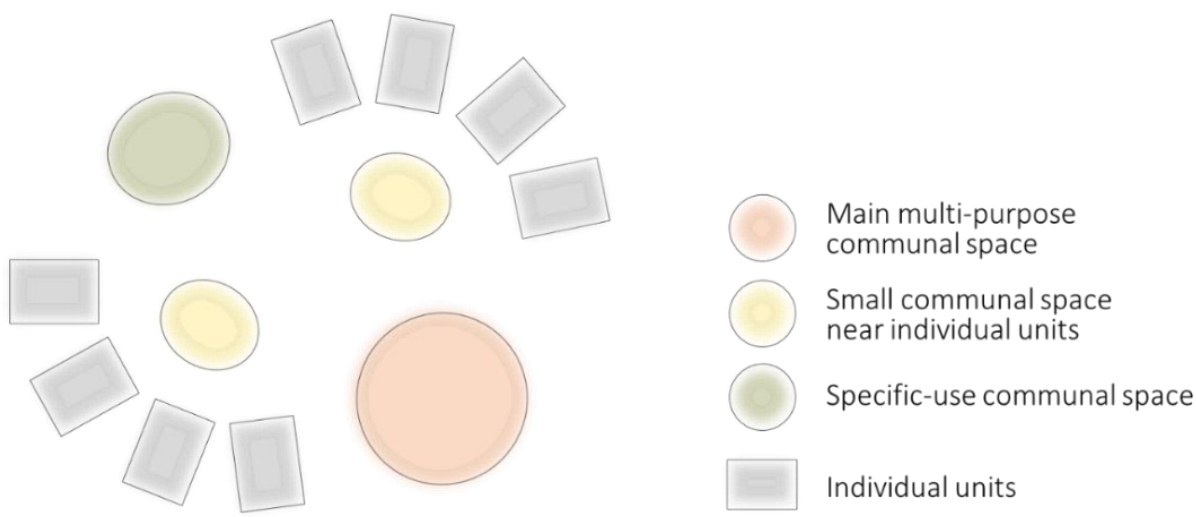

Figure 9-52: Deployment of three types of communal spaces

\section{Design}

Based on these concepts, as well as service models and building or unit types shown earlier, three models of complexes are designed here (Figure 9-53 - 9-56). 


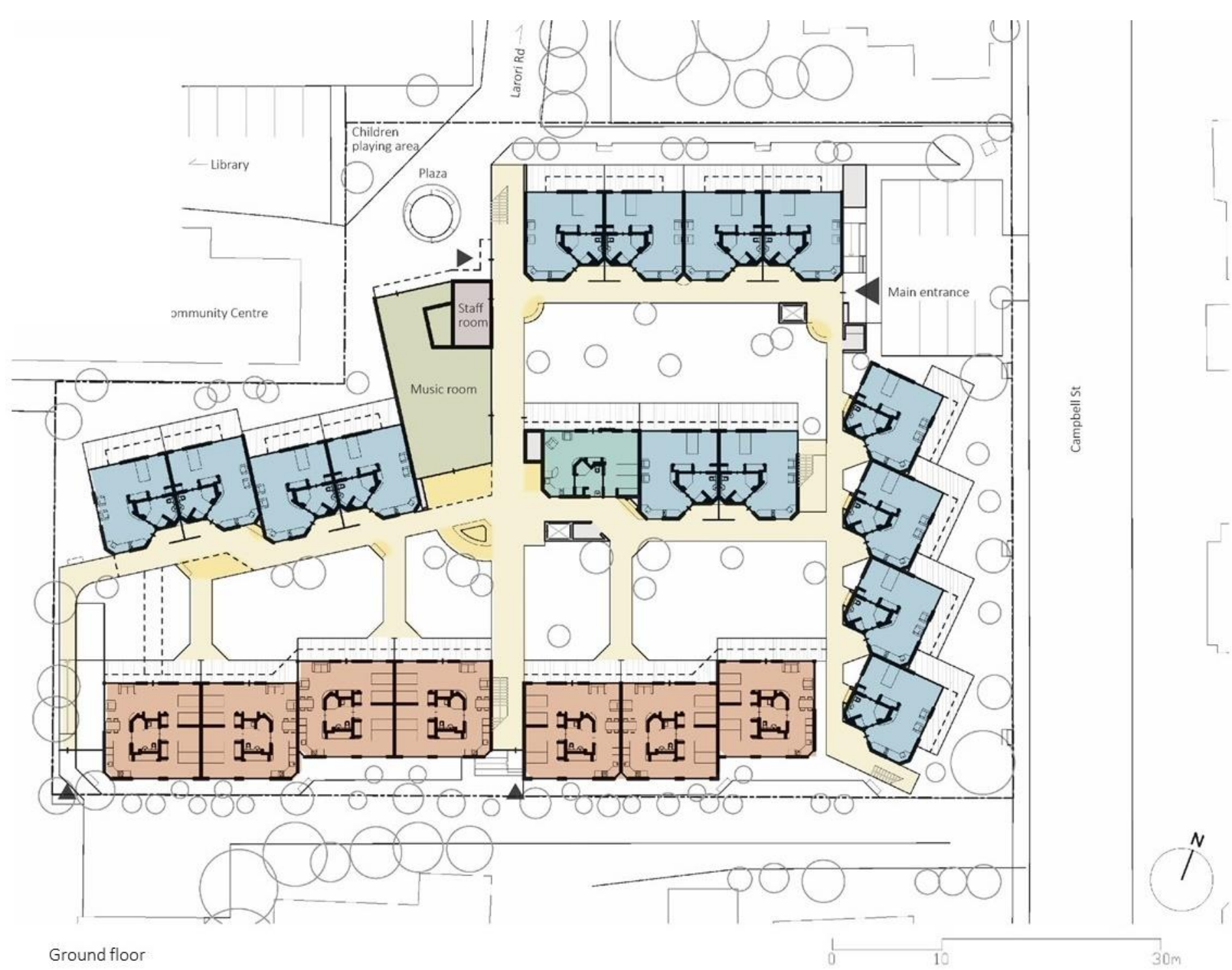

Individual units

2B Type

1B-A Type

1B-B Type

Communal spaces

Large communal space

Small communal space

Specific-use communal space

Pathway

Other

Other

Staff space

Figure 9-53: Rental housing, self-contained model - ground floor plan (22/49 units) 

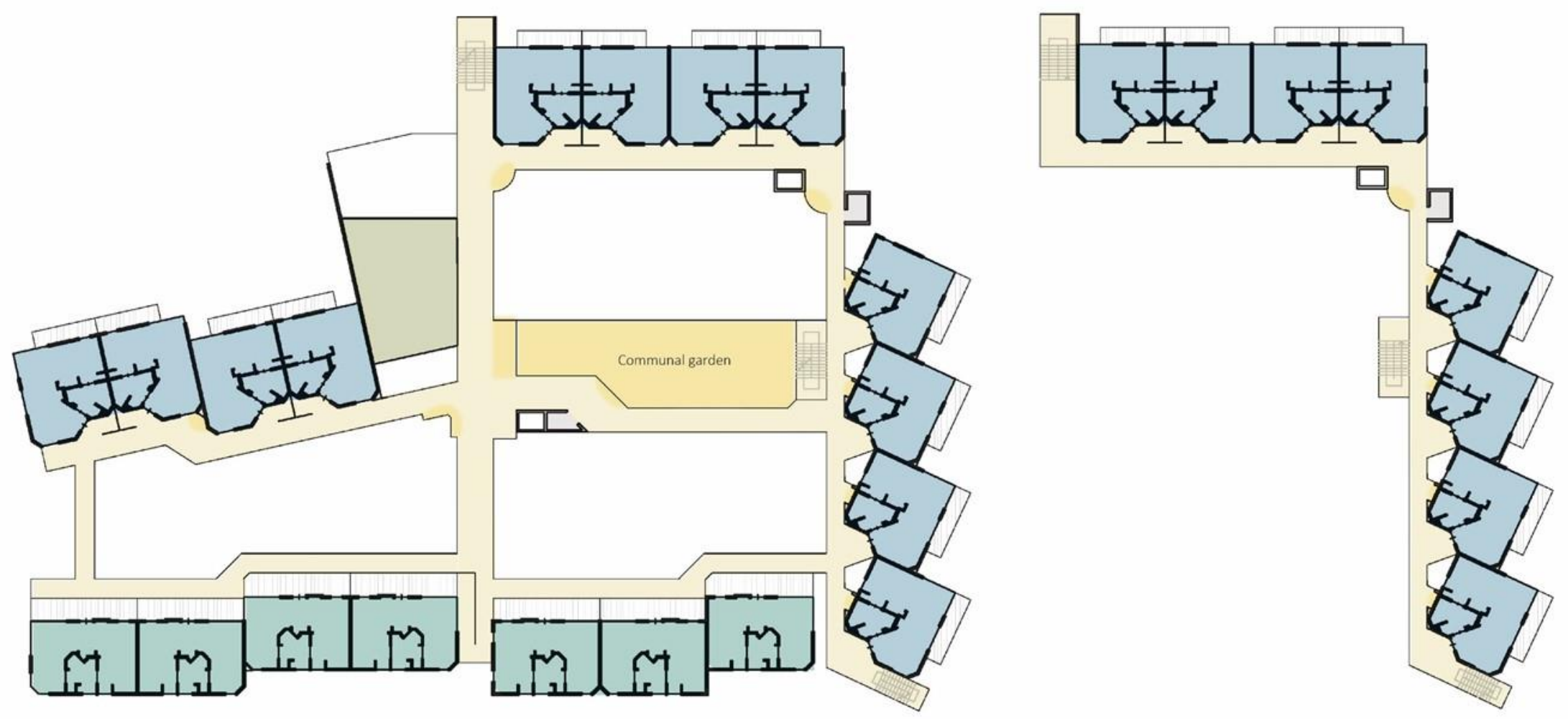

First floor

Second floor

Figure 9-54: Rental housing, self-contained model — first and second floor plans (27/49 units) 


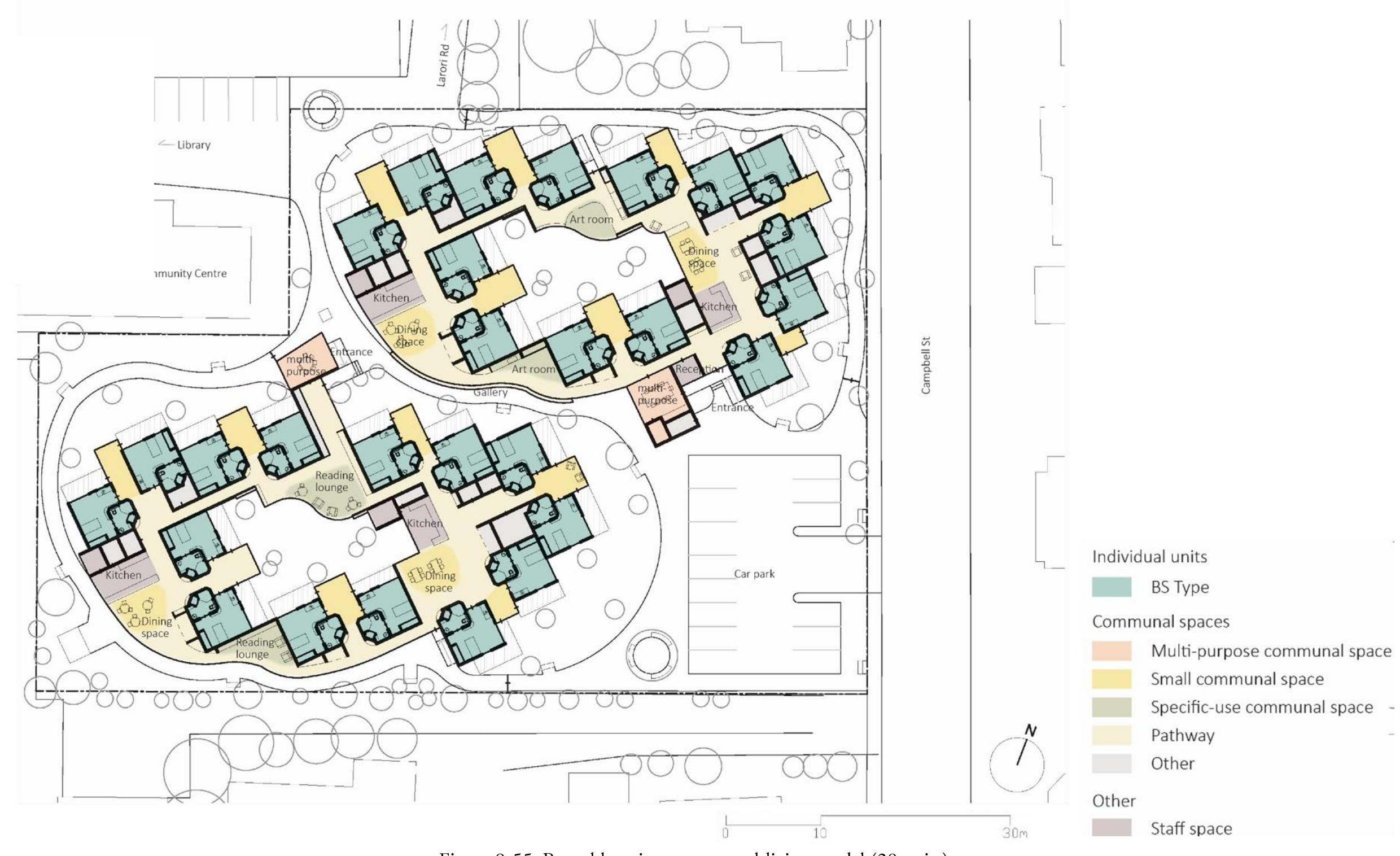

Figure 9-55: Rental housing, communal-living model (28 units) 


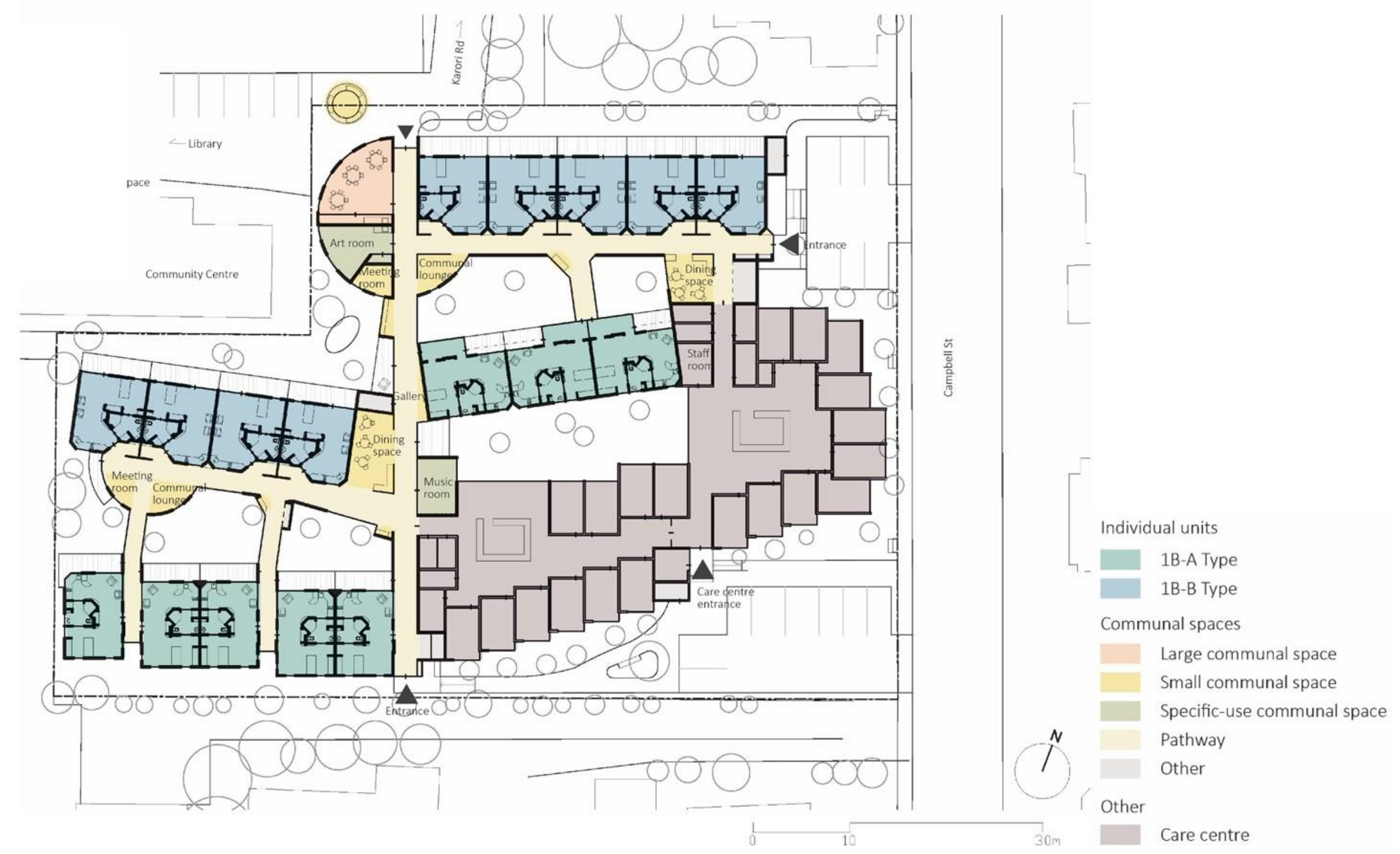

Figure 9-56: Retirement-village, supported-living model (17 units (excluding care units)) 


\section{Layout of units for greater privacy and connection among residents}

The units are laid out to maintain privacy and connection among residents. Where the loungeside of the unit faces to communal pathways, units are laid out at an angle (or enough distance is provided between them) to provide greater privacy for residents (Figures 9-57, 9-58). However, to facilitate communication between residents, views to each other's units are not completely blocked. Communal spaces with seating are provided between the units and pathways for residents to talk (Figures 9-59, 9-60).

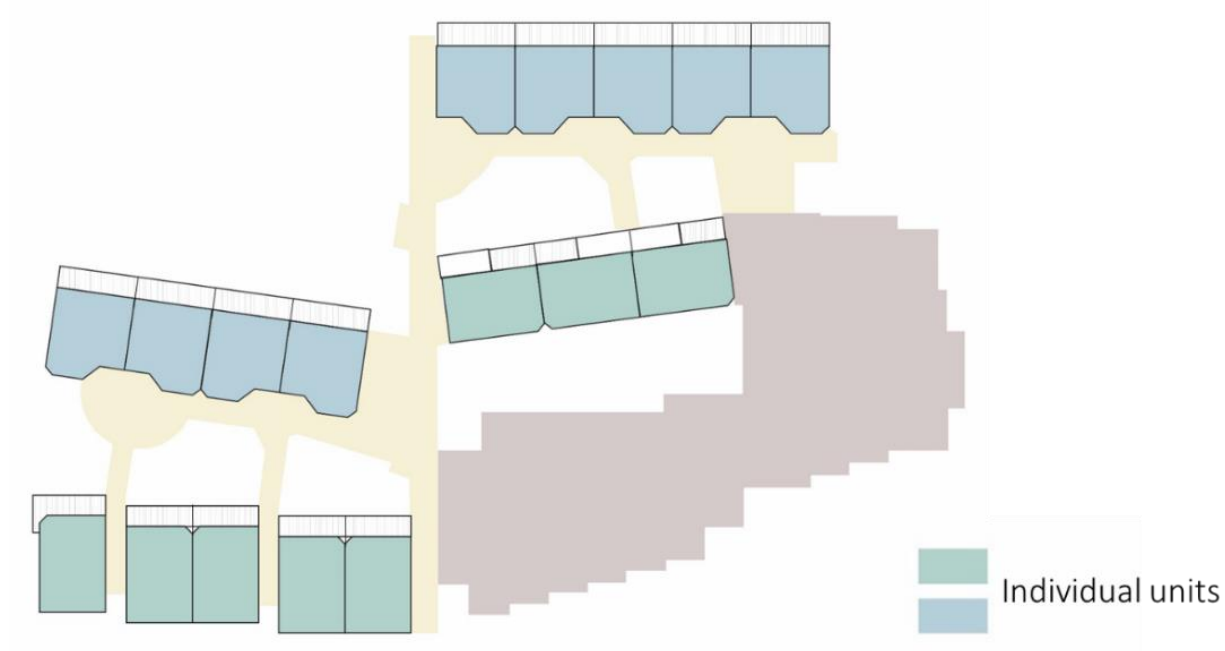

Figure 9-57: Layout of units at an angle

(Retirement-village supported-living model)

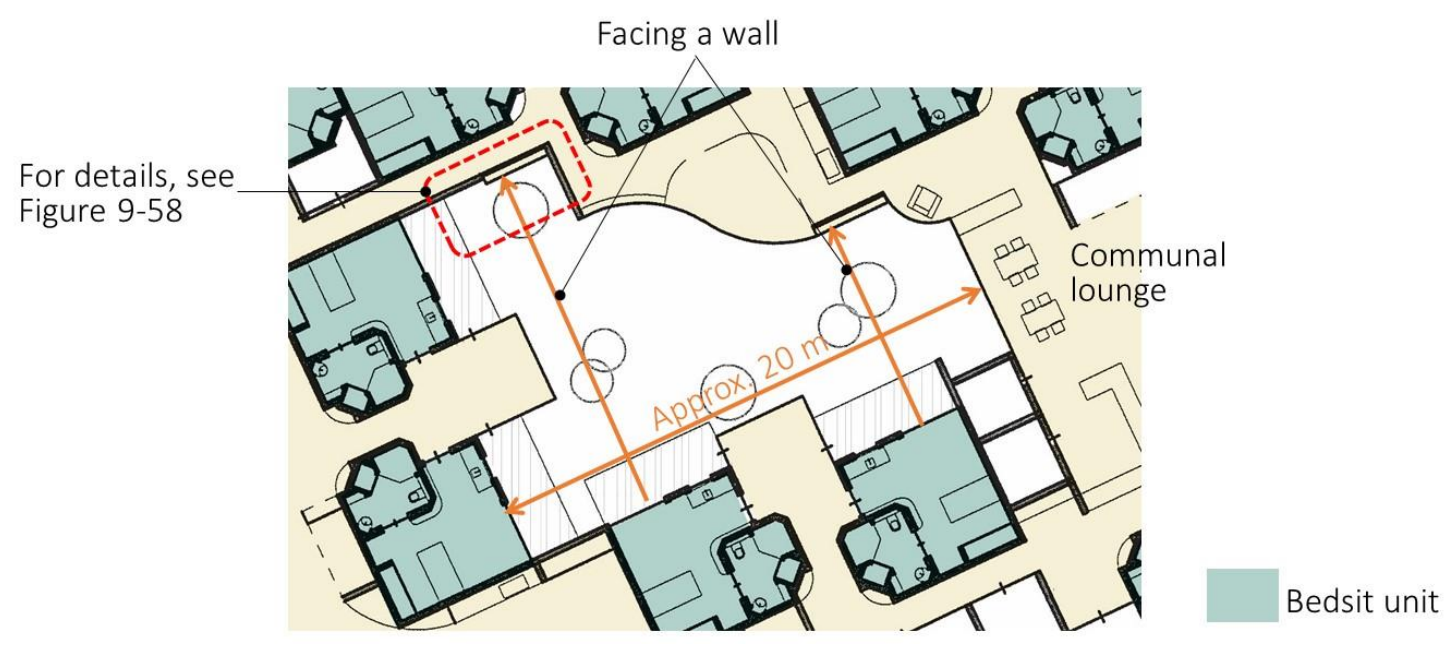

Figure 9-58: Provision of enough distance and the use of walls/glasses for maintaining resident privacy (Rental housing, communal-living model) 

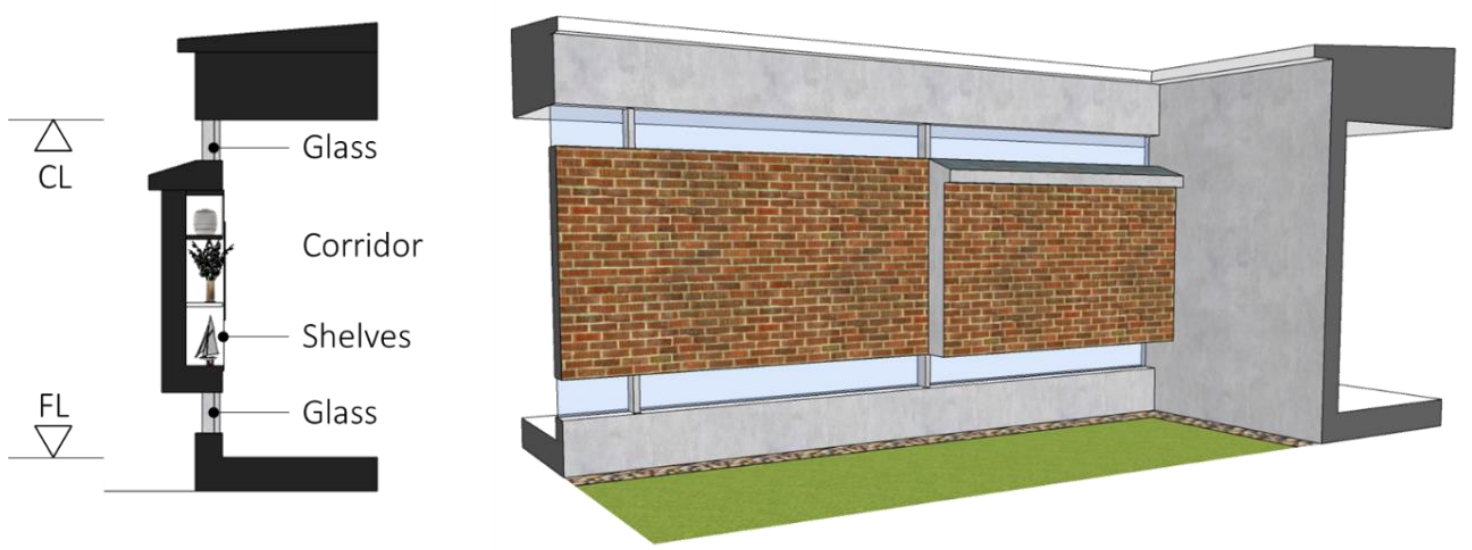

Figure 9-59: Strategy for maintaining resident privacy and a sense of connection 1 - the use of a wall/glass (The section of the wall (left) and the view from the unit side (right)) (Rental housing, communal-living model) 

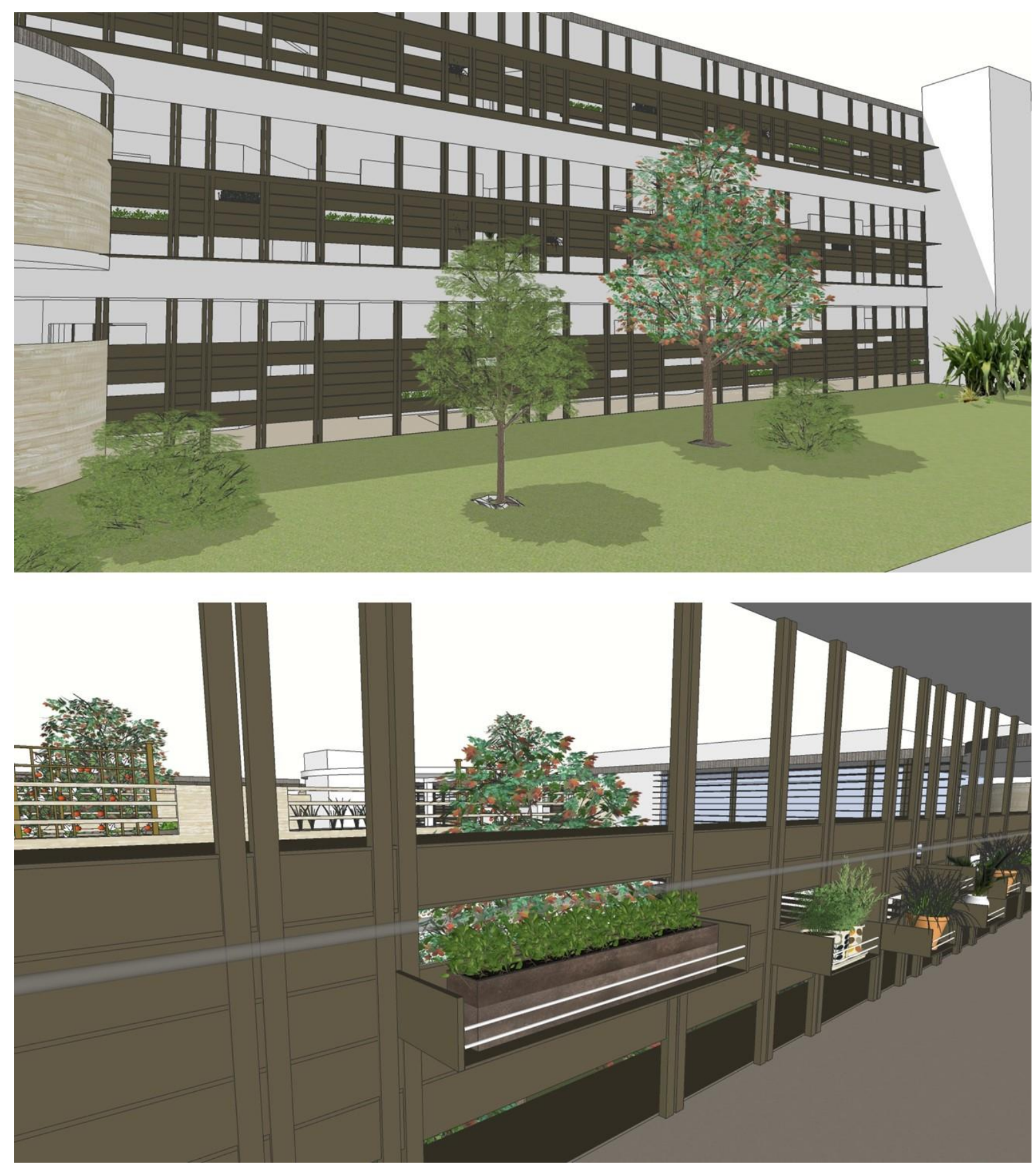

Figure 9-60: Strategy for maintaining resident privacy and a sense of connection 2 - the use of blind panels, level louvres and trees

- Elevation (above) and the view from the corridor (below) (Rental housing, self-contained model) 


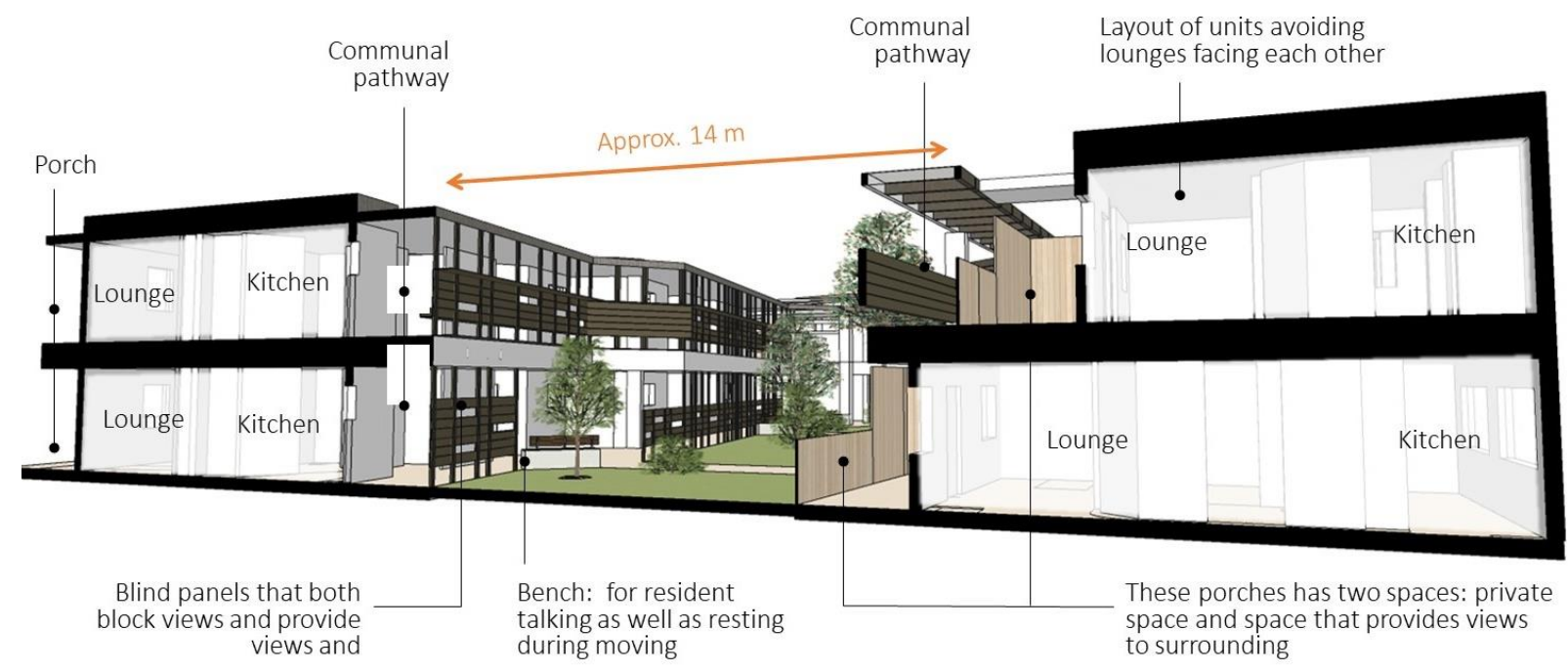

Figure 9-61: Section: relationships between units and communal pathways

— the views to the communal pathways can induce accidental communication between residents. (Rental housing, self-contained model)

\section{Space leading out from individual front doors}

The space leading out from individual front doors is provided with a buffer to enhance residents' privacy and sense of homeliness. Alcoves and passages with a longer distance can be used. This space is also useful to store mobility aids, such as a mobility scooter or a wheelchair (Figure 961). The BS-type units are laid out so that they can have a small private lounge that residents share with only another neighbour (Figure 9-62). Space for displaying personal items are also provided near units, which can increase the residents' sense of homeliness (Figure 9-63,9-64).

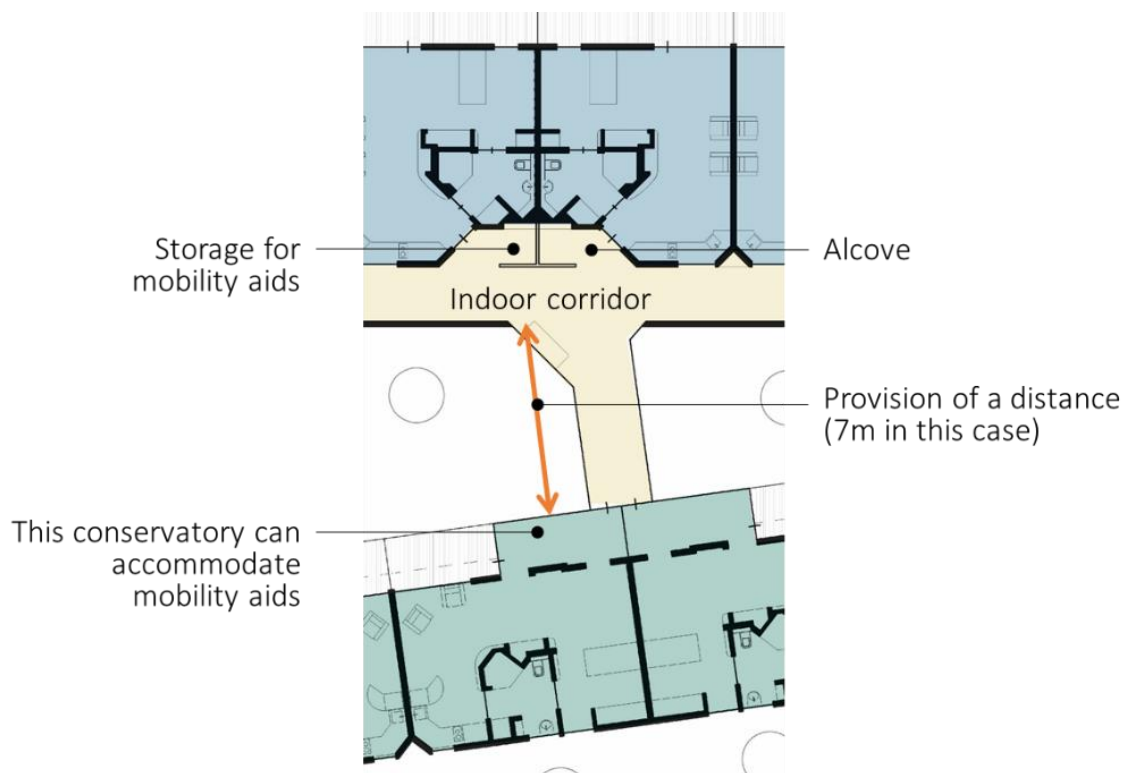

Figure 9-62: Unit entrance design

(Retirement-village supported-living model) 


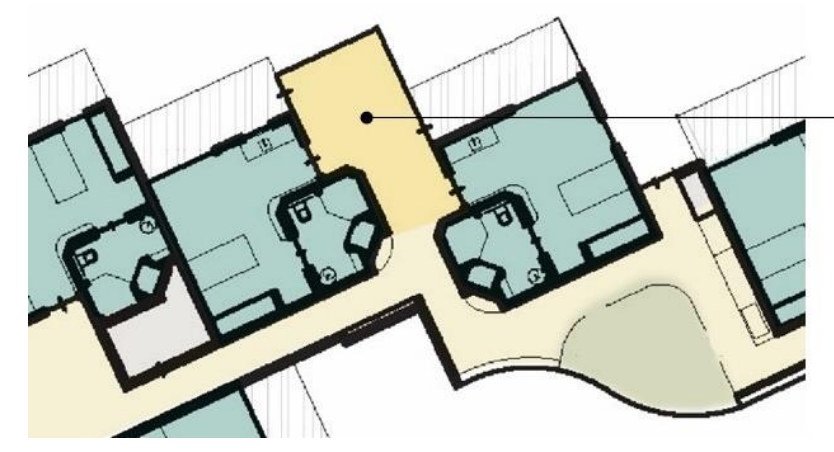

One small communal space is provided for two residents. This space can be used for meeting their private guests, as well as used as residents' own lounge.

Figure 9-63: Layout of private communal space

(Rental housing, communal-living model)

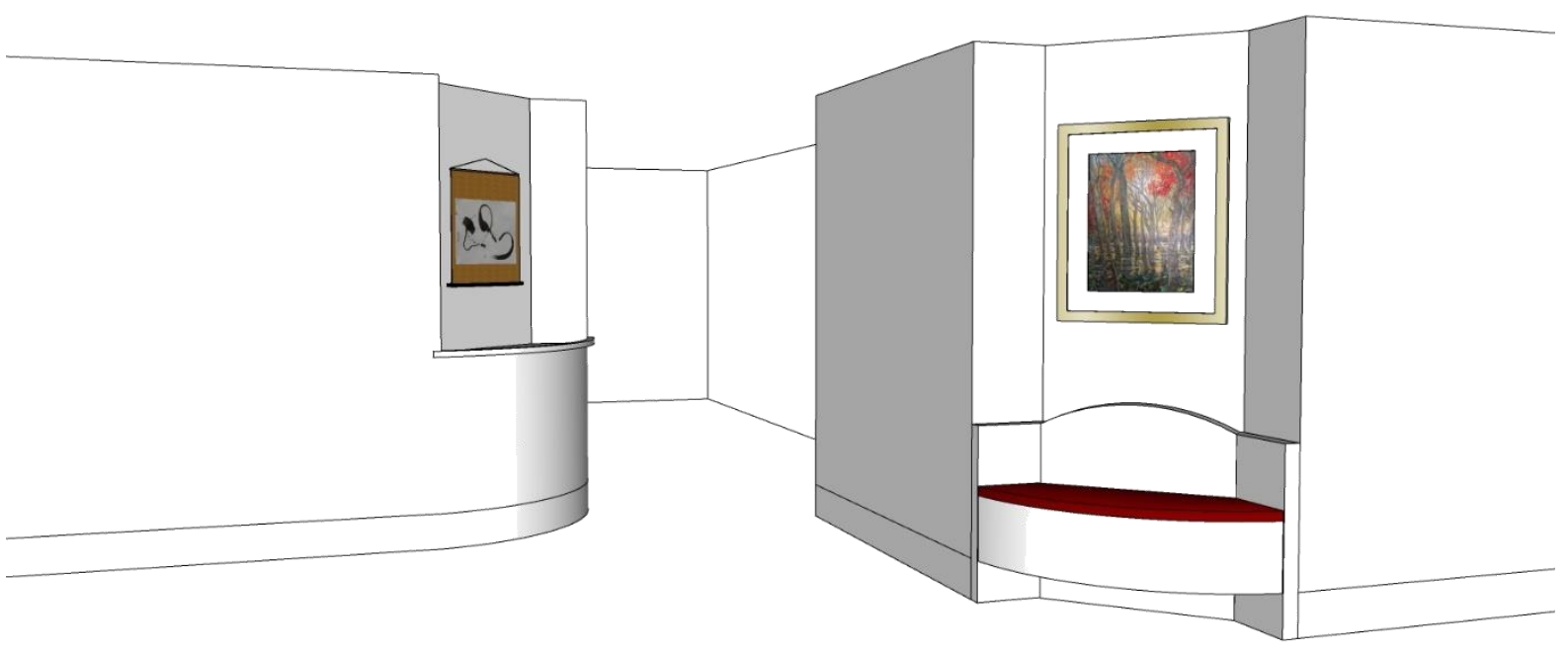

Figure 9-64: Space for displaying personal items at the entry of resident units

(Rental housing, communal-living model)

\section{Facilitating individual preferences}

Three complexes are designed to facilitate specific recreational activities, such as art, music, reading and gardening, which were enjoyed by some survey participants. Retirement-village supported-living units are clustered into two groups of units, with special activities facilitated by providing related special rooms (such as art and music rooms) (Figure 9-65). The Rental housing, communal-living model is made up of two themed clusters ('Art cluster' and 'Library cluster') (Figures 9-66, 9-67). The Rental housing, self-contained model facilitates gardening by providing communal garden, and music activities by the layout of the music room, which is set as a public facility, but allows the use by residents. Both facilities are located in the centre of the complex (Figure 9-68). 


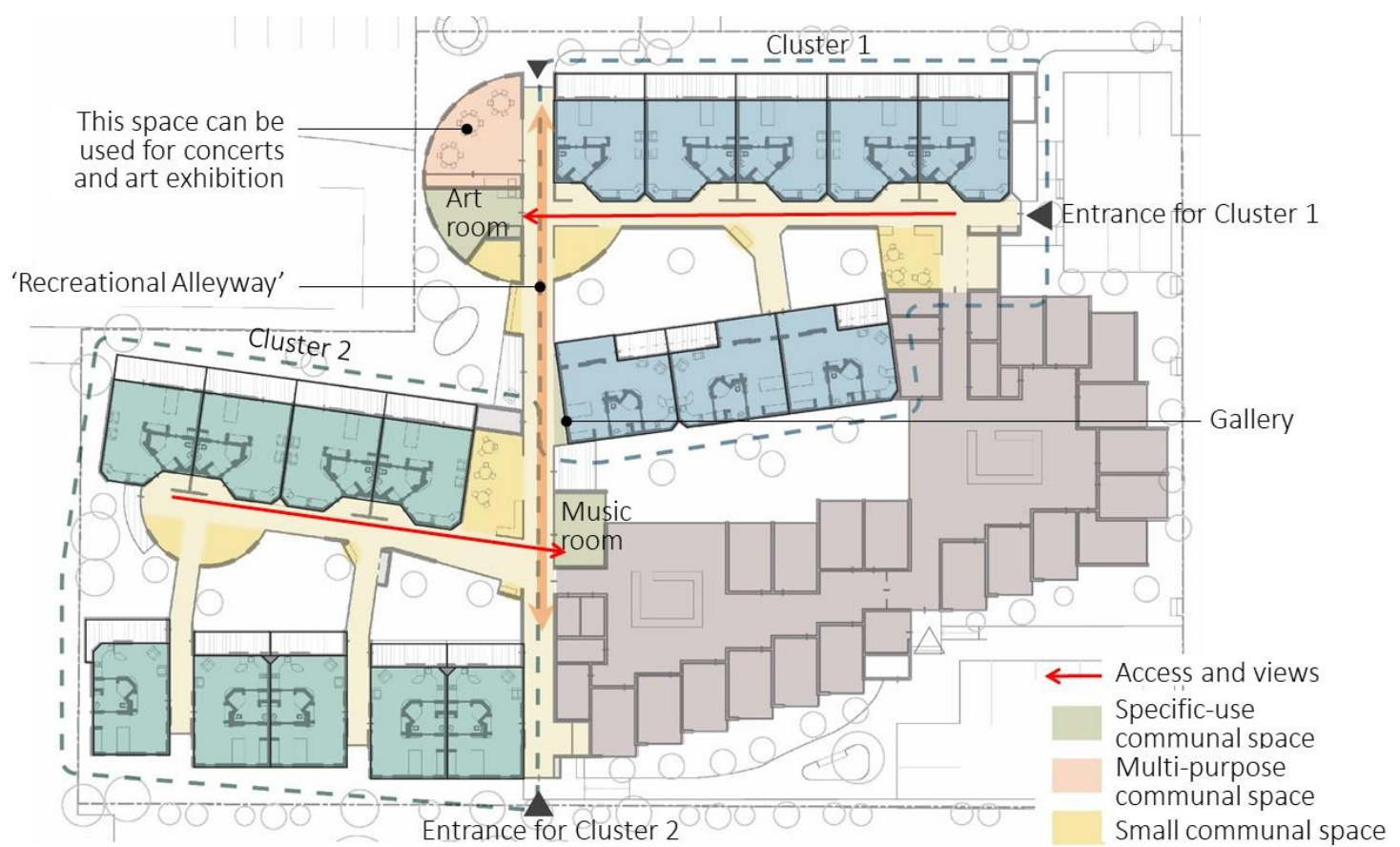

Figure 9-65: Clustering of units with specific-use communal spaces

(Retirement-village, supported-living model)

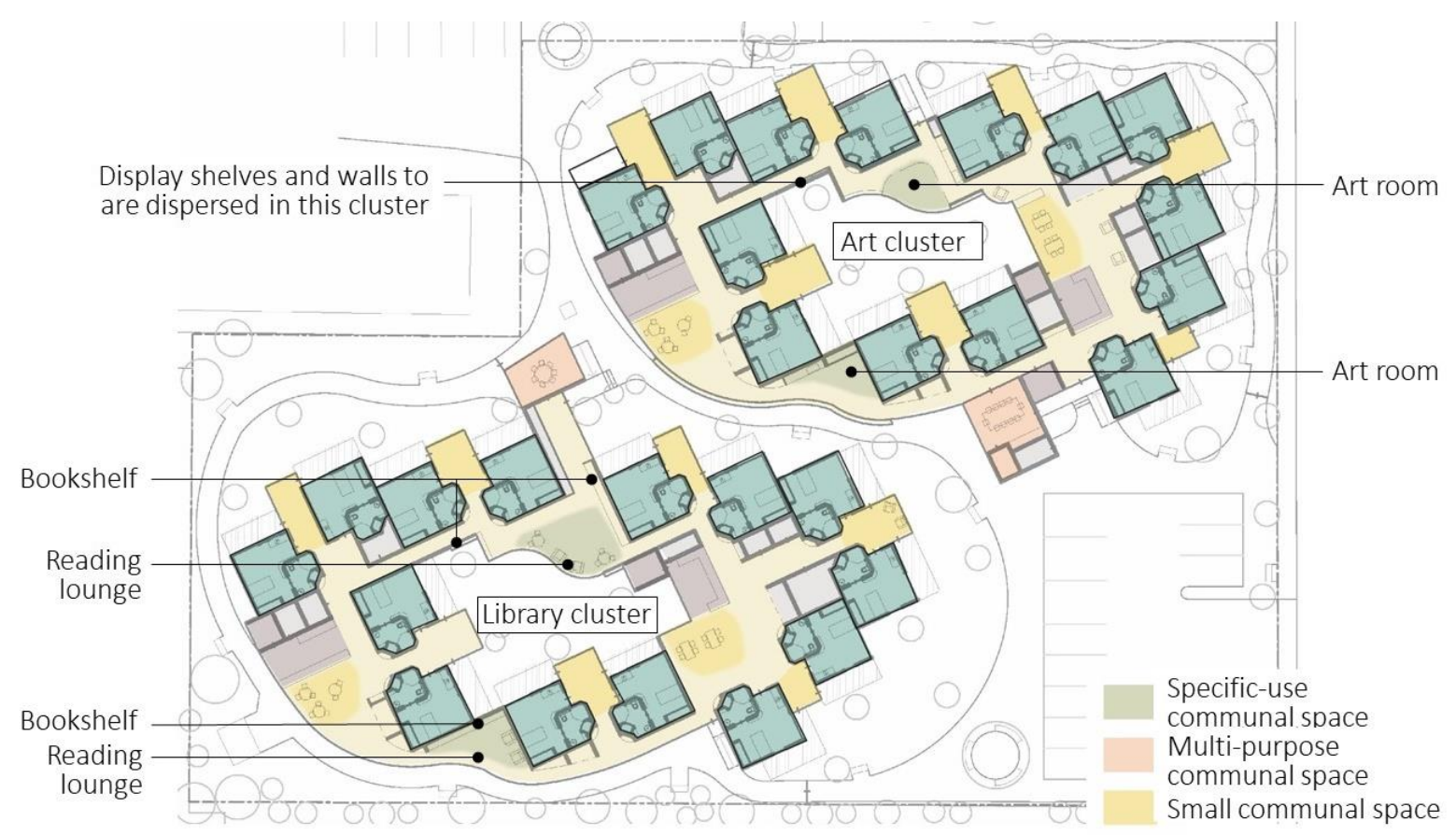

Figure 9-66: Clustering of units with specific-use communal spaces

(Rental housing, communal-living model) 


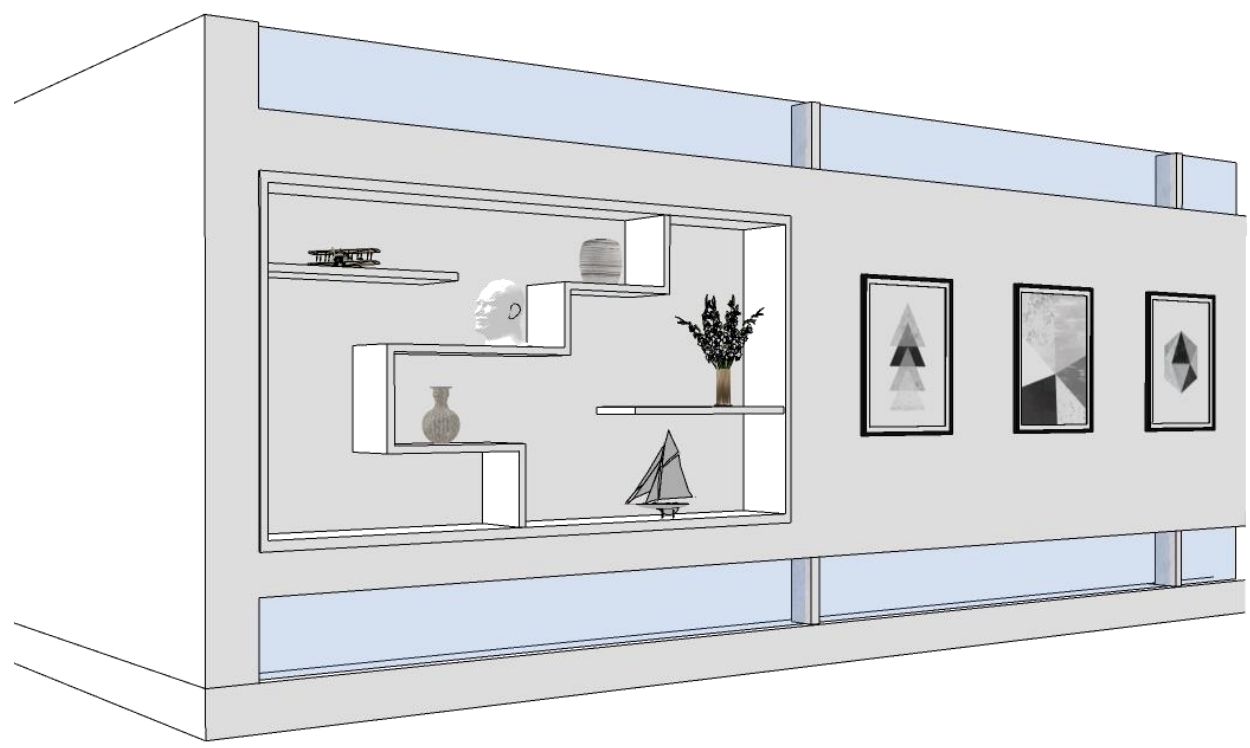

Figure 9-67: Example of display space for art work

(Rental housing, communal-living model)

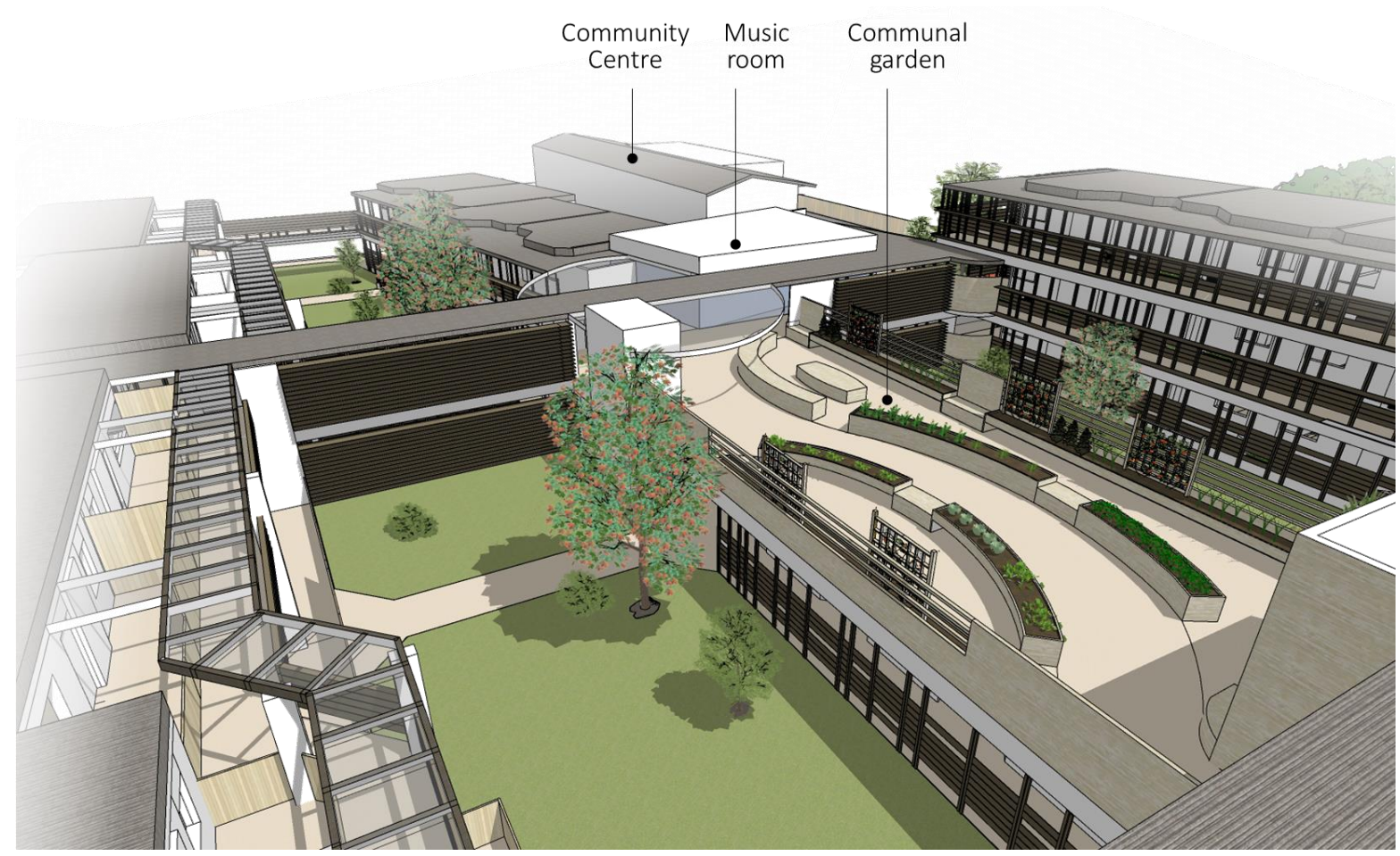

Figure 9-68: Layout of the music room and the communal garden in the centre of the complex (Rental housing, self-contained model)

Unit layout with respect to the staff space

In the Retirement-village supported-living model, the units are laid out with respect to the adjacent care centre. This care centre is designed with the assumption that it applies the Green House model (see also 8-2-3 'Unit layout'), where a small number of staff members oversee a small cluster of resident units. Residents in supported-living units are also looked after by these 
staff member. This model is applied because limiting the number of the staff changes leads to residents' greater sense of homeliness. The 69).

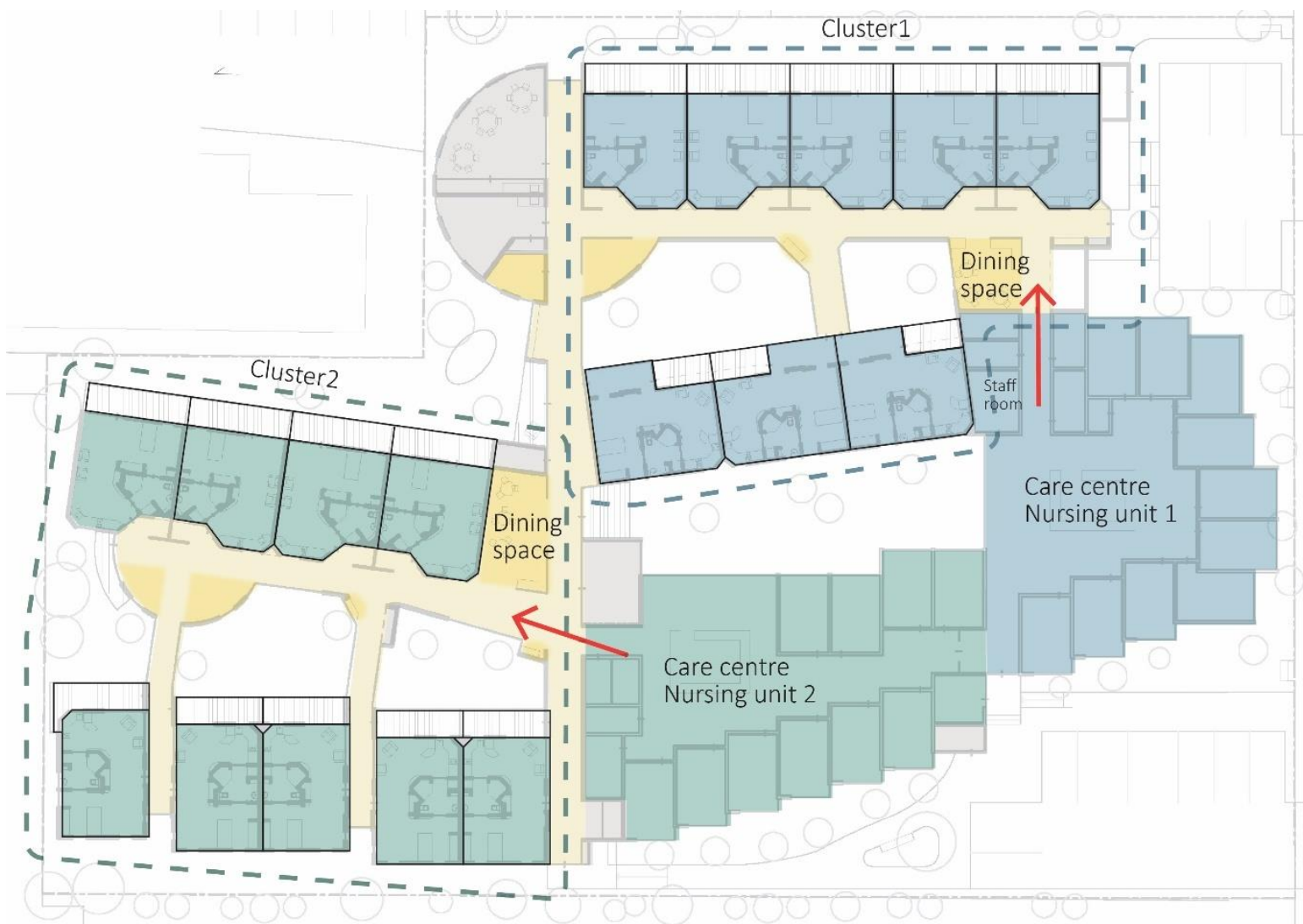

Figure 9-69: Unit layout with respect to the staff space and the care centre (Retirement-village supported-living model)

\section{Walkability}

The complex is designed to facilitate greater walkability. For example, sitting spaces are provided at intervals of less than $20 \mathrm{~m}$. Many walking routes are provided, from which residents can choose those suited for their mobility levels. Multiple entrances are provided so that residents can easily extend their walk to the outside of the complex (Figure 9-70). 


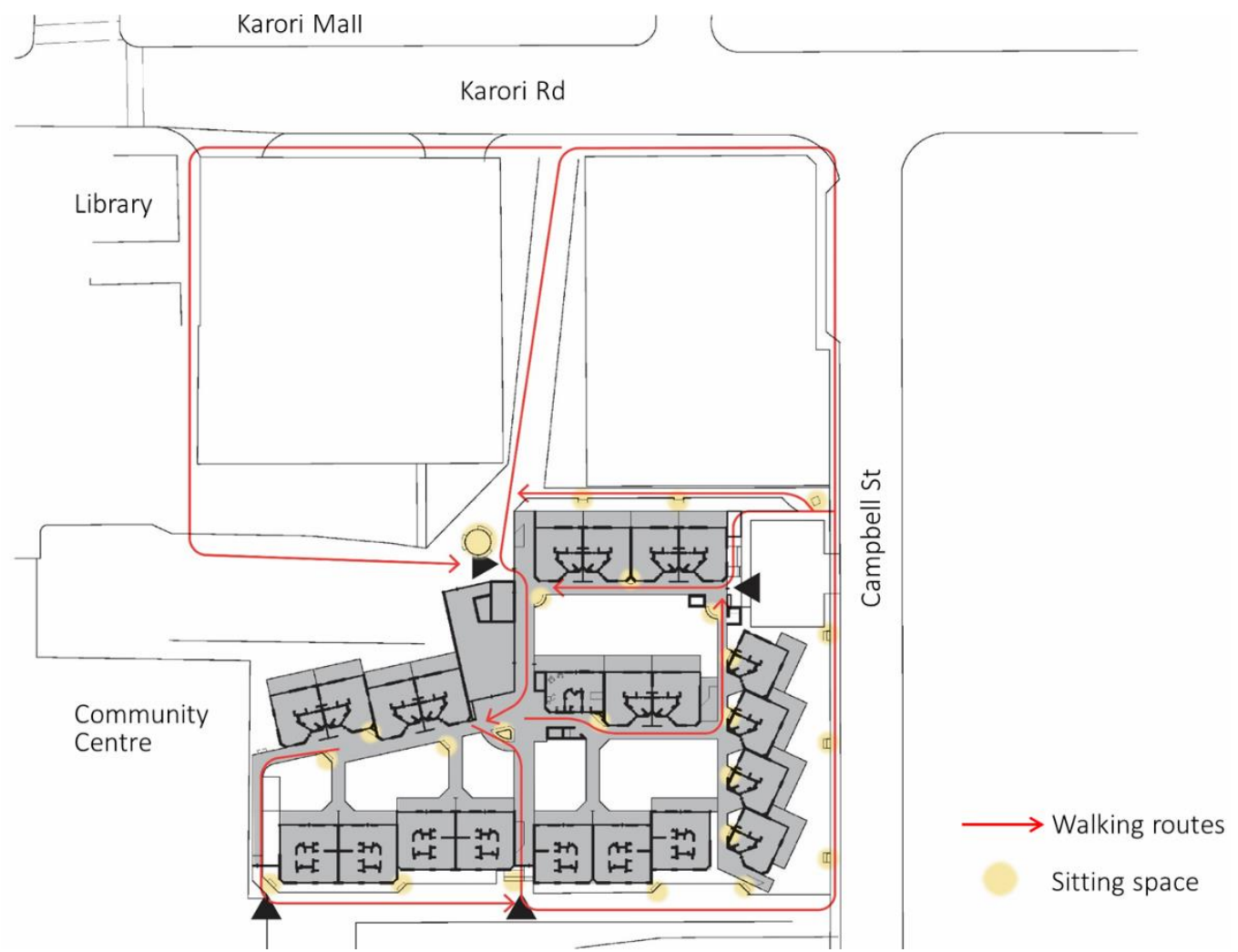

Figure 9-70: Various walking routes

(Rental housing, self-contained model)

Attractive features are provided to encourage residents to walk, such as a display space for artwork and bookshelves, as well as communal spaces and sitting spaces (Figure 9-71).

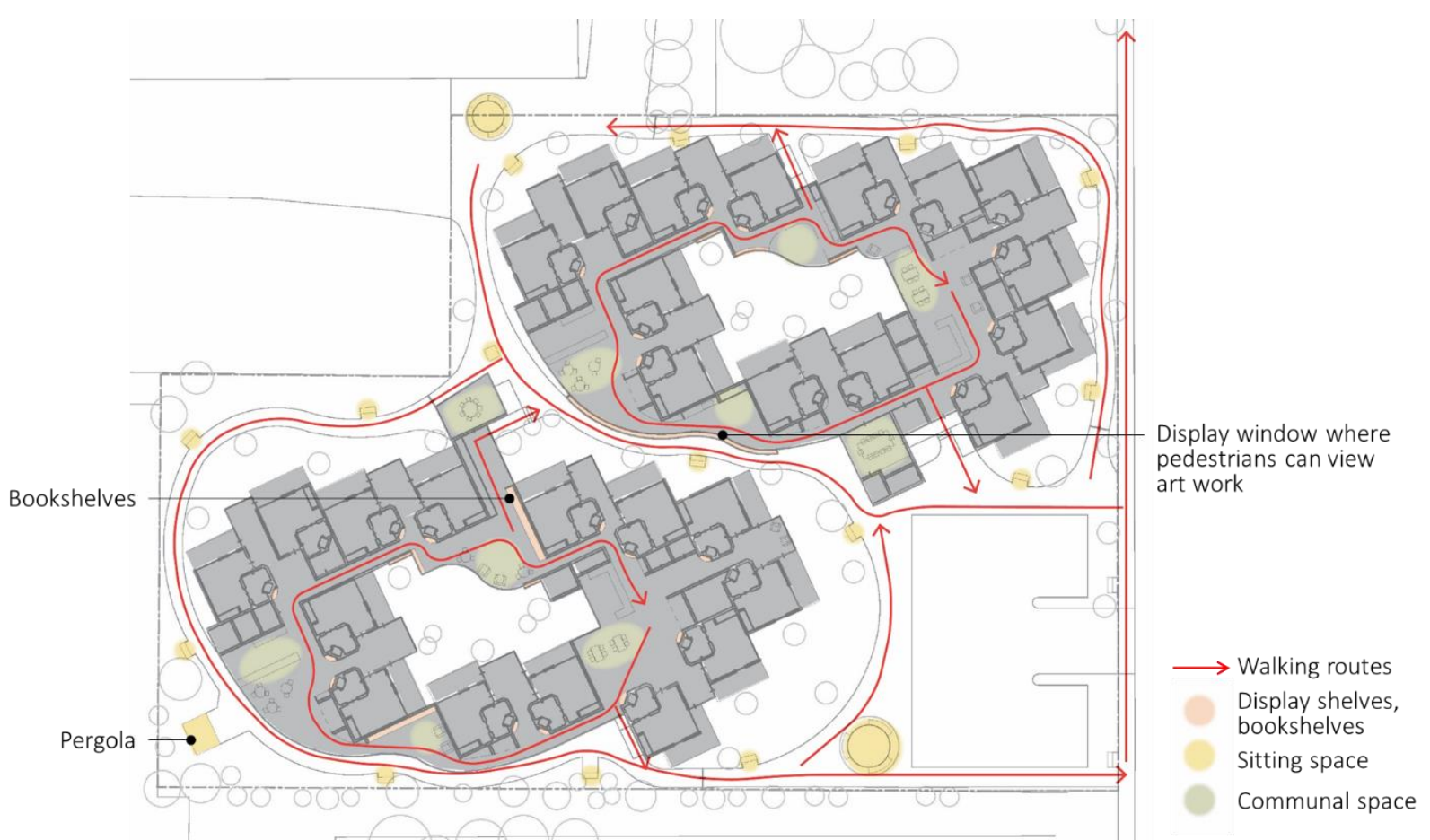

Figure 9-71: Layout of attracting features along communal pathways

(Rental housing, communal-living model) 
To facilitate a sense of connection to the wider community, communal space and outdoor pathways are laid out to facilitate the public to approach and use the space. In all models, the communal space that can be used by the public is located adjacent to the community centre. Public access is allowed through the site in two models of rental housing; however, the pathways for the public are limited for greater security and safety of residents (Figure 9-72).

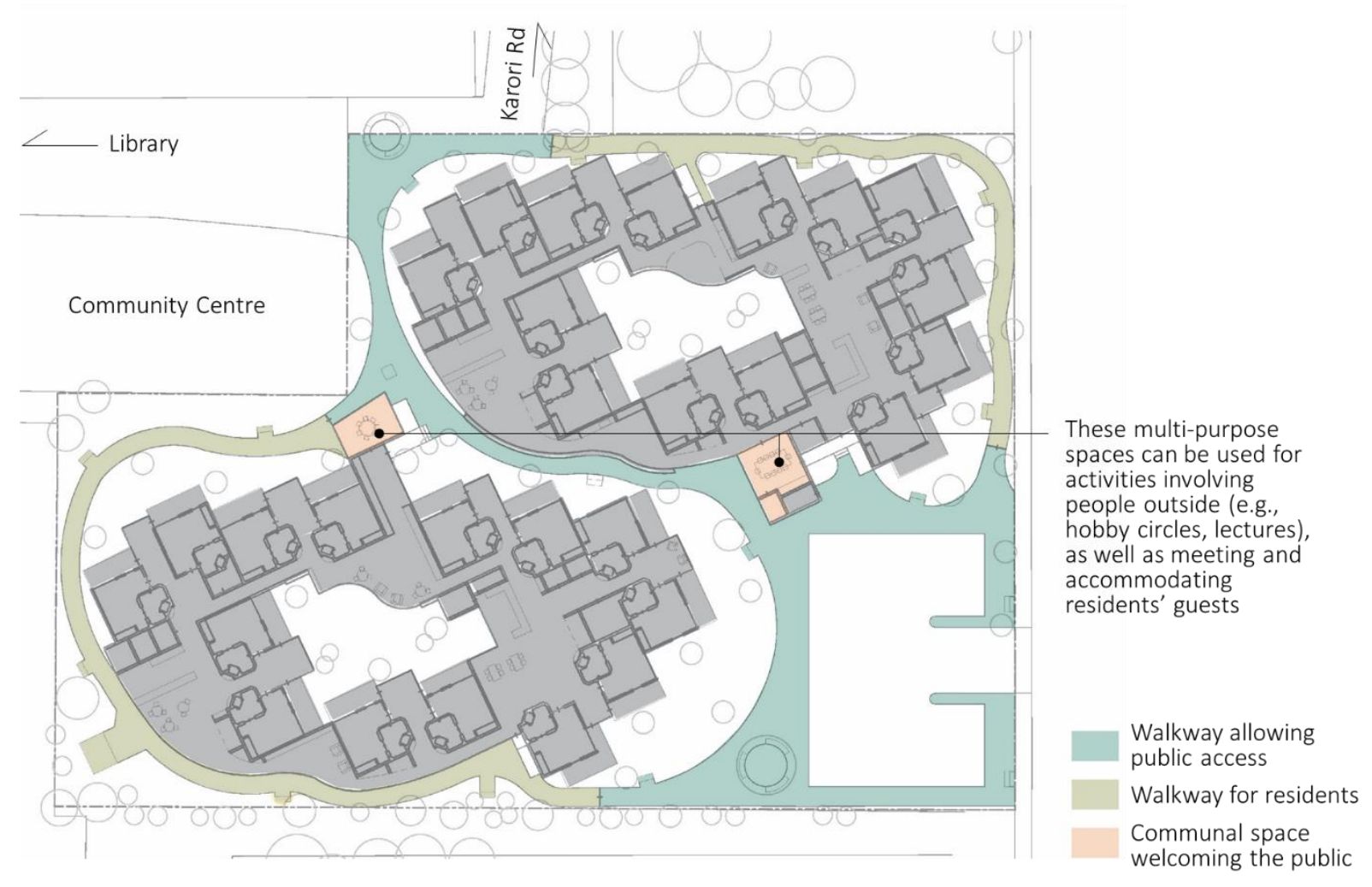

Figure 9-72: Definition of public access area and the layout of communal space welcoming the public (Rental housing, communal-living model)

The Rental housing, self-contained model is designed to facilitate the connection to the wider community by laying out the public facility (the music room), which is introduced in the centre of the complex. The plaza and the children playground laid out adjacent to the community centre can be easily accessed and viewed, which also contributes to a sense of connection (Figure 9-73). 


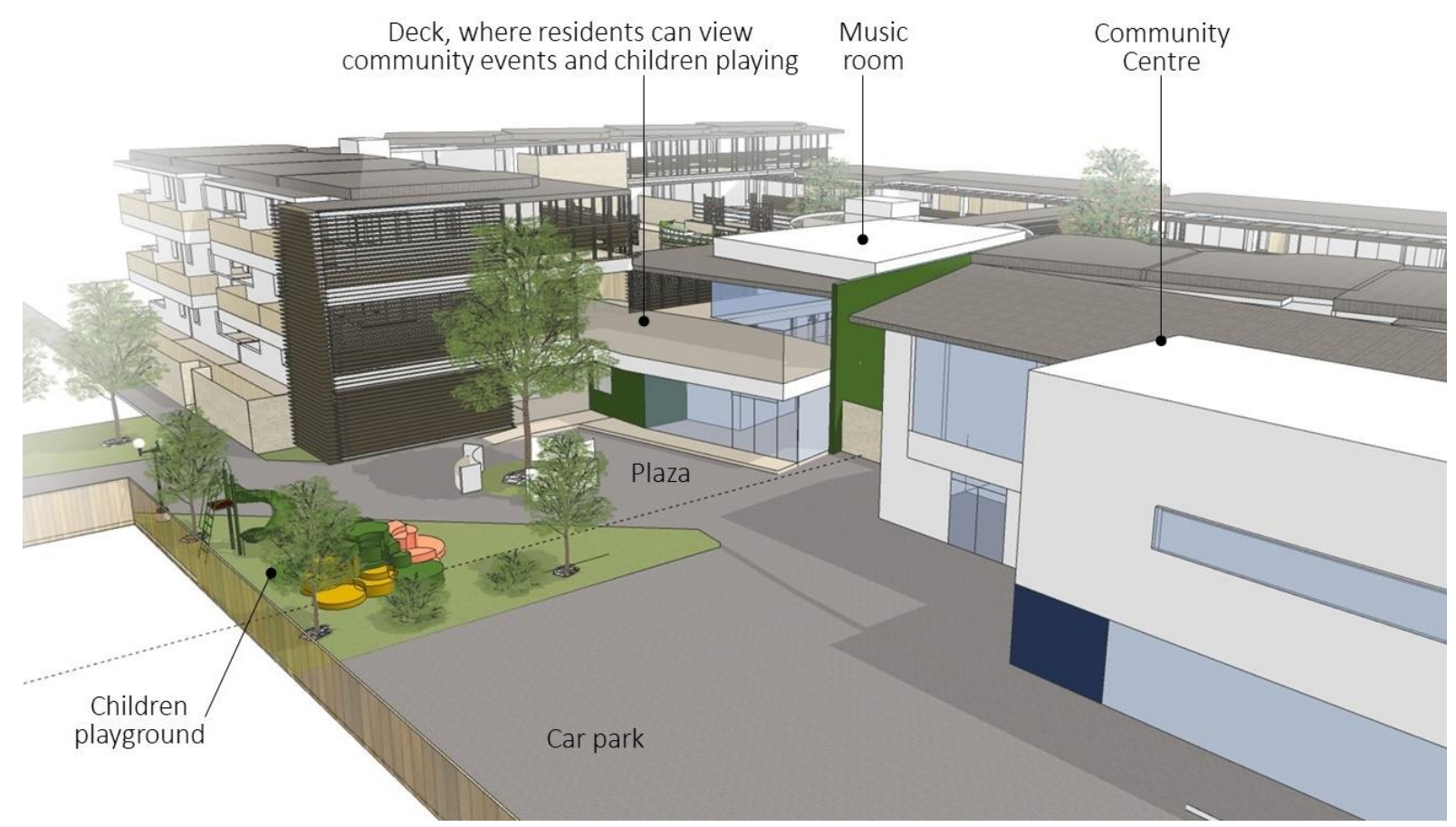

Figure 9-73: Connection to the wider community and children

(Rental housing, self-contained model)

\subsection{Discussion and conclusion}

This chapter has presented housing examples, in which the design framework developed in Chapter 9 has been implemented. This process has revealed some insights about the implementation potential for the framework. Particularly, there were concerns about potential conflicts and challenges in the requirements included. In the design, some conflicts can be solved through flexible design, such as providing options for partitioning spaces, as well as the use of movable or folding fixtures (e.g. the height-adjustable kitchen bench, the folding bench, the folding laundry rack). Some conflicting needs are met with a combination of strategies. For example, to allow views to the outside and maintain resident privacy from passers-by, the solutions include avoiding the lounges of individual units facing each other, avoiding parallel layout of units, the appropriate use of walls for passages near individual units, and the use of enclosures providing both views and privacy (such as trellis and blind panels). Solutions are also required to accommodate the preferences that vary by individual. The solutions in this chapter included the strategies of diversifying the design by clustering resident units (as seen in the design of Rental housing, communal model and Retirement-village, supported-living model) as well as providing spaces for facilitating activities such as gardening, viewing children and feeding birds (as seen in the design of Rental housing, self-contained model). The challenges in implementing 
the design framework can be overcome with careful considerations and creative strategies. This design framework, combined with such efforts of designers, has great potential to provide the highest QoL for the high-needs elderly.

This chapter only showed limited models of housing. However, the design of housing complexes differs by the service models of housing, which have an impact on the use of the type of units and the provision of communal spaces. The surrounding environments are also relevant to the design, having great potential to enhance resident QoL. These factors, as well as designers' creative solutions, will lead to unlimited possibilities in the design of housing.

It is also important to note that the design of housing and complexes of the elderly cannot be achieved without consideration of cost. In the design of individual dwellings, some considerations to reduce the cost, such as the possibility of replacing sliding doors with a roll screen and removing a glass screen from shelves, have been noted. However, there would be more aspects that require consideration for cost aspects both in the design of individual dwellings and complexes; for example, the avoidance of parallel layout of units would require more space or reduce the number of units supplied. Clustering of units with some amenities in each cluster contrasts with the conventional requirement for reducing the cost of providing 'the services centrally to avoid wasteful duplication' as mentioned in Guidelines for the planning of housing for senior citizens (WEL_HOPS, 2007). Greater consideration for the cost aspects would be essential to identify the successful models of housing. 


\section{CHAPTER 10: DISCUSSION AND CONCLUSION}

This thesis has explored the design of housing that improves the QoL of the high-needs elderly. This last chapter provides a brief summary and discusses the significance of this study and contribution to knowledge, as well as the limitation and the possible expansion of research.

The topic of this thesis is based in the dramatic extension of life expectancy and the increase in the ageing population in New Zealand, which has resulted, and will continue to result in greater care needs in later life. When people's impairments increase and their care needs increase, staying in the home in which they have lived often becomes difficult, both because of physical and psychological issues relating to the difficulty in maintenance and the changes of their spatial use of their home. In order to encourage the elderly to live independently (avoiding entering residential care), there is a growing demand for housing that provides support and suitable physical environments for those with high needs. However, in the housing type that provides the highest levels of care for such people to support their independent life in New Zealand, experiences of a low QoL were reported.

An extensive literature review was undertaken to seek architectural solutions to this problem. First, it explored the typical features of the QoL of the high-needs elderly, which was often reduced with their increasing care needs, and found the components of QoL that encompassed a wide range of aspects including the maintenance of personal identity and important relationships as well as the provision of care that facilitates autonomy and individual needs and activities that are interesting and meaningful to the individuals. Next, the review of domestic and international literature in a wide range of disciplines explored their QoL that relates to architecture and identified the main themes for QoL as independence and control, privacy, personal identity, activities, relationships and quality of care. Then, existing domestic and international frameworks for the design of housing for the elderly were examined for their applicability in the housing design that improves the QoL of those with high needs. Combining the outcomes of these two investigations, four main gaps were identified as; 1 ) limited focus on a wider range of aspects of QoL beyond independence and control, 2) a lack of knowledge for the spatial requirements for private space, which is especially important for those with high-needs, 3) a lack of knowledge for the design that facilitates the individuality, and 4) the lack of a framework for the design of housing for the high-needs elderly that improves a wide range of their QoL in New Zealand. In relation to these gaps, a lack of knowledge was identified in numerous specific design requirements as well. Given the significance and size of the gaps in knowledge and the 
absence of design frameworks that are useful for the design of housing that facilitates the QoL of the high-needs, developing an appropriate holistic design framework would be beneficial to the current New Zealand context. This research asks: 1) what is the optimal framework for the design of housing for the high-needs elderly in New Zealand that could improve a wide range of aspects of their QoL?' and 2) 'what are some desirable design solutions?'.

Information regarding the current housing options for the elderly in New Zealand, that was important for QoL and was not obtainable from the literature, was explored through primary research. Extensive questionnaires were conducted in three housing types — retirement villages, public-sector housing, private-sector rental housing for the elderly — in the Wellington Region (Phase 1), which established the models of care and the physical environment of housing and care requirements of elderly residents. They found that the design has great potential to help effectively accommodate necessary services and care for the elderly in housing. This survey was followed by a qualitative investigation of the QoL of elderly residents that related to physical environments (Phase 2). Taking an ethnographical approach, 30 elderly people were surveyed using semi-structured interviews and daytime observation as well as documentation of their physical environments. This generated very rich data about their QoL and was described in detail in the six main themes for QoL: 1) Control in daily basic activities, 2) Meaningful leisure activities, 3) Meaningful relationships, 4) Maintenance of possessions, 5) Comfort and 6) Quality of care. Based on the outcome of the survey, design elements were distilled and the relationships to QoL were established. Design requirements were defined for each design element (Phase 3). Through this process, the design framework for the design requirements that improve a wide variety of QoL of the high-needs elderly was established. Based on the design requirements, example models of housing were designed in Phase 4.

\subsection{Significance and contribution to knowledge}

The research addressed the research questions through the development of a holistic framework for the design of housing that improves the QoL of the high-needs elderly living independently and showed example models of housing design. Through this process, it has found new knowledge, including those addressing the gaps identified in Chapter 3. The significance also relates to the methodological originality and the possible expansion of research in the architectural discipline. Aspects of significance and contribution to knowledge are described below. 
Establishment of a holistic framework for housing design for the high-needs elderly that improves their QoL in New Zealand (addressing the research question and the gaps 1 \& 4) The review of design frameworks overseas and in New Zealand in Chapter 3 found that there is unevenness in addressing a range of aspects of QoL for the high-needs; particularly, there is no framework in New Zealand addressing other aspects than independence and control. In addition, the frameworks only included limited information on the spatial requirements for the highneeds, and some design solutions included were too ambiguous to be useful. In conclusion, there was no design framework suitable for the design of housing that could improve the QoL of the high-needs elderly in New Zealand. A review of extensive literature that includes fragmented information on the architectural influences on the QoL of the high-needs found similar gaps in knowledge. The creation of a framework for design of housing that could improve the QoL of the high-needs elderly in the New Zealand context is deemed beneficial, particularly given the increasing demand for housing the high-needs in New Zealand (Gap 4). Based on these gaps, this research has explored the relationships between a wide range of QoL aspects of this cohort and design elements in the physical environment through an ethnographical investigation. From the analysis, a holistic framework for the housing design that improves the QoL of the highneeds elderly was developed, identifying numerous design requirements and the influential aspects of QoL.

The development of this design framework also responds to the gap that there was a limited focus on a wider range of aspects of the QoL components other than the aspects of 'independence and control' (Gap 1). The design requirements included in the framework were related to a wide range of aspects of QoL, including: meaningful leisure activities, meaningful relationships, maintenance of possessions (which largely related to elderly people's identity) and quality of care, as well as 'control in daily basic activities'.

\section{Bringing new knowledge to the design requirements for greater QoL of the high-needs elderly, and to Universal Design}

The design framework developed includes new knowledge with regard to design requirements that provides greater QoL for the high-needs elderly, which also adds new insights to Universal Design. New knowledge generated has been categorised in five main areas for the high-needs elderly; 1) spatial requirements for private space, 2) design requirements that facilitate the individuality, 3) spatial requirements for accommodating caregivers, 4) design considerations for walker-frame users and 5) other.

1) Spatial requirements for private space of the high-needs elderly 
The Chapter 3 literature review identified that there is a lack of information regarding the spatial requirements for the private space of the high-needs elderly (Gap 2). Through close investigation of their living environments and questions relating to QoL, the research found a number of considerations relating to the design of individual dwellings. For example, existing literature suggested the importance of micro environments of sitting space for the high-needs elderly, but there was limited information useful for design (Hale et al., 2010). To address this gap, this research has identified key considerations for their sitting space. This space is important for residents to engage in activities even with limited mobility. Their activities are facilitated by the provision of space that can accommodate an armchair and level surfaces to accommodate their often-used items within reach, and for most people, the layout of an armchair with respect to the TV is essential. Activities of choice can be facilitated by the layout of an armchair with respect to a computer screen as well as the provision of separate space(s) for a table and a chair. The layout of an armchair with respect to the views to the outside is important for providing greater control of visitors as well as things to occupy them. The direction of the window and the sunlight with respect to an armchair should be considered also with respect to the location of a TV as well as other factors such as residents' sight impairments. This research has revealed the great importance of having access to the north sun for the sitting space for the high-needs - it was valued by all participants and, in many cases, determined the space that the residents stayed in which was addressed by the layout of all units with the lounge facing north in the design of complexes. The design of private space also has an impact on residents' ability to accommodate their important relationships, such as living as a couple and having personal guests, which has been suggested as valuable (Cooney et al., 2014; Kontos, 1998; Tompkins et al., 2012). However, there was limited information useful for design such as the number of people to accommodate and the type of relationships to accommodate as well as the spatial requirements. For this gap, this research clarified the spatial requirements to accommodate social gatherings; for example, the flexible design of space that can accommodate up to 10 providing the opportunity to continue to hold group meetings. Solutions to accommodate family members including small children have also been illustrated. The importance of belongings for the elderly has been stressed in the existing literature (Lewis et al., 2010; Cooney, 2012; Nord, 2013) but without detailed requirements in design except for the room shape suitable for accommodate furniture (e.g. rectangular). This research classified elderly residents' important possessions into some types and identified spatial requirements to accommodate them. For example, accommodating possessions requires consideration for the height that is reachable for the high-needs as suggested in the existing literature (Standards New Zealand, 2001), and the finding of this research has 
added the importance of accommodating a variety of items valuable for residents such as photographs, pictures and small items to display as well as furniture and enclosed storage, which requires careful design of wall space. The design of the kitchen and bathroom has a great implication for facilitating control in daily activities as has been suggested in existing literature (Burton et al., 2011; Iwarsson, 2005; Peace, 2017); however, this research has added the design requirements and potential solutions focusing on the micro environments of the elderly with limited mobility. This research has clarified that the design of every element of private space of the high-needs elderly, particularly their micro environments, has a great influence on QoL, and has shown the requirements to improve it.

2) Design requirements that facilitate the individuality of the high-needs elderly

Another gap identified was a lack of understanding of design requirements that facilitate the individuality of the high-needs elderly (Gap 3). For example, an existing design framework overseas acknowledges the importance of providing residents with interior space (e.g. the layout, finishes, decors) that suits their preferences, but there were no detailed requirements (WEL_HOPS, 2007). The investigation for the QoL in this research has found diverse individual preferences for the high-needs elderly, which provides the grounds for design that facilitates individuality. This diversity in the preference has resulted in differing requirements in potential designs and increased the difficult in meeting all the requirements. Through the design implementation in Chapter 9, solutions were explored, which included an adaptable design, a flexible design, and diversifying the design by resident preferences. The rental housing, communal-living model showed an example of diversifying the design by activities facilitated, using the strategy of clustering resident units.

3) Spatial requirements for accommodating caregivers

The study has also provided new insights regarding the design considerations for accommodating caregivers in the private home, particularly in the bathrooms. For example, the requirement for space for drying after showering that can accommodate the resident and their caregiver cannot be found in other literature, in contrast to the requirement for space for washing that can accommodate the two persons, which has been addressed in other literature and design frameworks (E. C. King \& Novak, 2017; Lewis et al., 2010; WEL_HOPS, 2007). This thesis also emphasises the importance of the provision and design of a shower enclosure as it has great implications for the safety of residents and caregivers, as well as improving resident privacy and dignity. This idea challenges the recommendation of a wet-area shower area with no solid 
enclosure in the NZ design frameworks such as NZS 4121 (Standards New Zealand, 2001) and Lifemark Design Standards (Lifetime Design Limited, 2012).

4) Design considerations for walker-frame users

New knowledge was also obtained for the requirements for those using mobility aids other than wheelchairs. This is particularly relevant to the New Zealand context, where the government provides assistance for equipment such as walker frames for the elderly (Office for Senior Citizens, 2013). These requirements were made clear through a comparison of the outcomes of this research in which the majority of participants were walker-frame users, and the existing knowledge such as the New Zealand Building Code and Lifemark Design Standards, which largely focus on the requirements for wheelchair users. For example, the requirements for the height of fixtures (e.g. door handles, light switches) for wheelchair users were not perfectly suitable for walker-frame users. Hinged doors were harder for walker-frame users to manoeuvre, which pointed out the greater significance of sliding doors. Considerations for greater walkability such as the distance between spaces for resting were also more important for those with or without mobility aids than wheelchair users. These considerations are important as many of the elderly requiring care and living on their own use walker frames, not a wheelchair. These requirements for walker-frame users contain new insights for the existing knowledge in the design for the disabled and Universal Design.

5) Design considerations for greater walkability of the high-needs elderly

Another area of new insights obtained through this research is the significance of walkability for those with high needs, and the requirements in the design of a housing complex to facilitate greater walkability for them. In this research, the investigation for the QoL of the high-needs elderly revealed their strong desire for keeping active and able, and walking was a popular activity for this purpose as well as for a change. As for the design requirements to facilitate their walkability, a review of literature for the QoL for the high-needs elderly that related to the physical environments in Chapter 3 identified information mainly relating to providing great accessibility such as eliminating level differences and provision of handrails. In the literature, only fragmented requirements were found for more actively facilitating walkability, such as the provision of sitting space at less than $30 \mathrm{~m}$ intervals (Lewis et al., 2010) and provision of attractive communal gardens (Lewis et al., 2010; WEL_HOPS, 2007). This research has identified requirements that facilitate active walking such as provision of a variety of walking routes that suit mobility levels (which could extend to the outside of the complex) and provision 
of wide walkways to facilitate walking with others, as well as various accessibility/safety requirements which include those for avoidance of road crossing on walking routes and speedbumps on roads in case they walk on the road. From the findings of this research, the sitting spaces should be provided at $20 \mathrm{~m}$ intervals for those with limited mobility, leg pains and/or breathlessness, which is more common among those with high needs and an enhancement to that suggested in an existing framework (Lewis et al., 2010). These new insights, combined with existing knowledge, in facilitating their autonomous walking activities, will advance the age-friendly communities that help the high-needs keep active and able.

\section{Application of an ethnographical investigation to explore the relationships between the QoL of the high-needs and physical environments}

This research applied an extensive qualitative investigation to explore the relationships between QoL and physical environments. More specifically, an ethnographical investigation was applied to investigate 30 elderly residents' QoL, which consisted of semi-structured interviews and daytime observation (seven consecutive hours) as well as the documentation of physical environments. This method has led to the capture of sensitive perceptions and feelings and their close relationships to the physical environments, which has provided important evidence for the design requirements for housing for the high-needs elderly. For example, this information could have not been obtained through focus groups. which have popularly been used in housing research (Hall, 2008; Howden-Chapman et al., 2000; Lewis et al., 2010) and in developing design frameworks for the elderly (Lewis et al., 2010). This is because focus groups do not involve close examination of the physical environments in relation to the QoL in the 'field.' The detailed description for the QoL and physical environments generated through this investigation can better inform designers and housing operators of the significance of the design requirements.

There have been some studies that used both interviews and observation that focused on the relationships between QoL and physical environments; however, no research applied these approaches for elderly individuals as extensively as this study. For example, Nord conducted interviews of care staff and elderly residents as well as observation of care tasks, to explore the architectural influence on resident privacy with regard to care; however, the number of residents interviewed was only 10, and the activities observed were limited to care activities (Nord, 2011). The application of the extensive qualitative method combining interviews and intimate observation for 30 people has contributed to capturing the holistic picture of QoL of individuals. 


\section{Contribution to research in architectural discipline}

QoL studies are a field of research that have increasingly attracted attention in various disciplines, and have been suggested as requiring multi-disciplinary approaches; however, QoL studies in architecture have been reported as scarce (Mohit, 2013; Robert W Marans \& Stimson, 2011). This thesis has focused on architectural design that improves the QoL and provided significant insights for the area of QoL studies. In particular, the method of this research contributes to the expansion of research methodologies in the architectural discipline in relation to QoL. Given the success in capturing the architectural influence on the resident QoL and in identifying architectural improvements, this method has also proved to be a useful means for Post Occupancy Evaluation (POE). POE refers to the assessment of how buildings are used to support their environmental performance and occupant wellbeing and productivity (Higher Education Funding Council for England, 2006; RIBA et al., 2017), which 'provides evidence of a wide range of environmental, social and economic benefits core to sustainability' (RIBA et al., 2017, p. 6). POE has been conducted in various building types, commonly on public buildings such as schools, but on housing as well (Orihuela \& Orihuela, 2014; Pretlove \& Kade, 2016). Increasingly, the significance of POE has been recognised internationally. While systematic approaches have been sought, a range of approaches and methods are currently used to carry out POE, depending on the focus of the particular outcome, and range from data monitoring and quantitative evaluation to the qualitative surveys of occupants (RIBA et al., 2017). This research has shown one method for POE to examine built environments that result in a great QoL of occupants using a qualitative survey. This method will be useful not only for the high-needs elderly but also in other contexts and can be used more widely as the demand for POE grows.

\section{Contribution to the government policy}

Housing has been one of the focus in the recent two policies for the ageing population in New Zealand (New Zealand Positive Ageing Strategy and Better Later Life - He Oranga Kaumātua 2019 to 2034), seeking to ensure that housing options that are adequate. These encourage the use of Universal Design features as well as the provision of a wide range of services (MSD, 2001, 2019). In particular, Better Later Life - He Oranga Kaumātua 2019 to 2034 notes one of the main functions of housing as 'being a place and base for us to enjoy life and stay connected (MSD, 2019)' including enjoying hobbies, welcoming visitors and providing hospitality, as well as receiving care. This research contributes to these policies by providing evidence and guidance for housing design for the elderly who require care. In particular, the implications encompass a wide range of aspects of QoL that can be afforded by housing for elderly residents, not only the independence and control aspects, which are the main focus of the conventional Universal 
Design features. In addition, the thesis has illuminated the diverse desire for active ageing by the high-needs elderly even with limitations in their mobility and needs for care, which is something that tends to be overlooked in positive ageing strategies. This included keeping active and able and hosting their guests, contributions to charities and others as well as continuing their familiar activities. To facilitate these desires, housing and their close communities have a great role to play. Similarly, the design of intimate environments has a great implication for facilitating autonomous activities while accommodating care, thereby supporting active life. This research provides beneficial insights for the policy for housing for the elderly that add a new view for active ageing of the high-needs, thereby informing better approaches to help the ageing population in New Zealand to maintain their independence and age-in-place successfully.

\subsection{Limitations}

This section critically examines the limitations of this research, which relate to the study sample as well as the limited integration with the technology and cost. These reflections, in turn, provides opportunities to identify possible focuses in future research.

First, some limitations of this study relate to the limitation in study participants. In Phase-2, the QoL of 30 elderly people was examined through an ethnographical study. Although this is a decent sample number for a qualitative study as noted in Chapter 6 , the number of sub-groups (e.g. for the type of mobility aids used, impairments/conditions and the ethnicity) might be expanded to more completely represent the sub-groups. Particularly, the type of mobility aids used had an impact on the spatial requirement. The study highlighted the design considerations for walker-frame users, which were the majority of the participants; however, requirements for those using other types of mobility aids, such as manual/electric wheelchairs and a mobility scooters, could not be sufficiently captured (there were only five wheelchair users and two mobility scooter users among 30 participants). This limitation could be overcome by combining the outcomes with existing knowledge on Universal Design, which contains various insights about the usability of wheelchair users; however, it would still be worth examining elderly wheelchair users with high needs, who typically have the limited arm strength and limited ability to move/control their own wheelchair. In order to accommodate the elderly with higher care needs in housing, it would be important to take account of the requirements for users of special equipment such as hoists and $\mathrm{O}_{2}$ cylinders, as included in a design framework overseas (Lewis et al., 2010). 
The type of impairments and conditions of participants also had an implication for the design requirements. This study identified some requirements specific to certain impairments/conditions such as arthritis (e.g. the requirements for the height of shelves, seats and fixtures), sight impairments (e.g. requirements for the layout of sitting space with respect to the window, requirements for sound insulation, requirements for roads and walkways), post-stroke conditions (e.g. requirements for the furniture layout suitable for semi paralysis, and those for eliminating level differences that affect manoeuvring mobility aids with one hand) and spinal conditions (requirements for the chair type); however, the number of people with each impairment/condition was small except for those with arthritis (the number of those with sight impairments, post-stroke conditions and spinal conditions was four, five and two respectively). Investigations focusing on each impairment/condition with a greater sample number would better capture the requirements specific to each cohort. With regard to the impairments of the elderly, it also is important to note that the scope of this research excluded those with cognitive impairments. Given that more than $10 \%$ of the elderly have cognitive impairments in New Zealand (Stats NZ, 2014b), this cohort cannot be disregarded. In addition, it has been reported that built environments have an impact on the ability of the cognitively impaired to lead an independent life (Fleming, Goodenough, Low, Chenoweth, \& Brodaty, 2016; Marquardt, Bueter, \& Motzek, 2014). EVOLVE includes specific design requirements for the dementia, which relates to improving visibility/ recognisability of the built environments, for example, through the use of colours and materials with a high contrast (Lewis et al., 2010). Research with a scope including these people would add important aspects of design consideration for the highneeds elderly.

Some of the limitations of this work and the possibilities for further research related to aspects of culture. The research could not look at diverse ethnicities that represent the population in New Zealand, as most participants of the Phase 2 survey were European New Zealanders (28 out of 30). As a result, this study well represents the QoL of European New Zealanders; however, to design housing for a wider population, it is important to consider the design requirements that facilitated the QOL for other ethnicities. Particularly, attention should be given to the design that can accommodate preferred activities and relationships for Maori and Pasifika, who have been reported to have different preferences in activities from European New Zealanders (V. A. Wright-St Clair et al., 2012) and more likely to live in an extended family compared to European New Zealanders (Jamieson et al., 2018). 
Notwithstanding the limitation above, this study is beneficial in that it has shown the design requirements for the majority of the elderly in New Zealand, who typically desire to live independently from their family to avoid being a burden even with the assistance of professional caregivers (Davey, 2006a, p. 135; HART, 2014). This can be applicable to similar contexts overseas, such as developed countries with individualist cultures. For example, the requirements for greater quality of care in showering may be applicable in such countries, where having formal caregivers' assistance in showering is commonplace. The possible expansion of research would be the inclusion of the case in which professional caregivers stay in a private house for longer hours including the night time. This would affect the required space for caregivers, as noted in a design framework overseas (Lewis et al., 2010; WEL_HOPS, 2007).

Another limitation of research to note is that the investigation of the housing studied in this research did not provide useful insights for the role of technology in improving the QoL of the high-needs elderly. For example, existing design frameworks overseas encourages the use of technologies such as those for greater control of climate and greater safety as part of solutions to improve QoL (Lewis et al., 2010; WEL_HOPS, 2007). For example, those with limited mobility could easily attain comfort by using climate control systems that are controllable with a remote control or smartphone app (e.g. control in natural/artificial light, ventilation and temperature), and systems with an automatic sensor would better suit those who have difficulty in finger movements. Alarm systems that can detect smoke, heat, water leak, flood and carbon monoxide with a remote response service. Technologies such as a video-entry phone and an opening system for the front door which can be remotely operated from inside the home would provide residents with great control in welcoming guests, which could eliminate the requirements in the interior layout that the front door should be within sight of the resident's sitting space for them to detect visitors' approaching, and close enough for the resident's voice 'come in' to reach visitors. The ultimate form of these technologies would be a smart home, which incorporate in a dwelling 'a communications network that connects the key electrical appliances and services, and allows them to be remotely controlled, monitored or accessed' (Housing Learning \& Improvement Network, 2003), and has been reported to greatly encourage the high-needs elderly to live independently. Incorporating a tele-medical system and the use of robotic technology for care for the elderly would change the models of care at home in the future, which will also change the requirements in the housing design. It would be essential to examine the implication of these rapidly developing technologies for their greater application in housing design. 
Finally, an important limitation that relates to the design of housing models is that they would require a further examination of cost for greater implementation in practice. Only limited consideration has been given for the cost aspect in this research, for example, consideration for this aspect in the design housing models included limiting the unit floor area so that it is within the range of the area of standard council flats as well as considerations for changing the type of partition to a cheaper type and removing some elements. However, the design requirements would require a refinement with a greater consideration for cost, given the great need of the current cohort for affordable housing including rental housing and/or social housing (Alan Johnson et al., 2018; Hon Phil Twyford, 2017; MSD, 2001, 2019).

The reflection of these limitations has identified some areas to investigate in future research. In the next section, a possible approach to expand this research will be explored, where these limitations will also be addressed.

\subsection{Expansion of research}

This research has established a holistic design framework for the design of housing for the highneeds elderly in New Zealand. The potential for the implementation of the framework has been tested through the design of example models of housing. However, given the limitations outlined in the previous section, further testing of the framework would be highly beneficial for confirming the effectiveness of the framework as well as examining the feasibility. This is a procedure also conducted in the development of existing design framework such as EVOLVE (Lewis et al., 2010) and Guidelines for the planning of housing for senior citizens (WEL_HOPS, 2007). For the design framework to be implemented widely by architects, designers and other stakeholders involved with the development of housing for the elderly, greater consideration is required for the cost-benefit analysis - the analysis for the level of QoL provided for the highneeds elderly with respect to the cost for implementing the design requirements. These examinations could also help capture the weight of the significance of the QoL for each individual, thereby prioritising the design requirements.

1) Obtaining feedback from experts involved in the design in practice

In order to make the framework more practical, feedback is required from experts such as architects, designers and quantity surveyors on the designs, mainly for technical and cost aspects. 
To obtain insights regarding the greater use of technology, feedback could also be obtained from various system providers. As required, the proto-types will be amended.

2) Testing the design with respect to the QoL of the high-needs

In order to test the effectiveness of the design in providing a higher QoL for the high-needs elderly, the QoL with respect to the design is investigated for study participants of this research as well as a wider sampling of the population to ensure adequate representation of the cohorts, who would include those using various types of mobility aids and equipment, those with various impairments/conditions, and those who live in an extended family home as well as those living separately from their family. In particular, expanding the sample to include those in diverse cultural groups will bring important information on housing design facilitating the diversity in the ageing population, which is also encouraged by the New Zealand policy (MSD, 2019).

\section{3) Quantitative and qualitative analysis}

Obtained data about the QoL and feedback from the experts should be analysed both quantitatively and qualitatively. The quantitative analysis for QoL scores with respect to various design aspects would be useful for examining the effectiveness of the design. The analysis with respect to various demographic groups would provide greater insights into suitable housing for each demographic or for advancing Universal Design that suits all the demographics. The analysis of QoL scores with respect to costs could provide useful information for prioritising the design considerations. On the other hand, qualitative data would provide important information on how the design considerations work as well as possible improvements.

\subsection{Concluding summary}

As the population ages there will be an increasing demand for housing that can accommodate those elderly people with high-care needs who wish to live at home. This study has examined the design that facilitates a wide range of QoL of the high-needs elderly living independently in New Zealand. Among investigations conducted in this project, the extensive qualitative survey of 30 elderly individuals as well as their caregivers enabled the capture of various QoL in detail, which lead to the establishment of the holistic design framework.

In the course of developing the framework, a number of design considerations/requirements were identified. While some of the requirements were consistent with the existing knowledge, the 
research found new insights into the design for greater QoL. Particularly, this research significantly contributed to the knowledge about the design of private space, which is important for the high-needs elderly. Greater attention is required to the micro-environment of the sitting space, kitchen and bathroom as well as the spatial layout to enhance the control of daily basic activities, and personal and social activities, of elderly residents. Elevation design of interior walls is also a key to facilitating the maintenance of possessions. Regarding both the private space and communal space, the requirements to facilitate individual preferences were identified, the solutions of which were explored and presented by the design of the housing. Particular consideration is required for some conflicting requirements, such as those in the unit size, layout, selection of materials and other spatial qualities. These can be resolved through flexible design and strategies of diversifying design as well as other careful considerations.

In the design of housing that provides a high QoL of residents, considerations are required for various elements of housing environments, from the micro level to the community level, which are complexly related to various aspects of their QoL. Research that has delved into the experiences and perceptions of elderly residents has informed us of the importance of a holistic approach for design that improves QoL. This is particularly crucial for the high-needs elderly seeking to maintain their independent life for as long as possible. 


\section{BIBLIOGRAPHY}

Abbeyfield New Zealand. (2016, 29 Jan. 2016). Abbeyfield. Retrieved from http://www.abbeyfield.co.nz/

Alan Johnson, Philippa Howden-Chapman, \& Shamubeel Eaqub. (2018). A Stocktake of New Zealand's Housing. New Zealand Government.

Altman, I. (1975). The Environment and Social Behavior : Privacy, Personal Space, Territory, Crowding Monterey, Calif.: Brooks/Cole Pub.

Anderzhon, J., Hughes, D., Judd, S., Kiyota, E., \& Wijnties, M. (2012). Design for Aging: International Case Studies of Building and Program. Hoboken, New Jersey: John Wiley \& Sons, Inc.

Arking, B. (2006). Biology of Aging : Observations and Principles. Cary, UNITED STATES: Oxford University Press.

Badkar, J., \& Manning, R. (2009). Paid Caregivers in New Zealand: Current Supply and Future Demand. New Zealand Population Review, 35, 113-127.

Biggs, S., Bernard, M., Kingston, P., \& Nettleton, H. (2000). Lifestyles of belief: Narrative and culture in a retirement community. Ageing and Society, 20, 649-672.

Bland, M. (2007). Betwixt and between: a critical ethnography of comfort in New Zealand residential aged care. Journal of Clinical Nursing, 16(5), 937-944. doi:10.1111/j.1365-2702.2006.01756.x

Bland, R. (1999). Independence, privacy and risk: Two contrasting approaches to residential care for older people. Ageing and Society, 19, 539-560.

Bocquet, V., Barre, J., Couffignal, S., apos, Incau, M., Delagardelle, C., . . Ruiz-Castell, M. (2018). Study design and characteristics of the Luxembourg European Health Examination Survey (EHES-LUX). BMC Public Health, 18.

Bonomi, A. E., Patrick, D. L., Bushnell, D. M., \& Martin, M. (2000). Validation of the United States' version of the World Health Organization Quality of Life (WHOQOL) instrument. Journal of Clinical Epidemiology, 53(1), 1-12. doi:https://doi.org/10.1016/S0895-4356(99)00123-7

Boström, G., Conradsson, M., Rosendahl, E., Nordström, P., Gustafson, Y., \& Littbrand, H. (2014). Functional capacity and dependency in transfer and dressing are associated with depressive symptoms in older people. Clinical Interventions in Aging, 9, 249-257.

Boyd, M., Broad, J., Kerse, N., Foster, S., von Randow, M., Lay-Yee, R., \& Connolly, M. (2011). Twenty-Year Trends in Dependency in Residential Aged Care in Auckland, New Zealand: A Descriptive Study. Journal of the American Medical Directors Association, 12(7), 535-540.

Boyd, M., Connolly, M., Kerse, N., Foster, S., Randow, M. v., Lay-Yee, R., . . Walters-Puttick, S. (2009). Changes in Aged Care Residents' Characteristics and Dependency in Auckland 1988 to 2008. Findings from OPAL 10/9/8 Older Persons' Ability Level Census. Auckland.

Brandi, J. M., Kelley-Gillespie, N., Liese, L. H., \& Farley, O. W. (2003). Nursing Home vs. Assisted Living. Journal of Housing For the Elderly, 18(1), 73-88. doi:10.1300/J081v18n01_05

BRANZ. (2011). Study Report SR 263 [2011]: Lifetime Housing - the Value Case. Porirua.

Bupa New Zealand. (2018). Bupa Medical Alarms are now owned and operated by Securely. Retrieved from http://www.bupa.co.nz/medical-alarms/

Burton, M., Reed, H., \& Chamberlain, P. (2011). Age-Related Disability and Bathroom Use. Journal of Integrated Care, 19(1), 37-43. doi:http://dx.doi.org/10.5042/jic.2011.0034

Callaghan, L., \& Towers, A.-M. (2014). Feeling in control: comparing older people's experiences in different care settings. Ageing and Society(8), 1427-1451.

Calman, K. C. (1984). Quality of Life in Cancer Patients: An Hypothesis. Journal of Medical Ethics, 10(3), 124-127.

Carr, A. J., Gibson, B., \& Robinson, P. G. (2001). Measuring Quality Of Life: Is Quality Of Life Determined By Expectations Or Experience? BMJ: British Medical Journal, 322(7296), 12401243. 
Clarkson, J., Keates, S., Coleman, R., \& Lebbon, C. (2003). Inclusive design : design for the whole population London; New York: Springer.

Community Housing Aotearoa. (2018a). A few terms explained. Retrieved from http://www.communityhousing.org.nz/new-zealand/housing-continuum/terms-explained

Community housing Aotearoa. (2018b). Member's Directory. Retrieved from http://www.communityhousing.org.nz/members/membersdirectory?keywords=\&region=wellington

Consumer NZ. (2013). Retirement villages. Retrieved from https://www.consumer.org.nz/articles/retirement-villages

Cooney, A. (2012). 'Finding home': a grounded theory on how older people 'find home' in long-term care settings. International Journal of Older People Nursing, 7(3), 188-199. doi:10.1111/j.1748-3743.2011.00278.x

Cooney, A., Dowling, M., Gannon, M. E., Dempsey, L., \& Murphy, K. (2014). Exploration of the meaning of connectedness for older people in long-term care in context of their quality of life: a review and commentary. International Journal of Older People Nursing, 9(3), 192-199. doi:10.1111/opn.12017

Crabtree, A., Rouncefield, M., \& Tolmie, P. (2012). Dispensing with Method. In Doing Design Ethnography (pp. 67-87). London: Springer London.

Crouch, C., \& Pearce, J. (2012). Doing Research in Design. London: Berg.

Custers, A. F. J., Westerhof, G. J., Kuin, Y., Gerritsen, D. L., \& Riksen-Walraven, J. M. (2012). Relatedness, autonomy, and competence in the caring relationship: The perspective of nursing home residents. Journal of Aging Studies, 26(3), 319-326. doi:http://dx.doi.org/10.1016/i.jaging.2012.02.005

Darton, R., Bäumker, T., Callaghan, L., \& Netten, A. (2011). Improving housing with care choices for older people: the PSSRU evaluation of extra care housing. Housing, Care and Support, 14(3), 77-82. doi:doi:10.1108/14608791111199741

Davey, J. (2006a). "AGEING IN PLACE": THE VIEWS OF OLDER HOMEOWNERS ON MAINTENANCE, RENOVATION AND ADAPTATION. Social Policy Journal of New Zealand(27), 128-141.

Davey, J. (2006b). RESEARCH ON AGEING IN NEW ZEALAND: PROGRESS, GAPS, AND THE POTENTIAL CONTRIBUTION OF EWAS RESEARCH.

Davey, J., \& Glasgow, K. (2006). Positive Ageing - A Critical Analysis. Policy Quarterly, 2.

Davey, J., Joux, V. d., Nana, G., \& Arcus, M. (2004). Accommodation Options for Older People in Aotearoa/New Zealand. Retrieved from http://repository.digitalnz.org/system/uploads/record/attachment/332/accommodation op tions for older people in aotearoa new zealand.pdf.

Davey, J., \& Keeling, S. (2004). COMBINING WORK AND ELDERCARE: A NEGLECTED WORK-LIFE BALANCE ISSUE. Paper presented at the Labour. Employment and Work in New Zealand.

Davey, J., Keeling, S., \& Zodgekar, A. (2010). Families, Ageing and Migration Indian Communities in Auckland, Wellington and Christchurch. WORKING PAPER 10/03.

Denscombe, M. (2014). The Good Research Guide: For small-scale social research projects (Fifth edition ed.). Berkshire, England: Open University Press.

Denzin, N. K., \& Lincoln, Y. S. (2000). The handbook of qualitative research (2nd ed.). Thousand Oaks, Calif.: Sage Publications.

Department of Building and Housing. (2011a). Compliance Document for New Zealand Building Code Clause G1 Personal Hygiene. Wellington: Department of Building and Housing.

Department of Building and Housing. Compliance Document for New Zealand Building Code: Clause D1 Access Routes, (2011b).

Department of Health. (2004). Extra Care Housing for Older People: An Introduction for Commissioners. London. 
Dias, G. N. F., Couceiro, M. S., Mendes, P., \& de Lurdes Almeida, M. (2017). Physical Activity Benefits in Active Ageing. In Active Ageing and Physical Activity: Guidelines, Functional Exercises and Recommendations (pp. 21-34). Cham: Springer International Publishing.

Dyck, I., Kontos, P., Angus, J., \& McKeever, P. (2005). The home as a site for long-term care: meanings and management of bodies and spaces. Health \& Place, 11(2), 173-185. doi:http://dx.doi.org/10.1016/i.healthplace.2004.06.001

Eakman, A. M. (2012). Measurement Characteristics of the Engagement in Meaningful Activities Survey in an Age-Diverse Sample. The American Journal of Occupational Therapy, 66(2), e2029.

Eakman, A. M., Carlson, M. E., \& Clark, F. A. (2010). The Meaningful Activity Participation Assessment: A Measure of Engagement in Personally Valued Activities. The International Journal of Aging and Human Development, 70(4), 299-317. doi:10.2190/AG.70.4.b

Eastman, P. (2013). Building Type Basics for Senior Living. In.

Eiser, C., \& Jenney, M. (2007). Measuring quality of life. Archives of disease in childhood, 92(4), 348350. doi:10.1136/adc.2005.086405

Ekelund, C., Dahlin-Ivanoff, S., \& Eklund, K. (2014). Self-determination and older people - A concept analysis. Scandinavian Journal of Occupational Therapy, 21(2), 116-124 119p. doi:10.3109/11038128.2013.853832

Eldernet Ltd. (2018). Eldernet. Retrieved from https://www.eldernet.co.nz/Home

Fleming, R., Goodenough, B., Low, L.-F., Chenoweth, L., \& Brodaty, H. (2016). The relationship between the quality of the built environment and the quality of life of people with dementia in residential care. Dementia, 15(4), 663-680. doi:10.1177/1471301214532460

Frances, M.-R. (2000). Combining methodological approaches in research: ethnography and interpretive phenomenology. Journal of Advanced Nursing, 31(1), 219-225. doi:doi:10.1046/j.1365-2648.2000.01243.x

Gabriel, Z., \& Bowling, A. (2004). Quality of Life in Old Age from the Perspectives of Older people. In A. Walker \& C. H. Hennessy (Eds.), Glowing Older: Quality of life in old age (pp. 14-34). Berkshire, England: Open University Press.

Galea, S., \& Tracy, M. (2007). Participation Rates in Epidemiologic Studies. Annals of Epidemiology, 17(9), 643-653. doi:https://doi.org/10.1016/i.annepidem.2007.03.013

Gillsjo, C., Schwartz-Barcott, D., \& von Post, I. (2011). Home: The place the older adult can not imagine living without. BMC Geriatrics, 11, 10.

Glasgow, K. (2013). A new old age? Exploring the values, attitudes and expectations of baby boomers and their implications for policy and practice in an ageing society. (Doctor of Philosophy in Social Policy), Victoria University of Wellington, Social Policy.

Goffman, E. (1968). Asylums: Essays on the social situation of mental patients and other inmates. Great Britain: Pelican Books.

Goodman, R. J., \& Smith, D. G. (1992). Retirement Facilities : Planning, Design, and Marketing. New York: Whitney Library of Design.

Gordon, C., \& Gaitz, C. M. (1976). Leisure and lives: Expressivity across the life span. In R. H. Binstock \& E. Shanas (Eds.), Handbook of aging and the social sciences (pp. 310-34l). New York: Van Nostrand Reinhold.

Granbom, M., Himmelsbach, I., Haak, M., Löfqvist, C., Oswald, F., \& Iwarsson, S. (2014). Residential normalcy and environmental experiences of very old people: Changes in residential reasoning over time. Journal of Aging Studies, 29, 9-19. doi:http://dx.doi.org/10.1016/j.jaging.2013.12.005

Grant Thornton New Zealand. (2010). Aged Residential Care Service Review.

Green Party of Aotearoa New Zealand and New Zealand Labour Party. (2010). A Report into Aged Care: What does the future hold for older New Zealanders?

Greenbrook, S. R. (2005). Village-People-The changing role of retirement villages in New Zealand's ageing society. (Master of Arts in Geography), The University of Auckland, 
Grimmer, K., Kay, D., Foot, J., \& Pastakia, K. (2015). Consumer views about aging-in-place. Clinical Interventions in Aging, 10, 1803+.

Gupta, R. (2014). Embedding Post-Occupancy Evaluation into Architectural Education: from Specialism to Mainstream. Paper presented at the 2nd Annual AAE Conference 2014: Living and Learning, Sheffield. https://aaeconference2014.wordpress.com/

Gusmano, M. K. (2004). Jacquelyn beth frank. the paradox of aging in place in assisted living / david barton smith. reinventing care: assisted living in New York city. Journal of Health Politics, Policy \& Law, 29(6), 1227-1234 1228p.

Haigh, R. (1993). The ageing process: a challenge for design. Applied Ergonomics, 24(1), 9-14. doi:https://doi.org/10.1016/0003-6870(93)90153-Z

Hale, B., Barrett, P., \& Gauld, R. (2010). The Age of Supported Independence: in Voices of In-home Care. In. Retrieved from http://www.springer.com/us/book/9789048188130

Hall, L. (2008). CHRANZ housing reports 2003 - 2007: A summary of the CHRANZ Reports in relation to the Auckland region. Auckland.

Hand, C., Retrum, J., Ware, G., Iwasaki, P., Moaalii, G., \& Main, D. S. (2017). Understanding Social Isolation Among Urban Aging Adults: Informing Occupation-Based Approaches. OTJR: Occupation, Participation and Health, 37(4), 188-198. doi:10.1177/1539449217727119

HART. (2014). Inclusion, Contribution and Connection: A study of the ageing in Aotearoa. Palmerston North: Massay University.

Hauge, Å. L. (2007). Identity and Place: A Critical Comparison of Three Identity Theories. Architectural Science Review, 50(1), 44-51. doi:10.3763/asre.2007.5007

Hauge, S., \& Heggen, K. (2008). The nursing home as a home: a field study of residents' daily life in the common living rooms. Journal of Clinical Nursing, 17(4), 460-467. doi:10.1111/j.13652702.2007.02031.x

Havighurst, R. J. (1961). The nature and value of meaningful free time activities. In R. W. Kleemier (Ed.), Aging and leisure (pp. 309-344). New York: Oxford University Press.

Hayward, C. R. (2012). A HOME AWAY FROM HOME? : THE TRANSITIONS OF OLDER PEOPLE WITHIN TWO NEW ZEALAND RETIREMENT VILLAGES. (MASTER OF ARTS IN SOCIOLOGY), THE UNIVERSITY OF CANTERBURY,

Higher Education Funding Council for England. (2006). Guide to Post Occupancy Evaluation.

HJ SIMS. (2015). Retirement Communities in New Zealand and the United Kingdom. Retrieved from http://www.hjsims.com/news-views/retirement-communities-in-new-zealand-and-theunited-kingdom/\#.VyQ11k1f2UI

HNZ. (2018). More thermal upgrades for homes in the Hutt. Retrieved from https://www.hnzc.co.nz/news/latest-news/more-thermal-upgrades-for-homes-in-the-hutt/

Holt-Lunstad, J. (2018). Why Social Relationships Are Important for Physical Health: A Systems Approach to Understanding and Modifying Risk and Protection. Annual Review of Psychology, 69(1), 437-458. doi:10.1146/annurev-psych-122216-011902

Hon Phil Twyford. (2017). The future of housing in New Zealand. Retrieved from https://www.beehive.govt.nz/release/new-housing-and-urban-development-ministry

Housing Learning \& Improvement Network. (2003). SMART HOME - A DEFINITION. UK: H. L. I. Network.

Howden-Chapman, P., Pene, G., Crane, J., Green, R., lupati, L., Prior, I., \& Teao, I. (2000). Open Houses and Closed Rooms: Tokelau Housing in New Zealand. Health Education \& Behavior, 27(3), 351-362. doi:10.1177/109019810002700309

Howell, K. (2016). SENIORS HOUSING: Issue Identification Paper. Auckland Council.

lan Page, \& Fung, J. (2008). Housing Typologies - Current Stock Prevalence. Auckland Retrieved from http://www.beaconpathway.co.nz/.

Innovation, t. H. o. A. P. P. f. (2009). HAPPI: Housing our Ageing Population Panel for Innovation. the UK Retrieved from https://www.housinglin.org.uk/Topics/type/The-Housing-our-AgeingPopulation-Panel-for-Innovation-HAPPI-Report-2009/. 
interRAI New Zealand. (2016a). interRAl in New Zealand. Retrieved from https://www.interrai.co.nz/ interRAI New Zealand. (2016b). National interRAI Data Analysis: Annual Report 2014/15. Wellington. Iwarsson, S. (2005). A Long-Term Perspective on Person-Environment Fit and ADL Dependence Among Older Swedish Adults. The Gerontologist, 45(3), 327-336. doi:10.1093/geront/45.3.327

Iwarsson, S., \& Isacsson, Å. (1997). Quality of life in the elderly population: an example exploring interrelationships among subjective well-being, ADL dependence, and housing accessibility. Archives of Gerontology and Geriatrics, 26(1), 71-83. doi:https://doi.org/10.1016/S01674943(97)00034-4

James, B., \& Saville-Smith, K. (2011). Retirement Villages Act 2003 Monitoring Project Residents' Perspectives. Auckland: R. Commissioner.

Jamieson, H. A., Gibson, H. M., Abey-Nesbit, R., Ahuriri-Driscoll, A., Keeling, S., \& Schluter, P. J. (2018). Profile of ethnicity, living arrangements and loneliness amongst older adults in Aotearoa New Zealand: A national cross-sectional study. Australasian Journal on Ageing, 37(1), 68-73. doi:doi:10.1111/ajag.12496

Jaye, C., Hale, B., Butler, M., McKechnie, R., Robertson, L., Simpson, J., .. Young, J. (2015). One of us: Stories from two New Zealand rest homes. Journal of Aging Studies, 35, 135-143. doi:http://dx.doi.org/10.1016/i.jaging.2015.08.010

JLL. (2017). New Zealand Retirement Village Database (NZRVD) November 2016: Whitepaper. Auckland.

John E. Ware, J., \& Sherbourne, C. D. (1992). The MOS 36-item short-form health survey (SF-36). Medical Care, 30(6), 473-483.

Jones, N. (2018). Lonely in New Zealand: A third of elderly spend their days alone. Retrieved from https://www.nzherald.co.nz/nz/news/article.cfm?c id=1\&objectid=12022462

Juárez-Cedillo, T., Jarillo-Soto, E., Sánchez-García, S., \& García-Peña, C. (2015). Qualitative Research. In C. García-Peña, L. M. Gutiérrez-Robledo, \& M. U. Pérez-Zepeda (Eds.), Aging Research Methodological Issues (pp. 47-58). Cham: Springer International Publishing.

Julie Hiromoto. (2015). Post Occupancy Evaluation Survey Report. Retrieved from https://www.som.com/ideas/research/post occupancy evaluation survey report

Katz, S., Ford, A. B., Moskowitz, R. W., Jackson, B. A., \& Jaffe, M. W. (1963). Studies of Illness in the Aged. The Index of AADL: A Standardised Measure of Biological and Psychosocial Function. JAMA, 185(12), 914-919.

Keeling, S. (2014). Later Life in Rental Housing. Policy Quarterly Special Issue - Ageing Population, 10(3), 54-59.

Kershaw, C., McIntosh, J., Marques, B., \& Cornwall, J. (2017). A potential role for outdoor, interactive spaces as a healthcare intervention for older persons. Perspectives in Public Health, 137(4), 212-213. doi:10.1177/1757913917709402

Kiata-Holland, E. J. (2010). 'All in a day's work': The life-world of older people in New Zealand rest homes. (Doctor of Philosophy in General Practice and Primary Health Care), The University of Auckland,

King, E. C., Holliday, P. J., \& Andrews, G. J. (2016). Care Challenges in the Bathroom:The Views of Professional Care Providers Working in Clients' Homes. Journal of Applied Gerontology, 1-23. doi:10.1177/0733464816649278

King, E. C., \& Novak, A. C. (2017). Effect of Bathroom Aids and Age on Balance Control During Bathing Transfers. American Journal of Occupational Therapy, 71(6), 1-9. doi:10.5014/ajot.2017.027136

King, N. (2004). Models of Extra Care and Retirement Communities. London: The Health and Social Care Change Agent Team.

Kitzinger, J., \& Barbour, R. S. (1999). Introduction: The Challenge and Promise of Focus Groups. In Developing Focus Group Research. London: SAGE Publications Ltd. Retrieved from 
https://methods.sagepub.com/book/developing-focus-group-research. doi:10.4135/9781849208857

Kiwaki, N., \& Arai, Y. (2015). Social Isolation of Elderly People in Japan: From the Viewpoint of Family Paradigm Changes. The Bulletin of the Faculty of Human Life Sciences, Fuji Women's University, 52, 65-74.

Klaassens, M., \& Meijering, L. (2015). Experiences of home and institution in a secured nursing home ward in the Netherlands: A participatory intervention study. Journal of Aging Studies, 34, 92 102. doi:http://dx.doi.org/10.1016/j.jaging.2015.05.002

Koehn, S. D., Mahmood, A. N., \& Stott-Eveneshen, S. (2016). Quality of Life for Diverse Older Adults in Assisted Living: The Centrality of Control. Journal of Gerontological Social Work, 59(7-8), 512-536. doi:10.1080/01634372.2016.1254699

Kontos, P. C. (1998). Resisting institutionalization: Constructing old age and negotiating home. Journal of Aging Studies, 12(2), 167-184. doi:http://dx.doi.org/10.1016/S08904065(98)90013-5

Koopman-Boyden, P., \& Moosa, S. (2014). Living Alone as a Lifestyle of Older People in New Zealand policy implications. Policy Quarterly Special Issue - Ageing Population, 10(3), 54-59.

Kopec, D., Sinclair, E. L. A., \& Matthes, B. (2010). Evidence Based Design: A Process for Research and Writing. NJ: Prentice Hall.

Krueger, R. A., \& Casey, M. A. (2000). Focus Group: A practical guide for applied research (3rd ed. ed.). Thousand Oaks, California: Sage Publication, Inc.

Kunduraci, A. C. (2017). Lighting Design for the Aging Eyes. International Journal of Science and Technology, 3(3), 185-194. doi:10.20319/mijst.2017.33.185194

La Grow, S., Yeung, P., Towers, A., Alpass, F., \& Stephens, C. (2013). The Impact of Mobility on Quality of Life Among Older Persons. Journal of Aging and Health, 25(5), 723-736. doi:10.1177/0898264313490198

Lawton, M. P., Altman, I., \& Wohlwill, J. F. (1984). Dimensions of Environment-Behavior Research. In I. Altman, M. P. Lawton, \& J. F. Wohlwill (Eds.), Elderly People and the Environment (pp. 115). Boston, MA: Springer US.

Lawton, M. P., \& Brody, E. M. (1970). Assessment of Older People: Self-Maintaining and Instrumental Activities of Daily Living. Nursing Research, 19(3), 278-278.

Lewis, A., Torrington, J., Barnes, S., Darton, R., Holder, J., McKee, K., ... Orrell, A. (2010). EVOLVE Tool - Evaluation of Older People's Living Environments. T. U. o. Sheffield Retrieved from https://www.housinglin.org.uk/Topics/type/EVOLVE-Tool-Evaluation-of-Older-PeoplesLiving-Environments/.

Li, W. W. (2011). Shifting Selves: Home beyond the House - A Study of Ageing, Housing and Wellbeing of Older Chinese Migrants to New Zealand (Doctoral thesis ), University of Waikato

Lifetime Design Limited. (2012). Lifemark Design Standards Handbook. In. Auckland: Lifetime Design Limited.

Lucas, R. (2016). Research Methods for Architecture. London: Laurence King Publishing.

M.B., M. S., K., B. D., M., R. E., \& Atatoa, C. P. E. (2012). In the 21st Century, what is an acceptable response rate? Aust N Z J Public Health, 36(2), 106-108. doi:doi:10.1111/j.17536405.2012.00854.x

Mace, R. L. (1998). Universal Design in Housing. Assistive Technology, 10(1), 21-28. doi:10.1080/10400435.1998.10131957

Magalhães, J. P. d. (2013). What Is Aging? Retrieved from http://www.senescence.info/aging definition.html\#Basic Definitions in Gerontology

Mahoney, F. I., \& Barthel, D. W. (1965). FUNCTIONAL EVALUATION: THE BARTHEL INDEX. Md State Med J, 14, 61-65. 
Marquardt, G., Bueter, K., \& Motzek, T. (2014). Impact of the Design of the Built Environment on People with Dementia: An Evidence-Based Review. HERD: Health Environments Research \& Design Journal, 8(1), 127-157. doi:10.1177/193758671400800111

Martin, A. (2005). Older adulthood, education and social change. (PhD), University of Auckland,

MBIE. Acceptable Solutions and Verification Methods For New Zealand Building Code Clause E2 External Moisture, (2014a).

MBIE. C/AS4 Acceptable Solution for Buildings with Public Access and Educational Facilities (Risk Group CA): For New Zealand Building Code Clause C1-C6 Protection from Fire, (2014b).

MBIE. Acceptable Solutions and Verification Methods For New Zealand Building Code Clause D1 Access Routes, (2016).

MBIE. C/AS3 Acceptable Solution for Buildings Where Care or Detention is Provided (Risk Group SI): For New Zealand Building Code Clause C1-C6 Protection from Fire, (2017).

McColgan, G. (2005). A Place to Sit: Resistance Strategies Used to Create Privacy and Home by People with Dementia. Journal of Contemporary Ethnography, 34(4), 410-433. doi:10.1177/0891241605275574

McLeod, S. (2013). What is Validity? Research Methods. Retrieved from https://www.simplypsychology.org/validity.html

Ministry of Business, I. a. E. Retirement Villages Code of Practice 2008: Variations included April 2017, (2017).

Ministry of Education. (2017). Post occupancy evaluations (POEs) of school building projects. Retrieved from https://www.education.govt.nz/school/property/state-schools/designstandards/post-occupancy-evaluations-of-school-building-projects/

$\mathrm{MOH}$. (2002). Health of Older People Strategy Wellington.

MOH. (2007). Older People's Health Chart Book 2006, Public Health Intelligence Monitoring Report No. 12. Wellington Retrieved from http://www.moh.govt.nz.

MOH. (2010). Trends in Service Design and New Models of Care: A Review. Wellington Retrieved from http://www.nationalhealthboard.govt.nz.

MOH. (2011). Needs Assessment and Support Services for Older People: What you need to know. Wellington Retrieved from http://www.moh.govt.nz.

MOH. (2012). Long-term Residential Care for Older People: What you need to know. Wellington Retrieved from www.health.govt.nz.

$\mathrm{MOH}$. (2013a). Independent Life Expectancy in New Zealand. Wellington: MOH Retrieved from www.health.govt.nz.

$\mathrm{MOH}$. (2013b). Residential care questions and answers. Wellington Retrieved from https://www.health.govt.nz/our-work/life-stages/health-older-people/long-termresidential-care/residential-care-questions-and-answers.

MOH. (2015). Independent life expectancy (ILE) 1996-2013 (Table). In. Wellington: MOH.

Mohit, M. A. (2013). Quality of Life in Natural and Built Environment - An Introductory Analysis. Procedia - Social and Behavioral Sciences, 101, 33-43. doi:https://doi.org/10.1016/j.sbspro.2013.07.176

Morales, E., Rousseau, J., \& Passini, R. (2012). Bathrooms in Retirement Residences: Perceptions and Experiences of Seniors and Caregivers. Physical \& Occupational Therapy In Geriatrics, 30(1), 1-21. doi:10.3109/02703181.2011.644656

Morse, J. M. (2000). Determining Sample Size. Qualitative Health Research, 10(1), 3-5. doi:10.1177/104973200129118183

MSD. (2001). The New Zealand Positive Ageing Strategy. Retrieved from http://www.msd.govt.nz/

MSD. (2019). Better Later Life - He Oranga Kaumātua 2019 to 2034. Retrieved from http://www.superseniors.msd.govt.nz/about-superseniors/ageing-population/better-laterlifereport/index.htm|\#Providinghousingchoicesandoptionssopeoplecanageinthecommunity110 $\underline{0}$ 
MSD. (n.d.-a). About social housing providers. Retrieved from http://housing.msd.govt.nz/information-for-housing-providers/providers/index.html

MSD. (n.d.-b). More housing for people in need. Retrieved from https://www.msd.govt.nz/aboutmsd-and-our-work/work-programmes/social-housing/more-social-housing.html

Murphy, K., Shea, E. O., \& Cooney, A. (2007). Quality of life for older people living in long-stay settings in Ireland. Journal of Clinical Nursing, 16(11), 2167-2177. doi:10.1111/j.13652702.2006.01865.x

Nakhodaeezadeh, M., Jafarabadi, M. A., Allahverdipour, H., Matlabi, H., \& Dehkordi, F. R. (2017). Home Environment and Its Relation with Quality of Life of Older People. Journal of Housing For the Elderly, 1-14. doi:10.1080/02763893.2017.1280583

Narayan, G. (2016). Dizziness and Syncope. In S. S. David (Ed.), Clinical Pathways in Emergency Medicine: Volume I (pp. 693-701). New Delhi: Springer India.

Nelson, I. (2002). CONTINUING CARE RETIREMENT COMMUNITIES. Encyclopedia of Aging. Retrieved from http://www.encyclopedia.com

New Zealand Property Investors' Federation. (2015). Rental housing for the older age group. Retrieved from https://www.nzpif.org.nz/news/view/57114

New Zealand Treasury. (2012). Long-term care and fiscal sustainability: DRAFT PAPER FOR THE LONG-TERM FISCAL EXTERNAL PANEL, Wellington.

Noell-Waggoner, E. (2017). Lighting and the Elderly. In R. Karlicek, Ching-Cherng, S. Zissis, \& R. Ma (Eds.), Handbook of Advanced Lighting Technology. Cham: Springer. doi:https://doi.org/10.1007/978-3-319-00176-0 50

Nord, C. (2011). Architectural space as a moulding factor of care practices and resident privacy in assisted living. Ageing \& Society, 31(06), 934-952. doi:doi:10.1017/S0144686X10001248

Nord, C. (2013). A day to be lived. Elderly peoples' possessions for everyday life in assisted living. Journal of Aging Studies, 27(2), 135-142. doi:http://dx.doi.org/10.1016/i.jaging.2012.12.002

Nordin, S., McKee, K., Wallinder, M., Koch, L., Wijk, H., \& Elf, M. (2017). The physical environment, activity and interaction in residential care facilities for older people: a comparative case study. Scand J Caring Sci, 31(4), 727-738. doi:doi:10.1111/scs.12391

NZG. Retirement Villages Act 2003, (2003).

NZG. (2015a). Age profile of Housing New Zealand tenants. Retrieved from: http://www.socialhousing.govt.nz/

NZG. (2015b). Overview of the Government's programme to improve social housing in New Zealand. Retrieved from http://www.socialhousing.govt.nz/.

NZG. (2018). Cooking and meals. Retrieved from https://www.govt.nz/browse/health-system/helpin-your-home/cooking-and-meals/

Office for Senior Citizens. (2013). Older New Zealanders: Healthy, independent, connected and respected. Wellington Retrieved from www.msd.govt.nz/oldernewzealanders.

Office for Seniors. (2019). Summary of Submissions to draft strategy: Better Later Life He Oranga Kaumātua 2019 to 2034. Wellington: O. f. Seniors.

Orihuela, P., \& Orihuela, J. (2014). Needs, Values and Post-occupancy Evaluation of Housing Project Customers: A Pragmatic View. Procedia Engineering, 85, 412-419. doi:https://doi.org/10.1016/i.proeng.2014.10.567

Orrell, A., McKee, K., Torrington, J., Barnes, S., Darton, R., Netten, A., \& Lewis, A. (2013). The relationship between building design and residents' quality of life in extra care housing schemes. Health \& Place, 21, 52-64. doi:http://dx.doi.org/10.1016/j.healthplace.2012.12.004

Parker, C., Barnes, S., McKee, K., Morgan, K., Torrington, J., \& Tregenza, P. (2004). Quality of life and building design in residential and nursing homes for older people. Ageing and Society, 24(6), 941-962. doi:10.1017/S0144686X04002387

Parsons, J. G. M., \& Parsons, M. J. G. (2012). The effect of a designated tool on person-centred goal identification and service planning among older people receiving homecare in New Zealand. 
Health \& Social Care in the Community, 20(6), 653-662. doi:10.1111/j.1365-

2524.2012.01081.x

Peace, S. (2017). Age-inclusive Design: A Challenge for Kitchen Living? In C. Bates, R. Imrie, \& K. Kullman (Eds.), Care and Design: bodies, buildings, cities / edited by Charlotte Bates (pp. 1832). Chichester, West Sussex, UK: John Wiley \& Sons, Ltd.

Peggy Koopman-Boyden, M. P. C., Judith Davey, Margaret Richardson. (2014). Making active ageing a reality: Maximising participation and contribution by older people.

Perkins, B., Hoglund, J. D., King, D., \& Cohen, E. (2004). Building Type Basics for Senior Living. Canada: John Wiley \& Sons, Inc.

Perkins, M. M., Ball, M. M., Whittington, F. J., \& Hollingsworth, C. (2012). Relational autonomy in assisted living: A focus on diverse care settings for older adults. Journal of Aging Studies, 26(2), 214-225. doi:http://dx.doi.org/10.1016/i.jaging.2012.01.001

Perl, L. (2010). Section 202 and Other HUD Rental Housing Programs for Low-Income Elderly Residents. Washington, DC: C. R. Service Retrieved from www.crs.gov.

Persson, T., \& Wästerfors, D. (2009). "Such Trivial Matters:" How staff account for restrictions of residents' influence in nursing homes. Journal of Aging Studies, 23(1), 1-11. doi:http://dx.doi.org/10.1016/i.jaging.2007.09.005

Povey, D. M., \& Harris, U. (2006). With My Boots On!: A survey of housing quality and preferences of a selected group of older people in Dunedin. Dunedin.

Pretlove, S., \& Kade, S. (2016). Post occupancy evaluation of social housing designed and built to Code for Sustainable Homes levels 3, 4 and 5. Energy and Buildings, 110, 120-134. doi:https://doi.org/10.1016/j.enbuild.2015.10.014

Proshansky, H. M., Fabian, A. K., \& Kaminoff, R. (1983). Place-identity: Physical world socialization of the self. Journal of Environmental Psychology, 3(1), 57-83. doi:https://doi.org/10.1016/S0272-4944(83)80021-8

Qualtrics. (2018). Qualtrics. Retrieved from https://www.qualtrics.com/au/

Reid, M. F. (2008). SUPPORTED INDEPENDENT ACCOMMODATION FOR OLDER NEW ZEALANDERS: A Review of Current Policy and Innovative Practice. (Master of Arts), Victoria University of Wellington,

Retirement Village Association of New Zealand. (2014). Your Questions Answered: Your life, your choice. In. Wellington: Retirement Village Association New Zealand.

RIBA, Hay, R., Bradbury, S., Dixon, D., Martindale, K., Samuel, F., \& Tait, A. (2017). Building Knowledge: Pathways to Post Occupancy Evaluation. London: RIBA.

RIBA Practice team, \& Morris, N. (2017). RIBA calls on government to commit to POE Retrieved from https://www.architecture.com/

Robert W Marans, \& Stimson, R. (2011). Investigating Quality of Urban Life Theory, Methods and Empirical Research. Dordrecht, New York: Springer.

Robertson, L., \& Fitzgerald, R. (2010). The Conceptualisation of Residential Home Environments: Implications for Occupational Therapy. The British Journal of Occupational Therapy, 73(4), 170-177. doi:10.4276/030802210x12706313443983

Robson, D., Nicholson, A.-M., \& Barker, N. (1997). Homes for the Third Age: A Design Guide for Extra Care Sheltered Housing. London: E \& FN SPON.

Salvation Army New Zealand. (2018). Housing for over 55s. Retrieved from http://www.salvationarmy.org.nz/

Sanders, J. (1997). CONTINUING CARE RETIREMENT COMMUNITIES: A BACKGROUND AND SUMMARY OF CURRENT ISSUES. Retrieved from https://aspe.hhs.gov/basicreport/continuing-care-retirement-communities-background-and-summary-current-issues.

Sarah Barnes. (2006). Space, choice and control, and quality of life in care settings for older people. Environment and Behavior, 38(5), 589-604.

Sarah Barnes, \& the Design in Caring Environments Study Group. (2002). The design of caring environments and the quality of life of older people. Ageing \& Society, 22, 775-789. 
Saville-Smith, K., James, B., \& Fraser, R. (2008). Older People's House Performance and Their Repair and Maintenance Practices: Analysis from a 2008 National Survey of Older People and Existing Darasets. Wellington.

Saville-Smith, K., James, B., Warren, J., \& Coleman, A. (2009). Older People's Housing Futures in 2050: Three Scenarios for an Ageing Society. Wellington: C. f. H. R. A. N. Zealand.

Schillmeier, M. (2017). Chapter Twelve Design and the Art of Care; Engaging the More than Human and Less than Inhuman. In C. bates, R. Imrie, \& K. Kullman (Eds.), Care and Design: Bodies, Buildings, Cities. Chichester, West Sussex, UK: John Wiley \& Sons, Ltd.

Schwarz, B., \& Brent, R. (1999). Aging, Autonomy and Architecture: Advances in Assisted Living. Baltimore: The Johns Hopkins University Press.

Seniorline. (2016). Rental Accommodation for Older People - Auckland. Retrieved from: http://www.adhb.govt.nz/seniorline/

Sharon Buckland. (2009). Not the Retiring Sort: New research into the intentions and aspirations of the New Zealand Baby Boomers. Auckland.

Shikder, S., Mourshed, M., \& Price, A. (2012). Therapeutic lighting design for the elderly: a review. Perspectives in Public Health, 132(6), 282-291.

Sikorska, E. (1999). Organizational Determinants of Resident Satisfaction With Assisted Living. The Gerontologist, 39(4), 450-456. doi:10.1093/geront/39.4.450

Sisters of Compassion Limited. (n.d.). Compassion Housing. Retrieved from https://compassion.org.nz/our-places/compassion-housing/

Sivignon, C. (2018). Elder abuse often linked to loneliness and isolation: Age Concern Nelson Tasman Retrieved from https://www.stuff.co.nz/life-style/104604550/elder-abuse-often-linked-toloneliness-and-isolation-age-concern-nelson-tasman

Smith, J. A., Flowers, P., \& Larkin, M. (2009). Interpretative Phenomenological Analysis. London: SAGE Publications Ltd.

Sok Teng, L. O. W., \& Thirumalaya, B. (2017). Physical activity level and fall risk among communitydwelling older adults. Journal of Physical Therapy Science, 29(7), 1121-1124.

St John. (n.d.). The only medical alarms monitored by St John. Retrieved from https://www.stjohn.org.nz/Medical-Alarms/

Stabell, A., Eide, H., Solheim, G. A., Solberg, K. N., \& Rustøen, T. (2004). Nursing home residents' dependence and independence. Journal of Clinical Nursing, 13(6), 677-686. doi:10.1111/j.1365-2702.2004.00942.x

Standards New Zealand. (2001). New Zealand Standard NZS 4121:2001 Design for Access and Mobility - Buildings and Associated Facilities. In. Wellington: Standards New Zealand.

Stats NZ. (2009). Migration and age. Wellington: Stats NZ.

Stats NZ. (2013). 2013 Census QuickStats about national highlights - tables. Retrieved from http://www.stats.govt.nz/.

Stats NZ. (2014a). 2013 QuickStats About Housing. Wellington: Stats NZ.

Stats NZ. (2014b). Disability Survey: 2013 -tables.

Stats NZ. (2014c). Frequently asked questions - Population statistics.

Stats NZ. (2014d). Subnational population projections, by age and sex, 2013(base)-2043. Wellington.

Stats NZ. (2015). 2013 Census QuickStats About people aged 65 and over. Wellington.

Stats NZ. (2016a). Income for all people by labour force status, sex, and age groups. Retrieved from http://nzdotstat.stats.govt.nz/

Stats NZ. (2016b). National population projections, by age and sex, 2016 (base) - 2068.

Stats NZ. (2018). New Zealand abridged period life table: 2015-17 (final). Retrieved from https://www.stats.govt.nz/information-releases/new-zealand-abridged-period-life-table201517-final

Stats NZ. (2019). Income by disability status, age groups and income source. Retrieved 3 Jan, 2019, from Stat NZ

Stats NZ. (n.d.). Life expectancy. Retrieved from https://www.stats.govt.nz/topics/life-expectancy 
Stephens, R. (2014). Focusing on the Future: a summary and critique of the 2013 retirement income report. Policy Quarterly Special Issue - Ageing Population, 10(3), 17-22.

Stock, R. (2018). People no longer want to live in fortress-like gated retirement villages. Retrieved from https://www.stuff.co.nz/business/money/106509545/people-no-longer-want-to-livein-fortresslike-gated-retirement-villages

Tamasese, T. K., Loudeen, T., \& Waldegrave, P. a. C. (2014). Pacific Perspectives on Ageing in New Zealand: Pacific-focussed Qualitative Research.

Te Pou - The National Centre of Mental Health Research, I. a. W. D. (2011). Older adults in New Zealand-Population changes, health, service use and workforce needs. Retrieved from www.tepou.co.nz.

Tester, S., Hubbard, G., Downs, M., MacDonald, C., \& Murphy, J. (2004). Frailty and institutional life. In A. Walker \& C. H. Hennessy (Eds.), Growing Older: Quality Of Life In Old Age: Extending quality of life. Maidenhead: Open University Press.

The Centre for Excellence in Universal Design. (2014). What is Universal Design. Retrieved from http://universaldesign.ie/What-is-Universal-Design/

the Department of Health. (2006). CARE STANDARDS ACT 2000 In Care Homes for Older People National Minimum Standards - and Care Homes Regulations (3rd ed.). Norwich: The Stationery Office

The Green House Project. (2017). The Green House Project: caring homes for meaningful lives. Retrieved from http://www.thegreenhouseproject.org/

The Housing Learning and Improvement Network. (2011). Section B, Paper B2: The design and build of successful extra care housing. In Strategic Housing for Older People: A Resource Pack. London: The Housing Learning and Improvement Network,.

The Housing Learning and Improvement Network. (n.d.). Extra care statistics. Retrieved from https://www.housinglin.org.uk/Topics/browse/HousingExtraCare/ExtraCareProvision/Statist ics/

The Royal Borough of Kensington and Chelsea. (2015). Older People's Housing Design Guidance. Retrieved from www.rbkc.gov.uk.

Theofilou, P. (2013). Quality of life: Definition and measurement. Europe's Journal of Psychology, 9(1), 150-162. doi:10.5964/ejop.v9i1.337

Toepoel, V. (2013). Ageing, Leisure, and Social Connectedness: How could Leisure Help Reduce Social Isolation of Older People? Social Indicators Research, 113(1), 355-372. doi:http://dx.doi.org/10.1007/s11205-012-0097-6

Tomah, A. N., Ismail, H. B., \& Abed, A. (2016). The concept of privacy and its effects on residential layout and design: Amman as a case study. Habitat International, 53, 1-7. doi:https://doi.org/10.1016/i.habitatint.2015.10.029

Tompkins, C. J., Ihara, E. S., Cusick, A., \& Park, N. S. (2012). “Maintaining Connections but Wanting More": The Continuity of Familial Relationships Among Assisted-Living Residents. Journal of Gerontological Social Work, 55(3), 249-261. doi:10.1080/01634372.2011.639439

Torrington, J., \& Bacon, V. (1997). A briefing and design guide: care homes for older people. Ageing and Society, 17(4), 479-480.

Torrington, J., Barnes, S., McKee, K., Morgan, K., \& Tregenza, P. (2004). The Influence of Building Design on the Quality of Life of Older People. Architectural Science Review(2), 193-197.

Turner, V. (1969). The ritual process : structure and anti-structure. Chicago: Aldine Pub. Co.

Twigg, J. (2002). Bathing - the Body and Community Care : The Body and Community Care (1). Florence, US: Routledge.

U.S. Department of Housing and Urban Development. (n.d.). Section 202 Supportive Housing for the Elderly Program. Retrieved from https://www.hud.gov/program offices/housing/mfh/progdesc/eld202 
United Nations Population Fund and HelpAge International. (2012). Ageing in the Twenty-First Century: A Celebration and A Challenge. New York, London: United Nations Population Fund and HelpAge International.

University of Kent and the Personal Social Services Research Unit. (2018). ASCOT: Adult Social Care Outcomes Toolkit. Retrieved from https://www.pssru.ac.uk/ascot/

Van Steenwinkel, I., Dierckx de Casterlé, B., \& Heylighen, A. (2017). How architectural design affords experiences of freedom in residential care for older people. Journal of Aging Studies, 41, 8492. doi:https://doi.org/10.1016/j.jaging.2017.05.001

Waldegrave, C. (2014). Income, assets, poverty and housing tenure. Summary report for the New Zealand Longitudinal Study of Ageing. Wellington.

Wang, D., \& Groat, L. N. (2013). Architectural Research Methods (2nd Edition). Somerset, NJ, USA: John Wiley \& Sons.

WEL_HOPS. (2007). Guidelines for the planning of housing for senior citizens. WEL_HOPS Retrieved from https://www.housinglin.org.uk/Topics/type/Guidelines-for-the-planning-of-housingfor-senior-citizens/.

Wellington City Council. (n.d.-a). Housing upgrades Retrieved from https://wellington.govt.nz/services/community-and-culture/housing-support/councilhousing/housing-upgrade

Wellington City Council. (n.d.-b). Marshall Court Retrieved from https://wellington.govt.nz/services/community-and-culture/housing-support/councilhousing/about-the-improvements/marshall-court

WHO. (1998). WHOQoL: User Manual. Geneva, Switzerland: W. H. Organisation.

WHO. (2004). A Glossary of Terms for Community Health Care and Services for Older Persons.

WHO. (2017). Global strategy and action plan on ageing and health. Switzerland.

Wiersma, E., \& Dupuis, S. L. (2010). Becoming institutional bodies: Socialization into a long-term care home. Journal of Aging Studies, 24(4), 278-291. doi:http://dx.doi.org/10.1016/j.jaging.2010.08.003

Wiles, J. L., Leibing, A., Guberman, N., Reeve, J., \& Allen, R. E. S. (2012). The Meaning of “Aging in Place" to Older People. Gerontologist, 52(3), 357-366 310p.

Wojgani, H., \& Hanson, J. (2007). Extra care housing: a paradigm shift. Paper presented at the Sixth International Space Syntax Symposium, Istanbul, Turkey.

Wright-St Clair, V. (2011). Being occupied with what matters in advanced age. Journal of Occupational Science, 19(1), 44-53.

Wright-St Clair, V. A., Kepa, M., Hoenle, S., Hayman, K., Keeling, S., Connolly, M., . . Kerse, N. (2012). Doing what's important: Valued activities for older New Zealand Māori and non-Māori. Australasian Journal on Ageing, 31(4), 241-246. doi:10.1111/j.1741-6612.2011.00583.x

Yeun, P., Good, G., O'Donoghue, K., Spence, S., \& Ros, B. (2017). What matters most to people in retirement villages and their transition to residential aged care. AOTEAROA NEW ZEALAND SOCIAL WORK, 29(4), 84-96.

Yeung, P., Good, G., O'Donoghue, K., Spence, S., \& Ros, B. (2017). What matters most to people in retirement villages and their transition to residential aged care. AOTEAROA NEW ZEALAND SOCIAL WORK, 29(4).

Young, I. M. (2005). A Room of One's Own: Old Age, Extended Care, and Privacy. In I. M. Young (Ed.), On Female Body Experience: "Throwing Like a Girl" and Other Essays Oxford University Press. 


\section{APPENDICES}

Appendix 1: Ethics approval memo, information sheets, consent forms and questionnaires

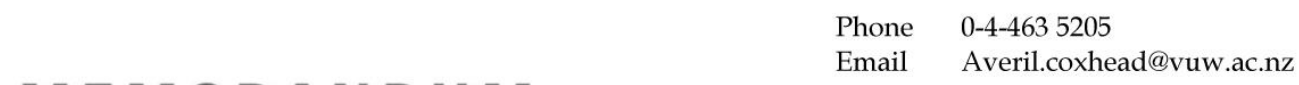

MEMORANDUM

\begin{tabular}{l|l}
\hline TO & Yukiko Kuboshima \\
\hline COPY TO & $\begin{array}{l}\text { Geoff Thomas } \\
\text { Jacqueline Mclntosh }\end{array}$ \\
\hline FROM & Dr Averil Coxhead, Acting Convener, Human Ethics Committee \\
\hline DATE & 23 May 2017 \\
\hline PAGES & 1 \\
\hline & $\begin{array}{l}\text { Ethics Approval: } 23243 \\
\text { Designing supported housing that improves quality of life of high- } \\
\text { dependency older people in New Zealand }\end{array}$ \\
\hline
\end{tabular}

Thank you for your request to amend your ethics approval. This has now been considered and the request granted.

Your application has approval until 31 October 2018. If your data collection is not completed by this date you should apply to the Human Ethics Committee for an extension to this approval.

Best wishes with the research.

Averil Coxhead,

Acting Convener, Victoria University Human Ethics Committee 


\title{
Designing supported housing which improves the quality of life Phase 1-1 Survey
}

\author{
INFORMATION SHEET FOR PARTICIPANTS
}

Thank you for your interest in this project. Please read this information. If you decide to participate, thank you. If you decide not to take part, thank you for considering my request.

\section{Who am I?}

My name is Yukiko Kuboshima and I am a PhD student in School of Architecture at Victoria University of Wellington.

\section{What is the aim of the project?}

This project aims to design a desirable model of supported housing for older people. This Phase 1-1 survey aims to clarify the models of care and physical environments of the existing supported housing. This research has been approved by the Victoria University of Wellington Human Ethics Committee [Approval number: 23243].

\section{How can you help?}

If you agree to take part, please proceed to the questionnaire. It will take 5-15 minutes. Please press $\mathrm{Ctrl}$ and click the link below to proceed to the survey.

Take the survey

\section{What will happen to the information you give?}

This research is confidential. I will not name you in any reports, and I will not include any information that would identify you. Only my supervisors and I will access the collected data. It will be kept securely and destroyed 3 years after the research ends.

\section{What will the project produce?}

The information from my research will be used in my PhD dissertation. You will not be identified in my report. I may also use the results of my research for conference presentations, and academic reports. I will take care not to identify you in any presentation or report. 


\section{If you accept this invitation, what are your rights as a research participant?}

You do not have to accept this invitation if you don't want to. If you do decide to participate, you have the right to:

- $\quad$ choose not to answer any questionnaire;

- $\quad$ withdraw from the survey up until two weeks after your submission of questionnaire;

- $\quad$ ask any questions about the survey at any time;

- be able to read the final report of this research by emailing me to request a copy.

If you have any questions or problems, who can you contact?

If you have any questions, either now or in the future, please feel free to contact either:

Student:

Name: Yukiko Kuboshima

Email: Yukiko.Kuboshima@vuw.ac.nz

\section{Supervisors:}

Name: Geoff Thomas

$\mathrm{R}$ o I e: Supervisor

School: School of Architecture

Phone: 044636247

Email: geoff.thomas@vuw.ac.nz

Name: Jacqueline Macintosh

$\mathrm{R}$ o I e: Supervisor

School: School of Architecture

Phone: 044636296

Email: jacqueline.mcintosh@vuw.ac.nz

\section{Human Ethics Committee information}

If you have any concerns about the ethical conduct of the research you may contact the Victoria University HEC Convener: Associate Professor Susan Corbett. Email susan.corbett@vuw.ac.nz or telephone+64-4-463 5480.

[I will include the information sheet in the invitation emails to respondents.] 


\section{Phase 1-1 Questionnaire on models of care and physical environments}

For operators of retirement villages

Consent

Thank you for your interest in this project.

Do you agree to take part in the survey?

I agree

Type of units

Q1-1. What type of units do you have?

Independent living units

Supported/assisted living units

Residential care (rest homes, specialist dementia units, long-term care hospitals, and/or a psycho-geriatric units)

Other (Please specify)

Independent living units

Q2-1. How many independent living units do you supply?

Q2-2. Of these, how many units are occupied by at least one person aged 75 and over?

Q2-3. How many independent living units have access and facilities for the disabled? (If unknown, put zero.)

Q2-4. What kind of common facilities are there for residents in independent living units? (Tick all that apply.)

Dining room/restaurant

Lounge/Dayroom (for multi-purpose)

Shared kitchen

Other (Please describe)

Q2-5. What kind of services do you provide to residents in independent living units? 


\begin{tabular}{|l|l|l|}
\hline & $\begin{array}{l}\text { Do you provide? } \\
\text { (Choose from Yes (without extra } \\
\text { cost), Yes (with extra cost), and No) }\end{array}$ & $\begin{array}{l}\text { Comment } \\
\text { (Optional) }\end{array}$ \\
\hline Visiting residents regularly & & \\
\hline Organising activities in communal areas & & \\
\hline Outing (including shopping trips) & & \\
\hline Transportation & & \\
\hline Emergency on call 24 hours a day & & \\
\hline Meals & & \\
\hline Laundry service & & \\
\hline $\begin{array}{l}\text { Assistance in household tasks (cleaning, } \\
\text { washing dishes, home maintenance } \\
\text { etc.) }\end{array}$ & & \\
\hline Shopping on behalf of residents & & \\
\hline Personal care & & \\
\hline Other & & \\
\hline
\end{tabular}

Supported/assisted living units

Q3-1. How many supported/assisted living units do you supply?

Q3-2. Of these, how many units are occupied by at least one person aged 75 and over?

Q3-3. How many supported/assisted living units have access and facilities for the disabled? (If unknown, put zero.)

Q3-4. What kind of common facilities are there for residents in supported/assisted living units? (Tick all that apply.)

Dining room/restaurant

Lounge/Dayroom (for multi-purpose)

Shared kitchen

Other (Please specify)

Q3-5. What kind of services do you provide to residents in supported/assisted living units? 


\begin{tabular}{|l|l|l|}
\hline & $\begin{array}{l}\text { Do you provide? } \\
\text { (Choose from Yes (without extra } \\
\text { cost), Yes (with extra cost), and } \\
\text { No) }\end{array}$ & $\begin{array}{l}\text { Comment } \\
\text { (Optional) }\end{array}$ \\
\hline Visiting residents regularly & & \\
\hline Organising activities in communal areas & & \\
\hline Outing (including shopping trips) & & \\
\hline Transportation & & \\
\hline Emergency on call 24 hours a day & & \\
\hline Meals & & \\
\hline Laundry service & & \\
\hline $\begin{array}{l}\text { Assistance in household tasks (cleaning, } \\
\text { washing dishes, home maintenance } \\
\text { etc.) }\end{array}$ & & \\
\hline Shopping on behalf of residents & & \\
\hline Personal care & & \\
\hline Other & & \\
\hline
\end{tabular}

Interest in the following survey

Q5. In the following phase, I will investigate how the residents and the staff use the space, after obtaining their consent. Are you interested in participating in this survey? (If you answer yes, I will send you an invitation letter.)

Yes

No

\section{For operators of private/public-sector housing}

\section{Consent}

Thank you for your interest in this project.

Do you agree to take part in the survey?

I agree

Type of units

Q1. What type of housing do you have?

Rental housing

Residential care (rest homes, specialist dementia units, long-term care hospitals, and/or a psycho-geriatric units)

Other (Please specify) 
Q2-1. How many rental housing units do you supply?

Q2-2. Of these, how many are occupied by people aged 75 and over?

Q2-3. How many rental housing units have access and facilities for the disabled? (If unknown put Zero.)

Q2-4. What kind of common facilities are there for residents in rental housing? (Tick all that apply.)

Dining room/restaurant

Lounge/Dayroom (for multi-purpose)

Shared kitchen

Other (Please describe)

Q2-5. What kind of services do you provide to residents in rental housing?

\begin{tabular}{|l|l|l|}
\hline & $\begin{array}{l}\text { Do you provide? } \\
\text { (Choose from Yes (without extra } \\
\text { cost), Yes (with extra cost), and No) }\end{array}$ & $\begin{array}{l}\text { Comment } \\
\text { (Optional) }\end{array}$ \\
\hline Visiting residents regularly & & \\
\hline Organising activities in communal areas & & \\
\hline Outing (including shopping trips) & & \\
\hline Transportation & & \\
\hline Emergency on call 24 hours a day & & \\
\hline Meals & & \\
\hline Laundry service & & \\
\hline $\begin{array}{l}\text { Assistance in household tasks (cleaning, } \\
\text { washing dishes, home maintenance } \\
\text { etc.) }\end{array}$ & & \\
\hline Shopping on behalf of residents & & \\
\hline Personal care & & \\
\hline Other & & \\
\hline
\end{tabular}

Interest in the following survey

Q3. In the following phase, I will investigate how the residents and the staff use the space, after obtaining their consent. Are you interested in participating in this survey? (If you answer yes, I will send you an invitation letter.)

Yes

No 


\section{Designing supported housing which improves the quality of life Phase 1-2 Survey}

\section{INFORMATION SHEET FOR PARTICIPANTS}

Thank you for your interest in this project. Please read this information. If you decide to participate, thank you. If you decide not to take part, thank you for considering my request.

\section{Who am I?}

My name is Yukiko Kuboshima and I am a PhD student in School of Architecture at Victoria University of Wellington.

\section{What is the aim of the project?}

This project aims to design a desirable model of supported housing for older people.

This research has been approved by the Victoria University of Wellington Human Ethics Committee [provide approval number].

\section{How can you help?}

If you agree to take part, please answer the questionnaire.

After answering the questionnaire, I will visit you to collect it.

\section{What will happen to the information you give?}

This research is confidential. I will not name you in any reports, and I will not include any information that would identify you. Only my supervisors and I will access the collected data. It will be kept securely and destroyed 3 years after the research ends.

\section{What will the project produce?}

The information from my research will be used in my PhD dissertation. You will not be identified in my report. I may also use the results of my research for conference presentations, and academic reports. I will take care not to identify you in any presentation or report.

\section{If you accept this invitation, what are your rights as a research participant?}

You do not have to accept this invitation if you don't want to. If you do decide to participate, you have the right to: 
- $\quad$ choose not to answer any questionnaire;

- $\quad$ withdraw from the survey up until two weeks after your submission of questionnaire; ask any questions about the survey at any time;

If you have any questions or problems, who can you contact?

If you have any questions, either now or in the future, please feel free to contact either:

Student:

Name: Yukiko Kuboshima

Email: Yukiko.Kuboshima@vuw.ac.nz

\section{Supervisors:}

Name: Geoff Thomas

R o l e: Supervisor

School: School of Architecture

Phone: 044636247

Email: geoff.thomas@vuw.ac.nz

Name: Jacqueline Macintosh

R o I e: Supervisor

School: School of Architecture

Phone: 044636296

Email: jacqueline.mcintosh@vuw.ac.nz

\section{Human Ethics Committee information}

If you have any concerns about the ethical conduct of the research you may contact the Victoria University HEC Convener: Associate Professor Susan Corbett. Email susan.corbett@vuw.ac.nz or telephone +64-4-463 5480. 


\section{Designing supported housing which improves the quality of life Phase 1-2 Survey}

\section{CONSENT TO SURVEY}

This consent form will be held for 5 years.

Researcher: Yukiko Kuboshima, School of Architecture, Victoria University of Wellington

- I have read the Information Sheet and I agree to take part in a questionnaire survey.

I understand that:

- I may withdraw from this study up to two weeks after the survey, and any information that I have provided will be returned to me or destroyed.

- $\quad$ The information I have provided will be destroyed 3 years after the research is finished.

- $\quad$ Any information I provide will be kept confidential to the researcher and the supervisors. I understand that the results will be used for a PhD report and a summary of the results may be used in academic reports and/or presented at conferences.

- My name will not be used in reports, nor will any information that would identify me.

- $\quad$ I am interested in participating in a study about housing, and I have added Yes $\square$ No my address below.

Signature of participant:

Date:

Contact details:

Name: Title:

Address:

Phone number:

Email address: 


\section{Questionnaire}

Thank you for choosing to participate in this survey. All your answers will be kept confidential.

Q1. Birth date

Q2. Are you

Q3. Ethnicity

European

$\square$ Maori

Asian
Day

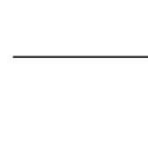

Month Year

Q4. What is your living arrangement?

Alone

With spouse/ partner only

$\square$ Other (Please specify)

Q5. Do you need assistance in personal hygiene (doing teeth, fitting false teeth, doing hair, shaving, washing face)?

$\square$ Yes

$\square$ Sometimes / Some of them

No

Q6. Do you need assistance in eating? (Preparing food not included)

Yes

$\square$ Sometimes / Need help with cutting, spreading butter, etc.

$\square$ No

Q7. Do you need assistance in moving from bed to chair/ wheelchair?

Yes

$\square$ Sometimes

$\square$ No 
Q8. Do you need assistance in dressing?

$\square$ Yes

$\square$ Sometimes

$\square$ No

Q9. Do you need assistance in bathing?

$\square$ Yes

$\square$ Sometimes/ Caregiver supervises

No

Q10. How do you need assistance in moving indoors?

Walk independently (may use sticks, walker flames, etc.)

$\square$ Move in wheelchair independently

$\square$ Walk with help of one person

$\square$ Walk with help of more than one person/ need assistance in moving in wheelchair

Q11. Do you receive any assistance (described in Q5 Q10) from family, relatives and/or friends?

Yes

$\square \quad$ No

$\square$ Not sure

Q12. Do you receive any assistance (described in Q5 Q10) from professional caregiver(s)?

Yes

No

Not sure

(If yes) How many days a week do they visit you? Days a week

Thank you for your cooperation. Please keep this questionnaire in your house until it is collected. 


\section{Designing supported housing which improves the quality of life Phase 2 \& 4 Surveys}

\section{INFORMATION SHEET FOR HOUSING OPERATORS}

Thank you for your interest in this project. Please read this. If you decide to participate, thank you. If you decide not to take part, thank you for considering my request.

\section{Who am I?}

My name is Yukiko Kuboshima and I am a PhD student in School of Architecture at Victoria University of Wellington.

\section{What is the aim of the project?}

This project aims to design a desirable model of supported housing for older people. The aims of each surveys are;

Phase 2-1: I will collect information on physical environments (common space only) to make plans.

Phase 2-2: I will interview and observe residents.

Phase 2-3: I will interview and/or observe the service providers (housing operator and caregivers).

Phase 4: I will have the feedback on the housing which I have redesigned in Phase 3

from residents and service providers through individual interviews and/or focus groups.

For the details of the Phase 2-2, 2-3 and 4 surveys, please see the attached information sheets for participants on each phase.

This research has been approved by the Victoria University of Wellington Human Ethics Committee [Approval number: 23243].

\section{How can you help?}

I would appreciate your cooperation in the following;

- I ask your permission to conduct these surveys described above. (For Phase 2-2, 2-3, and 4 surveys, I will obtain consent from individuals.)

- For Phase 2-1 survey, I ask you to provide the floor plans, if they are available, and ask permission to observe and take photos of common space, to make a plan. I will not collect the data on private rooms nor take photographs of any person. 
- $\quad$ choose not to answer any questionnaire;

- $\quad$ withdraw from the survey up until two weeks after your submission of questionnaire; ask any questions about the survey at any time;

\section{If you have any questions or problems, who can you contact?}

If you have any questions, either now or in the future, please feel free to contact either:

Student:

Name: Yukiko Kuboshima

Email: Yukiko.Kuboshima@vuw.ac.nz

\section{Supervisors:}

Name: Geoff Thomas

R o l e: Supervisor

School: School of Architecture

Phone: 044636247

Email: geoff.thomas@vuw.ac.nz

Name: Jacqueline Macintosh

R o I e: Supervisor

School: School of Architecture

Phone: 044636296

Email: jacqueline.mcintosh@vuw.ac.nz

\section{Human Ethics Committee information}

If you have any concerns about the ethical conduct of the research you may contact the Victoria University HEC Convener: Associate Professor Susan Corbett. Email susan.corbett@vuw.ac.nz or telephone +64-4-463 5480. 


\section{Designing supported housing which improves the quality of life Phase 2 \& 4 Surveys \\ CONSENT TO SURVEYS}

This consent form will be held for 5 years.

Researcher: Yukiko Kuboshima, School of Architecture, Victoria University of Wellington

- I have read the Information Sheet, and I agree to take part in the surveys.

I understand that:

- I may withdraw from this study up to two weeks after the survey, and any information that I have provided will be returned to me or destroyed.

- $\quad$ The information I have provided will be destroyed 3 years after the research is finished.

- $\quad$ Any information I provide will be kept confidential to the researcher and the supervisors. I understand that the results will be used for a PhD report and a summary of the results may be used in academic reports and/or presented at conferences.

- $\quad$ My name will not be used in reports, nor will any information that would identify me.

- I would like to receive a copy of the final report and have added my email Yes $\square$ No address below.

Signature of participant:

Date:

Contact details:

Name: Title:

Address:

Phone number:

Email address: 


\section{Designing supported housing which improves the quality of life Phase 2-2 \& 4 Survey}

\section{INFORMATION SHEET FOR PARTICIPANTS (RESIDENTS)}

Thank you for your interest in this project. Please read this information. If you decide to participate, thank you. If you decide not to take part, thank you for considering my request.

Who am I?

My name is Yukiko Kuboshima and I am a PhD student in School of Architecture at Victoria University of Wellington.

What is the aim of the project?

This project aims to design a desirable model of supported housing for older people. This research has been approved by the Victoria University of Wellington Human Ethics Committee [Approval number: 23243].

\section{How can you help?}

If you agree to take part,

- I will draw a plan of your private dwelling. It will take 1-2 hours.

- I will interview you about your sense of control, important activities, relationships, possessions, privacy and the desirable space for them. It will take about 1.5 hours and will be recorded.

- I will physically observe and take notes on your activities, the space for them and the time they take. This survey will be conducted from 9am to $6 \mathrm{pm}$ and will be audio-recorded.

- After I redesign housing, I will hold a focus groups or interview you for your feedback on my design. It will take 1.5 hours.

\section{What will happen to the information you give?}

This research is confidential. I will not name you in any reports, and I will not include any information that would identify you. Only my supervisors and I will access the collected data. I will be kept securely and destroyed 3 years after the research ends.

\section{What will the project produce?}


The information from my research will be used in my PhD dissertation. You will not be identified in my report. I may also use the results of my research for conference presentations, and academic reports. I will take care not to identify you in any presentation or report.

\section{If you accept this invitation, what are your rights as a research participant?}

You do not have to accept this invitation if you don't want to. If you do decide to participate, you have the right to:

- $\quad$ ask any questions about the study at any time;

- $\quad$ ask to stop the survey or withdraw from the survey at any time;

- $\quad$ withdraw from the survey up until two weeks after the survey;

- $\quad$ agree on another name for me to use rather than your real name; and

- be able to read the final report of this research by asking me at any time to request a copy.

\section{If you have any questions or problems, who can you contact?}

If you have any questions, either now or in the future, please feel free to contact either:

\section{Student:}

Name: Yukiko Kuboshima

Email:Yukiko.Kuboshima@vuw.ac.nz

\section{Supervisors:}

Name: Geoff Thomas

R o l e: Supervisor

School: School of Architecture

Phone: 044636247

Email: geoff.thomas@vuw.ac.nz

Name: Jacqueline Macintosh

$\mathrm{R}$ o I e: Supervisor

School: School of Architecture

Phone: 044636296

Email: jacqueline.mcintosh@vuw.ac.nz

\section{Human Ethics Committee information}

If you have any concerns about the ethical conduct of the research you may contact the Victoria University HEC Convener: Associate Professor Susan Corbett. Email susan.corbett@vuw.ac.nz or telephone +64-4-463 5480. 


\section{Designing supported housing which improves the quality of life Phase 2-2 \& 4 Survey \\ CONSENT TO SURVEY}

This consent form will be held for 5 years.

Researcher: Yukiko Kuboshima, School of Architecture, Victoria University of Wellington

- I have read the Information Sheet and the project has been explained to me.

- I agree to take part in the survey.

I understand that:

- I may withdraw from the survey up to two weeks after the survey, and any information that I have provided will be returned to me or destroyed.

- $\quad$ The information I have provided will be destroyed 3 years after the research is finished.

- $\quad$ Any information I provide will be kept confidential to the researcher and the supervisors. I understand that the results will be used for a PhD report and a summary of the results may be used in academic reports and/or presented at conferences.

- My name will not be used in reports, nor will any information that would identify me.

- I would like to receive a copy of the final report and have added my Yes $\square$ No address below.

Signature of participant:

Date:

Contact details:

Name: Title:

Address:

Phone number:

Email address: 


\title{
Designing supported housing which improves the quality of life Phase 2-3 \& 4 Survey
}

\author{
INFORMATION SHEET FOR PARTICIPANTS (CAREGIVERS)
}

Thank you for your interest in this project. Please read this information. If you decide to participate, thank you. If you decide not to take part, thank you for considering my request.

Who am I?

My name is Yukiko Kuboshima and I am a PhD student in School of Architecture at Victoria University of Wellington.

What is the aim of the project?

This project aims to design a desirable model of supported housing for older people. This Phase survey aims to clarify the spatial factors that contribute to staff's care for residents.

This research has been approved by the Victoria University of Wellington Human Ethics Committee [Approval number: 23243].

\section{How can you help?}

If you agree to take part,

- I will observe and take notes on your activities, their location and the time they take, for one partial day, while you are with the residents I am studying on.

- I will interview you about the care activities and the space for them. Interviews will take up to 1 hour and will be recorded.

- After I redesign housing, I will hold focus groups or interview you for your feedback on my design. It will take up to 1 hour.

\section{What will happen to the information you give?}

This research is confidential. I will not name you in any reports, and I will not include any information that would identify you. Only my supervisors and I will access the collected data. It will be kept securely and destroyed 3 years after the research ends.

\section{What will the project produce?}

The information from my research will be used in my PhD dissertation. You will not be identified in my report. I may also use the results of my research for conference presentations, and academic reports. I will take care not to identify you in any presentation or report. 


\section{If you accept this invitation, what are your rights as a research participant?}

You do not have to accept this invitation if you don't want to. If you do decide to participate, you have the right to:

- $\quad$ ask any questions about the study at any time;

- $\quad$ ask to stop the survey or withdraw from the study at any time;

- $\quad$ withdraw from the study up until two weeks after the survey;

- $\quad$ agree on another name for me to use rather than your real name; and

- be able to read the final report of this research by emailing me to request a copy.

If you have any questions or problems, who can you contact?

If you have any questions, either now or in the future, please feel free to contact either:

\section{Student:}

Name: Yukiko Kuboshima

Email:Yukiko.Kuboshima@vuw.ac.nz

\section{Supervisors:}

Name: Geoff Thomas

$\mathrm{R}$ o I e: Supervisor

School: School of Architecture

Phone: 044636247

Email: geoff.thomas@vuw.ac.nz

Name: Jacqueline Macintosh

$\mathrm{R}$ o I e: Supervisor

School: School of Architecture

Phone: 044636296

Email: jacqueline.mcintosh@vuw.ac.nz

\section{Human Ethics Committee information}

If you have any concerns about the ethical conduct of the research you may contact the Victoria University HEC Convener: Associate Professor Susan Corbett. Email susan.corbett@vuw.ac.nz or telephone +64-4-463 5480. 
Designing supported housing which improves the quality of life Phase 2-3 \& 4 Survey

\section{CONSENT TO SURVEY}

This consent form will be held for 5 years.

Researcher: Yukiko Kuboshima, School of Architecture, Victoria University of Wellington

- I have read the Information Sheet and I agree to take part in the survey.

I understand that:

- I may withdraw from this study up to two weeks after the survey, and any information that I have provided will be returned to me or destroyed.

- The information I have provided will be destroyed 3 years after the research is finished.

- $\quad$ Any information I provide will be kept confidential to the researcher and the supervisors. I understand that the results will be used for a PhD report and a summary of the results may be used in academic reports and/or presented at conferences.

- My name will not be used in reports, nor will any information that would identify me.

- I would like to receive a copy of the final report and have added my email Yes $\square$ No address below.

Signature of participant:

Name of participant:

Date:

Contact details: 


\section{Appendix 2: Data sheets in the Phase 2 survey}

- Example of coding data from semi-structured interviews

$\mathrm{I}=$ interviewer, $\mathrm{E}=$ Elderly person, $\mathrm{C}=$ Carer

\begin{tabular}{|c|c|c|c|c|}
\hline & Transcript & Explanatory Comments & Themes for Qol & Themes for Design \\
\hline I & $\begin{array}{l}\text { Okay, so you said you'd like the second bedroom, only that, yes? } \\
\text { What is your favorite space in this house? }\end{array}$ & & & \\
\hline $\mathbf{E}$ & $\begin{array}{l}\text { My favorite place, I suppose here because you can see out and } \\
\text { see what's going on and sometimes your visitors come in } \\
\text { backdoor too and I like to have some flowers. There aren't many } \\
\text { out at the moment but I like to see a bit of garden. }\end{array}$ & $\begin{array}{l}\text { Here = her armchair } \\
\text { With limited mobility, seeing outside } \\
\text { (what's going on) and seeing } \\
\text { flowers/ gardens are important } \\
\text { activities for her. She mentioned she } \\
\text { had spent a lot of time gardening }\end{array}$ & $\begin{array}{l}\text { Meaningful activity (seeing } \\
\text { activities outside, seeing } \\
\text { flowers/gardens) }\end{array}$ & $\begin{array}{l}\text { The layout of an } \\
\text { armchair with respect } \\
\text { to views to } \\
\text { outside/porch }\end{array}$ \\
\hline I & Where do you have meals? & & & \\
\hline $\mathbf{E}$ & have two meals here at the table. & \multirow{3}{*}{$\begin{array}{l}\text { The resident put a dining table in a } \\
\text { confined space. }\end{array}$} & \multirow[t]{3}{*}{ Control in dining } & \multirow[t]{3}{*}{ Space for dining table } \\
\hline 1 & At the table, here? & & & \\
\hline $\mathbf{E}$ & Yeah, that's breakfast and the evening meal. & & & \\
\hline $\mathbf{I}$ & I see, do you have any space that you don't like in this house? & & & \\
\hline E & No. & & & \\
\hline I & No? & & & \\
\hline $\mathbf{E}$ & I realize I'm lucky. A lot of people don't have anything like this. & & & \\
\hline I & $\begin{array}{l}\text { All right. Can you tell me your most important things, your } \\
\text { belongings, in this house? }\end{array}$ & & & \\
\hline $\mathbf{E}$ & Say a view out there. & $\begin{array}{l}\text { Views from her sitting space is most } \\
\text { important for her }\end{array}$ & $\begin{array}{l}\text { Meaningful activity (seeing } \\
\text { activities outside) }\end{array}$ & $\begin{array}{l}\text { The layout of an } \\
\text { armchair with respect } \\
\text { to views to } \\
\text { outside/porch }\end{array}$ \\
\hline I & ... Do you have any concern in privacy here? & & & \\
\hline $\mathbf{E}$ & In privacy, no. & & & \\
\hline I & No? & & & \\
\hline $\mathbf{E}$ & $\begin{array}{l}\text { Because I can shut these doors off. I wouldn't like to have just } \\
\text { the one room which if I shifted, it would be to a something } \\
\text { smaller. }\end{array}$ & \multirow{5}{*}{$\begin{array}{l}\text { She is anticipating the need to move } \\
\text { to a rest home, but wouldn't like a } \\
\text { rest home room, because she } \\
\text { wouldn't have a choice to shut the } \\
\text { door and because of the smaller } \\
\text { space. }\end{array}$} & \multirow[t]{5}{*}{$\begin{array}{l}\text { Privacy (the ability/choice } \\
\text { to shut the door) }\end{array}$} & \multirow[t]{5}{*}{ Door } \\
\hline I & You would have a concern? & & & \\
\hline $\mathbf{E}$ & I wouldn't have a choice there. & & & \\
\hline 1 & Okay, I see. The choice to shut the door? & & & \\
\hline E & Yeah. & & & \\
\hline
\end{tabular}

- Example of coding data from observation

\begin{tabular}{|c|c|c|c|c|c|c|c|}
\hline Time & Activities & Location & Posture & Resident remarks & Explanatory comments & Themes for QoL & Themes for design \\
\hline 34 & $\begin{array}{l}\text { Talk } \\
\text { Show me Msn news on PC }\end{array}$ & $\begin{array}{l}\text { Lounge, } \\
\text { Armchair }\end{array}$ & $\begin{array}{l}\text { Sit on } \\
\text { armchair }\end{array}$ & $\begin{array}{l}\text { 'It may be boring for you, but I'm } \\
\text { not bored' (I watch TV and PC for } \\
\text { news and games) } \\
\text { 'Id like to be occupied, you }\end{array}$ & $\begin{array}{l}\text { Being occupied is } \\
\text { important for the resident: }\end{array}$ & $\begin{array}{lr}\begin{array}{l}\text { Meaningful } \\
\text { activities }\end{array} \quad \begin{array}{l}\text { leisure } \\
\text { (doing }\end{array} \\
\text { multipler activities } \\
\text { (watching TV and PC)) }\end{array}$ & $\begin{array}{l}\text { The layout of armchair } \\
\text { with respect to TV and } \\
\text { PC screen }\end{array}$ \\
\hline 9.40 & $\begin{array}{l}\text { Show me LPs, and books on } \\
\text { Diana }\end{array}$ & $\begin{array}{l}\text { Bedroom } \\
\text { closet } \\
\text { (small) }\end{array}$ & Walk & $\begin{array}{l}\text { Referring to the LP player on the } \\
\text { TV shelf, In Christmas time, } \\
\text { isten to the music (Only } \\
\text { Christmas time, by himself? }\end{array}$ & $\begin{array}{l}\text { The resident has had a LP } \\
\text { player since he moved to } \\
\text { here. }\end{array}$ & $\begin{array}{l}\text { Meaningful leisure } \\
\text { activity (listening to the } \\
\text { music) }\end{array}$ & \\
\hline 42 & Talk about LP player & $\begin{array}{l}\text { Lounge, By } \\
\text { TV }\end{array}$ & Stand & & & & \\
\hline 45 & Sit down & $\begin{array}{l}\text { Lounge, } \\
\text { Armchair }\end{array}$ & $\begin{array}{l}\text { Sit on } \\
\text { armchair }\end{array}$ & & & & \\
\hline 50 & $\begin{array}{l}\text { Stand up and see outside by the } \\
\text { door, talk about activities } \\
\text { outside }\end{array}$ & $\begin{array}{l}\text { Lounge, by } \\
\text { door }\end{array}$ & Stand & & $\begin{array}{l}\text { Seeing activities outside is } \\
\text { important }\end{array}$ & $\begin{array}{lr}\text { Meaningful leisure } \\
\text { activity } & \text { (seeing } \\
\text { activities outside) }\end{array}$ & $\begin{array}{l}\text { The layout of Window } \\
\text { with respect to } \\
\text { activities outside }\end{array}$ \\
\hline 55 & $\begin{array}{l}\text { Beat and kill a cockroach, open } \\
\text { the door and throw it away }\end{array}$ & $\begin{array}{l}\text { Lounge, by } \\
\text { door }\end{array}$ & Stand & & & $\begin{array}{l}\text { Control in maintenance } \\
\text { of house }\end{array}$ & \\
\hline 56 & Sit down & $\begin{array}{l}\text { Lounge, } \\
\text { Armchair }\end{array}$ & $\begin{array}{l}\text { Sit on } \\
\text { armchair }\end{array}$ & & & & \\
\hline \multirow[t]{3}{*}{57} & Talk & & $\begin{array}{l}\text { Sit on } \\
\text { armchair }\end{array}$ & $\begin{array}{l}\text { ('Do you dose the curtain at } \\
\text { night?' } \\
\text { Yes, I don't in summer but do in } \\
\text { winter... it keeps the heat. It's } \\
\text { amazing...' }\end{array}$ & & Comfort (warmth) & Curtain \\
\hline & Look outside & $\begin{array}{l}\text { Lounge, } \\
\text { Armchair }\end{array}$ & $\begin{array}{l}\text { Sit on } \\
\text { armchair }\end{array}$ & Where has the car come from? & $\begin{array}{l}\text { Seeing activities outside is } \\
\text { important }\end{array}$ & $\begin{array}{lr}\text { Meaningful } & \text { leisure } \\
\text { activity } & \text { (seeing } \\
\text { activities outside) }\end{array}$ & $\begin{array}{l}\text { The layout of an } \\
\text { armchair with respect } \\
\text { to views to outside }\end{array}$ \\
\hline & Look at the laptop & $\begin{array}{l}\text { Lounge, } \\
\text { Armchair }\end{array}$ & $\begin{array}{l}\text { Sit on } \\
\text { armchair }\end{array}$ & Yesterday I won $\$ 10$ by Bingo.' & $\begin{array}{l}\text { Engagement with PC } \\
\text { games }\end{array}$ & $\begin{array}{l}\text { Meaningful leisure } \\
\text { activity (PC games) }\end{array}$ & $\begin{array}{l}\text { The layout of an } \\
\text { armchair with respect }\end{array}$ \\
\hline 10.07 & Play Slots (PC games) & $\begin{array}{l}\text { Lounge, } \\
\text { Armchair }\end{array}$ & $\begin{array}{l}\text { Sit on } \\
\text { armchair }\end{array}$ & $\begin{array}{l}\text { 'One thing... is that you can } \\
\text { practice without any money' }\end{array}$ & & & to the laptop \\
\hline 17 & $\begin{array}{l}\text { Stand up slowly, go to the } \\
\text { kitchen. Wipe cups }\end{array}$ & Kitchen & Stand & & Go to make a tea & Control in cooking & \\
\hline
\end{tabular}


- Example of plan drawings: sketch (above), digitised data (below)

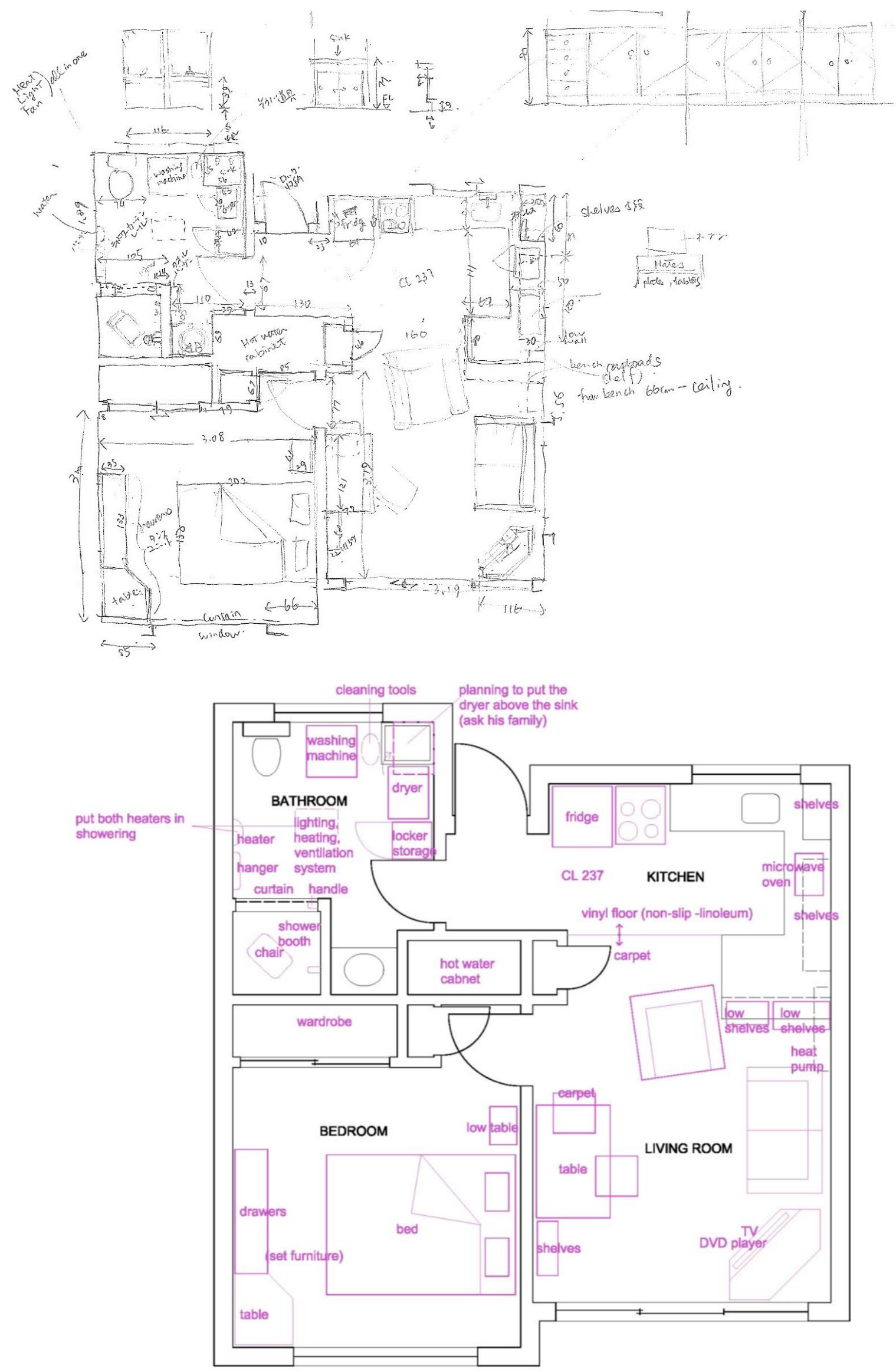




\section{Appendix 3: Individual preferences in activities and relationships}

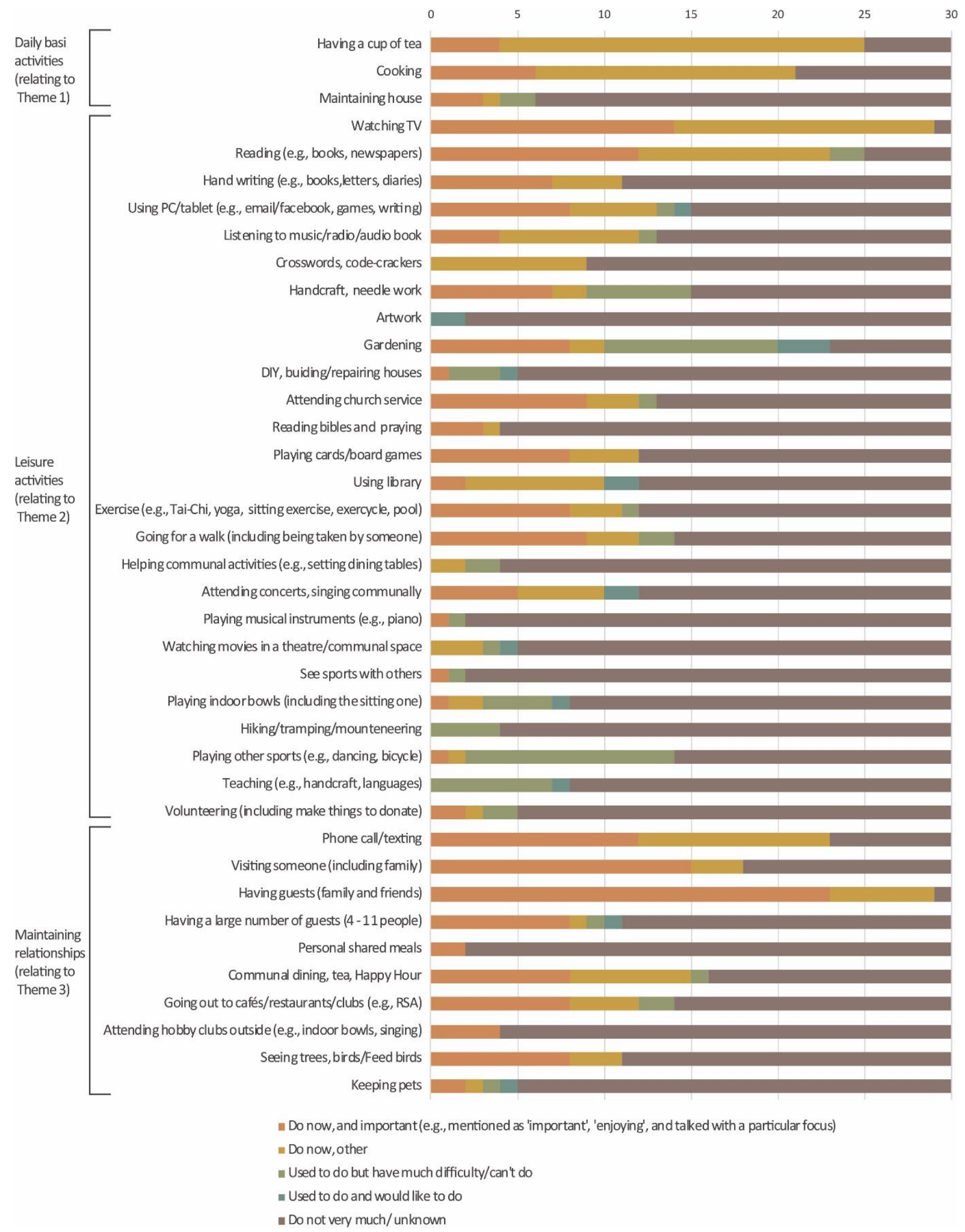




\section{Appendix 4: Analysis of relationships between QoL themes, design considerations and design elements}

\begin{tabular}{|c|c|c|c|}
\hline $\begin{array}{l}\text { Theme for } \\
\text { QoL }\end{array}$ & Sub-themes & Pesign considerations & Design elements \\
\hline \multirow{29}{*}{$\begin{array}{l}1-1 \\
\text { Control in } \\
\text { basic } \\
\text { movements }\end{array}$} & \multirow{21}{*}{ Moving/walking } & $\begin{array}{l}\text { Avoid level differences (e.g., step, a tiny level } \\
\text { difference) }\end{array}$ & Floor \\
\hline & & Avoid thresholds creating a level difference & Floor \\
\hline & & $\begin{array}{l}\text { Strategies required for water-tightness in level } \\
\text { entry (e.g., use of gutters) }\end{array}$ & Floor \\
\hline & & Avoid too steep and long slopes & Floor \\
\hline & & Avoid unstable ground (grass lawn etc.) & Floor \\
\hline & & Use floor materials with great grip & Floor \\
\hline & & $\begin{array}{l}\text { Choice of floor colours that do not show dirt by } \\
\text { wheels }\end{array}$ & Floor \\
\hline & & Firm fixturing of carpets to substrate materials & Floor \\
\hline & & $\begin{array}{l}\text { Ensuring firmness of walls, fixtures and furniture } \\
\text { (heavy chairs sofas) for use as things to hold } \\
\text { onto }\end{array}$ & $\begin{array}{l}\text { Things to hold onto, wall and } \\
\text { elevation design, fixtures }\end{array}$ \\
\hline & & Continuity of things to hold onto & Things to hold onto \\
\hline & & $\begin{array}{l}\text { Avoid unnecessary doors (e.g., between kitchen } \\
\text { and lounge, between the bathroom and } \\
\text { washroom) }\end{array}$ & Door \\
\hline & & Avoid hinged doors & Door \\
\hline & & $\begin{array}{l}\text { Sufficient space in front of doors for walker- } \\
\text { frame users }\end{array}$ & $\begin{array}{l}\text { Door, space for mobility aids, } \\
\text { unit size }\end{array}$ \\
\hline & & $\begin{array}{l}\text { Appropriate height of door handles for walker- } \\
\text { frame users }\end{array}$ & $\begin{array}{l}\text { Door, wall and elevation } \\
\text { design }\end{array}$ \\
\hline & & Type of door hook (e.g., magnet hook) & Door \\
\hline & & Sufficient passage width for wheelchair users & Passage, unit size \\
\hline & & $\begin{array}{l}\text { Sufficient space for turning around with a walker } \\
\text { frame }\end{array}$ & Space for mobility aids \\
\hline & & $\begin{array}{l}\text { Layout of spaces to avoid turning around in small } \\
\text { areas }\end{array}$ & $\begin{array}{l}\text { Planar relationships between } \\
\text { spaces }\end{array}$ \\
\hline & & Avoid wall corners & Passage \\
\hline & & $\begin{array}{l}\text { Providing accessibility in the storage for mobility } \\
\text { aids }\end{array}$ & $\begin{array}{l}\text { Storage, space for mobility } \\
\text { aids }\end{array}$ \\
\hline & & Space for a mobility scooter to turn around & $\begin{array}{l}\text { Storage, space for mobility } \\
\text { aids }\end{array}$ \\
\hline & \multirow{5}{*}{$\begin{array}{l}\text { Sitting down, crouching/bending } \\
\text { and getting up }\end{array}$} & Avoid too low seats (e.g., $400 \mathrm{~mm}$ high) & $\begin{array}{l}\text { Things to sit on, wall and } \\
\text { levation design }\end{array}$ \\
\hline & & Choice of furnishing with armrests & $\begin{array}{l}\text { Things to sit on, things to } \\
\text { hold onto }\end{array}$ \\
\hline & & $\begin{array}{l}\text { Height of electrical outlets and shelves that are } \\
\text { within reach }\end{array}$ & $\begin{array}{l}\text { Fixtures, storage, wall and } \\
\text { elevation design }\end{array}$ \\
\hline & & $\begin{array}{l}\text { Provision of storage for long items (e.g., picker } \\
\text { uppers) within reach }\end{array}$ & Storage \\
\hline & & Firm level surfaces proximity to sitting space & Things to hold onto \\
\hline & \multirow{3}{*}{ Arm/hand movements } & $\begin{array}{l}\text { Height of shelves and cupboards that are within } \\
\text { reach }\end{array}$ & $\begin{array}{l}\text { Wall and elevation design, } \\
\text { storage }\end{array}$ \\
\hline & & $\begin{array}{l}\text { Design of drawer handles for those with limited } \\
\text { hand strength }\end{array}$ & Storage \\
\hline & & $\begin{array}{l}\text { Choice of tap handle types for those with limited } \\
\text { hand strength }\end{array}$ & Fixtures \\
\hline \multirow{2}{*}{\multicolumn{2}{|c|}{$\begin{array}{l}1-2 \\
\text { Control in going to the toilet }\end{array}$}} & Proximity of sitting space and the toilet & $\begin{array}{l}\text { Planar relationships between } \\
\text { spaces, sitting space, } \\
\text { bathroom/toilet }\end{array}$ \\
\hline & & Proximity of the bedroom and the toilet & $\begin{array}{l}\text { Planar relationships between } \\
\text { spaces, bedroom, } \\
\text { bathroom/toilet }\end{array}$ \\
\hline
\end{tabular}




\begin{tabular}{|c|c|c|c|}
\hline & & $\begin{array}{l}\text { Route to/from the toilet not requiring to change } \\
\text { directions }\end{array}$ & Passage, bathroom/toilet \\
\hline & & Layout of toilets in communal space & $\begin{array}{l}\text { Bathroom/toilet, indoor } \\
\text { communal areass }\end{array}$ \\
\hline & & $\begin{array}{l}\text { Avoid level differences at the entry of shower } \\
\text { space }\end{array}$ & Bathroom/toilet, floor \\
\hline & & $\begin{array}{l}\text { Gradient of slope that does not cause users fears } \\
\text { for safety }\end{array}$ & Bathroom/toilet, floor \\
\hline & & yype and location of drain for rapid drainage & Bathroom/toilet, floor \\
\hline & & Type of shower enclosure that limits water flow & Bathroom/toilet, fixture \\
\hline & & $\begin{array}{l}\text { Fixtures to support residents in moving, sitting } \\
\text { down and standing up (e.g., grab bars, handrails) }\end{array}$ & $\begin{array}{l}\text { Bathroom/toilet, things to } \\
\text { hold onto, fixtures }\end{array}$ \\
\hline & & $\begin{array}{l}\text { Location of an emergency alarm within reach } \\
\text { following a fall }\end{array}$ & $\begin{array}{l}\text { Bathroom/toilet, fixtures, } \\
\text { wall and elevation design }\end{array}$ \\
\hline $\begin{array}{l}1-3 \\
\text { Control in sh }\end{array}$ & lowering & $\begin{array}{l}\text { Appropriate location of a shower mixer lever ano } \\
\text { shower hose }\end{array}$ & Bathroom/toilet, fixtures \\
\hline & & $\begin{array}{l}\text { Appropriate height of the vanity counter for } \\
\text { wheelchair users }\end{array}$ & Bathroom/toilet, fixtures \\
\hline & & Appropriate size and shape of rooms & $\begin{array}{l}\text { Bathroom/toilet, things to } \\
\text { hold onto, unit size }\end{array}$ \\
\hline & & $\begin{array}{l}\text { Installation of a folding chair (may not be } \\
\text { required) }\end{array}$ & $\begin{array}{l}\text { Bathroom/toilet, things to sit } \\
\text { on }\end{array}$ \\
\hline & & $\begin{array}{l}\text { Appropriate space for chairs (washing area and } \\
\text { drying area) }\end{array}$ & $\begin{array}{l}\text { Bathroom/toilet, things to sit } \\
\text { on }\end{array}$ \\
\hline & & $\begin{array}{l}\text { Space for a dressing table adjacent to the } \\
\text { bathroom (typically for female residents) }\end{array}$ & $\begin{array}{l}\text { wall and elevation design, } \\
\text { bedroom }\end{array}$ \\
\hline & & Height of space for a microwave oven & $\begin{array}{l}\text { Kitchen/dining space, wall } \\
\text { and elevation design }\end{array}$ \\
\hline & & Installation of hot plates & $\begin{array}{l}\text { Kitchen/dining space, } \\
\text { fixtures }\end{array}$ \\
\hline & & $\begin{array}{l}\text { Installation of mini oven, normal oven, double } \\
\text { bven }\end{array}$ & $\begin{array}{l}\text { Kitchen/dining space, } \\
\text { fixtures }\end{array}$ \\
\hline & & $\begin{array}{l}\text { low cooker/rice cooker: on the bench, } \\
\text { proximity to the sink and storage for ingredients } \\
\text { (e.g., rice) }\end{array}$ & Kitchen/dining space \\
\hline & & $\begin{array}{l}\text { Toaster, sandwich maker: proximity to } \\
\text { fridge/freezer (toast, spread, cheese etc.) and } \\
\text { bther storage for ingredients }\end{array}$ & Kitchen/dining space \\
\hline & & $\begin{array}{l}\text { Proximity to the electric kettle, sink/tap, storage } \\
\text { (cup, tea bags, coffee, spoon), fridge (milk) }\end{array}$ & Kitchen/dining space \\
\hline & Prenaring meals & $\begin{array}{l}\text { Fixtures improving safety in pouring boiled water } \\
\text { (e.g., table/bench height, boiling tap) }\end{array}$ & $\begin{array}{l}\text { Kitchen/dining space, } \\
\text { fixtures, wall and elevation } \\
\text { design }\end{array}$ \\
\hline $\begin{array}{l}\text { Control in } \\
\text { cooking and }\end{array}$ & & $\begin{array}{l}\text { Proximity to space for the special cutting board } \\
\text { and knives }\end{array}$ & Kitchen/dining space \\
\hline dining & & $\begin{array}{l}\text { Bench/table height for cooking while seated } \\
\text { (including cutting) }\end{array}$ & $\begin{array}{l}\text { Kitchen/dining space, } \\
\text { fixtures, wall and elevation } \\
\text { design }\end{array}$ \\
\hline & & $\begin{array}{l}\text { Bench/table depth for performing tasks and } \\
\text { storing tools and ingredients }\end{array}$ & $\begin{array}{l}\text { Kitchen/dining space, } \\
\text { fixtures, storage }\end{array}$ \\
\hline & & Continuity of bench/table & $\begin{array}{l}\text { Kitchen/dining space, } \\
\text { fixtures }\end{array}$ \\
\hline & & Shelves within reach from sitting space & $\begin{array}{l}\text { Kitchen/dining space, } \\
\text { storage, planar relationships } \\
\text { between spaces }\end{array}$ \\
\hline & & Proximity to fridge, cupboards & $\begin{array}{l}\text { Kitchen/dining space, } \\
\text { storage, planar relationships } \\
\text { between spaces }\end{array}$ \\
\hline & & $\begin{array}{l}\text { Chair type (e.g., swivel chair hel ps changing the } \\
\text { direction and moving between spaces) }\end{array}$ & $\begin{array}{l}\text { Kitchen/dining space, things } \\
\text { to sit on }\end{array}$ \\
\hline & Corning morle & Kitchen passage width & Space for mobility aids \\
\hline & Carrying meals & Threshold between different materials & Floor \\
\hline
\end{tabular}




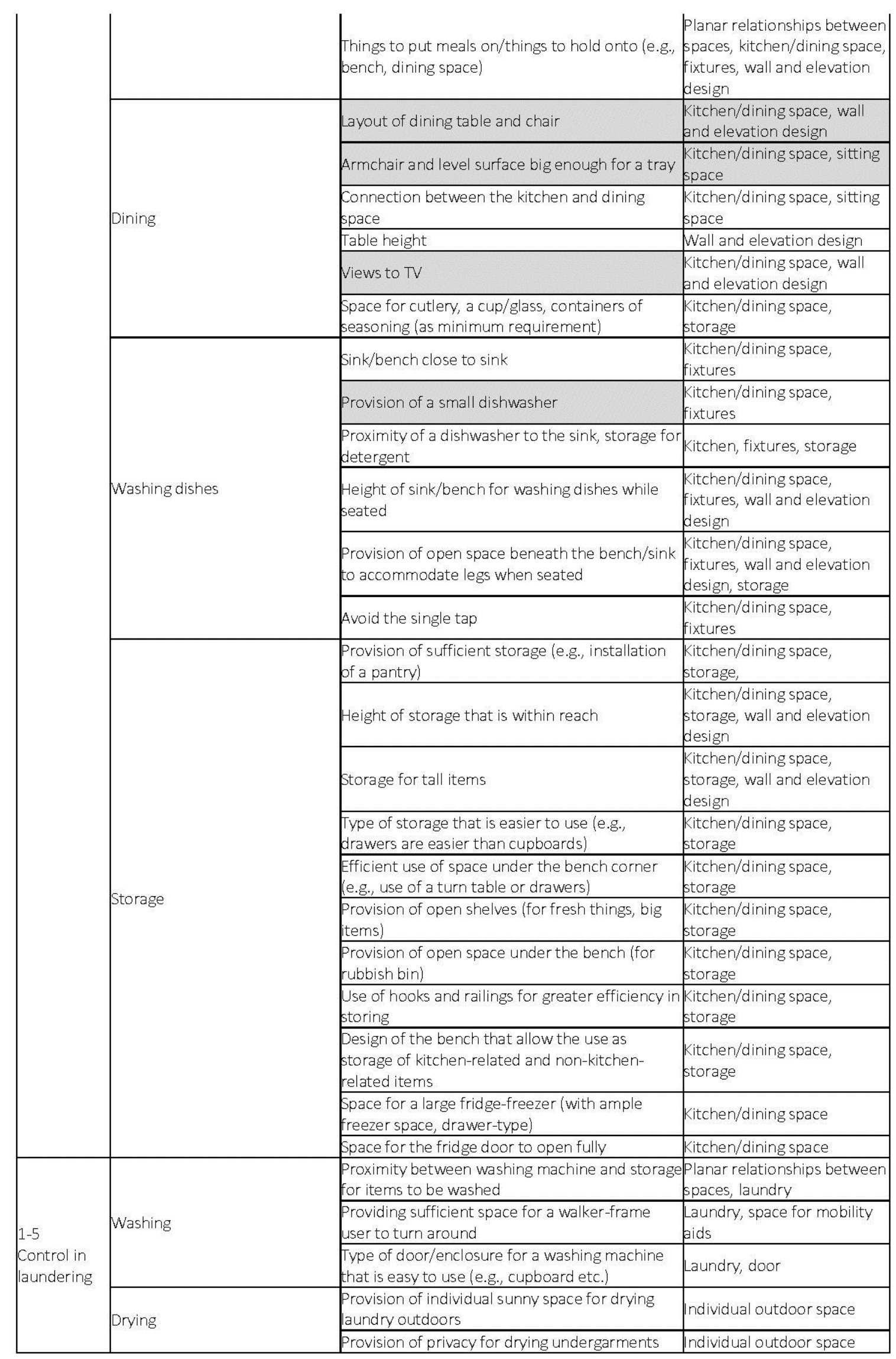




\begin{tabular}{|c|c|c|c|}
\hline & & $\begin{array}{l}\text { Proximity between clotheslines and the washing } \\
\text { machine }\end{array}$ & $\begin{array}{l}\text { Planar relationships between } \\
\text { spaces, laundry, Individual } \\
\text { outdoor space }\end{array}$ \\
\hline & & $\begin{array}{l}\text { Height of clotheslines that is within reach (for a } \\
\text { seated person) }\end{array}$ & $\begin{array}{l}\text { Individual outdoor space, } \\
\text { wall and elevation design }\end{array}$ \\
\hline & & $\begin{array}{l}\text { Provision of appropriate space for drying } \\
\text { indoors, such as sufficient space for a laundry } \\
\text { rack and space for hanging ropes (e.g., in a } \\
\text { garage, bedroom, spare room) }\end{array}$ & Extra rooms, unit size \\
\hline & & Installation of rail heater in the bathroom & Bathroom/toilet, fixtures \\
\hline & & Height of the clothes dryer within reach & $\begin{array}{l}\text { Laundry, wall and elevation } \\
\text { design }\end{array}$ \\
\hline & & $\begin{array}{l}\text { Provision of space for a clothes dryer (in } \\
\text { individual houses or in communal space) }\end{array}$ & $\begin{array}{l}\text { Laundry, indoor communal } \\
\text { areas }\end{array}$ \\
\hline \multirow{7}{*}{$\begin{array}{l}1-6 \\
\text { Control in } \\
\text { maintaining } \\
\text { the home }\end{array}$} & \multirow{2}{*}{$\begin{array}{l}\text { Ability to maintain the whole } \\
\text { house }\end{array}$} & Unit size that is easy to maintain & unit size \\
\hline & & Garden size that is easy to maintain & Individual outdoor space \\
\hline & \multirow{3}{*}{$\begin{array}{l}\text { Keeping various elements of the } \\
\text { house clean }\end{array}$} & Choice of floor materials that are easy to clean & Floor \\
\hline & & Choice of floor colours that don't show stains & Floor \\
\hline & & $\begin{array}{l}\text { Design of bumps for slip resistance and ease of } \\
\text { cleaning }\end{array}$ & Bathroom/toilet, floor \\
\hline & \multirow[t]{2}{*}{ Taking rubbish out } & $\begin{array}{l}\text { Provision of accessibility for the communal } \\
\text { rubbish/recycling point (e.g., Avoid level } \\
\text { differences, avoid a hinged door) }\end{array}$ & $\begin{array}{l}\text { Communal pathways, } \\
\text { communal space }\end{array}$ \\
\hline & & $\begin{array}{l}\text { Provision of temporary storage for } \\
\text { rubbish/recycling (e.g., under bench, garage) }\end{array}$ & $\begin{array}{l}\text { Storage, kitchen/dining } \\
\text { space, extra rooms }\end{array}$ \\
\hline \multirow{3}{*}{$\begin{array}{l}1-7 \\
\text { Control in } \\
\text { shopping }\end{array}$} & \multirow[t]{2}{*}{ Carrying shopping items } & Proximity between the garage and the kitchen & $\begin{array}{l}\text { Planar relationships between } \\
\text { spaces, kitchen/dining space, } \\
\text { extra rooms }\end{array}$ \\
\hline & & $\begin{array}{l}\text { Sufficient garage width for a walker frame when } \\
\text { moving beside the car }\end{array}$ & $\begin{array}{l}\text { Extra rooms, unit size, space } \\
\text { for mobility aids }\end{array}$ \\
\hline & Pn-site shop & Provision of on-site shops (including a cafe) & indoor communal areas \\
\hline \multirow{3}{*}{\multicolumn{2}{|c|}{$\begin{array}{l}1-8 \\
\text { Control in collecting mail }\end{array}$}} & $\begin{array}{l}\text { Sufficient space in front of the letterbox for } \\
\text { those with mobility aids }\end{array}$ & Individual outdoor space \\
\hline & & Height of a letterbox that is within reach & Individual outdoor space \\
\hline & & $\begin{array}{l}\text { Provision of accessibility for the letterbox (e.g., } \\
\text { avoid level differences, avoid hinged door) }\end{array}$ & Communal pathways \\
\hline \multirow{13}{*}{$\begin{array}{l}2-1 \\
\text { Continuing } \\
\text { familiar } \\
\text { activities }\end{array}$} & \multirow{4}{*}{ Religious activities } & $\begin{array}{l}\text { Ease of access to the church in the community } \\
\text { (proximity, accessibility }\end{array}$ & $\begin{array}{l}\text { Communal pathways, floor, } \\
\text { door }\end{array}$ \\
\hline & & $\begin{array}{l}\text { Use of communal space for church-related } \\
\text { activities (e.g., communion, bible study) }\end{array}$ & indoor communal areas \\
\hline & & $\begin{array}{l}\text { Layout of communal space in proximity from } \\
\text { individual units }\end{array}$ & indoor communal areas \\
\hline & & $\begin{array}{l}\text { Ease of access to communal space (e.g., } \\
\text { accessibility) }\end{array}$ & $\begin{array}{l}\text { Communal pathways, floor, } \\
\text { door }\end{array}$ \\
\hline & \multirow{2}{*}{ Handcrafts/Needlework/Artwork } & $\begin{array}{l}\text { Space for desks for specific uses (table for } \\
\text { heedlework and storage for tools (drawers)) }\end{array}$ & wall and elevation design \\
\hline & & Special room for art activities in communal area & indoor communal areas \\
\hline & \multirow{3}{*}{ Gardening } & $\begin{array}{l}\text { Size and type of gardens suitable for their ability } \\
\text { (e.g., garden beds/pots, several sections/only in } \\
\text { the porch) }\end{array}$ & Individual outdoor space \\
\hline & & Height of beds/space for pots & $\begin{array}{l}\text { Individual outdoor space, } \\
\text { wall and elevation design }\end{array}$ \\
\hline & & $\begin{array}{l}\text { Proximity of storage for gardening tools to } \\
\text { gardens }\end{array}$ & $\begin{array}{l}\text { Individual outdoor space, } \\
\text { storage }\end{array}$ \\
\hline & \multirow{4}{*}{ Reading } & $\begin{array}{l}\text { Layout of an armchair with respect to the north- } \\
\text { facing window }\end{array}$ & Sitting space \\
\hline & & Provision of light & Sitting space \\
\hline & & Adjacency to (book) shelves & $\begin{array}{l}\text { Sitting space, wall and } \\
\text { elevation design }\end{array}$ \\
\hline & & Provision of bookshelves & indoor communal areas \\
\hline
\end{tabular}




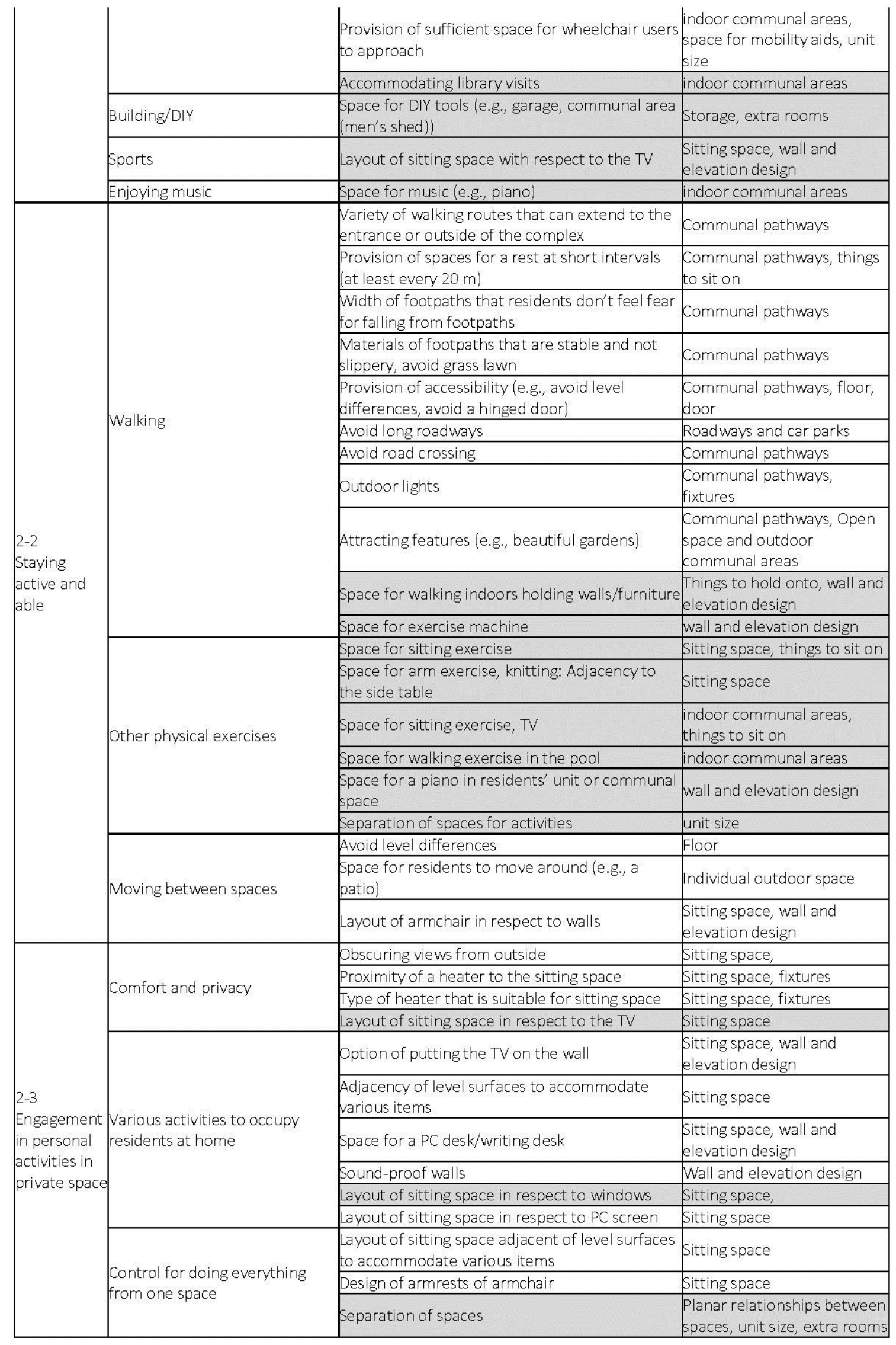




\begin{tabular}{|c|c|c|c|}
\hline & $\begin{array}{l}\text { Using different spaces for } \\
\text { different activities }\end{array}$ & indoor communal areas & \multirow[b]{2}{*}{ Storage, unit size } \\
\hline \multicolumn{2}{|c|}{$\begin{array}{l}2-4 \\
\text { Contribution }\end{array}$} & $\begin{array}{l}\text { Space for helping staff, teaching } \\
\text { handcraft/needlework }\end{array}$ & \\
\hline \multirow{4}{*}{\multicolumn{2}{|c|}{$\begin{array}{l}3-1 \\
\text { Living with spouse }\end{array}$}} & Sufficient ward robe space for two & Extra rooms \\
\hline & & $\begin{array}{l}\text { Provision of separate space for greater privacy } \\
\text { among a couple }\end{array}$ & Door \\
\hline & & $\begin{array}{l}\text { Provision of a partition that can be kept both } \\
\text { open and closed }\end{array}$ & Door \\
\hline & & $\begin{array}{l}\text { Layout of sitting space in respect to windows for } \\
\text { the views to visitors coming }\end{array}$ & Sitting space \\
\hline \multirow{14}{*}{$\begin{array}{l}3-2 \\
\text { Having } \\
\text { personal } \\
\text { guests }\end{array}$} & \multirow{3}{*}{ Welcoming guests } & Layout of sitting space in proximity to the door & $\begin{array}{l}\text { Sitting space, planar } \\
\text { relationships between } \\
\text { spaces, door }\end{array}$ \\
\hline & & Provision of individual external doors & Door \\
\hline & & Provision of space for extra chairs & $\begin{array}{l}\text { Storage, wall and elevation } \\
\text { design, indoor communal } \\
\text { areas }\end{array}$ \\
\hline & \multirow{8}{*}{ Entertaining guests } & Dining table (including folding table) & Kitchen/dining space \\
\hline & & Café & indoor communal areas \\
\hline & & Separation of the lounge and the bedroom & $\begin{array}{l}\text { Planar relationships between } \\
\text { spaces, unit size }\end{array}$ \\
\hline & & $\begin{array}{l}\text { Provision of extendable space from inside to } \\
\text { putside (e.g., indoor/outdoor, } \\
\text { private/communal) }\end{array}$ & $\begin{array}{l}\text { Planar relationships between } \\
\text { spaces, indoor communal } \\
\text { areas, unit size }\end{array}$ \\
\hline & & $\begin{array}{l}\text { Separation of space for guests from other space } \\
\text { in communal space }\end{array}$ & indoor communal areas \\
\hline & & Views to children & $\begin{array}{l}\text { Sitting space, extra rooms, } \\
\text { open space and outdoor } \\
\text { communal areas, }\end{array}$ \\
\hline & & $\begin{array}{l}\text { Provision of separate space for children from } \\
\text { residents' space indoors or outdoors }\end{array}$ & $\begin{array}{l}\text { Extra rooms, open space and } \\
\text { butdoor communal areas }\end{array}$ \\
\hline & & Choice of having a guest room & Extra rooms, unit size \\
\hline & \multirow{3}{*}{ Having overnight guests } & $\begin{array}{l}\text { Design for greater flexibility in use (e.g., } \\
\text { insulation of garage) }\end{array}$ & Extra rooms \\
\hline & & $\begin{array}{l}\text { Space for an extra bed or sofa bed for guests } \\
\text { (e.g., in the lounge) }\end{array}$ & Sitting space \\
\hline & & $\begin{array}{l}\text { Accommodating not too many units (less than } \\
200 \text { units) }\end{array}$ & Complex size \\
\hline \multirow{12}{*}{$\begin{array}{l}3-3 \\
\text { Relationships } \\
\text { with other } \\
\text { residents }\end{array}$} & \multirow{2}{*}{$\begin{array}{l}\text { Familiarity and sense of } \\
\text { homeliness }\end{array}$} & $\begin{array}{l}\text { Size of communal space that can accommodate } \\
\text { all residents }\end{array}$ & indoor communal areas \\
\hline & & Provision of space for organised activities & indoor communal areas \\
\hline & \multirow{9}{*}{ Socialising in organized activities } & $\begin{array}{l}\text { Provision of space for having meals in their own } \\
\text { unit (even where meals are served in communal } \\
\text { dining room) }\end{array}$ & $\begin{array}{l}\text { Kitchen/dining space, unit } \\
\text { size }\end{array}$ \\
\hline & & $\begin{array}{l}\text { Proximity between the unit and indoor } \\
\text { communal areas }\end{array}$ & indoor communal areas \\
\hline & & $\begin{array}{l}\text { Provision of spaces for a rest on the way to } \\
\text { communal space }\end{array}$ & $\begin{array}{l}\text { Communal pathways, things } \\
\text { to sit on }\end{array}$ \\
\hline & & Door weight & Door \\
\hline & & $\begin{array}{l}\text { Layout of sitting space in respect to the views to } \\
\text { butside }\end{array}$ & Sitting space, \\
\hline & & Space for residents to talk near resident units & Individual outdoor space \\
\hline & & $\begin{array}{l}\text { Provision of small communal spaces proximity to } \\
\text { resident units }\end{array}$ & indoor communal areas \\
\hline & & $\begin{array}{l}\text { Provision of space for activities attracting other } \\
\text { residents (e.g., playing musical instruments) }\end{array}$ & $\begin{array}{l}\text { indoor communal areas, } \\
\text { Individual outdoor space }\end{array}$ \\
\hline & & $\begin{array}{l}\text { Distance and angle between units for great } \\
\text { resident privacy }\end{array}$ & Unit layout \\
\hline & Privacy and connection & $\begin{array}{l}\text { Distance between windows for sense of } \\
\text { connection }\end{array}$ & Unit layout \\
\hline
\end{tabular}




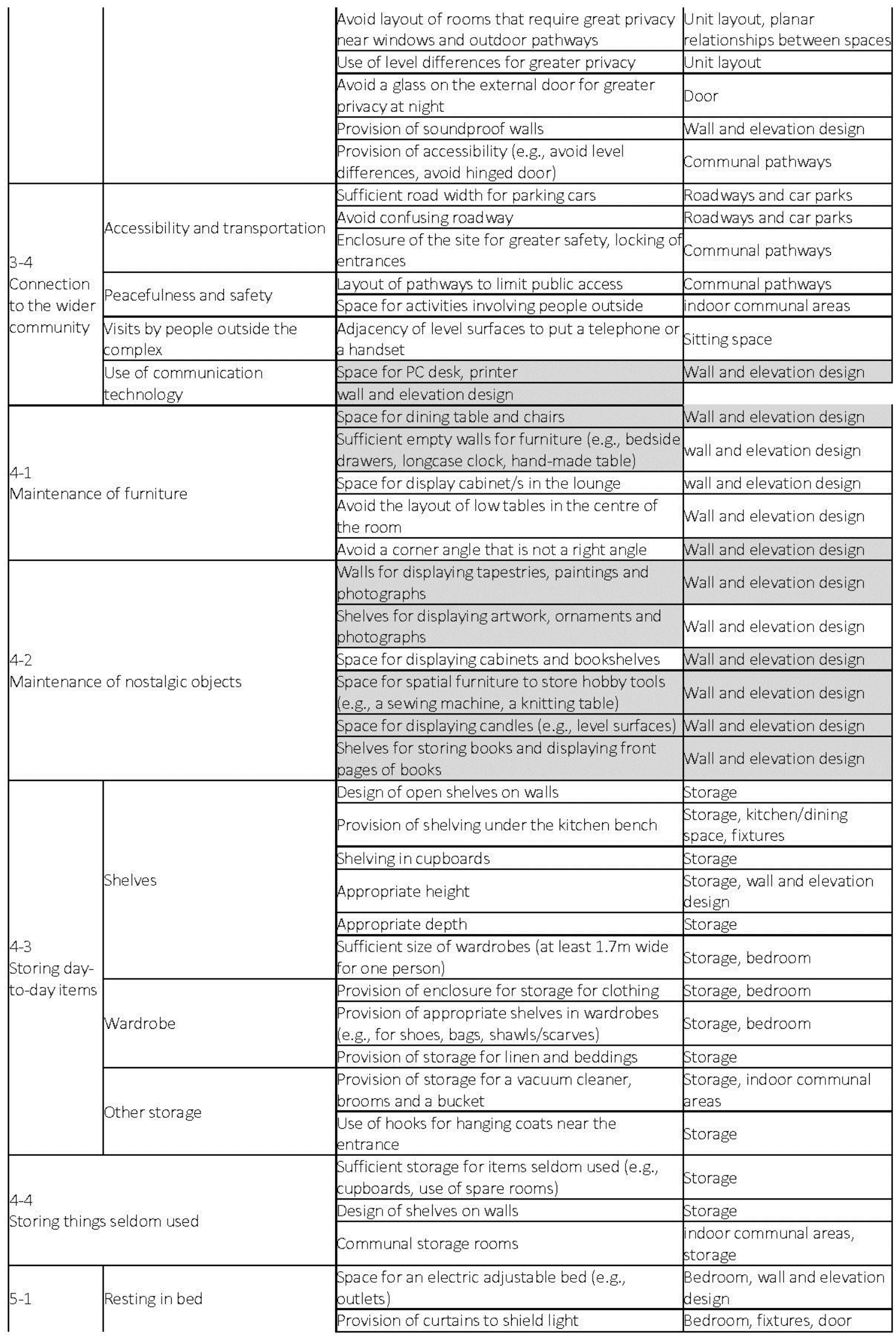




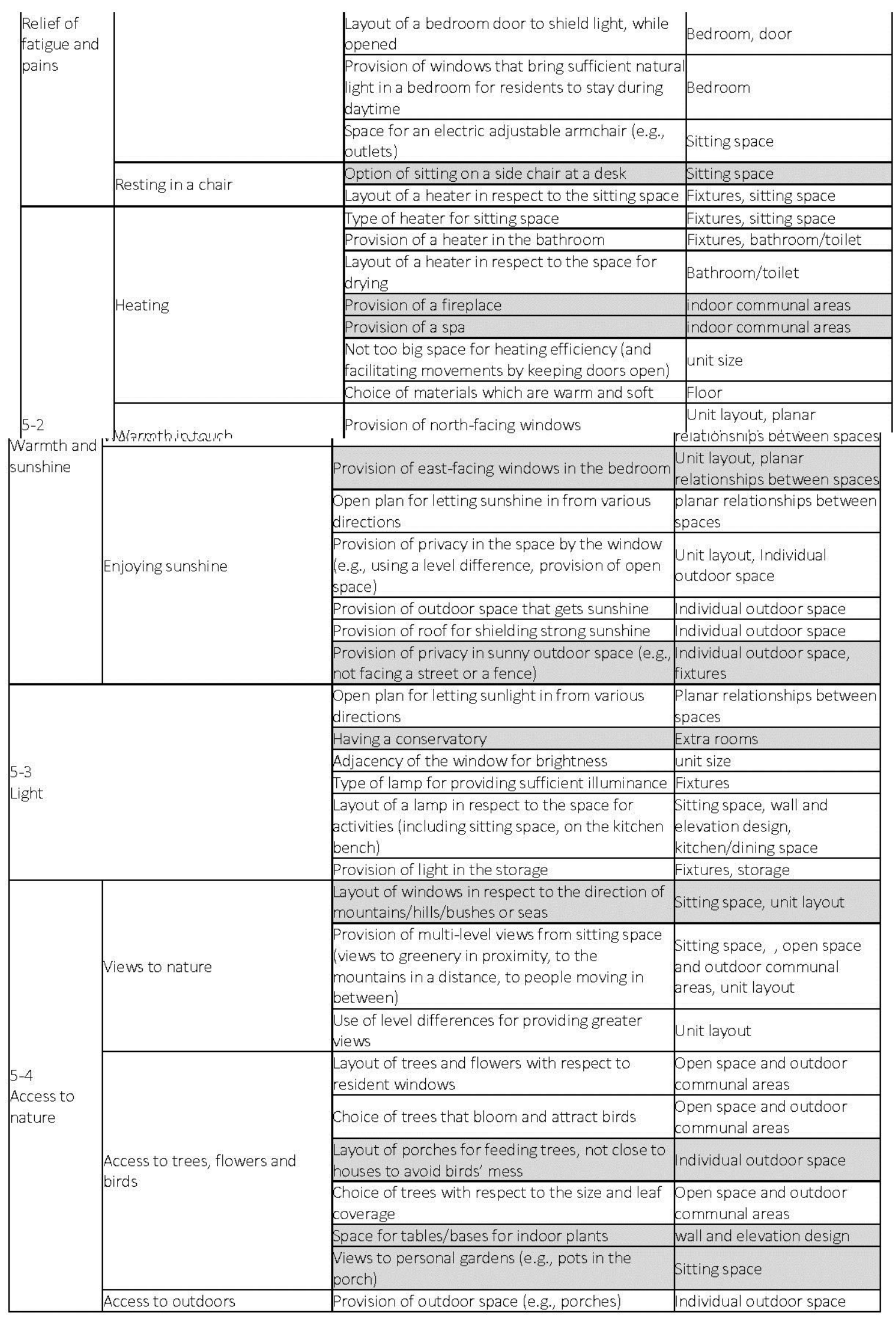




\begin{tabular}{|c|c|c|c|}
\hline & & $\begin{array}{l}\text { Provision of privacy in outdoor space (e.g., } \\
\text { enclosures) }\end{array}$ & $\begin{array}{l}\text { Individual outdoor space, } \\
\text { fixtures }\end{array}$ \\
\hline \multirow{15}{*}{$\begin{array}{l}6-1 \\
\text { Quality of } \\
\text { care in } \\
\text { assisted } \\
\text { showering }\end{array}$} & \multirow{8}{*}{ Ease of care activities } & $\begin{array}{l}\text { Size of space sufficient to accommodate both } \\
\text { the resident and the caregiver }\end{array}$ & Bathroom/toilet, unit size \\
\hline & & Avoid a level difference at the entry & Bathroom/toilet, floor \\
\hline & & Provision of a hose to the shower head & Bathroom/toilet, fixtures \\
\hline & & $\begin{array}{l}\text { Opening direction that didn't affect caregiver } \\
\text { movements }\end{array}$ & Bathroom/toilet, door \\
\hline & & Sufficient space for a caregiver to dry a resident & Bathroom/toilet, unit size \\
\hline & & $\begin{array}{l}\text { Adjacency of spaces for dry items such as clothes } \\
\text { and shoes }\end{array}$ & Bathroom/toilet \\
\hline & & $\begin{array}{l}\text { Sufficient space for a walker frame that doesn't } \\
\text { block caregiver movements }\end{array}$ & $\begin{array}{l}\text { Bathroom/toilet, space for } \\
\text { mobility aids, unit size }\end{array}$ \\
\hline & & $\begin{array}{l}\text { Space for a chair both for the wet space and for } \\
\text { the dry space }\end{array}$ & $\begin{array}{l}\text { Bathroom/toilet, things to sit } \\
\text { on }\end{array}$ \\
\hline & \multirow{3}{*}{ Safety in care } & Type of enclosure to limit the water flow & Bathroom/toilet, fixtures \\
\hline & & Type/location of drain & Bathroom/toilet, floor \\
\hline & & Things to hold onto by both hands & $\begin{array}{l}\text { Bathroom/toilet, things to } \\
\text { hold onto }\end{array}$ \\
\hline & \multirow{4}{*}{$\begin{array}{l}\text { Independence, privacy and } \\
\text { dignity }\end{array}$} & $\begin{array}{l}\text { Enclosure to limit the spread of steam } \\
\text { (particularly in small bathroom) }\end{array}$ & Bathroom/toilet, fixtures \\
\hline & & Enough ventilation & Bathroom/toilet \\
\hline & & Provision of a hose to the shower head & Bathroom/toilet, fixtures \\
\hline & & Material to obscure caregiver views & Bathroom/toilet, fixtures \\
\hline \multirow{3}{*}{\multicolumn{2}{|c|}{$\begin{array}{l}\text { 6-2 } \\
\text { Quality of care in household tasks }\end{array}$}} & $\begin{array}{l}\text { Proximity of spaces for care activities such as a } \\
\text { kitchen, a laundry and the space for drying } \\
\text { laundry }\end{array}$ & $\begin{array}{l}\text { Planar relationships between } \\
\text { spaces }\end{array}$ \\
\hline & & Sufficient bench/sink space for dirty dishes & $\begin{array}{l}\text { Kitchen/dining space, } \\
\text { fixtures }\end{array}$ \\
\hline & & Space for a wheelchair user to turn around & $\begin{array}{l}\text { Kitchen/dining space, space } \\
\text { for mobility aids }\end{array}$ \\
\hline \multirow{5}{*}{$\begin{array}{l}6-3 \\
\text { Quality of } \\
\text { care from } \\
\text { on-site staff }\end{array}$} & \multirow{2}{*}{ Efficient care provision } & $\begin{array}{l}\text { Proximity between individual units and the staff } \\
\text { office }\end{array}$ & Unit layout \\
\hline & & $\begin{array}{l}\text { Proximity to staff station(s) (e.g., layout of } \\
\text { multiple sub stations) }\end{array}$ & Unit layout \\
\hline & \multirow{2}{*}{$\begin{array}{l}\text { Staff visits/surveillance and } \\
\text { resident privacy }\end{array}$} & Views to staff approaching before their arrival & Sitting space \\
\hline & & Ease of shutting a door properly & Door \\
\hline & Familiarity of the staff & $\begin{array}{l}\text { Small number of units for greater } \\
\text { reliability/familiarity of the staff }\end{array}$ & Complex size, unit layout \\
\hline
\end{tabular}




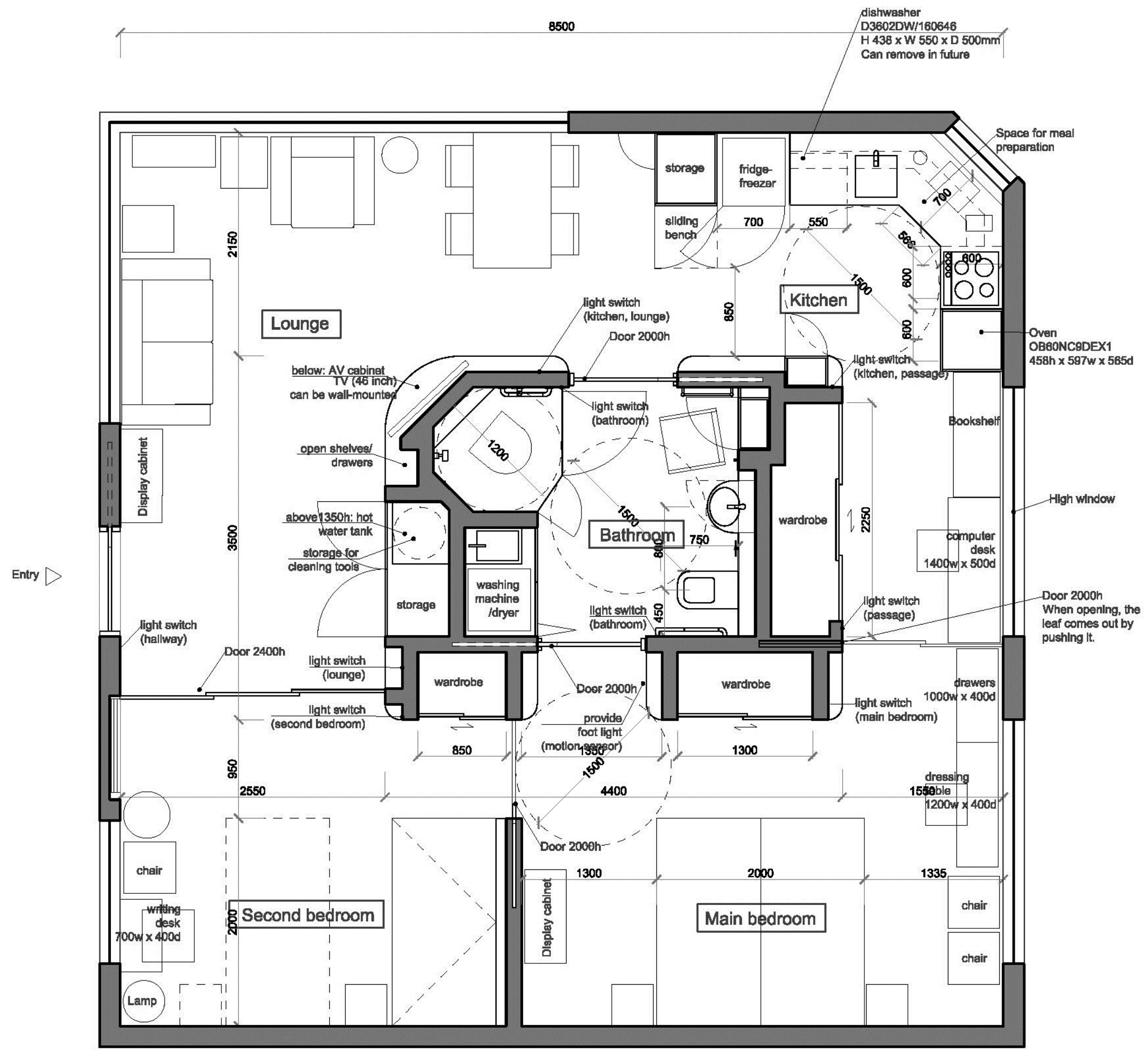

2B UNIT

$72.8 \mathrm{~m} 2$

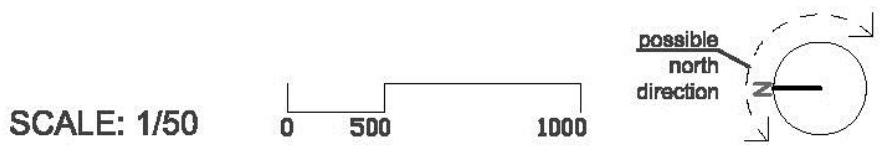

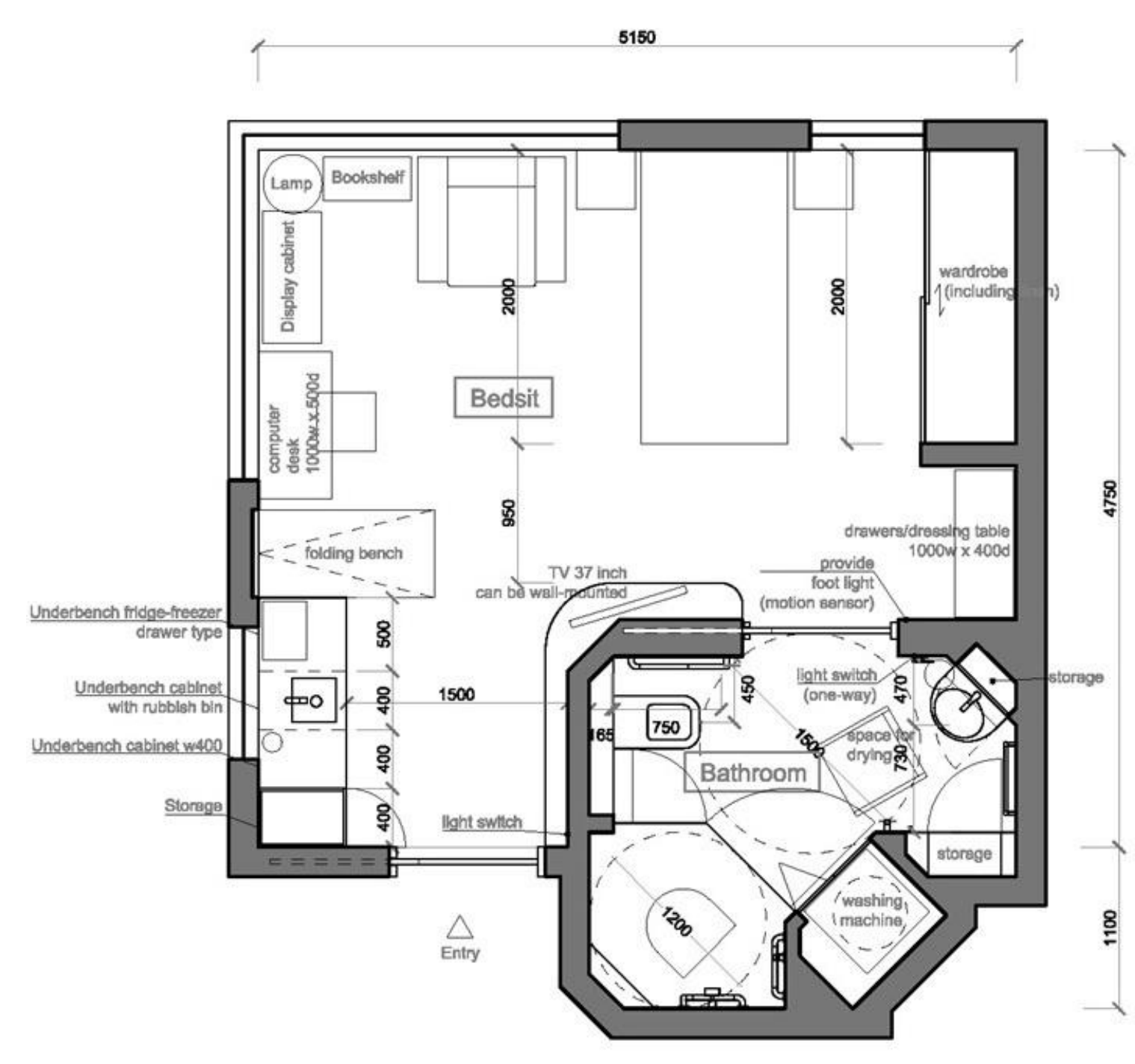

BS UNIT

$27.1 \mathrm{~m} 2$ 

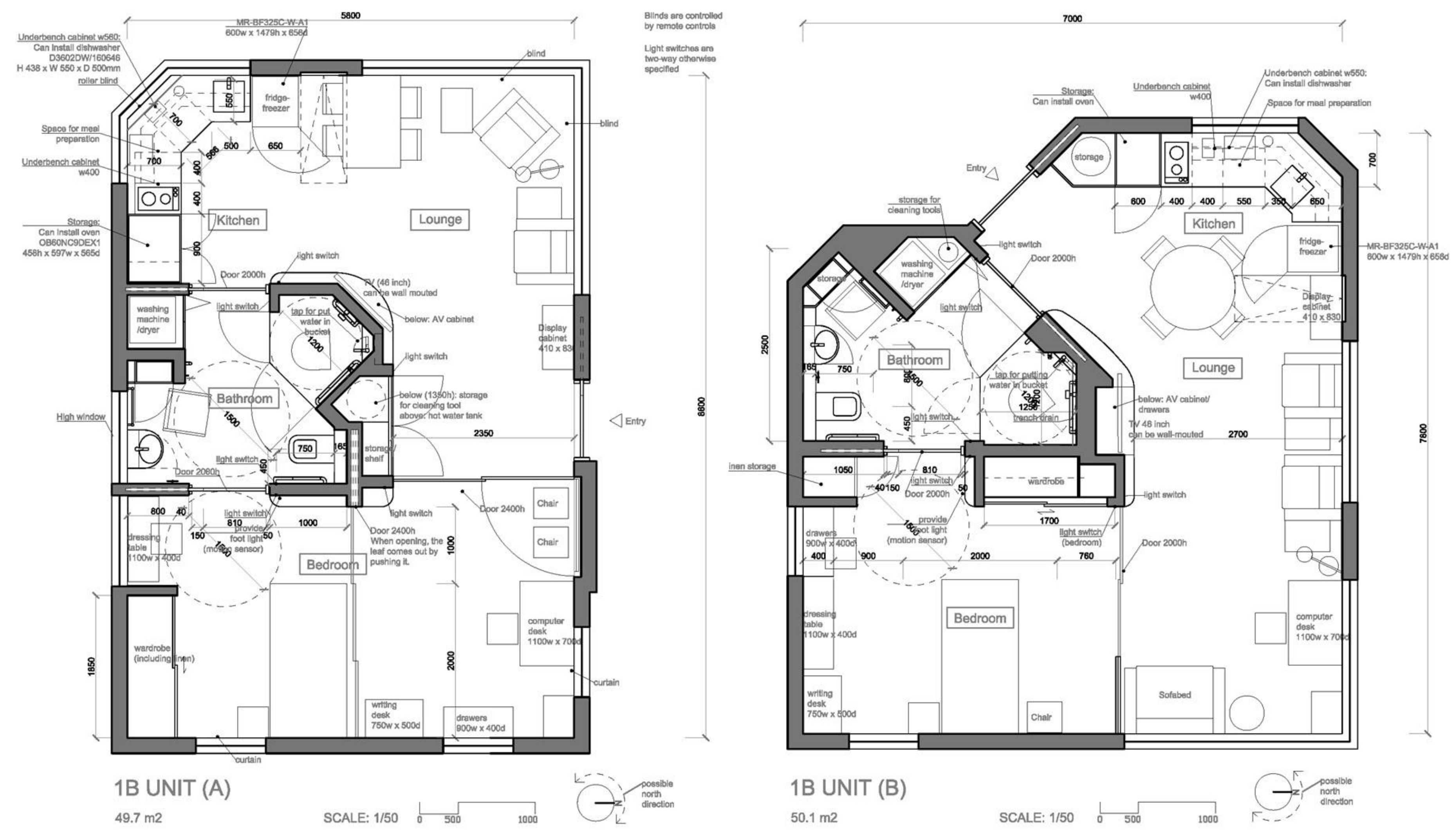
Patterns of adjoining units and window layout

1B-A UNIT

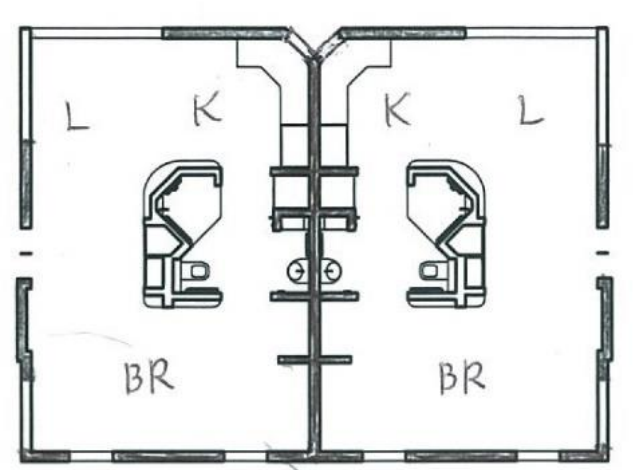

SEMI-DETACHED TYPE 1
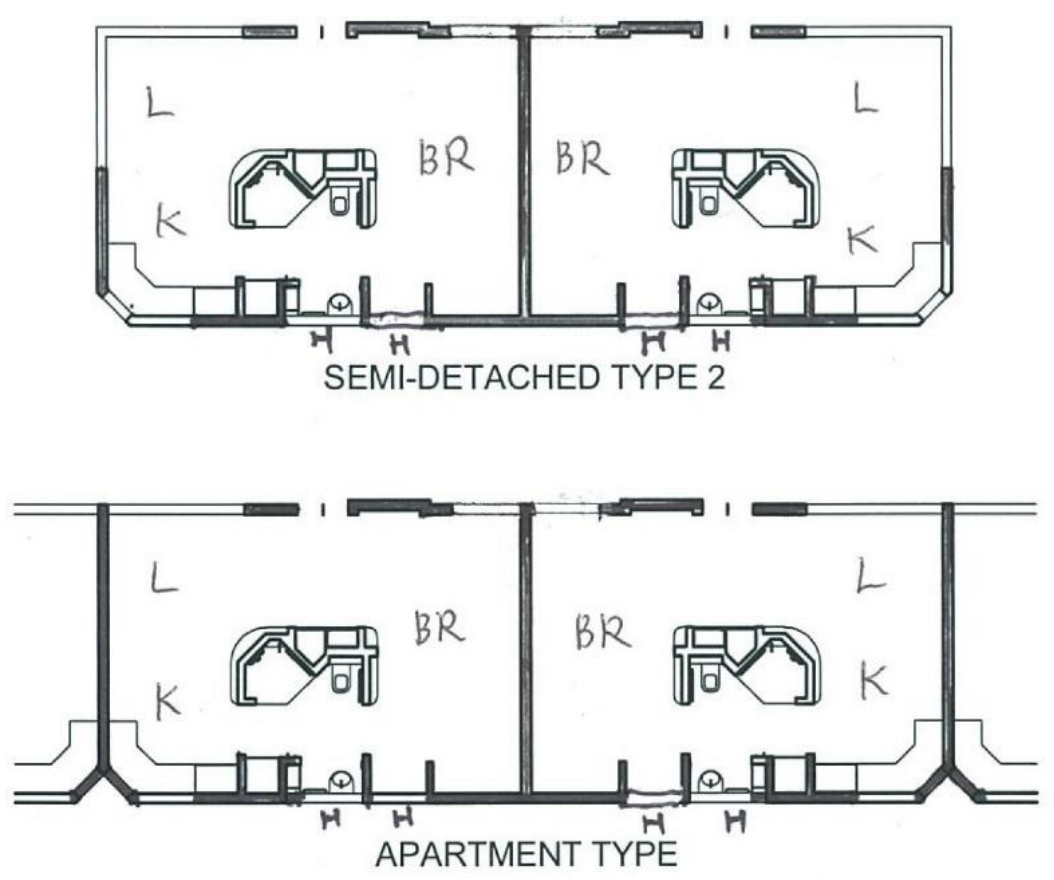

1B-B UNIT

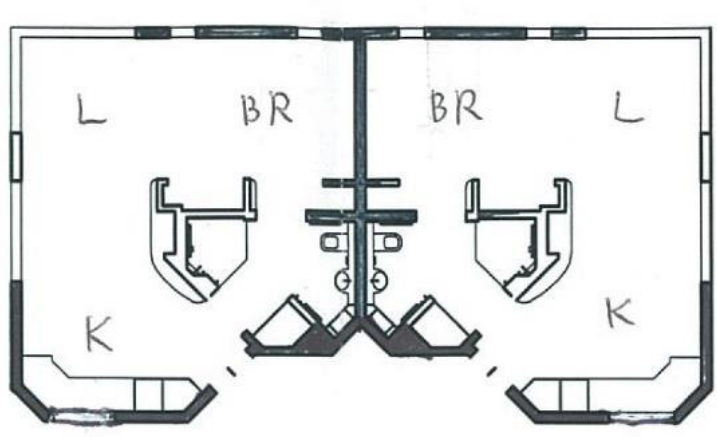

SEMI-DETACHED TYPE

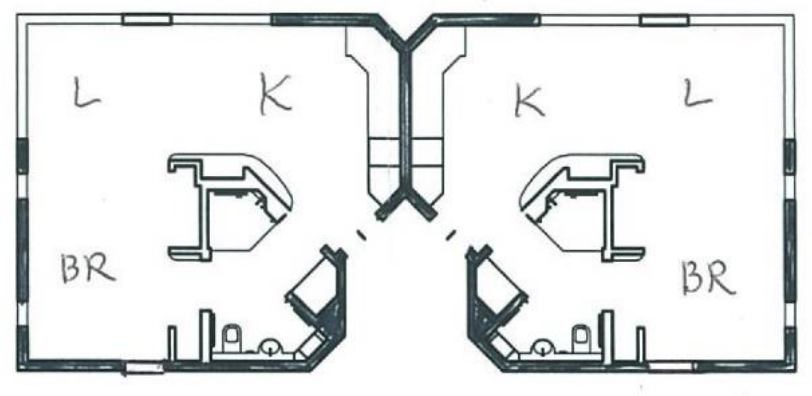

SEMI-DETACHED TYPE 2

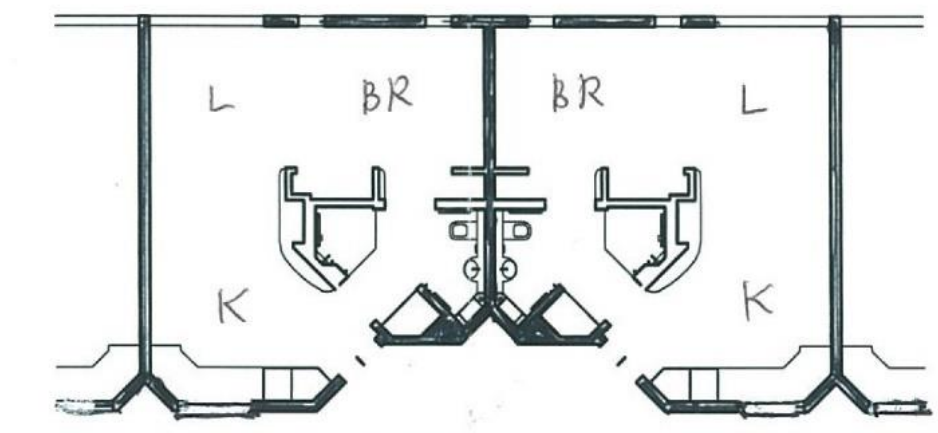

APARTMENT TYPE 1

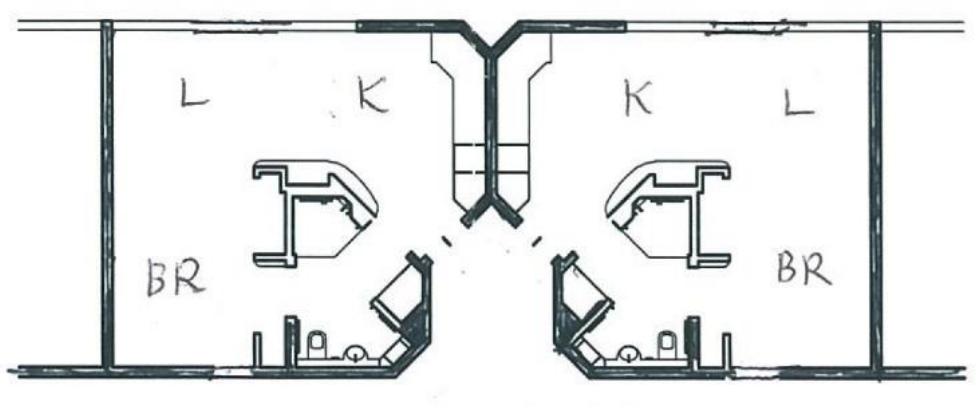

APARTMENT TYPE 2
2B UNIT

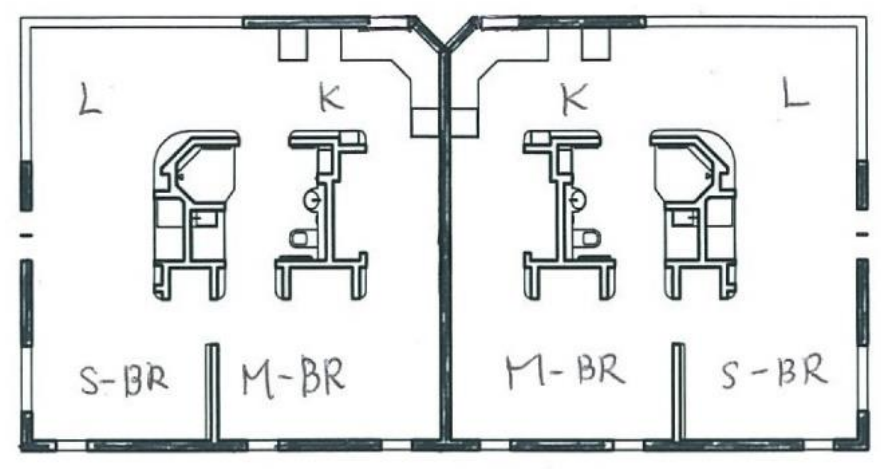

SEMI-DETACHED TYPE
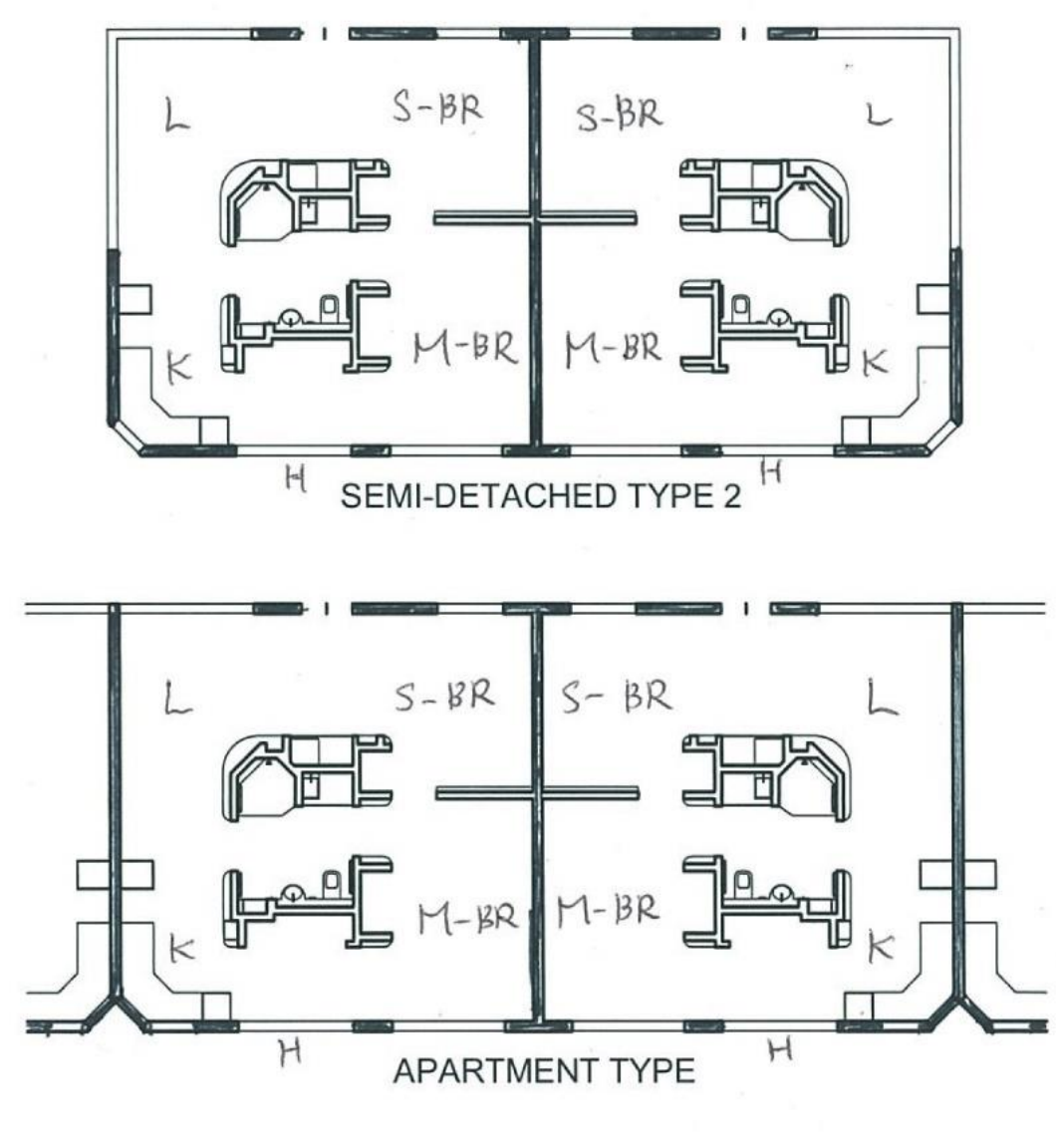

$H=$ high window 


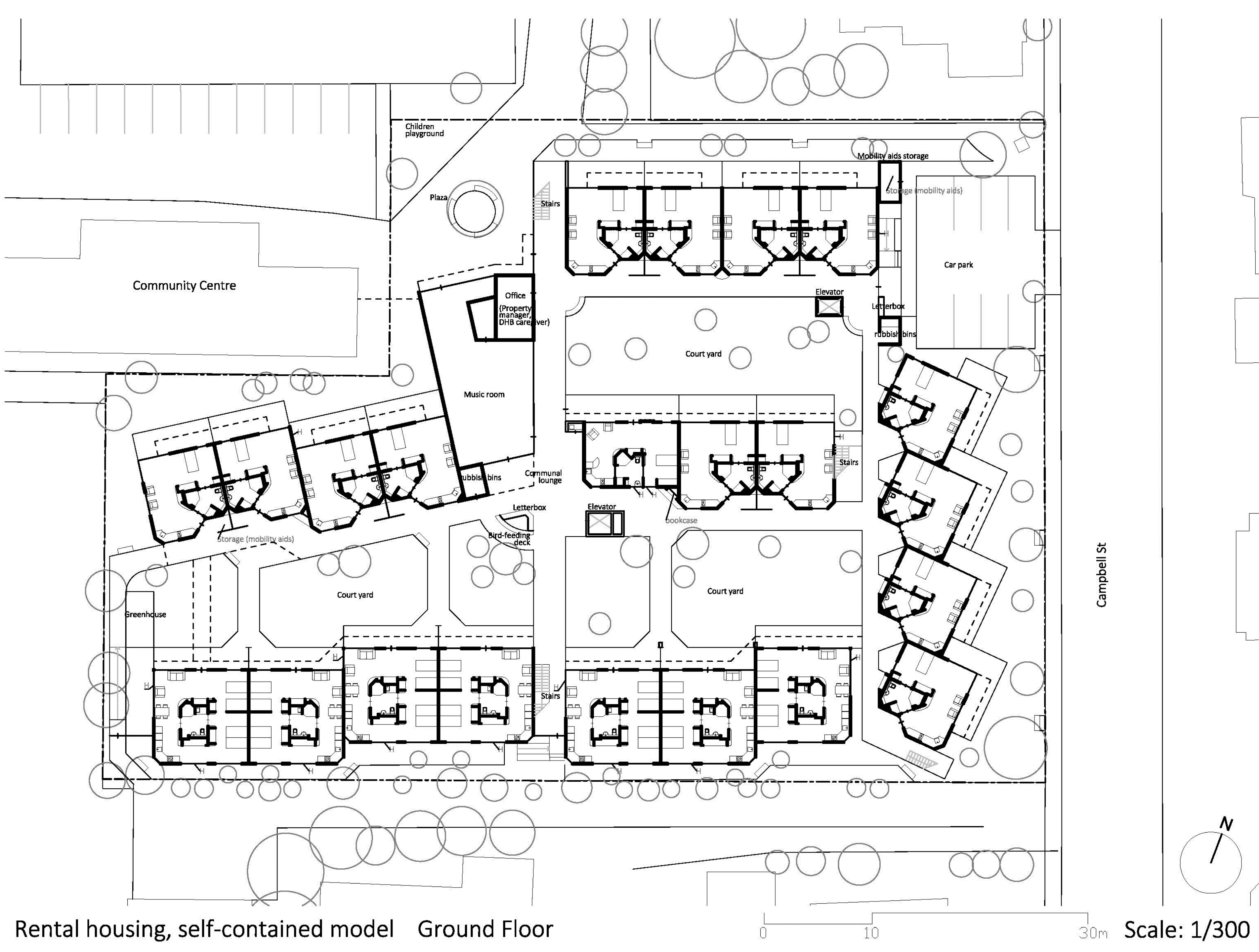




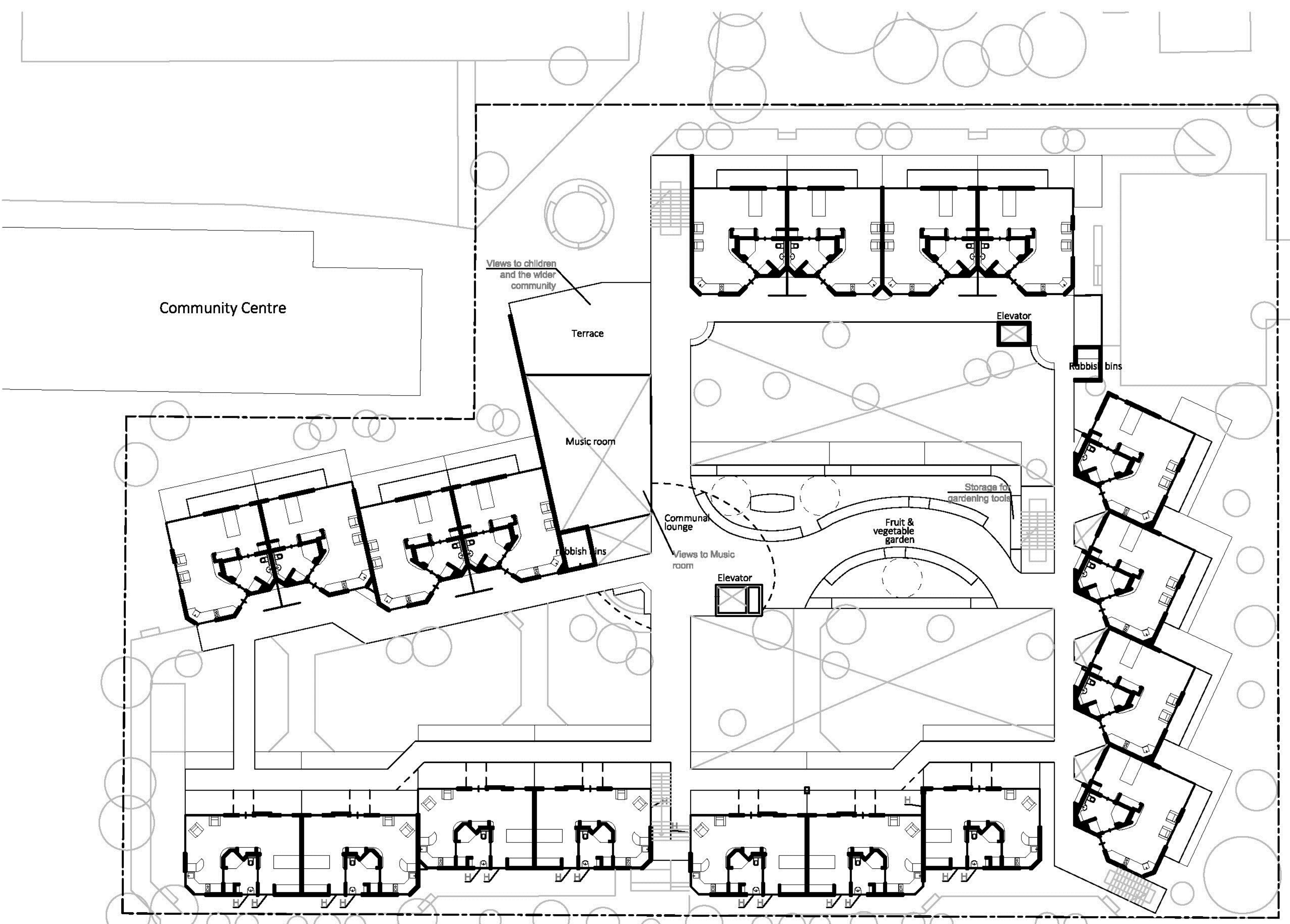

Rental housing, self-contained model First Floor

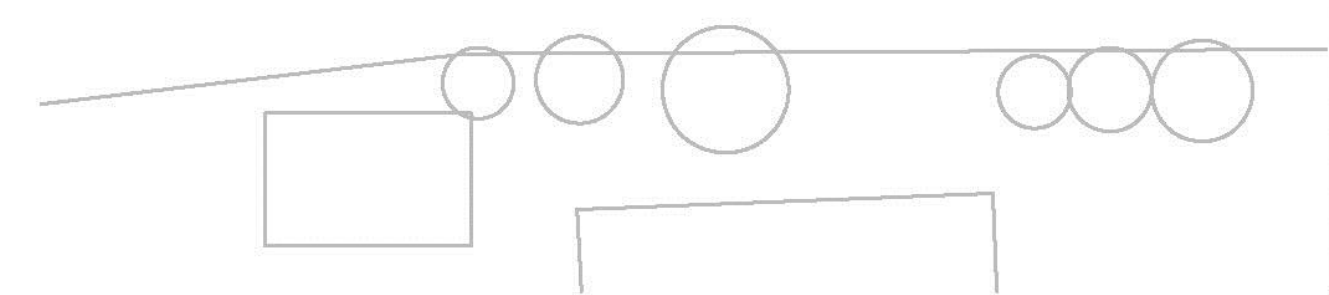




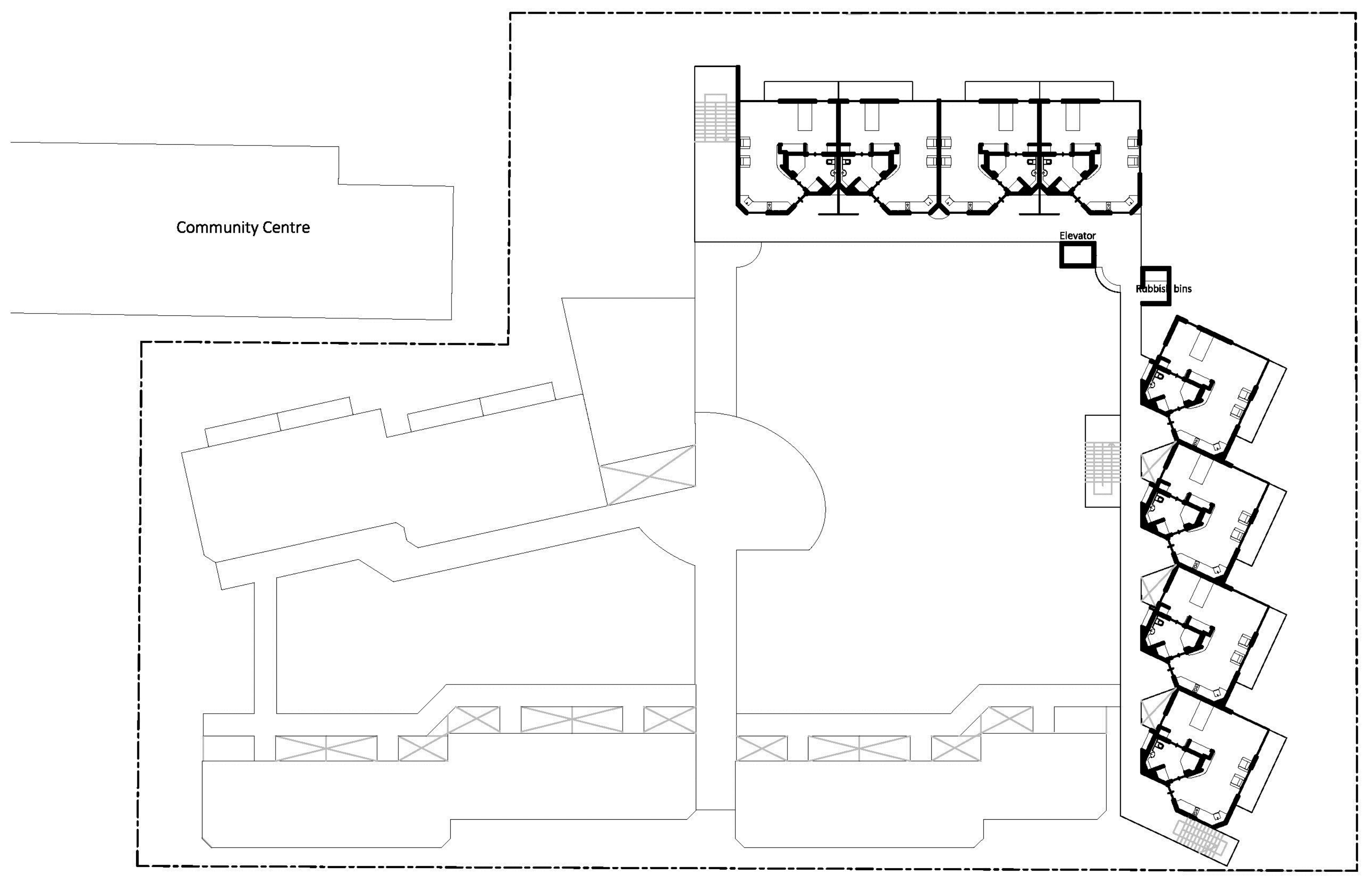




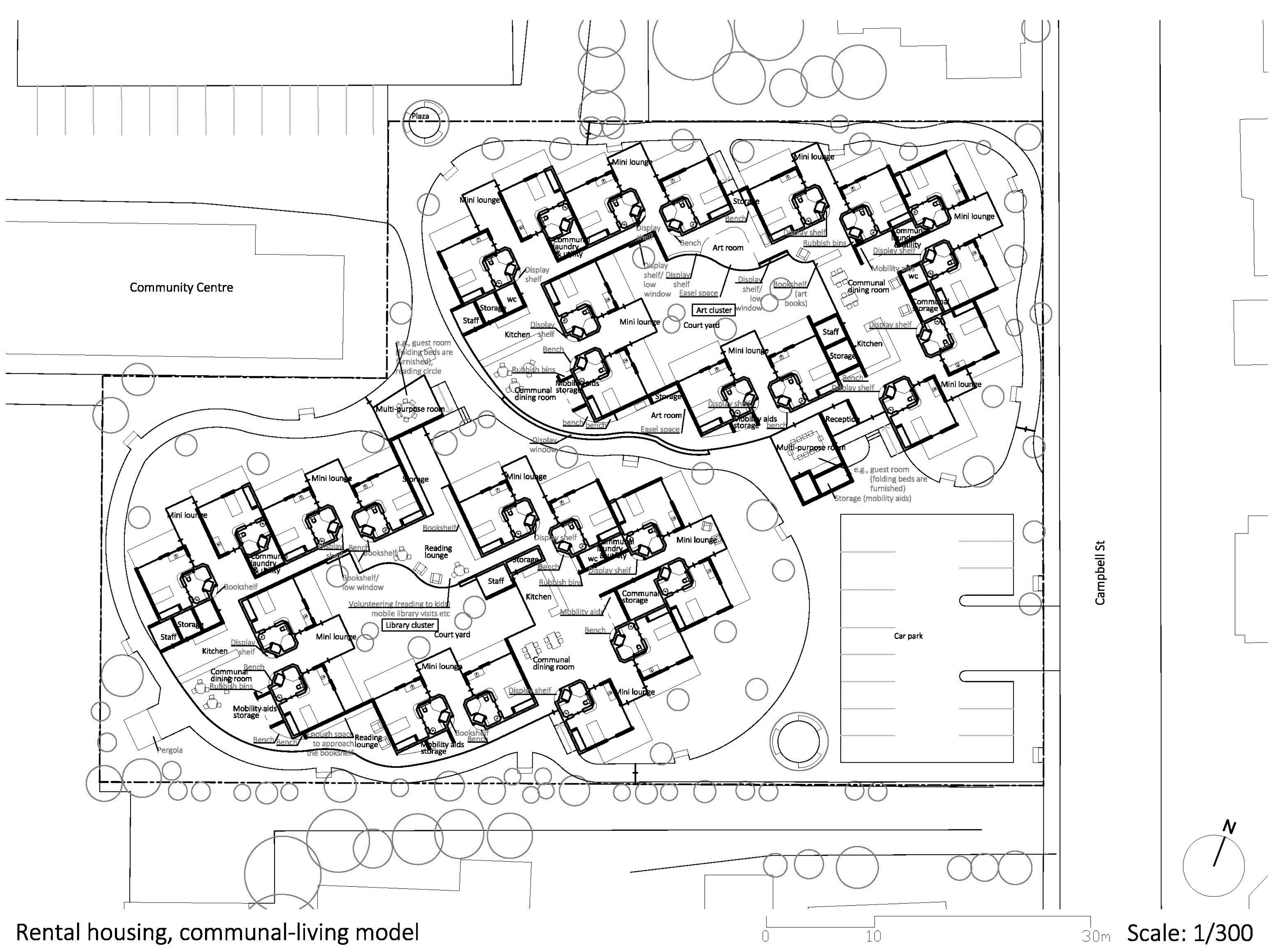




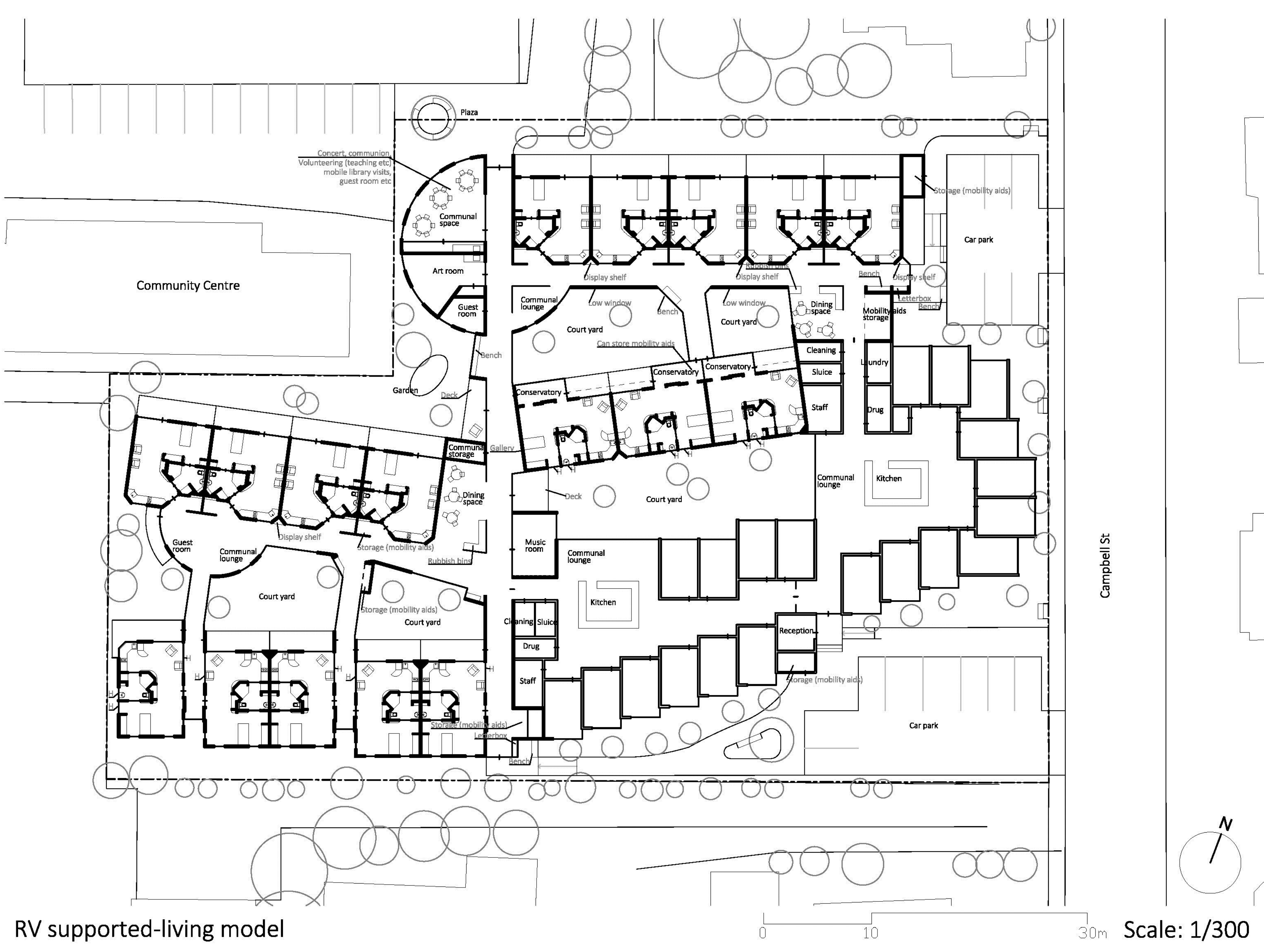


